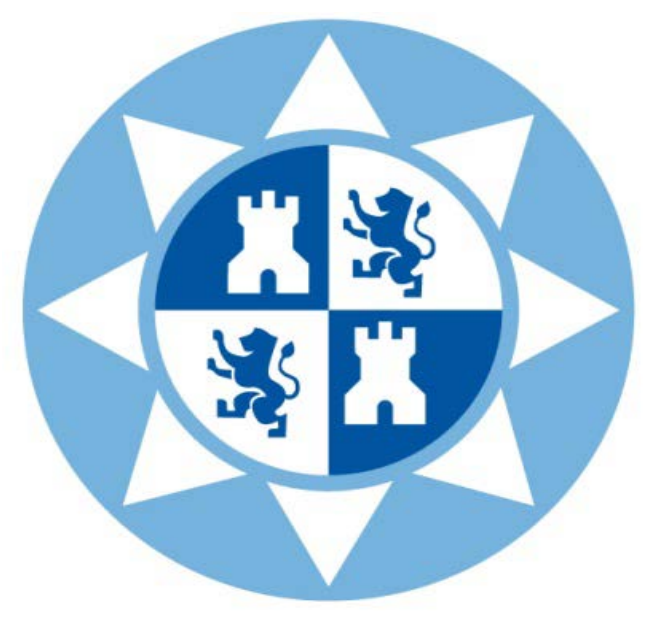

Universidad Politécnica de Cartagena Departamento de Ingeniería Civil

\title{
Metodología numérica y experimental para el diseño de los cuencos de disipación en el sobrevertido de presas de fábrica
}

D. José María Carrillo Sánchez 


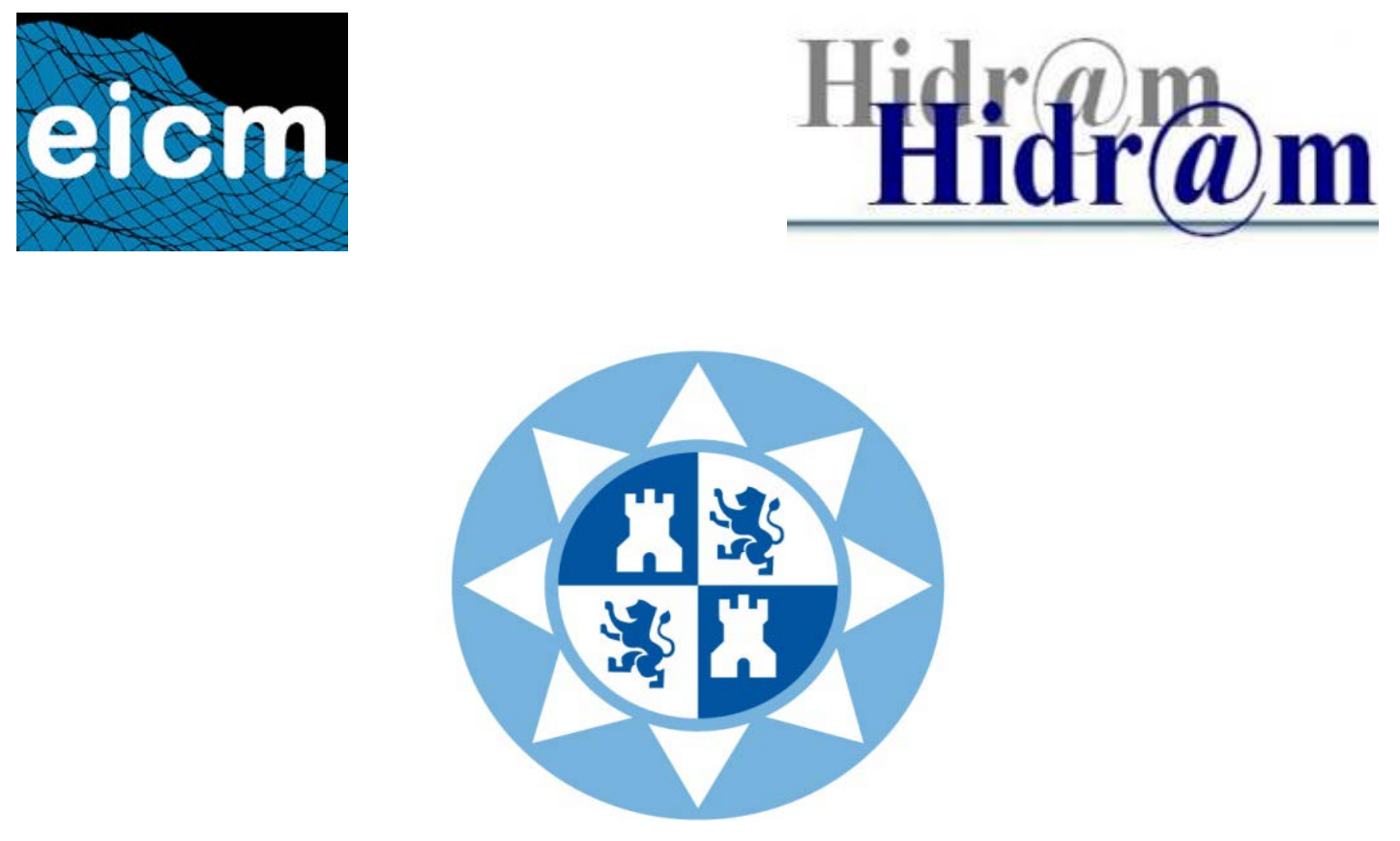

Universidad Politécnica de Cartagena Departamento de Ingeniería Civil

\title{
Metodología numérica y experimental para el diseño de los cuencos de disipación en el sobrevertido de presas de fábrica
}

\author{
D. José María Carrillo Sánchez
}

\author{
Director \\ Dr. Luis G. Castillo Elsitdié
}





\section{AGRADECIMIENTOS}

En primer lugar quiero expresar mi agradecimiento a mi director de tesis, Dr. Luis G. Castillo Elsitdié, por el esfuerzo y tiempo dedicado, así como por haber conseguido trasmitirme su entusiasmo por la investigación y el trabajo bien hecho.

También quiero agradecer el interés mostrado durante el desarrollo de esta Tesis por el Dr. Antonio Tomás Espín y D. Manuel Alcaraz Aparicio, director del Departamento de Ingeniería Civil y director de la Escuela Técnica Superior de Ingeniería de Caminos, Canales y Puertos y de Ingeniería de Minas, respectivamente.

A los compañeros del grupo Hidr@m con los que he tenido el placer de compartir despacho, laboratorio y publicaciones. Quisiera destacar el apoyo recibido por el Dr. Álvaro Sordo-Ward, D. Antonio Blázquez Vidal, D. Juan Tomás García Bermejo y Dña. María Dolores Marín Martín.

Asimismo, quiero expresar mi agradecimiento por la ayuda financiera recibida del Ministerio de Economía y Competitividad y el Fondo Europeo de Desarrollo Regional (FEDER) a través del proyecto "Aireación natural de flujos en el vertido libre por coronación de presas y su difusión en cuencos de disipación de energía hidráulica" (BIA2011 -28756-C03-02), y a la Universidad Politécnica de Cartagena por darme la oportunidad de disfrutar de una beca-contrato de formación de profesorado universitario.

Finalmente, agradecer a mi familia por su paciencia y ánimos hasta la culminación de la Tesis Doctoral. 



\section{ÍNDICE}

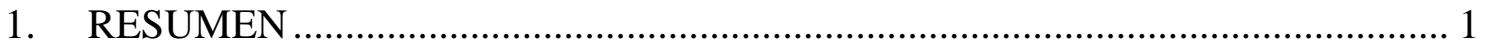

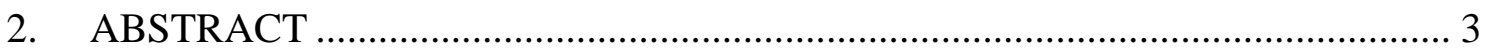

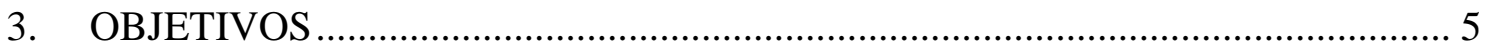

4. ESTADO DEL ARTE …………………………………………………….... 7

4.1. Sobrevertido de presas ................................................................................... 7

4.2. Características de la lámina vertiente ………………………………….......... 9

4.2.1. Trayectoria de la lámina vertiente................................................................... 9

4.2.2. Aireación de la lámina................................................................................ 12

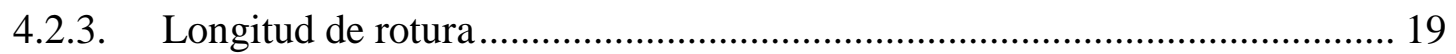

4.3. Flujo dentro del cuenco................................................................................. 22

4.3.1. Geometría del chorro dentro del colchón..................................................... 22

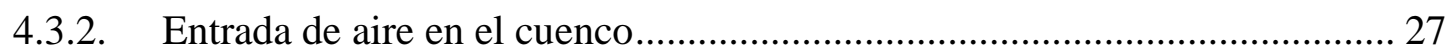

4.3.3. Condiciones en el cuenco en función del calado aguas abajo....................... 32

4.3.4. Presiones medias en el punto de estancamiento............................................. 34

4.3.5. Fluctuación de las presiones en el punto de estancamiento ........................... 41

4.3.6. Distribución de presiones en el entorno del punto de estancamiento ........... 45

4.3.7. Presiones extremas instantáneas en el punto de estancamiento ..................... 49

4.3.8. Distribución de velocidades en el cuenco .................................................... 52

4.3.9. Pérdida de energía en el cuenco .................................................................. 56

5. METODOLOGÍA EXPERIMENTAL ................................................................ 57

5.1. Infraestructura de laboratorio..................................................................... 57

5.1.1. Consideraciones en un modelo físico de laboratorio ...................................... 65

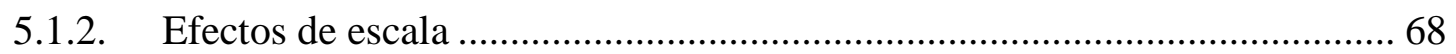

5.2. Transductores de presión ............................................................................. 70

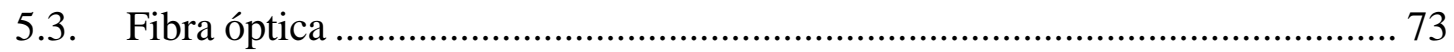

5.4. Velocímetro acústico Doppler ........................................................................ 75 


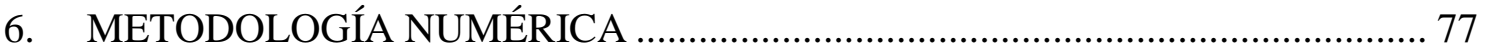

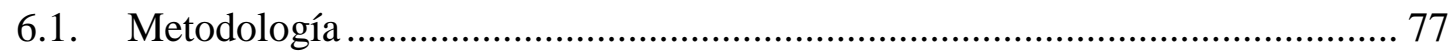

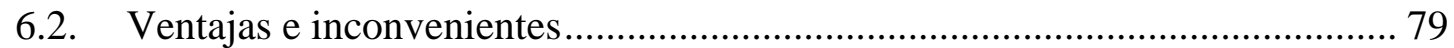

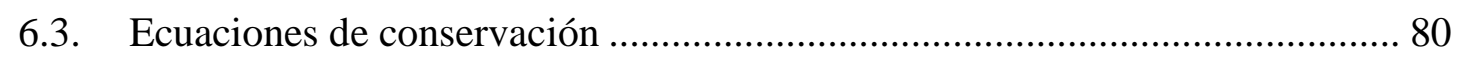

6.3.1. Ecuación de continuidad o de conservación de masa .................................. 81

6.3.2. Ecuación de conservación de la cantidad de movimiento............................ 81

6.3.3. Ecuación de conservación de la energía.................................................... 82

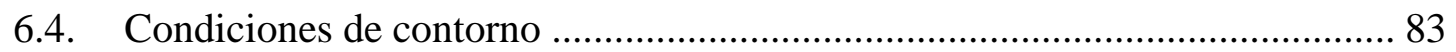

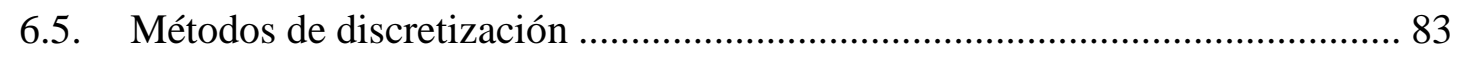

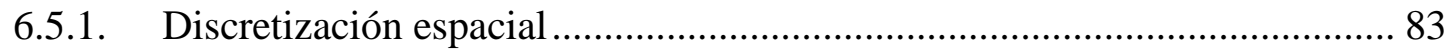

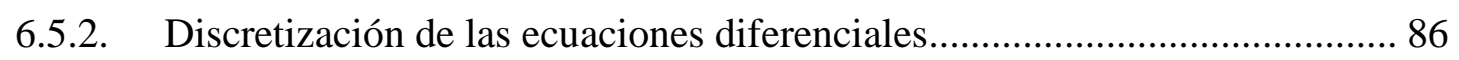

6.5.3. Formulación diferencial y formulación integral........................................ 87

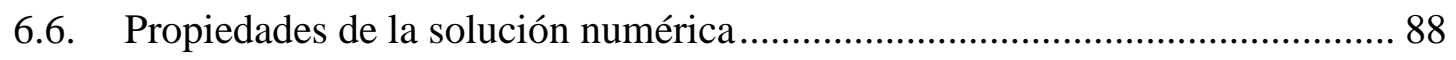

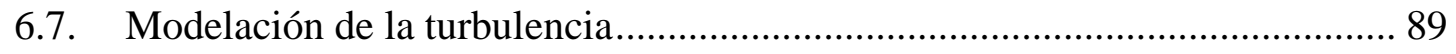

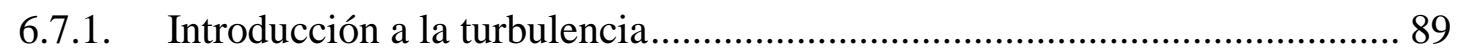

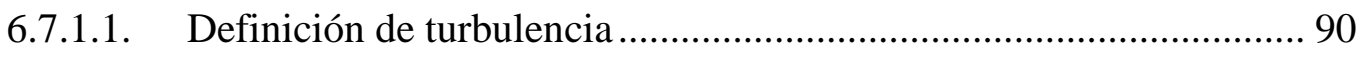

6.7.1.2. Las escalas de Kolmogorov............................................................ 92

6.7.1.3. Descripción espectral y Ley $-5 / 3$ de Kolmogorov ............................ 93

6.7.1.4. Ecuaciones de Navier-Stokes promediado de Reynolds ................... 95

6.7.2. Modelos de turbulencia de viscosidad de remolino ................................... 98

6.7.2.1. Modelos de turbulencia de dos ecuaciones ..................................... 100

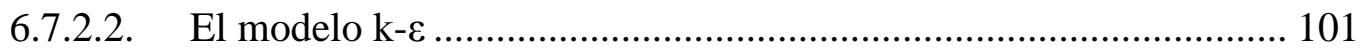

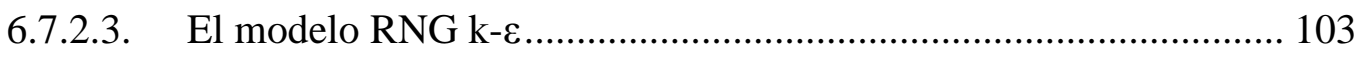

6.7.2.4. Modelos basados en k- $\omega$................................................................ 104

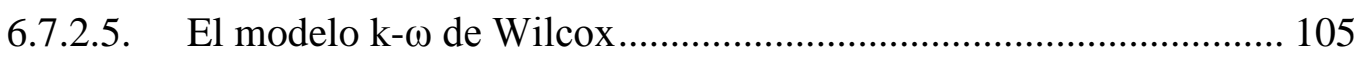

6.7.2.6. El modelo k- $\omega$ Baseline (BSL).................................................. 106

6.7.2.7. El modelo Shear Stress Transport (SST) ..................................... 108

6.7.3. Modelos de turbulencia de tensiones de Reynolds..................................... 109

6.7.3.1. Modelos de tensiones de Reynolds basados en omega ................... 111

6.7.4. El modelo de Simulación de Gran Remolino (LES) ................................. 113 
6.7.5. Comparación de los modelos de turbulencia................................................. 115

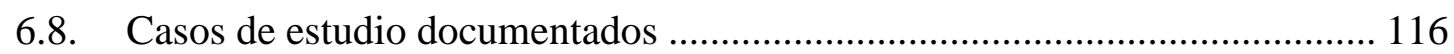

6.8.1. Validación de los modelos numéricos en aliviaderos ................................... 116

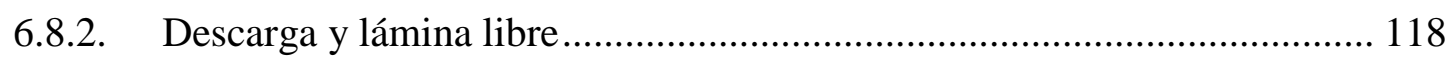

6.8.3. Análisis del impacto de la lámina de agua .................................................. 124

6.8.4. Distribución de presiones y cortantes.......................................................... 125

6.8.5. Análisis del canal de descarga y del cuenco de disipación .......................... 127

6.8.6. Simulación de chorros turbulentos ........................................................... 129

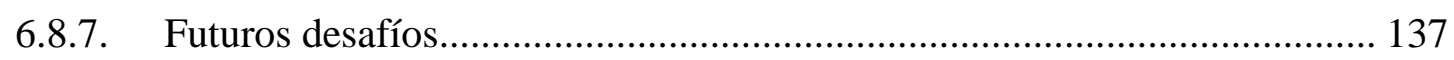

7. ANÁLISIS Y DISCUSIÓN DE RESULTADOS .............................................. 139

7.1. Campaña de laboratorio ............................................................................ 139

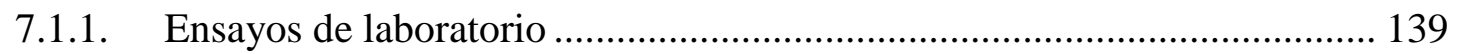

7.1.2. Análisis general de los registros de presiones ............................................ 141

7.1.3. Metodología Paramétrica ............................................................................. 144

7.1.4. Coeficientes de presión dinámica media.................................................. 145

7.1.5. Coeficientes de presión dinámica fluctuante.............................................. 148

7.1.6. Coeficientes de presión dinámica extrema.................................................. 152

7.1.7. Distribución de presiones alrededor del punto de estancamiento ............... 154

7.1.8. Colchón aguas arriba del punto de estancamiento ....................................... 159

7.1.9. Velocidades en el cuenco de amortiguación .............................................. 160

7.1.10. Disipación de energía en el cuenco de amortiguación ........................... 162

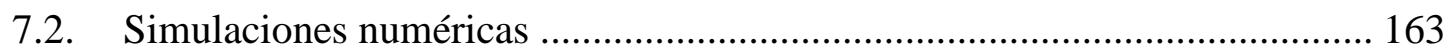

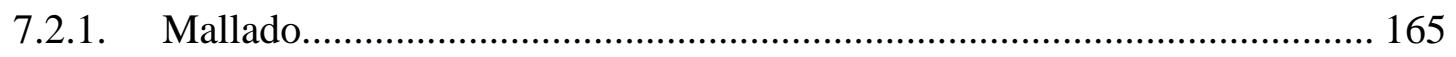

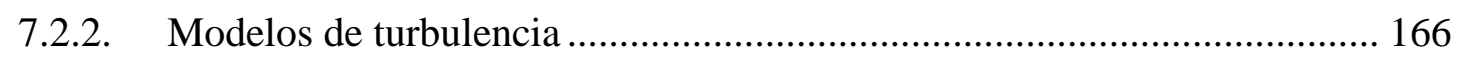

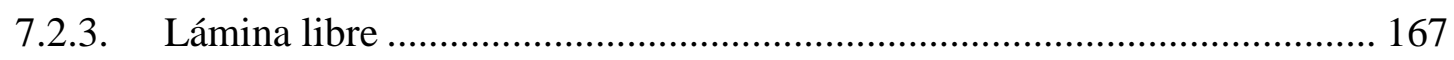

7.2.4. Estudios previos de la metodología CFD .................................................... 172

7.2.4.1. Análisis de un aliviadero de presa hidrodinámico ........................... 172

7.2.4.2. Análisis bidimensional de un aliviadero de vertido libre................. 173 
7.2.5. Análisis tridimensional de aliviaderos de vertido libre y cuencos de

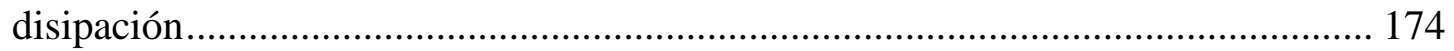

7.2.5.1. Primera aproximación tridimensional .......................................... 176

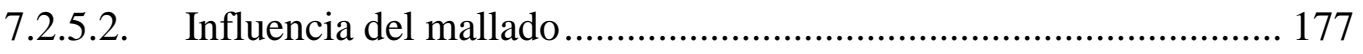

7.2.5.3. Análisis de la turbulencia del chorro ............................................ 179

7.2.5.4. Estudio de la aireación y la velocidad del chorro............................. 184

7.2.5.5. Estudio del chorro + cuenco de disipación .................................... 195

7.2.5.5.1. Análisis en función del tamaño del mallado................................... 195

7.2.5.5.2. Análisis en función de la turbulencia inicial.................................. 197

7.2.5.5.3. Análisis de la influencia del factor de escala ................................ 201

7.2.5.5.4. Análisis de la influencia del modelo de turbulencia ...................... 207

7.2.5.5.5. Estudio sistemático .................................................................... 214

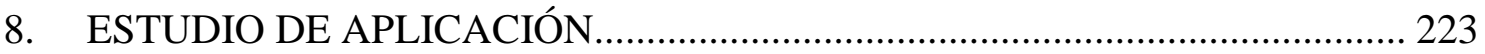

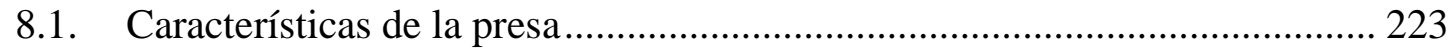

8.2. Estudio de la socavación con fórmulas empíricas …................................... 224

8.3. Estudio de socavación con el método semiempírico de fluctuaciones de presión

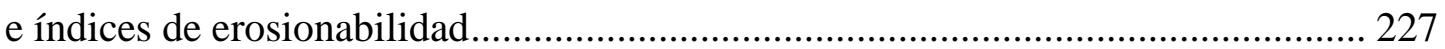

8.4. Modelación matemática tridimensional de la socavación .............................. 231

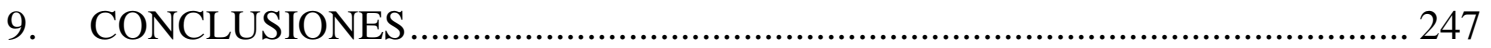

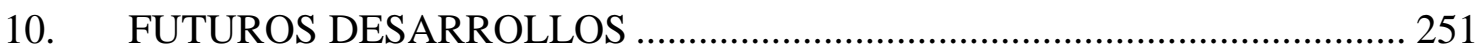

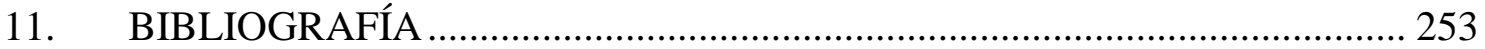




\section{ÍNDICE DE FIGURAS}

Figura 1. Sobrevertido por coronación actuando como vertedero de pared gruesa. ........ 7

Figura 2. Perfil de la lámina de agua completamente aireada en un vertedero bidimensional (Bazin 1896; Scimemi, 1930, obtenido de Bos, 1989)........................... 9

Figura 3. Error en la estimación de la trayectoria del chorro vertiente en función del número de Froude (Wahl et al., 2008)......................................................................... 10

Figura 4. Descripción de la lámina vertiente y notación empleada................................ 12

Figura 5. Chorro en la atmósfera y en el cuenco de disipación (Ervine y Falvey, 1987).

Figura 6. Dependencia de la expansión lateral del chorro con la intensidad de turbulencia actuando en el orificio de salida (Ervine y Falvey, 1987).......................... 15

Figura 7. Aliviaderos en vertido libre de presas bóveda (adaptado de Castillo, 1989).. 18

Figura 8. Variación de las intensidades turbulentas a lo largo de la línea central del chorro: $\square, u^{\prime} ; \circ, v^{\prime} ; \Delta, w^{\prime} ;--, v^{\prime}, \mathrm{AR}=10$, Sfeir (1976); - $-v^{\prime}, \mathrm{AR}=38$, Gutmark y Wygnanski (1976). (Krothapalli et al., 1981).

Figura 9. Variación de la longitud de rotura para distintas intensidades turbulentas iniciales. Chorro circular con una tobera de 25 mm de diámetro (Ervine y Falvey, 1987).

Figura 10. Expansión lateral de un chorro bidimensional (adaptado de Bollaert, 2002).

Figura 11. Esquemática de difusión del chorro (adaptado de Albertson et al., 1950). .. 24

Figura 12. Desarrollo de la capa límite en la solera del cuenco de disipación y evolución de la sobrepresión $\Delta p$ y velocidad $v$ a lo largo de la solera obtenido por Cola (1966), citado por Felderspiel (2011).....

Figura 13. Difusión e impacto de un chorro bidimensional sobre una superficie plana y lisa: a) Principales regiones de chorro; b) Distribuciones de velocidad y presión en cada región (Beltaos y Rajaratnam, 1973). 26

Figura 14. Máxima tasa de entrada de aire en diferentes estructuras hidráulicas en función de la velocidad del chorro $V_{j}$. Variación del exponente n con la velocidad del chorro (Ervine, 1998, citado por Bollaert, 2002). 29

Figura 15. Condiciones de flujo en el cuenco de disipación en función del calado de aguas abajo (Cui Guang Tao et al., 1986, adaptado de Castillo, 1989). 32 
Figura 16. Presión dinámica media en función de la relación $Y / D_{j}$ (Bollaert y Schleiss, 2003).

Figura 17. Coeficiente de presión dinámica media como función de la velocidad del chorro incidente $V_{j}$ (Bollaert y Schleiss, 2003). .......................................................... 38

Figura 18. Coeficiente de presión dinámica media (Castillo, 2006)............................ 40

Figura 19. Esquema de los cuatro diferentes tipos de chorros (Castillo, 2007)............. 42

Figura 20. Presión dinámica fluctuante en función de $y / D_{j}$ (Bollaert y Schleiss, 2001). 43

Figura 21. Coeficientes de presión dinámica fluctuante (Castillo, 2006).

Figura 22. Zonas del flujo del chorro dentro del colchón de agua (Xu Duo-Ming, 1983, citado por Castillo, 1989).

Figura 23. Distribución de presiones dinámicas medias propuestas por diferentes autores (Castillo, 1989).

Figura 24. Variación del coeficiente de presión dinámica media con la distancia radial al eje del chorro (Ervine et al., 1997).

Figura 25. Distribución radial del coeficiente de presión dinámica media (Bollaert, 2002).

Figura 26. Distribución radial del coeficiente de presión dinámica fluctuante (Bollaert, 2002).

Figura 27. Presiones dinámicas máximas en el punto de estancamiento en función de la altura del cochón (Castillo, 1989).

Figura 28. Amplitudes máximas de presión en el punto de estancamiento en función de la altura del cochón (Castillo, 1989) 50

Figura 29. Semiamplitudes de presión en el punto de estancamiento $\left(A_{\text {sup }} / H\right.$ y $\left.A_{\text {inf }} / H\right)$ en función de la relación $Y / B_{j}$ (Castillo, 1989)... 51

Figura 30. Coeficiente de presión dinámica extrema máxima, $C_{p}{ }^{+}$(Castillo, 2007). .... 51

Figura 31. Coeficiente de presión dinámica extrema mínima, $C_{p}^{-}$(Castillo, 2007)....... 51

Figura 32. Definición esquemática del perfil de velocidades medias en un resalto sumergido (adaptado de Wu y Rajaratnam, 1995).

Figura 33. Distribuciones típicas de fracción de vacío $C$, velocidad adimensional y frecuencia de burbujas adimensional $F_{a b}$ en resaltos hidráulicos (Chanson y Brattberg, 2000). 54

Figura 34. Perfil de velocidad media horizontal de un chorro plano (Lin et al., 2012). 55 
Figura 35. Variación de la longitud característica $\delta_{l}$ en resaltos sumergidos (Wu y Rajaratnam, 1995).

Figura 36. Infraestructura de laboratorio para el estudio de chorros turbulentos y bifásicos. 57

Figura 37. Canal de entrada en la infraestructura de laboratorio. 58

Figura 38. Vertedero rectangular de cresta delgada con contracción (Bos, 1989)......... 59

Figura 39. Valores de $K_{b}$ como función de $b_{d} / B_{1}$, obtenidos por Kindsvater y Carter (1957), citado en Bos (1989). 61

Figura 40. Cuenco de amortiguación y canal de desagüe en la infraestructura de

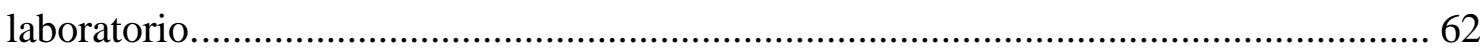

Figura 41. Vertedero triangular de pared delgada (Bos, 1989)....................................... 63

Figura 42. Coeficiente de descarga efectivo $C_{e}$ en vertederos triangulares con escotadura de $90^{\circ}$ (Bos, 1989). 64

Figura 43. Aforo por vertedero triangular y comprobación de su curva de gasto. 64

Figura 44. Corrección debida a la capa límite para los resultados experimentales de una familia de modelos (Naudascher, 2000).

Figura 45. a) Efecto del perfil de velocidades en el canal de aproximación; b) sobre la distribución de presiones en la cresta de un vertedero hidrodinámico (Naudascher, 2000) 70

Figura 46. Sensor de presión y tarjeta de adquisición de datos. 71

Figura 47. Software de adquisición de datos en entorno LabVIEW. 72

Figura 48. Distribución de sensores de presión en el eje central de la solera del cuenco de amortiguación.

Figura 49. Equipo de fibra óptica, osciloscopio para controlar la señal y sonda registrando en el cuenco de amortiguación. ................................................................ 73

Figura 50. Conversión de la señal analógica en digital en el equipo de fibra óptica. .... 74

Figura 51. Señal aire agua en un equipo de dos canales de fibra óptica. ........................ 75

Figura 52. Transmisión de la señal del equipo Doppler................................................... 76

Figura 53. Velocímetro acústico Doppler en el cuenco de disipación. ............................ 76

Figura 54. Tipologías de elementos: a) hexaédrico; b) cuña; c) pirámide; d) tetraedro (Carrillo y Castillo, 2011)............................................................................................ 85

Figura 55. Espectro de energía para un flujo turbulento (Wilcox, 2010)........................ 94 
Figura 56. Interrelación entre el mundo real, un modelo matemático y un modelo informatizado

Figura 57. Comparación del perfil de la lámina de agua y de las presiones sobre la cresta de un aliviadero hidrodinámico, considerando $H_{e} / H_{d}=0.50$ (Bouhadji, 2004) 118

Figura 58. Coeficiente de descarga en función de la carga para un vertedero de cresta elíptica con $P_{l} / H_{d}=2.0$ (Edwards, 2006). 120

Figura 59. Curva de descarga y perfil superficial para dos cargas distintas de la presa de Limestone (Chanel y Doering, 2007). 122

Figura 60. Coeficiente de descarga para un vertedero de pared delgada frente a la relación carga total sobre vertedero/altura de vertedero $\left(H_{t} / t_{w}\right)$ (Reda, 2011).

Figura 61. Comparación de los perfiles de velocidades horizontales en la cresta de un aliviadero circular, considerando diferentes modelos de turbulencia (Rahimzadeh et al., 2012).

Figura 62. Comparación de las distribuciones de presiones en la cresta de un aliviadero circular, considerando diferentes modelos de turbulencia (Rahimzadeh et al., 2012). 123

Figura 63. Comparación entre la distribución de presiones medida y calculada en la solera del cuenco (Weilin et al., 2002). 124

Figura 64. Comparación de la distribución de presiones a lo largo del centro del vano y de la pila en el aliviadero de la presa Hume, Nueva Gales del Sur (Ho et al., 2003). . 126 Figura 65. Comparación de presiones relativas obtenidas en modelo físco y modelo numérico para diversas sumergencias (Savage et al., 2009). 127

Figura 66. Tasa de disipación de la energía cinética turbulenta en la presa Porto Colombia, Brasil, para un caudal de $6000 \mathrm{~m}^{3} / \mathrm{s}$ (Amorin et al., 2004). 128

Figura 67. Perfiles de velocidad para varias posiciones, para un flujo de $R_{e}=44000$ : a) $x / h=4$, b) $x / h=6$, c) $x / h=10$, a) $x / h=19$ (Tota, 2009).

Figura 68. Perfil de energía cinética turbulenta para chorro plano: a) Modelo de turbulencia $k-\varepsilon$; b) Modelo de turbulencia RNG $k-\varepsilon$. (Aziz et al., 2008). 130

Figura 69. Perfil de energía cinética turbulenta para chorro circular: a) Modelo de turbulencia $k$ - $\varepsilon$; b) Modelo de turbulencia RNG $k$ - $\varepsilon$.(Aziz et al., 2008). 130

Figura 70. Presión normalizada simulada en la dirección radial sobre una placa plana frente a los resultados de Leach et al. (1996) (Guha et al., 2010).

Figura 71. Distribución de velocidades para $x / D=100,200$ y 300 y comparación con los resultados de Rajaratnam et al. (1994) (Guha et al., 2010). 132 
Figura 72. Comparación de los perfiles de velocidad medidos y calculados para el chorro plano: —— modelo $k-\omega$; - - - modelo $k-\varepsilon$; . . . modelo RNG $k-\varepsilon$; Bradbury (1965); Heskestad (1965) (Wilcox, 2010).

Figura 73. Comparación de los perfiles de velocidad medidos y calculados para el chorro circular: — modelo $k-\omega$; - - - modelo $k-\varepsilon$; · . . · modelo RNG $k-\varepsilon$; Wygnanski y Fiedler (1969); Rodi (1975) (Wilcox, 2010). 133

Figura 74. Comparación de los perfiles de velocidad medidos y calculados para el chorro radial: — modelo $k-\omega$; - - - modelo $k-\varepsilon$; . . . modelo RNG $k-\varepsilon$; Witze y Dwyer (1976) (Wilcox, 2010).

Figura 75. Comparación de los resultados de diferentes modelos de turbulencia con datos experimentales (Balabel et al., 2011). 134

Figura 76. Variación de la velocidad en la línea central del chorro (Aziz y Khan, 2011). 135

Figura 77. Perfiles de energía cinética turbulenta para distintas secciones (Aziz y Khan, 2011). 135

Figura 78. Distribución radial de la velocidad axial para flujo unifásico (Kendil et al., 2012). 136

Figura 79. Forma de la estela para diferentes valores del coeficiente de la fuerza de sustentación $C_{L}$, considerando una concentración de aire $\alpha_{G}=5 \%$ (Kendil et al., 2012).

Figura 80. Patrones de presiones de los cuatro tipos de chorros rectangulares: Chorro no desarrollado y colchón no efectivo $\left(H / L_{b} \leq 1\right.$ e $\left.Y / B_{j} \leq 5.5\right)$; Chorro no desarrollado y colchón efectivo $\left(H / L_{b} \leq 1\right.$ e $\left.Y / B_{j}>5.5\right)$; Chorro desarrollado y colchón no efectivo $\left(H / L_{b}>1\right.$ e $\left.Y / B_{j} \leq 5.5\right)$; Chorro desarrollado y colchón efectivo $\left(H / L_{b}>1\right.$ e $\left.Y / B_{j}>5.5\right)$. (adaptado de Castillo et al., 2014). 141

Figura 81. Función de distribución de probabilidad comparada con una distribución de Gauss. Chorros no desarrollados $\left(H / L_{b} \leq 1\right)$. Chorros desarrollados $\left(H / L_{b}>1\right)$. Colchón no efectivo $\left(Y / B_{j} \leq 5.5\right)$. Colchón efectivo $\left(Y / B_{j}>5.5\right)$.

Figura 82. Función de densidad de probabilidad comparada con una distribución de Gauss. Chorros no desarrollados $\left(H / L_{b} \leq 1\right)$. Chorros desarrollados $\left(H / L_{b}>1\right)$. Colchón no efectivo $\left(Y / B_{j} \leq 5.5\right)$. Colchón efectivo $\left(Y / B_{j}>5.5\right)$. (Castillo et al., 2014).

Figura 83. Esquema de parámetros relevantes en la formulación de la Metodología Paramétrica para análisis del sobrevertido de presas. 144

Figura 84. Coeficientes de presión dinámica media en la solera del cuenco de disipación y disipación de energía para $Y \leq 5.5 B_{j}$ (Castillo et al., 2014). 146 
Figura 85. Coeficientes de presión dinámica media para diferentes formas de chorros y entradas de aire (Castillo et al., 2014).

Figura 86. Variación del coeficiente de presión dinámica fluctuante con la relación colchón de agua/espesor del chorro (Castillo et al., 2014).

Figura 87. Variación del coeficiente de presión dinámica fluctuante con la longitud de rotura del chorro (Castillo et al., 2014). 151

Figura 88. Variación del coeficiente de presión dinámica fluctuante máximo con el colchón de agua (Castillo et al., 2014).

Figura 89. Variación del coeficiente de presión dinámica fluctuante mínimo con el colchón de agua (Castillo et al., 2014).

Figura 90. Distribución de presiones dinámicas medias alrededor del punto de estancamiento en función del parámetro $b_{\text {distrib }}$ (Castillo et al., 2014). 155

Figura 91. Distribución de presiones dinámicas medias alrededor del punto de estancamiento en función de la relación $x / Y$ 156

Figura 92. Distribución de presiones dinámicas medias alrededor del punto de estancamiento en función de la relación $x / x_{\max }$ (Castillo et al., 2014). 157

Figura 93. Distribución de presiones dinámicas fluctuantes alrededor del punto de

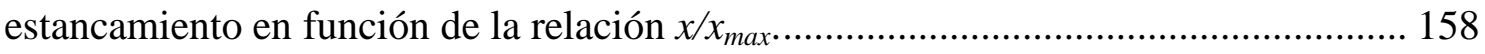

Figura 94. Coeficiente de pérdida de energía $\beta$ en función de la relación $Y / P_{l}$ (Castillo et al., 2014).

Figura 95. Perfil adimensional de velocidad media horizontal en el cuenco de disipación. 160

Figura 96. Variación de la longitud característica $\delta_{l}$ en resaltos sumergidos. 161

Figura 97. Pérdida de energía relativa en resaltos sumergidos en función del $\mathrm{n}^{0}$ de Froude. 162

Figura 98. Pérdida de energía relativa en resaltos sumergidos en función de la relación $y_{3} / B_{j}$ 163

Figura 99. Transitorio de de la presión total simulada en el punto de estancamiento del cuenco de disipación 164

Figura 100. Esquema conceptual del método FAVOR. 166

Figura 101. Interfaz del flujo bifásico aire-agua en un aliviadero de vertido libre...... 169

Figura 102. Lámina libre del chorro rectangular $\left(q=0.058 \mathrm{~m}^{2} / \mathrm{s}, H=2.27 \mathrm{~m}, \quad h=\right.$ $0.095 \mathrm{~m}, Y=0.17 \mathrm{~m}$ ): (a) Observación en laboratorio; (b) Resuelto con ANSYS CFX. 
Figura 103. Adaptación del mallado en la zona de lámina libre (Carrillo y Castillo, 2010). 172

Figura 104. Comparación de resultados obtenidos en el centro del vano del aliviadero para la situación de Nivel Máximo Normal del embalse con $H_{d}=4$ m (Carrillo y Castillo, 2010). 173

Figura 105. Perfil de la simulación bidimensional de un aliviadero de vertido libre

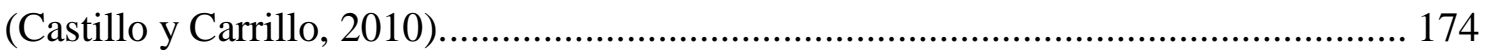

Figura 106. Esquema de las condiciones de contorno.................................................. 175

Figura 107. Comparación del vertido libre en laboratorio con la simulación numérica (Castillo y Carrillo, 2011). 176

Figura 108. Simulación de la lámina de vertido libre: a) Mallado tetraédrico; b) Mallado hexaédrico (Carrillo y Castillo, 2011)....................................................................... 177

Figura 109. Trayectoria de la lámina libre (Carrillo y Castillo, 2011)......................... 179

Figura 110. Sección de control de velocidades turbulentas en el modelo de laboratorio (Carrillo y Castillo, 2011). 180

Figura 111. Velocidades medias y turbulentas para $q=0.058 \mathrm{~m}^{2} / \mathrm{s}$ y $h=0.095 \mathrm{~m}$ (Carrillo y Castillo, 2011). 181

Figura 112. Velocidades y velocidades turbulentas para $q=0.037 \mathrm{~m}^{2} / \mathrm{s}$ y $h=0.073 \mathrm{~m}$ (Carrillo y Castillo, 2011). 181

Figura 113. Velocidades medias y turbulentas para $q=0.023 \mathrm{~m}^{2} / \mathrm{s}$ y $h=0.047 \mathrm{~m}$ (Carrillo y Castillo, 2011). 181

Figura 114. Evolución de la intensidad de turbulencia $T_{u}$ y del coeficiente del parámetro de turbulencia $K_{\varphi}$ para distintos caudales (Carrillo y Castillo, 2011). 182

Figura 115. Evolución de la intensidad de turbulencia del chorro vertiente en función de la intensidad turbulenta en la condición de entrada (Castillo et al., 2014). 182

Figura 116. Coeficiente del parámetro turbulento $K_{\varphi}$ en función de la intensidad turbulenta en la condición inicial (Castillo et al., 2014). 183

Figura 117. Lámina libre del chorro para $q=0.058 \mathrm{~m}^{2} / \mathrm{s}$ : a) ANSYS CFX; b) FLOW-3D (Castillo y Carrillo, 2012)..................................................................................... 184

Figura 118. Velocidad en el chorro para $q=0.058 \mathrm{~m}^{2} / \mathrm{s}$ (Castillo y Carrillo, 2012). .. 185

Figura 119. Fracción volumétrica de agua en el chorro $\left(r_{\text {agua }}\right)$ para $q=0.058 \mathrm{~m}^{2} / \mathrm{s}$ (Castillo y Carrillo, 2012). 186 
Figura 120. Lámina libre del chorro obtenida con FLOW-3D $\left(q=0.037 \mathrm{~m}^{2} / \mathrm{s}, H=2.30\right.$ $\mathrm{m}, h=0.073 \mathrm{~m}, Y=0.12 \mathrm{~m}$ ): a) Simulación con RNG; b) Simulación con $k-\varepsilon$ (Castillo y Carrillo, 2012). 186

Figura 121. Lámina libre del chorro para $q=0.023 \mathrm{~m}^{2} / \mathrm{s}$ : a) ANSYS CFX; b) FLOW-3D con mallado de $0.005 \mathrm{~m}$; c) FLOW-3D con mallado de $0.003 \mathrm{~m}$ (Castillo y Carrillo, 2012). 187

Figura 122. Fracción volumétrica de agua $r_{\text {agua }}$ para $q=0.023 \mathrm{~m}^{2} / \mathrm{s}$ : a) FLOW-3D con mallado de $0.005 \mathrm{~m}$; b) FLOW-3D con mallado de $0.003 \mathrm{~m}$; c) Detalle de la rotura del chorro (Castillo y Carrillo, 2012). 188

Figura 123. Velocidad en el chorro para $q=0.023 \mathrm{~m}^{2} / \mathrm{s}$ (Castillo y Carrillo, 2012). .. 188 Figura 124. Fracción volumétrica de agua en el chorro $r_{\text {agua }}$ para $q=0.023 \mathrm{~m}^{2} / \mathrm{s}$ (Castillo y Carrillo, 2012). 189

Figura 125. Orientación del chorro sobre el esquema de diferencias finitas (Castillo y Carrillo, 2012). 189

Figura 126. Chorro alineado con la malla en FLOW-3D (Castillo y Carrillo, 2012). . 190 Figura 127. Chorro oblicuo a la malla en FLOW-3D (Castillo y Carrillo, 2012)........ 191 Figura 128. Fracción volumétrica de agua en el chorro vertiente simulada con FLOW3D.

Figura 129. Energía cinética turbulenta en el chorro vertiente simulada con FLOW-3D.

Figura 130. Vectores de velocidad en un resalto hidráulico simulado con FLOW-3D.193 Figura 131. Energía cinética turbulenta en un resalto hidráulico simulado con FLOW3D. 194

Figura 132. Fracción volumétrica de aire introducido en un resalto hidráulico simulado con FLOW-3D. 194

Figura 133. Comparación de resultados de laboratorio y numéricos en un resalto hidráulico: a) Sección a 0.05 m del inicio del resalto; a) Sección a 0.20 m del inicio del resalto. 195

Figura 134. Velocidad del flujo en el chorro vertiente en función del tamaño de los elementos del mallado: a) $q=0.058 \mathrm{~m}^{2} / \mathrm{s}, h=0.095 \mathrm{~m}$; b) $q=0.037 \mathrm{~m}^{2} / \mathrm{s}, h=0.073 \mathrm{~m}$ (Castillo et al., 2014). 196

Figura 135. Fracción volumétrica de aire en el chorro vertiente en función del tamaño de los elementos del mallado: a) $q=0.058 \mathrm{~m}^{2} / \mathrm{s}, h=0.095 \mathrm{~m}$; b) $q=0.037 \mathrm{~m}^{2} / \mathrm{s}, h=$ $0.073 \mathrm{~m}$ (Castillo et al., 2014). 196 
Figura 136. Mallado hexaédrico en ANSYS CFX: a) Vista general del modelo; b) Detalle del aliviadero; c) detalle del punto de incidencia y del punto de estancamiento. 198

Figura 137. Distribución de presiones en el punto de estancamiento para $q=0.058 \mathrm{~m}^{2} / \mathrm{s}$, $H=2.27 \mathrm{~m}, h=0.095 \mathrm{~m}$ e $Y=0.17 \mathrm{~m}$ (Castillo y Carrillo, 2012)..... 199

Figura 138. Esquema de variables en vertido libre. 200

Figura 139. Calado sobre vertedero $y_{b}$ para distintas relaciones de escala...... 204

Figura 140. Espesor del chorro incidente $B_{j}$ para distintas relaciones de escala. 204

Figura 141.Velocidad del chorro incidente $V_{j}$ para distintas relaciones de escala. ...... 205

Figura 142. Distancia horizontal al punto de estancamiento $X_{\text {imp }}$ para distintas relaciones de escala. 205

Figura 143. Coeficiente de presión dinámica media $C_{p}$ para distintas relaciones de escala. Caso $Y / B_{j}=1.30$. 206

Figura 144. Calado bajo el chorro $Y_{u}$ para distintas relaciones de escala. 207

Figura 145. Distribución de presiones medias en torno al punto de estancamiento..... 211 Figura 146. Distribución de presiones medias en torno al punto de estancamiento en función de las formulaciones clásicas: a) $q=0.058 \mathrm{~m}^{2} / \mathrm{s}, h=0.095 \mathrm{~m}, H=2.27 \mathrm{~m}, Y=$ $0.17 \mathrm{~m}$; b) $q=0.037 \mathrm{~m}^{2} / \mathrm{s}, h=0.073 \mathrm{~m}, H=2.30 \mathrm{~m}, Y=0.12 \mathrm{~m}$.

Figura 147. Distribución de presiones medias en torno al punto de estancamiento en función de la relación $x / Y$ : a) $q=0.058 \mathrm{~m}^{2} / \mathrm{s}, h=0.095 \mathrm{~m}, H=2.27 \mathrm{~m}, Y=0.17 \mathrm{~m}$; b) $q$ $=0.037 \mathrm{~m}^{2} / \mathrm{s}, h=0.073 \mathrm{~m}, H=2.30 \mathrm{~m}, Y=0.12 \mathrm{~m}$

Figura 148. Distribución de presiones medias en torno al punto de estancamiento en función de la relación $x / x_{\text {max }}$ : a) $q=0.058 \mathrm{~m}^{2} / \mathrm{s}, h=0.095 \mathrm{~m}, H=2.27 \mathrm{~m}, Y=0.17 \mathrm{~m}$; b) $q=0.037 \mathrm{~m}^{2} / \mathrm{s}, h=0.073 \mathrm{~m}, H=2.30 \mathrm{~m}, Y=0.12 \mathrm{~m}$.

Figura 149. Perfiles de velocidades medias horizontales en el cuenco de disipación aguas abajo del punto de estancamiento: a) $q=0.058 \mathrm{~m}^{2} / \mathrm{s}, H=2.27 \mathrm{~m}, Y=0.17 \mathrm{~m}$; b) q $=0.058 \mathrm{~m}^{2} / \mathrm{s}, H=2.20 \mathrm{~m}, Y=0.25 \mathrm{~m}$; c) $q=0.058 \mathrm{~m}^{2} / \mathrm{s}, H=2.10 \mathrm{~m}, Y=0.35 \mathrm{~m} . . .215$

Figura 150. Perfiles de velocidades medias verticales en el cuenco de disipación aguas abajo del punto de estancamiento: a) $q=0.058 \mathrm{~m}^{2} / \mathrm{s}, H=2.27 \mathrm{~m}, Y=0.17 \mathrm{~m}$; b) $q=$ $0.058 \mathrm{~m}^{2} / \mathrm{s}, H=2.20 \mathrm{~m}, Y=0.25 \mathrm{~m}$; c) $q=0.058 \mathrm{~m}^{2} / \mathrm{s}, H=2.10 \mathrm{~m}, Y=0.35 \mathrm{~m}$........ 216

Figura 151. Perfiles de energía cinética turbulenta $K$ en el cuenco de disipación aguas abajo del punto de estancamiento: a) $q=0.058 \mathrm{~m}^{2} / \mathrm{s}, H=2.27 \mathrm{~m}, Y=0.17 \mathrm{~m}$; b) $q=$ $0.058 \mathrm{~m}^{2} / \mathrm{s}, H=2.20 \mathrm{~m}, Y=0.25 \mathrm{~m}$; c) $q=0.058 \mathrm{~m}^{2} / \mathrm{s}, H=2.10 \mathrm{~m}, Y=0.35 \mathrm{~m} \ldots \ldots . .216$ 
Figura 152. Perfiles de velocidades medias horizontales en el cuenco de disipación aguas abajo del punto de estancamiento: a) $q=0.037 \mathrm{~m}^{2} / \mathrm{s}, H=2.30 \mathrm{~m}, Y=0.12 \mathrm{~m}$; b) $q$ $=0.037 \mathrm{~m}^{2} / \mathrm{s}, H=2.20 \mathrm{~m}, Y=0.24 \mathrm{~m}$; c) $q=0.037 \mathrm{~m}^{2} / \mathrm{s}, H=2.12 \mathrm{~m}, Y=0.33 \mathrm{~m} . . .217$

Figura 153. Perfiles de velocidades medias verticales en el cuenco de disipación aguas abajo del punto de estancamiento: a) $q=0.037 \mathrm{~m}^{2} / \mathrm{s}, H=2.30 \mathrm{~m}, Y=0.12 \mathrm{~m}$; b) $q \quad=$ $0.037 \mathrm{~m}^{2} / \mathrm{s}, H=2.20 \mathrm{~m}, Y=0.24 \mathrm{~m}$; c) $q=0.037 \mathrm{~m}^{2} / \mathrm{s}, H=2.12 \mathrm{~m}, Y=0.33 \mathrm{~m} . \ldots \ldots . .217$

Figura 154. Perfiles de energía cinética turbulenta $K$ en el cuenco de disipación aguas abajo del punto de estancamiento: a) $q=0.037 \mathrm{~m}^{2} / \mathrm{s}, H=2.30 \mathrm{~m}, Y=0.12 \mathrm{~m}$; b) $q \quad q=$ $0.037 \mathrm{~m}^{2} / \mathrm{s}, H=2.20 \mathrm{~m}, Y=0.24 \mathrm{~m}$; c) $q=0.037 \mathrm{~m}^{2} / \mathrm{s}, H=2.12 \mathrm{~m}, Y=0.33 \mathrm{~m} . \ldots \ldots . .218$

Figura 155. Perfiles de velocidades medias horizontales en el cuenco de disipación aguas abajo del punto de estancamiento: a) $q=0.023 \mathrm{~m}^{2} / \mathrm{s}, H=2.29 \mathrm{~m}, Y=0.12 \mathrm{~m}$; b) $\mathrm{q}$ $=0.020 \mathrm{~m}^{2} / \mathrm{s}, H=2.18 \mathrm{~m}, Y=0.22 \mathrm{~m}$; c) $q=0.023 \mathrm{~m}^{2} / \mathrm{s}, H=2.10 \mathrm{~m}, Y=0.30 \mathrm{~m} . \ldots 218$

Figura 156. Perfiles de velocidades medias verticales en el cuenco de disipación aguas abajo del punto de estancamiento: a) $q=0.023 \mathrm{~m}^{2} / \mathrm{s}, H=2.29 \mathrm{~m}, Y=0.12 \mathrm{~m}$; b) $q \quad=$ $0.020 \mathrm{~m}^{2} / \mathrm{s}, H=2.18 \mathrm{~m}, Y=0.22 \mathrm{~m}$; c) $q=0.023 \mathrm{~m}^{2} / \mathrm{s}, H=2.10 \mathrm{~m}, Y=0.30 \mathrm{~m} \ldots \ldots . .219$

Figura 157. Perfiles de energía cinética turbulenta $K$ en el cuenco de disipación aguas abajo del punto de estancamiento: a) $q=0.023 \mathrm{~m}^{2} / \mathrm{s}, H=2.29 \mathrm{~m}, Y=0.12 \mathrm{~m}$; b) $q \quad q=$ $0.020 \mathrm{~m}^{2} / \mathrm{s}, H=2.18 \mathrm{~m}, Y=0.22 \mathrm{~m}$; c) $q=0.023 \mathrm{~m}^{2} / \mathrm{s}, H=2.10 \mathrm{~m}, Y=0.30 \mathrm{~m} . \ldots \ldots . .219$

Figura 158. Entrada de aire en el cuenco de disipación observada en laboratorio $(q=$ $\left.0.058 \mathrm{~m}^{2} / \mathrm{s}, H=2.27 \mathrm{~m}, Y=0.17 \mathrm{~m}\right)$. 220

Figura 159. Distribución de velocidades obtenidas aguas abajo del punto de estancamiento con simulaciones numéricas. 221

Figura 160. Distribución de velocidades obtenidas aguas abajo del punto de estancamiento con simulaciones numéricas y mediciones de laboratorio.................... 221

Figura 161. Presa y cerrada del proyecto Paute-Cardenillo (Ecuador). ........................ 223

Figura 162. Esquema de socavación en la presa Paute-Cardenillo (Castillo y Carrillo, 2014). 225

Figura 163. Detalle de la rotura del chorro en la simulación de un vano del aliviadero de vertido libre obtenido con FLOW-3D. 232

Figura 164. Detalle de la rotura del chorro de un vano del aliviadero. 233

Figura 165. Mallado del aliviadero de vertido libre de la presa Paute-Cardenillo y análisis de sensibilidad en FLOW-3D (Castillo y Carrillo, 2014). 233

Figura 166. Registro de presiones en el punto de estancamiento. 235

Figura 167. Perfil longitudinal y transversal de la socavación debida al aliviadero de vertido libre. 236 
Figura 168. Mallado de los desagües intermedios. 237

Figura 169. Funcionamiento conjunto de los dos desagües intermedios (caudal total de $\left.1760 \mathrm{~m}^{3} / \mathrm{s}\right)$. Vista en planta. 237

Figura 170. Perfil longitudinal de las velocidades en el cuenco erosionado. 238

Figura 171. Perfil del cuenco de socavación generado en la capa aluvial por el desagüe intermedio. 239

Figura 172. Planta del cuenco de socavación generado en la capa aluvial por el desagüe intermedio. 239

Figura 173. Sección trasversal del cuenco de socavación generado por el desagüe intermedio. 240

Figura 174. Localización de los cuencos erosionados generados en los distintos escenarios. 240

Figura 175. Cuenco pre-excavado propuesto y su disposición en el terreno. 241

Figura 176. Funcionamiento del cuenco pre-excavado para el caudal de $700 \mathrm{~m}^{3} / \mathrm{s}$ descargado por el aliviadero superior.

Figura 177. Distribución de presiones en solera del cuenco pre-excavado para el caudal de $700 \mathrm{~m}^{3} / \mathrm{s}$ descargado por el aliviadero de superficie.

Figura 178. Distribución de velocidades dentro del cuenco pre-excavado para un caudal de $1760 \mathrm{~m}^{3} / \mathrm{s}$ que pasa por los desagües intermedios.

Figura 179. Vectores de velocidades dentro del cuenco pre-excavado para un caudal de $1760 \mathrm{~m}^{3} / \mathrm{s}$ que pasa por los desagües intermedios. Vista en perfil.

Figura 180. Vectores de velocidades dentro del cuenco pre-excavado para un caudal de $1760 \mathrm{~m}^{3} / \mathrm{s}$ que pasa por los desagües intermedios. Vista en planta. 244

Figura 181. Distribución de presiones en la solera del cuenco pre-excavado para un caudal de $1760 \mathrm{~m}^{3} / \mathrm{s}$ que pasa por los desagües intermedios. 244

Figura 182. Funcionamiento del cuenco pre-excavado para un caudal de $4737.91 \mathrm{~m}^{3} / \mathrm{s}$ por los desagües de fondo. 245

Figura 183. Vectores de velocidades dentro del cuenco pre-excavado para un caudal de $4737.91 \mathrm{~m}^{3} / \mathrm{s}$ por los desagües de fondo. 245

Figura 184. Funcionamiento del cuenco pre-excavado para un caudal de $7197.91 \mathrm{~m}^{3} / \mathrm{s}$ (aliviadero de superficie + desagüe de medio fondo + desagüe de fondo). 246

Figura 185. Distribución de presiones en la solera del cuenco pre-excavado para un caudal de $7197.91 \mathrm{~m}^{3} / \mathrm{s}$ (aliviadero de superficie + desagüe de medio fondo + desagüe de fondo). 246 
ÍNDICE DE FIGURAS 


\section{ÍNDICE DE TABLAS}

Tabla 1. Expresiones de longitud de rotura para chorro rectangular y circular. 21

Tabla 2. Coeficiente $K$ de la longitud del núcleo del chorro según diversos autores (Bollaert y Schleiss, 2003). Complementado.

Tabla 3. Principales tres mecanismos de entrada de aire en el punto de impacto del chorro en el cuenco de disipación y su correspondiente tasa de aireación por unidad de ancho del chorro (Ervine, 1998, citado por Bollaert, 2002). 28

Tabla 4. Expresiones para el cálculo en flujo bidimensional del calado aguas arriba del impacto del chorro en función de la altura de caída $H$ y del calado crítico $y_{c}$ (Chanson, 1995).

Tabla 5. Coeficientes $C$ y $K$ de la formulación general de presiones dinámicas medias (Castillo, 2002). 36

Tabla 6. Parámetros de la ecuación del coeficiente de presión dinámica media en función de la longitud de rotura (Castillo, 2006). 40

Tabla 7. Coeficientes del polinomio de regresión para diferentes intensidades turbulentas (Bollaert, 2002).

Tabla 8. Coeficientes para el cálculo de la presión dinámica fluctuante (Castillo,2006).

Tabla 9. Parámetros de las ecuaciones para el cálculo de las presiones dinámicas medias (Castillo, 1989).

Tabla 10. Expresiones para el cálculo de perfiles de velocidad longitudinal

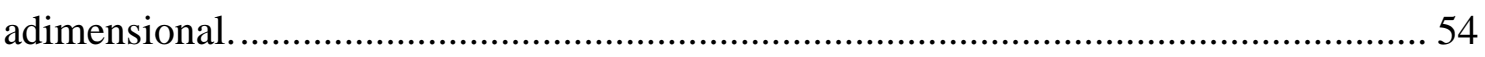

Tabla 11. Valores de $C_{e}$ como función de las relaciones $b_{c} / B_{1}$ y $h / P_{l}$ (Bos, 1989)........ 61

Tabla 12. Valores recomendados para vertederos triangulares (Bos, 1989)................... 62

Tabla 13. Factores de escala de diversas variables.

Tabla 14. Comparación entre la formulación integral y la formulación diferencial en un problema de CFD.

Tabla 15. Comparación de los requisitos para una simulación con modelos RANS y LES (Menter, 2012).

Tabla 16. Comparación de los modelos de turbulencia RANS. 115 
Tabla 17. Comparación de la descarga obtenida en un aliviadero tridimensional (Ho et al., 2003)

Tabla 18. Comparación de la descarga obtenida por Hager y Schwalt (1994) en un aliviadero cresta ancha con simulaciones de CFD (Hargreaves et al., 2007). 121

Tabla 19. Ratios de expansión de flujo de corte libre para modelos de dos ecuaciones (Wilcox, 2010)

Tabla 20. Lista de limitaciones habituales y sugerencias para investigaciones futuras en CFD (Ho y Riddette, 2010).

Tabla 21. Resumen de la fiabilidad de los modelos numéricos en la modelación hidráulica (ICOLD, 2001).

Tabla 22. Rango de las condiciones de ensayo en laboratorio (valores mínimos y máximos)

Tabla 23. Parámetros del coeficiente de presión dinámica media cuando $Y>5.5 B_{j}$ (Castillo et al., 2014).

Tabla 24. Parámetros para el cálculo del coeficiente de presión dinámica fluctuante (Castillo et al., 2014). 150

Tabla 25. Variación del valor máximo del coeficiente de presión dinámica fluctuante para diferentes formas de chorro en función de la relación colchón de agua/espesor del chorro (Castillo et al., 2014). 150

Tabla 26. Parámetros de la distribución de la presión dinámica media alrededor del punto de estancamiento (Castillo et al., 2014).

Tabla 27. Ajustes para el cálculo de la presión dinámica fluctuante alrededor del punto de estancamiento. 158

Tabla 28. Número de elementos en las simulaciones del aliviadero de vertido libre y tiempo de cálculo requerido (Carrillo y Castillo, 2011). 178

Tabla 29. Comparativa de parámetros representativos de la teoría de vertido libre (Carrillo y Castillo, 2011). 178

Tabla 30. Velocidades turbulentas en función de la turbulencia en la condición de entrada para distintas alturas $\left(q=0.058 \mathrm{~m}^{2} / \mathrm{s}, h=0.095 \mathrm{~m}\right.$ ). (Castillo et al., 2014).... 183 Tabla 31. Número de elementos en función del tamaño del elemento de mallado empleado. 195

Tabla 32. Presión media en el punto de estancamiento en función del tamaño del elemento del mallado. 
Tabla 33. Comparación de las principales variables medidas y calculadas con diferentes turbulencias en la condición de entrada para $q=0.058 \mathrm{~m}^{2} / \mathrm{s}, H=2.27 \mathrm{~m}, h=0.095 \mathrm{~m}$ e $Y=0.17 \mathrm{~m}$ (Castillo y Carrillo, 2012). 200

Tabla 34. Factores de escala con la ley de Froude para diferentes variables. 201

Tabla 35. Comparación de las principales variables medidas y calculadas (a escala de laboratorio $q=0.058 \mathrm{~m}^{2} / \mathrm{s}, Y=0.03 \mathrm{~m}$ ). 202

Tabla 36. Comparación de las principales variables medidas y calculadas (a escala de laboratorio $\left.q=0.058 \mathrm{~m}^{2} / \mathrm{s}, Y=0.17 \mathrm{~m}\right)$. 202

Tabla 37. Comparación de las principales variables medidas y calculadas (a escala de laboratorio $\left.q=0.058 \mathrm{~m}^{2} / \mathrm{s}, Y=0.25 \mathrm{~m}\right)$. 202

Tabla 38. Comparación de las principales variables medidas y calculadas (a escala de laboratorio $\left.q=0.058 \mathrm{~m}^{2} / \mathrm{s}, Y=0.35 \mathrm{~m}\right)$. 203

Tabla 39. Comparación de las principales variables medidas y calculadas $(q=0.058$

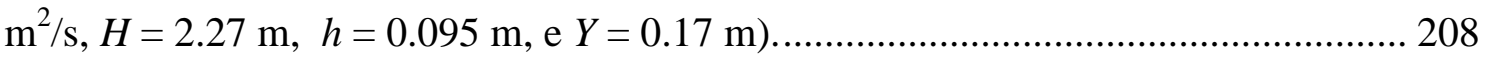

Tabla 40. Comparación de las principales variables medidas y calculadas $(q=0.037$

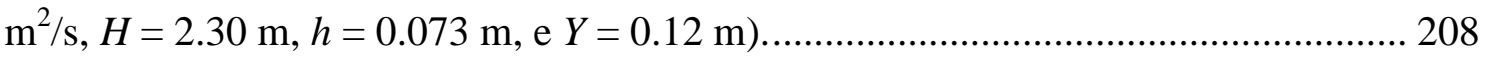

Tabla 41. Comparación de las principales variables medidas y calculadas $(q=0.023$

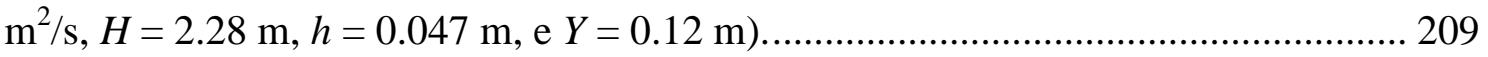

Tabla 42. Estudio sistemático con CFD de presiones en el punto de estancamiento... 215

Tabla 43. Coeficientes de formulaciones de socavación simplificadas (Castillo y Carrillo, 2014). 225

Tabla 44. Formulaciones de socavación generales (Castillo y Carrillo, 2014). 226

Tabla 45. Cálculo de la socavación del aliviadero de vertido libre y del desagüe de medio fondo con las formulaciones empíricas. Valores medios y dispersión de +/- 1 desviación estándar (Castillo y Carrillo, 2014).

Tabla 46. Parámetros del índice de erosionabilidad (adaptado de Annandale, 2006).. 228

Tabla 47. Datos de entrada y valores calculados en la metodología semiempírica...... 229

Tabla 48. Índices de erosionabilidad $K$ y umbral de la potencia de escurrimiento $P_{\text {roca }}$ en el perfil estratigráfico correspondiente a la zona aguas abajo de la presa PauteCardenillo. 230

Tabla 49. Cálculo de la socavación del aliviadero de vertido libre en función del índice de erosionabilidad de los materiales. 230

Tabla 50. Cálculo de la socavación del desagüe de medio fondo en función del índice de erosionabilidad de los materiales. 231 
Tabla 51. Análisis de sensibilidad en FLOW-3D para el aliviadero de vertido libre de la presa Paute-Cardenillo.

Tabla 52. Comparación de presiones y $\mathrm{C}_{\mathrm{p}}$ en la presa Paute-Cardenillo considerando un colchón de agua de $2 \mathrm{~m}$. 


\section{LISTA DE SÍMBOLOS}

\section{Caracteres latinos}

\begin{tabular}{|c|c|}
\hline A & Área \\
\hline$a, b$ & $\begin{array}{l}\text { Parámetros del coeficiente de presión dinámica media de la Metodología } \\
\text { Paramétrica }\end{array}$ \\
\hline$a_{i}$ & Coeficiente empleado en la discretización de las ecuaciones \\
\hline$B_{1}$ & Ancho del canal de aproximación \\
\hline$B_{g}$ & Ancho del chorro por condiciones gravitatorias \\
\hline$B_{i}$ & $\begin{array}{l}\text { Ancho del chorro en la condición inicial (sección transversal localizada en la cota } \\
z=-h \text {, siendo } z \text { la componente vertical con origen en el labio del aliviadero y } \\
\text { sentido ascendente) }\end{array}$ \\
\hline$B_{j}$ & Ancho del chorro en la sección del chorro antes del impacto con el colchón \\
\hline$b_{c}$ & Ancho del labio del vertedero \\
\hline$b_{\text {distrib }}$ & Ancho de la región de influencia de la presión \\
\hline$b_{j}$ & Ancho transversal del chorro incidente \\
\hline$C_{d}$ & Coeficiente de descarga en vertederos \\
\hline$C_{i}$ & Concentración de aire del chorro en la entrada al colchón \\
\hline$C_{p}$ & Coeficiente de presión dinámica media \\
\hline$C_{r}$ & Coeficiente de la densidad relativa \\
\hline$D$ & Diámetro del chorro circular. Diámetro medio del bloque \\
\hline$D E$ & $\begin{array}{l}\text { Energía disipada por aireación durante la caída y el calado de recirculación aguas } \\
\text { arriba del punto de estancamiento }\end{array}$ \\
\hline$D_{0}$ & Diámetro del chorro en la salida de la tobera \\
\hline$D_{c}$ & Diámetro del chorro circular no aireado debido a condiciones gravitatorias \\
\hline$D_{j}$ & Ancho del chorro circular incidente en el colchón \\
\hline$F_{D}$ & Número de Froude al cuadrado en el colchón de agua \\
\hline$F_{i}$ & Número de Froude en la condición inicial \\
\hline$F_{j}$ & Número de Froude en la condición incidente \\
\hline$f_{D}$ & Frecuencia Doppler en equipo ADV \\
\hline$f_{e}$ & Frecuencia de una onda acústica emitida en equipo ADV \\
\hline$f_{r}$ & Frecuencia de una onda reflejada en un equipo ADV \\
\hline $\overrightarrow{f_{m}}$ & Vector de fuerzas másicas \\
\hline$f(u)$ & Vector de flujo \\
\hline$g$ & Aceleración de la gravedad \\
\hline$H$ & $\begin{array}{l}\text { Diferencia entre la cota de la lámina libre aguas arriba del aliviadero y la } \\
\text { superficie libre en el cuenco }\end{array}$ \\
\hline$H_{e}$ & Carga total sobre el vertedero \\
\hline$H_{d}$ & Carga de diseño del perfil hidrodinámico del vertedero \\
\hline
\end{tabular}




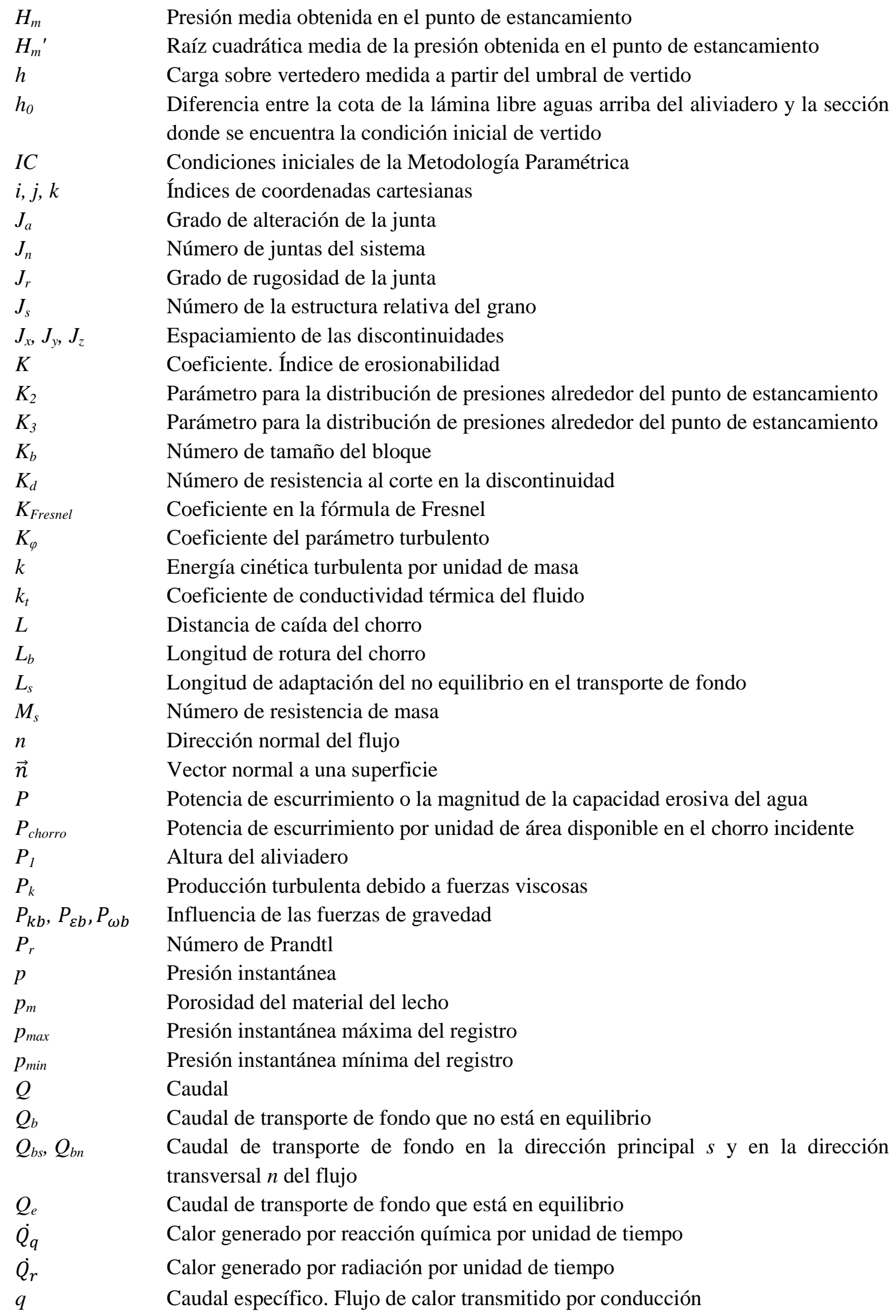


$q_{a e}$

$\dot{q}_{q}$

$\dot{q}_{r}$

$R_{e}$

$R_{g}$

$R^{2}$

$S$

$S_{E}$

$S_{i j}$

$S_{M}$

$S$

$T$

$T_{u}$

$t$

$t_{G i}$

$U$

$U_{0}$

$u, v, w$

$u^{\prime}, v^{\prime}, w^{\prime}$

$u_{\tau}$

$V$

$V_{D}$

$V_{i}$

$V_{j}$

$V_{0}$

$\bar{V}^{\prime}$

$W_{e}$

$X_{\text {imp }}$

$x, z$

$x^{*}, z^{*}$

$x_{\max }$

Y

$Y_{u}$

$y_{3}$

$y_{4}$

$y_{b}$

$y_{c}$

$y_{k}$

$Z$

$Z_{b}$

Tasa específica de entrada de aire

Calor generado por reacción química por unidad de tiempo y de volumen

Calor generado por radiación por unidad de tiempo y de volumen

Número de Reynolds

Constante de un gas

Coeficiente de correlación

Término fuente, tensor velocidad de deformación media

Fuente de energía

Tensor velocidad de deformación instantánea

Fuente de cantidad de movimiento

Dirección principal del flujo

Temperatura

Intensidad de turbulencia, $T_{u}=\bar{V}^{\prime} / V$

Tiempo

Tiempo durante el que la sonda de fibra óptica está en fase gaseosa

Vector velocidad

Velocidad a la salida de la tobera

Componentes de la velocidad instantánea en la dirección $x, y, z$

Componentes de la fluctuante instantánea en la dirección $x, y, z$

Velocidad de fricción

Velocidad media

Velocidad media en el colchón de agua

Velocidad media del chorro en la condición inicial (sección transversal localizada en la cota $z=-h$, siendo $z$ la componente vertical con origen en el labio del aliviadero y sentido ascendente)

Velocidad media del chorro en la sección del chorro antes del impacto con el colchón de agua

Velocidad media en la sección del umbral de vertido

Raíz cuadrática media de la velocidad

Número de Weber

Distancia horizontal del aliviadero al punto de impacto

Componentes horizontal y vertical

Ejes coordenados considerando que el origen del sistema está en el borde del vertedero

Extensión longitudinal máxima del área influenciada por la capa de corte turbulenta del chorro incidente

Calado en el cuenco de disipación

Calado del colchón de agua aguas arriba del punto de incidencia del chorro

Calado en el cuenco aguas arriba del resalto sumergido

Calado en el cuenco aguas abajo del resalto sumergido

Calado del flujo a su paso sobre el labio del vertedero de pared delgada

Calado crítico

Distancia para el desarrollo del chorro en un cuenco de amortiguación

Parámetro líquido

Cambio en el lecho debido al transporte de sedimentos 


\section{Caracteres griegos}

$\alpha_{b s}, \alpha_{b n} \quad$ Cosenos de los vectores de dirección que determinan las componentes del transporte de fondo en las direcciones $s$ y $n$

$\alpha_{t} \quad$ Difusividad térmica turbulenta

$\beta \quad$ Coeficiente de pérdida de energía

$\beta_{i} \quad$ Relación entre aire y agua de un chorro compacto entrando en el colchón de agua

$\Gamma_{t} \quad$ Difusividad turbulenta

$\gamma \quad$ Peso específico del agua

$\gamma_{r} \quad$ Peso específico de la roca

$\Delta t \quad$ Intervalo de paso

$\delta_{1} \quad$ Ángulo de disminución del núcleo interno del chorro

$\delta_{2} \quad$ Ángulo de expansión externa del chorro

$\delta_{i j} \quad$ Función delta de Kronecker

$\delta_{l} \quad$ Longitud característica del campo de velocidades medias en el resalto hidráulico (calado donde $V_{x}=V_{\text {máx }} / 2$ )

$\delta_{\text {máx }} \quad$ Calado donde se obtiene la velocidad máxima en el perfil de velocidades horizontales del resalto hidráulico

$\varepsilon \quad$ Tasa de disipación de la turbulencia

$\theta \quad$ Ángulo del chorro incidente en el cuenco de disipación

$\mu \quad$ Viscosidad (dinámica) molecular

$\mu_{t} \quad$ Viscosidad de remolino o viscosidad turbulenta

$\mu_{w} \quad$ Viscosidad cinemática

$\xi \quad$ Expansión lateral por efecto de la turbulencia

$\eta_{i} \quad$ Índice de refracción del material $i$

$\rho \quad$ Densidad

$\rho_{r} \quad$ Densidad de la roca

$\sigma \quad$ Desviación estándar

$\sigma_{w a} \quad$ Tensión superficial

$\tau \quad$ Tensor de tensiones

$\tau^{\prime} \quad$ Tensor de esfuerzos viscosos

$v_{t} \quad$ Viscosidad cinemática turbulenta

$\Phi \quad$ Variable dependiente genérica

$\Phi_{i j} \quad$ Tensor de presión-deformación

$\phi \quad$ Parámetro para la distribución de presiones alrededor del punto de estancamiento. Ángulo de fricción residual o interna del material granular

$\varphi \quad$ Parámetro turbulento de la Metodología Paramétrica, $\varphi=K_{\varphi}\left(\bar{V}^{\prime} / V\right)$

$\omega \quad$ Frecuencia turbulenta 


\section{$\underline{\text { Abreviaturas }}$}

ADV

CFD

CEDEX

CNEGP

FAVOR

$\mathrm{SPH}$

ICOLD

PDF

PIV

RANS

RQD

RMS

RSM

RNG

SMC

SPT

SST

UCS

USACE

USBR

VOF

WES
Acoustic Doppler Velocimeter (Velocímetro Acústico por efecto Doppler)

Computacional Fluid Dynamics (Dinámica de Fluidos Computacional)

Centro de Estudios y Experimentación de Obras Públicas

Comité Nacional Español de Grandes Presas

Fractional Area/Volume Obstacle Representation

Smoothed-Particle Hydrodynamics software

International Commission on Large Dams (Comité Internacional de Grandes Presas)

Probability Density Function (función de densidad de probabilidad)

Particle Image Velocimetry (Equipos con velocidad con imagen de partículas)

Reynolds Averaged Navier-Stokes Equations (Promediado de Reynolds de las ecuaciones de Navier-Stokes)

Rock Quality Designation

Root Mean Square (raíz cuadrática media)

Reynolds Stress Model (Modelo de Tensiones de Reynolds o Modelo de Turbulencia de cierre de segundo orden)

Renormalization Group Analysis of the Navier-Stokes equations Turbulence Model

Second Moment Closure (Modelo de Tensiones de Reynolds o Modelo de Turbulencia de cierre de segundo orden)

Standard Penetration Test (test de penetración estándar)

Shear Stress Transport Turbulence Model

Uniaxial Compression Srength (resistencia a comprensión no confinada)

United States Army Corp of Engineers

United Stated Bureau of Reclamation

Volume of Fluid method

Waterways Experimental Station 


\section{RESUMEN}

El incremento en la magnitud de las avenidas de diseño por efecto del cambio climático han promovido a nivel mundial, la revaluación de la capacidad de los aliviaderos y de los escenarios de operación de las grandes presas. Diversas investigaciones han demostrado que la capacidad actual de muchos aliviaderos es inadecuada, surgiendo la posibilidad de que las presas lleguen a desbordarse durante los eventos extremos.

El aumento de la capacidad de los aliviaderos resulta con frecuencia caro y en ocasiones técnicamente inviable. En estos casos, los responsables de las presas se podrían plantear el desbordamiento como una estrategia adicional de operación durante estos eventos extremos. Esta situación crea nuevos escenarios en las acciones sobre la presa, surgiendo interrogantes sobre los problemas de erosión y socavación aguas abajo. En el caso específico de las presas de fábrica, la socavación en los estribos y/o en el cauce del río puede ocurrir cuando el caudal que desborda impacta sobre los diferentes materiales que constituyen la cimentación de la presa (Wahl et al., 2008). Para evaluar los requerimientos de protección de estas áreas afectadas, se requiere hacer una comparación entre el potencial hidráulico erosivo y la resistencia a la erosión de los diferentes materiales (Castillo, 2011).

En este contexto, las principales agencias de gestión del agua de Estados Unidos (Bureau of Reclamation, Cuerpo de Ingenieros, etc.) vienen estudiando desde hace algunos años los efectos que los eventos extremos pueden producir en la infraestructura de regulación y evacuación de avenidas, con el objeto de reforzar adecuadamente las presas existentes y, así, evitar su destrucción (Annandale, 2006; Wahl et al., 2008).

Las formulaciones existentes que cuantifican las acciones hidrodinámicas por efecto del desbordamiento de presas han sido obtenidas principalmente a partir de medidas sistematizadas de presiones instantáneas en diferentes laboratorios de hidráulica. Destacan las investigaciones en la Universidad de Glasgow (Reino Unido) (Ervine y Falvey, 1987; Ervine et al., 1997), la Escuela Técnica Federal de Lausanne (Bollaert, 2002, Bollaert y Schleiss, 2003; Manso et al., 2005; y Felderspiel, 2011); así como en la Universidad Politécnica de Cataluña y Universidad Politécnica de Cartagena (Castillo, 1989, 1990, 2002, 2006, 2007; Armengou, 1991; Castillo et al., 1991, 1996, 1999, 2007; Puertas, 1994; Puertas y Dolz, 2002, 2005; y Castillo y Carrillo, 2011, 2012, 2013).

Sin embargo, la caracterización del potencial hidráulico erosivo (acciones debidas a los chorros turbulentos bifásicos) no está suficientemente analizada. Se considera necesario avanzar en su conocimiento con más estudios, tanto en modelos (físicos y 
numéricos) como en prototipos, caracterizando simultáneamente presiones, velocidades y fenómenos de aireación.

En esta Tesis se ha llevado a cabo una investigación sobre la localización y la cuantificación de la energía residual del chorro incidente durante el desbordamiento por coronación en las presas de fábrica. En la investigación se ha utilizado tecnología tanto de tipo experimental como numérico para definir la trayectoria del chorro vertiente, caracterizar la zona de impacto, determinar la disipación de energía en el aire por fenómenos de aireación y atomización, así como la disipación de energía por los fenómenos de difusión e impacto del chorro en el cuenco disipador o zona de recepción. 


\section{ABSTRACT}

The increase of the design floods due to climate change has promoted the revaluation of the spillways capacity and the operation scenarios of large dams throughout the world. Various research have shown that the current capacity of some spillways is inadequate. The studies show that many dams could overflow during extreme events.

Increasing the capacity of the spillways is difficult and expensive. Sometimes, the increase is technically impossible. In these cases, dam owners may consider acceptable when their dam overflows as it is a strategy to obtain additional discharge during extreme events. This creates new scenarios for actions related to the discharge. There are questions about the scour downstream from the dam. In the specific case of concrete dams, scour at the abutments and/or the riverbed can occur when the jet overflowing over the dam impacts on the foundation of the dam (Wahl et al., 2008). To evaluate the protection requirements of these affected areas, it is required to make a comparison between the hydraulic potential of scour of the water and the erosion resistance of the materials (Castillo, 2011).

In this context, the main water management agencies of the United States (Bureau of Reclamation, Corps of Engineers, etc.) have been studying for some years the effects that extreme events may generate in both, the regulatory infrastructures and the flood evacuation. The objective is to strengthen existing dams and, thus, to prevent their destruction (Annandale, 2006, Wahl et al., 2008.).

The formulae that quantify hydrodynamic actions of dam overflow have mainly been obtained from systematic measurements of instantaneous pressures in different hydraulic laboratories. This is highlighted in research done at the University of Glasgow (UK) (Ervine and Falvey, 1987; Ervine et al., 1997), the Federal School of Lausanne (Bollaert, 2002, Bollaert and Schleiss, 2003; Manso et al., 2005; and Felderspiel, 2011), as well as at the Universidad Politécnica de Cataluña and Universidad Politécnica Cartagena (Castillo, 1989, 1990, 2002, 2006, 2007; Armengou, 1991; Castillo et al., 1991, 1996, 1999, 2007; Puertas, 1994; Puertas and Dolz, 2002, 2005; and Castillo and Carrillo, 2011, 2012, 2013).

Nevertheless, the hydraulic potential of scour (actions due to the two-phase turbulent jets) has not been sufficiently analysed. Further studies in models (physical and numerical) and in prototypes should be done simultaneously characterising pressures, velocity and air entrainment.

In this Thesis, an investigation has been carried out about the localisation and the quantification of the residual energy of the incident jet during the overflow of concrete dams. During the research, technology experimental and numerical, was used to define 
the trajectory of the falling jet, characterize the impact zone, determine the energy dissipation in the air due to aeration and spray phenomena, the dissipation energy for diffusion phenomena, and the impact of the jet on the stilling basin. 


\section{OBJETIVOS}

El objetivo principal de la Tesis es mejorar la caracterización hidrodinámica de las acciones actuantes en el cuenco de disipación de energía de un aliviadero de vertido libre a partir de medidas de laboratorio, y apoyándonos en simulaciones numéricas de Dinámica de Fluidos Computacional (CFD).

Con tal fin, se han realizado diversas labores interrelacionadas:

1. Cuantificación de los diferentes índice turbulentos para el caso de vertido por coronación en presas de fábrica, a través de medidas de velocidades y concentraciones de aire, en diferentes situaciones de flujo y alturas de caída.

2. Determinación de la longitud de rotura del chorro en el caso de chorro rectangular o de vertido libre.

3. Mejora de las formulaciones existentes para la cuantificación de las acciones hidrodinámicas (media, fluctuante y extremas) en la zona de impacto del chorro.

4. Análisis del flujo en el seno del cuenco de amortiguación.

5. Calibración, contraste, simulación y validación de modelos numéricos de tipo Euleriano (CFD).

Los objetivos específicos marcados son:

- Medición, análisis y tratamiento de velocidades y tasas de aireación en distintas secciones del chorro vertiente.

- Medición, análisis y tratamiento de presiones instantáneas en la solera del cuenco de amortiguación.

- Medición, análisis y tratamiento del campo de velocidades instantáneas y energía cinética en el cuenco de disipación.

- Medición, análisis y tratamiento de la geometría del conjunto chorro + cuenco de disipación.

- Validar, actualizar y mejorar la metodología empírica existente.

Los datos de laboratorio se compararan con simulaciones numéricas, empleando dos programas comerciales de CFD con diferente enfoque:

- ANSYS CFX, basado en esquemas de volúmenes finitos.

- FLOW-3D, basado en esquemas de diferencias finitas. 


\section{ESTADO DEL ARTE}

\subsection{Sobrevertido de presas}

Como una primera aproximación, el sobrevertido de una presa se puede analizar como el paso del flujo sobre un vertedero de cresta ancha en descarga libre (Wahl et al., 2008), tal y como se observa en la Figura 1.

Un vertedero de cresta ancha es una estructura de flujo superior con una cresta horizontal sobre la cual las líneas de corriente son prácticamente rectas paralelas. Para obtener esta situación, la longitud de la cresta del vertedero en la dirección del flujo , $L$, debe estar relacionada con la carga de energía total sobre la cresta del vertedero, $h$, cumpliendo las siguientes relaciones:

- $h / L \leq 15$. Para anchos mayores, las pérdidas de energía sobre el vertedero no son despreciables.

- $L / h \geq 2 / 3$. De este modo existe una suave curvatura de las líneas de corriente sobre la cresta, pudiendo asumirse distribución de presiones hidrostática al menos en una corta distancia.

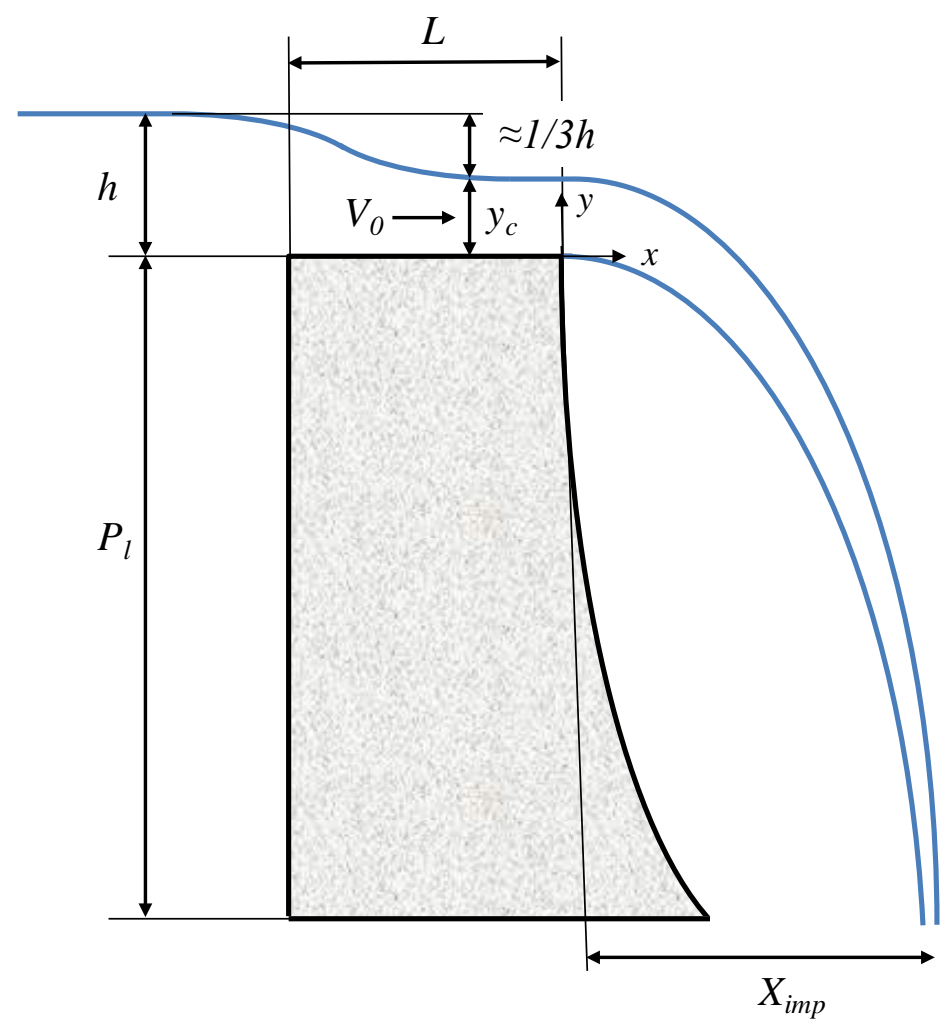

Figura 1. Sobrevertido por coronación actuando como vertedero de pared gruesa. 
Si la coronación de la presa es suficientemente ancha, existirá un punto en el que el flujo presenta condiciones críticas, donde se alcanza la velocidad crítica $V_{c}$ :

$$
V=V_{c}=\sqrt{g y_{c}}
$$

siendo $y_{c}$ el calado crítico. Aplicando la ecuación de Bernoulli entre una sección aguas arriba de la coronación y la sección de calado crítico sobre la coronación, y despreciando las pérdidas, se tiene:

$$
\frac{V_{1}^{2}}{2 g}+h+P_{l}=\frac{V_{c}^{2}}{2 g}+y_{c}+P_{l}
$$

donde $P_{l}$ es la altura de la presa.

Sustituyendo $V_{c}$, el calado crítico queda:

$$
y_{c} \cong \frac{2}{3}\left(h+\frac{V_{1}^{2}}{2 g}\right)
$$

Despreciando la carga de velocidad en la sección situada aguas arriba, se tiene:

$$
y_{c} \cong \frac{2}{3} h
$$

Cuando la cresta de la presa es más estrecha que $3 h_{\text {sobrevertido, la situación tiende }}$ hacia el caso de vertedero de cresta delgada (Wahl et al., 2008).

Esta condición se cumple cuando la condición $h / L>15$. En laboratorio, la longitud de la cresta en la dirección del flujo debe ser menor o igual de $0.002 \mathrm{~m}$, con lo cual no se produce adherencia de la lámina a su paso por el vertedero, formándose una zona aireada incluso con una carga mínima de $0.03 \mathrm{~m}$.

En este tipo de vertederos, el chorro de sobrevertido no está en contacto con el cuerpo del mismo, produciéndose una zona de aire bajo la lámina vertiente. Si la zona inferior no está suficientemente aireada, se crea una subpresión que genera que el chorro se adhiera a la pared delgada e incrementa la curvatura de la lámina, generando un incremento del coeficiente de descarga.

La Figura 2 muestra el perfil de la lámina de agua sobre un vertedero de pared delgada completamente aireado correspondiente a los resultados obtenidos por Bazin (1896) y Scimemi (1930), citados por Bos (1989). Se observa que el calado sobre el labio es superior al crítico (línea $O D=0.85 h$ ), alcanzándose el calado crítico aguas abajo del labio del aliviadero, concretamente en el punto de mayor cota (línea $C E=$ 0.65h). 


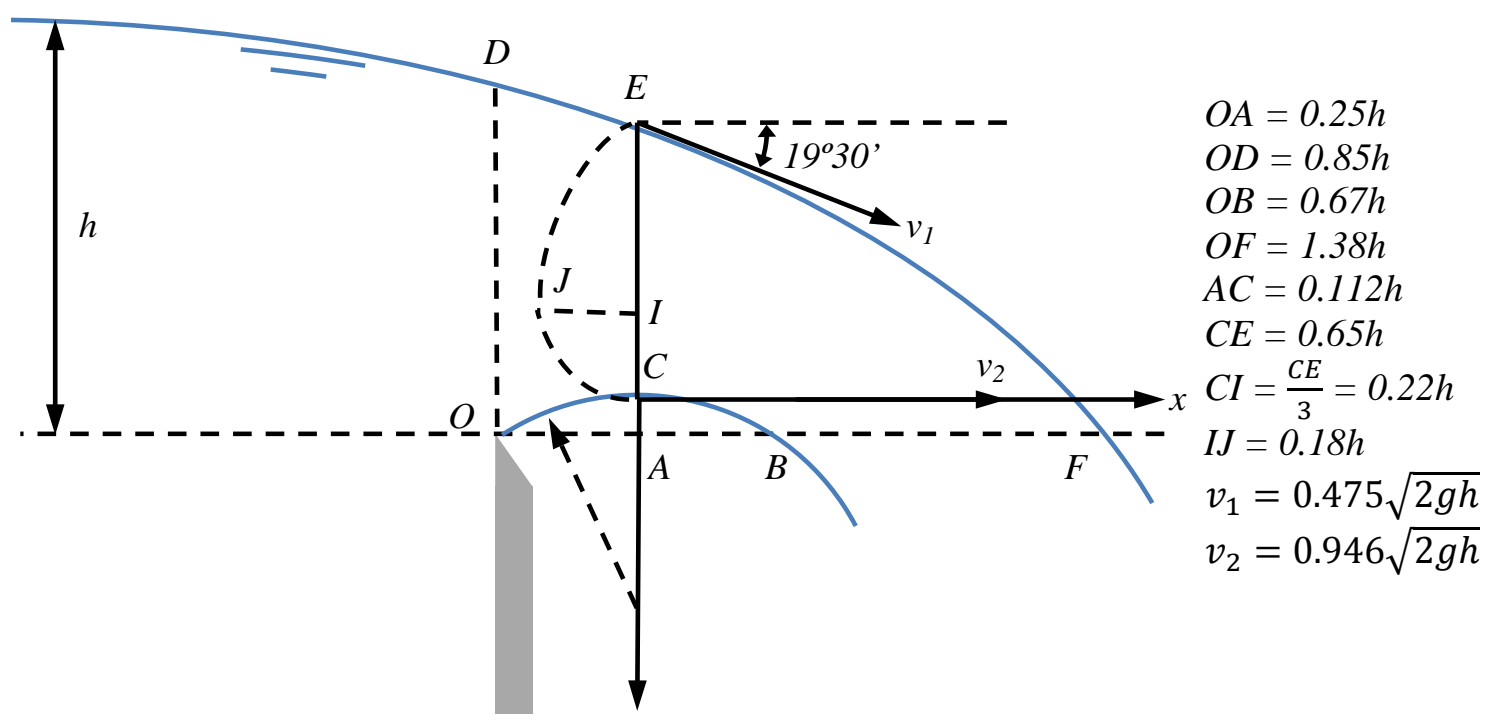

Figura 2. Perfil de la lámina de agua completamente aireada en un vertedero bidimensional (Bazin 1896; Scimemi, 1930, obtenido de Bos, 1989).

Tras el paso por el vertedero se produce una ligera ascensión para descender posteriormente hacia el cuenco, alcanzándose el máximo aproximadamente a una sobreelevación de $0.112 \mathrm{~h}$ y una distancia horizontal de $0.25 \mathrm{~h}$, lo que concuerda con los resultados de Creager (1917). En el punto de máxima cota se obtiene el calado equivalente al crítico (línea $C E=0.65 h$ ).

La forma aproximada de la cara inferior de la lámina define la geometría de un aliviadero de perfil hidrodinámico. De este modo, la forma de esta curva ha sido modelada mediante dos arcos de circunferencias en el perfil obtenido por el USBR.

\subsection{Características de la lámina vertiente}

\subsubsection{Trayectoria de la lámina vertiente}

En el caso de que la coronación de la presa sea suficientemente ancha, la trayectoria de la lámina se puede obtener con las fórmulas de lanzamiento usadas en aliviaderos de canales abiertos. El USBR considera la siguiente formulación para el caso de sobrevertidos por coronación en distintas ediciones de su libro Small Dams (2007):

$$
y=x \tan \theta-\frac{x^{2}}{4 K\left(d+h_{v}\right) \cos ^{2} \theta}
$$


donde $\theta$ es el ángulo del lanzamiento, $d$ es el calado del flujo, $h_{v}$ es la carga de velocidad (carga sobre vertedero $h=d+h_{v}$ ) y $K$ es un factor de ajuste con valor menor o igual a 1.0 (habitualmente se sugiere entre 0.75 y 0.90 ) para el cálculo de la trayectoria real del chorro de sobrevertido.

Según Wahl et al. (2008), la obtención de esta fórmula no está bien documentada. Al comparar la ecuación (5) con la formulación de lanzamiento parabólico, se observa que el denominador de la ecuación (5) contiene un término adicional de calado, $d$, por lo que obtiene un lanzamiento más aplanado de lo que teóricamente es posible. El calado $d$ no está claramente identificado por lo que puede llevar a confusión. Esto puede conducir a subestimar el potencial erosivo del chorro cerca de la estructura o a sobreestimar la zona que requiere protección frente erosión. El error cometido al considerar $d+h_{v}$ en vez de $h_{v}$ es $\left(2+F^{2}\right) / F^{2}$, siendo $F$ el número de Froude. La Figura 3 muestra el error cometido en función del número de Froude.

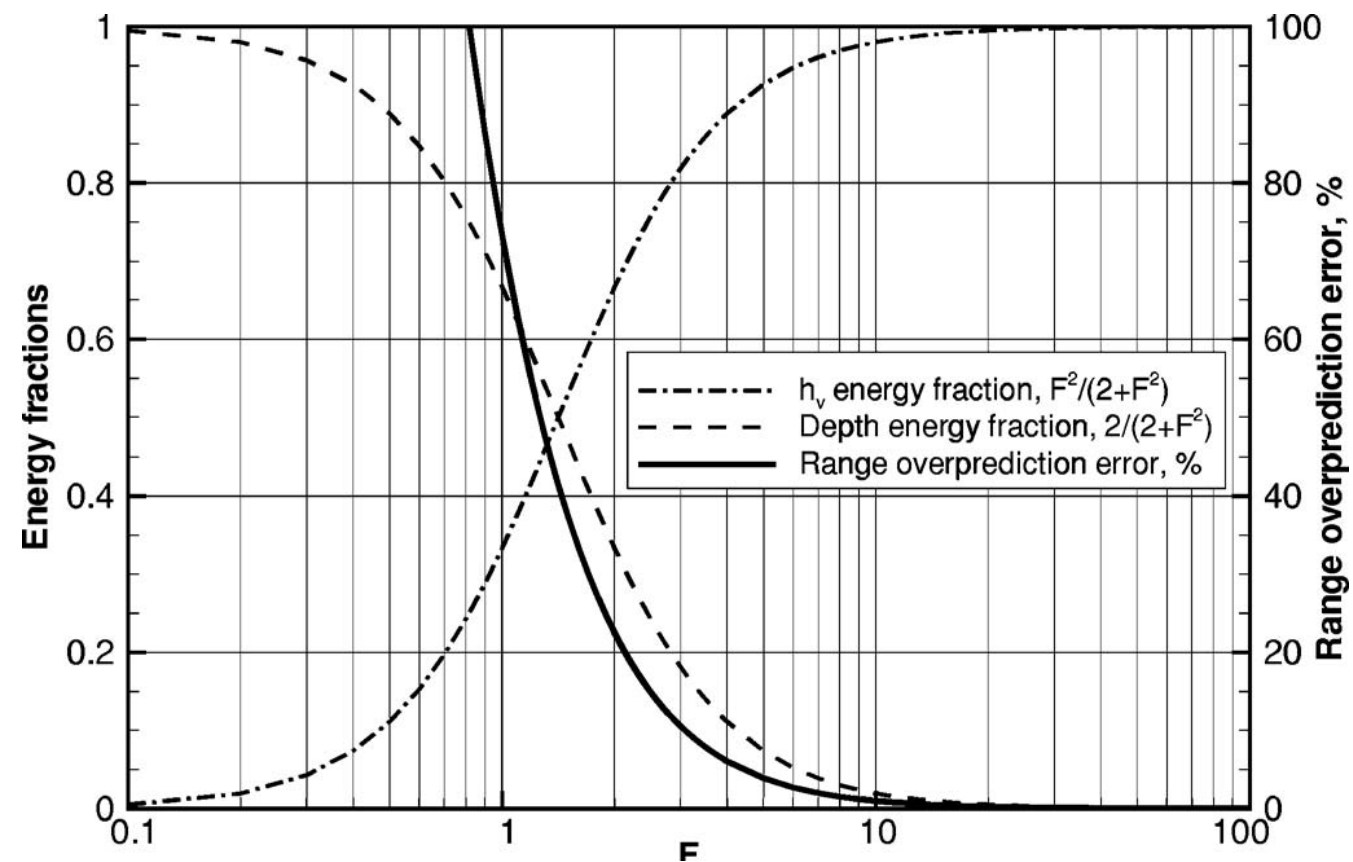

Figura 3. Error en la estimación de la trayectoria del chorro vertiente en función del número de Froude (Wahl et al., 2008).

La distancia de lanzamiento obtenida con la ecuación (5) sobreestima la longitud del alcance entre el 10 y el $70 \%$ cuando se tienen números de Froude de 4 y 1 , respectivamente. De este modo, el error es muy grande para números de Froude bajos, y llega a ser importante hasta valores de Froude de 10. La ecuación (5) parece haber sido desarrollada para flujos con grandes números de Froude, donde el error es relativamente pequeño. En estas condiciones, podría haber sido difícil descubrir el error en los experimentos realizados debido a los efectos de la aireación, la expansión lateral, 
la resistencia del aire y las dificultades existentes para realizar una medición precisa de la trayectoria (Wahl et al., 2008).

En la práctica, se puede emplear la ecuación (5) utilizando un factor $K$ inferior a la unidad para obtener un resultado concordante a la formulación de lanzamiento parabólico, aunque el significado físico del parámetro $K$ nunca se ha establecido. Annandale (2006) propone un valor de $K=0.75$. Según Wahl et al. (2008), el mejor resultado se obtiene con $K=0.652$, puesto que la carga de velocidad sobre un vertedero grueso es $0.652 \mathrm{~h}$ asumiendo que no se producen pérdidas. Cuando se emplean las ecuaciones correctas de la trayectoria, el factor $K$ debería estar próximo a la unidad.

En caso de considerar un aliviadero de pared delgada sin contracciones laterales, se pueden aplicar diferentes expresiones. La formulación de Scimemi (1930), citado por Castillo (1989), es una de las más conocidas, siendo válida siempre que no existan efectos de escala:

- Borde inferior:

$$
\frac{y}{h}=\left(\frac{\frac{x}{h}-0.10}{1.550}\right)^{2}+0.062 \frac{x}{h}-0.186 \text { para } \frac{x}{h}>0.50
$$

- Eje de la lámina:

$$
\frac{y}{h}=\left(\frac{\frac{x}{h}+1.00}{2.155}\right)^{2.33}-1 \text { para } \frac{x}{h}>2.00
$$

- Borde superior:

$$
\frac{y}{h}=\left(\frac{\frac{x}{h}-0.70}{1.420}\right)^{2} \quad \text { para } \frac{x}{h}>1.40
$$

donde $x$ es el eje horizontal con sentido positivo en la dirección del flujo e $y$ el eje vertical orientado hacia abajo y origen en el labio del vertedero (Figura 4).

Creager (1917) propone otra formulación para el eje central de la lámina vertiente:

$$
\left(\frac{x^{\prime}}{h}\right)^{2}=2.732 \frac{y^{\prime}}{h}
$$


donde el eje $x^{\prime}$ está desplazado una distancia 0.056h hacia aguas arriba, mientras que el eje $y^{\prime}$ se desplaza una distancia 0.344 h sobre el umbral del vertedero (Figura 4).

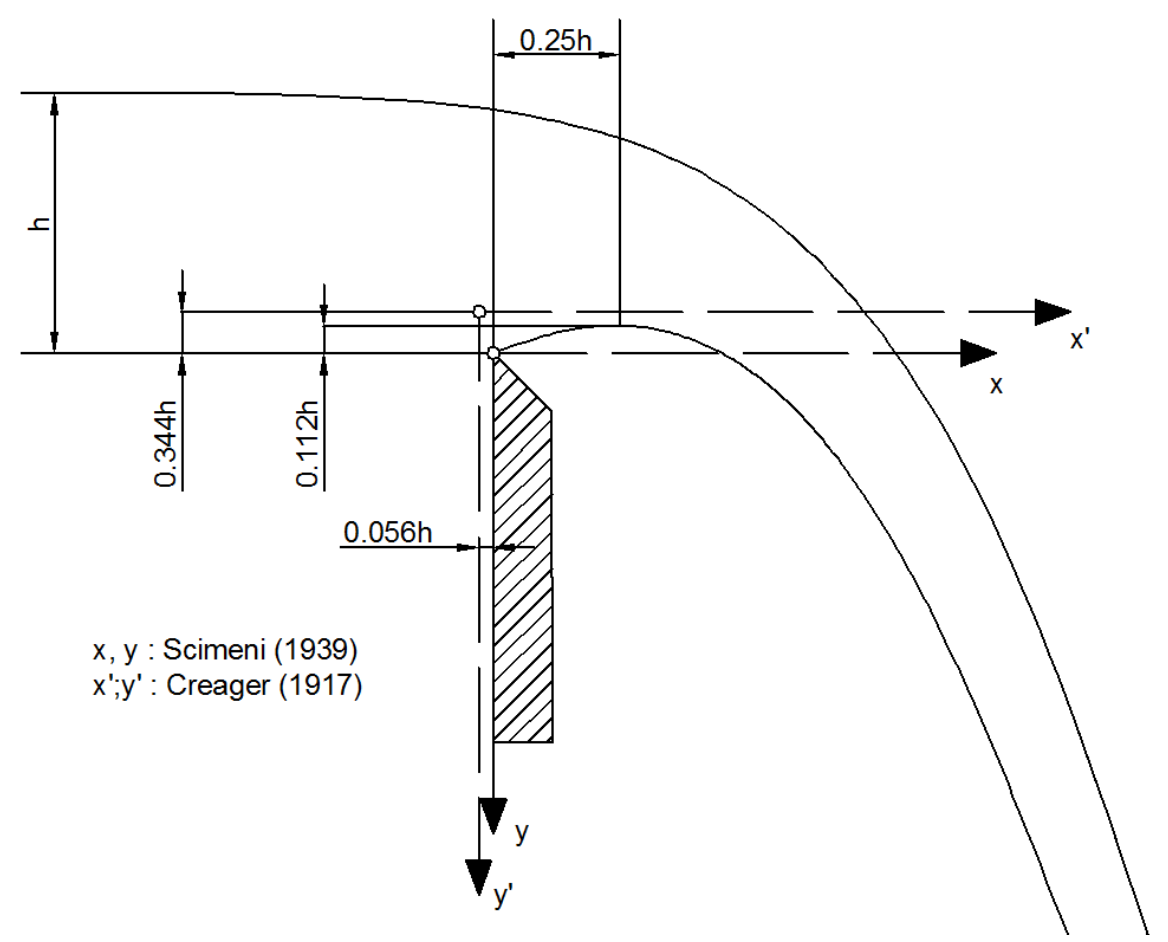

Figura 4. Descripción de la lámina vertiente y notación empleada.

\subsubsection{Aireación de la lámina}

El perfil teórico de la lámina se ve afectado por fenómenos en los que la turbulencia del chorro y la tensión superficial tienen una gran importancia, sin olvidar el efecto generado por el viento. Durante la caída se produce la aireación del chorro, lo que origina una disminución del núcleo central compacto al tiempo que aumenta la fracción de agua atomizada.

En la caída del chorro se producen dos efectos opuestos. Por un lado se obtiene estabilidad por efecto de la contracción debida a la gravedad. Por otro lado, la turbulencia interna contrarresta la contracción, generando una expansión lateral que distorsiona el borde del chorro, pudiendo producir su disgregación y rotura.

Ervine y Falvey (1987) realizaron el estudio de chorros circulares turbulentos aireados y no aireados. Sus principales resultados se resumen en la Figura 5. 


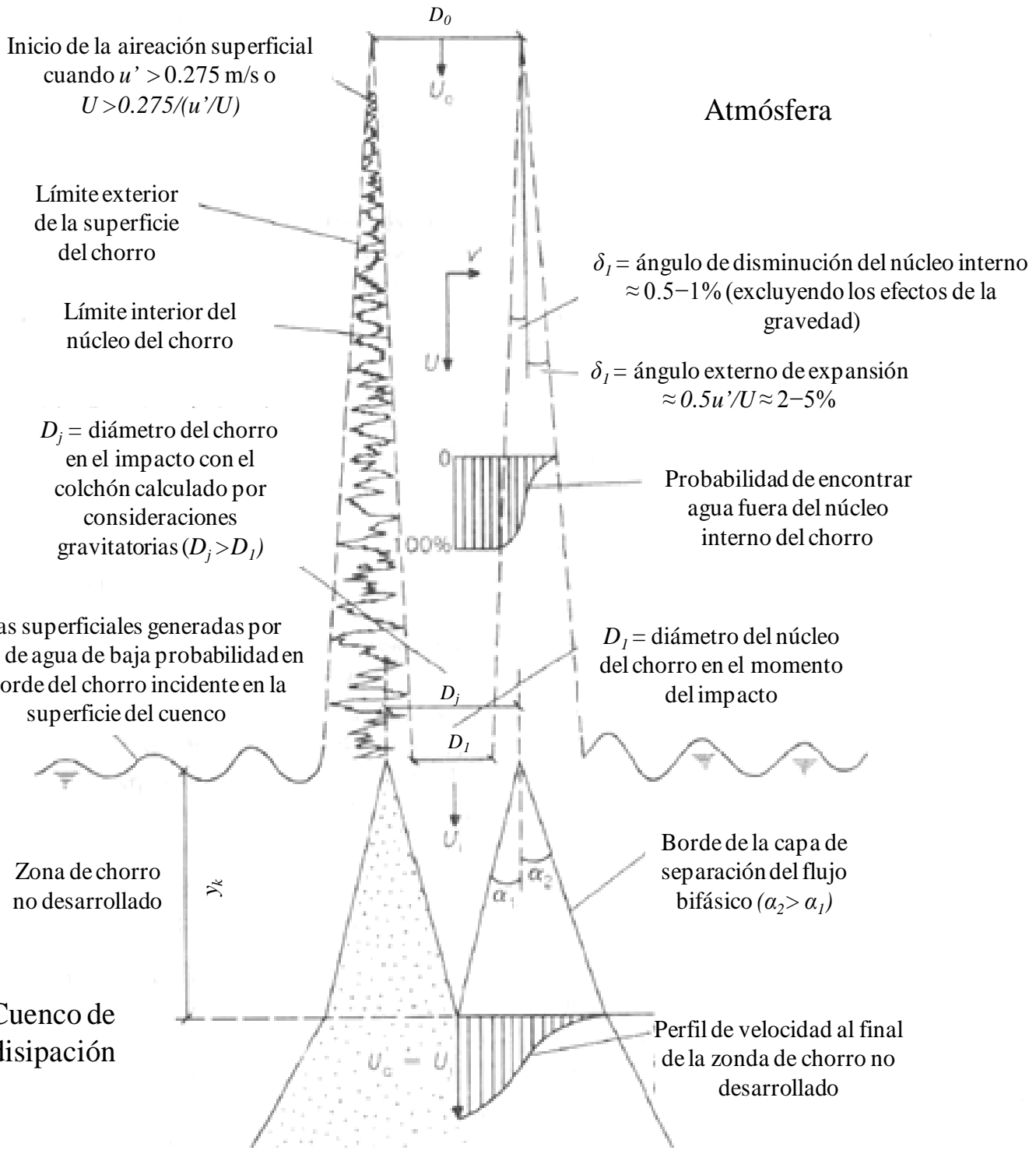

Figura 5. Chorro en la atmósfera y en el cuenco de disipación (Ervine y Falvey, 1987).

Castillo (1989) considera que el vuelo del chorro se puede dividir en cinco fases diferenciadas:

- Chorro turbulento no aireado.

Se produce una caída libre del agua con una intensidad de turbulencia inicial que depende de las condiciones en el vertedero y de la forma de aproximación al mismo, hasta el momento en que se inicia la aireación del chorro.

- Aireación del chorro.

La velocidad relativa entre el chorro turbulento y el aire provoca que la turbulencia aumente. Llega un momento en que los esfuerzos provocados por la turbulencia en la superficie del chorro vencen a la tensión superficial, produciéndose la rotura de la superficie, lo que permite que se introduzcan 
burbujas de aire. Ervine y Falvey (1987) y Ramos (1988) consideran que este fenómeno se inicia cuando el chorro alcanza una velocidad de $5.5-7.0 \mathrm{~m} / \mathrm{s}$ en condiciones de turbulencia inicial baja (4-5\%). Con el fin de favorecer la disipación de energía en el chorro vertiente, se puede acelerar el proceso de aireación y atomización aumentando la turbulencia por métodos artificiales (CNEGP, 1997).

- Chorro turbulento aireado.

Aumenta la velocidad del chorro a la vez que se produce el desprendimiento de partículas de agua de un modo esporádico. Las gotas separadas son frenadas por el rozamiento del aire hasta alcanzar su velocidad de caída límite. El efecto de introducción del aire en el chorro apenas elimina energía al flujo.

- Atomización del chorro.

Aumenta la zona aireada y atomizada a medida que el chorro desciende, mientras la zona no aireada situada en la parte central de la lámina va disminuyendo hasta desaparecer. Las partículas de agua cercanas a la superficie se van desprendiendo de un modo continuado. Se considera que se ha alcanzado la atomización de la lámina cuando una parte importante del chorro se ha separado del núcleo del mismo. Según Ervine y Falvey (1987), este proceso se produce cuando el chorro alcanza una velocidad del orden de $20-30 \mathrm{~m} / \mathrm{s}$.

- Chorro atomizado.

El chorro está formado por grandes gotas de agua que se van disgregando en gotas más pequeñas debido al rozamiento con la atmósfera.

La intensidad de turbulencia en las condiciones iniciales $T_{u}$, es el parámetro que gobierna los efectos de la turbulencia en el chorro, la cual se puede definir como:

$$
T_{u}=\bar{V}^{\prime} / U
$$

donde $\bar{V}^{\prime}$ es la raíz cuadrática media de la velocidad transversal y $U$ es la velocidad media en el eje del chorro.

La velocidad del chorro sin pérdidas de energía se puede obtener a partir de las condiciones gravitatorias:

$$
v_{j}=\sqrt{v_{0}^{2}+2 g H}
$$

donde $v_{0}$ es la velocidad inicial y $H$ es la altura de caída.

Lewis (1996), citado por Bollaert (2002), considerando el arrastre aerodinámico determina la velocidad del chorro una vez que se ha producido la rotura: 


$$
v_{j}=\sqrt{v_{0}^{2}+2 g H}-\sqrt{3 C_{d}\left(\frac{\rho_{i}}{\rho_{w}}\right)\left(\frac{H}{d}\right) v_{0}^{2}}
$$

donde $C_{d}$ es el coeficiente de arrastre ( 0.5 en caso de esferas circulares), $\rho_{i}$ es la densidad de la mezcla y $\rho_{w}$ es la densidad del agua.

Las condiciones de desintegración del chorro circular han sido estudiadas por Ervine y Falvey (1987) y por Ervine et al. (1997). Obtienen que la intensidad de la turbulencia inicial es el parámetro más importante en la longitud de rotura debido a que determina el grado de expansión lateral del chorro $\delta_{2}$, y por continuidad controla la velocidad de disminución del núcleo interno del chorro $\delta_{1}$ (Figura 6). La turbulencia $T_{u}$ puede relacionarse con el ángulo de la expansión lateral exterior del chorro, la velocidad media del chorro en la salida de la tobera $U_{0}$, y la distancia longitudinal recorrida desde la tobera de salida del chorro $X$, mediante la siguiente expresión:

$$
\delta_{2} / X=0.38 \overline{V^{\prime}} / U_{0}=0.38 T_{u}
$$

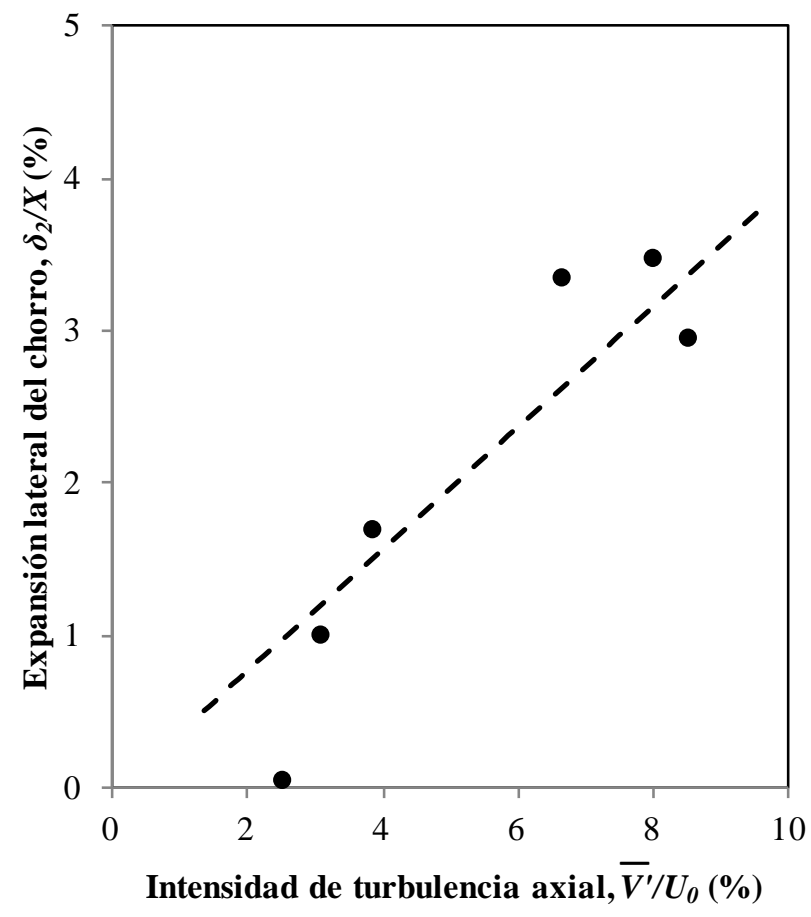

Figura 6. Dependencia de la expansión lateral del chorro con la intensidad de turbulencia actuando en el orificio de salida (Ervine y Falvey, 1987).

De un modo similar, obtienen la tasa de disminución del núcleo interno del chorro $\delta_{1}$. Basándose en las mediciones de la probabilidad de encontrar agua en el borde del 
chorro, consideraron que $\delta_{1}$ puede aproximarse de un modo razonable a un 15-20\% del ángulo de expansión lateral del chorro. Para una intensidad turbulenta entre 5 y 8\%, obtienen los siguientes ángulos:

$$
\frac{\delta_{1}}{X} \cong 0.5-1.0 \% ; \quad \frac{\delta_{2}}{X} \cong 3.0-4.0 \%
$$

De este modo, aunque el chorro parezca desde fuera extremadamente aireado y presente una superficie rugosa, puede existir un núcleo no aireado grande.

Davies (1972) considera que las perturbaciones de la superficie turbulenta aumentan linealmente con la raíz cuadrada de la distancia de caída del chorro $L$. Ervine et al. (1997) confirmaron la suposición de Davies y obtuvieron una formulación para calcular la expansión lateral del chorro $\xi$, y el diámetro del chorro no aireado debido a condiciones gravitatorias $D_{c}$ :

$$
\begin{gathered}
\xi=\frac{1.14 T_{u} U^{2}}{g}\left[\sqrt{\frac{2 L}{D_{0} F_{0}^{2}}+1}-1\right] \\
D_{c}=D_{0} \sqrt{\frac{U_{0}}{U}}
\end{gathered}
$$

donde $g$ es la aceleración de la gravedad, $L$ es la distancia de caída, $U_{0}$ es la velocidad a la salida de la tobera, $D_{0}$ es el diámetro del chorro en la salida de la tobera y $U$ es la velocidad en un determinado punto. $F_{0}$ es el número de Froude en la tobera obtenido como:

$$
F_{0}=\frac{U_{0}}{\sqrt{g D_{0}}}
$$

De este modo, la expansión lateral del chorro $\xi$, se produce a una velocidad que depende de la intensidad de turbulencia inicial. Las partículas del chorro que se mueven perpendicularmente a la dirección del flujo debido a la energía cinética lateral tienden a superar la restricción impuesta por la tensión superficial.

Asumiendo una turbulencia isotrópica, se puede obtener una estimación de las condiciones a cumplir para que la partícula de agua salga de la superficie libre aireada (Ervine y Falvey, 1987):

$$
U=\frac{0.275}{\bar{V}^{\prime} / U}=\frac{0.275}{T_{u}}
$$


donde $U$ es la velocidad del chorro a la que se inicia la aireación.

Esta expresión no es estrictamente exacta puesto que se basa en la energía cinética total de las fluctuaciones turbulentas. Falvey y Ervine (1988) señalan que un análisis adecuado debería tener en cuenta únicamente la energía turbulenta contenida en el rango de tamaños de burbujas de aire arrastradas por el chorro.

Castillo (2006), considera que puede obtenerse el espesor de un chorro rectangular $B_{j}$, aplicando la siguiente expresión:

$$
B_{j}=B_{g}+B_{s}=B_{g}+2 \xi
$$

donde $B_{g}$ es el ancho por condiciones gravitatorias, obtenido como:

$$
B_{g}=\frac{q}{\sqrt{2 g H}}
$$

mientras que $B_{s}=2 \xi$ es el ancho por expansión lateral del chorro en la atmósfera, el cual se puede obtener como:

$$
\xi=2 \varphi \sqrt{h_{0}}\left[\sqrt{H}-\sqrt{h_{0}}\right]
$$

siendo $\varphi=k_{\varphi} T_{u}{ }^{*}$ el parámetro turbulento en la condición inicial, considerada a una distancia vertical $h$ bajo el umbral del vertedero (Figura 7). $h_{0}=2 h$ es el doble de la carga sobre vertedero $h$, y $H$ es la diferencia de alturas entre la lámina de agua en el embalse y en el cuenco de disipación. $T_{u}{ }^{*}$ es la intensidad de turbulencia definida en las condiciones iniciales. $k_{\varphi}=\bar{V}^{\prime} / w^{\prime}$ es el coeficiente del parámetro turbulento, donde $w^{\prime}$ es la velocidad turbulenta vertical.

Para caudales de laboratorio $\left(q<0.25 \mathrm{~m}^{2} / \mathrm{s}\right)$, propone:

$$
T_{u}^{*}=\frac{q^{0.43}}{I C}
$$

siendo IC las condiciones iniciales de vertido con dimensiones $\left[\mathrm{L}^{0.86} \mathrm{~T}^{-0.43}\right.$, definidas como:

$$
I C=\frac{14.95 g^{0.50}}{K^{1.22} C_{d}^{0.19}}
$$

donde $K \approx 0.85$ es un parámetro adimensional y $C_{d} \approx 2.1$ es el coeficiente de descarga del aliviadero de perfil hidrodinámico de dimensiones $\left[\mathrm{L}^{0.5} \mathrm{~T}^{-1}\right]$. 


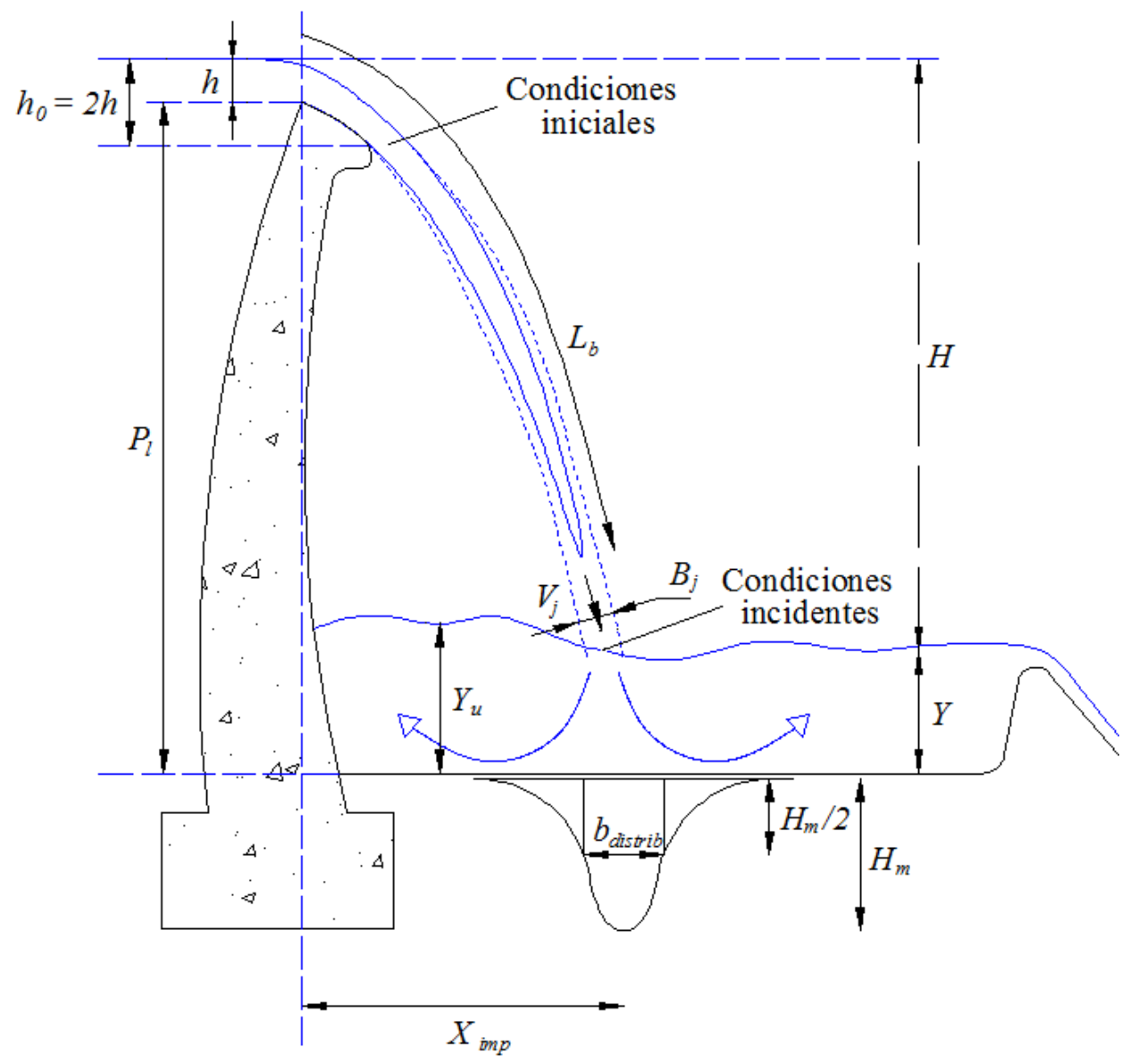

Figura 7. Aliviaderos en vertido libre de presas bóveda (adaptado de Castillo, 1989).

Ervine y Falvey (1987) obtuvieron las relaciones entre las componentes de la velocidad turbulenta transversal en chorros circulares aireados y unidimensionales como $u^{\prime}=w^{\prime}, y u^{\prime}=0.38 v^{\prime}$. De este modo, la raíz cuadrática media (RMS) de la componente turbulenta en la línea de corriente puede obtenerse como:

$$
\overline{v^{\prime}}=\sqrt{u^{\prime 2}+v^{\prime 2}+w^{\prime 2}}=1.14 v^{\prime}
$$

La expansión lateral queda:

$$
\xi=k T_{u} V_{i} t=1.14 v^{\prime} t
$$

Krothapalli et al. (1981) midieron las velocidades turbulentas transversales en un chorro bidimensional de aire (Figura 8). En la proximidad de la tobera de salida, las componentes máximas turbulentas fueron $u^{\prime} \approx 0.95 v^{\prime}, \mathrm{y} w^{\prime} \approx 0.78 v^{\prime}$, quedando la raíz cuadrática media de la componente turbulenta en la línea de corriente como: 


$$
\overline{v^{\prime}}=\sqrt{u^{\prime 2}+v^{\prime 2}+w^{\prime 2}}=1.59 v^{\prime}
$$

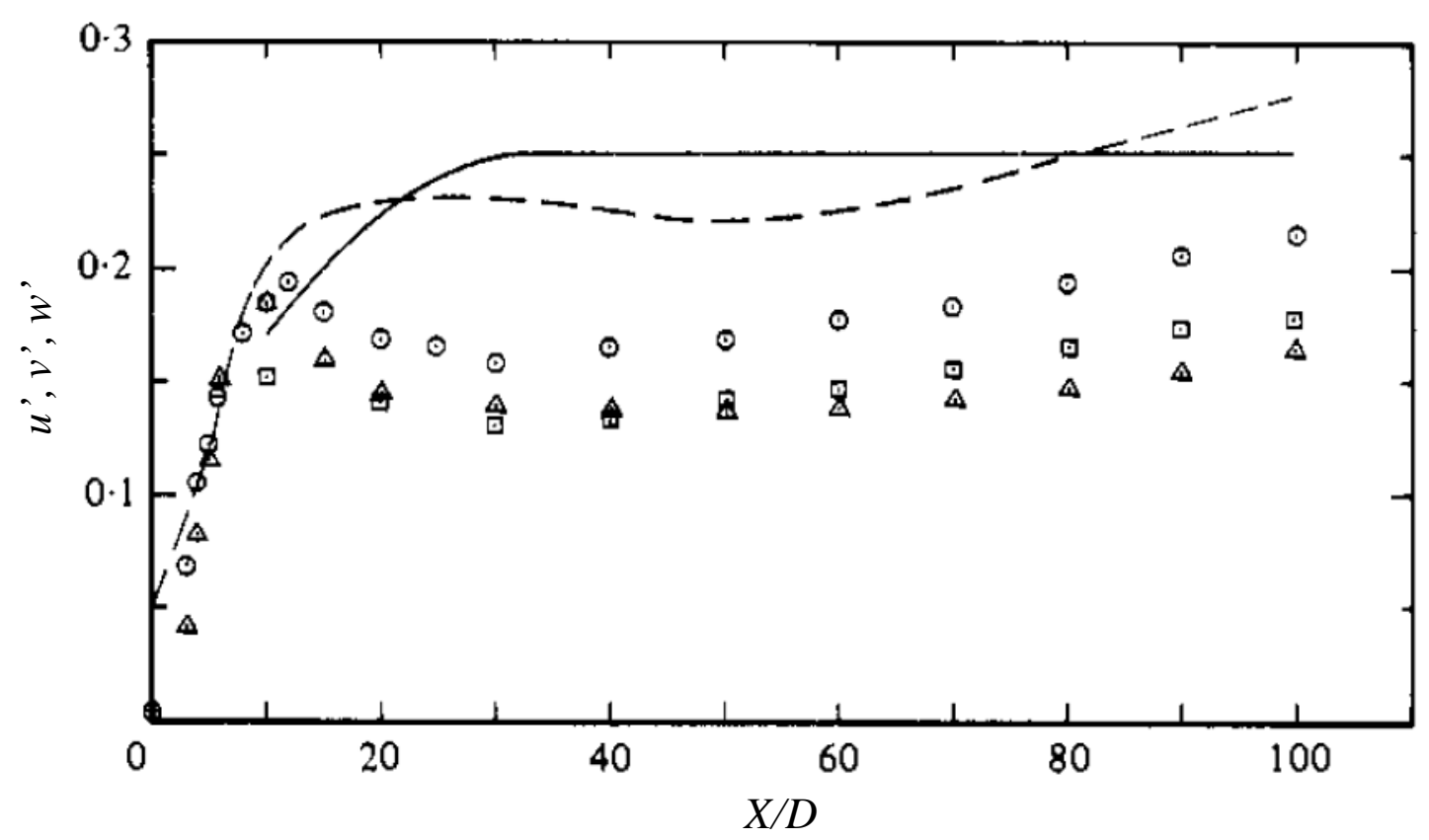

Figura 8. Variación de las intensidades turbulentas a lo largo de la línea central del chorro: $\square$, $u^{\prime} ; \circ, v^{\prime} ; \Delta, w^{\prime} ;--, v^{\prime}, A R=10$, Sfeir (1976); —, v', AR = 38, Gutmark y Wygnanski (1976). (Krothapalli et al., 1981).

Sin embargo, el chorro analizado por los autores fue sobre un eje unidimensional y no consideró el flujo bifásico aire-agua.

En el caso particular de chorros bifásicos rectangulares, el chorro es aireado y tridimensional. Por este motivo, la raíz cuadrática media de la velocidad debería están en algún lugar entre $1.14 v^{\prime}$ y $1.59 v^{\prime}$. Castillo y Carrillo (2013), apoyándose en simulaciones numéricas, consideran que $k_{\varphi} \rightarrow 1.24$ en el caso de flujo tridimensional.

\subsubsection{Longitud de rotura}

En el caso de que el chorro tenga suficiente distancia de caída, las perturbaciones turbulentas de la superficie pueden ser suficientemente grandes como para penetrar en el núcleo compacto, e incluso llegar a desaparecer. La longitud de rotura $L_{b}$ es la distancia a partir de la cual se ha producido la disgregación completa del chorro, desapareciendo el núcleo compacto.

Ervine y Falvey (1987) analizaron chorros circulares obtenidos con una tobera de $25 \mathrm{~mm}$ de diámetro e intensidades turbulentas entre 0.3 y $8.0 \%$. En los resultados 
mostrados Figura 9 se observa que la longitud de rotura varía hasta 4 veces en función de la turbulencia inicial (de una relación $L_{b} / D \approx 50$ para $T_{u}=0.08$ hasta una relación $L_{b} / D \approx 220$ para $\left.T_{u}=0.003\right)$.

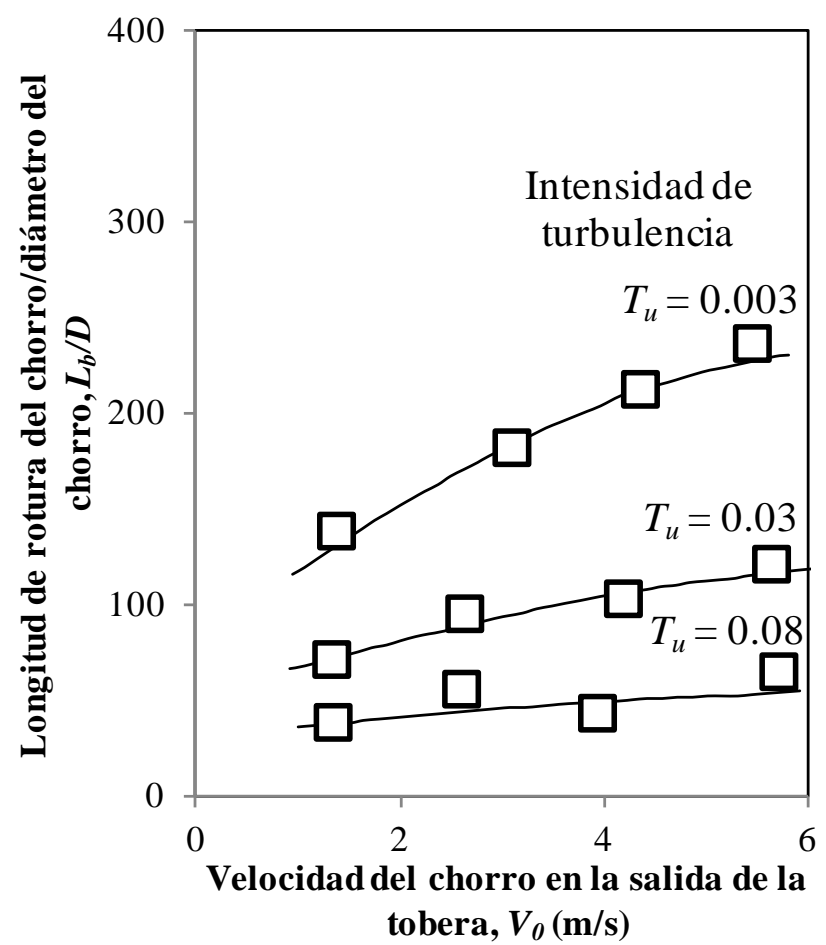

Figura 9. Variación de la longitud de rotura para distintas intensidades turbulentas iniciales. Chorro circular con una tobera de 25 mm de diámetro (Ervine y Falvey, 1987).

Basándose en la expansión lateral del chorro debida a la turbulencia inicial de la ecuación (13), y en la contracción del chorro debida a la gravedad, Ervine et al. (1997) desarrollan una expresión teórica para obtener la longitud de rotura de chorros circulares con diferentes $T_{u}$ :

$$
C^{2}=\frac{1}{\sqrt{\frac{2 L_{b}}{D_{i} F^{2}}+1}\left(\sqrt{\frac{2 L_{b}}{D_{i} F^{2}}+1}-1\right)^{2}}
$$

donde $C$ es el parámetro turbulento definido como:

$$
C=1.14 T_{u} F^{2}
$$

Los resultados de esta fórmula casan con los obtenidos en chorros circulares a escala de laboratorio por Baron (1949) con una turbulencia estimada del 3\%, McKeogh 
(1978) con una turbulencia de 0.3\% y Withers (1991) con diferentes niveles de turbulencia.

La Tabla 1 recoge distintas formulaciones obtenidas para chorros planos y circulares, donde las variables $W_{e}, R_{e}$ y $F$ hacen referencia a los números de Weber, Reynolds y Froude al inicio del chorro, respectivamente. $Q$ es el caudal de descarga en $\mathrm{m}^{3} / \mathrm{s}$ y $q$ es el caudal específico en $\mathrm{m}^{2} / \mathrm{s}, D_{i}$ y $D_{j}$ son los diámetros en la condición inicial y en el punto de impacto con el cuenco de amortiguación, y $B_{i}$ es el espesor del chorro en la condición inicial.

\begin{tabular}{|l|c|c|l|}
\hline \multicolumn{1}{|c|}{ Tipo de chorro } & $\boldsymbol{L}_{\boldsymbol{b}}$ & $\boldsymbol{T}_{\boldsymbol{u}}$ & \multicolumn{1}{|c|}{ Autor } \\
\hline Chorro circular & $1.7 \frac{W_{e}}{\left(10^{-4} R_{e}\right)^{\frac{5}{8}}}$ & $3.0 \%$ & Baron (1949) \\
\hline Lámina rectangular & $6 q^{0.39}$ & - & Horeni (1956) \\
\hline Chorro circular & $60 Q^{0.39}$ & $0.3 \%$ & \\
& $17.4 Q^{0.31}$ & $3.0 \%$ & Ervine, McKeogh y Elsawy (1980) \\
\hline Chorro circular & $\begin{array}{c}4.1 Q^{0.20} \\
(50-100) D_{j}\end{array}$ & $\begin{array}{c}3.0-8.0 \% \\
(200-300) D_{j}\end{array}$ & $\begin{array}{l}0.003 \% \\
\text { Chorvine y Falvey (1987) }\end{array}$ \\
\hline Cámina rectangular & $0.85 \frac{B_{i} F^{2}}{\left(K_{\varphi} T_{u} F^{2}\right)^{0.82}}$ & $<3.0 \%$ & Castillo (2006) \\
\hline
\end{tabular}

Tabla 1. Expresiones de longitud de rotura para chorro rectangular y circular.

El análisis dimensional muestra que el arrastre de aire de un chorro en la atmósfera se rige por la gravedad, la tensión superficial y los efectos de turbulencia inicial, siendo una combinación de fenómeno de Froude, Weber y Reynolds. El correcto estudio en modelo a escala debería simular todos estos fenómenos, lo que resulta imposible.

Según Bollaert (2002), en caso de ser capaces de representar con precisión la intensidad de la turbulencia en prototipo, se podría realizar una simulación adecuada de la entrada de aire en modelos a escala de Froude. Además, el modelo debería ser lo suficientemente grande como para alcanzar la velocidad en que se inicia el arrastre de aire, haciendo que los efectos de tensión superficial sean pequeños. Mientras que estos 
últimos aspectos puede ser obtenidos fácilmente en un modelo físico de tamaño considerable, el contenido espectral de energía de los remolinos turbulentos no se puede escalar (Falvey y Ervine, 1988). Este problema implica que se deben considerar velocidades de chorro de prototipo o cercanas a prototipo con el fin de obtener el contenido espectral del chorro con precisión, lo que representa una gran limitación en la realización de estudios en modelos de laboratorio.

\subsection{Flujo dentro del cuenco}

\subsubsection{Geometría del chorro dentro del colchón}

El estudio de la geometría de los chorros incidentes en un colchón de agua ha sido estudiado por diversos autores.

La entrada de aire en el chorro en la fase de vuelo es de suma importancia puesto que define la geometría del chorro antes del impacto y provoca la entrada de aire en el cuenco de disipación. El diámetro del núcleo no aireado en la zona de impacto influye directamente en la distancia de desarrollo del chorro en el seno del cuenco. El diámetro máximo del chorro aireado define la zona de la solera que estará bajo la influencia directa del impacto del chorro.

La difusión de un chorro bidimensional a través de un medio en reposo se resolvió en primer lugar a partir de ecuaciones básicas de la mecánica de fluidos. El concepto fundamental de un chorro con campo de velocidad uniforme que penetra en un medio en reposo se basa en la expansión progresiva de la interfaz debido al intercambio de cantidad de movimiento, la cual está compuesta de partículas del propio chorro y de partículas del medio circundante.

El fenómeno se caracteriza por un aumento de la sección transversal total del chorro ( $\alpha_{2}$ incrementa el ancho del chorro) a la vez que se reduce la región central no alterada por el medio ( $\alpha_{1}$ reduce el ancho del núcleo). La zona en la que se produce la disminución del núcleo del chorro hasta su total desaparición se denomina región de desarrollo del chorro, mientras que la región de chorro desarrollado o establecido es aquella en la que el núcleo ha desaparecido (Figura 10).

La longitud de la zona de desarrollo $y_{K}$ depende del ángulo interno de difusión $\alpha_{1}$, el cual se ve alterado por la entrada de aire en el chorro y la turbulencia inicial. $\alpha_{1}$ está en torno a $4.5^{\circ}$ para chorros sumergidos, y es aproximadamente $8^{\circ}$ en el caso de chorros de alta turbulencia y aireados, siendo esta última la situación habitual en modelos y prototipos (Ervine y Falvey, 1987). Según Hartung y Häusler (1973), el ángulo de contracción del núcleo compacto es ligeramente más reducido $\left(\alpha_{1} \approx 4-6^{\circ}\right)$. 


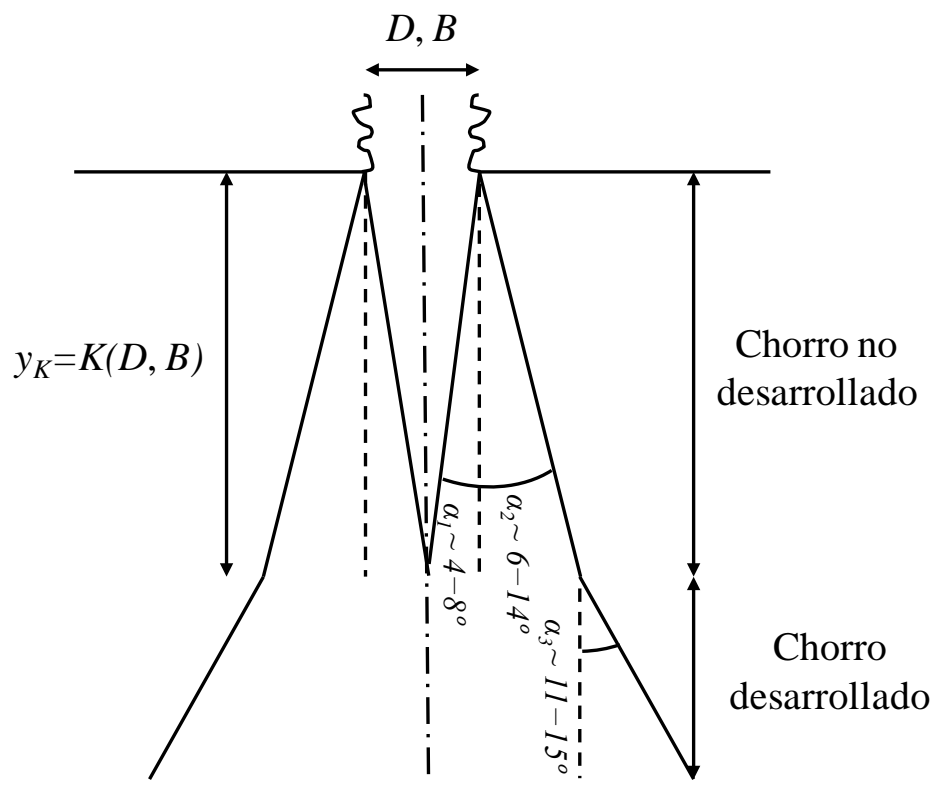

Figura 10. Expansión lateral de un chorro bidimensional (adaptado de Bollaert, 2002).

Suponiendo que existe presión hidrostática, se puede considerar que el núcleo del chorro viaja a una velocidad constante. A lo largo del eje central del chorro en la región no desarrollada, los perfiles de velocidad mantienen la forma cuando se grafican con una escala de longitud adecuada (por ejemplo, la anchura correspondiente a la mitad de la velocidad máxima). Esta característica ha sido utilizada para predecir los campos de velocidad media y presión media.

En la Tabla 2 se presentan los resultados teóricos y experimentales obtenidos por distintos autores sobre la distancia de desarrollo del chorro. La longitud de desarrollo del chorro $y_{K}$ se obtiene como $K$ veces el diámetro del chorro $D$ para chorros circulares o $K$ veces la anchura del chorro $B$ en el caso de chorros rectangulares (Figura 10).

Según esta tabla, la distancia necesaria para que desaparezca el núcleo del chorro está entre 5.2 y 8.3 en caso de chorro rectangular no aireado previamente, mientras que se reduce a entre 3.2 y 6.4 si el chorro rectangular esté previamente aireado.

De acuerdo con la experiencia de Albertson et al. (1950), el análisis cuantitativo de la velocidad de un chorro se hace considerando que la distribución es gausiana en la zona de chorro desarrollado, mientras que en la zona del chorro no alterada por los remolinos se tiene $v_{\text {máx }}=v_{0}$ (Figura 11$)$.

Para la zona de desarrollo del chorro, considera la siguiente expresión:

$$
\frac{v_{x}}{v_{0}}=e^{\frac{-\left(y+\sqrt{\pi} C_{1} \frac{x}{2}-\frac{B}{2}\right)^{2}}{2\left(C_{1} x\right)^{2}}}
$$


donde $v_{0}$ es la velocidad del agua al penetrar en el colchón, $B$ es la anchura inicial del chorro y $C_{1}=\sigma / x$ es un coeficiente que relaciona la desviación típica $\sigma$ con la distancia recorrida.

\begin{tabular}{|l|c|l|l|}
\hline \multicolumn{1}{|c|}{ Autor } & $\boldsymbol{K}$ & \multicolumn{1}{c|}{ Tipo de chorro } & \multicolumn{1}{c|}{ Análisis } \\
\hline Albertson et al. (1950) & 5.2 & Rectangular & Difusión de chorro y experimental \\
& 6.2 & Circular & Difusión de chorro y experimental \\
\hline Homma (1953) & 4.8 & Circular & Experimental \\
\hline Cola (1965) & 7.18 & Rectangular & Leyes de conservación y experimental \\
\hline Poreh y Hefez (1967) & 9 & Circular & Difusión de chorro \\
\hline Hartung y Häusler & 5 & Circular aireado & Estimación del ángulo de difusión \\
(1973) & 5 & Rectangular aireado & Estimación del ángulo de difusión \\
\hline Beltaos y Rajaratnam & 8.26 & Rectangular & Cantidad de movimiento del chorro \\
(1973, 1974) & $5.8-7.4$ & Circular & Cantidad de movimiento del chorro \\
\hline Franzetti y Tanda (1984) & 4.7 & Circular aireado & Difusión de chorro y experimental \\
& 6.03 & Circular & Difusión de chorro y experimental \\
\hline Chee y Yuen (1985) & 3.3 & Circular aireado & Análisis dimensional \\
\hline Cui Guang Tao et al. & 6.35 & Rectangular aireado & Basado en Aki (1969) y experimental \\
$(1985)$ & & & \\
\hline Ervine y Falvey (1987) & 4 & Circular aireado & Cantidad de movimiento y experimental \\
& 6.2 & Circular & Experimental \\
\hline Castillo (1989) & 7.2 & Rectangular aireado & Experimental \\
\hline Armengou (1991) & 3.19 & Rectangular aireado & Experimental \\
\hline Bormann y Julien (1991) & 3.24 & Rectangular aireado & Coeficiente de difusión del chorro \\
\hline Ervine et al. (1997) & $4-5$ & Circular aireado & Experimental \\
\hline Castillo (2006) & 4.0 & Rectangular aireado & Experimental \\
\hline Castillo et al. (2014) & 5.5 & Rectangular aireado & Experimental \\
\hline
\end{tabular}

Tabla 2. Coeficiente K de la longitud del núcleo del chorro según diversos autores (Bollaert y Schleiss, 2003). Complementado.

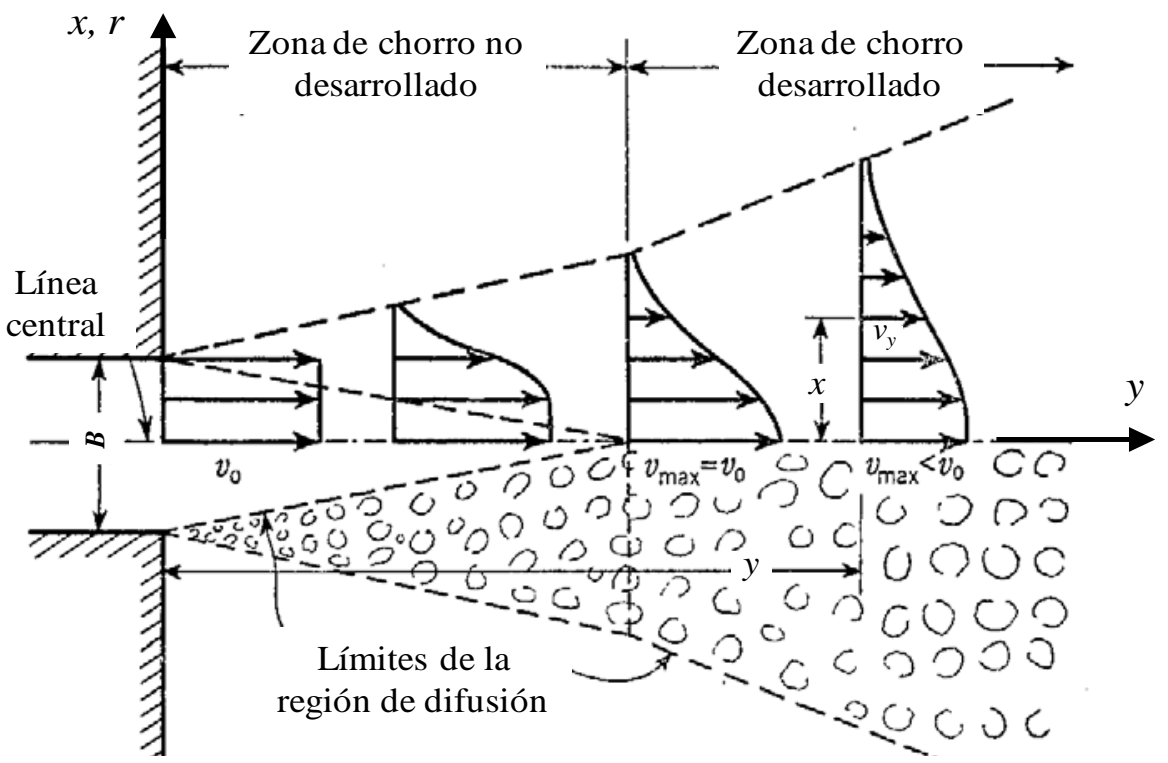

Figura 11. Esquemática de difusión del chorro (adaptado de Albertson et al., 1950). 
La velocidad máxima se obtiene siempre a lo largo del eje del chorro. Mediante ajuste experimental, Albertson et al. obtuvieron un valor de $C_{1} \approx 0.109$ válido para cualquier chorro bidimensional.

Según Hartung y Häusler (1973), al inicio de la zona de flujo desarrollado únicamente se ha disipado un $20 \%$ de la energía inicial en chorros rectangulares y un $30 \%$ en circulares. Para un chorro circular se tiene una disipación del 85\% a una distancia $20 D_{j}$. Sin embargo, según Cola (1965), citado por Hartung y Häusler (1973), en un chorro rectangular se requiere una distancia $40 B_{j}$ para alcanzar una disipación del $70 \%$.

Este análisis fundamental de difusión no tiene en cuenta la existencia de límites que modifican la distribución de la presión hidrostática y hacen que el problema sea dependiente de la gravedad. La existencia de una superficie rígida hace que las expresiones anteriores no tengan una completa validez. A medida que el chorro avanza en el cuenco, la superficie rígida dificulta su descenso provocando que la trayectoria se modifique hacia una dirección horizontal (chorro de pared). El cambio de dirección provoca que la energía cinética se transforma en potencial, originando una sobrepresión en el fondo del cuenco.

Cola (1965), citado por Castillo (1989), experimentalmente obtuvo que los límites influyen en el campo de velocidad uniforme cuando la distancia recorrida por el chorro es $x=0.724 Y$, siendo $Y$ el calado en el colchón, siempre y cuando se cumpla:

$$
Y>5.2 \frac{B_{j}}{\left(1-\eta_{A}\right)}
$$

donde $\eta_{A} \approx 0.276$ un coeficiente experimental. Por tanto, la deflexión del chorro se inicia a una distancia $0.276 Y$ del fondo.

La Figura 12 muestra la evolución de la distribución de presiones y de la velocidad a lo largo de la solera del cuenco de disipación a partir del punto de estancamiento.

Beltaos y Rajaratnam (1973, 1974), y Beltaos (1976) realizaron un estudio completo del impacto de chorros planos y circulares sobre una superficie plana y suave. Calibraron experimentalmente expresiones teóricas obtenidas mediante análisis dimensional, las ecuaciones de conservación y teoría de difusión de chorros bidimensionales. Los autores consideran tres zonas: la región el chorro sin afección, la región de impacto con la superficie y la zona de chorro de pared (Figura 13). La región de impacto representa la zona en la que la superficie de impacto influye en el campo de flujo uniforme del chorro incidente (aproximadamente un 30\% de la profundidad total del agua). 


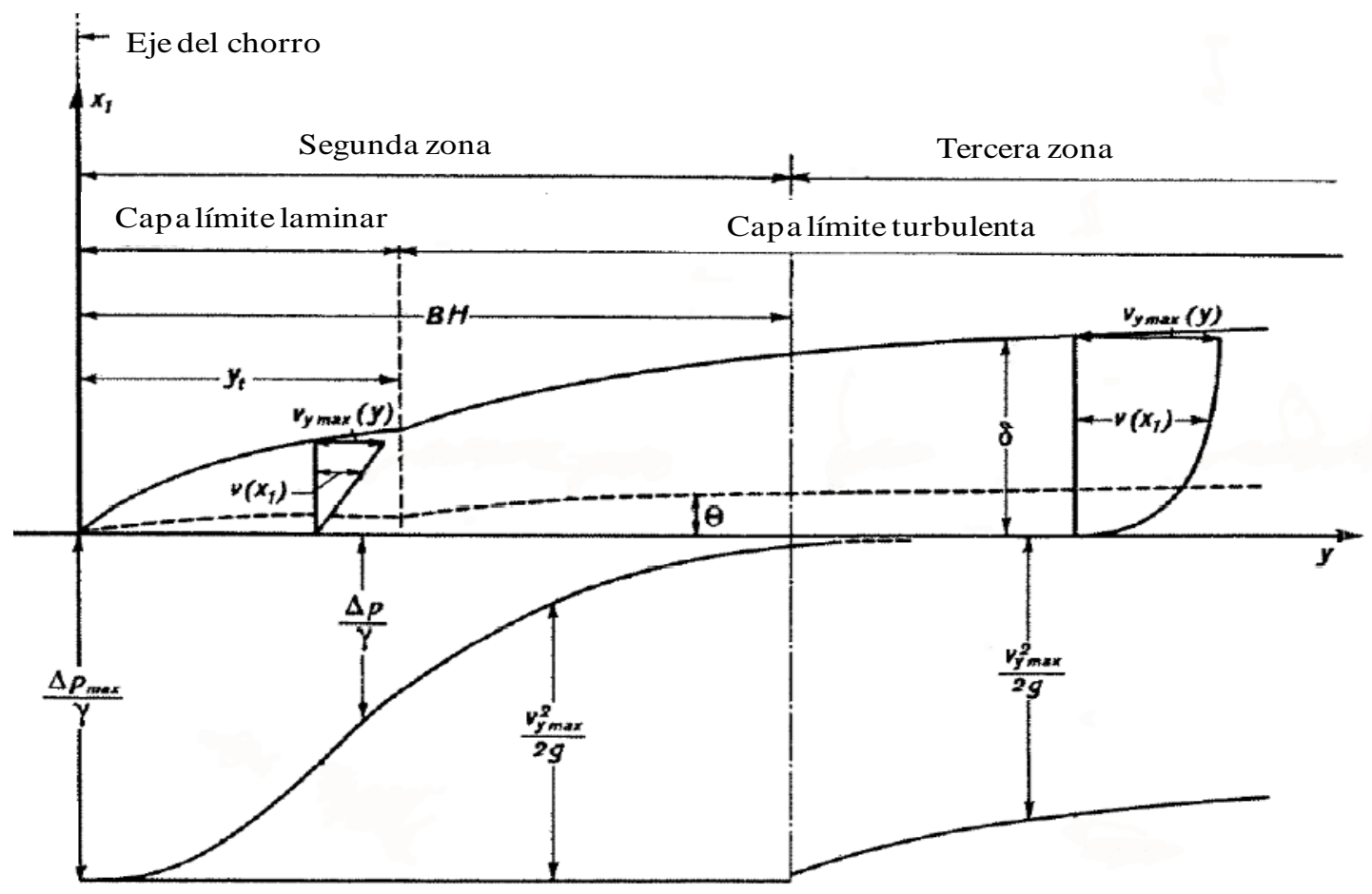

Figura 12. Desarrollo de la capa límite en la solera del cuenco de disipación y evolución de la sobrepresión $\Delta p$ y velocidad $v$ a lo largo de la solera obtenido por Cola (1966), citado por Felderspiel (2011).

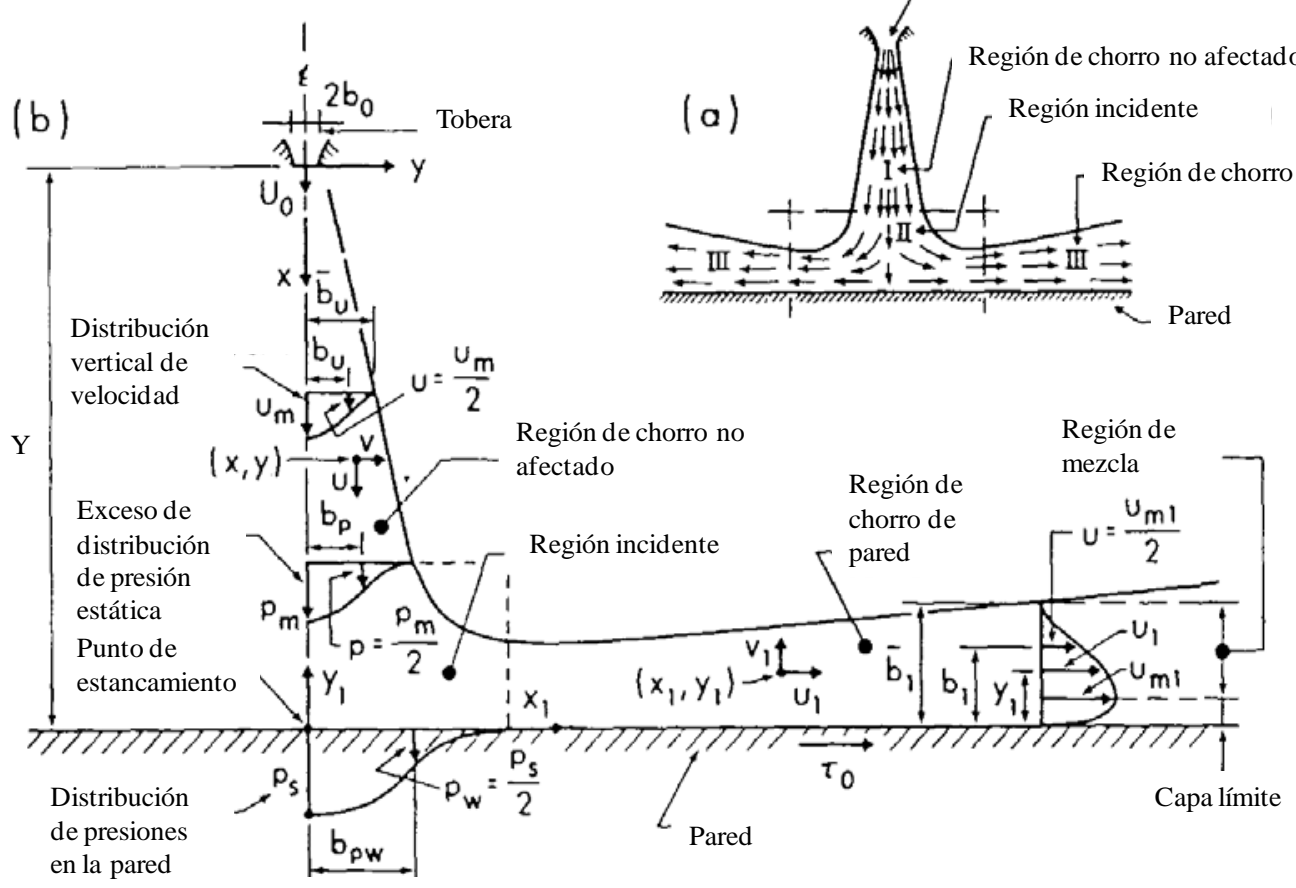

Figura 13. Difusión e impacto de un chorro bidimensional sobre una superficie plana y lisa: a) Principales regiones de chorro; b) Distribuciones de velocidad y presión en cada región (Beltaos y Rajaratnam, 1973). 


\subsubsection{Entrada de aire en el cuenco}

La entrada de aire en el cuenco de disipación está gobernada por la incidencia de un chorro de alta velocidad en un ambiente en relativa calma. El chorro introduce el aire capturado en la capa turbulenta de expansión del mismo, donde se producen fenómenos de deceleración y difusión lateral.

Ervine (1998), citado por Bollaert (2002), distingue tres mecanismos de entrada de aire, recogidos en la Tabla 3:

- Perturbaciones de la superficie turbulenta del chorro incidente. La entrada de aire puede deberse a diversos motivos como son remolinos turbulentos, inestabilidades o vorticidad longitudinal. Se asume que el tamaño de las turbulencias está relacionado con $V_{j}^{2} / 2 g$, donde $V_{j}$ es la velocidad media del chorro en el momento del impacto con el colchón de agua. De este modo, la tasa de aireación por unidad de ancho del chorro queda $q_{a} \sim V_{j}{ }^{3} / g$. En términos de velocidad turbulenta, la altura de las fluctuaciones turbulentas se pueden expresar como ${\overline{w^{\prime}}}^{2} / 2 g$, donde $\overline{w^{\prime}}$ es la velocidad turbulenta transversal del chorro.

- Aire introducido por una delgada capa generada en torno a la superficie de un chorro liso (no alterado por la aireación). Al incidir el chorro, se genera un espacio entre el chorro y el flujo del recirculación del colchón cuyo espesor es proporcional a $V_{j}^{1 / 2}$. Este mecanismo es sólo significante cuando la velocidad del chorro es superior a $5 \mathrm{~m} / \mathrm{s}$.

- Aireación desde la superficie libre. Este fenómeno puede producirse tanto en la superficie del cuenco receptor como aguas arriba del punto de impacto, sobre todo cuando se tienen chorros de alta velocidad. La aireación es debida a la existencia de grandes perturbaciones generadas por la intensa turbulencia y vorticidad en el cuenco receptor.

En situaciones prácticas, la entrada de aire en el cuenco es una combinación de estos mecanismos. Cada mecanismo tiene un umbral diferente que requiere ser superado, y una relación particular con la velocidad del chorro. La importancia de cada uno no es conocida exactamente, por lo que no se puede obtener una relación de tasa de aireación en el cuenco de disipación acertada.

El mismo estudio obtuvo que para un espesor superior a $30 \mathrm{~mm}$, la tasa de aireación se hace independiente del espesor del chorro. 


\begin{tabular}{|c|c|c|}
\hline $\begin{array}{c}\text { Mecanismo de } \\
\text { aireación }\end{array}$ & $\begin{array}{c}\text { Tasa de aireación por unidad de } \\
\text { ancho del chorro }\end{array}$ \\
\hline $\begin{array}{c}\text { Perturbaciones de la } \\
\text { superficie del chorro }\end{array}$ & & \\
\hline Capa límite del aire & \\
\hline Capa esponjada en la &
\end{tabular}

Tabla 3. Principales tres mecanismos de entrada de aire en el punto de impacto del chorro en el cuenco de disipación y su correspondiente tasa de aireación por unidad de ancho del chorro (Ervine, 1998, citado por Bollaert, 2002).

Ervine (1998) graficó la máxima tasa de aireación $q_{a}$ como función de la velocidad del chorro $V$ (Figura 14). Obtuvo que el exponente $n$ en la relación $q_{a} \sim V_{j}{ }^{n}$ varía entre 8.0 y 9.0 para chorros con velocidades de $2-3 \mathrm{~m} / \mathrm{s}$, mientras que para velocidades de 10-15 m/s el exponente pasa a valores entre 1.5-2.0. De este modo, la relación clásica $q_{a} \sim V_{j}^{3}$ es sólo válida para chorros con velocidades en el rango de $3-6 \mathrm{~m} / \mathrm{s}$, donde es aplicable el primer mecanismo. Con mayores velocidades, el exponente decrece indicando un aumento de la influencia de los mecanismos segundo y tercero, lo que indica que la extrapolación de la relación clásica $q_{a} \sim V_{j}^{3}$ de modelo a prototipo no es válida.

Mediante regresión polinómica, Ervine (1998), citado por Bollaert (2002), obtuvo una aproximación del caudal de aire para un rango de velocidades entre 1.5 y $15.0 \mathrm{~m} / \mathrm{s}$, válida para diámetros del chorro mayores de 30 mm, cuya precisión se estima en $\pm 30 \%$ :

$$
q_{a}=0.00002\left(V_{j}-1\right)^{3}+0.0003\left(V_{j}-1\right)^{2}+0.0074\left(V_{j}-1\right)-0.0058
$$



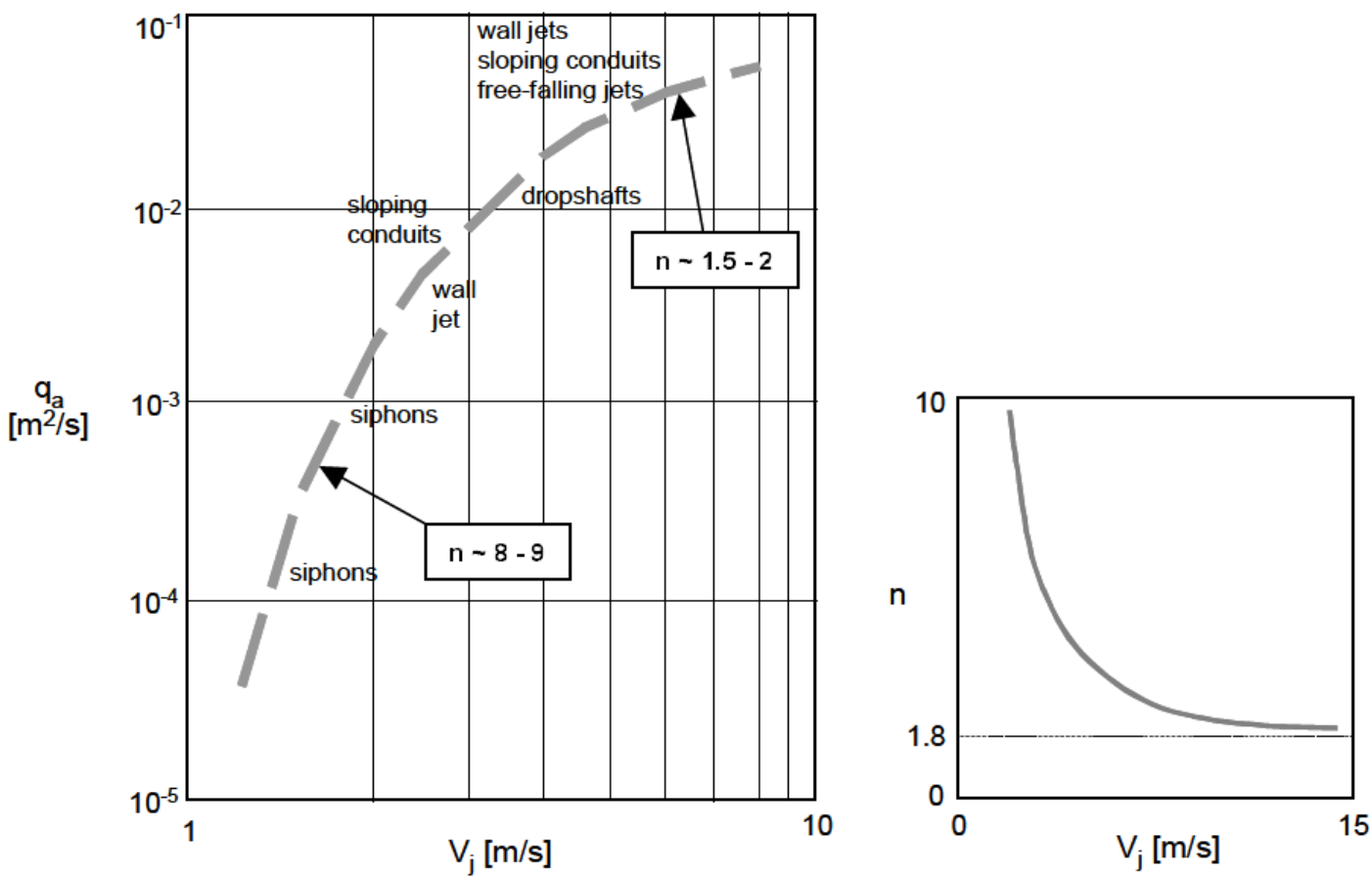

Figura 14. Máxima tasa de entrada de aire en diferentes estructuras hidráulicas en función de la velocidad del chorro $V_{j}$. Variación del exponente $n$ con la velocidad del chorro (Ervine, 1998, citado por Bollaert, 2002).

Chanson (1996) estudió los diferentes mecanismos de entrada de aire para chorros de baja y alta velocidad. Para bajas velocidades, el agua del cuenco de disipación no es capaz de seguir las ondulaciones de la superficie del chorro, produciéndose la entrada intermitente de pequeñas bolsas de aire. Con chorros de alta velocidad (4-12 m/s), se produce la entrada de una delgada lámina de aire debido a los esfuerzos de corte en la superficie del chorro que provocan la entrada de bolsas de aire alargadas. Falvey y Ervine (1988) analizaron estos dos mecanismos y propusieron una velocidad de transición entre ambos alrededor de $10 \mathrm{~m} / \mathrm{s}$.

Kiger y Duncan (2012) recopilan los últimos modelos de entrada de aire debidos a la incidencia de chorros en el cuenco de disipación. Las correlaciones vienen dadas en forma de relación caudal de aire/caudal líquido entrante, con la dependencia explícita de la velocidad del chorro en el impacto:

- Para El Hammoumi et al. (2002), la relación adimensional es:

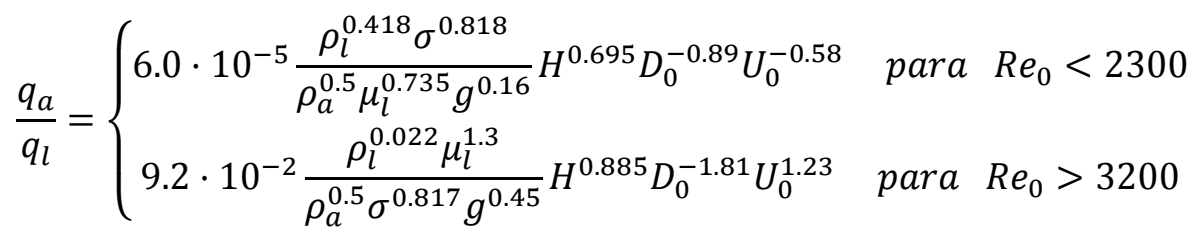


donde $\sigma$ es la tensión superficial, $\rho$ es la densidad, $\mu$ es la viscosidad dinámica, $g$ es la aceleración de la gravedad y $H$ es la altura de caída desde la tobera al cuenco receptor (los subíndices $l$ y $a$ indican líquido y aire, respectivamente). $U_{0}, D_{0}$ y $R e_{0}$ son la velocidad, diámetro y número de Reynolds en la condición de aguas arriba.

- Brattberg y Chanson (1998) consideran la siguiente expresión:

$$
\frac{q_{a}}{q_{l}}= \begin{cases}7.7 \cdot 10^{-4}\left(\frac{H}{D_{j}}-1.04\right)\left(\frac{U_{j}-U_{e}}{\sqrt{g D_{j}}}\right)^{1.8} & \text { para } U_{e}<U_{j}<4 \mathrm{~m} / \mathrm{s} \\ 2.0 \cdot 10^{-3}\left(\frac{H}{D_{j}}-1.04\right)\left(\frac{U_{j}-U_{e}}{\sqrt{g D_{j}}}+9.3\right) & \text { para } 4<U_{j}<8 \mathrm{~m} / \mathrm{s}\end{cases}
$$

siendo $U_{e}$ la velocidad crítica mínima para que se produzca la entrada de aire, mientras que $U_{j}$ y $D_{j}$ son la velocidad y el diámetro con que incide el chorro en el cuenco.

- Ma et al. (2009) deducen la siguiente expresión:

$$
\frac{q_{a}}{q_{l}}=K_{2} \cdot\left\{\begin{array}{lr}
\frac{U_{j}^{2}}{g D_{j}} & \text { para } 1<\frac{U_{j}}{U_{e}}<\frac{U_{t}}{U_{e}} \\
\left(\frac{U_{t}^{2}}{g D_{j}}\right)^{\frac{3}{4}}\left(\frac{U_{j}^{2}}{g D_{j}}\right)^{\frac{1}{4}} & \text { para } \frac{U_{t}}{U_{e}}<\frac{U_{j}}{U_{e}}
\end{array}\right.
$$

donde $K_{2}$ es una constante de ajuste y $U_{t}=4.5 \mathrm{~m} / \mathrm{s}$ es la velocidad de transición entre las dos situaciones.

Chanson y Manasseh (2003), y Cummings y Chanson (1997), citados por Kiger y Duncan (2012), obtuvieron diversos comportamientos para condiciones de entrada del aire en el agua en el caso de baja viscosidad:

- Para velocidades bajas $\left(U_{e} \approx 0.7 \mathrm{~m} / \mathrm{s}\right)$, la entrada de aire se produce de modo intermitente como pequeñas burbujas alrededor de la zona de incidencia que parecen corresponder a largas perturbaciones propagadas por la superficie del chorro.

- Para velocidades relativamente elevadas $\left(U_{e} \approx 1.0 \mathrm{~m} / \mathrm{s}\right)$, se forma una cavidad inestable con localización aleatoria alrededor del perímetro de la zona de impacto que introduce grandes cantidades de aire.

- Con mayores velocidades $\left(U_{e} \approx 3.5\right.$ a $5.0 \mathrm{~m} / \mathrm{s}$ ), se forma una cavidad separada alrededor de todo el perímetro y el aire es introducido por la rotura de la cavidad. 
En este sentido, los autores desarrollaron una correlación adimensional para la velocidad en que se inicia la entrada de aire:

$$
\frac{U_{e} \mu}{\sigma}=0.04\left[1+3.375 \exp \left(-70 \frac{u^{\prime}}{U_{j}}\right)\right]
$$

siendo $u^{\prime}$ la velocidad fluctuante.

Tendencias similares se pueden encontrar para relaciones entre la velocidad y la longitud del chorro. Van de Sande y Smith (1976), citados por Kiger y Duncan (2012), obtuvieron:

$$
\frac{q_{a}}{q_{l}} \propto\left\{\begin{array}{cc}
\rho_{l} U_{j}^{2} & \text { parachorros con } \frac{H}{L_{b}}>1 \\
\frac{H^{1 / 3} U_{j}}{D_{j}^{1 / 2}} & \text { para chorros con } \frac{H}{L_{b}}<1
\end{array}\right.
$$

donde $L_{b}$ es la longitud de rotura del chorro.

La condición de chorros largos obtiene una escala de velocidades similares a las condiciones de baja velocidad de Ma et al. (2009), y de Brattberg y Chanson (1998), lo que es algo significante para este último dada su corta longitud de chorro $\left(H / D_{j}<13\right)$. La condición de chorro corto se corresponde con la condición de transición propuesta por Brattberg y Chanson (1998), y por El Hammoumi et al. (2002), pero con una menor dependencia de la longitud del chorro.

En el mismo artículo, los autores analizan la formulación propuesta por Bin (1993), válida para un rango de longitudes de chorros $H / D_{0}<13$, obtenida con tubos largos cilíndricos $\left(L_{t u b o} / D_{0}>10\right)$ :

$$
\frac{q_{a}}{q_{l}}=0.04\left(\frac{H}{D_{0}}\right)^{0.4}\left(\frac{U_{j}^{2}}{g D_{j}}\right)^{0.28}
$$

Esta correlación sobreestima sistemáticamente el ratio de entrada para toberas cortas, que además muestra una fuerte dependencia de las variables independientes (Bin, 1993). En comparación con los trabajos más recientes, esta forma es muy similar al régimen de transición mostrado en la ecuación (34), que muestra además una dependencia al número de Froude $\left(\mathrm{Fr}^{0.25}\right)$.

Pese a los esfuerzos realizados por diversos autores, el mecanismo de entrada de aire en el cuenco no es todavía bien conocido, por lo que no se puede obtener una acertada relación de la tasa de aireación y su distribución en el cuenco de disipación en función de la relación altura de caída/longitud de rotura del chorro en las distintas tipologías de chorros incidentes. 


\subsubsection{Condiciones en el cuenco en función del calado aguas abajo}

El comportamiento del agua en el interior del cuenco puede clasificarse en función del calado aguas abajo del punto de impacto del chorro. Cui Guang Tao et al. (1986), citados por Castillo (1989) y Puertas (1994) proponen una clasificación basada en tres tipos de condiciones observadas en un modelo bidimensional (Figura 15):

- Tipo 1. El calado de aguas abajo $Y$ es inferior al calado conjugado correspondiente a un resalto hidráulico. En esta condición, no se genera un colchón eficaz de agua en el cuenco, sino que existe una zona de flujo en régimen rápido. Aguas abajo del punto de impacto puede generarse un resalto hidráulico libre cuya sección inicial depende de las condiciones a la salida del cuenco. Aguas arriba del impacto se crea un colchón de agua $Y_{u}$ superior al calado de aguas abajo, el cual contribuye a la amortiguación del chorro. Este hecho ya fue deducido por Moore (1943) mediante la aplicación de la ecuación de conservación de la cantidad de movimiento.

- Tipo 2. El calado de aguas abajo $Y$ es superior al calado conjugado correspondiente a un resalto hidráulico, por lo que se forma un resalto sumergido que alcanza el punto de impacto. Debido a los remolinos que se generan aguas arriba y aguas abajo del punto de impacto, el perfil de la lámina libre se curva hacia abajo en las cercanías del chorro. Para que se forme este tipo de flujo se debe cumplir $Y_{c 0} / B_{j}<20$, donde $y_{c 0}$ es el calado en el cuenco en la zona de impacto y $B_{j}$ es el espesor del chorro a la entrada al cuenco (Aki, 1969, citado por Cui Guang Tao et al., 1986).

- Tipo 3. Cuando $Y_{c 0} / B_{j}>20$, no se produce una curvatura apreciable en el perfil del agua en el cuenco puesto que la influencia de los remolinos no es suficiente para condicionar los niveles en el cuenco $\left(Y_{c 0} \approx Y\right)$.

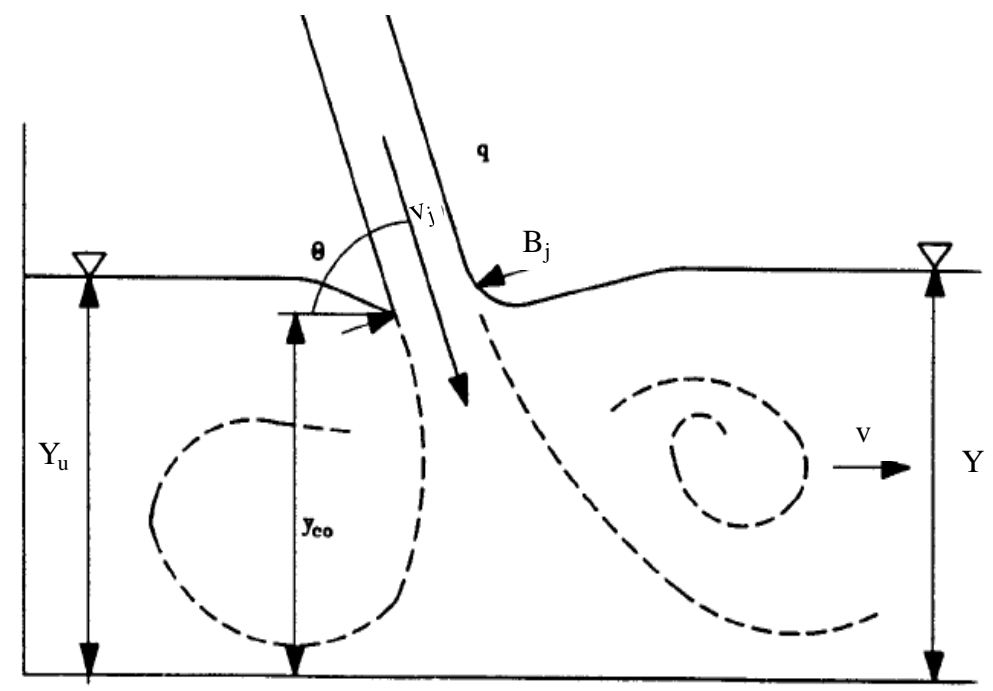

Figura 15. Condiciones de flujo en el cuenco de disipación en función del calado de aguas abajo (Cui Guang Tao et al., 1986, adaptado de Castillo, 1989). 
En prototipo, se tiende a garantizar las condiciones tipo 2 o 3 para que el colchón de agua eficaz ayude a amortiguar la energía cinética del chorro.

Moore (1943) realizó ensayos utilizando caídas desde gradas de 0.15 y $0.45 \mathrm{~m}$ de altura, obteniendo la siguiente expresión para el calado aguas arriba del punto de incidencia del chorro cuando no se forma un resalto al pie del punto de impacto:

$$
\frac{Y_{u}}{y_{c}}=\sqrt{\left(\frac{Y}{y_{c}}\right)^{2}+2\left(\frac{y_{c}}{Y}\right)-3}
$$

donde $y_{c}$ es el calado crítico e $Y$ el calado de aguas abajo del punto de incidencia.

Para unas condiciones de flujo sin colchón efectivo aguas abajo del punto de impacto (configuración tipo 1), obtuvo que la máxima energía disipada es del orden del $65 \%$, lo que ocurre con valores bajos de caudal específico. En caso de contar con un colchón de agua, se producirá una disipación de energía suplementaria debido a los remolinos turbulentos que se forman.

Otros autores relacionan el calado aguas arriba del punto de impacto con la atura de caída desde un escalón $H$ (Tabla 4).

\begin{tabular}{|c|c|c|}
\hline Autor & Análisis & Expresión \\
\hline Rand (1955) & $\begin{array}{c}\text { Modelo físico } \\
\text { Para } 0.045<y_{d} / H<1\end{array}$ & $\frac{Y_{u}}{H}=\left(\frac{y_{c}}{H}\right)^{0.66}$ \\
\hline Gill (1979) & $\begin{array}{c}\text { Modelo físico } \\
\text { Para } 0.075<y_{c} / H<0.45\end{array}$ & $\frac{Y_{u}}{H}=1.067\left(\frac{y_{c}}{H}-0.0016\right)^{0.697}$ \\
\hline Rajaratnam y Chamani (1995) & $\begin{array}{c}\text { Solución de ecuaciones } \\
\text { no lineales }\end{array}$ & $\frac{Y_{u}}{H}=1.107\left(\frac{y_{c}}{H}\right)^{0.719}$ \\
\hline Chanson (1995) & $\begin{array}{c}\text { Solución de ecuaciones } \\
\text { no lineales }\end{array}$ & $\frac{Y_{u}}{H}=0.998\left(\frac{y_{c}}{H}\right)^{0.675}$ \\
\hline
\end{tabular}

Tabla 4. Expresiones para el cálculo en flujo bidimensional del calado aguas arriba del impacto del chorro en función de la altura de caída $\mathrm{H}$ y del calado crítico $y_{c}$ (Chanson, 1995).

Rand (1955), citado por Castillo (1989), obtuvo el calado aguas arriba del punto de impacto en función del caudal específico $q$ y la diferencia de cotas entre la solera del cuenco y el umbral del vertedero de pared delgada $\Delta z$ en caso de que se forme un resalto hidráulico libre aguas abajo del vertedero:

$$
Y_{u}=\Delta z\left(\frac{q^{2}}{g \Delta z^{3}}\right)^{0.22}
$$


La relación adimensional $q^{2} / g \Delta z^{3}$ se conoce como número de caída. Si el resalto de aguas abajo es sumergido, se espera que el calado de aguas arriba sea similar al calado de aguas abajo (condición de flujo tipo 2 o 3).

Cui Guang Tao et al. (1986) aplicaron el teorema de la cantidad de movimiento para calcular el calado del colchón aguas arriba en función del calado aguas abajo en el caso de flujos del tipo 2 o 3.

$$
\rho q v_{0} \cos \theta_{0}+\frac{1}{2} \rho g Y_{u}^{2}=\rho q v+\frac{1}{2} \rho g Y^{2}+\beta_{0} \rho q v_{0} \cos \theta_{0}
$$

El último término de esta expresión tiene en cuenta la pérdida de energía producida por la entrada del chorro en el cuenco.

Despejando la relación entre calados, y considerando $\beta=1-\beta_{0}$, se obtiene:

$$
\frac{Y_{u}}{Y}=\sqrt{1-2 F\left[\left(\frac{v_{0}}{v}\right) \beta \cos \theta_{0}-1\right]}
$$

$\beta_{0}$ es un coeficiente al que se le puede atribuir el sentido físico de tener en cuenta las pérdidas de carga que se producen dentro del volumen de control. El coeficiente $\beta$ se encuentra entre 0.5 y 0.7 para un flujo bidimensional, pudiendo considerarse un valor medio de 0.6. $F=v^{2} / g y$ es el cuadrado del número de Froude en la sección de aguas abajo del punto de impacto. Castillo y Carrillo (2011) consideran que $\beta \approx 0.55$ para el caso tridimensional.

Según Puertas (1994), Cui Guang Tao et al. trabajan con grandes colchones de agua (32.5, 43.5 y 54.9 m para alturas de caída entre 187 y $165.1 \mathrm{~m}$ en prototipo). El ajuste propuesto por los autores parece ofrecer distribuciones de presiones muy elevadas y longitudes de disipación demasiado altas, no llegando a la atenuación total ni siquiera con el mayor colchón de agua. Puesto que no se indican las dimensiones reales del modelo reducido, no se puede saber el posible efecto de escala de la tensión superficial en la mayoración de las presiones, aunque cabe pensar en chorros muy compactos.

\subsubsection{Presiones medias en el punto de estancamiento}

La incidencia del chorro y su deflexión provocan un incremento en la presión que soporta la solera del cuenco de amortiguación debido a la variación en la cantidad de movimiento del chorro (se pasa de un chorro casi ortogonal a la solera, a uno paralelo a la misma). Al ser un proceso dinámico (la presión es variable en el tiempo), se puede descomponer en la parte de presión media temporal y la parte de fluctuación respecto de la media. Asimismo, dado que el colchón ejerce una presión constante (hidrostática) 
sobre la solera, se puede distinguir entre presión media y presión dinámica media, esta última obtenida al restar la componente hidrostática a la presión media.

El punto de estancamiento es el punto en que toda la energía cinética se transforma en potencial, obteniendo los mayores valores de la presión (punto de velocidad nula).

Los valores máximos y mínimos de la presión no se miden simultáneamente, sino que corresponden a los valores extremos de mediciones suficientemente largas. La presión definida de este modo constituye un límite físico superior de las condiciones dinámicas existentes, por lo que el método de presiones extremas a menudo resulta en un diseño conservador (Bollaert, 2002).

Armengou (1991) propone que la presión en el punto de estancamiento se puede obtener mediante la siguiente expresión:

$$
\Delta p_{\text {máx }}=C \rho \frac{V_{j}^{2}}{2} \frac{B_{j}}{Y}=\frac{C q \gamma H^{2}}{Y \sqrt{2 g H^{3}}}
$$

La presión en zonas alejadas del punto de estancamiento responde a la fórmula general, inicialmente propuesta por Cola:

$$
\Delta p(x)=\Delta p_{\text {máx }} e^{-K\left|\frac{x}{Y}\right|^{2}}
$$

donde $\rho$ y $\gamma$ son la densidad y el peso específico del agua, $V_{j}$ y $B_{j}$ la velocidad y el ancho del chorro incidente, e $Y$ el colchón en el cuenco de disipación. $C$ y $K$ son parámetros para ajustar las ecuación a los métodos propuestos por los diversos autores. Castillo (2002) resume y homogeneíza las distintas formulaciones en la Tabla 5.

Las expresiones permiten calcular los valores de la presión en distintos puntos de la solera del cuenco de amortiguación. Existe una gran divergencia entre los resultados obtenidos por los diferentes autores, lo que está relacionado con la diferente naturaleza de los ensayos realizados en modelo reducido que no están suficientemente explicados en muchos casos, particularmente en lo que se refiere a la aireación de la lámina (Castillo, 1989).

Las presiones dinámicas pueden generarse por el impacto directo del núcleo del chorro en condiciones de calados pequeños en el cuenco de disipación, o por impactos de flujos turbulentos con relaciones entre el calado del cuenco y el espesor del chorro $\left(y / B_{j}\right)$ mayores a 4-6 según la teoría de chorros bidimensionales recogida en la Tabla 2. Los patrones de presiones de estos dos tipos de impactos son diferentes.

El impacto del núcleo genera un valor constante y elevado de la presión media con bajas fluctuaciones, mientras que el impacto del chorro desarrollado puede describirse como un conjunto de remolinos de diferente tamaño que se encuentran recirculando en 
el cuenco. Estos remolinos producen grandes regiones fluctuantes con valores bajos de presión media.

\begin{tabular}{|c|c|c|c|c|c|}
\hline Autor & $C$ & $\boldsymbol{K}$ & $\begin{array}{c}\text { Características } \\
\text { del ensayo }\end{array}$ & $\begin{array}{l}\text { Tipo de } \\
\text { ensayo }\end{array}$ & Observaciones \\
\hline $\begin{array}{l}\text { Cola } \\
(1966)\end{array}$ & 7.18 & 40.51 & $\begin{array}{l}B_{j}=12-24 \mathrm{~mm} \\
Y=0.165-0.835 \mathrm{~m} \\
V_{j}=1.3-4.8 \mathrm{~m} / \mathrm{s}\end{array}$ & $\begin{array}{l}\text { Agua } \\
\text { Chorro } \\
\text { sumergido (sin } \\
\text { aireación) } \\
\text { Bidimensional } \\
\text { Simétrico }\end{array}$ & \\
\hline Aki (1969) & 5.06 & 12.56 & & & \\
\hline $\begin{array}{l}\text { Hartung y } \\
\text { Häusler } \\
\text { (1973) }\end{array}$ & 5 & 19.60 & $\begin{array}{l}\text { Teórico } \\
\text { Longitud de } \\
\text { desintegración del } \\
\text { chorro }=5 B_{j}\end{array}$ & $\begin{array}{l}\text { Agua } \\
\text { Colchón } \\
\text { ilimitado }\end{array}$ & $\begin{array}{l}\text { Si se considera chorro } \\
\text { turbulento rugoso, se } \\
\text { obtiene } C=3.56 ; K=9.92 \\
\text { (Ervine y Falvey, } 1987 \text { ) }\end{array}$ \\
\hline $\begin{array}{l}\text { Bealtos } \\
(1976)\end{array}$ & 8 & 42.01 & $\begin{array}{l}B_{j}=0.224 \mathrm{~cm} \\
Y / B_{j}=45.5-68.2\end{array}$ & $\begin{array}{l}\text { Aire } \\
\text { Bidimensional } \\
\text { Distintos } \\
\text { ángulos }\end{array}$ & $\begin{array}{l}\text { Comprueba el ajuste de } \\
\text { la expresión teórica de } \\
\text { Schauer y Eustis (1963) }\end{array}$ \\
\hline $\begin{array}{l}\text { Cui Guang } \\
\text { Tao et al. } \\
\text { (1985) }\end{array}$ & $5.2-6.35$ & 12.56 & $\begin{array}{l}\text { Modelo sin escala } \\
\text { conocida } \\
\text { En prototipo: } \\
Q=80 \mathrm{~m}^{3} / \mathrm{s} \\
H=165-187 \mathrm{~m} \\
Y=32.5-54.9 \mathrm{~m} \\
V_{0}=1.3-4.8 \mathrm{~m} / \mathrm{s}\end{array}$ & $\begin{array}{l}\text { Agua } \\
\text { Bidimensional } \\
\text { no simétrico } \\
\text { Modelo } \\
\text { reducido de } \\
\text { presa bóveda }\end{array}$ & $\begin{array}{l}\text { Se desconocen los } \\
\text { posibles efectos de escala }\end{array}$ \\
\hline $\begin{array}{l}\text { Armengou } \\
(1991)\end{array}$ & 3.19 & 25.00 & $\begin{array}{l}Q<50 \mathrm{l} / \mathrm{s} \\
H=1.8-5.5 \mathrm{~m} \\
Y<1.2 \mathrm{~m} \\
V_{j}=6-10.4 \mathrm{~m} / \mathrm{s} \\
* H / L_{b}=0.4-2.73\end{array}$ & $\begin{array}{l}\text { Agua } \\
\text { Bidimensional } \\
\text { No simétrico } \\
\text { Chorro aireado }\end{array}$ & $\begin{array}{l}\text { Primeros valores } \\
\text { obtenidos en la puesta a } \\
\text { punto de una instalación } \\
\text { experimental }\end{array}$ \\
\hline $\begin{array}{l}\text { Puertas } \\
\text { (1994) }\end{array}$ & 3.88 & 2 & $\begin{array}{l}Q<86 \mathrm{l} / \mathrm{s} \\
H=1.85-5.45 \mathrm{~m} \\
Y=0.08-0.80 \mathrm{~m} \\
V_{j}=6-10.4 \mathrm{~m} / \mathrm{s} \\
* H / L_{b}=0.4-2.73\end{array}$ & $\begin{array}{l}\text { Agua } \\
\text { Bidimensional } \\
\text { No simétrico } \\
\text { Chorro aireado }\end{array}$ & $\begin{array}{l}\text { Válida para } \\
Y \geq 0.368 q^{0.50} H^{0.25} \\
\text { (colchón efectivo) } \\
\text { En otro caso, el } \\
\text { exponente de la ecuación } \\
\text { (43) es } m=0.5\end{array}$ \\
\hline $\begin{array}{l}\text { Ervine et } \\
\text { al. (1997) }\end{array}$ & $\begin{array}{l}38.4(1- \\
\left.C_{i}\right)(D / Y)\end{array}$ & $\begin{array}{l}25 \text { para } \\
Y / D<4 \\
30 \text { para } \\
Y / D>4\end{array}$ & $\begin{array}{l}Q<63 \mathrm{l} / \mathrm{s} \\
H=0.51-2.63 \mathrm{~m} \\
Y=0.10-0.50 \mathrm{~m} \\
V_{j}=4-25 \mathrm{~m} / \mathrm{s} \\
* * H / L_{b}<0.5\end{array}$ & $\begin{array}{l}\text { Agua } \\
\text { Chorro circular } \\
\text { Chorro aireado }\end{array}$ & $\begin{array}{l}\text { En el diámetro } D \text { se } \\
\text { considera el término de } \\
\text { expansión lateral debida } \\
\text { a la turbulencia }\end{array}$ \\
\hline
\end{tabular}

${ }^{*} H_{b}=$ longitud de rotura del chorro para chorro rectangular (Horeni, 1956): $H_{b} \approx 6 q^{0.32}$.

**Chorro circular (Ervine et al., 1997): $H_{b} / D_{0} F_{0}^{2}=1.05 / C^{0.82} ; \quad C=1.14 T_{u} F_{0}^{2}=$ $\beta_{i} /\left(1+\beta_{i}\right)=$ concentración de aire inicial en la condición incidente; $T_{u}=v^{\prime} / V=$ intensidad turbulenta del chorro; $\beta_{i}=Q_{a} / Q_{w}=K_{1}\left[1-V_{\min } / V\right] \sqrt{H / D} ; K_{1}=0.2$ para chorro con baja turbulencia; $K_{1}=0.4$ para chorro con muy alta turbulencia; $V_{\min } \approx 1 \mathrm{~m} / \mathrm{s}=$ velocidad mínima para que se produzca la entrada de aire; $Q_{a}=$ caudal de aire; $Q_{w}=$ caudal de agua; $D_{0}=$ diámetro inicial del chorro; $D=$ diámetro del chorro; $D_{c}=$ diámetro del núcleo del chorro; $F_{0}=$ número de Froude inicial; $D=D_{c}+2 \xi ; \xi=\left(1.14 T_{u} U_{0}^{2} / g\right)\left[\sqrt{2 H_{b} /\left(D_{0} F_{0}^{2}\right)}-1\right] ; \xi=$ expansión lateral.

Tabla 5. Coeficientes $C$ y K de la formulación general de presiones dinámicas medias (Castillo, 2002). 
De este modo, según Castillo (2007), los chorros rectangulares no desarrollados ( $H$ $<L_{b}$ ) con colchones poco profundos muestran registros de presiones con patrones constantes de picos de presiones similares por encima y por debajo de la media, que disminuyen a medida que aumenta el colchón. En el caso de chorros rectangulares desarrollados $\left(H>L_{b}\right)$ se registran presiones más fluctuantes, aumentando el fenómeno con la altura de caída y con el colchón de agua.

Diversos autores han obtenido expresiones para el cálculo del coeficiente de presión dinámica media $C_{p}$ en la línea central del chorro a partir del estudio de la difusión de chorros bidimensionales. La Figura 16 recoge los resultados de 11 estudios diferentes realizados en chorros rectangulares y circulares, en los que se obtiene el coeficiente adimensional presión dinámica media en función de la relación entre el calado del colchón y el ancho del chorro $Y / D_{j}$. La región de impacto del núcleo del chorro se considera hasta 5 veces el ancho inicial en chorros que impactan con el colchón y hasta 7 veces en chorros sumergidos.

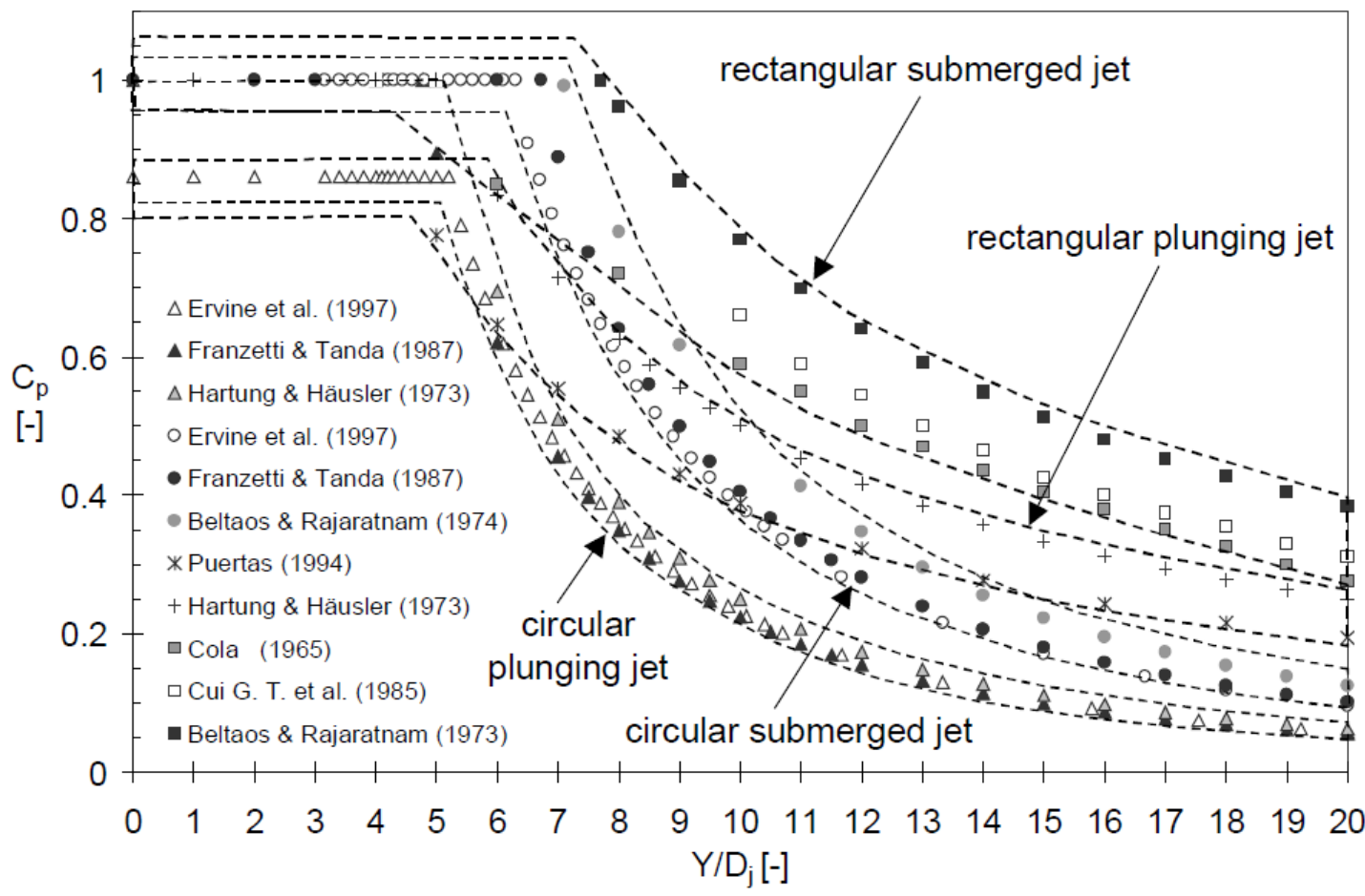

Figura 16. Presión dinámica media en función de la relación $Y / D_{j}$ (Bollaert y Schleiss, 2003).

Sin embargo, la Figura 16 genera ciertas dudas. En este sentido, los resultados de Cola (1956) parecen no coincidir con la formulación propuesta por el autor, mientras que Puertas (1994) no presenta en su estudio ninguna curva de ajuste como la descrita. 
Debido a los efectos de aireación y expansión lateral del chorro, los chorros aireados presentan un valor máximo de $C_{p}$ entre 0.8 y 0.9 , en vez de 1.0. Se observa además que los chorros circulares tienen un descenso más pronunciado de $C_{p}$ en la zona de chorro desarrollado (relaciones $Y / D_{j}>4-6$ ). Esto puede deberse a la definición del ancho del chorro incidente $B_{j}$ y/o al hecho de que la difusión en chorros circulares ocurre radialmente (en todas direcciones), mientras que en chorros rectangulares ocurre longitudinalmente (unidireccionalmente).

Bollaert y Schleiss (2003) obtuvieron una dependencia de $C_{p}$ a la velocidad $V_{j}$ cuando se tienen frecuencias turbulentas bajas $(<1 \mathrm{~Hz})$, provocando una disminución de $C_{p}$ con la disminución de $V_{j}$ (Figura 17). Este descenso es menos acusado cuando se tiene un chorro desarrollado antes del impacto. La dependencia de $C_{p}$ a los valores de $V_{j}$ está sobretodo influenciada por frecuencias turbulentas bajas. Los autores consideran que este efecto está probablemente exagerado en laboratorio en comparación con el funcionamiento en prototipo.

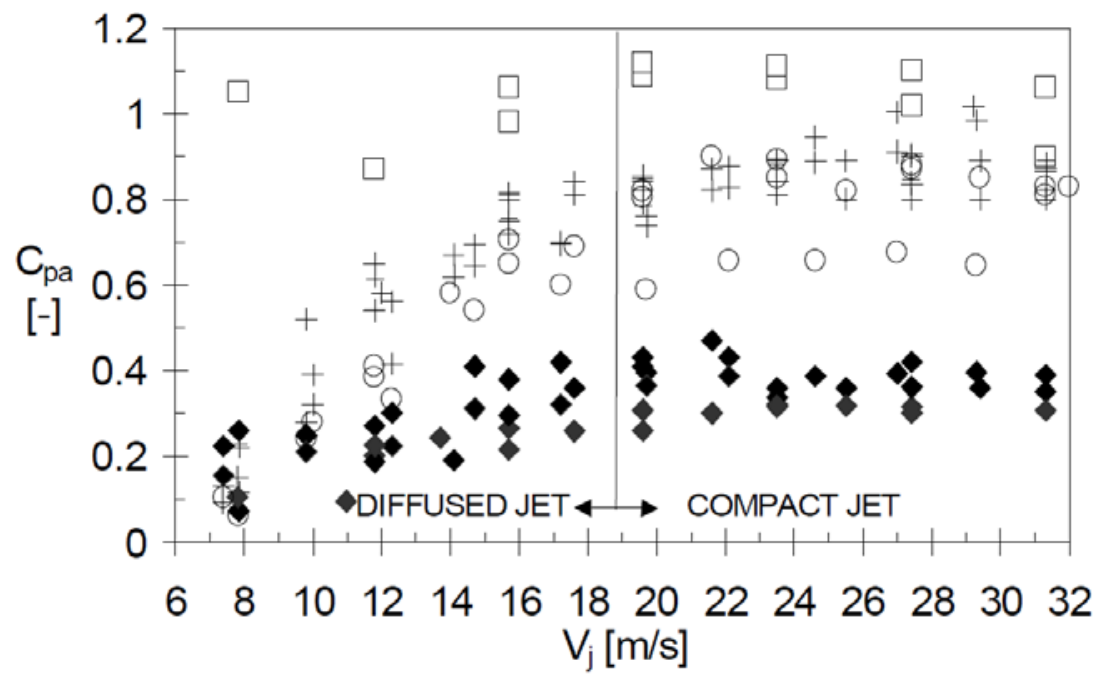

\begin{tabular}{|c|c|c|c|c|c|c|c|c|}
\hline $\mathrm{N}^{\circ}$ & Type & \multicolumn{5}{|c|}{ Geometrical ratio } & Jet velocity & Symbol \\
\hline 1 & core jet & 0 & $<$ & $\mathrm{Y} / \mathrm{D}_{\mathrm{j}}$ & $<$ & $4-6$ & for all $\mathrm{V}_{\mathrm{j}}$ & + \\
\hline 2 & developed jet & $4-6$ & $<$ & $\mathrm{Y} / \mathrm{D}_{\mathrm{j}}$ & $<$ & $20-40$ & for all $\mathrm{V}_{\mathrm{j}}$ & $\bullet$ \\
\hline 3 & transitional jet & $\begin{array}{l}0 \\
4-6\end{array}$ & $\begin{array}{l}< \\
<\end{array}$ & $\begin{array}{l}\mathrm{Y} / \mathrm{D}_{\mathrm{j}} \\
\mathrm{Y} / \mathrm{D}_{\mathrm{j}}\end{array}$ & $\begin{array}{l}< \\
<\end{array}$ & $\begin{array}{l}4-6 \\
20-40\end{array}$ & $\begin{array}{l}\text { for } 15 \mathrm{~m} / \mathrm{s}<\mathrm{V}_{\mathrm{j}} \\
\text { for } \mathrm{V}_{\mathrm{j}}<15 \mathrm{~m} / \mathrm{s}\end{array}$ & 0 \\
\hline
\end{tabular}

Figura 17. Coeficiente de presión dinámica media como función de la velocidad del chorro incidente $V_{j}$ (Bollaert y Schleiss, 2003).

Ervine et al. (1997) consideran que el coeficiente de presión dinámica media para relaciones $H / L_{b}<0.5$ puede obtenerse como: 
- $\quad$ Para $Y / D_{j}<4-5$ :

$$
C_{p}=0.86
$$

- Para $Y / D_{j}>4-5$ :

$$
C_{p}=38.4\left(1-C_{i}\right)\left(\frac{D_{j}}{Y}\right)^{2}
$$

donde $C_{i}$ es la concentración de aire del chorro en la entrada al colchón, obtenida como:

$$
C_{i}=\frac{\beta_{i}}{1+\beta_{i}}
$$

siendo $\beta_{i}$ la relación entre aire y agua de un chorro compacto entrando en el colchón de agua (Ervine, 1976). Cuando $\beta_{i} \approx 0.99, C_{i} \rightarrow 0.50$. La relación $\beta_{i}$ se obtiene como:

$$
\beta_{i}=\frac{Q_{a}}{Q_{w}}=K_{1}\left[1-\frac{U_{m}}{U_{j}}\right] \sqrt{\frac{L}{D_{j}}}
$$

donde $Q_{a}$ es el caudal de aire entrante, $Q_{w}$ el caudal de agua, $U_{m}$ la velocidad mínima requerida para que se produzca la entrada de aire por impacto de un chorro $(\approx 1 \mathrm{~m} / \mathrm{s}), \mathrm{y}$ $L$ la distancia de caída del chorro. $K_{1}$ varía entre 0.2 para chorros circulares de turbulencia baja a 0.4 para chorros de elevada turbulencia.

Castillo (2006) propone diferentes curvas para obtener $C_{p}$ en función de los valores $Y / B_{j}$ y $H / L_{b}$. Para colchones no efectivos (zona de establecimiento del flujo, $Y \leq 4 B_{j}$ ), considera la siguiente expresión:

$$
C_{p}=0.36\left(H / L_{b}\right)^{-1.04}
$$

Esta expresión tiene un límite máximo, $C_{p}=0.78$ cuando $H / L_{b} \leq 0.5$.

Para colchones efectivos $\left(Y>4 B_{j}\right)$, Castillo aplica la siguiente expresión:

$$
C_{p}=\frac{H_{m}-Y}{V_{j}^{2} / 2 g}=a e^{-b\left(Y / B_{j}\right)}
$$

donde $H_{m}$ e $Y$ son la presión media registrada en el punto de estancamiento y el calado en el cuenco de amortiguación. $V_{j}$ y $B_{j}$ son la velocidad y el espesor del chorro en la sección previa al impacto con el colchón. Los parámetros $a$ y $b$ de las leyes exponenciales se obtienen de la Tabla 6. 


\begin{tabular}{|c|c|c|c|}
\hline $\mathbf{H} / \mathbf{L}_{\boldsymbol{b}}$ & $\mathbf{a}$ & $\mathbf{b}$ & $\boldsymbol{C}_{\boldsymbol{p}}\left(\mathbf{Y} / \boldsymbol{B}_{\boldsymbol{j}} \leq \mathbf{4}\right)$ \\
\hline$<0.5$ & 0.98 & 0.070 & 0.78 \\
$0.5-0.6$ & 0.92 & 0.079 & 0.69 \\
$0.6-0.8$ & 0.65 & 0.067 & 0.50 \\
$1.0-1.3$ & 0.65 & 0.174 & 0.32 \\
$1.5-1.9$ & 0.55 & 0.225 & 0.22 \\
$2.0-2.3$ & 0.50 & 0.250 & 0.18 \\
$>2.3$ & 0.50 & 0.400 & 0.10 \\
\hline
\end{tabular}

Tabla 6. Parámetros de la ecuación del coeficiente de presión dinámica media en función de la longitud de rotura (Castillo, 2006).

En la Figura 18 se comparan los resultados de estas ecuaciones con los obtenidos por diversos investigadores.

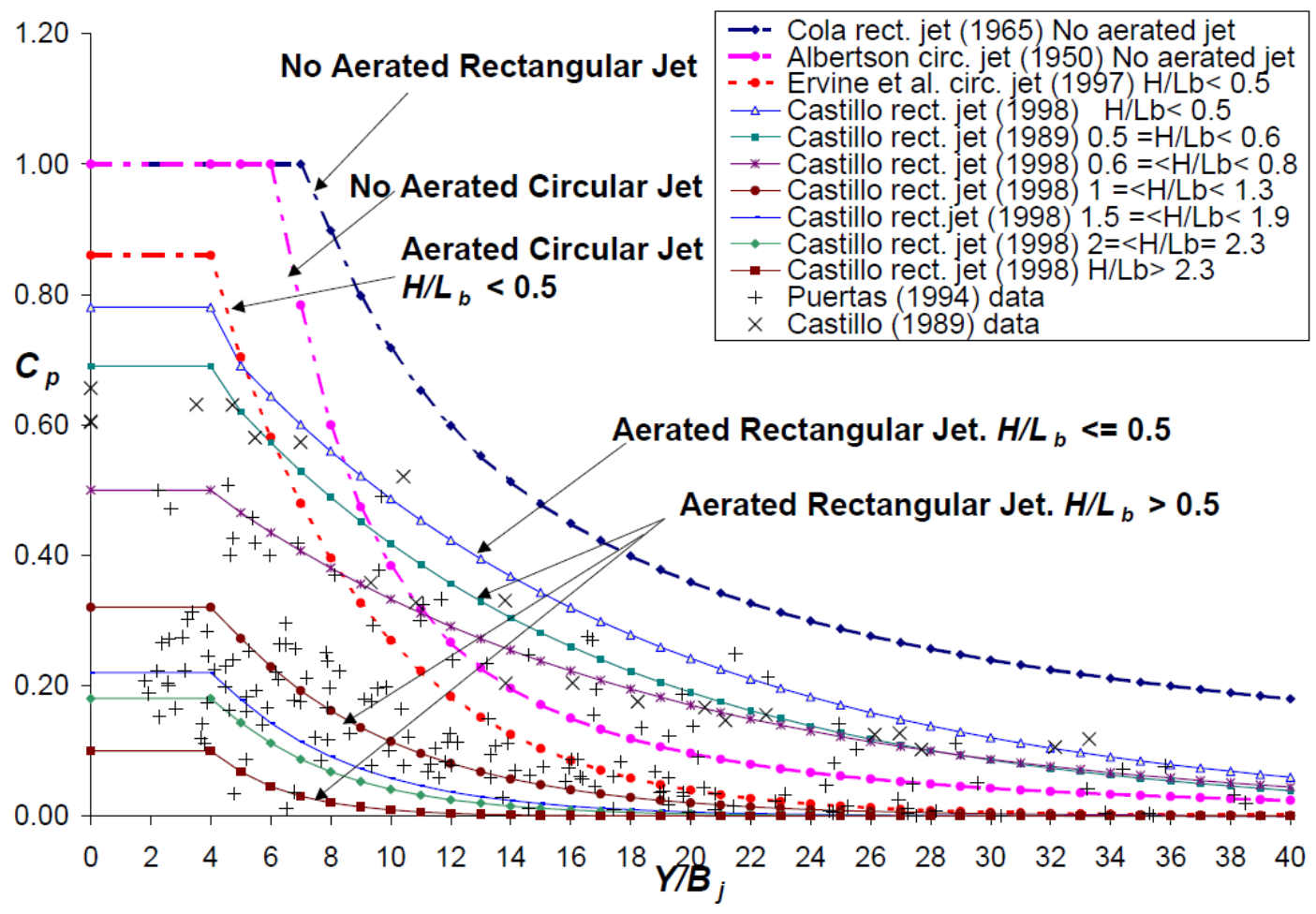

Figura 18. Coeficiente de presión dinámica media (Castillo, 2006).

Se observa que los chorros rectangulares pierden más energía en el aire que los circulares puesto que el mayor coeficiente de presión dinámica media obtenido $\left(C_{p}=\right.$ $0.78)$ es inferior al valor correspondiente al caso de chorros circulares $\left(C_{p}=0.86\right)$.

Además, la desintegración del núcleo del chorro se produce a una profundidad aproximada de cuatro veces la anchura del chorro incidente, generándose a partir de ese punto una reducción considerable de la presión por efecto de la difusión en el cuenco, que es más elevada en el caso de chorros circulares. 
Se debe tener en cuenta que los valores del coeficiente de presión dinámica media obtenidos en laboratorio están del lado de la seguridad, debido a la dificultad que entraña reproducir la aireación natural de los chorros de vertido libre en una instalación de laboratorio (las presiones son mayores que las que se obtendrían en medidas de prototipo).

\subsubsection{Fluctuación de las presiones en el punto de estancamiento}

La presión en la solera del cuenco es una variable aleatoria, lo que da lugar a su análisis estadístico a partir de la media, los valores máximo y mínimo, etc. Como norma general, la varianza es mayor cuando no existe un colchón de agua, mientras que a partir de un cierto espesor el efecto dinámico es despreciable.

Lencastre (1961), citado por Puertas (1994), distingue entre la microescala de turbulencia, que genera vórtices de pequeño tamaño que disipan una gran cantidad de energía, y la macroescala de turbulencia, en la que se engloban los vórtices de gran tamaño. Los pequeños vórtices tendrán frecuencias altas, mientras que los vórtices de mayor tamaño estarán asociados a bajas frecuencias. La zona intermedia es donde se disipa la mayor energía. El rango de mayores energías está asociado a las frecuencias entre 1 y 4 Hz. En este sentido, Castillo (1989) obtuvo que los vórtices de gran tamaño (del orden de las dimensiones del colchón) son los que controlan el movimiento general del agua.

Armengou (1991) obtiene:

$$
H_{m}^{\prime}=0.74 H_{m}
$$

donde $H_{m}{ }^{\prime}$ y $H_{m}$ son la raíz cuadrática media de la presión y la presión media registrada en el punto de estancamiento.

En la literatura no se produce una clara distinción entre aireación y atomización del chorro, mezclándose ambos conceptos. Castillo (2007) considera cuatro tipos de registros de presiones en función de que el chorro aireado haya alcanzado la atomización, y de su longitud de desarrollo en función del calado en el cuenco de disipación (Figura 19).

En el caso de chorros no desarrollados $\left(H<L_{b}\right)$ con colchones de agua poco profundos, las presiones se ajustan a una distribución normal y los registros muestran picos de presiones similares a ambos lados de la media, los cuales disminuyen al aumentar el colchón de agua en el cuenco. Cuando el chorro incide tras haber alcanzado la longitud de rotura $\left(H>L_{b}\right)$, los registros de presiones son más fluctuantes, aumentando con la atura de caída y con el colchón de agua. 

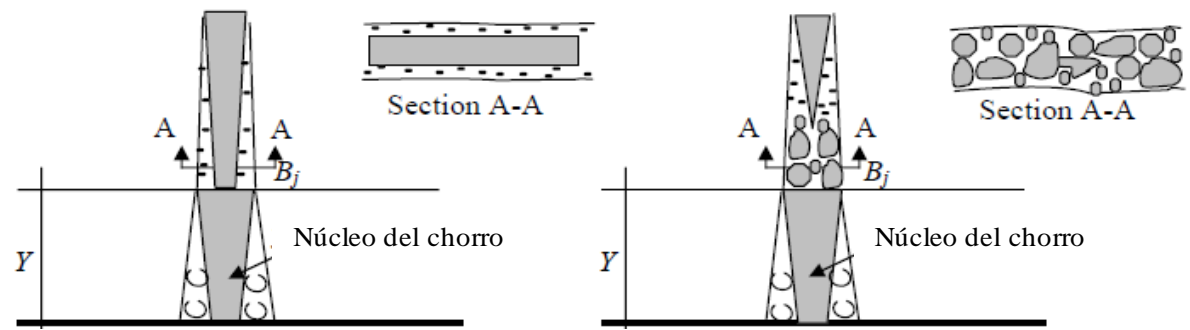

a) Chorro no desarrollado, $H / L_{b}<1$

b) Chorro desarrollado, $H / L_{b}>1$

Colchón no efectivo, $Y / B_{j}<4$

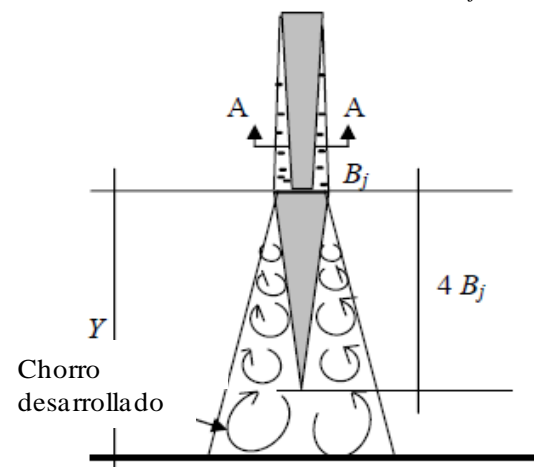

c) Chorro no desarrollado, $H / L_{b}<1$ Colchón efectivo, $Y / B_{j}>4$

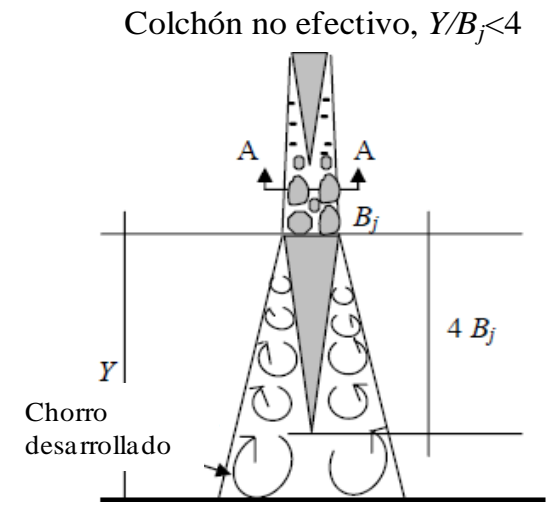

d) Chorro desarrollado, $H / L_{b}>1$ Colchón efectivo, $Y / B_{j}>4$

Figura 19. Esquema de los cuatro diferentes tipos de chorros (Castillo, 2007).

La Figura 20 muestra los resultados de presión dinámica fluctuante $C_{p}{ }^{\prime}$ obtenidos por diversos autores, la cual se estima como la relación entre la raíz cuadrática media de la presión en el punto de estancamiento $H_{m}$ ' y la energía cinética del chorro incidente $V_{j}^{2} / 2 g$. La raíz cuadrática media se ve influenciada por tres efectos: la intensidad turbulenta inicial del chorro $T_{u}$, el grado de longitud de rotura del chorro y los efectos de la aireación.

En la figura se observa que se requiere un calado mínimo para desarrollarse la energía contenida en los remolinos de modo que los efectos de difusión llegan a ser predominantes al incrementarse el calado, salvo para los datos del chorro con una gran oblicuidad (entre 40 y $50^{\circ}$ con la horizontal) obtenidos en chorros circulares por $\mathrm{Xu}$ Duo-Ming (1983). Posteriormente se produce un descenso casi lineal de la turbulencia.

Bollaert (2002) realiza mediciones con velocidades superiores a $20 \mathrm{~m} / \mathrm{s}$. El autor considera que el valor de la raíz cuadrática media puede considerarse independiente de la velocidad. Además, considera que los resultados están exentos de efectos de escala, representando resultados de prototipos. De este modo, obtiene un polinomio de tercer grado para calcular el coeficiente de presión dinámica fluctuante de chorros circulares:

$$
C_{p}^{\prime}=0.0022\left(Y / D_{j}\right)^{3}-0.0079\left(Y / D_{j}\right)^{2}+0.0716\left(Y / D_{j}\right)+0.0583
$$




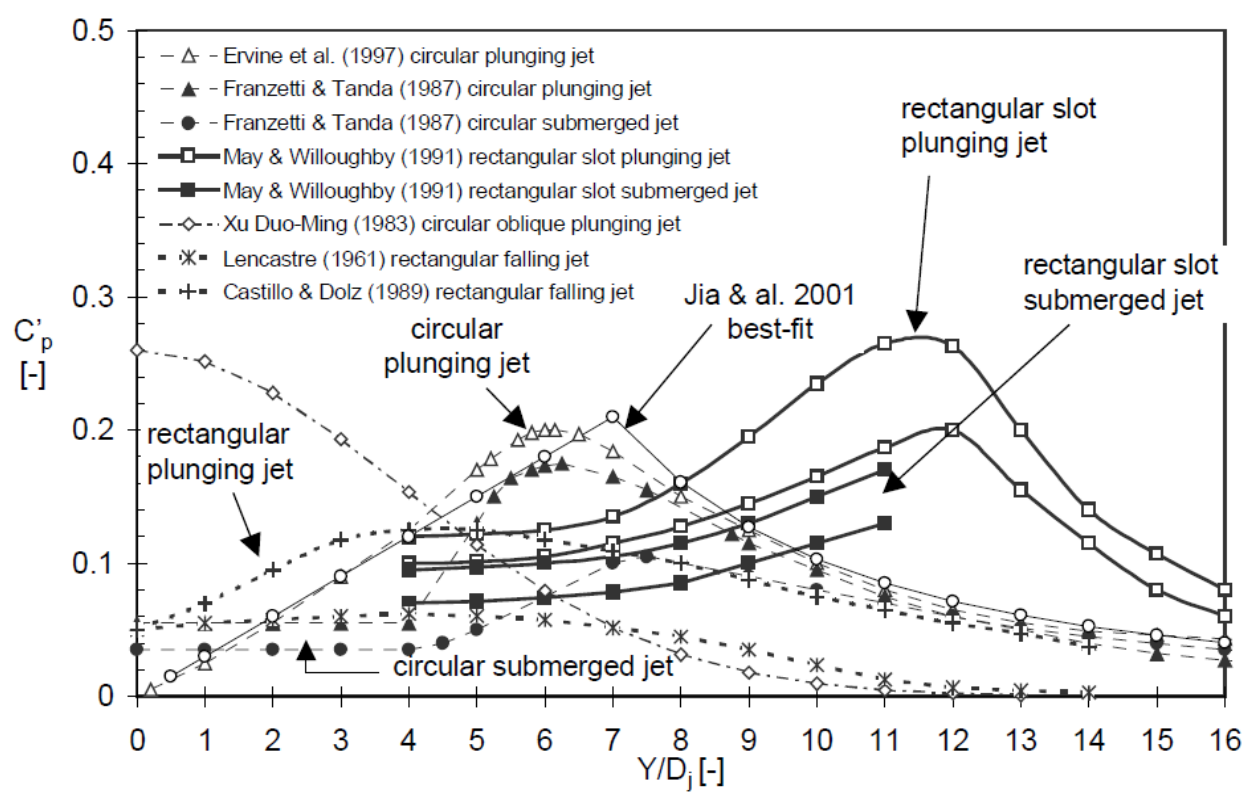

Figura 20. Presión dinámica fluctuante en función de y/D $D_{j}$ (Bollaert y Schleiss, 2001).

El primer y cuarto coeficiente de la ecuación (51) se modifican en función de la turbulencia inicial según la Tabla 7.

\begin{tabular}{|c|c|c|c|c|c|}
\hline Tu (\%) & $\boldsymbol{a}_{\mathbf{1}}$ & $\boldsymbol{a}_{\mathbf{2}}$ & $\boldsymbol{a}_{\mathbf{3}}$ & $\boldsymbol{a}_{\mathbf{4}}$ & Tipo de chorro \\
\hline$<1$ & 0.0022 & -0.0079 & 0.0716 & 0 & Compacto \\
$1-3$ & 0.00215 & -0.0079 & 0.0716 & 0.050 & Intermedio \\
$3-5$ & 0.00215 & -0.0079 & 0.0716 & 0.100 & Ondulante \\
$>5$ & 0.00215 & -0.0079 & 0.0716 & 0.150 & Muy ondulante \\
\hline
\end{tabular}

Tabla 7. Coeficientes del polinomio de regresión para diferentes intensidades turbulentas (Bollaert, 2002).

Castillo (2006) propone dos expresiones para calcular la presión dinámica fluctuante en chorros rectangulares, en función de las relaciones $H / L_{b}$ e $Y / B_{j}$.

- Si $Y / B_{j}<14$, considera un ajuste polinómico:

$$
C_{p}^{\prime}=a\left(Y / B_{j}\right)^{3}+b\left(Y / B_{j}\right)^{2}+c\left(Y / B_{j}\right)+d
$$

- $\quad$ Si $Y / B_{j} \geq 14$, aplica un ajuste potencial:

$$
C_{p}{ }^{\prime}=a\left(Y / B_{j}\right)^{b}
$$


donde los distintos coeficientes se recogen en la Tabla 8.

\begin{tabular}{|c|c|c|c|c|l|}
\hline \multicolumn{5}{|c|}{ Ajuste polinómico. Válido si $\mathbf{Y} / \boldsymbol{B}_{\boldsymbol{j}}<\mathbf{1 4}$} \\
\hline $\boldsymbol{H} / \mathbf{L}_{\boldsymbol{b}}$ & $\boldsymbol{a}$ & $\boldsymbol{b}$ & $\boldsymbol{c}$ & $\boldsymbol{d}$ & \multicolumn{1}{c|}{ Tipo de chorro } \\
\hline$\leq 1.4$ & 0.0003 & -0.0104 & 0.0900 & 0.083 & Compacto-Desarrollado-Desintegrado \\
$1.5-2$ & 0.0003 & -0.0094 & 0.0745 & 0.050 & Desarrollado-Desintegrado \\
$>2$ & 0.0002 & -0.0061 & 0.0475 & 0.010 & Desarrollado-Desintegrado \\
\hline \multicolumn{5}{|c|}{ Ajuste potencial. Válido si $\mathbf{Y} / \boldsymbol{B}_{\boldsymbol{j}} \geq \mathbf{1 4}$} \\
\hline $\boldsymbol{H} / \mathbf{L}_{\boldsymbol{b}}$ & $\boldsymbol{a}$ & $\boldsymbol{b}$ & Tipo de chorro \\
\hline$\leq 1.4$ & 5.30 & -1.405 & Compacto-Desarrollado-Desintegrado \\
$1.5-2$ & 3.14 & -1.422 & Desarrollado-Desintegrado \\
$>2$ & 1.50 & -1.500 & Desarrollado-Desintegrado \\
\hline
\end{tabular}

Tabla 8. Coeficientes para el cálculo de la presión dinámica fluctuante (Castillo,2006).

La Figura 21 muestra los resultados obtenidos por Bollaert (2002) para chorros circulares y diferentes intensidades turbulentas, junto a los resultados experimentales en chorros rectangulares de Castillo (1989) y Puertas (1994) y con el ajuste anterior. Se observa que el coeficiente de presión dinámica fluctuante máximo se obtiene para una relación $Y / B_{j} \approx 5$, lo que concuerda con el ajuste propuesto por Bollaert.

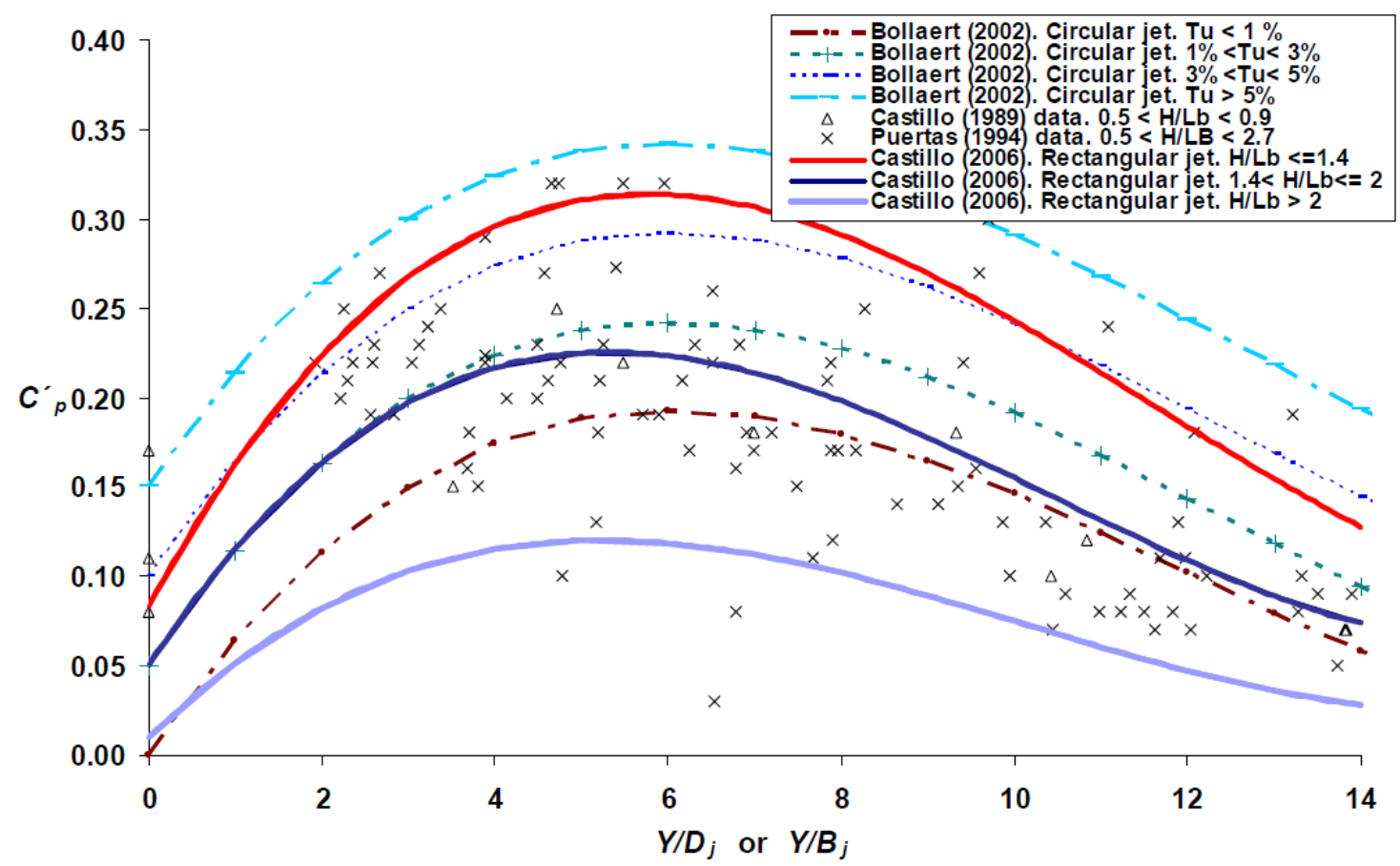

Figura 21. Coeficientes de presión dinámica fluctuante (Castillo, 2006). 


\subsubsection{Distribución de presiones en el entorno del punto de estancamiento}

Hartung y Häusler (1973) consideran que las presiones sobre una superficie perpendicular al chorro siguen una distribución normal.

Sin embargo, en sobrevertidos de presas la distribución de las presiones medias en la solera no es simétrica ya que el eje del chorro incide con una cierta inclinación. De este modo, aguas arriba del punto de estancamiento existe una zona de presión menor que aguas abajo.

A pesar de que la distribución de presiones no es simétrica, las leyes propuestas por los diferentes autores son de tipo simétrico exponencial, lo cual puede ser una buena aproximación ya que el ángulo del chorro a la entrada del cuenco está próximo a $90^{\circ}$ (Figura 22).

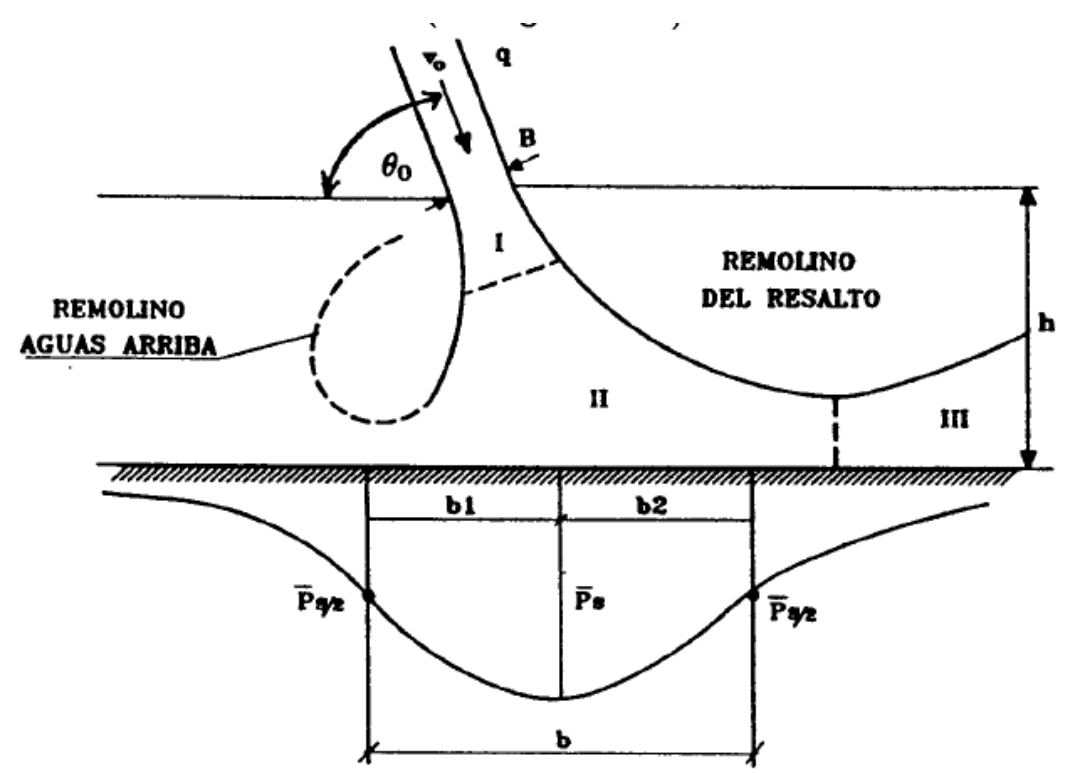

Figura 22. Zonas del flujo del chorro dentro del colchón de agua (Xu Duo-Ming, 1983, citado por Castillo, 1989).

Se define el valor $b$ como un factor de escala que cuantifica el ancho hasta el cual existe influencia del chorro, medido a partir del punto de estancamiento. Se determina como el valor de la abscisa $x$ que se obtiene cuando la presión es igual a la mitad de la presión media en el punto de estancamiento $\left(p=H_{m} / 2\right)$.

Castillo (1989) recopila los resultados obtenidos por diversos autores para el cálculo de la distribución de presiones en la solera del cuenco. Considera que las diferencias observadas entre las distintas publicaciones pueden deberse al empleo de diferentes metodologías de trabajo y condiciones de ensayo, algunos de los cuales no 
están bien documentados. Para comparar las distintas metodologías, aplica dos expresiones en función de la distancia de influencia, $b$, o de la altura del colchón, $Y$ :

$$
\begin{aligned}
& \frac{p}{H_{m}}=e^{-\Phi(x / b)} \\
& \frac{p}{H_{m}}=e^{-\Phi^{\prime}(x / Y)}
\end{aligned}
$$

siendo $p$ la presión dinámica media en la solera, $H_{m}$ la presión dinámica media en el punto de impacto, $b$ el ancho del punto de influencia, $x$ la abscisa media a partir del punto de estancamiento, e $Y$ la altura del colchón de agua. Los parámetros $\Phi$ y $\Phi^{\prime}$ se obtienen de la Tabla 9.

\begin{tabular}{|l|c|c|c|c|}
\hline \multicolumn{1}{|c|}{ Autor } & $\boldsymbol{\Phi}$ & $\boldsymbol{\Phi}^{\prime}$ & $\boldsymbol{b}$ & $\boldsymbol{y}_{\boldsymbol{K}}$ \\
\hline Cola (1966) & 0.685 & 40.51 & $0.13 Y$ (Beltaos) & \\
\hline Aki (1969) & 0.212 & 12.56 & $0.13 Y$ (Beltaos) & \\
\hline Hartung y Häusler (1973) & 0.664 & 12.56 & $0.23 Y$ (Xu Duo-Ming) & \\
\hline Beltaos (1976) & 0.688 & 40.71 & $0.13 Y$ (Beltaos) & $7.2 B$ (Castillo) \\
\hline
\end{tabular}

Tabla 9. Parámetros de las ecuaciones para el cálculo de las presiones dinámicas medias (Castillo, 1989).

La Figura 23 muestra el adecuado ajuste obtenido con los datos experimentales al considerar las expresiones de Bealtos, Cola, Hartung y Häusler con $y_{K}=7.20 B$, y Aki con $b=0.23 Y$.

Las ecuaciones de Aki con $b=0.23 Y$ y de Hartung y Häusler con $y_{K}=5 B$ obtienen valores de envolventes superiores a las presiones dinámicas medias, sobreestimando los valores tanto en magnitud como en longitud de influencia.

Ervine et al. (1997) consideraron que la variación del coeficiente de presión dinámica media en la dirección longitudinal $C_{p}(x)$ puede ser obtenida con una expresión general de tipo:

$$
\frac{C_{p}(x)}{C_{p}}=e^{-K_{2}\left(\frac{x}{Y}\right)^{2}}
$$


donde $x$ es la distancia horizontal al punto de estancamiento e $Y$ el colchón de agua en el cuenco. El parámetro $K_{2}$ varía entre 30 para calados poco profundos $\left(Y / D_{j} \leq 4\right)$ hasta 50 para colchones grandes (Figura 24).

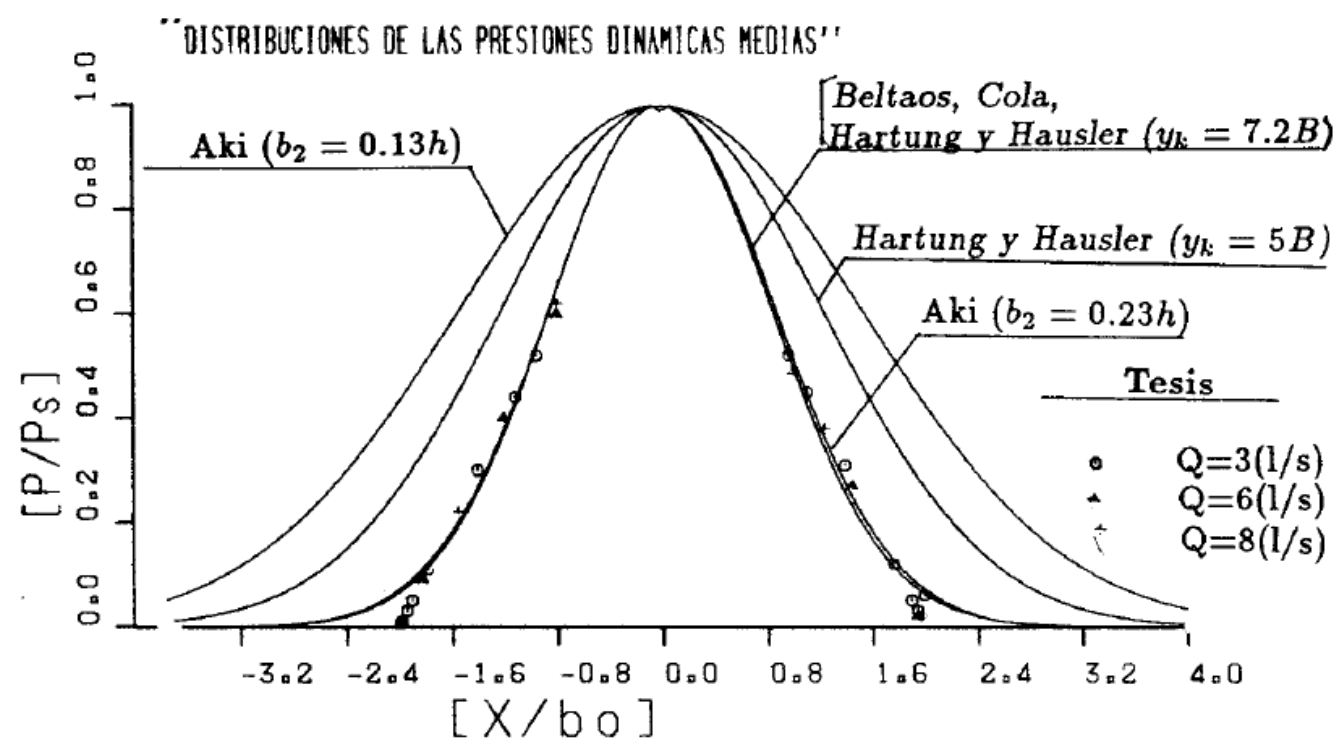

Figura 23. Distribución de presiones dinámicas medias propuestas por diferentes autores (Castillo, 1989).

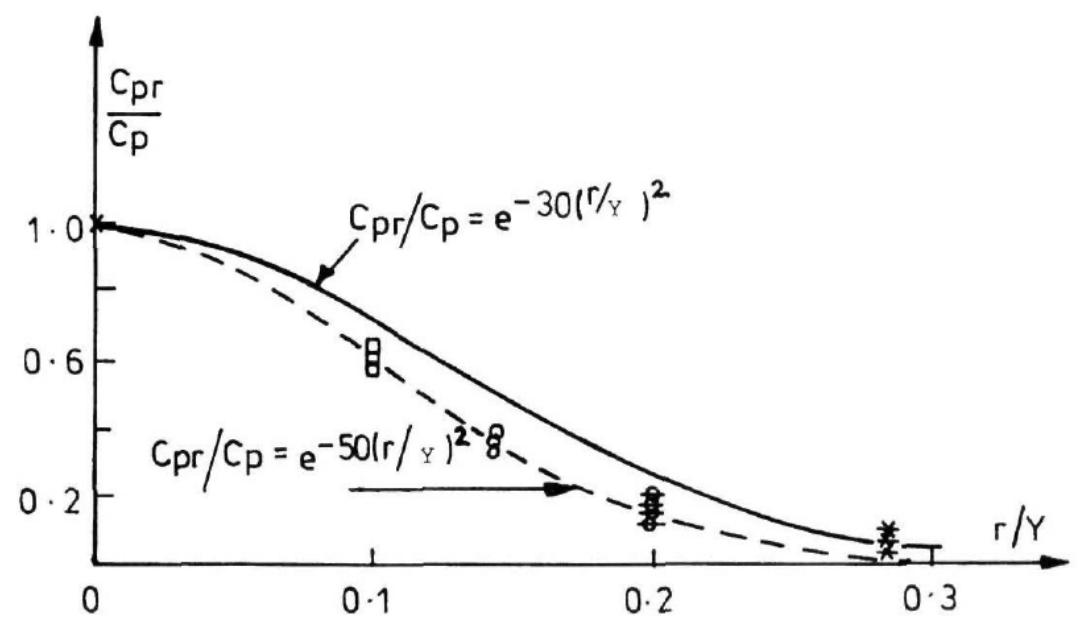

Figura 24. Variación del coeficiente de presión dinámica media con la distancia radial al eje del chorro (Ervine et al., 1997).

De un modo similar, Bollaert (2002) consideró que la distribución longitudinal del coeficiente de presión dinámica media $C_{p}(x)$ puede ser obtenida en función de la relación adimensional $x / x_{\max }$ : 


$$
\frac{C_{p}(x)}{C_{p}}=e^{-K_{3}\left(\frac{x}{x_{\max }}\right)^{2}}
$$

donde $x_{\max }$ es la extensión longitudinal máxima del área influenciada por la capa de corte turbulenta del chorro incidente. Este parámetro se define como $x_{\max }=0.5 D_{j}+$ $0.25 Y$. El valor de $0.25 Y$ se obtiene de la expansión del chorro dentro del colchón de agua en el cuenco (según McKeogh y Elsawy, 1980, la expansión está gobernada por un ángulo exterior de $13-14^{\circ}$ ).

Para chorros circulares, el parámetro $K_{3}$ varía entre 3 para colchones poco profundos y 6 para mayores colchones de agua (Figura 25).

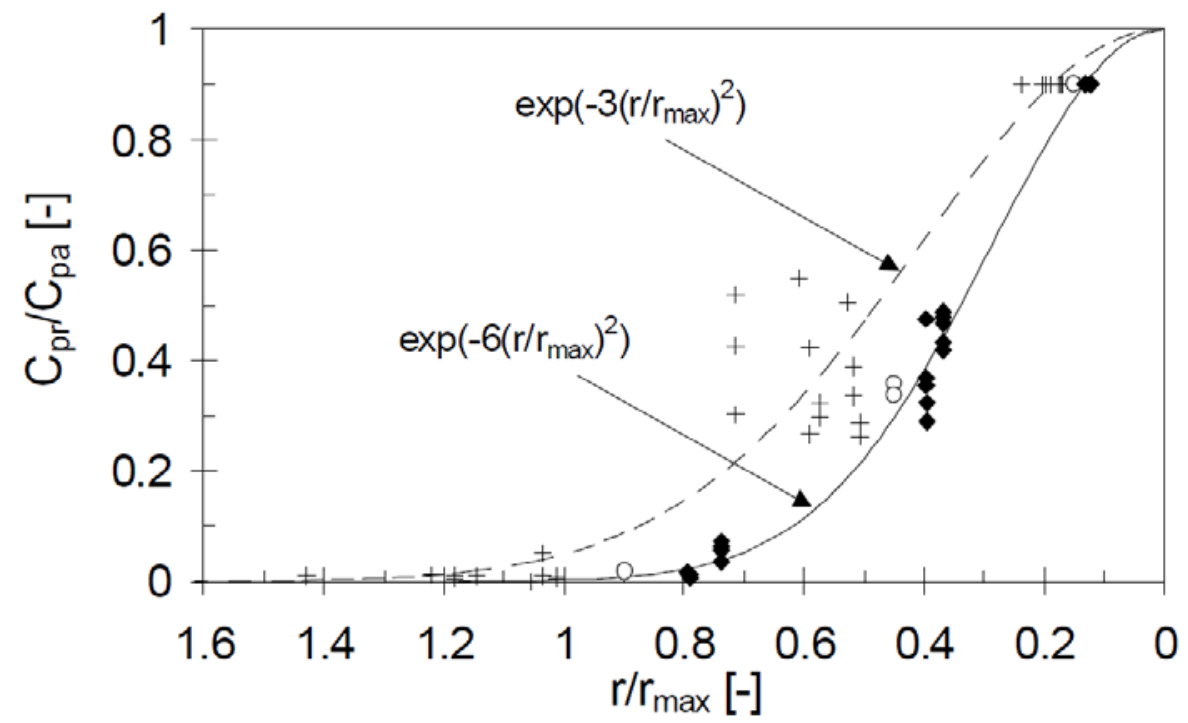

Figura 25. Distribución radial del coeficiente de presión dinámica media (Bollaert, 2002).

Bollaert (2002) también obtuvo relaciones para la distribución longitudinal del coeficiente de presión dinámica fluctuante $C_{p}^{\prime}(x)$ en chorros circulares (Figura 26):

- Para chorros desarrollados:

$$
\frac{C_{p}^{\prime}(x)}{C_{p}^{\prime}}=e^{-3\left(\frac{x}{x_{\max }}\right)^{2}}
$$

- Para chorros compactos con $x>0.5 x_{\text {máx: }}$

$$
\frac{C_{p}^{\prime}(x)}{C_{p}^{\prime}}=e^{-3\left(\frac{x}{x_{\max }}-0.5\right)^{2}}
$$


- Para chorros compactos con $x<0.5 x_{\text {máx: }}$

$$
\frac{C_{p}^{\prime}(x)}{C_{p}^{\prime}}=1
$$

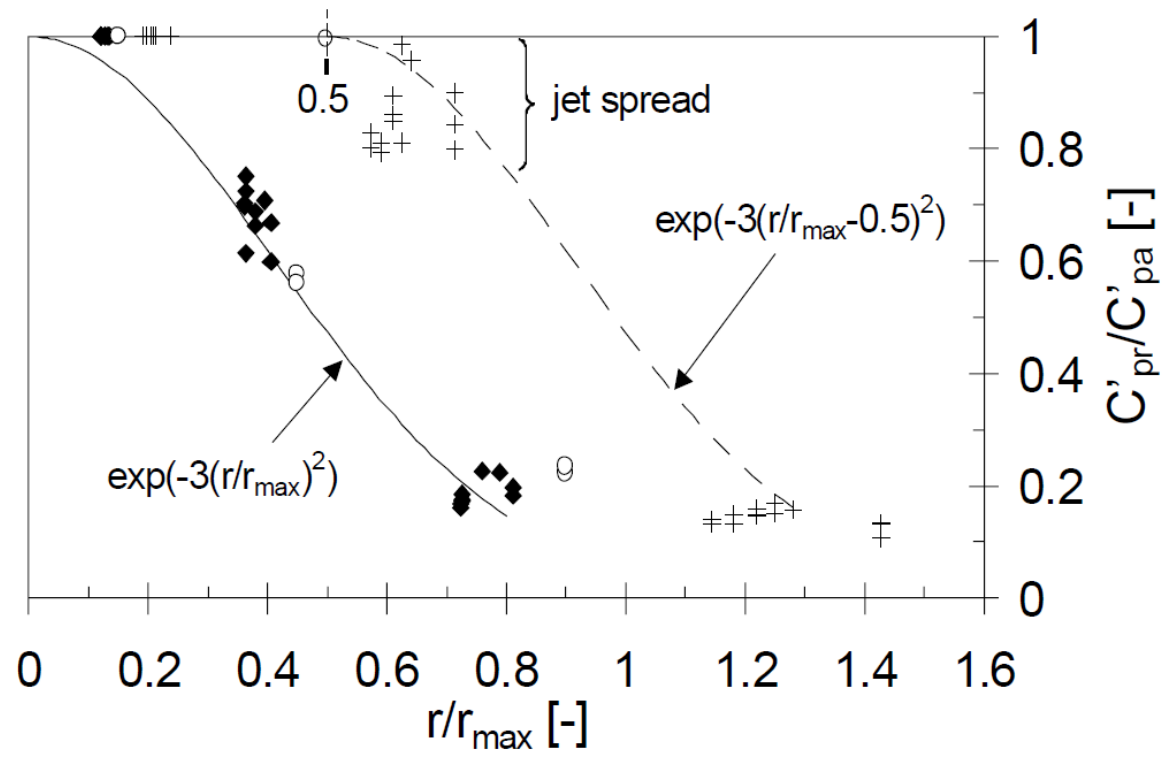

Figura 26. Distribución radial del coeficiente de presión dinámica fluctuante (Bollaert, 2002).

\subsubsection{Presiones extremas instantáneas en el punto de estancamiento}

Según Lencastre (1961), citado por CNEGP (1997), los máximos valores de fluctuación se dan para una relación $Y / B_{j}<11.4$, llegando a valer 2.8 veces el valor de la presión media. Las máximas fluctuaciones se reducen notablemente para valores $21.1<Y / B_{j}<$ 30.2 y desaparecen prácticamente para colchones de espesor superior.

Ervine et al. (1997) obtuvieron valores extremos de hasta 4 veces la raíz cuadrática media de la presión para valores positivos y hasta 3 veces para valores negativos, lo que concuerda con el sesgo positivo que normalmente se observa en resaltos hidráulicos.

Respecto a los valores de presiones instantáneas extremas, diversos autores han encontrado valores entre 2 y 4 veces el correspondiente a su raíz cuadrática media (Bollaert y Schleiss, 2001).

Estos valores extremos se deben considerar con precaución debido a los ensayos relativamente cortos. Toso y Bowers (1988), citado por Bollaert (2002) encontraron en un resalto hidráulico que los valores extremos eran el doble en un registro de 24 horas que en uno de 10 minutos. 
Cui Guang Tao et al. (1985), citado por Castillo (1989), deducen la siguiente expresión:

$$
\frac{P_{m a ́ x}}{H}=0.68^{\left(\frac{Y}{y_{c}}-1.5\right)} ; \quad \frac{2 A_{m}}{H}=0.73^{\left(\frac{Y}{y_{c}}\right)}
$$

que representan el valor máximo en el punto de estancamiento y la amplitud máxima de las fluctuaciones, siendo $2 A_{m}=P_{\text {máx }}-P_{\min }$, la amplitud máxima de la presión. $P_{\text {máx }} y$ $P_{\min }$ son la presión instantánea máxima y mínima del registro. $Y$ es el calado en el cuenco de disipación, mientras que $y_{c}$ es el calado crítico.

Castillo (1989) obtuvo que la expresión que relaciona $P_{\text {máx }}$ con $H$, ecuación (61), es válida para los valores $Y / y c \geq 1.5$ medidos en su tesis, mientras que una parábola se ajustaría mejor para el intervalo $0 \leq Y / y c \leq 1.5$, aunque se requerirían más ensayos (Figura 27). Para la relación $2 A_{m} / H$ obtuvo un adecuado ajuste de sus datos en el intervalo $Y / y c \geq 2.0$, mientras que se requeriría otro tipo de ajuste en el intervalo $0 \leq$ $Y / y c \leq 2.0$ (Figura 28).

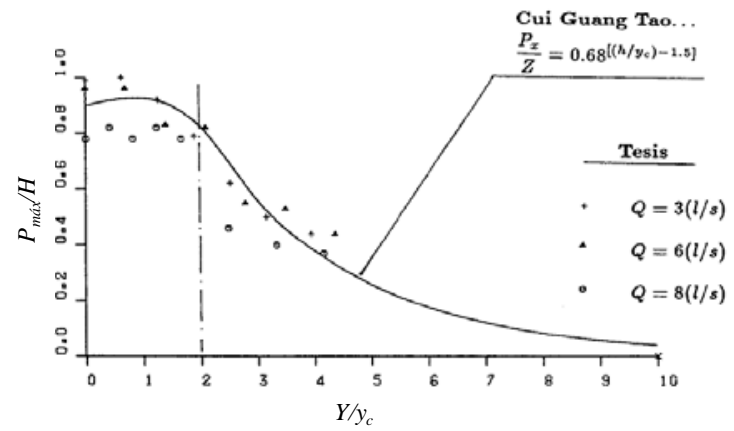

Figura 27. Presiones dinámicas máximas en el punto de estancamiento en función de la altura del cochón (Castillo, 1989).

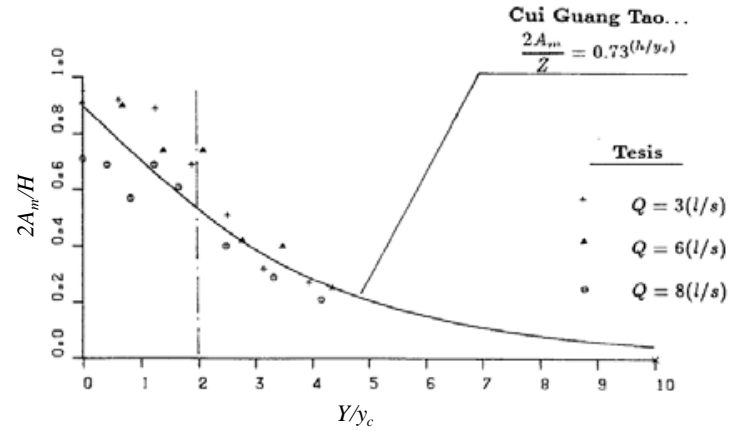

Figura 28. Amplitudes máximas de presión en el punto de estancamiento en función de la altura del cochón (Castillo, 1989).

Definiendo las semiamplitudes superiores e inferiores como la diferencia de la presión máxima y mínima con respecto a la presión media $\left(A_{\text {sup }}=P_{\text {máx }}-H_{m}\right.$ y $A_{\text {inf }}=H_{m}$ - $P_{\text {mín }}$ ), al adimensionalizarlas respecto de la altura neta de caída $H$, Castillo (1989) obtuvo una dependencia débil de las mismas respecto al caudal, siendo posible ajustar una sola curva para cada familia de semiamplitudes (Figura 29).

Las semiamplitudes superiores $A_{\text {sup }}$ alcanzan los valores máximos para relaciones $Y / B_{j}<6$, pudiendo obtenerse hasta un $55 \%$ de la altura neta de caída $H$. Para $Y / B_{j}>6$, las semiamplitudes disminuyen rápidamente hasta llegar a ser del orden del $10 \%$ de la carga total del embalse. 


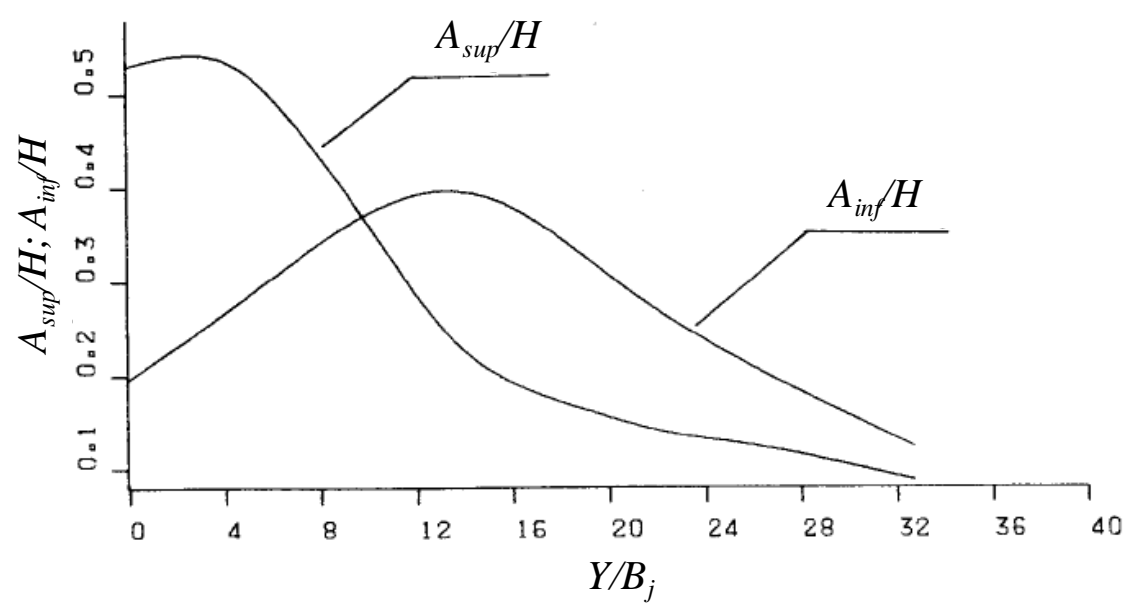

Figura 29. Semiamplitudes de presión en el punto de estancamiento $\left(A_{\text {sup }} / H\right.$ y $\left.A_{\text {inf }} / H\right)$ en función de la relación $Y / B_{j}$ (Castillo, 1989).

Las semiamplitudes inferiores $A_{\text {inf }}$ se inician con valores del orden del $20 \%$ de la carga del embalse, obteniendo los valores máximos en el intervalo $12<Y / B_{j}<18$, con valores en torno al $40 \%$ de la carga. Para relaciones mayores, disminuyen hasta llegar a valores del orden del $10 \%$ de $H$.

A partir de los valores de presiones extremas instantáneas de los registros, $P_{\text {máx }} \mathrm{y}$

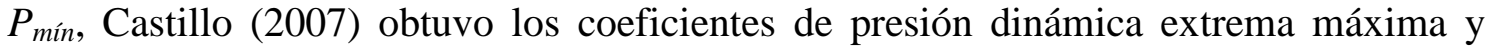
mínima $C_{p}^{+}$y $C_{p}^{-}$(Figura 30 y Figura 31, respectivamente).

$$
\begin{aligned}
& C_{p}^{+}=\frac{P_{\text {máx }}-H_{m}}{V^{2} / 2 g} \\
& C_{p}^{-}=\frac{H_{m}-P_{\text {min }}}{V^{2} / 2 g}
\end{aligned}
$$

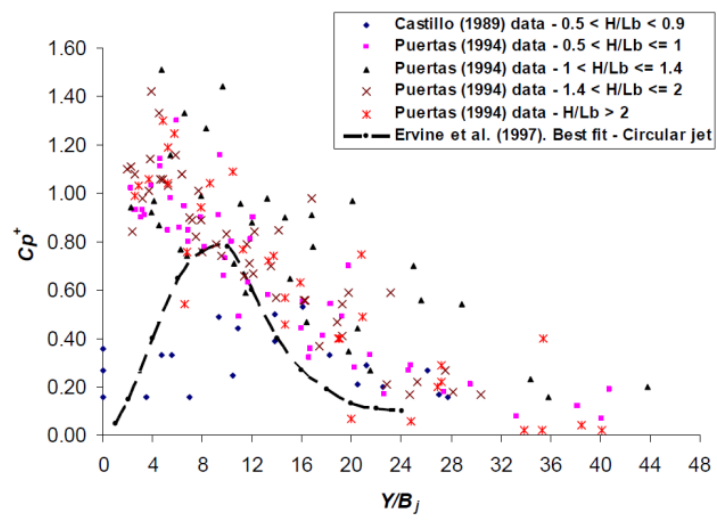

Figura 30. Coeficiente de presión dinámica extrema máxima, $C_{p}^{+}$(Castillo, 2007).

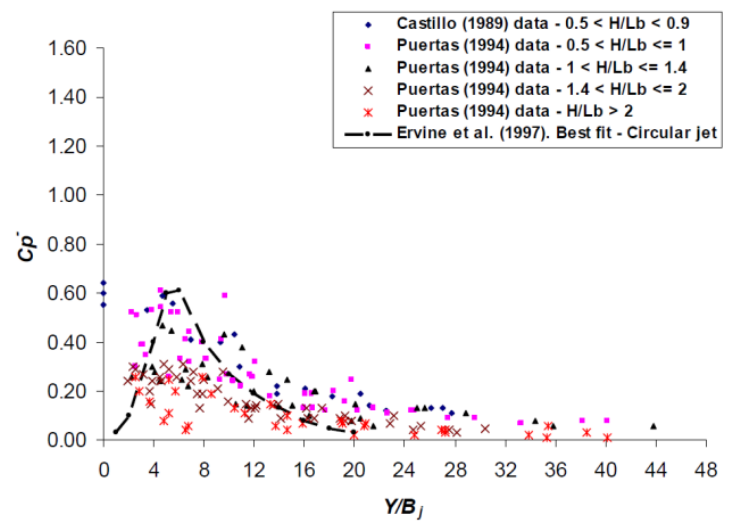

Figura 31. Coeficiente de presión dinámica extrema mínima, $C_{p}^{-}$(Castillo, 2007). 
Los valores obtenidos no permitieron realizar ningún tipo de agrupamiento.

Se observa que los valores de $C_{p}{ }^{+}$son en general superiores a los comentados en la bibliografía existente de chorros circulares (Ervine et al., 1997, Bollaert y Schleiss, 2003). Sin embargo, los valores extremos negativos concuerdan con la bibliografía.

\subsubsection{Distribución de velocidades en el cuenco}

Considerando que $V_{j}$ es la velocidad del chorro incidente en el colchón de agua, $B_{j}$ el espesor del chorro, y $\beta$ el ángulo que forma el chorro incidente con la horizontal, a partir del análisis dimensional de las ecuaciones de Navier-Stokes promediadas de Reynolds, se pueden definir las características básicas del flujo principal en un cuenco de disipación (Rajaratnam, 1976, citado por Liu et al., 1998):

$$
\begin{gathered}
\frac{\partial u_{j}}{\partial x_{j}}=0 \\
\frac{\partial u_{i} u_{j}}{\partial x_{j}}+\frac{\partial u_{i}}{\partial x_{i}}=\frac{1}{\rho} \frac{\partial}{\partial x_{j}}\left(\tau_{i j}-\rho \overline{u_{\imath}^{\prime} u_{\jmath}^{\prime}}\right)
\end{gathered}
$$

donde $u_{i}$ es la componente de la velocidad media en el eje $i, \rho$ la densidad del agua, $\tau$ el tensor de esfuerzos viscosos, $\mathrm{y}-\rho \overline{u_{\imath}^{\prime} u_{\jmath}^{\prime}}$ las tensiones de Reynolds.

Si en cada sección transversal del resalto sumergido se considera la máxima velocidad media de la sección del chorro $V_{\text {máx }}$ y la altura $\delta_{l}$ en la que la velocidad $V=$ $V_{\text {máx }} / 2$ como escala característica del campo de velocidades medias en el chorro (Figura 32), a partir de relaciones de semejanza se puede obtener:

$$
\frac{V}{V_{\text {máx }}}=f(\eta) ; \quad \frac{\tau}{\rho u_{\text {máx }}^{2}}=S(\eta) ; \quad \eta=\frac{y}{\delta_{l}}
$$

Para un chorro plano, usando la conservación de cantidad de movimiento en la dirección principal del flujo $x$ junto a las ecuaciones anteriores, se puede obtener:

$$
V_{\text {máx }} \propto 1 / \sqrt{x} ; \quad \delta_{l} \propto x
$$

Para un chorro sumergido en un colchón finito, usando la conservación de masa en la dirección del flujo principal, Guo (1982) obtuvo las siguientes relaciones:

$$
V_{\text {máx }} \propto 1 / x ; \quad \delta_{l} \propto x
$$




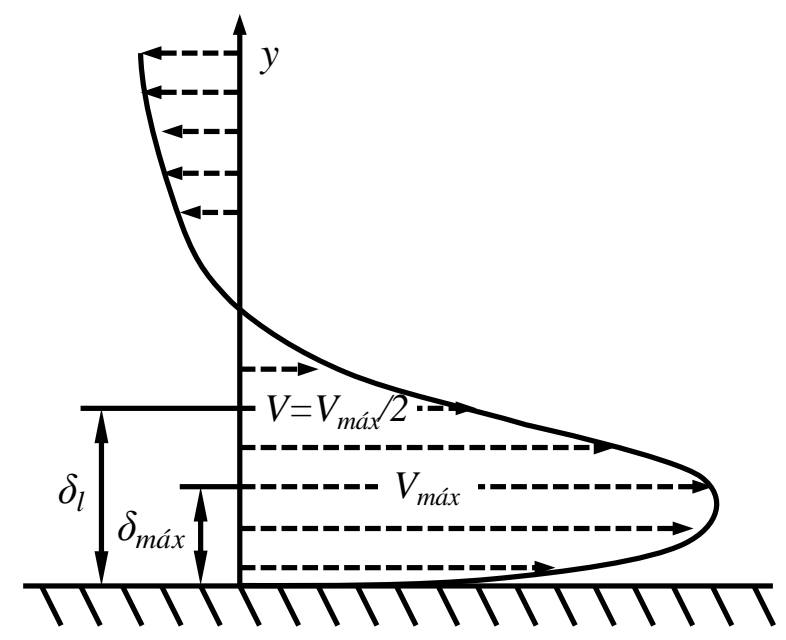

Figura 32. Definición esquemática del perfil de velocidades medias en un resalto sumergido (adaptado de Wu y Rajaratnam, 1995).

Considerando un chorro incidente en un cuenco de amortiguación, las escalas características se pueden relacionar con la zona de sumergencia del chorro como:

$$
\frac{V_{\text {máx }}}{V_{j}} \propto\left(\frac{x}{B_{j}}\right)^{-m} ; \quad \frac{\delta_{l}}{B_{j}} \propto \frac{x}{B_{j}}
$$

donde el exponente $m$, con valores entre 0.50 y 1.00, está relacionado con el colchón $Y$, y $B_{j}$ es el espesor del chorro incidente.

Para obtener el perfil de velocidad media adimensional del chorro, diversos autores proponen expresiones de ajuste (Tabla 10).

Chanson y Bratteberg (2000) utilizaron una sonda de Pitot para analizar la zona inferior de resaltos libres (zona poco aireada) y una sonda de conductividad en la zona superior (Figura 33). Los resultados muestran las velocidades horizontales adimensionalizadas con los dos equipos $V / U_{1}$, la concentración de aire $C$, y la frecuencia de las burbujas $f_{a b}$, frente al calado adimensionalizado. La curva de velocidad adimensional muestra un buen ajuste en la zona inferior con la fórmula propuesta por Ohtsu et al. (1990), mientras que para relaciones $V / V_{\text {máx }}<0.40$ el flujo está más aireado y existe una mayor dispersión.

Usando la función error, Lin et al. (2012) obtuvieron un ajuste del 97\% a sus datos. La curva de ajuste propuesta se comparó también con los resultados de Rajaratnam (1965) y de Chanson y Brattberg (2000). Los valores de Rajaratnam mostraron una tendencia similar, mientras que las mediciones de Chanson y Brattberg tuvieron una mayor dispersión alrededor de la curva de ajuste propuesta (Figura 34). 


\begin{tabular}{|l|c|}
\hline \multicolumn{1}{|c|}{ Autor } & Expresión \\
\hline $\begin{array}{l}\text { Görtler (1942), } \\
\text { citado por Liu et al. (1998) }\end{array}$ & $\frac{V}{V_{\text {máx }}}=1-\tanh ^{2}\left(0.881 \frac{y}{\delta_{l}}\right)$ \\
\hline Rajaratnam (1976) & $\frac{V}{V_{\text {máx }}}=e^{-0.693\left(\frac{y}{\delta_{l}}\right)^{2}}$ \\
\hline $\begin{array}{l}\frac{V}{V_{\text {máx }}}=1.48\left(\frac{y}{\delta_{1}}\right)^{1 / 7}\left(1-\operatorname{erf}\left(0.68 \frac{y}{\delta_{l}}\right)\right) \\
\text { citado por Chanson y Brattberg (2000) }\end{array}$ & $\frac{V}{V_{\text {máx }}}=2\left(5 \frac{y}{\delta_{l}} e^{\left.1-5 \frac{y}{\delta_{l}}\right)}\right)^{0.12}$ \\
\hline Ohtsu et al. (1990) & $\frac{V}{V_{\text {máx }}}=e^{-0.5\left(1.765 \frac{y-\delta_{\text {máx }}}{\delta_{l}}\right)^{2}}$ \\
& $\frac{V}{V_{\text {máx }}}=e^{-0.5\left(1.814 \frac{y-\delta_{\text {máx }}}{\delta_{l}}\right)^{2}}$ \\
\hline Lin et al. (2012) & $\frac{V}{V_{\text {máx }}}=2.3\left(\frac{y}{\delta_{l}}\right)^{0.42}\left(1-\operatorname{erf}\left(0.886 \frac{y}{\delta_{l}}\right)\right)$ \\
\hline
\end{tabular}

NOTA: $\operatorname{erf}(u)=\frac{2}{\sqrt{\pi}} \int_{0}^{u} e^{-t^{2}} d t$ es la función error.

Tabla 10. Expresiones para el cálculo de perfiles de velocidad longitudinal adimensional.

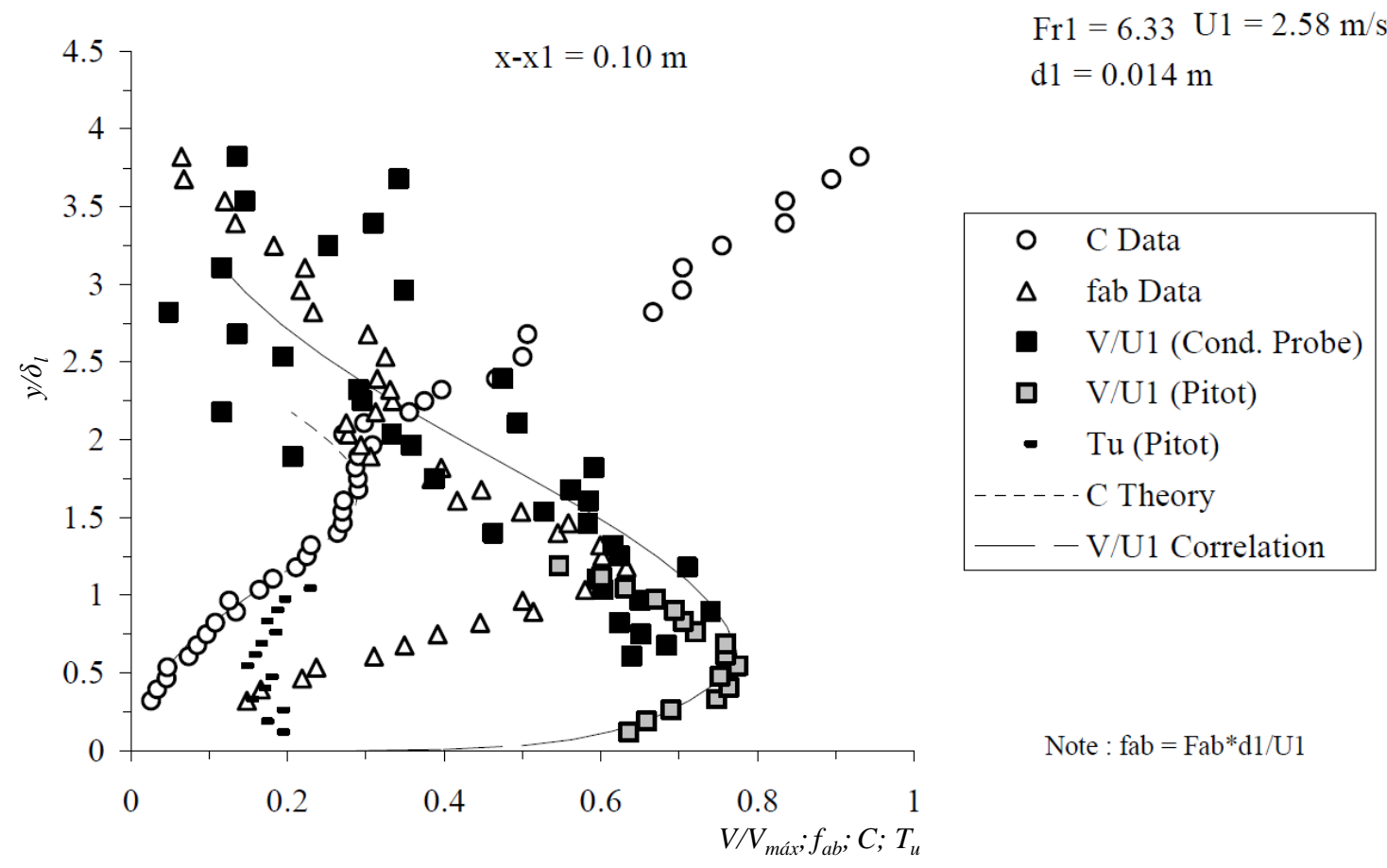

Figura 33. Distribuciones típicas de fracción de vacío $C$, velocidad adimensional y frecuencia de burbujas adimensional $F_{a b}$ en resaltos hidráulicos (Chanson y Brattberg, 2000). 


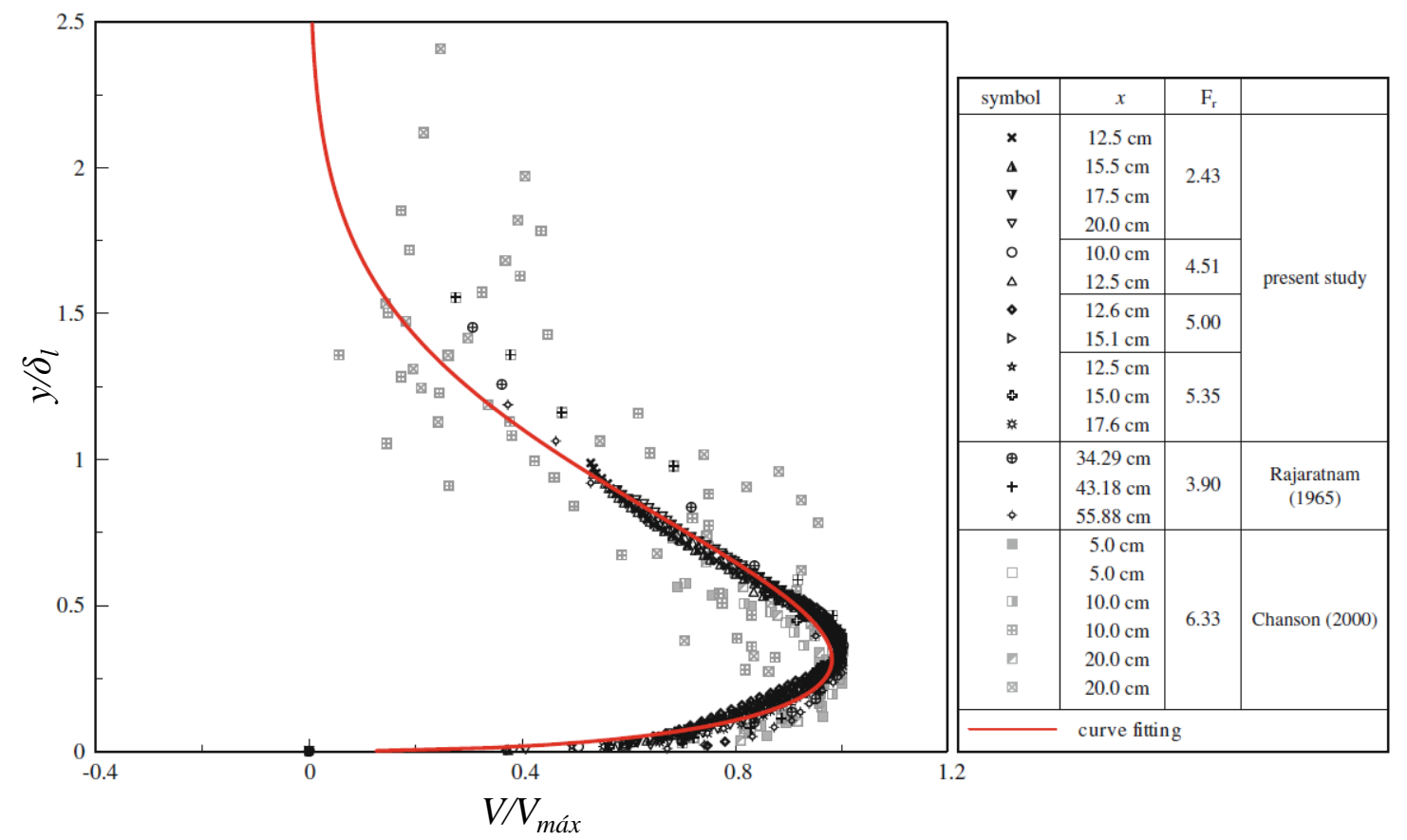

Figura 34. Perfil de velocidad media horizontal de un chorro plano (Lin et al., 2012).

Wu y Rajaratnam (1995) analizaron la longitud característica $\delta_{l}$ que define el campo de velocidades en resaltos sumergidos en función de la distancia al inicio del resalto, adimensionalizados en función del ancho del chorro que entra en el resalto $B_{j}$ (Figura 35). Obtuvieron que la mayoría de las observaciones en resaltos sumergidos caían en la banda \pm 1 desviación estándar de la línea que delimita los chorros de pared, mientras que sólo los datos cercanos al final del resalto mostraban un crecimiento del espesor de la capa de mezcla.

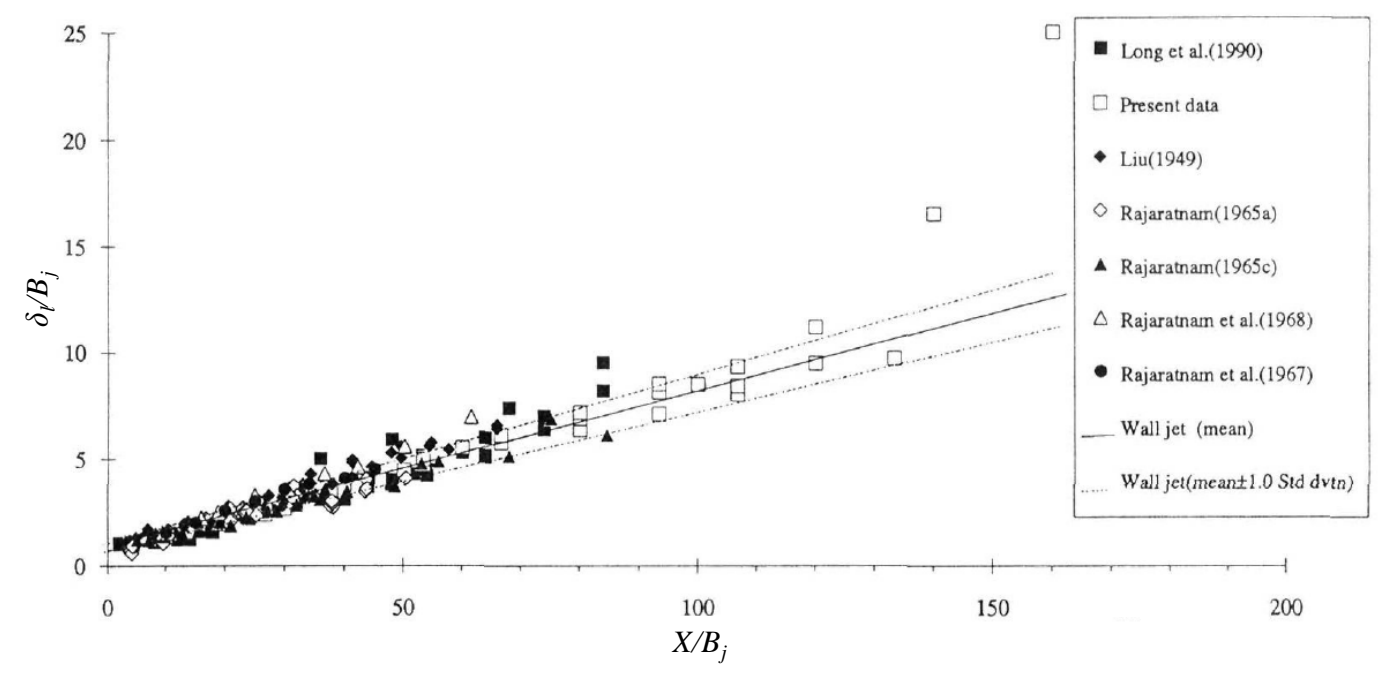

Figura 35. Variación de la longitud característica $\delta_{l}$ en resaltos sumergidos (Wu y Rajaratnam, 1995). 


\subsubsection{Pérdida de energía en el cuenco}

En muchas aplicaciones la función principal del resalto hidráulico es la disipación de energía. En un canal horizontal, el cambio de la energía entre las secciones de aguas arriba y aguas abajo del resalto sumergido se obtiene como:

$$
H_{L}=\left(\frac{V_{j}^{2}}{2 g}+y_{3}\right)-\left(\frac{V_{4}^{2}}{2 g}+y_{4}\right)
$$

donde $y_{3}$ e $y_{4}$ son los calados en el cuenco de disipación aguas arriba y aguas abajo del resalto sumergido formado al pie del punto de incidencia del chorro.

Usando la ecuación (70) junto con la ecuación de continuidad, la pérdida de energía puede ser obtenida mediante la expresión:

$$
\frac{H_{L}}{H_{0}}=\frac{2\left(\frac{y_{3}}{y_{0}}-\frac{y_{4}}{y_{0}}\right)+\left(1-\frac{1}{\left(y_{4} / y_{0}\right)^{2}}+F_{0}^{2}\right)}{2\left(y_{3} / y_{0}\right)+F_{0}^{2}}
$$

Cuando $y_{3} / y_{0}=1$, y $F_{0}=F_{1}$, se tiene la expresión de resaltos hidráulicos libres. 


\section{METODOLOGÍA EXPERIMENTAL}

\subsection{Infraestructura de laboratorio}

El Laboratorio de Hidráulica de la Universidad Politécnica de Cartagena cuenta con una infraestructura para el estudio de chorros turbulentos y bifásicos aire-agua (Figura 36), la cual permite analizar la disipación de energía de chorros turbulentos rectangulares.

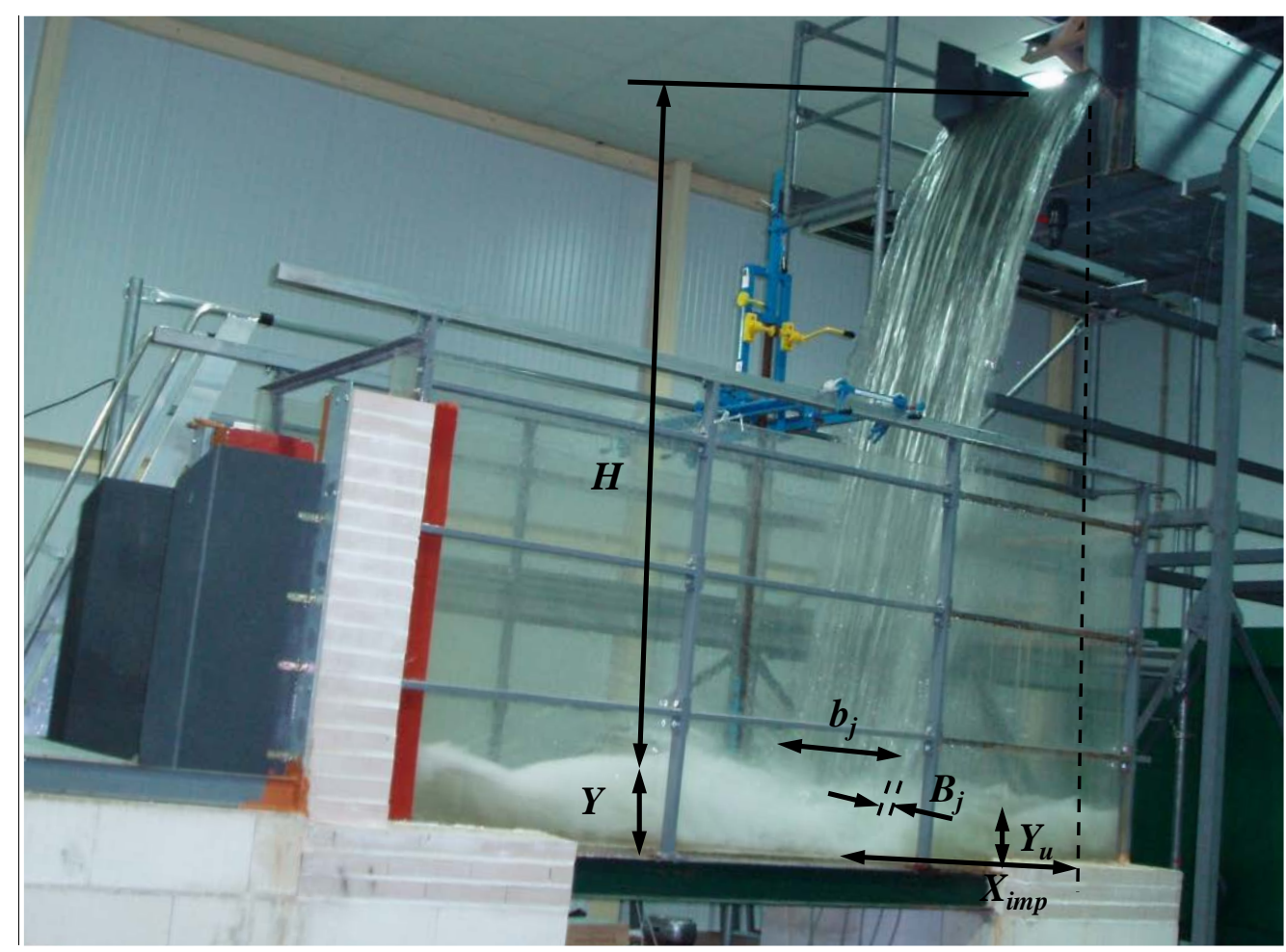

Figura 36. Infraestructura de laboratorio para el estudio de chorros turbulentos y bifásicos.

Un mecanismo móvil permite variar la altura de descarga entre 1.70 y $4.00 \mathrm{~m}$, mientras que el caudal de ensayo puede ser regulado mediante una válvula de mariposa entre 10 y 150 l/s.

El canal de entrada, donde se han dispuesto diferentes sistemas de disipación y orientación de las líneas de corriente, tiene $3.00 \mathrm{~m}$ de longitud y $0.95 \mathrm{~m}$ de ancho. El vertedero es un perfil de cresta delgada con $0.85 \mathrm{~m}$ de ancho y $0.37 \mathrm{~m}$ de altura respecto la base del canal de entrada (Figura 37). 

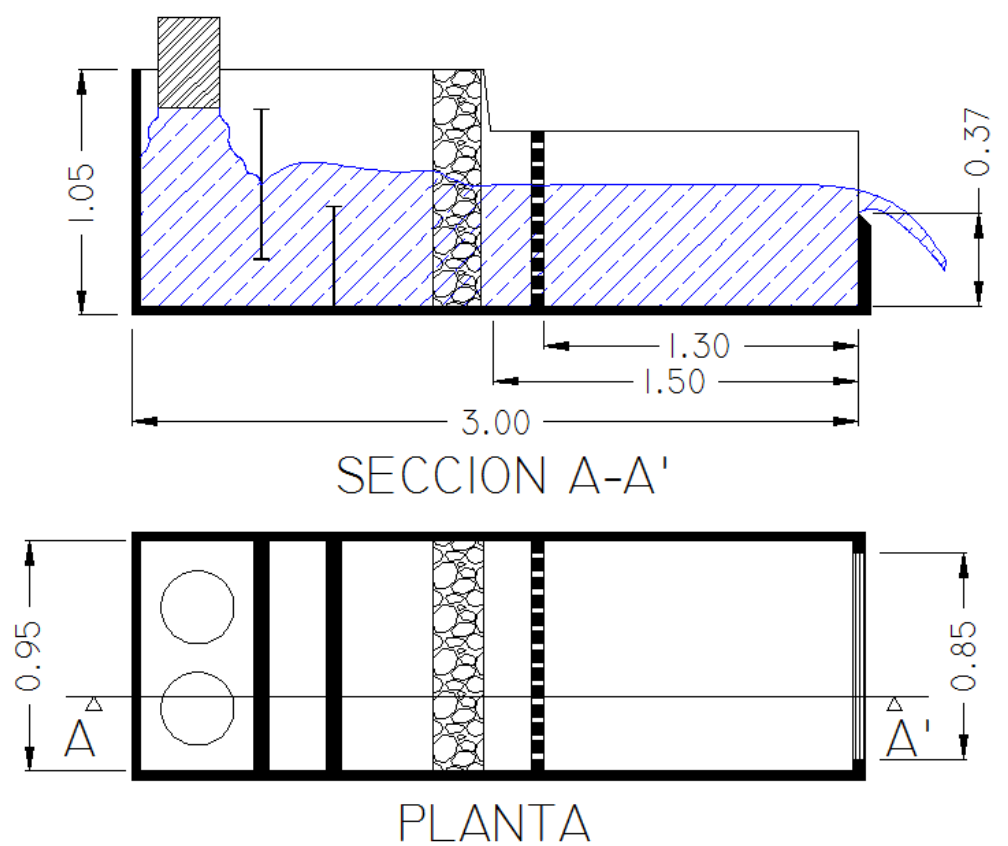

Figura 37. Canal de entrada en la infraestructura de laboratorio.

Los vertederos planos rectangulares se pueden agrupar en tres tipos:

- Vertederos de contracción total. Son vertederos que tienen un canal de aproximación con los muros y la solera suficientemente alejados de los límites del vertedero, de modo que no generan una influencia significativa de los límites del canal de aproximación en la contracción de la lámina.

- Vertederos de ancho total. Son vertederos que se extienden a lo largo de todo el ancho del canal de aproximación $\left(B_{1} / b_{c}=1.0\right)$. Son conocidos como vertederos de Rehbock.

- Vertedero de contracción parcial. Son vertederos con contracción en los cuales la proximidad de los muros o del fondo del canal de aproximación influyen de modo no despreciable en la lámina vertiente.

En general, los tres tipos de vertederos rectangulares deberían estar localizados en un canal de aproximación rectangular. Si el canal de aproximación es suficientemente largo $\left(B_{1}\left(h+P_{l}\right) \geq 10 b_{c} h\right)$ para poder considera que la velocidad es despreciable, y el vertedero es de contracción total, la forma del canal de aproximación no influye en la forma de la lámina vertiente.

El vertedero totalmente contraído debe cumplir las siguientes limitaciones: 


$$
\begin{aligned}
& B_{1}-b_{c} \geq 4 h \\
& h / P_{l} \leq 0.5 \\
& h / b_{c} \leq 0.5 \\
& 0.07 m \leq h \leq 0.60 m \\
& b_{c} \geq 0.30 \mathrm{~m} \\
& P_{l} \geq 0.30 \mathrm{~m}
\end{aligned}
$$

donde $B_{1}$ es el ancho del canal de aproximación, $b_{c}$ es el ancho del labio del vertedero, $h$ es la carga sobre el vertedero y $P_{l}$ es la altura del labio del vertedero. Las distintas variables se muestran en la Figura 38.

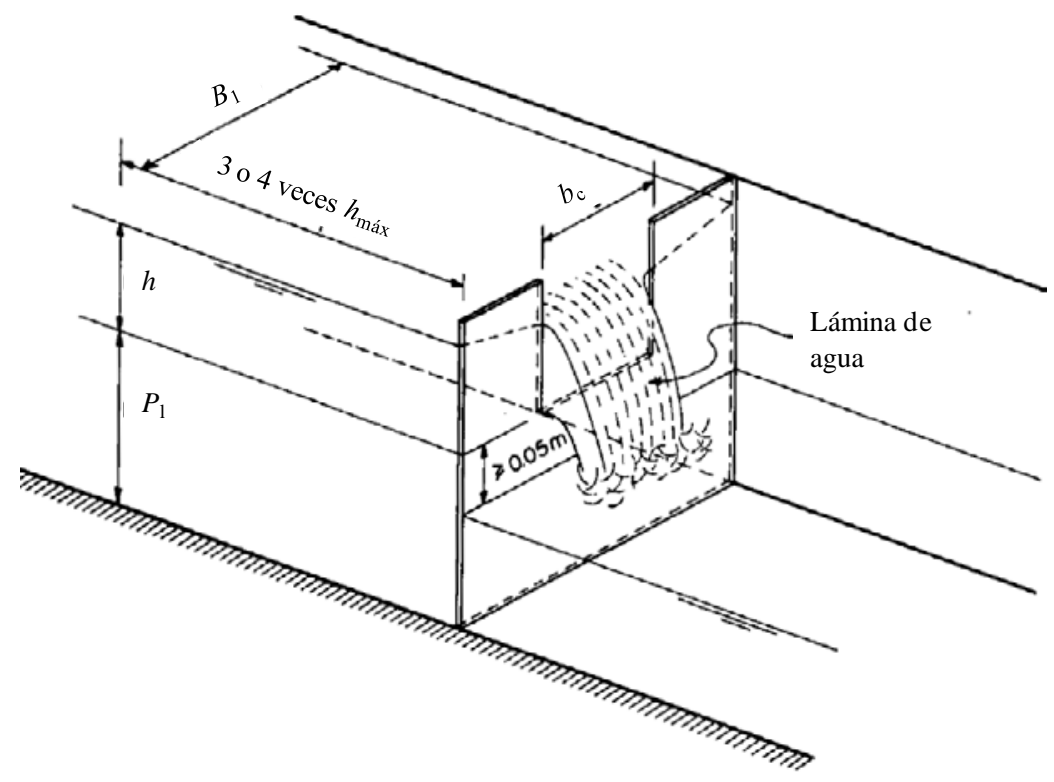

Figura 38. Vertedero rectangular de cresta delgada con contracción (Bos, 1989).

Con las dimensiones de la infraestructura no se cumple la relación $B_{1}-b_{c} \geq 4 h$, por lo que se tiene un vertedero de contracción parcial.

Para obtener los mejores resultados en la medición del caudal con vertederos planos rectangulares se recomienda:

- El plano del vertedero debe ser normal al flujo y la cara aguas arriba perfectamente vertical, plana y lisa.

- La superficie de la cresta debe ser perfectamente horizontal. 
- La cara de aguas abajo debe estar biselada si el espesor de la placa es superior a 2 milímetros, formando un ángulo de al menos 45 grados con la superficie en un vertedero con hendidura rectangular.

- Si el vertedero tiene contracciones, estas deberán estar a una distancia mínima de $0.30 \mathrm{~m}$.

- La altura desde el fondo del canal debe ser $0.30 m \leq P_{l} \geq 2 h$.

- La carga sobre el vertedero debe mediarse en un punto situado por lo menos a cuatro veces la carga máxima hacia aguas arriba.

- Los lados de un canal rectangular sobre el nivel de la cresta de un vertedero de ancho total deberían extenderse al menos $0.3 h_{1}$ máx aguas abajo de la cresta del vertedero.

- La altura debe ser superior a 3 veces a sobreelevación. En caso contrario, la descarga del aliviadero varía fuertemente con la sobreelevación (USBR, 2007). También se supondrá que la sobreelevación es superior a $4 \mathrm{~cm}$, de modo que se puede despreciar el efecto de la tensión superficial (D'Alpaos y Ghetti, 1984).

Planteando la ecuación de Bernoulli entre un punto situado aguas arriba del vertedero y otro situado aguas abajo, y despreciando la carga de velocidad aguas arriba, se llega a la ecuación de descarga:

$$
Q_{\text {teórico }}=\frac{2}{3} b_{c} \sqrt{2 g} h^{3 / 2}
$$

Para calcular el caudal real, se introduce el coeficiente de descarga $C_{d}$, quedando:

$$
Q_{\text {real }}=C_{d} \frac{2}{3} b_{c} \sqrt{2 g} h^{3 / 2}
$$

donde $C_{d}=f(R e, W e, h)$, siendo Re el número de Reynolds y We el número de Weber.

Para aplicar a esta ecuación al vertedero de contracción parcial, se considera la modificación propuesta por Kindsvater y Carter (1957), citados por Bos (1989).

$$
Q=C_{e} \frac{2}{3} b_{e} \sqrt{2 g} h_{e}^{3 / 2}
$$

donde el ancho efectivo $b_{e}=b_{c}+K_{b}$, y la carga efectiva $h_{e}=h+K_{h}$. Los coeficientes $K_{b}$ y $K_{h}$ representan los efectos combinados de diversos fenómenos atribuidos a tensión superficial y viscosidad. $C_{e}$ es el coeficiente de descarga efectiva empleado en la ecuación (75), el cual está en función de las relaciones $b_{c} / B_{1}$ y $h / P_{l}$ recogidas en la Tabla 11. En general, se recomienda un valor constante de $K_{h}=0.001 \mathrm{~m}$, mientras que $K_{b}$ se obtiene empíricamente en función de la relación $b_{c} / B_{1}$ (Figura 39). 


\begin{tabular}{|c|c|}
\hline $\boldsymbol{b}_{\boldsymbol{c}} \boldsymbol{B}_{1}$ & $\boldsymbol{C}_{\boldsymbol{e}}$ \\
\hline 1.0 & $0.602+0.075 \mathrm{~h} / \mathrm{P}_{l}$ \\
\hline 0.9 & $0.599+0.064 \mathrm{~h} / \mathrm{P}_{l}$ \\
\hline 0.8 & $0.597+0.045 \mathrm{~h} / \mathrm{P}_{l}$ \\
\hline 0.7 & $0.595+0.030 \mathrm{~h} / \mathrm{P}_{l}$ \\
\hline
\end{tabular}

\begin{tabular}{|c|c|}
\hline $\boldsymbol{b}_{\boldsymbol{c}} / \boldsymbol{B}_{1}$ & $\boldsymbol{C}_{\boldsymbol{e}}$ \\
\hline 0.6 & $0.593+0.018 \mathrm{~h} / \mathrm{P}_{l}$ \\
\hline 0.5 & $0.592+0.011 \mathrm{~h} / \mathrm{P}_{l}$ \\
\hline 0.4 & $0.591+0.0058 \mathrm{~h} / \mathrm{P}_{l}$ \\
\hline 0.3 & $0.590+0.0020 \mathrm{~h} / \mathrm{P}_{l}$ \\
\hline
\end{tabular}

\begin{tabular}{|c|c|}
\hline $\boldsymbol{b}_{\boldsymbol{c}} / \boldsymbol{B}_{1}$ & $\boldsymbol{C}_{\boldsymbol{e}}$ \\
\hline 0.2 & $0.589-0.018 \mathrm{~h} / \mathrm{P}_{l}$ \\
\hline 0.1 & $0.588-0.0021 \mathrm{~h} / \mathrm{P}_{l}$ \\
\hline 0 & $0.587-0.0023 \mathrm{~h} / \mathrm{P}_{l}$ \\
\hline
\end{tabular}

Tabla 11. Valores de $C_{e}$ como función de las relaciones $b_{c} / B_{1}$ y h/P $P_{l}$ (Bos, 1989).

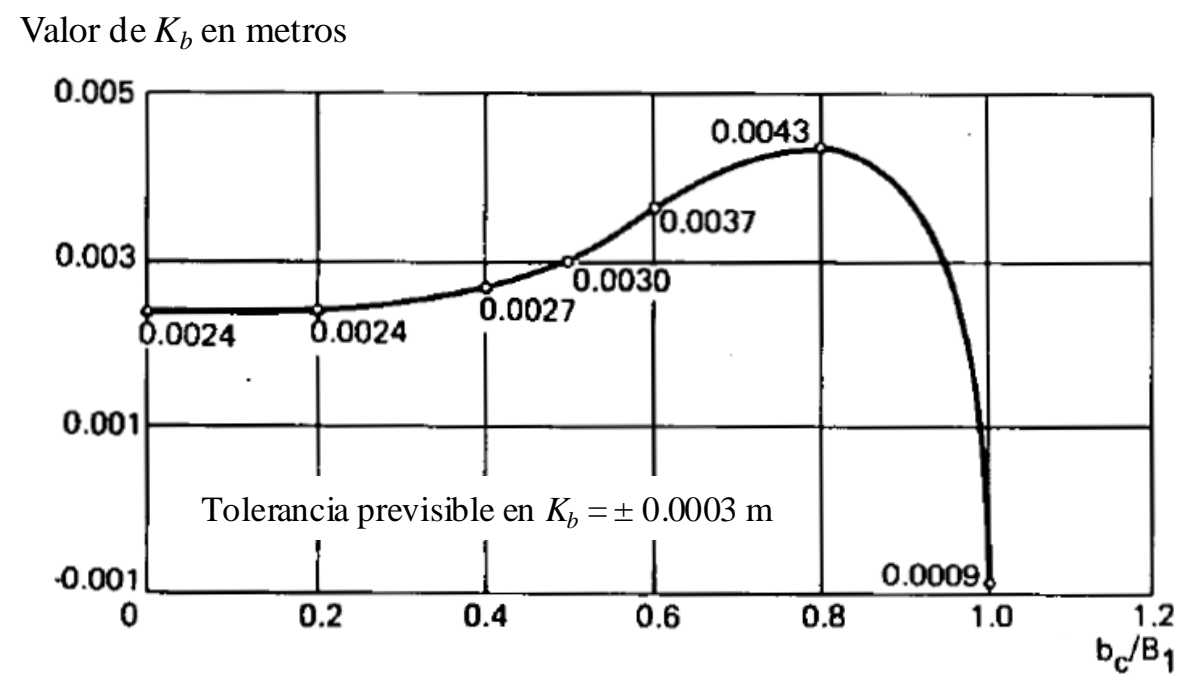

Figura 39. Valores de $K_{b}$ como función de $b_{d} B_{1}$, obtenidos por Kindsvater y Carter (1957), citado en Bos (1989).

El cuenco de disipación permite regular diferentes colchones de agua. Tiene una altura de $1.30 \mathrm{~m}$, un ancho de $1.05 \mathrm{~m}$ y una longitud de $3.00 \mathrm{~m}$ (Figura 40). Las paredes y la solera están hechas de metacrilato con el fin de poder observar los fenómenos que ocurren en el seno del cuenco. En el eje de simetría que atraviesa la solera del cuenco longitudinalmente se han dispuesto transductores de presión instantánea, separados a distancias regulares de $5 \mathrm{~cm}$ entre ejes.

La velocidad instantánea y la energía cinética turbulenta el flujo se registran en el canal de entrada y en el cuenco de disipación mediante velocímetros acústicos por efecto Doppler (Acoustic Doppler Velocimeter, ADV).

La velocidad media y las tasas de aireación en el chorro vertiente y en el cuenco de disipación se obtienen mediante un equipo de fibra óptica. 

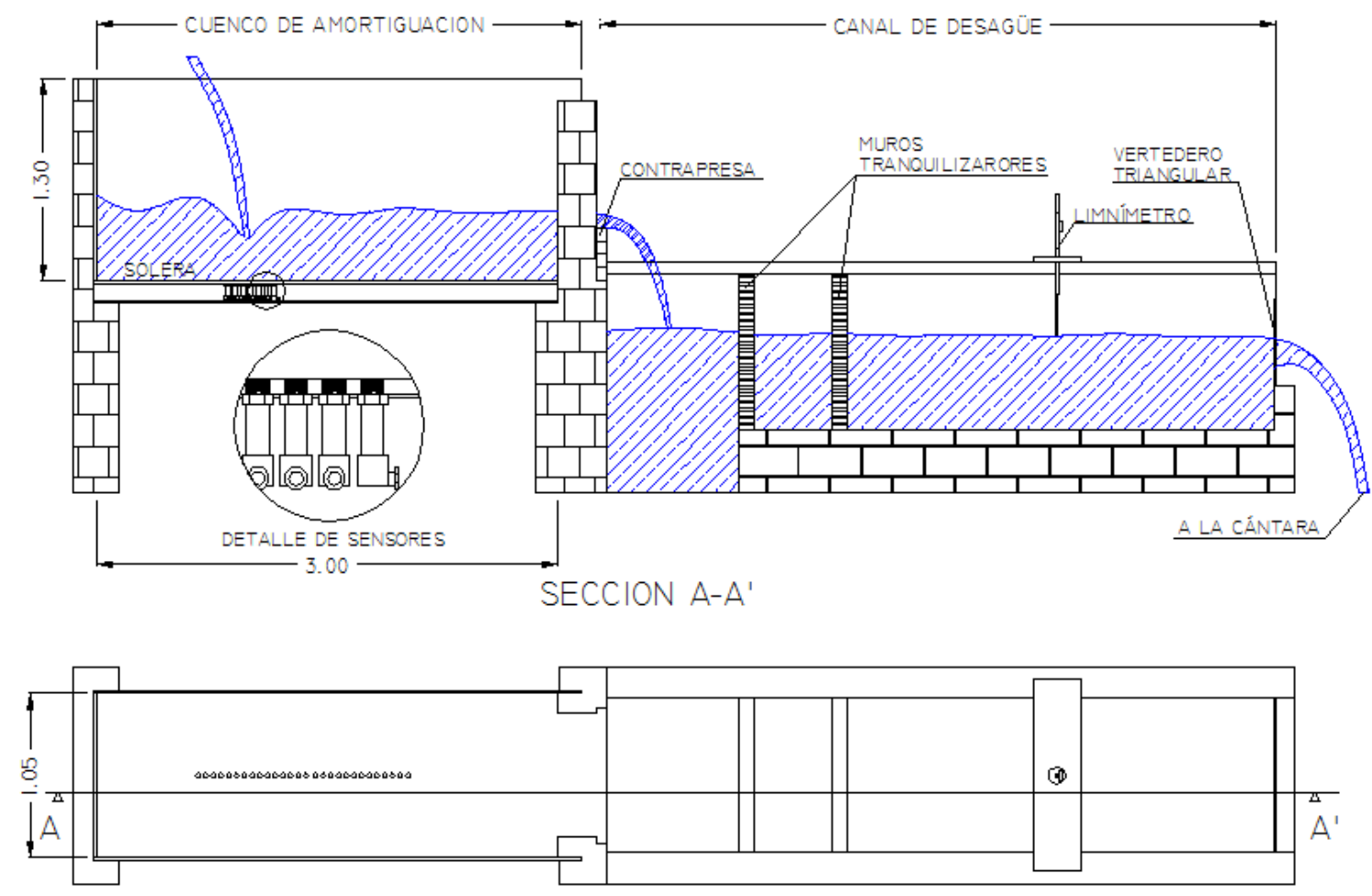

PLANTA

Figura 40. Cuenco de amortiguación y canal de desagüe en la infraestructura de laboratorio.

Para conocer el ancho en la dirección transversal del chorro incidente $b_{j}$, se emplean fotografías tomadas desde aguas abajo. Para cada caudal específico, se digitaliza la imagen para obtener los anchos transversales en diferentes cotas.

El caudal circulante se mide mediante un vertedero triangular tipo V-notch de ángulo de $90^{\circ}$, localizado aguas abajo del cuenco de disipación . Para el funcionamiento adecuado del vertedero triangular, se deben cumplir unos determinados rangos definidos en la Tabla 12, donde las variables vienen descritas en la Figura 41.

\begin{tabular}{|l|l|}
\hline \multicolumn{1}{|c|}{$\begin{array}{c}\text { Vertedero con } \\
\text { contracción parcial }\end{array}$} & \multicolumn{1}{c|}{$\begin{array}{c}\text { Vertedero con } \\
\text { contracción completa }\end{array}$} \\
\hline$h_{1} / p_{l} \leq 1.2$ & $h_{1} / p_{l} \leq 0.4$ \\
$h_{1} / B_{1} \leq 0.4$ & $h_{1} / B_{1} \leq 0.2$ \\
$0.05 \mathrm{~m}<h_{1} \leq 0.60 \mathrm{~cm}$ & $0.05 \mathrm{~m}<h_{1} \leq 0.38 \mathrm{~m}$ \\
$p_{l} \geq 10 \mathrm{~cm}$ & $p_{l} \geq 45 \mathrm{~cm}$ \\
$B_{1} \geq 60 \mathrm{~cm}$ & $B_{1} \geq 90 \mathrm{~cm}$ \\
\hline
\end{tabular}

Tabla 12. Valores recomendados para vertederos triangulares (Bos, 1989). 


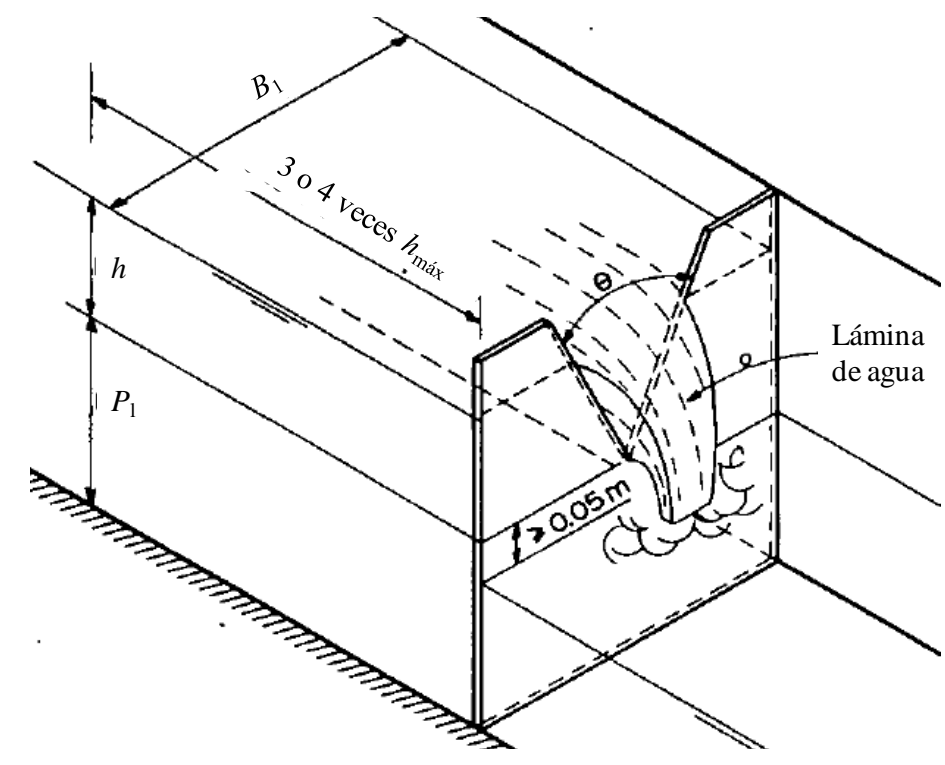

Figura 41. Vertedero triangular de pared delgada (Bos, 1989).

Para obtener la descarga de vertederos triangulares de pared delgada, tanto con contracción completa como con contracción parcial, se puede emplear la expresión propuesta por Kindsvater y Carter (1957), citados por Bos (1989):

$$
Q=C_{e} \frac{8}{15} \sqrt{2 g} \tan \frac{\theta}{2} h_{e}^{2.5}
$$

donde $\theta$ es el ángulo de la escotadura y $h_{e}$ la energía especifica, obtenida como $h_{1}+$ $K_{h}$ (para $\theta=90^{\circ}, K_{h} \approx 0.80 \mathrm{~mm}$ ). $C_{e}$ es el coeficiente de descarga efectivo que depende de los parámetros $h_{1} / p_{1}, p_{l} / B_{1}, \theta$. Su estimación para un ángulo de escotadura $\theta=90^{\circ} \mathrm{y}$ vertedero de contracción parcial se determina mediante la Figura 42. La formulación anterior tiene una precisión entre 1.0 y 2.0\%. Considerando que el canal de descarga tiene $1.01 \mathrm{~m}$ de ancho, y que la altura del vértice del aliviadero se encuentra a $0.40 \mathrm{~m}$, se obtiene la siguiente expresión:

$$
Q_{\text {teórica }}=1.3489 h^{2.4833}
$$

siendo $h$ la carga sobre vertedero.

La precisión del vertedero se comprobó mediante el método de área-velocidad utilizando un equipo ADV aguas arriba del vertedero triangular. Para cada caudal se registraron velocidades en 25 puntos uniformemente distribuidos en la sección de control. La curva de descarga obtenida en el aforo es:

$$
Q_{\text {aforo }}=0.9448 h^{2.2361}
$$


Para los caudales ensayados (entre 0.017 y $0.054 \mathrm{~m}^{3} / \mathrm{s}$ ) las diferencias entre el caudal obtenido en el vertedero y con el equipo Doppler están por debajo del 5\% del caudal circulante. A partir del caudal de $0.07 \mathrm{~m}^{3} / \mathrm{s}$, las curvas tienden a separarse, obteniéndose una diferencia del 13\% para el caudal de $0.160 \mathrm{~m}^{3}$ /s (Figura 43).

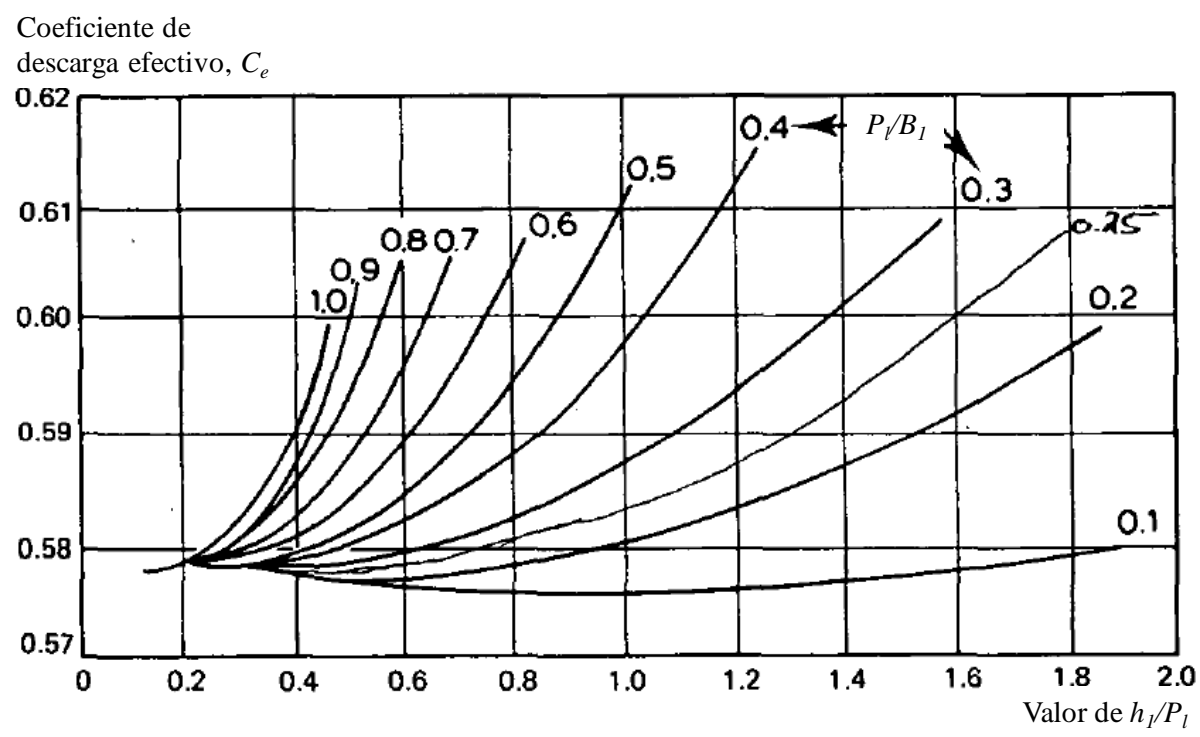

Figura 42. Coeficiente de descarga efectivo $C_{e}$ en vertederos triangulares con escotadura de $90^{\circ}$ (Bos, 1989).
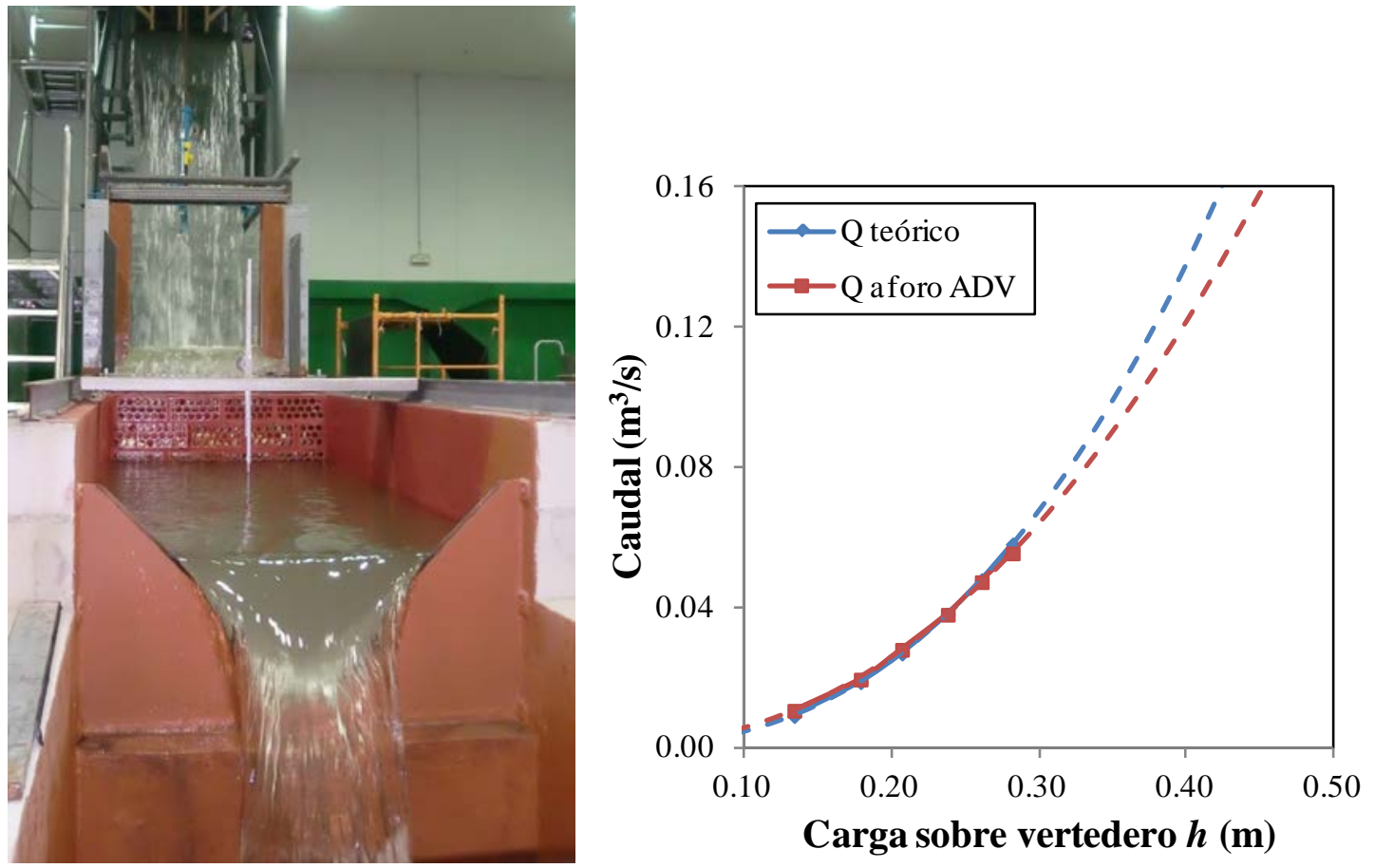

Figura 43. Aforo por vertedero triangular y comprobación de su curva de gasto. 


\subsubsection{Consideraciones en un modelo físico de laboratorio}

Se consideran dos sistemas físicos, el modelo a escala y el sistema real o prototipo, los cuales presentan comportamientos semejantes respecto a determinados fenómenos. Con ciertas limitaciones, las medidas efectuadas sobre algunas magnitudes físicas en el modelo permiten anticipar el comportamiento del sistema real mediante la extrapolación de modelo a prototipo.

Cuando se construyen los modelos físicos a escala, al reducir el tamaño se producen efectos de escala, como es el caso de la gravedad, que afecta por igual en la realidad como en el modelo de laboratorio. De este modo, se trabaja con fuerzas dominantes.

Se suele designar con el término genérico de efectos de escala a las discrepancias sistemáticas que se presentan entre los valores medidos en los ensayos con modelos a diferentes escalas de un mismo prototipo, aun cuando se puede demostrar, con base en las consideraciones del análisis dimensional, que las discrepancias obedecen en realidad a que no todos los parámetros pueden ser tomados en cuenta, simultanea y adecuadamente, en los ensayos realizados en modelo.

Dada la enorme dificultad y peligrosidad de instrumentar un prototipo, las mediciones de laboratorio se han llevado a cabo en una infraestructura física de tamaño relativamente pequeño. Para extrapolar los resultados a dimensiones de prototipo se debe considerar una ley de semejanza o de similitud.

Una adecuada ley de semejanza tiene que conservar los valores relativos de las diferentes características físicas que influyen en el movimiento del fluido. La elección de la mayoría de las variables de interés se determinará no sólo en función de la forma de la estructura, sino también de sus límites de validez y fiabilidad.

En los problemas de mecánica de fluidos que se presentan en los estudios de obras hidráulicas, las principales fuerzas a considerar son: fuerzas de inercia $F_{i}$, gravedad $F_{g}$, viscosidad $F_{\mu}$, tensión superficial $F_{\sigma s l}$, elasticidad $F_{e}$, y presión $F_{p}$.

El teorema $\Pi$ de Buckingham considera diferentes números adimensionales que relacionan las características físicas con las fuerzas de inercia. De esta manera, se tiene:

- Número de Newton, $N_{e}$ : relación entre las fuerzas de presión y las fuerzas de inercia.

- Número de Froude, $F_{r}$ : relación entre las fuerzas inerciales y las fuerzas gravitacionales.

- Número de Reynolds, $R_{e}$ : relación entre las fuerzas inerciales y las fuerzas de viscosidad.

- Número Weber, $W_{e}$ : relación entre las fuerzas inerciales y las fuerzas de tensión superficial. 
- Número de Cauchy o Mach, $C_{a}$ : relación entre las fuerzas inerciales y las fuerzas elásticas.

Con el fin de que los resultados del modelo físico puedan ser considerados como representativos de un prototipo, se deben satisfacer determinadas leyes de semejanza. Se consideran tres leyes de semejanza diferentes:

- Geométrica, $k_{l}$ : Permite relacionar las dimensiones del modelo con las del prototipo a través de transformaciones geométricas.

- Cinemática, $k_{t}$ : Añadiendo a las relaciones geométricas una escala de tiempos, permite relacionar las velocidades y aceleraciones de modelo y prototipo.

- Dinámica, $k_{F}$ : Amplía las anteriores con las escalas de fuerzas existentes en el fenómeno de estudio.

Si se cumplen las leyes de semejanza geométrica, cinemática y dinámica, se cumple la semejanza mecánica. De este modo, las fuerzas actuantes sobre los distintos modelos a escala que actúan en los puntos homogéneos serán proporcionales:

$$
k_{N_{e}}=k_{F_{r}}=k_{R_{e}}=k_{W_{e}}=k_{C_{a}}=1
$$

La segunda Ley de Newton establece que el vector suma de fuerzas exteriores a un elemento es igual a la reacción actuante contra ellas de la masa del elemento:

$$
\sum F_{\text {ext }}=F_{i}=F_{g}+F_{\sigma s l}+F_{e}+F_{\mu}+F_{p}
$$

Por tanto, en la semejanza entre modelo y prototipo debería cumplirse la relación:

$$
\frac{\left(F_{i}\right)_{p}}{\left(F_{i}\right)_{m}}=\frac{\left(F_{g}+F_{\sigma s l}+F_{e}+F_{\mu}+F_{p}\right)_{p}}{\left(F_{g}+F_{\sigma s l}+F_{e}+F_{\mu}+F_{p}\right)_{m}}
$$

La relación entre fuerzas de presión se deja como variable dependiente, pues no se suele utilizar en la determinación de la escala de los ensayos. El resto de las fuerzas presentan el problema de la inexistencia de un fluido que verifique simultáneamente las igualdades de fuerzas de viscosidad, de tensión superficial y de elasticidad; salvo que el modelo sea igual al prototipo.

Por tanto, no es posible obtener semejanza total entre modelo y prototipo, empleándose semejanzas parciales. Puesto que no todas las fuerzas actuantes tienen la misma importancia, se buscan leyes de semejanza en las que se reproduzcan 
adecuadamente las fuerzas dominantes (de gravedad y presión), admitiendo una cierta desviación de las secundarias.

Si se considera $k_{l}$ la escala geométrica del modelo $\left(L_{p}=k_{l} \cdot L_{m}\right)$, y puesto que se mantiene la gravedad $\left(g_{p} / g_{m}=1\right)$, a partir de las relaciones de semejanza, en la Tabla 13 se obtienen parámetros de escala entre prototipo (realidad) y modelo (laboratorio):

\begin{tabular}{|c|c|}
\hline Escala & Factor de escala \\
\hline Longitud & $k_{l}$ \\
\hline Tiempo & $\sqrt{k_{l}}$ \\
\hline Velocidad & $\sqrt{k_{l}}$ \\
\hline Aceleración & 1 \\
\hline Caudal & $k_{l}^{5 / 2}$ \\
\hline Fuerza & $k_{l}{ }^{3}$ \\
\hline Presión & $k_{l}$ \\
\hline
\end{tabular}

Tabla 13. Factores de escala de diversas variables.

Cuando predominan las fuerzas de gravedad, se tendrá:

$$
\left(F_{i}\right)_{p} /\left(F_{i}\right)_{m}=\left(F_{g}\right)_{p} /\left(F_{g}\right)_{m}
$$

o lo que es lo mismo:

$$
\left(\frac{v^{2}}{g L}\right)_{m}=\left(\frac{v^{2}}{g L}\right)_{p}
$$

siendo $V$ la velocidad típica del fenómeno, $g$ la aceleración de la gravedad y $L$ la longitud típica del fenómeno.

La raíz cuadrada de esta relación se conoce como Número de Froude, $F_{r}$. Su constancia en modelo y prototipo constituye la Ley de Semejanza de Froude:

$$
F_{r_{\text {modelo }}}=F_{r_{\text {prototipo }}}=\frac{v}{\sqrt{g L}}
$$

Cuando el agua circula, tanto la fricción hidráulica, como la gravedad y la inercia pueden ser factores determinantes. Sin embargo, para flujos en un canal abierto, a menudo el flujo es completamente turbulento, en cuyo caso la fricción hidráulica es independiente del número de Reynolds. Para este caso, los números de Froude idénticos 
aseguran la semejanza dinámica. Cuando un líquido de alta viscosidad fluye en un canal o cuando el agua fluye a un número de Reynolds relativamente bajo, el efecto del número de Reynolds no puede ser despreciado.

En chorros de caída libre, diversos autores consideran que algunos efectos tienen poca importancia:

- La compresibilidad del agua no suele tener importancia puesto que la velocidad del flujo es muy pequeña en comparación con la velocidad del sonido en el medio (el Número de Mach, relación entre la velocidad $V$ y la velocidad del sonido en el medio $a$, es muy pequeño).

- La viscosidad tiende a ser despreciada debido a que la turbulencia es suficiente para no considerar las tensiones viscosas. D'Alpaos y Ghetti (1984) consideran que las tensiones viscosas sólo condicionan la región próxima al vertedero. Los autores proponen una carga de agua superior a $4 \mathrm{~cm}$ aguas arriba del vertedero para asegurar que los efectos viscosos no influyen en la geometría de la napa.

Aceptando que la aireación es un efecto de escala que no se puede resolver, se suelen aplicar leyes de semejanza de Froude (ley de semejanza de modelos eulerianos). En estas condiciones, las presiones debidas al impacto de un chorro cuya velocidad ha sido adquirida por efectos gravitacionales mantienen una relación de proporcionalidad.

\subsubsection{Efectos de escala}

Naudascher (2000) recopila los efectos de escala que aparecen en el coeficiente de descarga de estructuras de flujo superior:

- Influencia del número de Reynolds. La Figura 44 muestra el resultado de la conversión modelo-prototipo $\left(R_{e} \rightarrow \infty\right)$ de los datos de ensayos en dos modelos de tamaño diferente, realizado por Bretschneider (1971). El efecto de la capa límite que origina la viscosidad se calcula aproximándolo al caso de un flujo uniforme equivalente sobre una placa plana con longitud $L=0.5 h_{0}$, siendo $h_{0}$ la carga de diseño sobre el vertedero (Naudascher, 1984).

A pesar de esta simplificación, el resultado aparentemente justifica el procedimiento de extrapolación. De este modo, los datos de los dos modelos extrapolados para $R e \rightarrow \infty$ (datos sin efecto de la capa limite) coinciden. Estos resultados fueron también comprobados por Varschney (1977) mediante una investigación sobre una familia de cinco modelos de un vertedero de descarga superior. 
Se puede afirmar que los valores del coeficiente de descarga $C_{q}$ obtenidos en ensayos de modelos no requieren de corrección debida a la capa límite para casos en que el número de Reynolds en laboratorio cumpla:

$$
R e_{L}=L \sqrt{g H}>5 \cdot 10^{5}
$$

siendo $H$ la carga total y $L$ la dimensión definida en la Figura 44.

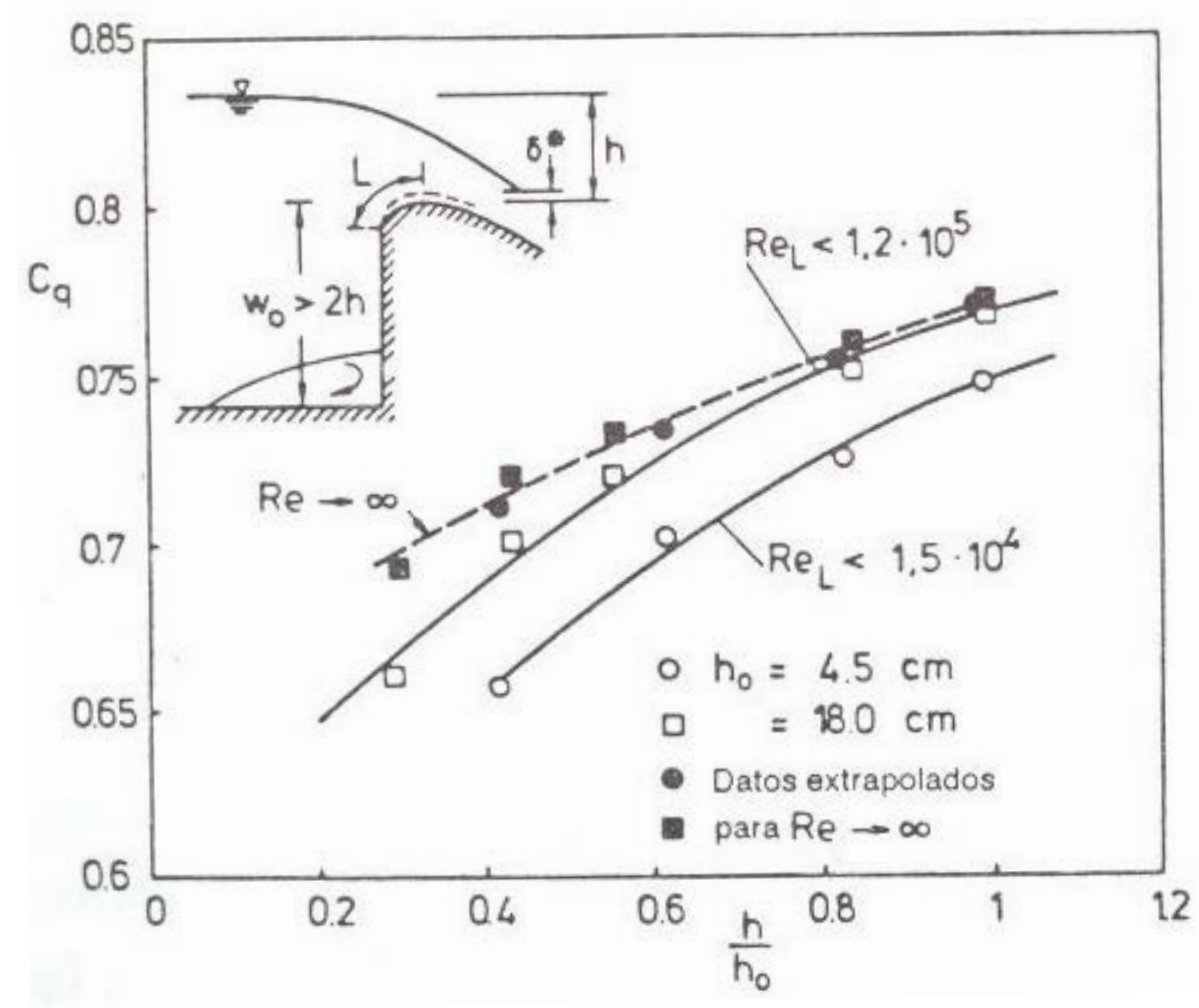

Figura 44. Corrección debida a la capa límite para los resultados experimentales de una familia de modelos (Naudascher, 2000).

- Influencia del número de Weber. La influencia simultanea de la tensión superficial y de la viscosidad sobre el flujo en un vertedero de pared delgada fue investigada por Ranga Raju y Asawa (1977). Considerando el número de Weber $W_{e}=h / \sqrt{\sigma / \gamma}$, siendo $\sigma$ la tensión superficial y $\gamma$ el peso específico, y el número de Reynolds $R_{e}=h \sqrt{g h} / v$, con $v$ la viscosidad cinemática, entonces la acción combinada de las dos fuerzas se hace notar cuando:

$$
R e^{0,2} \cdot W e^{1,2}<900
$$


o cuando $h<11 \mathrm{~cm}$ para agua a $20^{\circ} \mathrm{C}$. Rehbock da como límite para el mismo caso $h=9 \mathrm{~cm}$. Para cargas menores de este valor, el efecto de la tensión superficial y de la viscosidad sobre el caudal es mayor del $1 \%$. Por su parte, D'Alpaos y Ghetti (1984) suponen que se puede despreciar el efecto de la tensión superficial cuando la sobreelevación $h$ es superior a $4 \mathrm{~cm}$.

- Influencia de las condiciones del flujo de aproximación. Cuanto menor sea el ancho de un canal, mayor será el efecto de las perturbaciones debidas a las paredes laterales del canal de aproximación sobre el coeficiente de descarga y las características del flujo. La influencia perturbadora más importante constituye la aparición de remolinos laterales.

Poggensee (1942) estudió la influencia de la distribución de velocidades en el canal de aproximación (Figura 45), siendo su efecto importante sobre la distribución de presiones y el caudal cuando la relación $h / w_{0}$ del flujo sobre el vertedero satisface la condición $h / w_{0}>0.67$, donde $w_{0}$ es la altura del vertedero.

a)

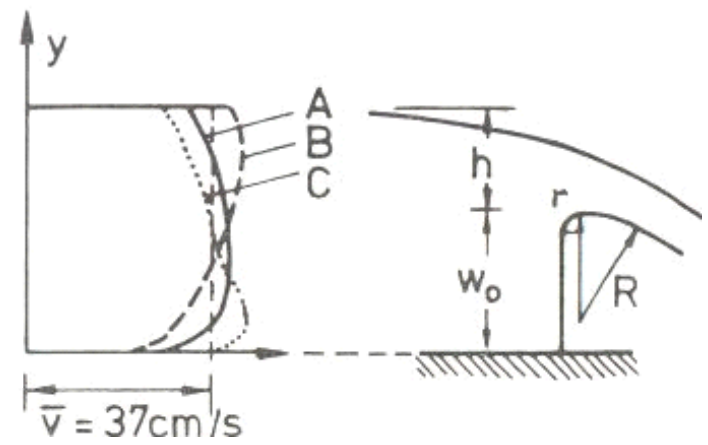

b)

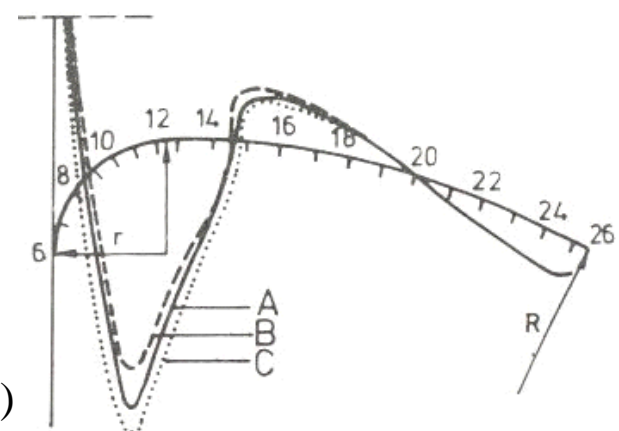

Figura 45. a) Efecto del perfil de velocidades en el canal de aproximación; b) sobre la distribución de presiones en la cresta de un vertedero hidrodinámico (Naudascher, 2000).

En lo referente a la aireación, se produce un efecto de escala importante, difícil de resolver. Se debe aceptar que las presiones en modelo serán más grandes que las que existen en prototipo. De este modo, un experimento que no considera aireación (se desprecian la viscosidad y la tensión superficial) quedará del lado de la seguridad.

\subsection{Transductores de presión}

El sistema de adquisición de datos se compone de una tarjeta DAQCard (Data AcQuisition Card) Tipo 6024 serie E para PCMCIA, suministrada por National Instrument. Este dispositivo es un módulo de adquisición de datos multifuncional de alta velocidad. Cuenta con 16 entradas analógicas SE (una única entrada) u 8 entradas 
analógicas DI (diferenciales) con una resolución de 12 bits y una velocidad máxima de adquisición de 200 ksamples/s (Figura 46).

Para obtener las presiones instantáneas se han utilizado transductores de presión GE DRUCK, modelo UNIK-5000 (Figura 46). Los sensores se encuentran en la solera del cuenco de disipación, en el plano de simetría de la infraestructura de chorros turbulentos. El rango de medición de los sensores varía entre -200 y +800 mbar, con una precisión de $\pm 0.04 \%$ del fondo de escala. Este tipo de sensores cuenta con un diafragma de metal en contacto con el fluido a medir. La transferencia de la fuerza se lleva a cabo a través de un fluido aceitoso no compresible que se dispone entre el diafragma de metal y un sensor piezorresistivo, protegido por una pantalla metálica. El sensor piezorresistivo transforma la presión ejercida en voltaje, el cual es recogido por la tarjeta de adquisición.

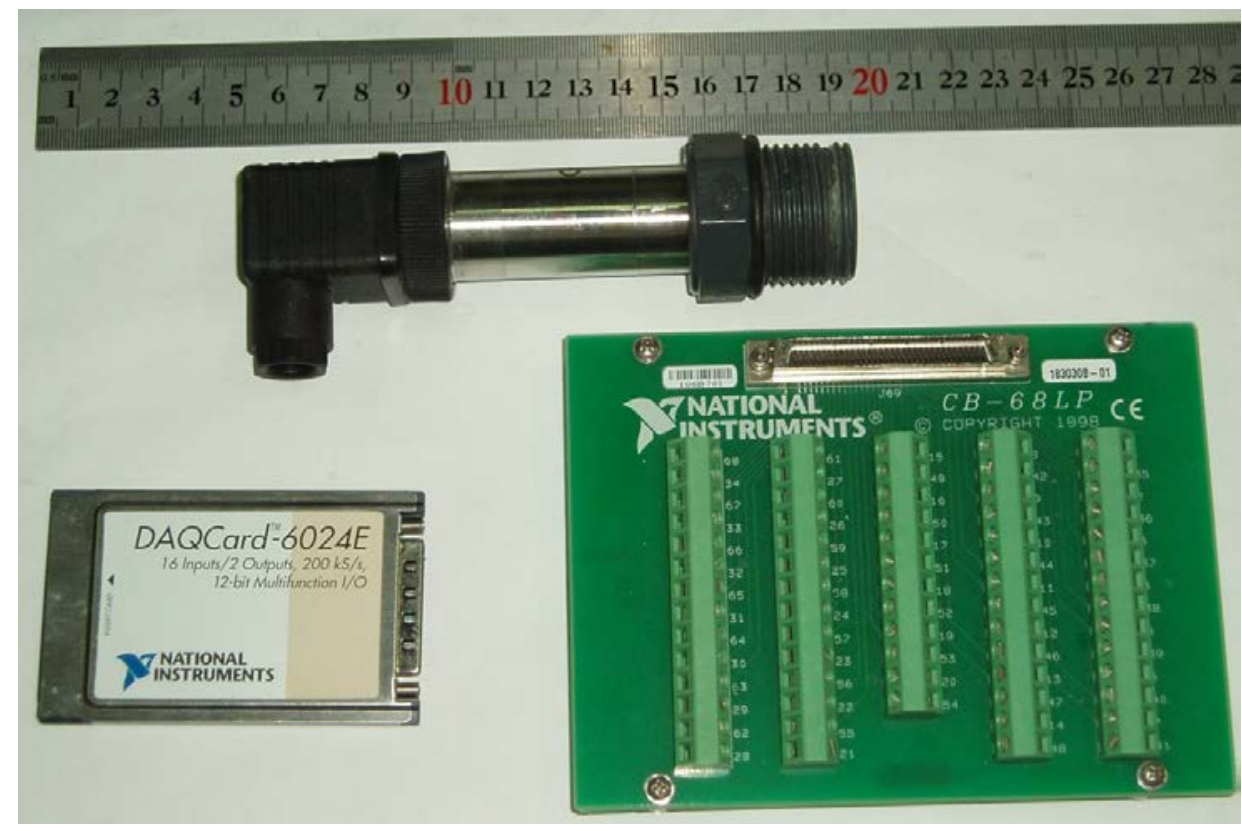

Figura 46. Sensor de presión y tarjeta de adquisición de datos.

Para el uso del dispositivo se ha desarrollado un software que se ejecuta en el entorno de LabVIEW (Figura 47).

Tras realizar una calibración estática, la precisión de los transductores de presión se estima en $\pm 0.01 \mathrm{~m}$.

Los registros de presiones instantáneas se obtienen considerando una tasa de frecuencia de $20 \mathrm{~Hz}$ y un tiempo de muestreo de 360 s. En cada medición, se utilizan 7 sensores al mismo tiempo: uno situado en el punto de estancamiento, 3 aguas arriba del punto de estancamiento, y 3 aguas abajo (Figura 48). 


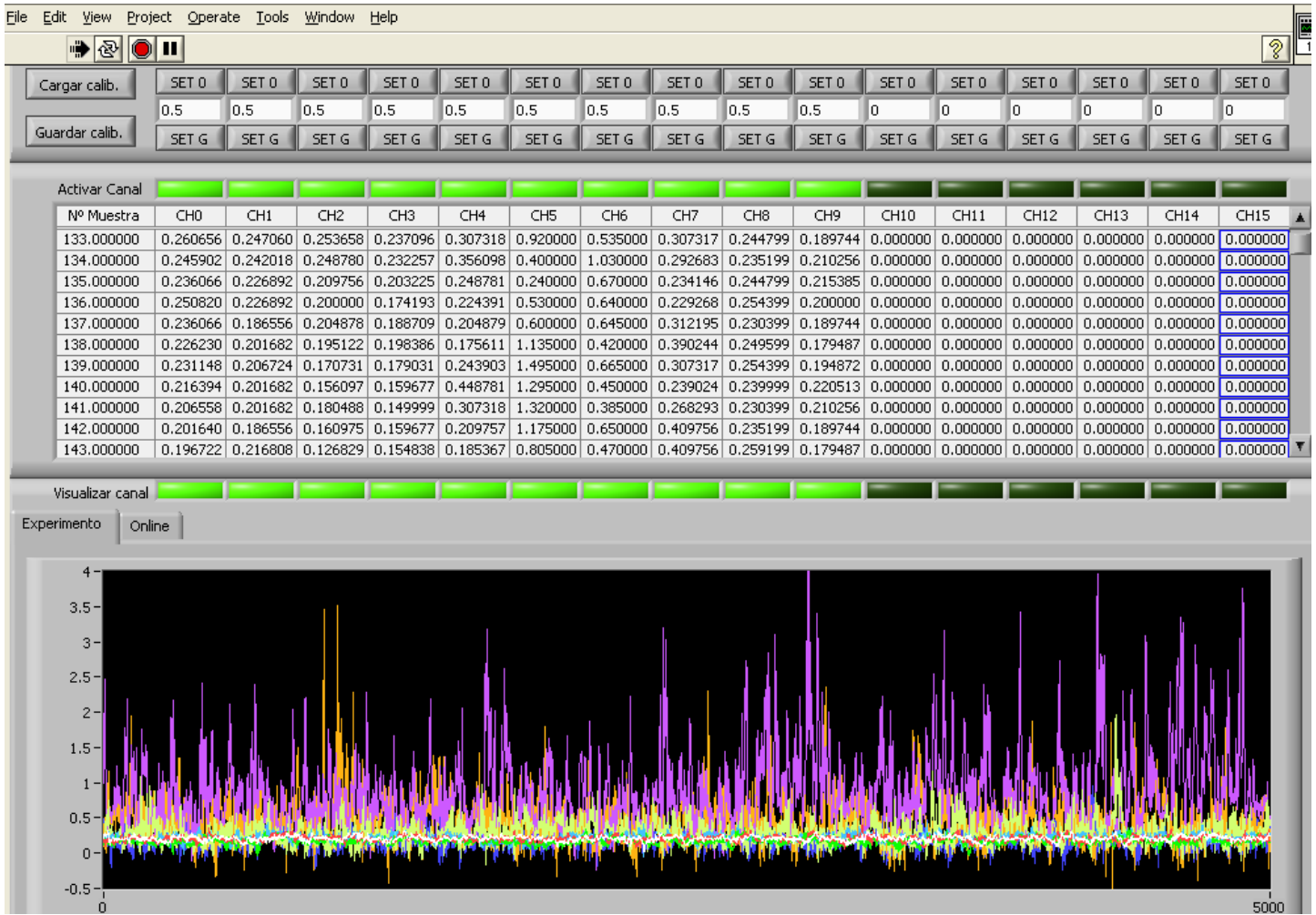

Figura 47. Software de adquisición de datos en entorno LabVIEW.

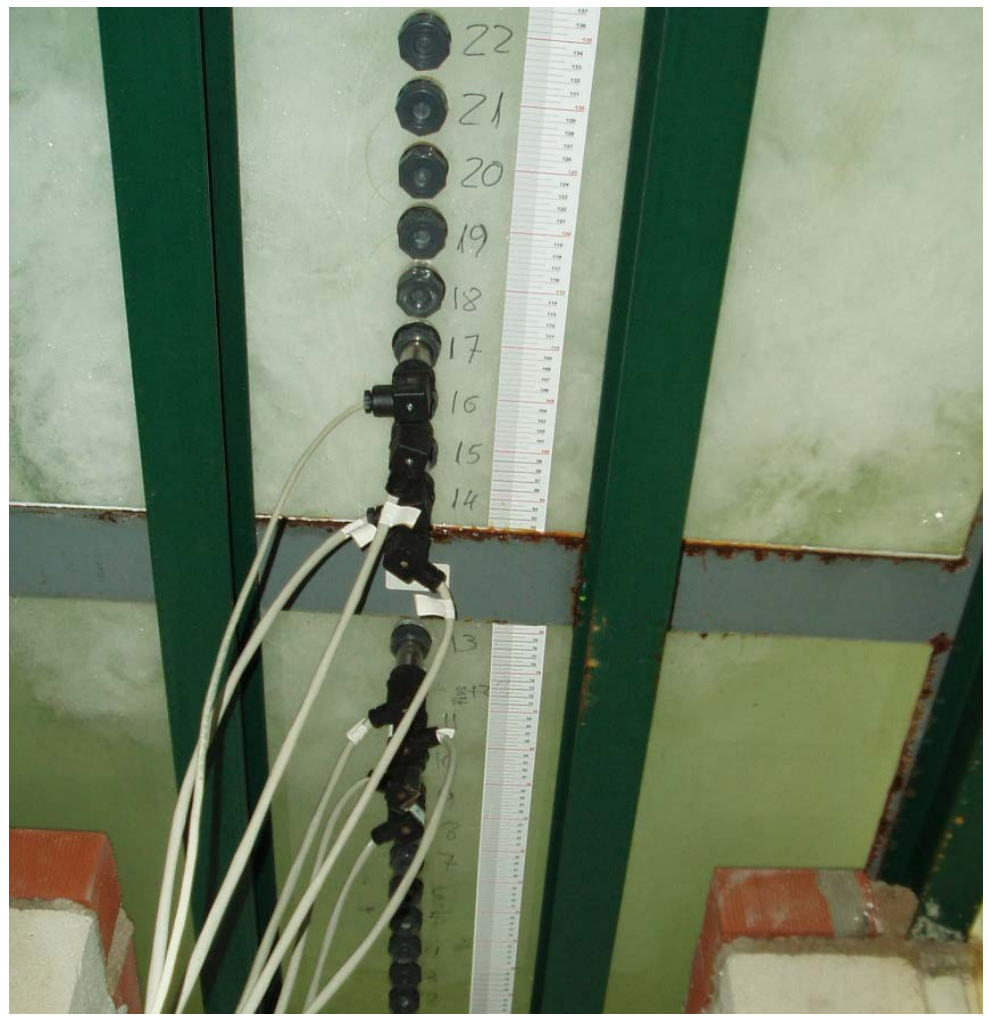

Figura 48. Distribución de sensores de presión en el eje central de la solera del cuenco de amortiguación. 


\subsection{Fibra óptica}

Para medir la velocidad media y las tasas de aireación en las distintas secciones del chorro vertiente se ha utilizado un equipo de fibra óptica de la casa RBI-instrumentation (Figura 49). Este equipo permite la medición de flujos bifásicos agua-aire con velocidades hasta $20 \mathrm{~m} / \mathrm{s}$.
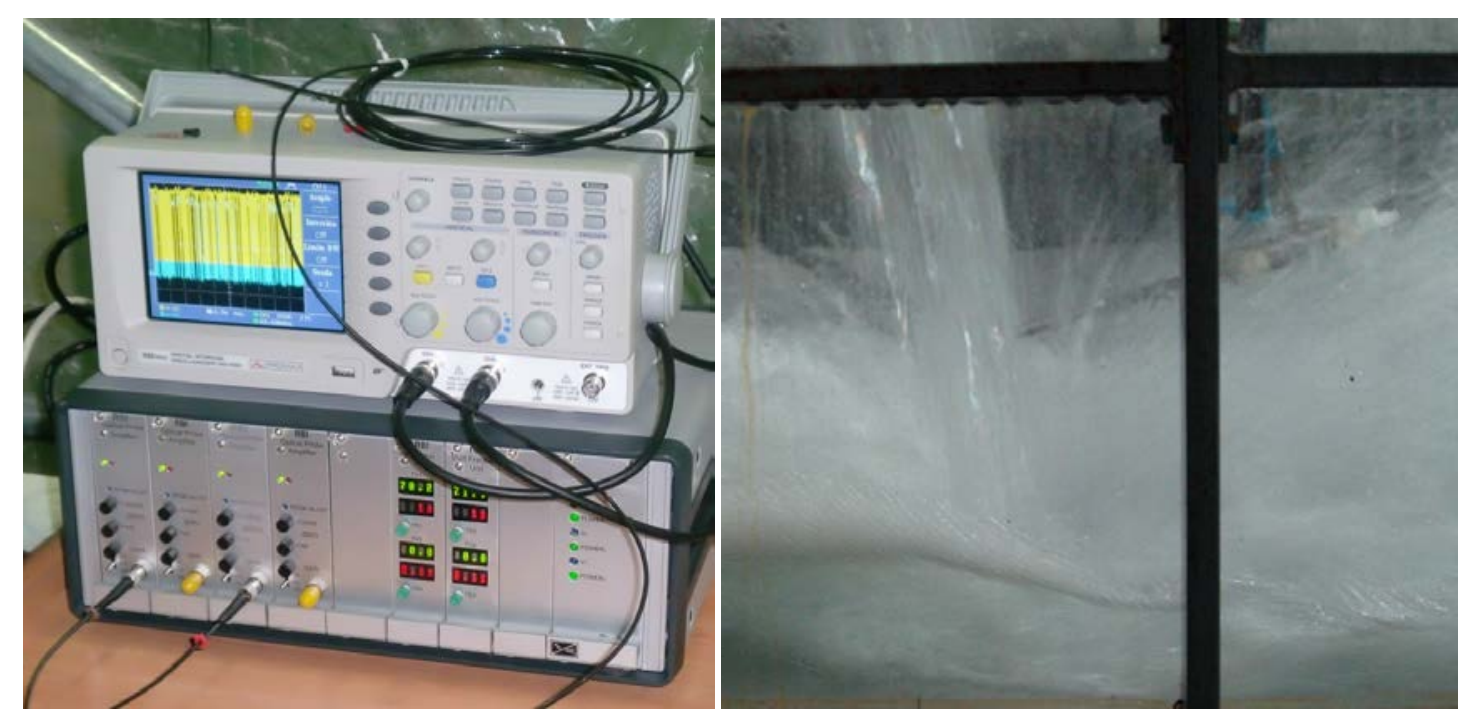

Figura 49. Equipo de fibra óptica, osciloscopio para controlar la señal y sonda registrando en el cuenco de amortiguación.

La sonda se compone de dos cables de fibra óptica de $200 \mathrm{~mm}$, insertadas en tubos de acero inoxidable de $820 \mathrm{~mm}$. Las puntas se encuentran separadas una distancia fija conocida.

Cada sonda funciona de forma independiente. Un haz de luz infrarroja se transmite a las puntas de las sondas a través de la fibra. La luz reflejada retorna a través de la misma fibra en la dirección opuesta. La luz incidente se separa mediante un prisma de Descartes, y un fotodiodo convierte la señal en voltaje. El tiempo de respuesta del circuito optoelectrónico es inferior a $5 \mu$ s.

La intensidad de la luz reflejada $R$ depende de la geometría de la sonda, del índice de refracción del líquido que rodea la parte sensible del sensor $\eta_{\text {fluido }}\left(\eta_{\text {agua }}=1.33\right.$; $\eta_{\text {aire }}=$ $1.00)$, y del índice de refracción del material de la sonda $\eta_{\text {sonda }}\left(\eta_{\text {sonda }}=1.42\right)$, los cuales están relacionados a través de la fórmula de Fresnel:

$$
R=K_{\text {Fresnel }}\left(\frac{\eta_{\text {fluido }}-\eta_{\text {sonda }}}{\eta_{\text {fluido }}+\eta_{\text {sonda }}}\right)^{2}
$$


La subida y la bajada de la señal de la sonda corresponde, respectivamente, a la llegada y la salida de la fase gaseosa a la punta del sensor. La diferencia de tiempo entre estas dos transiciones, requiere el uso de dos umbrales distintos debido a la deformación de las interfaces inducida por fenómenos hidrodinámicos locales (Figura 50). Las señales son tratadas en orden inverso para obtener el segundo nivel inferior al primero y evitar tiempos de permanencia artificiales. Siguiendo los resultados de Boes y Hager (1998), los valores de umbrales se establecen en 1.0 y 2.5 V.

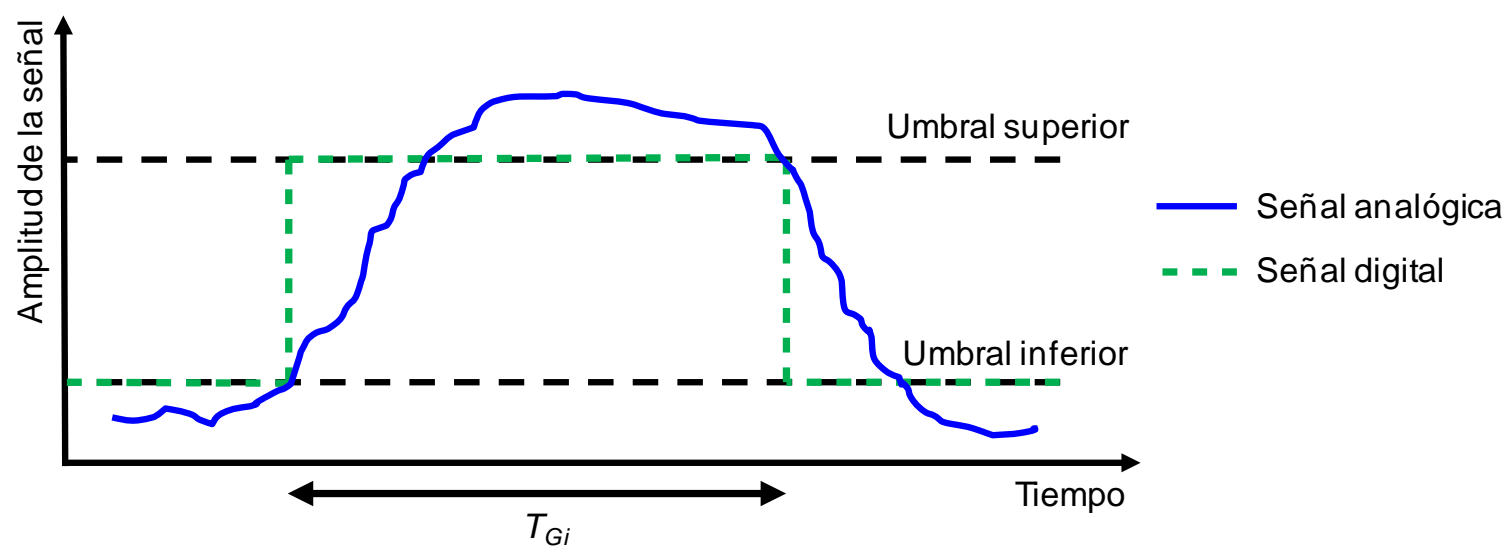

Figura 50. Conversión de la señal analógica en digital en el equipo de fibra óptica.

La fracción de vacío se define como la relación entre el tiempo total en que la sonda está en el gas $\left(\Sigma t_{G i}\right)$ y el tiempo de duración del experimento $t$ (Figura 51). La incertidumbre relativa respecto a la fracción de vacío se estima alrededor del 15\% (Stutz y Reboud, 1997).

$$
\text { Fracción de vacío }=\sum_{i=1}^{n} \frac{t_{G_{i}}}{t}
$$

La velocidad media del fluido se estima a partir del tiempo que tarda una interfaz aire-agua en pasar de una punta de la sonda a la otra (Figura 51). El tiempo promedio entre las dos señales se deduce usando una técnica de correlación cruzada de las señales recogidas por cada punta. Para el caso de flujo permanente, la precisión de la velocidad se estima en un $\pm 10 \%$ en un rango de velocidades inferior a $20 \mathrm{~m} / \mathrm{s}$ (Stutz y Reboud, 1997).

$$
V=\frac{d}{t_{\text {desfase }}}
$$




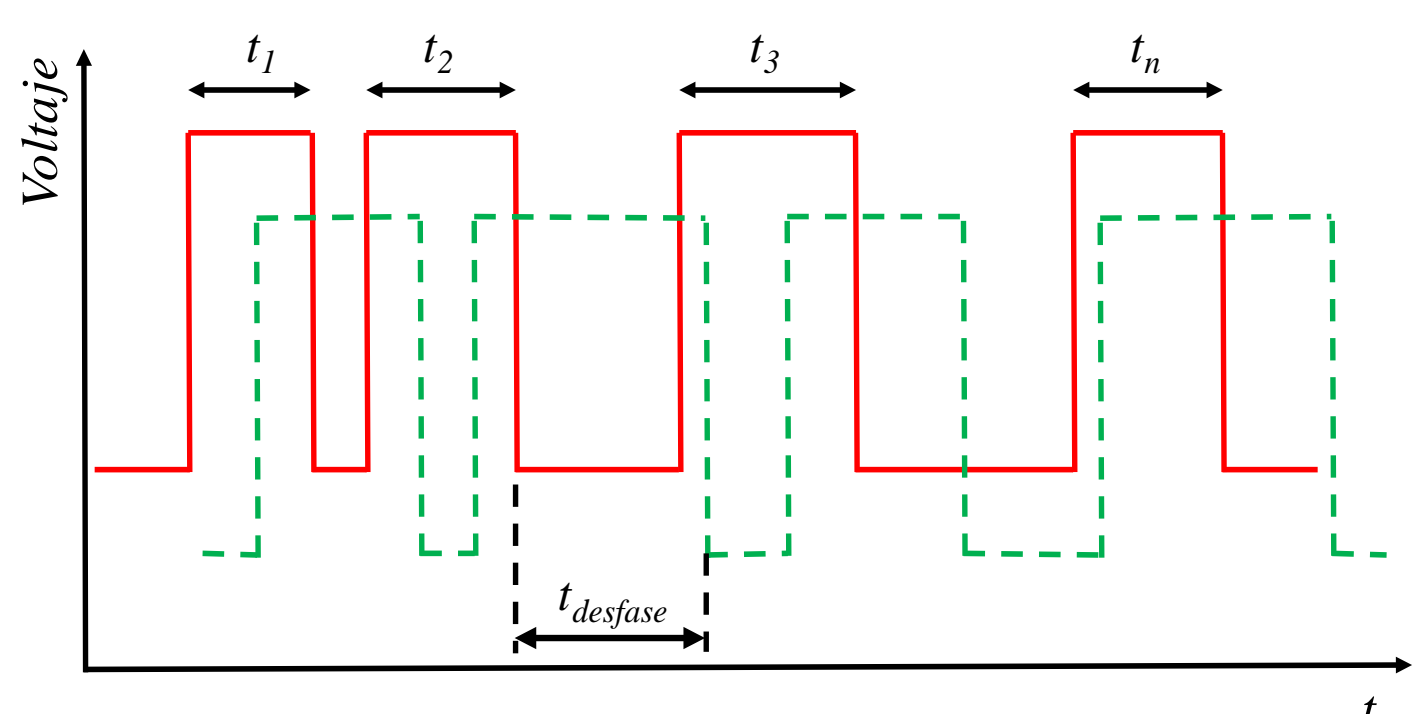

Figura 51. Señal aire agua en un equipo de dos canales de fibra óptica.

\subsection{Velocímetro acústico Doppler}

Los instrumentos de medición de velocidad por efecto Doppler (ADV) son muy fiables para caracterizar velocidades instantáneas en flujos laminares y turbulentos sin la presencia de aire, puesto que el agua constituye el elemento fundamental de la transmisión del sonido.

Generalmente consisten en un emisor rodeado de 2 a 4 receptores, cada uno de ellos midiendo una proyección del vector velocidad. El emisor genera una onda acústica a una frecuencia $f_{e}$, con velocidad del sonido $c$ y longitud de onda $\lambda_{e}=c / f_{e}$ que se propaga a través del líquido. La onda se dispersa por las partículas que se mueven con la velocidad del fluido $u$, y, finalmente, es detectada por los receptores (Figura 52). Debido a la velocidad $u$, la longitud de la onda acústica reflejada $\lambda_{r}$ y su frecuencia $\lambda_{r}=$ $c / f_{r}$ difieren de la emitida. La frecuencia Doppler se define como la variación de frecuencia de la onda acústica inducida por las partículas en movimiento $f_{D}=f_{r}-f_{e}$. La relación entre la frecuencia Doppler $f_{D}$ y las proyecciones de la velocidad $u$ recibidas a lo largo del eje del emisor y de los ejes receptores, $u_{e}$ y $u_{r}$, es $f_{D}=f_{c} / c\left(u_{e}+u_{r}\right)$.

Los equipos ADV miden la velocidad de las partículas que se mueven con el fluido, en lugar de la velocidad del fluido. Dado que estas partículas siguen el movimiento del fluido con un desfase inercial despreciable, se puede asumir que su velocidad es idéntica a la velocidad del fluido. 


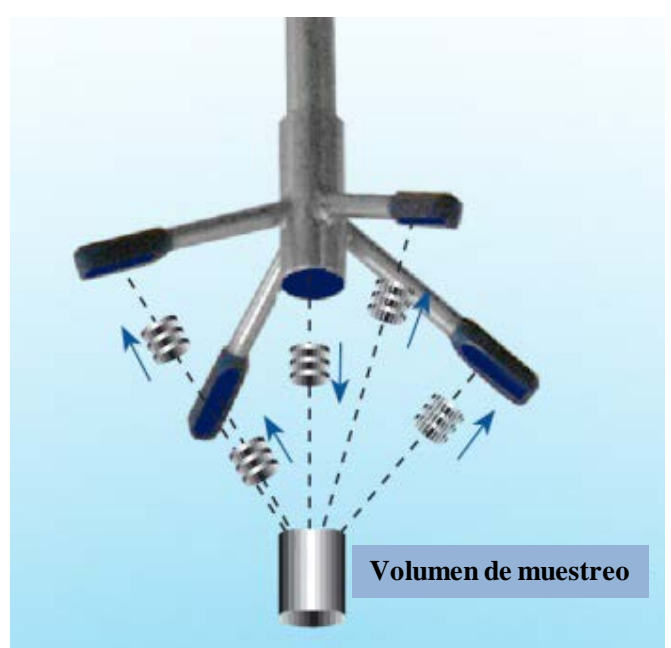

Figura 52. Transmisión de la señal del equipo Doppler.

Considerando el rango de velocidad $\pm 0.30 \mathrm{~m} / \mathrm{s}$ y una frecuencia de muestreo de 10 $\mathrm{Hz}$, el equipo ADV de la casa Nortek AS es capaz de medir el campo de velocidades promediadas en el tiempo con una precisión de $\pm 0.002 \mathrm{~m} / \mathrm{s}$.

La energía cinética turbulenta se midió en la condición de entrada del modelo numérico (0.50 m aguas arriba del vertedero de pared delgada).

A continuación, el equipo se ha empleado para medir los perfiles de velocidad en distintas secciones del cuenco de amortiguación (Figura 53).
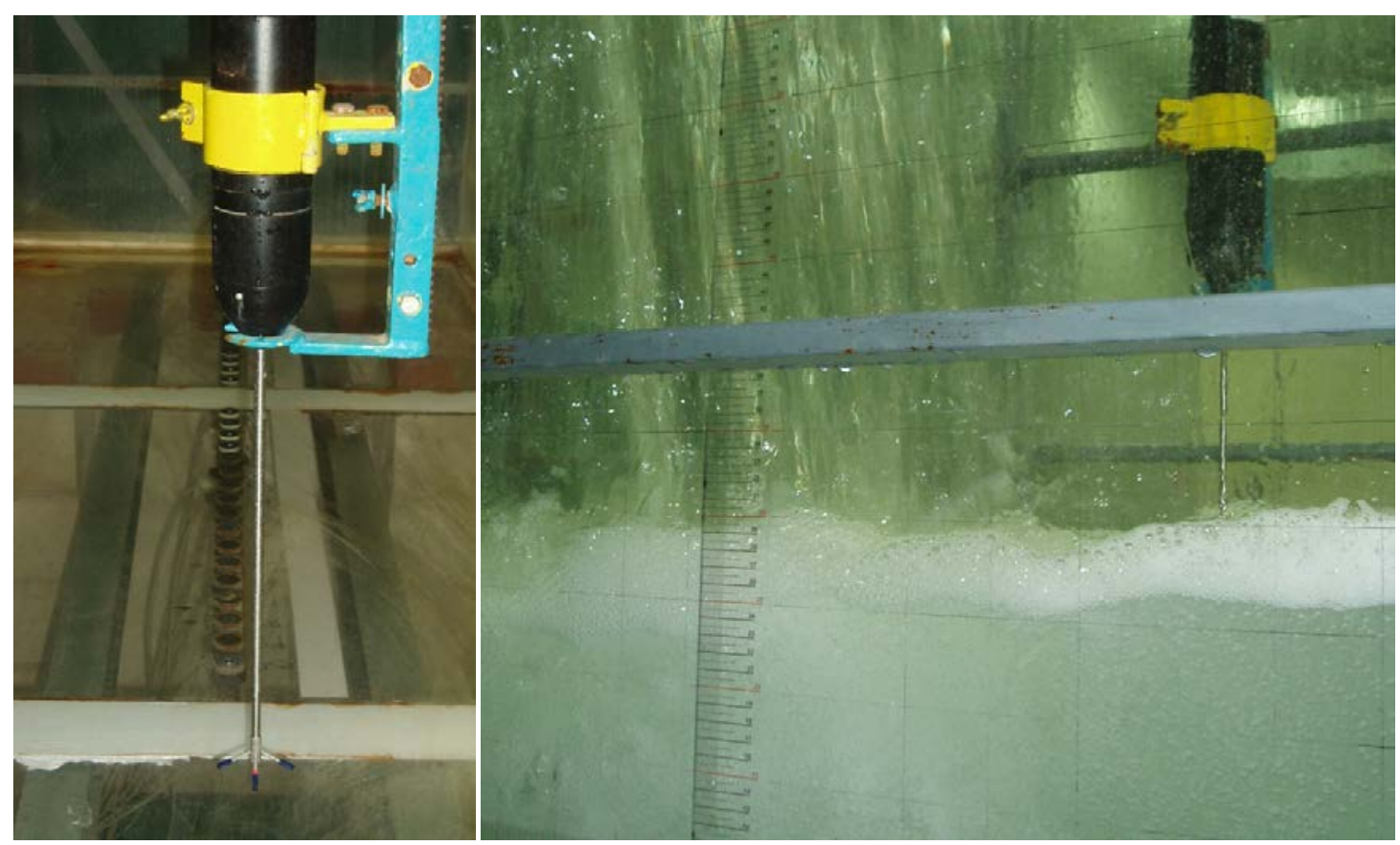

Figura 53. Velocímetro acústico Doppler en el cuenco de disipación. 


\section{METODOLOGÍA NUMÉRICA}

La metodología de Dinámica de Fluidos Computacional (Computational Fluid Dynamics, CFD) es, junto a la investigación experimental y al desarrollo teórico analítico, el tercer método de análisis de problemas de Mecánica de Fluidos. Los tres métodos están íntimamente relacionados, y se complementan entre sí.

En la metodología CFD, el flujo de cualquier fluido se resuelve a partir de unas ecuaciones generales de conservación fuertemente acopladas, que se obtienen de la aplicación a una partícula fluida de los siguientes principios y ecuaciones:

- Principio de conservación de la masa $\rightarrow$ Ecuación de la continuidad.

- Principio de conservación de la cantidad de movimiento o segunda ley de Newton $\rightarrow$ Ecuaciones de Navier-Stokes (si se tienen en cuenta los términos viscosos y de disipación de energía) o ecuaciones de Euler (si se desprecian).

- Principio de conservación de la energía $\rightarrow$ Ecuación de la energía.

- Ecuación de estado o de comportamiento del fluido.

- Ecuaciones constitutivas del medio.

La resolución de las ecuaciones diferenciales proporciona las tres componentes de velocidad en cualquier punto, para cualquier instante de tiempo $t, u(x, y, z, t), v(x, y, z, t)$ $\mathrm{y} w(x, y, z, t)$, junto con las distribuciones de presión $p(x, y, z, t)$, densidad, $\rho(x, y, z, t) \mathrm{y}$ temperatura, $T(x, y, z, t)$.

Sin embargo, la simulación numérica no puede resolver con éxito problemas en los que los principios físicos no hayan sido formulados de manera correcta, como por ejemplo, en la simulación de flujos turbulentos, donde se utilizan modelos aproximados que incluyen constantes empíricas. De este modo, los resultados numéricos obtenidos están sujetos a la exactitud del modelo de turbulencia empleado, así como a la conveniencia de la aplicación del modelo al problema concreto que se quiere resolver.

Puesto que la mayoría de las ecuaciones de conservación a resolver son no lineales, los valores de las variables se corrigen secuencialmente utilizando un proceso iterativo equivalente a un avance secuencial con pequeños intervalos de tiempo, donde el número de iteraciones depende de la complejidad del problema.

\subsection{Metodología}

En los paquetes existentes de CFD se deben especificar las condiciones del problema que se va a resolver, así como ciertos parámetros de resolución para que el programa 
consiga encontrar una solución correcta del problema. Los pasos genéricos que hay que seguir son (Blanco, 2007):

- Especificación de la geometría del problema. Es preciso introducir los contornos del dominio del problema. Este proceso es relativamente simple cuando el modelo es bidimensional, aunque puede ser realmente complejo cuando el modelo es tridimensional.

- Creación del mallado o celdas en las que van a ser calculadas todas las variables. El mallado puede ser estructurado o no estructurado. El mallado estructurado está formado por rectángulos o paralelepípedos ordenados en una matriz, que pueden deformarse para adaptarse a las superficies curvas de la geometría. El mallado no estructurado está habitualmente formado por triángulos o tetraedros $\mathrm{y}$, en cualquier caso, no tiene un orden matricial. Este último tipo de mallado es mucho más sencillo de ajustar a formas complejas que el anterior, aunque es más costoso de calcular.

- Definición de los modelos que se van a utilizar: modelos de turbulencia, de radiación, de flujo bifásico, de multiespecie, etc. Estos modelos deben estar implementados en el software.

- Especificación de las propiedades del fluido: viscosidad, densidad, propiedades térmicas..., etc. El programa debe ser capaz de manejar las ecuaciones de variación de estas propiedades con la temperatura, la presión, etc.

- Imposición de las condiciones de contorno que controlan los valores de ciertas variables en los límites del dominio. Básicamente, se imponen valores fijos de la presión, velocidad y temperatura, o de sus gradientes. También hay que imponer condiciones para las variables de turbulencia que haya, según el modelo que se esté utilizando.

- Introducción de las condiciones iniciales. En el caso de un problema no estacionario, hace falta definir unos valores de las variables para todos los puntos del dominio, desde los cuales empezará a calcular las soluciones de los sucesivos pasos temporales. En el caso de un problema estacionario, es preciso introducir unos valores iniciales de las variables, de los cuales partirá el proceso iterativo.

- Control de los parámetros que afectan a la resolución numérica del problema. Se puede actuar sobre el proceso iterativo definiendo los tipos de discretización, variando los factores de relajación, y fijando los criterios de finalización de las iteraciones.

- Proceso de cálculo. Se monitoriza la convergencia y se suele controlar la evolución de las variables en algunos puntos.

- Análisis de la solución. Comprobación de que la resolución es correcta y estudio de los resultados. La gran cantidad de datos generados hace necesario el uso de sistemas de postproceso gráficos. 


\subsection{Ventajas e inconvenientes}

Diversas fuentes destacan las siguientes ventajas de la modelación numérica:

- Bajo coste. Por ejemplo, Chandler et al. (2003), citados por Ho et al. (2006), consideran que el coste de la simulación numérica está en torno al 20 - 25\% del coste de un modelo físico para la presa de Winenhoe (Brisbane, Australia).

- Pueden estudiarse configuraciones y variaciones distintas de un mismo problema con un tiempo de calculo relativamente corto. Generalmente, resulta sencillo cambiar geometrías y condiciones de contorno.

- La solución ofrece un información completa y detallada sobre el dominio de interés, mientras que en la experimentación sólo es posible obtenerla en unos pocos puntos.

- Las condiciones reales se implementan de una forma relativamente sencilla, de modo que pueden simularse problemas complejos que en la experimentación deberían tener que simplificarse para lograr mediciones fiables.

Una causa común de los errores de los modelos son de tipo numérico. Se trata de una pérdida de precisión debido al modo en que los paquetes de CFD resuelven las ecuaciones, que es especialmente peligroso cerca de las condiciones de contorno del mallado. Según Hirsch (2007), los errores numéricos que reducen la precisión de los resultados sin romper la simulación pueden ser debidos a:

- La discretización del espacio y del tiempo.

- Soluciones implícitas que no convergen, en especial para la presión.

- Pérdida de precisión debida a redondeos.

- Inadecuada o inexacta geometría y topografía. En geometrías complejas los costos computacionales pueden ser del mismo orden que los costes experimentales.

- Condiciones de contorno incorrectas o incompatibles.

- Errores del usuario en parámetros que afectan a la resolución numérica del problema.

- Simplificaciones y aproximaciones en modelos, especialmente las relacionadas con cierres de la turbulencia, flujos multifásicos, etc. En estos casos para los que no se ha desarrollado un modele matemático fiable, es posible estar simulando un fenómeno que no ocurre en la realidad física, por lo que se requiere la comparación de los resultados numéricos con datos experimentales semejantes para validar la solución numérica.

- Errores en el código implementado en el paquete de CFD. 


\subsection{Ecuaciones de conservación}

Las ecuaciones generales de la Mecánica de Fluidos son ecuaciones de conservación de las propiedades fluidas, aplicadas a un sistema cerrado denominado volumen fluido $V_{f}$.

Estas ecuaciones pueden expresarse en forma diferencial y en forma integral. En forma diferencial, las ecuaciones de conservación se aplican a un elemento infinitesimal de fluido, pero lo suficientemente grande como para contener un número grande de moléculas (hipótesis de medio continuo). En forma integral, las ecuaciones de conservación se aplican a un volumen fluido fijo el cual es atravesado por el fluido, o móvil (o deformable) que se desplaza con el fluido.

Cualquier problema de mecánica de fluidos puede resolverse a partir de las ecuaciones diferenciales de conservación (con las correspondientes condiciones iniciales y de contorno, y las ecuaciones de comportamiento necesarias), que determinan la densidad, la presión, las tres componentes de la velocidad y la temperatura en cada punto, en función de la posición $x, y, z, \mathrm{y}$ del tiempo $t$. Sin embargo, contienen términos en derivadas parciales segundas, y están fuertemente acopladas entre sí, por lo que tienen solución analítica únicamente en casos muy particulares.

Pueden efectuarse una serie de observaciones sobre las ecuaciones generales de la Mecánica de Fluidos (Zamora, 2008):

- Constituyen un sistema fuertemente acoplado, muy difícil de resolver analíticamente.

- Los esfuerzos viscosos $\bar{\tau}^{\prime}$ son función únicamente de los gradientes de velocidad (los coeficientes de viscosidad se pueden considerar constantes en la mayoría de las ocasiones, o bien dependen sólo de la temperatura).

- Se trata de un sistema de cinco ecuaciones (conservación de masa, conservación de cantidad de movimiento en cada dirección y conservación de la energía) con seis incógnitas $(u, v, w, p, \rho, T)$, que se completa con la ecuación de estado. Para un líquido se puede suponer que la densidad $\rho=$ cte. Para un gas se puede considerar que existe comportamiento perfecto, cumpliéndose $p / \rho=R_{g} T$, donde $R_{g}$ es la constante del gas.

- Aunque las ecuaciones de Navier-Stokes son las de cantidad de movimiento, esta denominación se extiende usualmente a las ecuaciones de continuidad y de conservación de la energía.

- El sistema de ecuaciones se completa con las condiciones iniciales y de contorno. Las condiciones iniciales en problemas transitorios deben especificar las variables en un instante inicial de partida. Las condiciones de contorno son físicas, y están impuestas por el entorno real. 


\subsubsection{Ecuación de continuidad o de conservación de masa}

La ley de Lomonosov-Lavoisier considera que la masa de un sistema cerrado permanece constante, independientemente de los procesos que se produzcan dentro del sistema. La ecuación de conservación de masa expresa la masa de un volumen de control fijo como:

$$
\frac{d}{d t} \int_{V_{f}(t)} \rho d V=\int_{V_{0}} \frac{\partial \rho}{\partial t} d V+\int_{S_{0}} \rho(\vec{v} \cdot \vec{n}) d S=0
$$

donde $S_{0}$ es la superficie que delimita a un volumen fluido fijo $V_{0}$ y $\vec{n}$ es el vector normal con sentido hacia fuera de la superficie. La integral sobre $V_{f}(t)$ es muy difícil de calcular directamente, por lo que es preciso buscar volúmenes fijos o volúmenes de control adecuados que permitan resolver los problemas.

En forma diferencial, la ecuación de continuidad queda:

$$
\frac{\partial \rho}{\partial t}+\nabla \cdot(\rho \vec{v})=0
$$

\subsubsection{Ecuación de conservación de la cantidad de movimiento}

La segunda ley de Newton considera que la variación por unidad de tiempo de la cantidad de movimiento de una masa fluida es igual a la resultante instantánea de las fuerzas (de superficie y de volumen o másicas) que actúan sobre esa masa fluida. En forma integral puede expresarse como:

$$
\frac{d}{d t} \int_{V_{f}(t)} \rho \vec{v} d V=\int_{V_{0}} \frac{\partial}{\partial t}(\rho \vec{v}) d V+\int_{S_{0}} \rho \vec{v}(\vec{v} \cdot \vec{n}) d S=\int_{V_{0}} \rho \overrightarrow{f_{m}} d V+\int_{S_{0}}(\vec{n} \bar{\tau}) d S
$$

donde $\overrightarrow{f_{m}}$ es el vector de fuerzas másicas y $\bar{\tau}$ es el tensor de esfuerzos, que puede dividirse en:

$$
\bar{\tau}=-p \vec{n}+\bar{\tau}^{\prime}
$$

siendo $\bar{\tau}^{\prime}$ el tensor de esfuerzos viscosos y $p$ la presión que soporta el volumen fluido.

La forma diferencial de la ecuación de conservación de la cantidad de movimiento puede ponerse como:

$$
\frac{\partial\left(\rho u_{i}\right)}{\partial t}+\nabla \cdot\left(\rho u_{i} \vec{v}\right)=-\frac{\partial p}{\partial x_{i}}+\frac{\partial \tau_{i i}^{\prime}}{\partial x_{i}}+\frac{\partial \tau_{i j}^{\prime}}{\partial x_{j}}+\frac{\partial \tau_{i k}^{\prime}}{\partial x_{k}}+\rho \overrightarrow{f_{m}}
$$


Estas expresiones reciben el nombre de ecuaciones de Navier-Stokes.

Los esfuerzos viscosos pueden ponerse en función del campo de velocidades:

$$
\tau_{i i}^{\prime}=2 \mu\left(S_{i j}-\frac{1}{3} \delta_{i j} S_{i j}\right)=\mu\left[\left(\frac{\partial u_{i}}{\partial x_{j}}+\frac{\partial u_{j}}{\partial x_{i}}\right)-\frac{2}{3} \delta_{i j} \frac{\partial u_{k}}{\partial x_{k}}\right]
$$

siendo $\mu$ el coeficiente de viscosidad dinámica (molecular), $\delta_{i j}$ la delta de Kronecker y $S_{i j}$ el tensor de velocidad de deformación instantánea:

$$
S_{i j}=\frac{1}{2}\left(\frac{\partial u_{i}}{\partial x_{j}}+\frac{\partial u_{j}}{\partial x_{i}}\right)
$$

Los fluidos newtonianos son aquellos en los que los esfuerzos viscosos son proporcionales a los gradientes de velocidad que los producen. El agua es un fluido newtoniano, por lo que su comportamiento se ajusta a las ecuaciones anteriores.

\subsubsection{Ecuación de conservación de la energía}

La primera ley de la termodinámica considera que la variación de las energías interna y cinética en una masa fluida se debe al trabajo de las fuerzas de superficie y de las fuerzas másicas, y al calor comunicado por unidad de tiempo. En forma integral, la ecuación de conservación de la energía puede ponerse:

$$
\begin{aligned}
\frac{d}{d t} \int_{V_{f}(t)} \rho\left(e+\frac{v^{2}}{2}\right) d V & \\
& =\int_{V_{0}} \overrightarrow{f_{m}} \cdot \vec{v} d V+\int_{S_{0}}(\vec{n} \cdot \vec{\tau}) \vec{v} d S+\int_{V_{0}}\left(\dot{q}_{r}+\dot{q}_{q}\right) d V-\int_{S_{0}} \vec{q} \cdot \vec{n} d S
\end{aligned}
$$

donde $e$ es la energía interna y $\vec{q}$ es el flujo de calor transmitido por conducción. $\dot{q}_{r}$ y $\dot{q}_{q}$ son los calores por unidad de tiempo y por unidad de volumen transmitidos por radiación y por reacción química, respectivamente.

En forma diferencial, utilizando la ley de Fourier para expresar el flujo de calor por conducción en función de los gradientes de temperatura, la ecuación de conservación de la energía queda: 


$$
\begin{aligned}
\frac{\partial}{\partial t}\left[\rho\left(e+\frac{v^{2}}{2}\right)\right] & +\nabla \cdot\left[\rho\left(e+\frac{v^{2}}{2}\right) \vec{v}\right] \\
& =\frac{\partial}{\partial x}\left(k_{t} \frac{\partial T}{\partial x}\right)+\frac{\partial}{\partial y}\left(k_{t} \frac{\partial T}{\partial y}\right)+\frac{\partial}{\partial z}\left(k_{t} \frac{\partial T}{\partial z}\right)-\frac{\partial(u p)}{\partial x}-\frac{\partial(v p)}{\partial y} \\
& -\frac{\partial(w p)}{\partial z}+\frac{\partial\left(u \tau^{\prime}{ }_{x x}\right)}{\partial x}+\frac{\partial\left(u \tau^{\prime}{ }_{y x}\right)}{\partial y}+\frac{\partial\left(u \tau^{\prime}{ }_{z x}\right)}{\partial z}+\frac{\partial\left(v \tau_{x y}^{\prime}\right)}{\partial x} \\
& +\frac{\partial\left(v \tau_{y y}^{\prime}\right)}{\partial y}+\frac{\partial\left(v \tau^{\prime}{ }_{z y}\right)}{\partial z}+\frac{\partial\left(w \tau^{\prime}{ }_{x z}\right)}{\partial x}+\frac{\partial\left(w \tau^{\prime}{ }_{y z}\right)}{\partial y}+\frac{\partial\left(w \tau^{\prime}{ }_{z z}\right)}{\partial z} \\
& +\rho \overrightarrow{f_{m}} \cdot \vec{v}+\rho\left(\dot{Q}_{r}+\dot{Q}_{q}\right)
\end{aligned}
$$

donde $k_{t}$ es el coeficiente de conductividad térmica del fluido. $\dot{Q}_{r} \mathrm{y}{\dot{Q_{q}}}_{\mathrm{g}}$ son las potencias caloríficas totales transmitidas por radiación y por reacción química sobre el volumen de control.

\subsection{Condiciones de contorno}

Las condiciones de contorno pueden agruparse esquemáticamente en:

- Condición de Dirichlet. Especifica la variable dependiente $\Phi$ a lo largo de un contorno.

- Condición de Neumann. Especifica la derivada de la variable dependiente $\Phi$ a lo largo de un contorno.

- Condición de Robin. Especificación mixta de un valor de $\Phi$ y de una derivada de $\Phi$. Esta condición no es frecuente en Mecánica de Fluidos.

\subsection{Métodos de discretización}

\subsubsection{Discretización espacial}

Las ecuaciones que gobiernan el flujo por lo general, salvo en casos muy simples, no son susceptibles de soluciones analíticas. Por lo tanto, se debe realizar la discretización del dominio en pequeños subdominios denominados celdas (compuestos por formas geométricas primitivas como hexaedros y tetraedros en 3D, y cuadriláteros y triángulos en 2D) que conforman la malla. Las ecuaciones son discretizadas y resueltas dentro de cada uno de estos subdominios, utilizando uno de los tres sistemas existentes para resolver la forma aproximada del sistema de ecuaciones: volúmenes finitos, elementos finitos o diferencias finitas. 
El tipo de discretización espacial depende del tipo de discretización de las ecuaciones empleado. De este modo, el método de las diferencias finitas, que discretiza las ecuaciones de Navier-Stokes en forma diferencial, requiere una malla estructurada de puntos en los que se almacenan las variables del flujo. En el método de los volúmenes finitos se definen puntos de control donde se almacenan las variables en cada volumen generado. Los puntos están situados en el centro del elemento en el caso del método de volúmenes finitos, o en los vértices de las celdas en el método de diferencias finitas.

Se debe tener cuidado para asegurar la continuidad adecuada de la solución a través de las interfaces comunes entre los sub-dominios, de modo que las soluciones aproximadas dentro de las diversas partes se puedan unir para dar una imagen completa de todo el dominio.

El proceso de obtención de una malla adecuada ha sido considerado un cuello de botella en el proceso de análisis debido a la falta de un procedimiento totalmente automático. De este modo, el acceso a un buen paquete de generación del mallado y la experiencia son vitales para el éxito de la simulación.

Muchos algoritmos de mallado empiezan delimitando las superficies del dominio antes de llenar el interior con nodos de la malla. En este tipo de algoritmos, la generación de un mallado de calidad en la superficie es primordial.

Las clasificaciones usuales de mallados se basan en la conectividad o en el tipo de elementos que las conforman.

\section{Clasificación basada en la conectividad}

\section{- Mallados estructurados}

Se caracterizan por la ordenación regular de los elementos que pueden ser expresados como una matriz de dos o tres dimensiones, lo que restringe al empleo de elementos cuadriláteros en el caso bidimensional o hexaedros en estudios tridimensionales. En estos mallados, cada punto de la malla está inequívocamente identificado por los índices $i, j, k$ en coordenadas cartesianas. La regularidad de la conectividad permite ahorrar espacio ya que las relaciones entre elementos son definidas por la disposición de almacenamiento. Una clasificación adicional puede ser considerada en función de si el mallado es cartesiano o curvilíneo.

\section{- Mallados no estructurados}

Se caracterizan por una conectividad irregular que no es fácilmente expresable en forma de matriz. Permite utilizar cualquier elemento que el programa pueda 
resolver, ofreciendo gran flexibilidad en el tratamiento de geometrías complejas. La principal ventaja es que los elementos se pueden generar automáticamente independientemente de la complejidad del dominio, aunque en la práctica se deben determinar unos parámetros adecuados para obtener una malla de buena calidad.

En comparación con los mallados estructurados, los requisitos de almacenamiento suelen ser mucho mayores ya que la conectividad entre elementos vecinos debe ser explícitamente almacenada.

\section{- Mallados híbridos}

Un mallado híbrido contiene partes estructuradas y partes no estructuradas. El término mixto se aplica generalmente a los mallados que contienen elementos asociados con mallados estructurados y no estructurados (presumiblemente almacenada de una manera no estructurada).

\section{Clasificación basada en elementos}

Dependiendo del tipo de análisis y requisitos del programa, las mallas generadas pueden ser bidimensionales (triángulos o cuadriláteros) o tridimensionales. Algunas opciones de conectividad limitan los tipos de elementos presentes, por lo que existe un cierto solapamiento entre la clasificación basada en la conectividad y basada en el elemento base.

Los nodos de malla tridimensionales no están obligados a residir en un solo plano, siendo los elementos más habituales en mallas 3D los hexaedros, tetraedros, pirámides cuadradas y triángulos extrusionados en forma de cuña o prismas triangulares, delimitados por elementos 2D (Figura 54).

a)

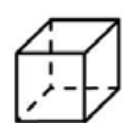

b)

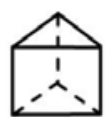

c)

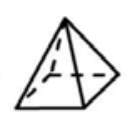

d)

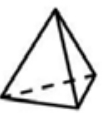

Calidad del elemento

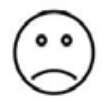

Obtención manual Vs. obtención automática
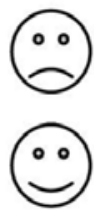

Figura 54. Tipologías de elementos: a) hexaédrico; b) cuña; c) pirámide; d) tetraedro (Carrillo y Castillo, 2011).

Los errores numéricos son un problema potencial en todos los paquetes de CFD, aunque hay modos de evitarlos. Sin tener en cuenta el código, una de las mayores 
fuentes de errores numéricos es la calidad de la malla. Las siguientes reglas ayudan a minimizar los errores de difusión (Hirsch, 2007):

- Colocar las condiciones de contorno de la malla, incluyendo las condiciones entre bloques de malla contiguos, tan lejos como sea razonablemente posible en áreas con grandes gradientes, incluyendo zonas de recirculación, resaltos hidráulicos y geometrías complejas.

- Refinar la malla donde se espere que existan grandes gradientes. Las regiones con grandes cambios en las propiedades del flujo a veces necesitan tamaños de celda menores para mantener la precisión de la solución.

- Evitar distorsiones en las relaciones de aspecto de los elementos. El cubo es la relación de aspecto más estable, aunque cuando la dirección del flujo es principalmente la horizontal, el tamaño vertical del elemento se puede reducir para obtener una mejor distribución de las velocidades.

- Evitar ensanchamientos discontinuos de la malla.

- Evitar discontinuidades entre los tamaños de celdas en las interfaces entre bloques. Cuando dos bloques de malla lindan, la relación de aspecto máxima entre las caras de las celdas debe ser 2:1. Además, las caras de las celdas deben se múltiplos exactos entre ellas, y se deben alinear perfectamente para evitar difusiones de cantidad de movimiento artificiales causadas por la interpolación en la condición de contorno.

\subsubsection{Discretización de las ecuaciones diferenciales}

La solución numérica de una ecuación diferencial consiste en un conjunto de valores a partir de los que se puede determinar la distribución de la variable dependiente $\Phi$ dentro del dominio considerado. La variación de $\Phi$ se suele representar mediante un polinomio en $x$ :

$$
\Phi=a_{o}+a_{1} x+a_{2} x^{2}+\ldots+a_{m} x^{m}
$$

El procedimiento numérico para encontrar un numero finito de coeficientes $a_{0}, a_{1}$, $a_{2}, \ldots a_{m}$, hace posible evaluar $\Phi$ para cualquier valor de $x$.

La mayoría de los métodos clásicos de discretización parten de valores desconocidos de una variable dependiente en un numero finito de localizaciones (nodos de la malla), que cubren todo el dominio de interés. El procedimiento consiste en encontrar un conjunto de ecuaciones algebraicas para dichos valores y en la utilización de un algoritmo para resolver el sistema de ecuaciones.

Para obtener los valores de la variable dependiente $\Phi$ en los nodos de malla se discretiza la distribución de dicha variable. Las ecuaciones algebraicas que tratan los 
valores desconocidos de $\Phi$ en los puntos de la malla (ecuaciones discretizadas), se derivan de la ecuación diferencial para $\Phi$. En esta derivación se emplea una función de distribución (o función de interpolación) que describe cómo varía $\Phi$ entre los nodos de la malla. La discretización sistemática del espacio y del tiempo, y de las variables dependientes, permite sustituir la ecuación diferencial de conservación por ecuaciones algebraicas, resolubles con cierta facilidad.

La estructura de una ecuación discretizada es una expresión algebraica que relaciona los valores de la variable dependiente en un grupo de nodos de la malla. El valor de $\Phi$ en un nodo dado sólo está afectado por los valores de los nodos adyacentes. De este modo, la solución será tanto mas exacta cuanto más nodos de malla existan, ya que las diferencias de la propiedad $\Phi$ se hacen muy pequeñas y los detalles sobre las hipótesis empleadas para la definición de la función pierden toda importancia. La forma clásica de una ecuación discretizada puede ser la siguiente:

$$
a_{i j} \Phi_{i j}=a_{i+1, j} \Phi_{i+1, j}+a_{i-1, j} \Phi_{i-1, j}+a_{i, j+1} \Phi_{i, j+1}+a_{i, j-1} \Phi_{i, j-1}
$$

siendo $a_{i j}, a_{i+1, j}, \ldots$ coeficientes distintos para cada uno de los nodos, $y \Phi_{i j}, \Phi_{i+1, j}, \ldots$ los valores correspondientes de la variable de interés.

Debido a las distintas funciones locales de interpolación empleadas y a los diferentes métodos de derivación, pueden encontrarse distintas ecuaciones discretizadas para una misma ecuación diferencial,.

El método de volúmenes finitos es el más utilizado en general, ya que aplica las ecuaciones de conservación a cada uno de los volúmenes finitos en los que se divide el dominio sin necesidad de suponer una forma de la solución a priori. Este método ha alcanzado soluciones correctas en múltiples problemas de ingeniería.

Existen enfoques alterativos, de carácter más básico, como proponer soluciones aproximadas basadas en soluciones analíticas exactas, como el lema de Riemann de flujo transitorio no viscoso y unidireccional.

\subsubsection{Formulación diferencial y formulación integral}

Las ecuaciones generales de conservación pueden aplicarse a un elemento diferencial de fluido (forma diferencial), o a un volumen fluido (forma integral).

Los métodos basados en una formulación diferencial se utilizaron en el inicio de los programas CFD, mientras que actuantemente se tiende a los métodos integrales. En la Tabla 14 se exponen las características fundamentales de las dos formulaciones (Kotate y Hijikata, 1993, citados por Zamora, 2008). 


\begin{tabular}{|c|c|}
\hline Formulación integral & Formulación diferencial \\
\hline Las propiedades se conservan exactamente & $\begin{array}{c}\text { Las propiedades se conservan } \\
\text { aproximadamente }\end{array}$ \\
\hline $\begin{array}{l}\text { Propiedades promediadas en un volumen } \\
\text { de control }\end{array}$ & Propiedades físicas locales \\
\hline Cantidades globales difíciles de tratar & Cantidades específicas fáciles de tratar \\
\hline Cálculo de las superficies complicada & Discretización de ecuaciones complicada \\
\hline Volúmenes finitos y elementos finitos & $\begin{array}{l}\text { Diferencias finitas } \\
\end{array}$ \\
\hline
\end{tabular}

\subsection{Propiedades de la solución numérica}

Las ecuaciones diferenciales en forma conservativa tienen la propiedad de que todos los coeficientes de los términos que incluyen derivadas son constantes o sus derivadas no aparecen en la ecuación. Sin embargo, una formulación numérica basada en la forma no conservativa (truncamiento de las ecuaciones diferenciales en polinomios de Taylor) puede originar dificultades numéricas en situaciones donde los coeficientes pueden ser discontinuos.

Los esquemas que cumplen en su versión discretizada el principio de conservación de forma exacta (salvo errores de redondeo), para cualquier tamaño de malla sobre un volumen finito arbitrario que contenga un cierto número de nodos, se dice que poseen la propiedad conservativa.

Todo esquema consistente debe cumplir aproximadamente el estado de conservación sobre grandes regiones del dominio.

Las soluciones numéricas deben estar dentro de unos limites apropiados. De este modo, cantidades que no pueden ser físicamente negativas, tales como la densidad o la energía cinética turbulenta, deben ser siempre positivas; otras, tales como la concentración de aire $C$, deben estar comprendidas entre 0 y $100 \%$.

Los esquemas de primer orden producen soluciones acotadas, mientras que los esquemas de mas alto orden pueden producir soluciones no acotadas, que suelen tener problemas de estabilidad y convergencia.

Los modelos matemáticos de fenómenos físicos muy complejos, tales como flujos turbulentos, combustión o flujos multifásicos, deben ser fiables, que garanticen que se simula la realidad con suficiente aproximación. Es fundamental que los modelos no terminen simulando procesos físicamente imposibles, puesto que el modelo numérico tenderá a reproducir las posibles inconsistencias físicas del modelo analítico. 


\subsection{Modelación de la turbulencia}

\subsubsection{Introducción a la turbulencia}

La turbulencia consiste en fluctuaciones en el tiempo y en el espacio dentro del campo fluido. Es un proceso complejo, principalmente debido a que se produce en las tres direcciones del espacio, es inestable y presenta muchas escalas fluctuantes aparentemente aleatorias, lo que ha motivado la caracterización de este tipo de flujos bajo un enfoque estadístico.

Para escalas suficientemente pequeñas y velocidades suficientemente bajas (número de Reynolds no demasiado grande), las ecuaciones de movimiento de un fluido viscoso tienen un buen comportamiento, con soluciones estables o permanentes. Estos flujos están dominados por la difusión viscosa de la vorticidad y la cantidad de movimiento. El movimiento se denomina laminar y se puede observar experimentalmente y en la naturaleza.

Con mayores números de Reynolds, las fuerzas de inercia del fluido llegan a ser significantes comparadas con las fuerzas viscosas, y el movimiento laminar se vuelve inestable. Aparecen fluctuaciones rápidas de velocidad y presión, y el movimiento se convierte en tridimensional e inestable (movimiento turbulento).

En principio, las ecuaciones de Navier-Stokes describen los flujos laminar y turbulento sin necesidad de información adicional. Sin embargo, los flujos turbulentos con números de Reynolds reales abarcan un amplio rango de longitudes turbulentas y tiempos de escala. En su resolución, generalmente, se requieren de longitudes de escala mucho menores que el menor mallado que puede ser usado de modo práctico en un análisis numérico.

Para poder predecir los efectos de la turbulencia, muchas investigaciones se han centrado en métodos que hacen uso de modelos de turbulencia. Los modelos de turbulencia han sido específicamente desarrollados para tener en cuenta los efectos de la turbulencia sin recurrir a un mallado prohibitivamente fino y a la simulación numérica directa (Direct Numerical Simulation, DNS).

La primera idea de modelización mediante un modelo de ecuaciones de transporte surge de Kolmogorov (1941). Muchos de los resultados postulados por Prandtl (teoría de la capa límite, idea de la longitud de mezcla turbulenta), von Kármán (Ley universal de la capa límite) y Kolmogorov (energía cinética turbulenta, frecuencia de turbulencia), han sido de una importancia vital en la comprensión y en la simulación numérica de la turbulencia.

Sin embargo, ninguno de los modelos de turbulencia está aceptado como el mejor para todo tipo de problemas. La elección del modelo depende de consideraciones como 
la precisión deseada, los recursos informáticos, el tiempo disponible, o la práctica establecida para cada tipo de problema.

\subsubsection{Definición de turbulencia}

No existe una definición clara de flujo turbulento. Wilcox (2010) realiza diversas reflexiones referentes a la turbulencia:

- Definición básica. von Kármán (1937), citando a Taylor, definió la turbulencia de la siguiente manera:

"Turbulence is an irregular motion which in general makes its appearance in fluids, gaseous or liquid, when they flow past solid surfaces or even when neighboring streams of the same fluid flow past or over one another."

Diferentes autores consideran que el término irregular motion es demasiado impreciso. Un movimiento irregular es un movimiento que es típicamente aperiódico y que no puede describirse como una función sencilla dependiente del espacio y del tiempo. Un movimiento irregular también podría ser debido a las condiciones iniciales. Según Wilcox, el problema de esta definición es que existen flujos no turbulentos que puede ser descritos como irregulares.

Un movimiento turbulento es irregular en el sentido de que puede ser descrito por las leyes de probabilidad. Las propiedades instantáneas en un flujo turbulento son extremadamente sensibles a las condiciones iniciales, pero no sus promediados estadísticos. Hinze (1975) formula la siguiente definición:

"Turbulent fluid motion is an irregular condition of flow in which the various quantities show a random variation with time and space coordinates, so that statistically distinct average values can be discerned."

Diversos investigadores se refieren al movimiento de remolino, que es un movimiento local donde aparecen remolinos turbulentos de un amplio rango de tamaños que originan la mezcla del fluido y la aparición de tensiones turbulentas efectivas, las cuales pueden ser muy grandes en comparación con los valores del flujo laminar.

- Inestabilidad. El análisis de las soluciones de las ecuaciones de Navier-Stokes muestra que la turbulencia se desarrolla como una inestabilidad del flujo laminar. Matemáticamente hablando, en un fluido real las inestabilidades son principalmente debidas a la interacción entre los términos no lineales inerciales y los términos viscosos de las ecuaciones de Navier-Stokes. La interacción es muy compleja, ya que es rotacional, tridimensional y dependiente del tiempo. 
- No linealidad. La no linealidad de las ecuaciones de Navier-Stokes conduce a la interacción entre las fluctuaciones de diferentes direcciones y longitudes de onda. La vorticidad es el principal proceso físico que propaga el movimiento en una amplia gama de longitudes de onda. La turbulencia a gran escala lleva la mayor parte de la energía y es el principal responsable de la difusividad y las tensiones. A su vez, los remolinos más grandes generan los vórtices que componen los remolinos más pequeños, disminuyendo la energía en ellos. Finalmente, la energía se disipa por viscosidad en las menores longitudes de onda.

- Aspectos estadísticos. La turbulencia se caracteriza por fluctuaciones aleatorias que exigen el uso de métodos estadísticos para analizarlo. Aunque este aspecto no es realmente un problema desde la perspectiva ingenieril, las operaciones de promedio temporal originan términos de las ecuaciones del movimiento que no pueden ser determinadas a priori.

- Fenómeno continuo. En principio, sabemos que la dependencia temporal, la continuidad tridimensional y las ecuaciones de Navier-Stokes contienen toda la física de un flujo turbulento. Que esto es así se deduce del hecho de que la turbulencia es un fenómeno continuo. Tennekes y Lumley (1983) consideran:

"Even the smallest scales occurring in a turbulent flow are ordinarily far larger than am molecular length scale. "

Sin embargo, las menores escalas de la turbulencia son muchos órdenes de magnitud más pequeñas que las escalas más grandes. Además, la relación de escala de menor a mayor disminuye rápidamente a medida que aumenta el número de Reynolds. Para hacer una simulación numérica exacta (es decir, una solución tridimensional completamente dependientes del tiempo) de un flujo turbulento, deben ser resueltas todas las escalas físicas relevantes.

Si bien se están haciendo avances, los ordenadores de principio del siglo XXI no tienen suficiente memoria y velocidad para resolver cualquier problema de flujo turbulento de interés práctico.

- Estructura de la vorticidad. La vorticidad en un flujo turbulento es en sí misma tridimensional, por lo que las líneas de vórtice en el flujo no son paralelas. Esta tridimensionalidad inherente significa que no hay aproximaciones bidimensionales satisfactorias para determinar los detalles de flujos turbulentos puesto que los flujos turbulentos deben ser de tridimensionales, incluso cuando el movimiento promedio es bidimensional. La mayor parte de la vorticidad en un flujo turbulento reside en los remolinos más pequeños.

- Escalas de la turbulencia y la cascada de turbulencias. La turbulencia consiste en un espectro continuo de escalas que van de mayor a menor. Un remolino turbulento se puede considerar como un movimiento de rotación local 
cuya dimensión característica es la escala de turbulencia local. Los remolinos se solapan en el espacio, de modo que los más grandes transportan otros más pequeños. La turbulencia tiene un proceso en cascada por el cual, cuando la turbulencia disminuye, la energía cinética se transfiere de los remolinos más grandes a los más pequeños. En última instancia, los remolinos más pequeños disipan la energía en forma de calor a través de la acción de la viscosidad molecular, y la velocidad a la que se disipan está controlada por la velocidad a la que reciben la energía de los remolinos más grandes. Por lo tanto, los flujos turbulentos son siempre disipativos.

- Grandes remolinos y mezcla turbulenta. La turbulencia está dominado por remolinos. La aproximación de los grandes remolinos hacia la interfaz entre la región turbulenta y el fluido no turbulento distorsiona la interfaz entre ambas regiones, pudiendo llegar hasta distancias del orden de 30 veces el ancho de la corriente (Bradshaw, 1972). Por lo tanto, el estado de un flujo turbulento en una posición determinada depende de la situación aguas arriba y no puede ser únicamente especificada en términos del tensor tensión-deformación local, como en el flujo laminar.

- Mejora de la difusividad. La difusión turbulenta mejora la transferencia de masa, cantidad de movimiento y energía, siendo varios órdenes de magnitud mayor que en los flujos laminares.

\subsubsection{Las escalas de Kolmogorov}

Se considera la turbulencia como un fenómeno del continuo. El proceso en cascada presente en todos los flujos turbulentos implica una transferencia de la energía cinética turbulenta de los grandes remolinos a los remolinos más pequeños. Puesto que los movimientos de pequeña escala tienden a ocurrir en una escala de tiempo pequeña, se puede suponer que es independiente de la dinámica relativamente lenta de los grandes remolinos y de las propiedades medias del flujo.

Según la Teoría del equilibrio universal de Kolmogorov (1941), los remolinos más pequeños deben estar en un estado en que la tasa a la que reciben la energía de los remolinos más grandes es casi igual a la tasa a la que los remolinos más pequeños disipan la energía en forma de calor. Por lo tanto, el movimiento en las escalas más pequeñas debería dependen sólo de:

- El ratio con el que suministran la energía los remolinos más grandes, $\varepsilon=-d k / d t$ $\left[\mathrm{L}^{2} / \mathrm{T}^{3}\right]$.

- La viscosidad cinemática, $v\left[\mathrm{~L}^{2} / \mathrm{T}\right]$.

Las escalas de Kolmogorov de longitud $(\eta)$, tiempo $(t)$ y velocidad $(v)$ son: 


$$
\eta \equiv\left(v^{3} / \varepsilon\right)^{1 / 4}, \quad t \equiv(v / \varepsilon)^{1 / 2}, \quad v \equiv(v \varepsilon)^{1 / 4}
$$

\subsubsection{Descripción espectral y Ley $-5 / 3$ de Kolmogorov}

Una representación espectral es una descomposición de Fourier en números de onda $\kappa$ o en su equivalente en longitudes de onda $\lambda=2 \pi / \kappa$. En consecuencia, la densidad de energía espectral o la función espectral de energía $E(\kappa)$ está relacionada con la transformada de Fourier de la energía cinética turbulenta $k$.

Si $E(\kappa) d \kappa$ es la energía cinética turbulenta $k$ contenida entre los números de onda $\kappa$ y $\kappa+d \kappa$, se puede considerar:

$$
k=\int_{0}^{\infty} E(\kappa) d \kappa
$$

donde $k$ es la energía cinética por unidad de masa de la velocidad turbulenta fluctuante.

Puesto que la turbulencia está fuertemente influenciada por los remolinos grandes, se espera que $E(\kappa)$ sea función de la longitud característica de los grandes remolinos $l$ y del tensor velocidad de deformación media $S$ que incrementa la turbulencia mediante la interacción directa del flujo y los remolinos grandes. Además, dado que la turbulencia es siempre disipativa, se espera que $E(\kappa)$ dependa de la viscosidad cinemática $v$ y del ratio con el que suministran la energía los remolinos más grandes $\varepsilon$. Por definición, también debe depender del número de onda $\kappa$. Para turbulencias con un elevado número de Reynolds, $k$ se puede expresar en términos de $\varepsilon$ y l (Taylor, 1935):

$$
\varepsilon \sim \frac{k^{3 / 2}}{l} \Rightarrow k \sim(\varepsilon l)^{2 / 3}
$$

l es la longitud de escala en que se basan la mayoría de los modelos de turbulencia, aunque aún no se ha cuantificado. En la mayoría de los análisis de los modelos de turbulencia se asume que hay una amplio rango de escalas, lo que implícitamente supone que $l$ es muy grande en comparación con la longitud de escala de Kolmogorov $(l \gg \eta)$.

Sustituyendo la estimación de $\varepsilon$ en la longitud de escala de Kolmogorov, queda:

$$
\frac{l}{\eta}=\frac{l}{\left(v^{3} / \varepsilon\right)^{1 / 4}} \sim \frac{l\left(k^{3 / 2} / l\right)^{1 / 4}}{v^{3 / 4}} \sim R e_{T}^{3 / 4} \quad \text { donde } \quad R e_{T}^{3 / 4}=\frac{k^{1 / 2} l}{v}
$$

$R e_{T}$ es el número de Reynolds turbulento. Por lo tanto, la condición $l \gg \eta$ se mantiene siempre y cuando se tengan altos números de Reynolds $\left(R e_{T} \gg 1\right)$. 
La existencia de una gran separación de escalas es la hipótesis fundamental de la teoría del equilibrio universal de Kolmogorov. Es decir, para números de Reynolds muy elevados, existe un rango de tamaños de remolino entre el tamaño más grande y el más pequeño para el cual el proceso en cascada es independiente de la energía contenida en los remolinos (de modo que $S$ y $l$ pueden ser ignorados), y de los efectos directos de la viscosidad molecular (por lo que $v$ puede ser ignorada). De este modo, existe una serie de números de onda en la que la energía transferida por los efectos inerciales es dominante, por lo cual $E(\kappa)$ depende sólo de $\varepsilon$ y $\kappa$. Por análisis dimensional:

$$
E(\kappa)=C_{K} \varepsilon^{2 / 3} \kappa^{-5 / 3} ; \quad \frac{1}{l} \ll \kappa \ll \frac{1}{\eta}
$$

donde $C_{K}$ es la constante de Kolmogorov.

Puesto que la transferencia inercial de energía es dominante, Kolmogorov identificó este rango de números de onda como el subrango inercial. La existencia del subrango inercial ha sido verificada por muchos experimentos y simulaciones numéricas. La Figura 55 muestra un espectro de energía típico de un flujo turbulento.

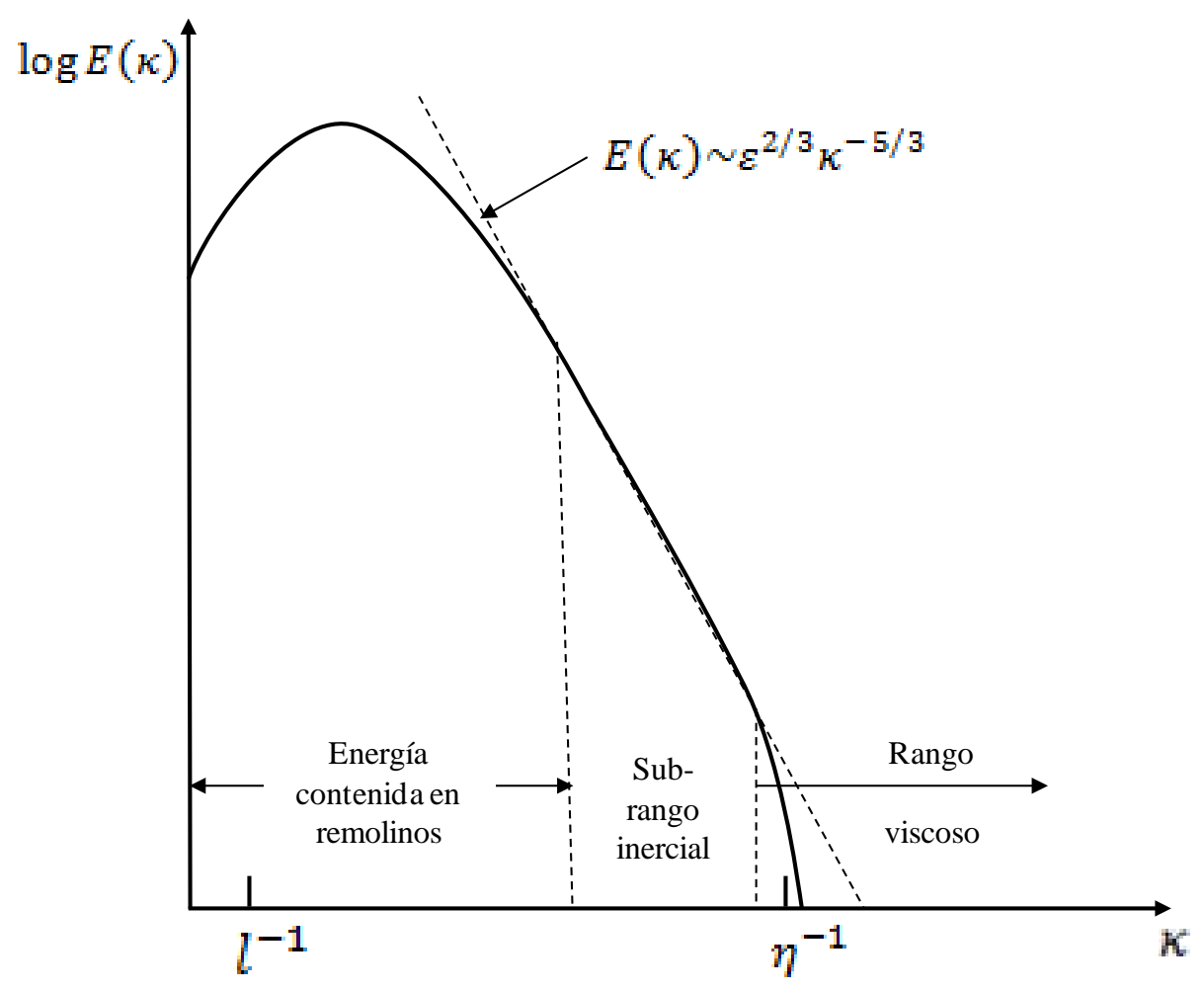

Figura 55. Espectro de energía para un flujo turbulento (Wilcox, 2010).

Aunque la ley -5/3 de Kolmogorov apenas se emplea en los modelos de turbulencia convencionales, tiene gran importancia en Simulación Numérica Directa (DNS), 
Simulación de Gran Remolino (LES), y Simulación de Remolino Independiente (DES). La ley -5/3 de Kolmogorov está tan afianzada que las predicciones teóricas o numéricas son vistas con escepticismo si la misma no puede reproducirse.

\subsubsection{Ecuaciones de Navier-Stokes promediado de Reynolds}

Cuando en un fenómeno se presentan tiempos de escala muy superiores a los tiempos de escala de las fluctuaciones turbulentas, se puede considerar que los flujos turbulentos presentan características medias, con una variación temporal adicional de la componente fluctuante. De este modo, cada componente $\Phi$ puede ser dividida en una componente media $\bar{\Phi}$, y una componente variable en el tiempo $\Phi^{\prime}$ :

$$
\Phi(\vec{x}, t)=\bar{\Phi}(\vec{x}, t)+\Phi^{\prime}(\vec{x}, t)
$$

La componente media viene dada por:

$$
\bar{\Phi}=\frac{1}{\Delta t} \int_{t}^{t+\Delta t} \Phi d t
$$

donde $\Delta t$ es una escala de tiempo grande en relación con las fluctuaciones turbulentas, pero pequeña en relación con la escala de tiempo empleada para resolver las ecuaciones.

En general, los modelos de turbulencia buscan modificar las ecuaciones de NavierStokes no permanentes (transitorias) mediante la introducción de promediados y cantidades fluctuantes, obteniendo las denominadas ecuaciones de Navier-Stokes promediado de Reynolds (RANS). Estas ecuaciones representan únicamente las cantidades de flujo medias, modelando los efectos de la turbulencia sin necesidad de resolver las fluctuaciones turbulentas.

Los modelos de turbulencia basados en las ecuaciones RANS son conocidos como modelos de turbulencia estadística, debido a que para obtener las ecuaciones se realizan promediados estadísticos.

Sustituyendo las cantidades promediadas en las ecuaciones de Navier-Stokes originales, los resultados de las ecuaciones de Navier-Stokes promediadas de Reynolds se muestran a continuación:

$$
\begin{gathered}
\frac{\partial \rho}{\partial t}+\frac{\partial}{\partial x_{j}}\left(\rho u_{j}\right)=0 \\
\frac{\partial \rho u_{i}}{\partial t}+\frac{\partial}{\partial x_{j}}\left(\rho u_{i} u_{j}\right)=-\frac{\partial \rho u_{i}}{\partial x_{i}}+\frac{\partial}{\partial x_{j}}\left(\tau_{i j}-\rho \overline{u_{\imath}^{\prime} u_{\jmath}^{\prime}}\right)+S_{M}
\end{gathered}
$$




$$
\begin{aligned}
\frac{\partial \rho h_{t o t}}{\partial t}-\frac{\partial p}{\partial t}+ & \frac{\partial}{\partial x_{j}}\left(\rho u_{j} h_{t o t}\right) \\
& =\frac{\partial}{\partial x_{j}}\left(\lambda \frac{\partial T}{\partial x_{j}}-\rho \overline{u_{\jmath}^{\prime} h}\right)+\frac{\partial}{\partial x_{j}}\left[u_{i}\left(\tau_{i j}-\rho \overline{u_{\imath}^{\prime} u_{\jmath}^{\prime}}\right)\right]+S_{E}
\end{aligned}
$$

siendo $\tau_{i j}$ el tensor de esfuerzos viscosos (que incluye las componentes normal y de las tensiones de corte). $S_{M}$ y $S_{E}$ son los términos fuente de las ecuaciones de cantidad de movimiento y energía respectivamente.

La ecuación de continuidad no ha sido alterada, pero las ecuaciones de cantidad de movimiento y de energía contienen las tensiones de Reynolds, $-\rho \overline{u_{\imath}{ }^{\prime} u_{\jmath}{ }^{\prime}}$, que son términos de flujo turbulento adicionales al flujo difusivo molecular. Estos términos surgen de los términos convectivos no lineales en las ecuaciones no promediadas, y reflejan el hecho de que el trasporte convectivo mejora la mezcla debido a las fluctuaciones de velocidades turbulentas, por encima de los efectos causados por fluctuaciones térmicas a nivel molecular.

La ecuación de la energía contiene un término de flujo turbulento adicional, $\rho \overline{u_{l}{ }^{\prime} h}$, comparada con la ecuación instantánea. El término $\frac{\partial}{\partial x_{i}}\left[u_{j}\left(\tau_{i j}-\rho \overline{u_{\imath}{ }^{\prime} u_{\jmath}^{\prime}}\right)\right]$ hace referencia al trabajo viscoso.

La energía total media del fluido por unidad de masa viene dada por:

$$
h_{\text {tot }}=h_{\text {stat }}+\frac{1}{2} U U+k
$$

donde $h_{\text {stat }}$ es la entalpía estática (cantidad de energía que un sistema puede intercambiar con su entorno) y $U$ es el vector velocidad. La entalpía estática se define en términos de energía interna del fluido y del estado del fluido.

En los modelos de turbulencia que calculan la energía cinética turbulenta $k$ (modelos $k$-épsilon, $k$-omega, etc.) se incluye el término de la energía cinética turbulenta dado por:

$$
k=\frac{1}{2} \overline{u_{l}^{\prime 2}}
$$

La simulación de las ecuaciones RANS reduce considerablemente los esfuerzos computacionales comparados con la Simulación Numérica Directa y son generalmente adoptados en los cálculos prácticos. Sin embargo, el procedimiento de promediado introduce incógnitas adicionales difíciles de determinar directamente denominadas tensiones turbulentas o de Reynolds, generando incógnitas adicionales. 
Al existir más incógnitas que ecuaciones, se plantea el problema de cierre de la turbulencia. Las tensiones (turbulentas) de Reynolds necesitan entonces ser modeladas mediante ecuaciones adicionales que permitan lograr el cierre del sistema. El cierre implica que existe un número suficiente de ecuaciones para todas las incógnitas, incluyendo el tensor de tensiones de Reynolds resultante del procedimiento de promediado. Las ecuaciones usadas para cerrar el sistema definen el tipo de modelo de turbulencia.

Algunas teorías clásicas para lograr el cierre de las ecuaciones son:

- Viscosidad turbulenta de Boussinesq. Propone expresar los esfuerzos turbulentos de modo análogo a los viscosos mediante una cierta viscosidad de remolino o turbulenta $\mu_{t}$ :

$$
\tau_{i j}^{\prime}=-\rho \overline{u_{\imath}^{\prime} u_{\jmath}^{\prime}}=\mu_{t}\left(\frac{\partial u_{i}}{\partial x_{j}}+\frac{\partial u_{j}}{\partial x_{i}}\right)
$$

En general $\mu_{t}$ no es uniforme para todo el campo fluido, por lo que posteriormente se introdujo la siguiente expresión de la viscosidad turbulenta:

$$
\mu_{t}=C \rho u_{m} l_{m}
$$

donde $C$ es una constante que depende del tipo de flujo, mientras que $u_{m}$ y $l_{m}$ son los valores característicos de la escala turbulenta. Puede considerarse que el transporte turbulento de la propiedad escalar $\phi$ es proporcional a los gradientes medios de esa misma propiedad $\left(\phi^{\prime}=f(\phi)\right)$ :

$$
-\rho \overline{u_{\imath}^{\prime} \phi^{\prime}}=\Gamma_{t} \frac{\partial \phi}{\partial x_{j}}
$$

siendo $\Gamma_{t}$ la difusividad turbulenta, que se asocia con el número de Prandtl turbulento como:

$$
\Gamma_{t}=\frac{\mu_{t}}{P r_{t}}
$$

donde $P r_{t}=v_{t} / \alpha_{t}$ es el número de Prandtl turbulento, siendo $v_{t}$ la viscosidad cinemática turbulenta y $\alpha_{t}$ la difusividad térmica turbulenta.

- Longitud de mezcla de Prandtl. Define el recorrido medio que hacen las partículas del flujo cuando transportan una determinada cantidad (movimiento, energía, etc.). La velocidad característica o de agitación es: 


$$
U_{m} \approx \mathrm{Cl}_{m}\left|\frac{\partial u_{i}}{\partial x_{j}}\right|
$$

donde $l_{m}$ es la longitud de mezcla, quedando:

$$
-\rho \overline{u_{\imath}^{\prime} u_{\jmath}^{\prime}} \approx \mu_{t} \frac{\partial u_{i}}{\partial x_{j}}
$$

siendo donde $l_{m}$ es la longitud de mezcla, quedando:

$$
\mu_{t} \approx \rho l_{m}^{2}\left|\frac{\partial u}{\partial y}\right|
$$

La viscosidad turbulenta $\mu_{t}$ es una propiedad del flujo, no del fluido, y puede modelarse de distintas formas.

- Hipótesis de semejanza de von Kármán. La longitud de mezcla se representa en función de los gradientes locales del campo de velocidades, donde $y$ es la distancia a la pared:

$$
l=\kappa y
$$

donde $\kappa=0.41$ es la constante de von Kármán, la cual ha sido determinada a partir de datos experimentales (aunque realmente no es constante). Incorporando la suposición anterior, el esfuerzo de Reynolds puede expresarse como

$$
\rho \overline{u^{\prime} v^{\prime}} \approx \rho \kappa^{2} y^{2}\left(\frac{\partial u}{\partial y}\right)^{2}=\rho \kappa^{2} y^{2}\left|\frac{\partial u}{\partial y}\right|\left(\frac{\partial u}{\partial y}\right)
$$

Se han desarrollado diferentes modelos basados en las ecuaciones promediadas de Navier-Stokes (RANS), que pueden ser empleados para aproximar los efectos de la turbulencia. Algunos son válidos para aplicaciones muy específicas, mientras que otros pueden aplicarse a varias clases de flujos con un grado razonable de confianza. Los modelos de turbulencia pueden ser en general divididos en dos clases: modelos de viscosidad de remolino y modelos de tensiones de Reynolds.

\subsubsection{Modelos de turbulencia de viscosidad de remolino}

La hipótesis de viscosidad de remolino de Boussinesq en que se basan estos modelos sugiere que la turbulencia se compone de pequeños remolinos que están continuamente formándose y desapareciendo. Supone que las tensiones de Reynolds se pueden 
relacionar con los gradientes de velocidad media y la viscosidad de remolino (turbulenta) mediante la hipótesis del gradiente de difusión, de manera análoga a la relación entre el tensor de tensiones y la tensión en flujos laminares Newtonianos:

$$
-\rho \overline{u_{\imath}^{\prime} u_{\jmath}^{\prime}}=\mu_{t}\left(\frac{\partial U_{i}}{\partial x_{j}}+\frac{\partial U_{j}}{\partial x_{i}}\right)-\frac{2}{3} \delta_{i j}\left(\rho k+\mu_{t} \frac{\partial U_{k}}{\partial x_{k}}\right)
$$

donde $\mu_{t}$ es la viscosidad de remolino o viscosidad turbulenta, que debe ser modelada y $\delta_{i j}$ es el delta de Kronecker.

La ecuación puede expresar fluctuaciones turbulentas en términos de funciones de las variables medias sólo si se conoce la viscosidad turbulenta $\mu_{t}$. Los modelos de turbulencia de dos ecuaciones, $k-\varepsilon$ y $k-\omega$, utilizan esta variable.

Con estas hipótesis, las ecuaciones de cantidad de movimiento con el promedio de Reynolds quedan:

$$
\frac{\partial \rho U_{i}}{\partial t}+\frac{\partial}{\partial x_{j}}\left(\rho U_{i} U_{j}\right)=-\frac{\partial p^{\prime}}{\partial x_{i}}+\frac{\partial}{\partial x_{j}}\left[\mu_{e f f}\left(\frac{\partial U_{i}}{\partial x_{j}}+\frac{\partial U_{j}}{\partial x_{i}}\right)\right]+S_{M}
$$

donde $S_{M}$ es la suma de las fuerzas másicas, y $\mu_{\text {eff }}$ es la viscosidad efectiva definida por:

$$
\mu_{\text {eff }}=\mu+\mu_{t}
$$

p' es la presión modificada, definida por:

$$
p^{\prime}=p+\frac{2}{3} \rho k+\frac{2}{3} \mu_{e f f} \frac{\partial U_{k}}{\partial x_{k}}
$$

El último término en la ecuación (125), $\frac{2}{3} \mu_{e f f} \frac{\partial U_{k}}{\partial x_{k}}$, tiene en cuenta la divergencia de la velocidad. Este término suele despreciarse en el caso de fluidos incompresibles.

El promedio de Reynolds de la ecuación de la energía se convierte en:

$$
\begin{aligned}
\frac{\partial \rho h_{t o t}}{\partial t}-\frac{\partial \rho}{\partial t}+ & \frac{\partial}{\partial x_{j}}\left(\rho U_{j} h_{t o t}\right) \\
& =\frac{\partial}{\partial x_{j}}\left(\lambda \frac{\partial T}{\partial x_{j}}+\frac{\mu_{t}}{P r_{t}} \frac{\partial h}{\partial x_{j}}\right)+\frac{\partial}{\partial x_{j}}\left[U_{i}\left(\tau_{i j}-\rho \overline{u_{\imath}^{\prime} u_{\jmath}^{\prime}}\right)\right]+S_{E}
\end{aligned}
$$

Aunque la transformación del término de difusión molecular puede ser inexacta si la energía depende de otras variables como la temperatura, el término de difusión turbulenta es correcto, sin perjuicio de la hipótesis de la difusividad de remolino. 
Además, como la difusión turbulenta es generalmente mucho mayor que la difusión molecular, los pequeños errores en el segundo pueden ser despreciables.

Del mismo modo, el promediado de Reynolds de las ecuación de transporte para las variables adicionales se convierte en:

$$
\frac{\partial \rho \phi}{\partial t}+\frac{\partial}{\partial x_{j}}\left(\rho U_{j} \phi\right)=\frac{\partial}{\partial x_{j}}\left[\left(\Gamma_{\phi}+\frac{\mu_{t}}{\sigma_{\phi}}\right) \frac{\partial \phi}{\partial x_{j}}\right]+S_{\phi}
$$

La diferencia entre los diversos modelos de viscosidad de remolino viene determinada por la forma en que se determinan la viscosidad de remolino y la difusividad de remolino (modelos $k-\varepsilon, k-\omega$, etc.).

\subsubsection{Modelos de turbulencia de dos ecuaciones}

Los modelos de turbulencia de dos ecuaciones son muy utilizados debido a que ofrecen un buen compromiso entre el esfuerzo computacional y la precisión numérica. Tanto la velocidad como la longitud de escala son resueltos usando ecuaciones de transporte independientes, una para la energía cinética turbulenta $k$ y otra para la tasa de disipación de energía cinética turbulenta $\varepsilon$, o para la frecuencia turbulenta o ritmo de disipación de energía específica $\omega$.

Los modelos de dos ecuaciones $k-\varepsilon$ y $k$ - $\omega$ usan la hipótesis del gradiente de difusión para relacionar las tensiones de Reynolds con los gradientes de la velocidad media y la viscosidad turbulenta. La viscosidad turbulenta se obtiene como producto de la velocidad turbulenta y la longitud de escala turbulenta.

En estos modelos, la longitud de escala de la velocidad turbulenta se calcula mediante la energía cinética turbulenta, que se obtiene a partir de la solución de sus ecuaciones de transporte. La longitud de escala turbulenta se estima mediante dos propiedades del campo de turbulencias (generalmente la energía cinética turbulenta y su ratio de disipación). El ratio de disipación de la energía cinética turbulenta se obtiene a partir de la solución de sus ecuaciones de trasporte.

Estos modelos consideran el concepto de energía cinética turbulenta. Se supone que la energía cinética total instantánea $E_{c}(t)$ de un flujo turbulento se obtienen como:

$$
E_{c}(t)=E_{c}+k
$$

siendo $E_{c}$ la energía cinética correspondiente al movimiento medio,

$$
E_{c}=\frac{1}{2}\left(U^{2}+V^{2}+W^{2}\right)
$$


y $k$ la correspondiente al movimiento turbulento.

$$
k=\frac{1}{2}\left(\overline{u^{\prime 2}}+\overline{v^{\prime 2}}+\overline{w^{\prime 2}}\right)
$$

\subsubsection{2. $\quad$ El modelo $k-\varepsilon$}

El modelo k-e ó k-épsilon (Harlow y Nakayama, 1968; Launder y Sharma, 1974) se considera el modelo estándar, siendo uno de los modelos más empleados en los códigos de CFD. Ha demostrado ser estable, numéricamente robusto y presentar buena capacidad de predicción. Para simulaciones de uso general, ofrece un buen compromiso en términos de precisión y robustez.

Aunque los modelos estándar de dos ecuaciones, como el modelo $k-\varepsilon$, proporcionan buenas predicciones para muchos flujos de interés ingenieril, hay aplicaciones para las que estos modelos no son adecuados. Entre ellas se encuentran:

- Flujos con separación de capa límite.

- Flujos con cambios repentinos en la velocidad media de deformación.

- Flujos de fluidos en rotación.

- Flujos sobre superficies curvas.

Los fundamentos teóricos del modelo $k-\varepsilon$ son:

- $\quad k$ es la energía cinética turbulenta, definida como la varianza de las fluctuaciones de velocidad. Tiene dimensiones de $\left[\mathrm{L}^{2} \mathrm{~T}^{-2}\right]$.

- $\varepsilon$ es la disipación de remolino turbulenta (el ratio a partir del cual las fluctuaciones de velocidad se disipan), y tiene dimensiones de $k$ por unidad de tiempo $\left[\mathrm{L}^{2} \mathrm{~T}^{-3}\right]$.

El modelo $k-\varepsilon$ introduce dos nuevas variables en el sistema de ecuaciones, por lo que la ecuación de continuidad queda:

$$
\frac{\partial \rho}{\partial t}+\frac{\partial}{\partial x_{j}}\left(\rho U_{j}\right)=0
$$

y la ecuación de cantidad de movimiento queda:

$$
\frac{\partial \rho U_{i}}{\partial t}+\frac{\partial}{\partial x_{j}}\left(\rho U_{i} U_{j}\right)=-\frac{\partial p^{\prime}}{\partial x_{i}}+\frac{\partial}{\partial x_{j}}\left[\mu_{e f f}\left(\frac{\partial U_{i}}{\partial x_{j}}+\frac{\partial U_{j}}{\partial x_{i}}\right)\right]+S_{M}
$$


donde $S_{M}$ es la suma de las fuerzas másicas, $\mu_{e f f}$ es la viscosidad efectiva debida a la turbulencia, y p' es la presión modificada, definida en la ecuación (125).

El modelo $k-\varepsilon$ está basado en el concepto de la viscosidad de remolino, de este modo:

$$
\mu_{\text {eff }}=\mu+\mu_{t}
$$

donde $\mu_{t}$ es la viscosidad turbulenta.

El modelo $k-\varepsilon$ asume que la viscosidad turbulenta está relacionada con la energía cinética turbulenta y la disipación mediante la relación:

$$
\mu_{t}=C_{\mu} \rho \frac{k^{2}}{\varepsilon}
$$

donde $C_{\mu}=0.09$ es una constante.

Los valores de $k$ y $\varepsilon$ vienen de las ecuaciones de trasporte para la energía cinética turbulenta y el ratio de disipación turbulenta:

$$
\begin{gathered}
\frac{\partial(\rho k)}{\partial t}+\frac{\partial}{\partial x_{j}}\left(\rho U_{j} k\right)=\frac{\partial}{\partial x_{j}}\left[\left(\mu+\frac{\mu_{t}}{\sigma_{k}}\right) \frac{\partial k}{\partial x_{j}}\right]+P_{k}-\rho \varepsilon+P_{k b} \\
\frac{\partial(\rho \varepsilon)}{\partial t}+\frac{\partial}{\partial x_{j}}\left(\rho U_{j} \varepsilon\right)=\frac{\partial}{\partial x_{j}}\left[\left(\mu+\frac{\mu_{t}}{\sigma_{\varepsilon}}\right) \frac{\partial \varepsilon}{\partial x_{j}}\right]+\frac{\varepsilon}{k}\left(C_{1 \varepsilon} P_{k}-C_{2 \varepsilon} \rho \varepsilon+C_{1 \varepsilon} P_{\varepsilon b}\right)
\end{gathered}
$$

$C_{1 \varepsilon}, C_{2 \varepsilon}, \sigma_{k}$ y $\sigma_{\varepsilon}$ son constantes, $P_{k}$ es la producción turbulenta debido a fuerzas viscosas, mientras que $P_{k b}$ y $P_{\varepsilon b}$ representan la influencia de las fuerzas de gravedad.

El significado físico de las expresiones de $k$ y $\varepsilon$ se pude resumir como:

$$
\begin{aligned}
& {\left[\begin{array}{c}
\text { Velocidad de } \\
\text { cambio de } k / \varepsilon
\end{array}\right]+\left[\begin{array}{c}
\text { Transporte de } k / \varepsilon \\
\text { por convección }
\end{array}\right]} \\
& =\left[\begin{array}{c}
\text { Transporte de } k / \varepsilon \\
\text { por difusión }
\end{array}\right]+\left[\begin{array}{c}
\text { Producción } \\
\text { de } k / \varepsilon
\end{array}\right]-\left[\begin{array}{c}
\text { Destrucción } \\
\text { de } k / \varepsilon
\end{array}\right]
\end{aligned}
$$

Para flujos incompresibles, el término $\partial U_{k} / \partial x_{k}$ es pequeño y el segundo término en el lado derecho de la ecuación anterior no contribuye significativamente a la producción. Para flujos compresibles, $\partial U_{k} / \partial x_{k}$ sólo es grande en regiones con gran divergencia de la velocidad, como en un choque.

Davidson (2011) recoge el modo en que se han obtenido las constantes del modelo de turbulencia: 
- Para la determinación de la constante $C_{\mu}$ se analizaron flujos simples donde la ecuación de la energía cinética turbulenta $k$ se puede simplificar y comparar con datos experimentales. La ecuación de $k$ se estudió en la parte logarítmica de la capa límite turbulenta donde se pueden despreciar los términos de convección y difusión.

- De manera similar se obtuvo el valor de la constante $C_{1 \varepsilon}$. La ecuación de $\varepsilon$ se analizó en la parte logarítmica de la capa límite turbulenta, donde el término de convección puede despreciarse, y se consideró que los términos de producción y disipación están en equilibrio $\left(P_{k}=\rho \varepsilon\right)$.

- Considerando que en una zona muy alejada de una perturbación el gradiente de la velocidad es muy pequeño. De este modo, se puede considerar $P_{k}=0, \mathrm{y}$ obtener $C_{2 \varepsilon}$.

- Las dos últimas constantes, $\sigma_{k}$ y $\sigma_{\varepsilon}$, han sido optimizadas por diversos autores mediante la aplicación del modelo a diversos flujos fundamentales tales como flujo en canales, tuberías, chorros, olas, etc.

De este modo, se obtienen los valores de las constantes $C_{\mu}, C_{1 \varepsilon}, C_{2 \varepsilon}, \sigma_{k}$ y $\sigma_{\varepsilon}(0.09$, 1.44, 1.92, 1.0 y 1.3, respectivamente).

\subsubsection{El modelo RNG $k-\varepsilon$}

El modelo RNG (Re-Normalisation Group) k- $\varepsilon$ (Yakhot y Orszag, 1986; Orszag et al., 1993) es una alternativa al modelo estándar $k-\varepsilon$ que ofrece una pequeña mejora respecto a éste. Se basa en un análisis grupal renormalizado de las ecuaciones de Navier-Stokes.

Para cuestiones prácticas, Ho et al. (2006) consideran que este modelo de turbulencia puede considerarse como el estándar en los estudios de hidráulica, aunque es necesario realizar su validación frente a prototipos.

Las ecuaciones de transporte para la generación de turbulencia y disipación son las mismas que las empleadas por el modelo $k-\varepsilon$ estándar, pero las constantes del modelo difieren, y la constante $C_{1 \varepsilon}$ es reemplazada por la función $C_{1 \varepsilon R N G}$ :

$$
\frac{\partial(\rho k)}{\partial t}+\frac{\partial}{\partial x_{j}}\left(\rho U_{j} k\right)=\frac{\partial}{\partial x_{j}}\left[\left(\mu+\frac{\mu_{t}}{\sigma_{k R N G}}\right) \frac{\partial k}{\partial x_{j}}\right]+P_{k}-\rho \varepsilon+P_{k b}
$$




$$
\begin{aligned}
\frac{\partial(\rho \varepsilon)}{\partial t}+\frac{\partial}{\partial x_{j}}( & \left.\rho U_{j} \varepsilon\right) \\
= & \frac{\partial}{\partial x_{j}}\left[\left(\mu+\frac{\mu_{t}}{\sigma_{\varepsilon R N G}}\right) \frac{\partial \varepsilon}{\partial x_{j}}\right] \\
& +\frac{\varepsilon}{k}\left(C_{1 \varepsilon R N G} P_{k}-C_{2 \varepsilon R N G} \rho \varepsilon+C_{1 \varepsilon R N G} P_{\varepsilon b}\right)
\end{aligned}
$$

donde

$$
\begin{gathered}
C_{1 \varepsilon R N G}=1.42-f_{\eta} \\
f_{\eta}=\frac{\eta\left(1-\frac{\eta}{4.38}\right)}{\left(1+\beta_{R N G} \eta^{3}\right)} \\
\eta=\sqrt{\frac{P_{k}}{\rho C_{\mu R N G} \varepsilon}}
\end{gathered}
$$

siendo $C_{\mu R N G}, C_{2 \varepsilon R N G}, \sigma_{k R N G}, \sigma_{\varepsilon R N G}$ y $\beta_{R N G}$ constantes $(0.085,1.68,0.7179,0.7179 \mathrm{y}$ 0.012, respectivamente). Los valores de las constantes se han obtenido de un modo similar que en el modelo $k-\varepsilon$.

\subsubsection{Modelos basados en $\mathbf{k}-\omega$}

En general, los modelos de turbulencia basados en $k-\varepsilon$ predicen el comienzo de la separación demasiado tarde y subestiman la cantidad separada bajo condiciones de gradientes de presión adversos. Para resolver este problema se han desarrollado diferentes modelos que obtienen una predicción mucho más precisa. En la actualidad, los modelos de dos ecuaciones más destacados en este área son los modelos basados en $k$ - $\omega$ de Menter (1994).

Una de las ventajas de la formulación del modelo $k-\omega$ es el tratamiento de las paredes próximas para el cálculo con números de Reynolds bajos. El modelo no implica las complejas funciones no lineales de amortiguación requeridas en el modelo $k-\varepsilon$ y es sin embargo más preciso y más robusto. Se desarrolló un nuevo tratamiento de pared para los modelos $k$ - $\omega$ que permite un cambio suave de la formulación de números de Reynolds bajos a una formulación de función de pared.

El modelo $k-\omega$ asume que la viscosidad turbulenta está relacionada con la energía cinética turbulenta y la frecuencia turbulenta mediante la relación: 


$$
\mu_{t}=\rho \frac{k}{\omega}
$$

La convergencia del modelo $k-\omega$ es similar a la del modelo $k-\varepsilon$. Puesto que los modelos zonales de $k$ - $\omega$ (BSL y SST) incluyen funciones de mezcla en la región cercana a la pared que dependen de la distancia a la pared, en las primeras iteraciones de las simulaciones se resuelve una ecuación adicional para calcular la distancia a la pared.

\subsubsection{5. $\quad$ El modelo $k-\omega$ de Wilcox}

Este modelo resuelve dos ecuaciones de transporte, una para la energía cinética turbulenta $k$ y otra para la frecuencia turbulenta $\omega$. El tensor de tensiones se calcula mediante el concepto de viscosidad de remolino. De este modo, la ecuación $k$ queda:

$$
\frac{\partial(\rho k)}{\partial t}+\frac{\partial}{\partial x_{j}}\left(\rho U_{j} k\right)=\frac{\partial}{\partial x_{j}}\left[\left(\mu+\frac{\mu_{t}}{\sigma_{k}}\right) \frac{\partial k}{\partial x_{j}}\right]+P_{k}-\beta^{\prime} \rho k \omega+P_{k b}
$$

y la ecuación $\omega$ es:

$$
\frac{\partial(\rho \omega)}{\partial t}+\frac{\partial}{\partial x_{j}}\left(\rho U_{j} \omega\right)=\frac{\partial}{\partial x_{j}}\left[\left(\mu+\frac{\mu_{t}}{\sigma_{\omega}}\right) \frac{\partial \omega}{\partial x_{j}}\right]+\alpha \frac{\omega}{k} P_{k}-\beta \rho \omega^{2}+P_{\omega b}
$$

donde $P_{k b}$ y $P_{w b}$ son los términos de turbulencia debida a la gravedad para la ecuación $\omega$.

Las constantes del modelo se obtienen de modo similar que en el modelo $k-\varepsilon$ :

$$
\begin{aligned}
& \beta^{\prime}=0.09 \\
& \alpha=5 / 9 \\
& \beta=0.075 \\
& \sigma_{k}=2 \\
& \sigma_{\omega}=2
\end{aligned}
$$

Las variables independientes, la densidad $\rho$ y el vector velocidad $U$ son tratados como cantidades conocidas. $P_{k}$ es el ratio de producción de turbulencia.

El tensor de tensiones de Reynolds, $-\rho \overline{u_{\imath}{ }^{\prime} u_{\jmath}{ }^{\prime}}$, se calcula como:

$$
-\rho \overline{u_{\imath}^{\prime} u_{\jmath}^{\prime}}=\mu_{t}\left(\frac{\partial U_{i}}{\partial x_{j}}+\frac{\partial U_{j}}{\partial x_{i}}\right)-\frac{2}{3} \delta_{i j}\left(\rho k+\mu_{t} \frac{\partial U_{k}}{\partial x_{k}}\right)
$$




\subsubsection{6. $\quad$ El modelo $k-\omega$ Baseline (BSL)}

El principal problema del modelo $k$ - $\omega$ de Wilcox es su fuerte sensibilidad a las condiciones de lámina libre. Dependiendo del valor especificado a la entrada para $\omega$, puede obtenerse una variación significativa en los resultados del modelo, lo que es indeseable.

Menter (1994) desarrolló un modelo de turbulencia que se comporta como una mezcla entre el modelo $k$ - $\omega$ cerca de los contornos sólidos y el modelo $k$ - $\varepsilon$ en las otras regiones. Consiste en una transformación del modelo $k-\varepsilon$ a la formulación $k-\omega$ y en su posterior sustitución en las ecuaciones correspondientes.

De este modo, el modelo de Wilcox queda multiplicado por una función de mezcla $F_{1}$ y el modelo $k$ - $\varepsilon$ es transformado por una función $1-F_{1}$, donde $F_{1}$ es igual a uno cerca de las superficies y decrece hasta cero fuera de la capa límite (que es función de la distancia a la pared). Por lo tanto, en el contorno de la capa límite y fuera de ella se emplea el modelo $k-\varepsilon$ estándar.

- Modelo de Wilcox:

$$
\begin{gathered}
\frac{\partial(\rho k)}{\partial t}+\frac{\partial}{\partial x_{j}}\left(\rho U_{j} k\right)=\frac{\partial}{\partial x_{j}}\left[\left(\mu+\frac{\mu_{t}}{\sigma_{k 1}}\right) \frac{\partial k}{\partial x_{j}}\right]+P_{k}-\beta^{\prime} \rho k \omega \\
\frac{\partial(\rho \omega)}{\partial t}+\frac{\partial}{\partial x_{j}}\left(\rho U_{j} \omega\right)=\frac{\partial}{\partial x_{j}}\left[\left(\mu+\frac{\mu_{t}}{\sigma_{\omega 1}}\right) \frac{\partial \omega}{\partial x_{j}}\right]+\alpha_{1} \frac{\omega}{k} P_{k}-\beta_{1} \rho \omega^{2}
\end{gathered}
$$

- Modelo $k-\varepsilon$ transformado:

$$
\begin{gathered}
\frac{\partial(\rho k)}{\partial t}+\frac{\partial}{\partial x_{j}}\left(\rho U_{j} k\right)=\frac{\partial}{\partial x_{j}}\left[\left(\mu+\frac{\mu_{t}}{\sigma_{k 2}}\right) \frac{\partial k}{\partial x_{j}}\right]+P_{k}-\beta^{\prime} \rho k \omega \\
\frac{\partial(\rho \omega)}{\partial t}+\frac{\partial}{\partial x_{j}}\left(\rho U_{j} \omega\right) \\
=\frac{\partial}{\partial x_{j}}\left[\left(\mu+\frac{\mu_{t}}{\sigma_{\omega 2}}\right) \frac{\partial \omega}{\partial x_{j}}\right]+2 \rho \frac{1}{\sigma_{\omega 2} \omega} \frac{\partial k}{\partial x_{j}} \frac{\partial \omega}{\partial x_{j}}+\alpha_{2} \frac{\omega}{\kappa} P_{k}-\beta_{2} \rho \omega^{2}
\end{gathered}
$$

Incluyendo los efectos de la gravedad, el modelo BSL queda:

$$
\frac{\partial(\rho k)}{\partial t}+\frac{\partial}{\partial x_{j}}\left(\rho U_{j} k\right)=\frac{\partial}{\partial x_{j}}\left[\left(\mu+\frac{\mu_{t}}{\sigma_{k 3}}\right) \frac{\partial k}{\partial x_{j}}\right]+P_{k}-\beta^{\prime} \rho k \omega+P_{k b}
$$




$$
\begin{aligned}
\frac{\partial(\rho \omega)}{\partial t}+\frac{\partial}{\partial x_{j}} & \left(\rho U_{j} \omega\right) \\
& =\frac{\partial}{\partial x_{j}}\left[\left(\mu+\frac{\mu_{t}}{\sigma_{\omega 3}}\right) \frac{\partial \omega}{\partial x_{j}}\right]+\left(1-F_{1}\right) 2 \rho \frac{1}{\sigma_{\omega 2} \omega} \frac{\partial k}{\partial x_{j}} \frac{\partial \omega}{\partial x_{j}}+\alpha_{3} \frac{\omega}{\kappa} P_{k} \\
& -\beta_{3} \rho \omega^{2}+P_{\omega b}
\end{aligned}
$$

El coeficiente $\alpha$ en el término de producción gravitacional $P_{\omega b}$ se reemplaza también por el nuevo coeficiente $\alpha_{3}$.

Los coeficientes del nuevo modelo son una combinación lineal de los coeficientes correspondientes a los modelos fundamentales:

$$
\Phi_{3}=F_{1} \Phi_{1}+\left(1-F_{1}\right) \Phi_{2}
$$

La formulación de la función de mezcla está basada en la distancia a la superficie más cercana y en las variables del flujo:

$$
F_{1}=\tanh \left[\left[\min \left(\max \left(\frac{\sqrt{k}}{\beta^{\prime} \omega y}, \frac{500 v}{y^{2} \omega}\right), \frac{4 \rho k}{C D_{k \omega} \sigma_{\omega 2} y^{2}}\right)\right]^{4}\right]
$$

donde $y$ es la distancia a la superficie más cercana, $v$ es la viscosidad cinemática, y:

$$
C D_{k \omega}=\max \left(2 \rho \frac{1}{\sigma_{\omega 2} \omega} \frac{\partial k}{\partial x_{j}} \frac{\partial \omega}{\partial x_{j}}, 1.0 \times 10^{-10}\right)
$$

Los valores de los coeficientes se obtienen de modo similar que en el modelo $k-\varepsilon$ :

$$
\begin{aligned}
& \beta^{\prime}=0.09 \\
& \alpha_{1}=5 / 9 \\
& \beta_{1}=0.075 \\
& \sigma_{k 1}=2 \\
& \alpha_{2}=0.44 \\
& \beta_{2}=0.0828 \\
& \sigma_{k 2}=1 \\
& \sigma_{\omega 2}=1 / 0.856
\end{aligned}
$$




\subsubsection{7. $\quad$ EI modelo Shear Stress Transport (SST)}

El modelo de transporte de tensiones de corte turbulentas (SST) basado en $k$ - $\omega$ fue diseñado para dar una elevada precisión del inicio y de la cantidad de flujo separado bajo gradientes de presión adversos, mediante la inclusión de los efectos de transporte en la formulación de la viscosidad de remolinos. Esto se traduce en la mejora de las predicciones de la separación del flujo. El rendimiento superior de este modelo ha sido demostrado en un gran número de estudios de validación (Bardina et al, 1997).

El modelo SST fue desarrollado para superar las deficiencias de los modelos $k-\omega \mathrm{y}$ BSL $k-\omega$. El modelo BSL combina las ventajas de los modelos de Wilcox y $k-\varepsilon$, pero continúa fallando en la adecuada predicción del inicio y la cantidad de separación de flujo en superficies lisas (Menter, 1994). La principal razón es que estos modelos no modifican el transporte del tensor de tensiones turbulentas, lo que da como resultado una sobreestimación de la viscosidad de remolino. El comportamiento adecuado del transporte puede ser obtenido mediante la limitación de la viscosidad de remolino:

$$
\begin{gathered}
v_{t}=\frac{a_{1} k}{\max \left(a_{1} \omega, S F_{2}\right)} \\
v_{t}=\frac{\mu_{t}}{\rho}
\end{gathered}
$$

$S$ es un invariante que mide la velocidad de deformación. $F_{2}$ es una función de mezcla similar a $F_{1}$ que restringe la zona de influencia de la capa límite, puesto que la hipótesis básica no es correcta para los flujos de corte libre:

$$
F_{2}=\tanh \left[\left[\max \left(\frac{2 \sqrt{k}}{\beta^{\prime} \omega y}, \frac{500 v}{y^{2} \omega}\right)\right]^{2}\right]
$$

El modelo SST se recomienda para las simulaciones de alta precisión de la capa límite. Las ecuaciones de transporte se calculan como:

$$
\begin{gathered}
\frac{\partial(\rho k)}{\partial t}+\frac{\partial}{\partial x_{j}}\left(\rho U_{j} k\right)=\frac{\partial}{\partial x_{j}}\left[\left(\mu+\frac{\mu_{t}}{\sigma_{k 3}}\right) \frac{\partial k}{\partial x_{j}}\right]+P_{k}-\beta^{\prime} \rho k \omega+P_{k b} \\
\frac{\partial(\rho \omega)}{\partial t}+\frac{\partial}{\partial x_{j}}\left(\rho U_{j} \omega\right) \\
=\frac{\partial}{\partial x_{j}}\left[\left(\mu+\frac{\mu_{t}}{\sigma_{\omega 3}}\right) \frac{\partial \omega}{\partial x_{j}}\right]+\left(1-F_{1}\right) 2 \rho \frac{1}{\sigma_{\omega 2} \omega} \frac{\partial k}{\partial x_{j}} \frac{\partial \omega}{\partial x_{j}}+\alpha_{3} \frac{\omega}{k} P_{k} \\
-\beta_{3} \rho \omega^{2}+P_{\omega b}
\end{gathered}
$$


donde $F_{1}$ y $F_{2}$ son las funciones de mezcla y $\beta^{\prime}=0.09$.

Los coeficientes se obtienen como combinación lineal:

$$
\Phi_{3}=\mathrm{F}_{1} \Phi_{1}+\left(1-\mathrm{F}_{1}\right) \Phi_{2}
$$

$\operatorname{con} \alpha_{1}=5 / 9, \beta_{1}=0.075, \sigma_{k 1}=2, \sigma_{\omega 1}=2, \alpha_{2}=0.44, \beta_{2}=0.0828, \sigma_{k 2}=1, \sigma_{\omega 2}=1 / 0.856$, obtenidas de modo similar que en el modelo $k-\varepsilon$.

Para flujos de corte libre, la formulación del modelo SST es idéntica al modelo $k-\varepsilon$.

\subsubsection{Modelos de turbulencia de tensiones de Reynolds}

Los modelos de turbulencia de dos ecuaciones (modelos basados en $k-\varepsilon$ y $k$ - $\omega$ ) obtienen buenas predicciones de las características y los fenómenos físicos de muchos flujos de interés. Sin embargo, en flujos donde el transporte turbulento o los efectos de la anisotropía son importantes, la hipótesis de viscosidad de remolino deja de ser válida y los resultados de los modelos de viscosidad de remolino pueden ser inexactos.

Frente a los modelos de viscosidad de remolino, los modelos de tensiones de Reynolds (Reynolds Stress Models, RSM) o de cierre de segundo orden (Second Moment Closure, SMC) reproducen flujos más complejos. Se debe considerar usar modelos de tensiones de Reynolds en los siguientes casos donde han demostrado mayor precisión que los modelos de viscosidad de remolino (ANSYS, 2010):

- Flujos de lámina libre con fuerte anisotropía, como por ejemplo una fuerte componente de remolino (vórtices).

- Flujos con cambios bruscos en la velocidad de deformación media.

- Flujos cuyos campos de deformación son complejos, y reproducen la naturaleza anisotrópica de la turbulencia en sí.

- Flujos con fuerte curvatura de las líneas de corriente.

- Flujos secundarios.

- Flujos generados por efectos de la gravedad.

Los modelos de tensiones de Reynolds están basados en las ecuaciones de transporte de todas las componentes del tensor de tensiones de Reynolds y la tasa de disipación. No usan la hipótesis de viscosidad de remolino, sino que resuelven cada una de las componentes de las tensiones de Reynolds individuales. Se caracterizan por un mayor grado de universalidad.

El inconveniente para esta flexibilidad es un alto grado de complejidad en el sistema de ecuaciones a resolver. El aumento del número de ecuaciones de transporte 
lleva asociado una reducción de la robustez numérica, requiriendo mayores esfuerzos computacionales, lo que impide a veces su uso en flujos complejos.

Teóricamente, la resolución de las tensiones anisotrópicas hace que los modelos de tensiones de Reynolds sean más adecuados para flujos complejos. Sin embargo, los resultados demuestran que en ocasiones no son superiores a los modelos de dos ecuaciones. Un ejemplo de esto es la delimitación de la capa límite de una pared, donde a pesar de su mayor grado de universalidad teórico, los modelos de tensión de Reynolds a menudo resultan inferiores a los modelos de dos ecuaciones (ANSYS, 2010).

Los modelos de tensiones de Reynolds algebraicos resuelven las ecuaciones algebraicas de las tensiones de Reynolds, mientras que los modelos de tensiones de Reynolds diferenciales resuelven las ecuaciones de transporte diferenciales individualmente para cada componente del tensor de Reynolds.

Las ecuaciones de cantidad de movimiento con promediado de Reynolds para la velocidad media son:

$$
\frac{\partial \rho U_{i}}{\partial t}+\frac{\partial}{\partial x_{j}}\left(\rho U_{i} U_{j}\right)-\frac{\partial}{\partial x_{j}}\left[\mu\left(\frac{\partial U_{i}}{\partial x_{j}}+\frac{\partial U_{j}}{\partial x_{i}}\right)\right]=-\frac{\partial p^{\prime \prime}}{\partial x_{i}}-\frac{\partial}{\partial x_{i}}\left(\rho \overline{u_{\imath}^{\prime} u_{\jmath}^{\prime}}\right)+S_{M_{i}}
$$

donde $p^{\prime \prime}$ es la presión modificada, $S_{M_{i}}$ es la suma de las fuerzas másicas, y $-\rho \overline{u_{\iota}{ }^{\prime} u_{\jmath}}$ es la contribución de las tensiones de Reynolds fluctuantes.

A diferencia de los modelos de viscosidad de remolino, la presión modificada no tiene la contribución de la turbulencia, estando relacionada con la presión estática (termodinámica) por:

$$
p^{\prime}=p+\frac{2}{3} \mu \frac{\partial U_{k}}{\partial x_{k}}
$$

En el modelo de tensiones diferencial, $-\rho \overline{u_{\imath}{ }^{\prime} u_{\jmath}{ }^{\prime}}$ satisface la ecuación del transporte. De este modo, se resuelve una ecuación de transporte individual para cada una de las seis componentes de las tensiones de Reynolds $-\rho \overline{u_{\imath}{ }^{\prime} u_{\jmath}{ }^{\prime}}$. La ecuación diferencial del transporte de tensiones de Reynolds es:

$$
\begin{gathered}
\frac{\partial \rho \overline{u_{\imath}^{\prime} u_{\jmath}^{\prime}}}{\partial t}+\frac{\partial}{\partial x_{k}}\left(U_{k} \rho \overline{u_{\imath}^{\prime} u_{\jmath}^{\prime}}\right)-\frac{\partial}{\partial x_{k}}\left(\left(\mu+\frac{2}{3} C_{S} \rho \frac{k^{2}}{\varepsilon}\right) \frac{\partial \overline{u_{\imath}^{\prime} u_{\jmath}^{\prime}}}{\partial x_{k}}\right) \\
=P_{i j}-\frac{2}{3} \delta_{i j} \rho \varepsilon+\Phi_{i j}+P_{i j, b}
\end{gathered}
$$


donde $P_{i j}$ y $P_{i j, b}$ son los términos de producción de turbulencia de corte y gravitacional de las tensiones de Reynolds respectivamente, $\Phi_{i j}$ es el tensor de presión-deformación y $C_{S}$ es una constante.

Los términos de turbulencia gravitacionales $P_{i j, b}$ también se tienen en cuenta en la contribución gravitacional en el término de presión y son controlados de modo análogo a como ocurre en los modelos $k-\varepsilon$ y $k-\omega$.

\subsubsection{Modelos de tensiones de Reynolds basados en omega}

Existen modelos de tensiones de Reynolds basados en la ecuación $\varepsilon$, aunque presentan algunas deficiencias para la simulación de la capa límite, como es la precisión de la predicción de la separación del flujo. Por otra parte, las formulaciones para bajos números de Reynolds de la ecuación $\varepsilon$ suelen ser complejas y difíciles de integrar, complicándose aun más cuando se combina con la formulación de un modelo de tensiones de Reynolds.

Los modelos de turbulencia de tensiones de Reynolds basados en la ecuación $\omega$ permiten un tratamiento más preciso cerca de la pared. De este modo, incluyen el tratamiento automático de pared desarrollado para los modelos basados en $k-\omega$.

El modelo de tensiones de Reynolds Base (BSL) se fundamenta en el modelo de dos ecuaciones BSL. Las ecuaciones para las tensiones de Reynolds quedan:

$$
\begin{aligned}
& \frac{\partial \rho \overline{u_{\imath}^{\prime} u_{\jmath}^{\prime}}}{\partial t}+\frac{\partial}{\partial x_{k}}\left(U_{k} \rho \overline{u_{\imath}^{\prime} u_{\jmath}^{\prime}}\right) \\
& \quad=P_{i j}-\frac{2}{3} \beta^{\prime} \rho \omega k \delta_{i j}+\Phi_{i j}+P_{i j, b}+\frac{\partial}{\partial x_{k}}\left(\left(\mu+\frac{\mu_{t}}{\sigma_{k}}\right) \frac{\partial \overline{u_{\imath}^{\prime} u_{\jmath}^{\prime}}}{\partial x_{k}}\right)
\end{aligned}
$$

La ecuación para $\omega$ queda:

$$
\begin{aligned}
\frac{\partial(\rho \omega)}{\partial t}+\frac{\partial}{\partial x_{k}} & \left(U_{k} \rho \omega\right) \\
& =\alpha_{3} \frac{\omega}{k} P_{k}+P_{\omega b}-\beta_{3} \rho \omega^{2}+\frac{\partial}{\partial x_{k}}\left[\left(\mu+\frac{\mu_{t}}{\sigma_{\omega 3}}\right) \frac{\partial \omega}{\partial x_{k}}\right] \\
& +\left(1-F_{1}\right) 2 \rho \frac{1}{\sigma_{2} \omega} \frac{\partial k}{\partial x_{k}} \frac{\partial \omega}{\partial x_{k}}
\end{aligned}
$$

Los coeficientes $\alpha$ y $\beta$ de la ecuación $\omega$, junto a los números de turbulencia de Prandtl $\sigma^{*}$ y $\sigma$, son obtenidos a partir de los valores de las dos constantes de 
transformación correspondientes a las constantes del modelo basado en $\omega$ y a las constantes del modelo basado en $\varepsilon$, transformadas a la formulación $\omega$ :

- Configuración 1 (zona SMC- $\omega)$ :

$$
\begin{aligned}
& \sigma_{1}^{*}=2 \\
& \sigma_{1}=2 \\
& \beta_{1}=0.075 \\
& \beta^{\prime}=0.09 \\
& \alpha_{1}=\frac{\beta}{\beta^{\prime}}-\frac{\kappa^{2}}{\sigma\left(\beta^{\prime}\right)^{0.5}}=0.553
\end{aligned}
$$

El valor de $\beta$ corresponde al modelo $k$ - $\omega$. La constante $\kappa$ de von Kármán tiene un valor aproximado de 0.41 .

- Configuración 2 (zona SMC-e):

$$
\begin{aligned}
& \sigma_{2}^{*}=1.0 \\
& \sigma_{2}=0.856 \\
& \beta_{2}=0.0828 \\
& \beta^{\prime}=0.09 \\
& \alpha_{2}=\frac{\beta}{\beta^{\prime}}-\frac{\kappa^{2}}{\sigma\left(\beta^{\prime}\right)^{0.5}}=0.44
\end{aligned}
$$

La combinación de los coeficientes se realiza por interpolación lineal suave con la misma función de peso $F_{1}$ que se emplea en el término de difusión transversal de la ecuación $\omega$ del modelo BSL (ecuación (163)).

La relación constitutiva para la correlación presión-tensión viene dada por:

$$
\begin{aligned}
\phi_{i j}=\beta^{\prime} C_{1} \rho \omega & \left(-\overline{u_{\imath}{ }^{\prime} u_{\jmath}{ }^{\prime}}+\frac{2}{3} k \delta_{i j}\right)-\hat{\alpha}\left(P_{i j}-\frac{2}{3} P \delta_{i j}\right)-\hat{\beta}\left(D_{i j}-\frac{2}{3} P \delta_{i j}\right) \\
& -\hat{\gamma} \rho \kappa\left(S_{i j}-\frac{1}{3} S_{k k} \delta_{i j}\right)
\end{aligned}
$$

donde el tensor de producción de las tensiones de Reynolds viene dado por:

$$
P_{i j}=-\rho \overline{u_{\imath}{ }^{\prime} u_{k}{ }^{\prime}} \frac{\partial U_{j}}{\partial x_{k}}-\rho \overline{u_{\jmath}^{\prime} u_{k}^{\prime}} \frac{\partial U_{i}}{\partial x_{k}} ; \quad P=0.5 P_{k k}
$$


El tensor $D_{i j}$, participante en la ecuación (171) del modelo presión-tensión, difiere del tensor de producción en los índices del producto escalar:

$$
D_{i j}=-\rho \overline{u_{\imath}{ }^{\prime} u_{k}{ }^{\prime}} \frac{\partial U_{k}}{\partial x_{j}}-\rho \overline{u_{\jmath}^{\prime} u_{k}{ }^{\prime}} \frac{\partial U_{k}}{\partial x_{i}}
$$

La viscosidad turbulenta en los términos de difusión se calcula de la misma manera que en el modelo $k-\omega$ de Wilcox:

$$
\mu_{T}=\rho \frac{k}{\omega}
$$

Los coeficientes del modelo son:

$$
\begin{aligned}
& \beta^{\prime}=0.09 \\
& \hat{\alpha}=\left(8+C_{2}\right) / 11 \\
& \hat{\beta}=\left(8 C_{2}-2\right) / 11 \\
& \hat{\gamma}=\left(60 C_{2}-4\right) / 55 \\
& C_{1}=1.8 \\
& C_{2}=0.52
\end{aligned}
$$

\subsubsection{El modelo de Simulación de Gran Remolino (LES)}

Los flujos turbulentos contienen una amplia gama de escalas de longitudes turbulentas y tiempos, con movimientos a gran escala que por lo general son más energéticos que los de pequeña escala. El modelo de Simulación de Gran Remolino (LES) es una aproximación que resuelve los movimientos fluctuantes a gran escala y utiliza modelos de turbulencia de escala sub-celdas para el movimiento de pequeña escala. El modelo de Smagorinsky (1963), aplicado con diferentes formulaciones de la función de amortiguación de la pared, es el más habitual.

El enfoque habitual para la predicción de flujos turbulentos es utilizar las ecuaciones de Navier-Stokes promediadas de Reynolds (RANS), que resuelven las cantidades promediadas en el tiempo. Sin embargo, hay algunas situaciones donde el enfoque no es adecuado, y deben ser adoptados los enfoques alternativos de Simulación de Gran Remolino (LES) o Simulación Numérica Directa (DNS). En estos métodos las ecuaciones que dependen del tiempo se resuelven para el movimiento de turbulencia, con la ausencia de aproximaciones y la resolución de todas las escalas relevantes (DNS), o las ecuaciones se filtran para eliminar longitudes de escala y tiempos muy 
reducidos (LES). Estos enfoques requieren mallados muy finos e intervalos de paso pequeños, sobre todo para flujos en las proximidades de las paredes, así como un gran número de intervalos de paso para generar correlaciones estadísticamente significativas para las componentes de las fluctuaciones de velocidad. Sin embargo, pueden dar detalles sobre la estructura de flujos turbulentos, como pueden ser las fluctuaciones de presión, que no se pueden obtenerse a partir de una formulación RANS.

Antes de iniciar una simulación LES, se debe considerar si es el método de solución más adecuada. Para números de Reynolds bajos, es decir $R_{e}<5000$, o cuando es importante ser capaz de resolver todas las escalas (por ejemplo, para la transición a flujo turbulento), se puede consideran el método DNS si se dispone una gran capacidad computacional. Para mayores números de Reynolds, el modelo LES podría ser una opción adecuada en los casos en que:

- El flujo es probable que sea inestable, con grandes escalas de agitación de una capa de corte o desprendimientos de vórtices.

- El flujo es probable que sea inestable con estructuras coherentes (ciclones).

- Hay simetrías en la geometría y en el flujo que podría causar ruptura de la simetría en bifurcaciones.

- El flujo es gravitacional, con grandes zonas inestables creadas por el calentamiento desde abajo, o por la existencia de un líquido más ligero bajo un líquido más pesado (flujo multifásico en tuberías inclinadas).

- Se sabe que no funciona el enfoque convencional RANS (por ejemplo debido a la existencia de turbulencia altamente anisotrópica).

- Se requiere una buena representación de la estructura turbulenta para procesos de pequeña escala, como micro-mezclas o reacciones en sustancias químicas.

- Se requieren otros datos fluctuantes (por ejemplo fuerzas fluctuante o ráfagas de viento).

- El usuario puede esperar hasta una semana para la obtención de los resultados, usando un equipo con 8 o 16 procesadores.

El uso del método LES en flujos de contornos de pared no se recomienda, debido a los requisitos de alta resolución y los tiempos de cálculo que implica.

Menter (2012) realiza la comparación de los requerimientos para resolver una turbina con $R_{e}=10^{5}-10^{6}$ aplicando modelos de turbulencia RANS y LES, la cual requiere resolver la turbulencia en las capas límite y la transición entre flujo laminar y turbulento (Tabla 15). Las simulaciones con LES requieren altas prestaciones de simulaciones numéricas que en muchos casos son inabordables en la actualidad. 


\begin{tabular}{|c|c|c|c|c|}
\hline Método & $\begin{array}{c}\text { Número de } \\
\text { celdas }\end{array}$ & $\begin{array}{c}\text { Número de } \\
\text { intervalos de paso }\end{array}$ & $\begin{array}{c}\text { Número de iteraciones en } \\
\text { cada intervalo de paso }\end{array}$ & $\begin{array}{c}\text { Relación de } \\
\text { CPU requerida }\end{array}$ \\
\hline RANS & $\sim 10^{6}$ & $\sim 10^{2}$ & 1 & 1 \\
\hline LES & $\sim 10^{9}$ & $\sim 10^{4}$ & 10 & $10^{6}$ \\
\hline
\end{tabular}

Tabla 15. Comparación de los requisitos para una simulación con modelos RANS y LES

(Menter, 2012).

\subsubsection{Comparación de los modelos de turbulencia}

En la Tabla 16 se realiza una comparación de las fortalezas y debilidades de los modelos de turbulencia RANS.

\begin{tabular}{|c|c|c|}
\hline Modelo & Fortalezas & Debilidades \\
\hline$k-\varepsilon$ & $\begin{array}{l}\text { Estable, numéricamente robusto, } \\
\text { económico, razonablemente preciso, } \\
\text { ampliamente utilizado. }\end{array}$ & $\begin{array}{l}\text { No es adecuado en flujos con separación de } \\
\text { capa límite, grandes gradientes de presión, } \\
\text { fuerte curvatura de las líneas de corriente, } \\
\text { remolinos y flujos de rotación. } \\
\text { Predice que la expansión lateral de los chorros } \\
\text { circulares es un } 25 \% \text { más rápida que en } \\
\text { chorros planos. }\end{array}$ \\
\hline RNG $k-\varepsilon$ & $\begin{array}{l}\text { Buen comportamiento en flujos } \\
\text { moderadamente complejos como chorros } \\
\text { incidentes, flujos de separación, } \\
\text { remolinos y flujos secundarios. }\end{array}$ & $\begin{array}{l}\text { Sujeto a las limitaciones debidas a la hipótesis } \\
\text { de viscosidad de remolino isotrópica. } \\
\text { El mismo problema que el modelo k- } \varepsilon \\
\text { estándar con chorros circulares y planos. }\end{array}$ \\
\hline$k-\omega$ & $\begin{array}{l}\text { Tratamiento de las paredes más robusto } \\
\text { y preciso. } \\
\text { Mejor predicción de flujos con } \\
\text { separación de capa límite. }\end{array}$ & $\begin{array}{l}\text { Fuerte sensibilidad a las condiciones de } \\
\text { lámina libre. } \\
\text { Sujeto a las limitaciones debidas a la hipótesis } \\
\text { de viscosidad de remolino isotrópica. }\end{array}$ \\
\hline BSL & $\begin{array}{l}\text { Mejor predicción de flujos con } \\
\text { separación de capa límite. } \\
\text { Modelo } k-\omega \text { cerca de los contornos } \\
\text { sólidos y } k-\varepsilon \text { en el resto de regiones. }\end{array}$ & $\begin{array}{l}\text { Continúa fallando en la predicción del inicio y } \\
\text { la cantidad de separación de la capa límite. } \\
\text { Sujeto a las limitaciones debidas a la hipótesis } \\
\text { de viscosidad de remolino isotrópica. }\end{array}$ \\
\hline SST & $\begin{array}{l}\text { Elevada precisión de inicio y de la } \\
\text { cantidad de flujo separado bajo } \\
\text { gradientes de presión adverso. } \\
\text { Recomendado para simulaciones de alta } \\
\text { precisión de la capa límite. }\end{array}$ & $\begin{array}{l}\text { Sujeto a las limitaciones debidas a la hipótesis } \\
\text { de viscosidad de remolino isotrópica. }\end{array}$ \\
\hline $\begin{array}{l}\text { Reynolds } \\
\text { Stress } \\
\text { Model }\end{array}$ & $\begin{array}{l}\text { Modelos físicamente más complejos: } \\
\text { flujos con fuerte anisotropía, cambios } \\
\text { bruscos de velocidad de deformación, } \\
\text { fuerte curvatura de las líneas de } \\
\text { corriente, flujos secundarios, flujos } \\
\text { generados por efecto de la gravedad. }\end{array}$ & $\begin{array}{l}\text { Menor robustez numérica, requiere más } \\
\text { esfuerzo computacional (2-3 veces superior), } \\
\text { ecuaciones de cantidad de movimiento y } \\
\text { turbulencia fuertemente acopladas. }\end{array}$ \\
\hline
\end{tabular}

\footnotetext{
Tabla 16. Comparación de los modelos de turbulencia RANS.
} 


\subsection{Casos de estudio documentados}

Se ha realizado una extensa revisión bibliográfica de casos de estudio documentados en ingeniería hidráulica de presas. Debido a que el uso de la metodología CFD en aplicaciones a aliviaderos es relativamente reciente, existen escasas referencias de estudios de aliviaderos de vertido libre bien documentados. Por este motivo, y con el fin de conocer el adecuado ajuste de la metodología en general, se han abarcado diversas tipologías de aliviaderos de presas.

A continuación se comentan los resultados obtenidos con distintos programas de CFD en el campo de la hidráulica de presas. Los estudios se centran en el estudio de caudales de descarga, lámina libre, velocidades y presiones, obtenidos con paquetes informáticos comerciales de diferencias finitas (FLOW-3D) y de volúmenes finitos (ANSYS CFX y FLUENT).

\subsubsection{Validación de los modelos numéricos en aliviaderos}

Debido a la complejidad de los fenómenos que se producen en un aliviadero (flujo bifásico, alta turbulencia, pérdida de energía, etc.), y a la gran variedad de tipologías y singularidad de las construcciones, la validación de los modelos resulta extremadamente importante para conocer el comportamiento real del flujo.

Cuando se habla de estudios con CFD, el Instituto Americano de Aeronáutica y Astronáutica (AIAA, 1998) define modelo (model) como una representación de un sistema físico o proceso destinado a aumentar nuestra habilidad para mejorar, predecir o controlar su comportamiento. El término modelación (modeling) se refiere al proceso de construcción o modificación de un modelo, mientras que simulación (simulation) es el uso del modelo (un modelo se usa en una simulación).

Asimismo, define modelo matemático o modelo conceptual (mathematical model o conceptual model) como toda la información, datos para la modelación matemática y ecuaciones matemáticas que describen el sistema físico o proceso de interés (se obtiene por el análisis y observación del sistema físico), mientras que un modelo de ordenador o modelo informatizado (computer model o computerized model) es un código que implementa un modelo matemático o conceptual.

Verificación (verification) es el proceso de determinar que la implementación de un modelo representa la descripción conceptual desarrollada y la solución del modelo. Validación (validation) es el proceso de determinar el grado en que un modelo es una representación precisa del mundo real desde la perspectiva del uso previsto del modelo.

La Figura 56 muestra la interrelación entre mundo real, modelo matemático o conceptual y modelo de ordenador. 


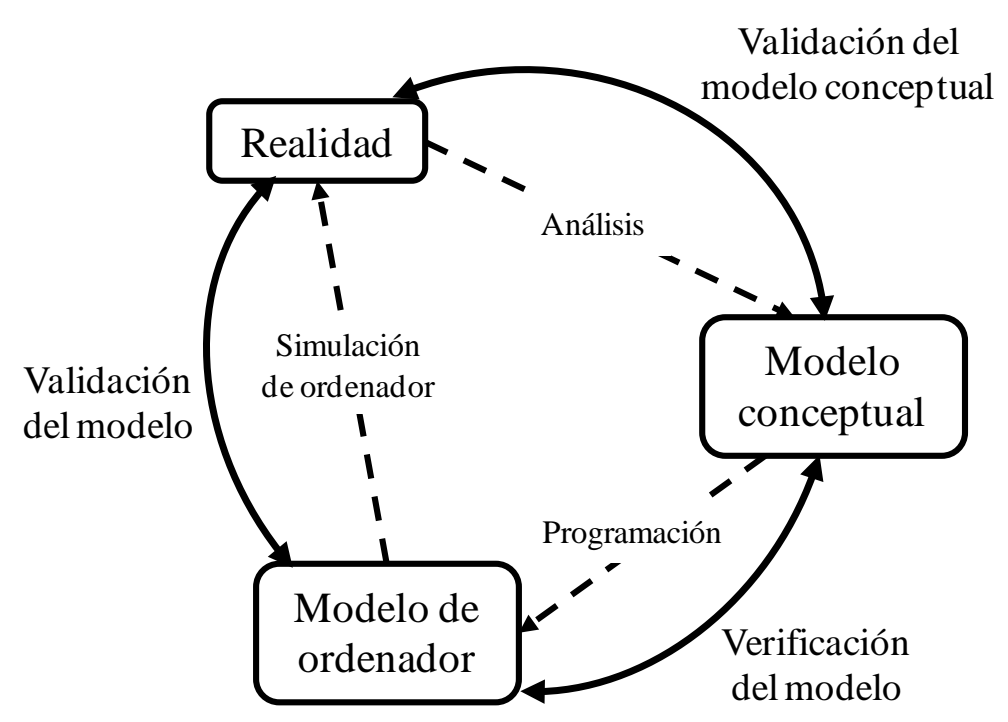

Figura 56. Interrelación entre el mundo real, un modelo matemático y un modelo informatizado.

Por tanto, un proceso de validación apropiado consiste en comparar los resultados calculados con aquellos tomados del sistema real bajo condiciones análogas de flujo. Desafortunadamente, es difícil encontrar estudios completos de mediciones en aliviaderos debido a los elevados costes involucrados y a que los eventos extremos son poco frecuentes. En la práctica, son escasas las veces en que es posible medir cuantitativamente el comportamiento hidráulico, existiendo sólo observaciones cualitativas a partir de videos y fotografías, lo que dificulta la validación apropiada.

El rendimiento de los aliviaderos se suele estudiar mediante modelos físicos. Sin embargo, debido a que los modelos físicos son una representación aproximada del mundo real, existen también muchos de los problemas que sufren los modelos numéricos. En este sentido, la habitual validación práctica de modelos de aliviaderos puede ser sólo considerada como pseudo-validación debido a la falta de mediciones en sistemas reales (Ho y Riddette, 2010).

En muchas de las validaciones se emplean resultados obtenidos en modelos físicos o gráficos de diseño publicados como los del US Army Corp of Engineers (USACE) y del United Stated Bureau of Reclamation (USBR). La información obtenida en estos gráficos de diseño se basaba en modelos físicos limitados a un determinado tipo de cresta de vertedero y unas condiciones particulares de aproximación, por lo que sólo pueden proporcionar una grosera validación para formas de cresta no estándar o condiciones de aproximación inusuales. 


\subsubsection{Descarga y lámina libre}

Savage y Johnson (2001) realizaron simulaciones bidimensionales de un aliviadero hidrodinámico con diferentes cargas de agua, empleando el programa FLOW-3D, y usando el modelo de turbulencia RNG. Las comparaciones frente al modelo físico obtuvieron errores en la descarga inferiores al $-1 \%$ cuando $H_{e} / H_{d}$ (carga total sobre el vertedero/carga de diseño del perfil hidrodinámico del vertedero) es mayor de $0.7, \mathrm{y}$ aumentando hasta $-6 \%$ cuando $H_{e} / H_{d}$ es mayor de 0.2 . Los resultados extrapolados del gráfico de USACE fueron similares a los del modelo numérico pero con una ligera menor precisión, mientras que los obtenidos de los resultados del USBR mostraban la mayor desviación frente al modelo físico, debido a que la cresta del USBR es ligeramente diferente. Los máximos errores relativos se obtuvieron para $H_{e} / H_{d}=0.07$, donde el modelo numérico calculó una variación del 12\%, mientras que el USACE y el USBR tienen variaciones del 22\%.

Bouhadji (2004) simuló con ANSYS CFX y el modelo de turbulencia SST la lámina libre y las presiones sobre la cresta de un aliviadero hidrodinámico con diferentes cargas $\left(H_{e} / H_{d}=0.50 ; 1.00 ; 1.33\right)$. Los resultados fueron muy similares a los resultados del USACE (Figura 57).
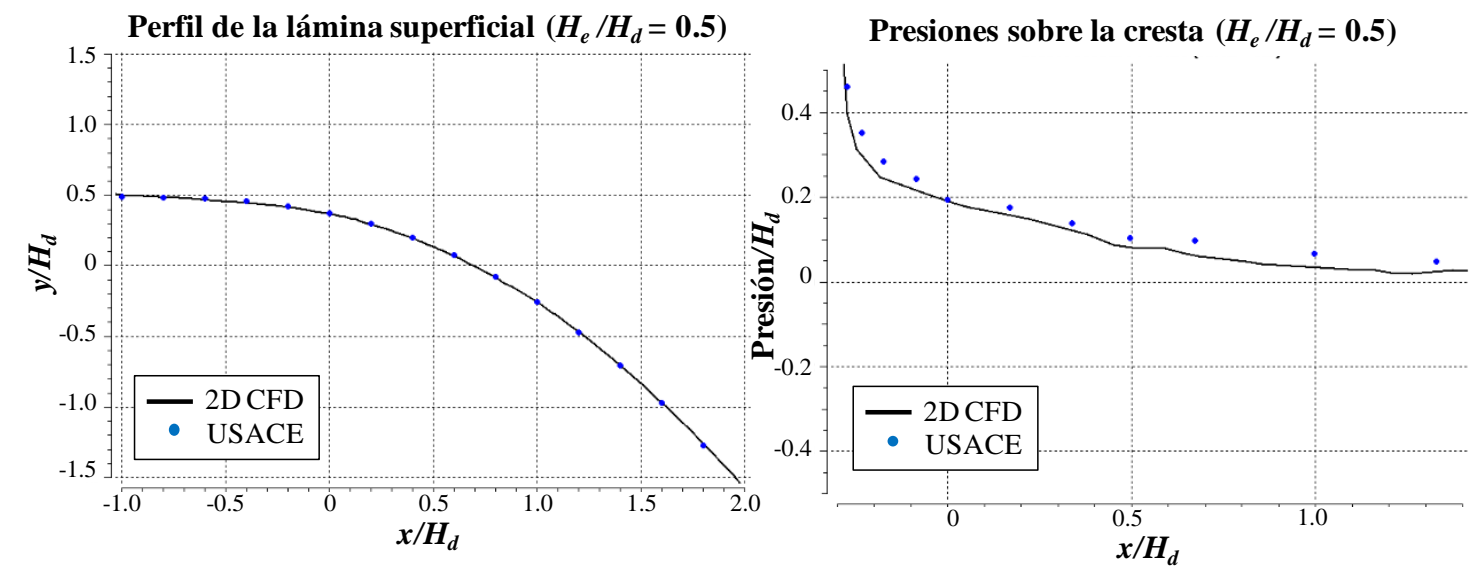

Figura 57. Comparación del perfil de la lámina de agua y de las presiones sobre la cresta de un aliviadero hidrodinámico, considerando $H_{e} / H_{d}=0.50$ (Bouhadji, 2004).

Ho et al. (2003) emplearon FLOW-3D para el estudio de aliviaderos en simulaciones bidimensionales y las compararon con fórmulas empíricas simplificadas. Aunque los perfiles de lámina libre en la cresta del aliviadero fueron muy similares, las simulaciones tendieron a sobreestimar la descarga entre un $17 \%$ cuando $H_{e} / H_{d}=1.33 \mathrm{y}$ un $10 \%$ cuando $H_{e} / H_{d}=0.50$. Posteriores estudios achacan las diferencias a haber realizado las simulaciones considerando flujo laminar y no viscoso. 
Ho et al. (2003) posteriormente estudiaron un aliviadero tipo con pilas obteniendo diferencias respecto a las ecuaciones empíricas del USACE entre $\pm 1.7 \%$ y $\pm 3.9 \%$ (Tabla 17). En la aplicación a la presa Búfalo (Victoria, Estados Unidos), las diferencias fueron de hasta un $3 \%$ respecto a las predicciones del USACE usando el modelo de turbulencia $k-\varepsilon$.

\begin{tabular}{|c|c|c|c|}
\hline$H_{e} / H_{d}$ & $\begin{array}{c}\text { Predicción empírica } \\
\left(\mathrm{m}^{3} / \mathrm{s} / \mathrm{m}\right)\end{array}$ & $\begin{array}{c}\text { Predicción CFD } \\
\left(\mathrm{m}^{3} / \mathrm{s} / \mathrm{m}\right)\end{array}$ & $\begin{array}{c}\text { Diferencia } \\
(\%)\end{array}$ \\
\hline 1.33 & 115.1 & 113.2 & 1.7 \\
\hline 1.00 & 70.2 & 70.1 & 0.1 \\
\hline 0.50 & 22.6 & 21.7 & 3.9 \\
\hline
\end{tabular}

Tabla 17. Comparación de la descarga obtenida en un aliviadero tridimensional (Ho et al., 2003).

Sarker y Rhodes (2004) simularon con FLUENT la lámina libre sobre un vertedero de pared gruesa, usando el modelo de turbulencia $k-\varepsilon$. Los resultados reprodujeron la tendencia observada en laboratorio a elevarse la lámina libre cerca de las paredes y a disminuir en la zona central de la sección transversal. Las máximas diferencias en el calado medio de la sección sobre el vertedero fueron de $1.5 \mathrm{~mm}$ (7.1\%), mientras que aguas arriba del mismo fueron de $0.3 \mathrm{~mm}(0.32 \%)$.

Edwards (2006) estudió el coeficiente de descarga $C_{d}$ usando dos códigos CFD (ANSYS CFX y FLOW-3D). La Figura 58 muestra que ambos códigos obtuvieron resultados de $C_{d}$ similares a los experimentos de Maynord (1985) para el rango de cargas de agua analizado. Los resultados numéricos tendían a sobreestimar la descarga en comparación con los datos del modelo físico.

Johnson y Savage (2006) y Savage et al. (2009) realizaron simulaciones de tres tipos de aliviaderos, considerando diferentes condiciones aguas abajo, obteniendo un error máximo en la descarga del 3.2\% respecto al caudal de los modelos físicos. Las simulaciones se realizaron con FLOW-3D empleando el modelo de turbulencia RNG.

Fuamba et al. (2006) realizaron la simulación tridimensional de la presa de Robert Bourassa, en Quebec (Canadá). Las diferencias entre las descargas simuladas y observadas fueron inferiores a $\pm 1.5 \%$.

Ho et al. (2006) y Ho y Riddette (2010) recopilan 14 casos de aliviaderos de Australia en los que comprobaron los resultados del coeficiente de descarga obtenido con FLOW-3D frente a resultados de modelos físicos o gráficos de diseño. En general, las diferencias fueron inferiores al $5 \%$ aunque existieron un par de casos con grandes diferencias que ocurrieron con baja carga y pequeños caudales, probablemente debido al uso de un mallado relativamente grande para esos caudales. La tendencia observada sobreestimaba un 3\% la descarga, lo que concuerda con los resultados de Gessler (2005) 
y de Yang y Johansson (1998), aun usando diferentes códigos de CFD y diferentes modelos bifásicos (Ho et al., 2006). Por otro lado, también obtuvieron un buen ajuste en aliviaderos en Morning Glory y de laberinto, así como en presiones sobre la cresta del aliviadero y la lámina libre, que pueden fluctuar dependiendo de la densidad del mallado.

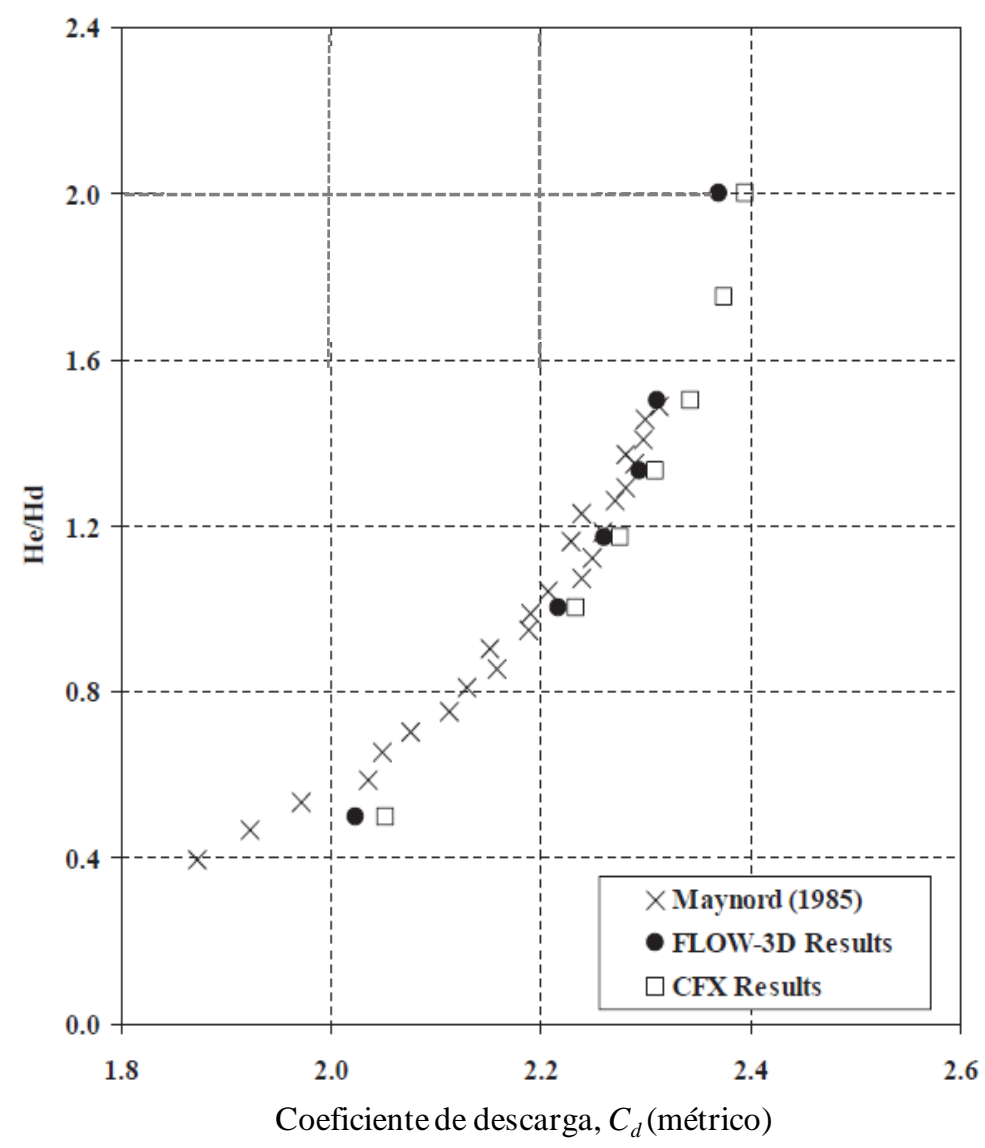

Figura 58. Coeficiente de descarga en función de la carga para un vertedero de cresta elíptica con $\mathrm{P}_{/} / \mathrm{H}_{d}=2.0$ (Edwards, 2006).

Kenny (2007) examinó los modelos $k-\varepsilon$ y $k-\omega$ (Shear Stress Transport) y el modelo de Tensiones de Reynolds, y encontró que existen insignificantes diferencias en la predicción del rango de caudales para un aliviadero de forma hidrodinámica. Asimismo, recomienda aplicar un espaciado de malla normalizado de $\sim 0.04 \% y_{c}$ para diseño conceptual y $0.013 \% y_{c}$ para diseños con detalle en el programa FLUENT.

Hargreaves et al. (2007) realizaron la validación de los resultados obtenidos en un vertedero de cresta ancha con FLUENT, usando el modelo de turbulencia RNG $k-\varepsilon, \mathrm{y}$ realizaron algunas comparaciones con otros modelos de turbulencia (estándar $k-\varepsilon \mathrm{y}$ RSM). La lámina libre así como la descarga fueron reproducidos con gran precisión por 
el modelo RNG (Tabla 18). Las presiones máximas simuladas sobre la superficie del vertedero fueron ligeramente inferiores a las medidas experimentales, divergiendo un 5\% la localización horizontal del valor máximo. La recirculación sobre el vertedero se reprodujo mejor con el modelo RSM, mientras que el modelo $k-\varepsilon$ obtuvo resultados bastante alejados.

\begin{tabular}{|c|c|c|c|}
\cline { 2 - 4 } \multicolumn{1}{c|}{} & \multirow{2}{*}{$h(\mathrm{~mm})$} & \multicolumn{2}{c|}{$Q(\mathrm{l} / \mathrm{s})$} \\
\cline { 3 - 4 } \multicolumn{1}{c|}{} & & Laboratorio & CFD \\
\hline 2D, Modelo RNG $k-\varepsilon$ & 50.9 & 8.25 & 8.27 \\
\hline 2D, Modelo RNG $k-\varepsilon$ & 60.7 & 10.90 & 10.84 \\
\hline 2D, Modelo RNG $k-\varepsilon$ & 84.4 & 17.81 & 17.65 \\
\hline 2D, Modelo RNG $k-\varepsilon$ & 108.4 & 25.98 & 25.74 \\
\hline 2D, Modelo RNG $k-\varepsilon$ & 139.2 & 37.59 & 37.49 \\
\hline 2D, Modelo RNG $k-\varepsilon$ & 178.0 & 54.83 & 54.42 \\
\hline 2D, Modelo RNG $k-\varepsilon$ & 204.7 & 68.07 & 67.38 \\
\hline 2D, Modelo estándar $k-\varepsilon$ & 204.7 & 68.07 & 69.58 \\
\hline 2D, Modelo RSM & 204.7 & 68.07 & 66.96 \\
\hline 3D, Modelo RNG $k-\varepsilon$ & 204.7 & 68.07 & 68.37 \\
\hline
\end{tabular}

Tabla 18. Comparación de la descarga obtenida por Hager y Schwalt (1994) en un aliviadero cresta ancha con simulaciones de CFD (Hargreaves et al., 2007).

Chanel y Doering $(2007,2008)$ compararon las simulaciones tridimensionales llevadas a cabo con el modelo de turbulencia RNG, con modelos físicos de aliviaderos hidrodinámicos de tres presas situadas en la provincia de Manitoba (Canadá). En la presa de Wuskwatim, FLOW-3D tendió a sobreestimar la descarga entre un 3.8 y un $10.0 \%$, aumentando conforme la carga de agua disminuía. Al reducir el tamaño de los elementos de malla de 1 a $0.5 \mathrm{~m}$ el error disminuyó. El empleo de una malla de $0.25 \mathrm{~m}$ redujo aun más el error, mejorando significativamente tanto el perfil superficial como las presiones, aunque aumentó sustancialmente el coste computacional. En la presa de Limestone, las mayores diferencias en la descarga pasaron de un $7.8 \%$ con un mallado de $1 \mathrm{~m}$ a un $2.8 \%$ con el mallado de $0.5 \mathrm{~m}$ (Figura 59).

La lámina libre y presiones obtenidas con una malla de $0.33 \mathrm{~m}$ concordaron con las mediciones de laboratorio. Para la presa de Conawapa, la reducción del tamaño de la malla tendió también a disminuir el caudal de descarga, aunque puesto que en este caso la descarga estaba subestimada, las diferencias aumentan. El error medio fue de $-5.2 \%$, aunque se obtuvieron diferencias de $-24.4 \%$ para la carga de $0.15 H_{d}$.

Gendzelevich et al. (2009) obtuvieron un buen ajuste de las simulaciones realizadas con FLOW-3D con observaciones fotográficas, así como con el modelo reducido del canal artificial Red River Floodway, en Winnipeg (Manitoba, Canadá). Las simulaciones se emplearon para analizar las dimensiones y diseñar estructuras de descarga considerando un incremento del $130 \%$ en el caudal de diseño. 

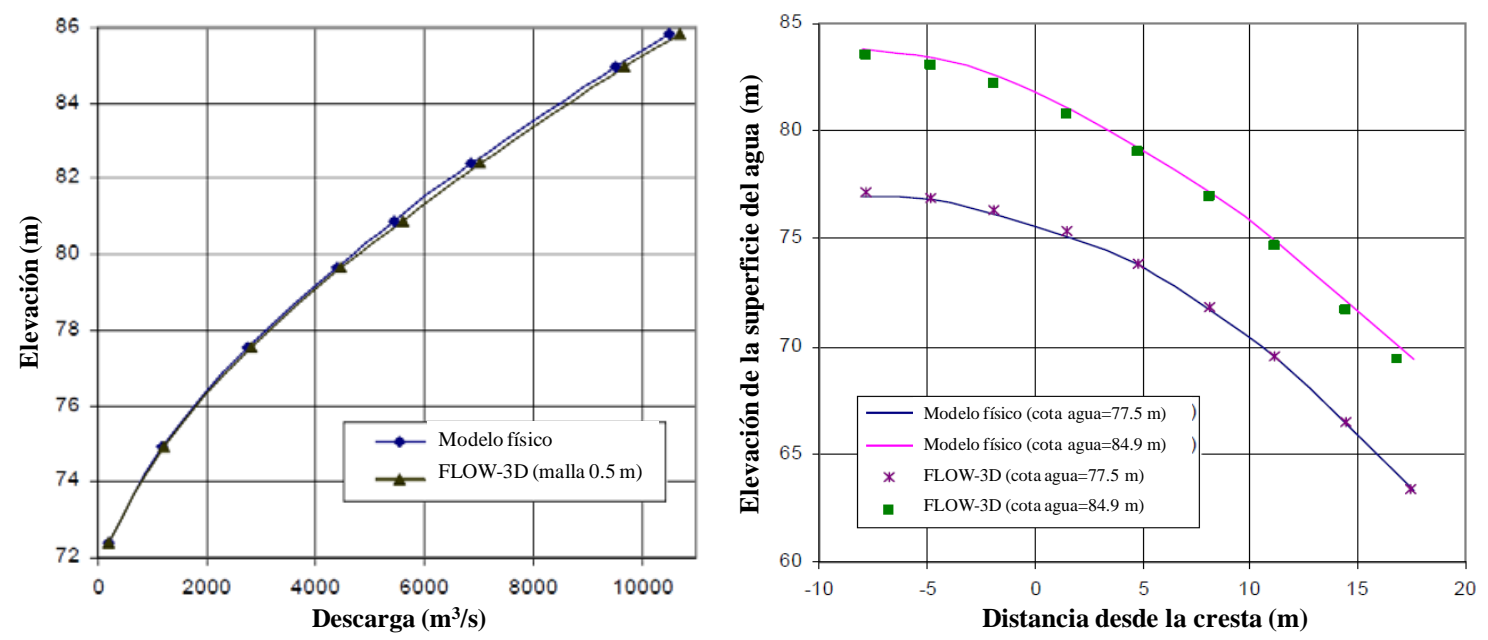

Figura 59. Curva de descarga y perfil superficial para dos cargas distintas de la presa de Limestone (Chanel y Doering, 2007).

Reda (2011) comparó el coeficiente de descarga de un vertedero de pared delgada simulado en FLOW-3D frente a resultados obtenidos de la ecuación teórica. Comprobó 4 alturas de pared del vertedero, con 11 caudales cada una (Figura 60). En todos los casos obtuvo una desviación máxima de $\pm 3 \%$ respecto al coeficiente teórico.

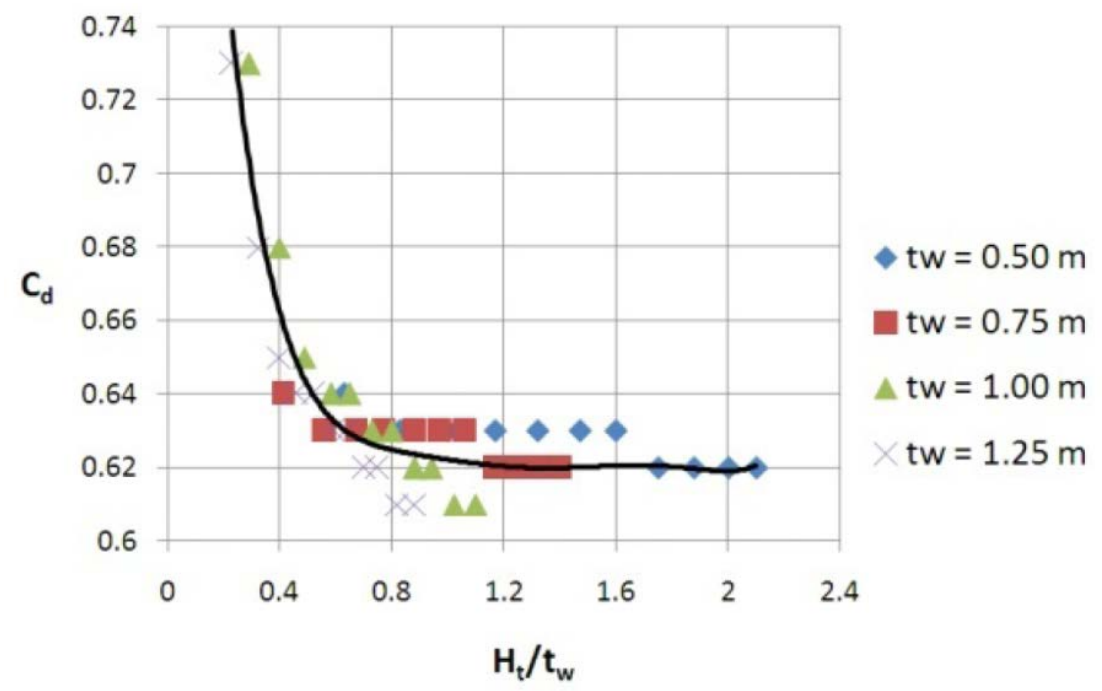

Figura 60. Coeficiente de descarga para un vertedero de pared delgada frente a la relación carga total sobre vertedero/altura de vertedero $\left(H_{t} / t_{w}\right)($ Reda, 2011).

Rahimzadeh et al. (2012) analizaron con FLUENT el flujo sobre vertederos circulares, empleando diversos modelos de turbulencia (estándar $k-\varepsilon$, RNG $k-\varepsilon$, realizable $k-\varepsilon$, estándar $k-\omega$, SST y RSM), obteniendo buenos resultados frente a un 
modelo físico. La elección del modelo de turbulencia no tuvo efecto en la predicción de la lámina libre. Las velocidades horizontales en el plano de la cresta obtuvieron buenos resultados respecto a los medidos (Figura 61), salvo con los modelos estándar $k-\varepsilon$, RNG $k-\varepsilon$, y estándar $k-\omega$. Las presiones en el eje vertical de la cresta obtenidas con los modelos estándar $(k-\varepsilon$ y $k-\omega)$ no mostraron un buen ajuste debido a la curvatura existente (Figura 62). Los mejores resultados se obtuvieron con el modelo de turbulencia RSM.

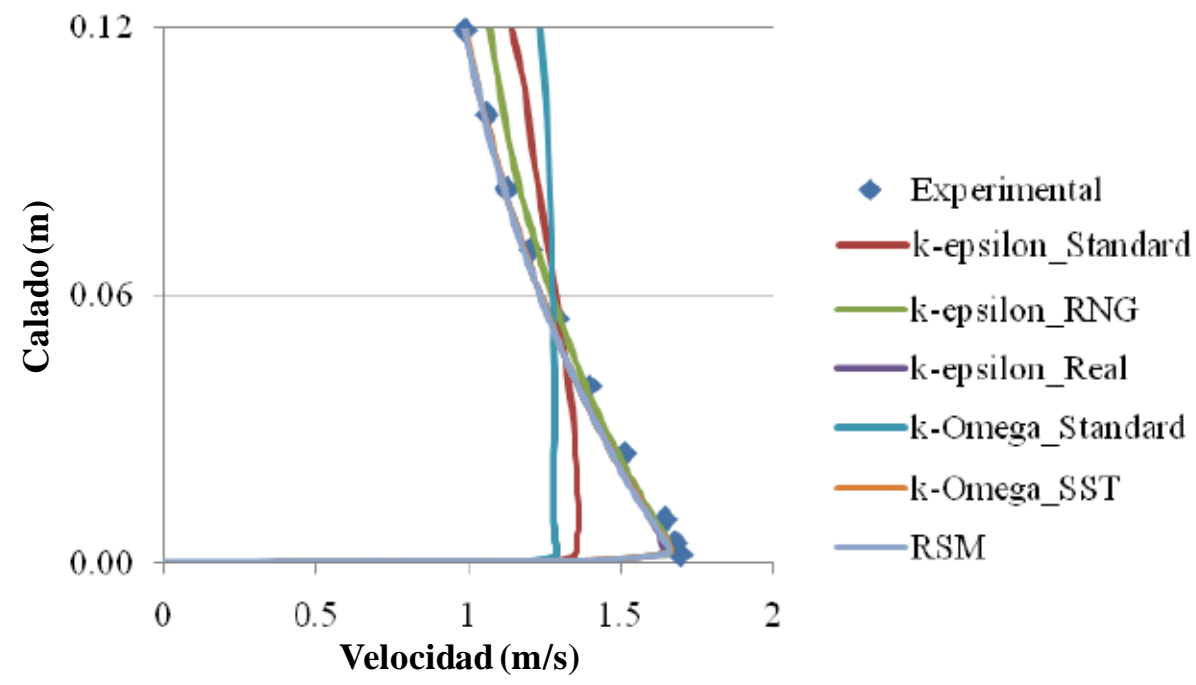

Figura 61. Comparación de los perfiles de velocidades horizontales en la cresta de un aliviadero circular, considerando diferentes modelos de turbulencia (Rahimzadeh et al., 2012).

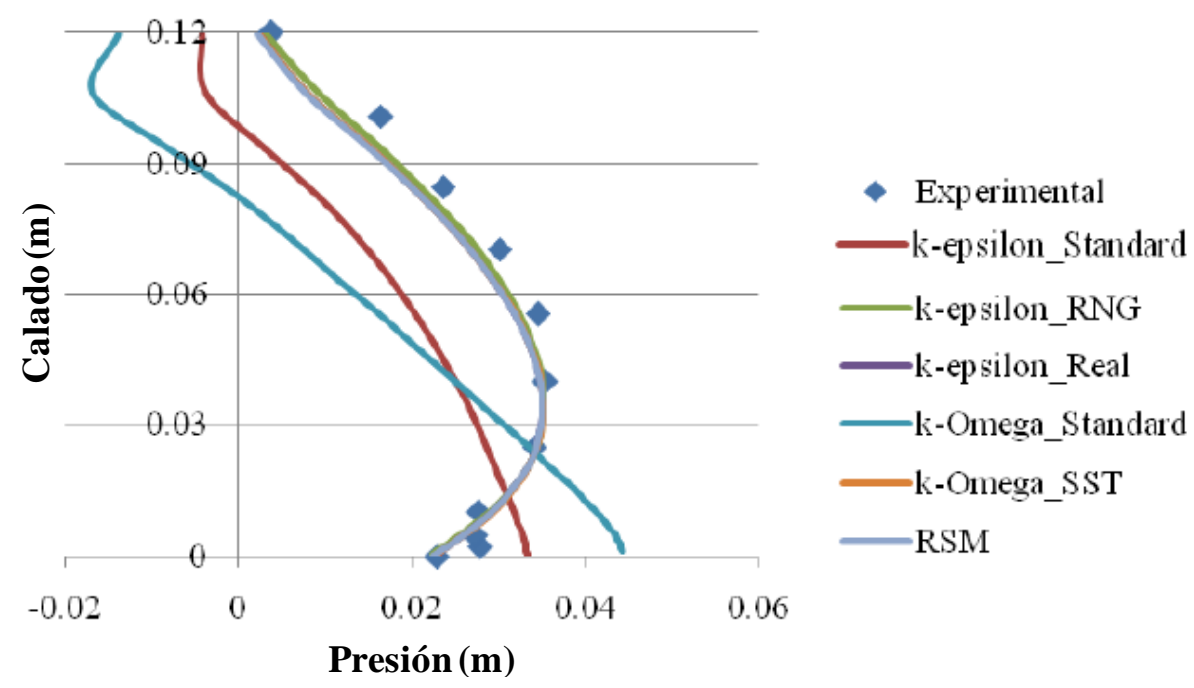

Figura 62. Comparación de las distribuciones de presiones en la cresta de un aliviadero circular, considerando diferentes modelos de turbulencia (Rahimzadeh et al., 2012). 


\subsubsection{Análisis del impacto de la lámina de agua}

Cook y Richmond (2001) obtuvieron buenos resultados con FLOW-3D en su estudio de validación del flujo sobre una grada considerando un caudal de $0.55 \mathrm{~m}^{3} / \mathrm{s}$, donde las peores predicciones fueron el calado (diferencia de $0.03 \mathrm{~m}$ ) y la velocidad (diferencia de $0.2 \mathrm{~m} / \mathrm{s}$ ) en el borde de la grada. En otro estudio, obtuvieron buenos resultados del perfil de la superficie del chorro, pero poca concordancia con los perfiles de velocidad de aguas abajo. Las relaciones RMS/velocidad media se diferenciaban entre un 7\% y un 87\%. Las diferencias las atribuyeron principalmente a la obtención de ángulos diferentes en los chorros, posiblemente debida a la falta de un modelo de entrada de aire, la turbulencia, dificultades en el escalado en el modelo físico o difusión numérica debido a una malla no estructurada.

Weilin et al. (2002) simularon las presas arco Xiaowan y Laxiwa (China), empleando el modelo de turbulencia $k-\varepsilon$ y el programa FLOW-3D, y realizaron la comparación con datos de modelos físicos obtenidos con esferas de Pitot de cinco huecos y sensores de presión. Para la simulación de los chorros, debido a que en el modelo físico no se apreciaba mucha aireación del chorro, reprodujeron el espesor del chorro entrante, calculado a partir del caudal, la velocidad y su ancho. Las presiones en la solera tuvieron un ajuste adecuado (Figura 63). Las velocidades aguas arriba y en la zona de impacto no se pudieron medir. Aguas abajo no se dan datos numéricos, aunque la tendencia del flujo parece ser reproducida. La energía turbulenta se concentró en la zona de impacto. No pudo ser comparada experimentalmente, pero puso de manifiesto que cuanto más concentrado es el chorro, más complicada resulta su disipación de energía.

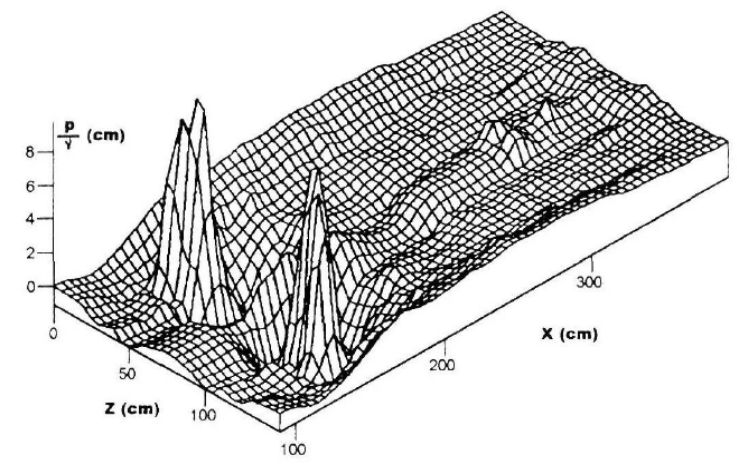

( $\Lambda$ ) The measured

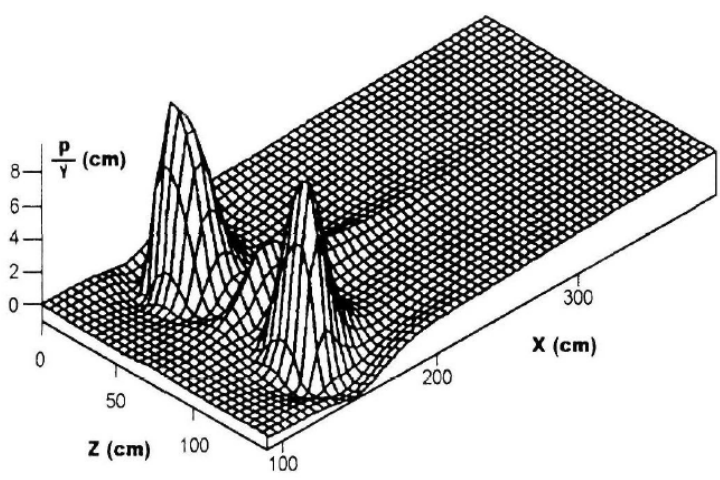

(B) The calculated

Figura 63. Comparación entre la distribución de presiones medida y calculada en la solera del cuenco (Weilin et al., 2002). 
Gessler y Rasmussen (2005) realizaron un estudio tridimensional de la presa de Smith Mountain en el río Roanoake (Virginia, Estados Unidos), validando los resultados frente a un modelo físico. El modelo predijo la trayectoria del chorro, el punto de impacto y el funcionamiento del aliviadero con caudales aproximadamente el doble del caudal de diseño. El coste del modelo numérico de validación se estimó en aproximadamente un $20 \%$ del coste de un modelo físico equivalente.

Lesleighter et al. (2008) simularon la trayectoria de un chorro tras un trampolín de lanzamiento, obteniendo una forma de la trayectoria más suave que la observada en el modelo físico, posiblemente debido a la gruesa resolución de la malla o a efectos del modelo de turbulencia en la separación del flujo.

\subsubsection{Distribución de presiones y cortantes}

Savage y Johnson (2001) realizaron simulaciones bidimensionales de un aliviadero hidrodinámico con $H_{d}=301 \mathrm{~mm}$ y relación altura de aliviadero/carga $P / H_{d}=2.7$, considerando tres cargas de agua $\left(H_{e} / H_{d}=0.51,0.82,1.20\right)$. Para ello emplearon el programa FLOW-3D, usando el modelo de turbulencia RNG. Las presiones sobre la cresta se compararon con un modelo físico y con los datos del USACE extrapolados, obteniéndose diferencias respecto al modelo físico inferiores a $5 \mathrm{~cm}$ en la rápida e inferiores a $10 \mathrm{~cm}$ en la curva del pie del aliviadero.

Cook et al. (2002) realizaron la comparación de la simulación numérica tridimensional de un cuenco de disipación con dientes frente a un modelo a escala 1:36. Los perfiles de presión obtenidos concordaron con la precisión de los piezómetros.

Ho et al. (2003) compararon las presiones obtenidas en un modelo bidimensional con los resultados del Water Experimental Station (WES) del USACE. Obtuvieron buena concordancia en los perfiles de presiones sobre el aliviadero, con una presión negativa ligeramente superior. Según los autores, algunas oscilaciones en la presión se debieron a efectos locales del mallado. Sin embargo, en este estudio no consideraron modelos de turbulencia, lo que pudo haber causado una sobreestimación de la velocidad y por tanto una subestimación de las presiones.

Ho et al. (2003) también estudiaron en un modelo tridimensional las presiones sobre la cresta de aliviaderos con pilas, obteniendo la tendencia y magnitud dada por el USACE, aunque alcanzando resultados ligeramente más negativos. En la validación frente a modelos a escala observaron que para el aliviadero de la presa de Warragamba (Nueva Gales del Sur, Australia) el uso de distintos modelos de turbulencia no mostraba variaciones significativas en las presiones de la cresta frente al modelo a escala. En la presa de Hume (Nueva Gales del Sur, Australia), las simulaciones reprodujeron la posible cavitación observada en modelo físico a escala 1:50. Los resultados 
subestimaron las presiones cerca del borde de la pila, aunque la tendencia fue reproducida. Las presiones inmediatamente detrás de la pila no coincidieron con las mediciones puesto que en esta zona del modelo se observaron fluctuaciones entre 4.57 m y la presión atmosférica (Figura 64).
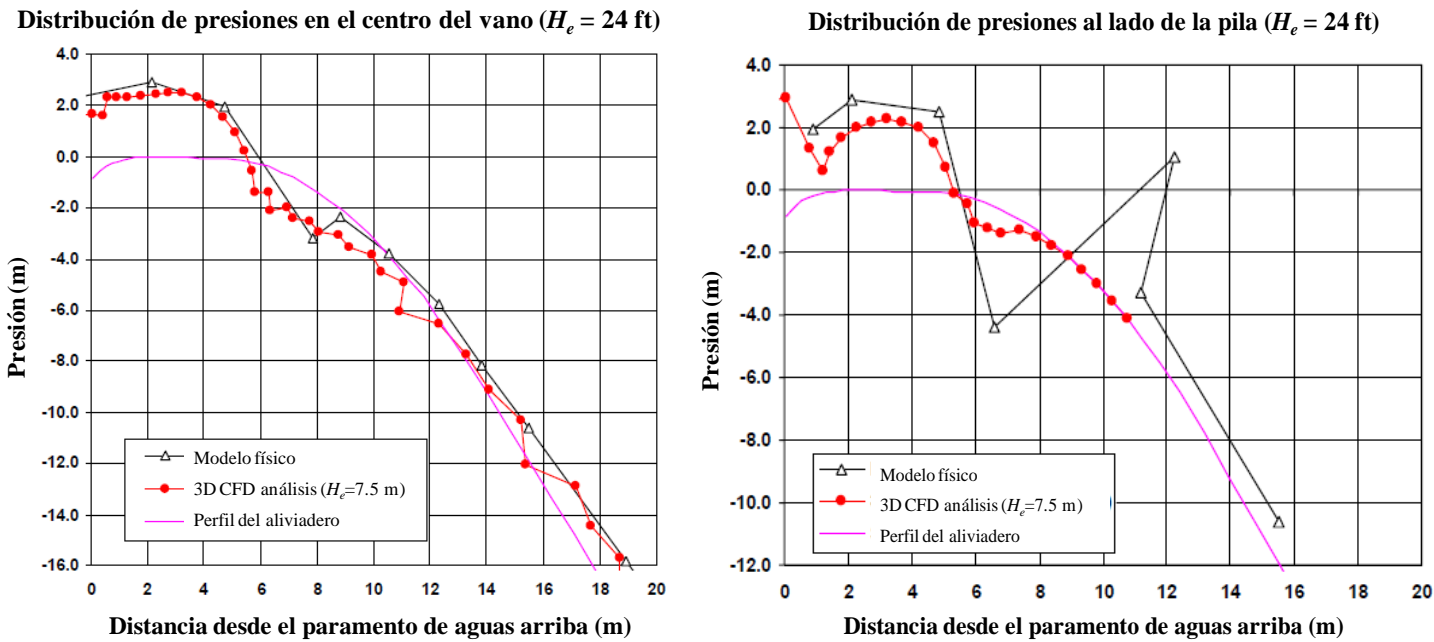

Figura 64. Comparación de la distribución de presiones a lo largo del centro del vano y de la pila en el aliviadero de la presa Hume, Nueva Gales del Sur (Ho et al., 2003).

Johnson y Savage (2006) y Savage et al. (2009) compararon tres modelos físicos de aliviaderos con simulaciones numéricas considerando diferentes condiciones aguas abajo. Los modelos numéricos reprodujeron adecuadamente las presiones aun cuando se disponía de un elevado nivel de agua aguas abajo. Las diferencias en presiones absolutas respecto al modelo físico fueron en todos los casos inferiores a $\pm 2.5 \mathrm{~cm}$ (para el aliviadero de $81 \mathrm{~cm}$ de altura), y siendo en general inferiores a $\pm 1 \mathrm{~cm}$ en la zona sumergida (Figura 65).

Groeneveld et al. (2007) analizaron la distribución de presiones, variación y magnitud dentro de los túneles de la presa Cabinet Gorge del río Clark Fork (Idaho, Estados Unidos). La validación frente a un modelo físico a escala obtuvo una concordancia razonable para las presiones medias y sus distribuciones. Sin embargo, la variación en las presiones debida a la turbulencia fue menor en el modelo numérico ( $\pm 0.91 \mathrm{~m}$ ) que en el modelo físico $( \pm 3.35 \mathrm{~m})$, de modo que se consideró más preciso el modelo físico debido a la pequeña longitud y tiempos de escala de los remolinos turbulentos.

Ho y Riddette (2010) realizaron la validación de la distribución de presiones sobre un vertedero de perfil trapezoidal, obteniendo presiones a lo largo del fondo del chorro con razonable precisión. 


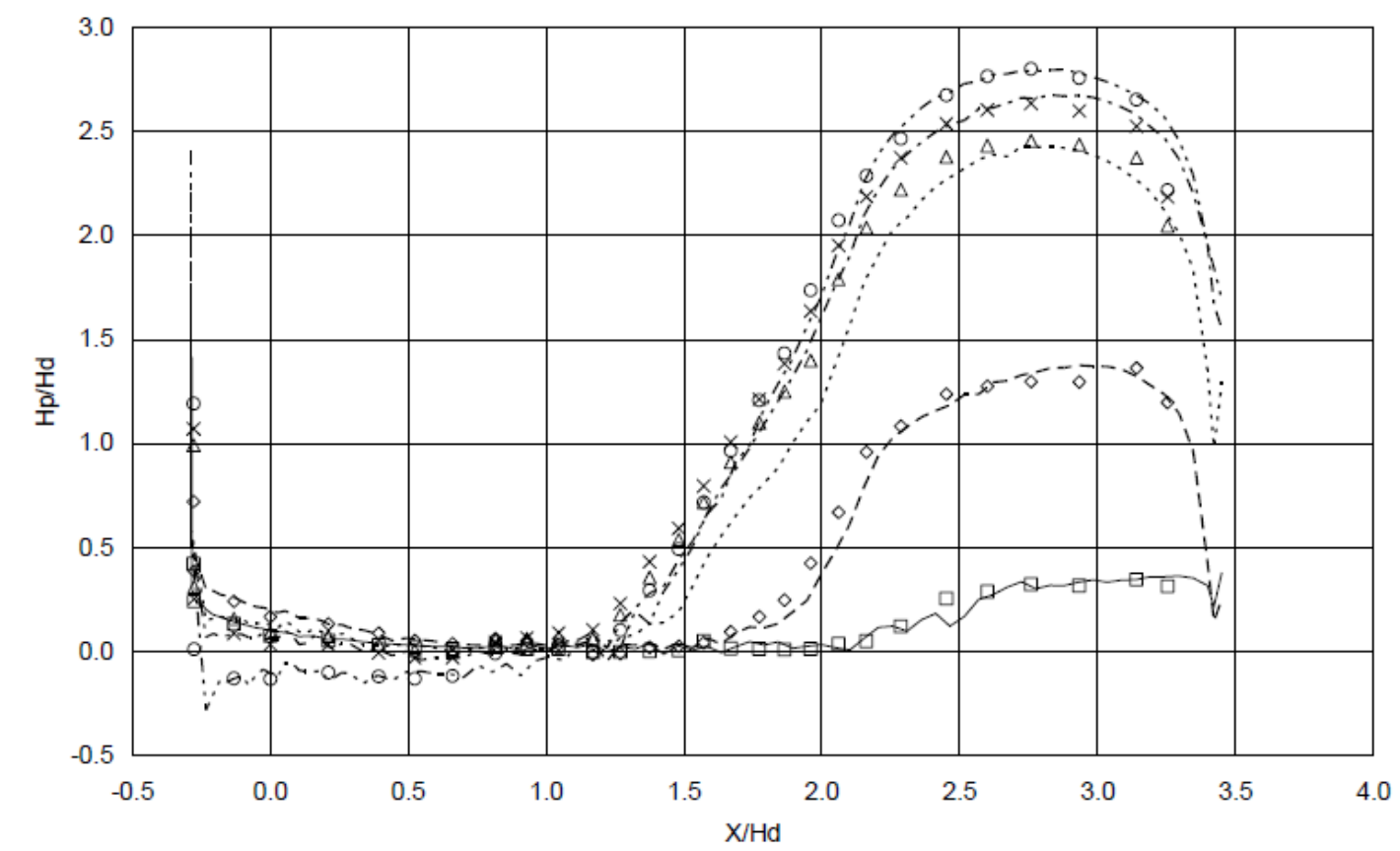

- Numerical Run \#1 - - - Numerical Run \#2 -..... Numerical Run \#3 - - - • Numerical Run \#4 - -.- Numerical Run \#5 口 Physical Run \#1 $\diamond$ Physical Run \#2 $\triangle$ Physical Run \#3 $\times$ Physical Run \#4 $\circ$ Physical Run \#5

Figura 65. Comparación de presiones relativas obtenidas en modelo físco y modelo numérico para diversas sumergencias (Savage et al., 2009).

\subsubsection{Análisis del canal de descarga y del cuenco de disipación}

Cook y Richmond (2001) realizaron la validación del canal de descarga de la central eléctrica Bonneville Second en el río Columbia (Oregón, Estados Unidos), obteniendo que el $46 \%$ de las velocidades estaban dentro de la banda $\pm 1 \sigma$ y el $99 \%$ dentro de la banda $\pm 2 \sigma$ de los valores medidos con doppler, siendo $\sigma$ la desviación estándar. El promedio de los errores de todos los RMS fue inferior al $20 \%$.

Cook et al. (2002) compararon los perfiles de velocidades obtenidas en una simulación tridimensional de un cuenco de disipación con dientes. El 73\% de las velocidades estaban dentro de la banda $\pm 1 \sigma$ y el $95.5 \%$ dentro del rango $\pm 2 \sigma$ del modelo físico. Los resultados de la simulación tridimensional mostraban una diferencia en velocidad media de $0.43 \mathrm{~m} / \mathrm{s}$ y un ajuste $R^{2}=0.81$ respecto al modelo a escala 1:80.

Amorin et al. (2004) analizaron el cuenco de disipación de resalto hidráulico de la presa Porto Colombia (Brasil), realizando la comparación de un modelo numérico bidimensional aplicando el modelo de turbulencia $k-\varepsilon$, con un modelo a escala 1:100 y con medidas en prototipo de la central eléctrica. En laboratorio comprobaron que el incremento de caudal originaba un incremento gradual de energía debida a la turbulencia y por tanto una mayor fluctuación de la presión. En las simulaciones, los valores mostraron grandes variaciones durante el establecimiento del resalto, mientras 
que una vez estabilizado mostraban pequeñas fluctuaciones en torno al valor promedio. También se observó que la energía turbulenta es máxima cerca del inicio del resalto, disminuyendo a lo largo del cuenco (Figura 66), lo que concuerda con los estudios de Marques et al. (1998). La distribución de presiones concordaba con el modelo a escala y las medidas in-situ. El modelo reprodujo la pauta de recirculación y la localización del resalto hidráulico observada en función de la descarga.

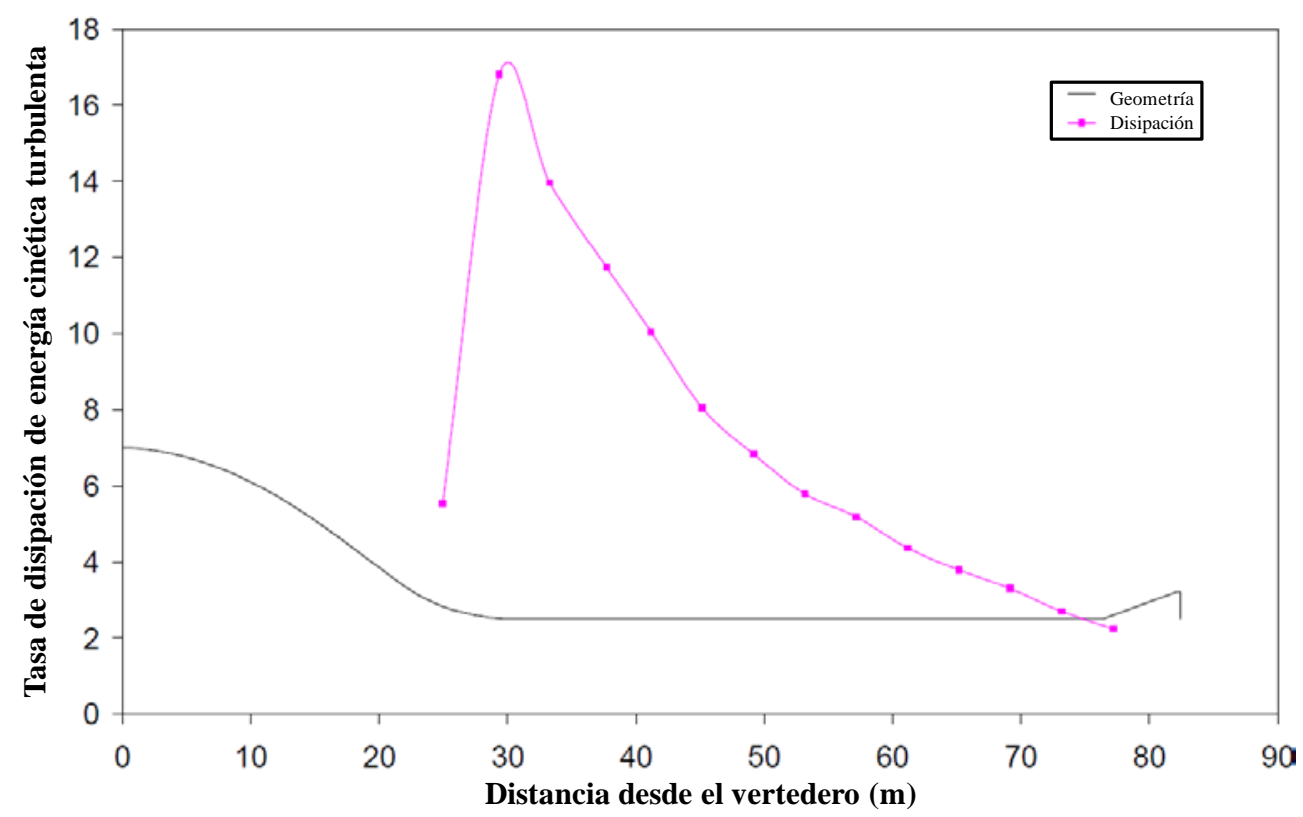

Figura 66. Tasa de disipación de la energía cinética turbulenta en la presa Porto Colombia, Brasil, para un caudal de $6000 \mathrm{~m}^{3} / \mathrm{s}$ (Amorin et al., 2004).

Bischof et al. (2004) investigaron con FLUENT el efecto que tienen las constantes de los modelos $k-\varepsilon$ y RNG aguas abajo de un escalón. El modelo estándar $k-\varepsilon$ tendía a subestimar la zona de recirculación, mientras que el modelo RNG la capturaba mejor. Usando el modelo estándar $k-\varepsilon$ con unos nuevos parámetros $\left(C_{1 \varepsilon}=2.289, C_{2 \varepsilon}=3.020\right.$, $\left.C_{\mu}=0.094, \sigma_{k}=0.690, \sigma_{\varepsilon}=0.615\right)$ obtuvieron una solución muy similar a la del modelo RNG. El análisis de sensibilidad de la evaluación de las derivadas de las componentes de la velocidad respecto de los parámetros reveló que el modelo $k$ - $\varepsilon$ tiende a ser más sensible a los parámetros turbulentos $C_{1 \varepsilon}$ y $C_{2 \varepsilon}$ que el modelo RNG, mientras que el modelo RNG es más sensible a $C_{\mu}$ que el modelo $k-\varepsilon$.

Raiford y Khan (2006) realizaron simulaciones bidimensionales con los modelos de turbulencia estándar $k$ - $\varepsilon$ y RNG para analizar la estructura interna en resaltos hidráulicos sumergidos. El modelo RNG obtuvo mejores resultados cerca de la solera. 
Tota (2009) estudió las características del flujo aguas abajo de un escalón de altura $h$, considerando dos flujos con distinto número de Reynolds. La solución numérica reprodujo las características esenciales del flujo, como la longitud de recirculación del flujo, la zona de flujo inverso y los parámetros medios del flujo con razonable precisión. Realizó además un estudio de sensibilidad del parámetro longitud de mezcla turbulenta en FLOW-3D, obteniendo con el modelo de turbulencia RNG que a partir del 14\% de longitud de mezcla los parámetros no se veían alterados, mientras que valores muy bajos calculaban resultados alejados respecto a los esperables (Figura 67).
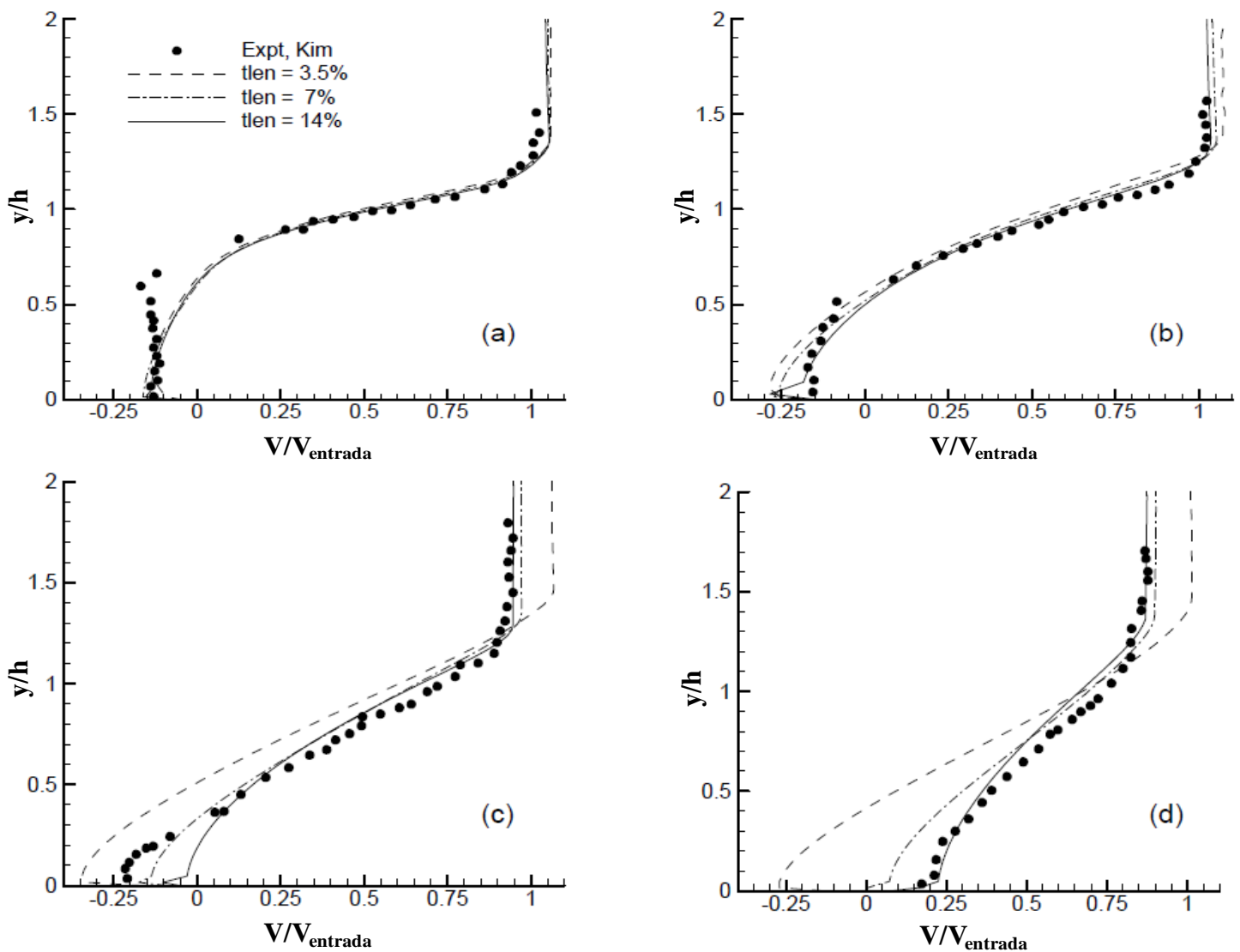

Figura 67. Perfiles de velocidad para varias posiciones, para un flujo de $R_{e}=44000:$ a) $\times / h=$ 4, b) $x / h=6, c) x / h=10, a) x / h=19$ (Tota, 2009).

\subsubsection{Simulación de chorros turbulentos}

Aziz et al. (2008) emplearon FLOW-3D con los modelos de turbulencia $k-\varepsilon$ y RNG para simular las características de chorros planos y circulares en descarga profunda y horizontal, donde no se tiene en cuenta el impacto con la lámina libre ni con elementos sólidos. En estas condiciones, analizaron la evolución de la velocidad media y la energía cinética del chorro y compararon sus resultados con estudios experimentales de diversos 
autores. En chorros planos, el modelo $k-\varepsilon$ obtuvo resultados ligeramente mejores en la predicción de la expansión del chorro, perfiles de velocidad y velocidad en el eje del chorro, mientras que el modelo RNG predijo mejor la energía cinética. En chorros circulares, el modelo $k-\varepsilon$ obtuvo mejores resultados en la predicción de la expansión del chorro y en la velocidad en el eje del chorro, aunque ninguno de los dos predijo adecuadamente la energía cinética, especialmente el modelo RNG.

En las Figura 68 se muestran los resultados de energía cinética turbulenta $k$, obtenidos con chorros planos en la sección $x / 2 b_{0}=50$, mientras que en la Figura 69 se observan los resultados de $k$ obtenidos con chorros circulares en la sección $x / d=61.5$, donde $b_{0}$ es la mitad del ancho del chorro inicial, $d$ es el diámetro de la tobera, $u_{m}$ es la velocidad en el eje del chorro, $x$ es el eje del chorro, $y$ es el eje transversal del chorro, $y$ $r$ es el diámetro del chorro circular.
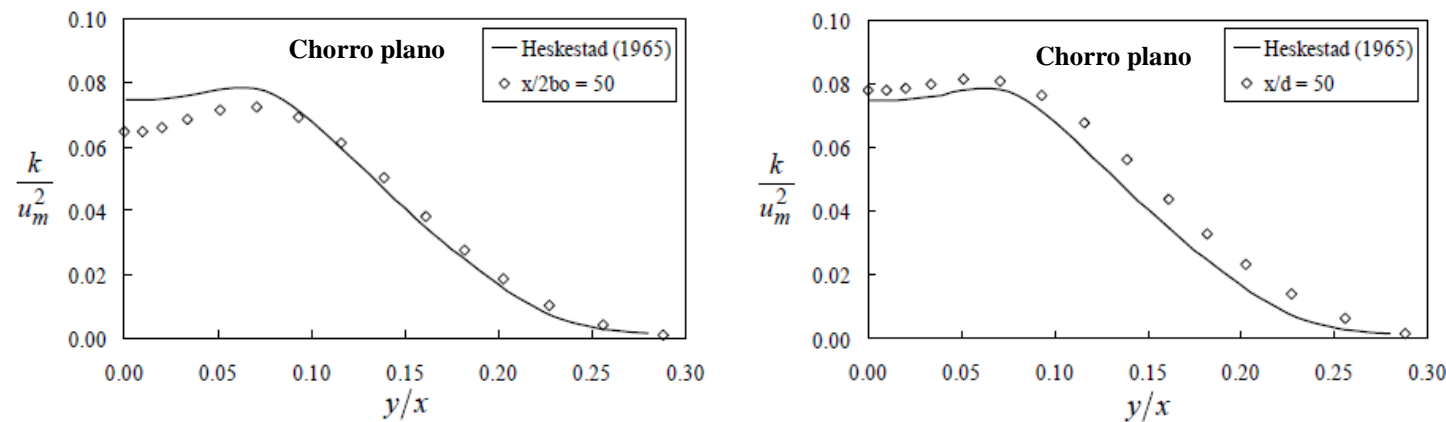

Figura 68. Perfil de energía cinética turbulenta para chorro plano: a) Modelo de turbulencia $k-\varepsilon$; b) Modelo de turbulencia RNG $k-\varepsilon$. (Aziz et al., 2008).
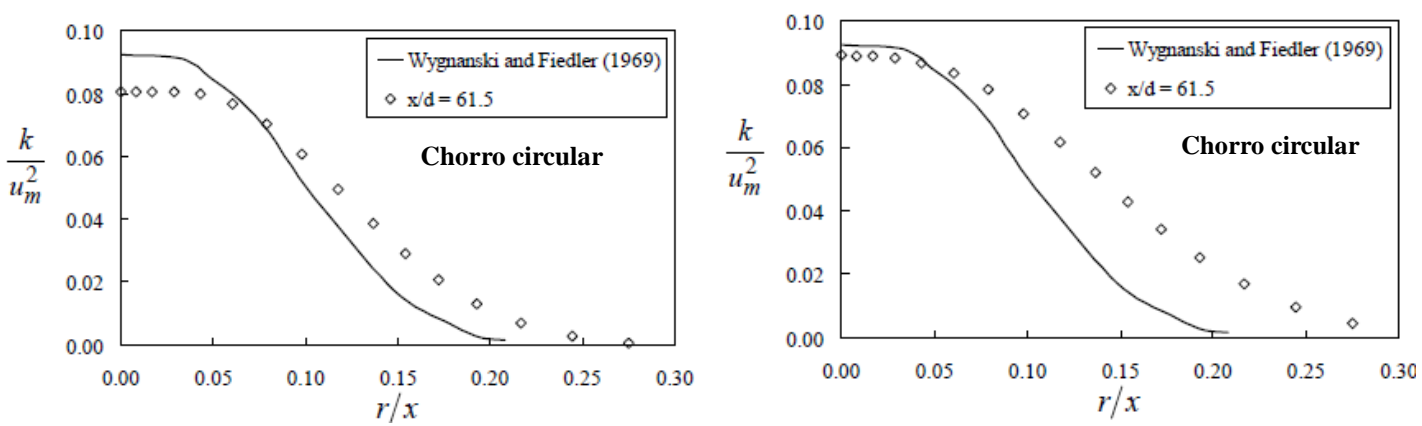

Figura 69. Perfil de energía cinética turbulenta para chorro circular: a) Modelo de turbulencia

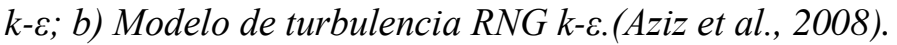

Guha et al. (2010) simularon con FLUENT y el modelo de turbulencia $k$ - $\varepsilon$ chorros turbulentos de alta velocidad de agua en el aire. Las simulaciones numéricas predijeron razonablemente las velocidades, presiones y distribución de la fracción volumétrica del 
agua, obteniendo gran precisión en las características de la línea central, pero subestimaron la velocidad y sobreestimando la fracción de agua en el borde del chorro.

En la Figura 70 se muestra la distribución de presiones obtenida sobre una placa perpendicular al chorro de alta velocidad en la sección $x / D=76$, siendo $x$ la distancia medida sobre el eje del chorro, $D$ el diámetro de la tobera y $r$ la distancia transversal hasta el eje del chorro. Los resultados numéricos coinciden adecuadamente en la línea central del chorro pero se desvían hacia el borde subestimando las presiones.

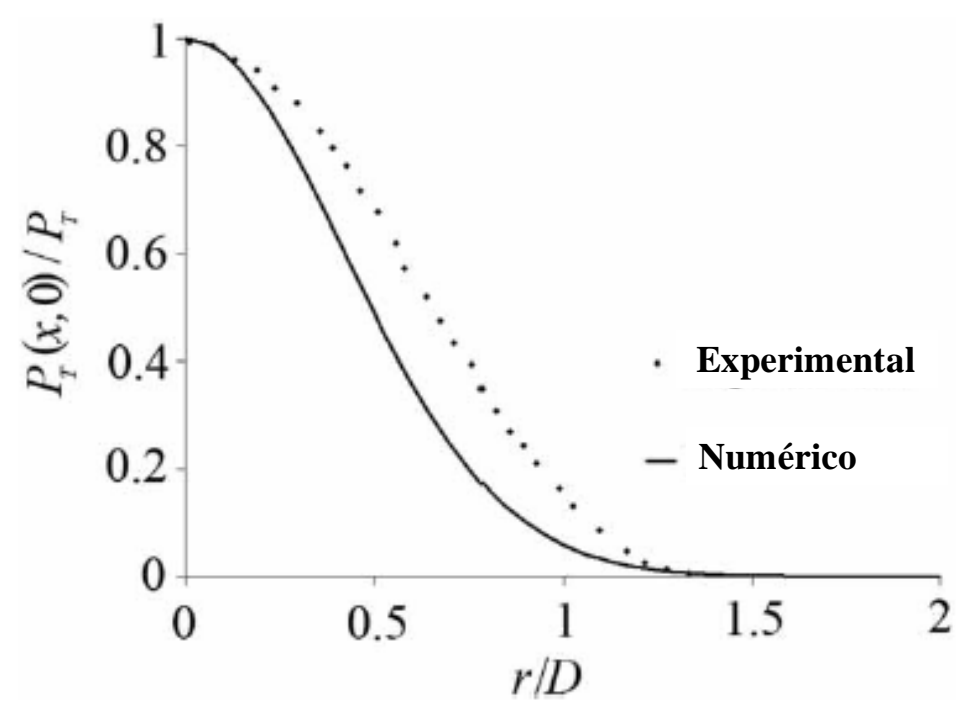

Figura 70. Presión normalizada simulada en la dirección radial sobre una placa plana frente a los resultados de Leach et al. (1996) (Guha et al., 2010).

La Figura 71 muestra la distribución de velocidades para las secciones $x / D=100$, 200 y 300. En comparación con los resultados de Rajaratnam et al. (1994), la distribución de velocidades obtiene buenos resultados hasta un ancho radial de 2-3 veces el diámetro inicial $D$. Fuera de esta región, la zona de mezcla del agua es más importante. La zona de mezcla exterior está formada por gotas de agua espaciadas. Con el tamaño de mallado empleado, la aproximación Euleriana no es capaz de capturar el fenómeno adecuadamente puesto que no reproduce el fenómeno físico de formación de gotas dispersas.

Wilcox (2010) analizó el ratio de expansión de diferentes chorros calculados con varios modelos de turbulencia de dos ecuaciones. Para chorros planos, circulares y radiales, consideró que el ratio de expansión es el valor de $y / x$ donde la velocidad es la mitad de la que tiene el chorro en la línea central. La Tabla 19 compara los valores de los perfiles de velocidad medidos y calculados con distintos modelos de turbulencia. 


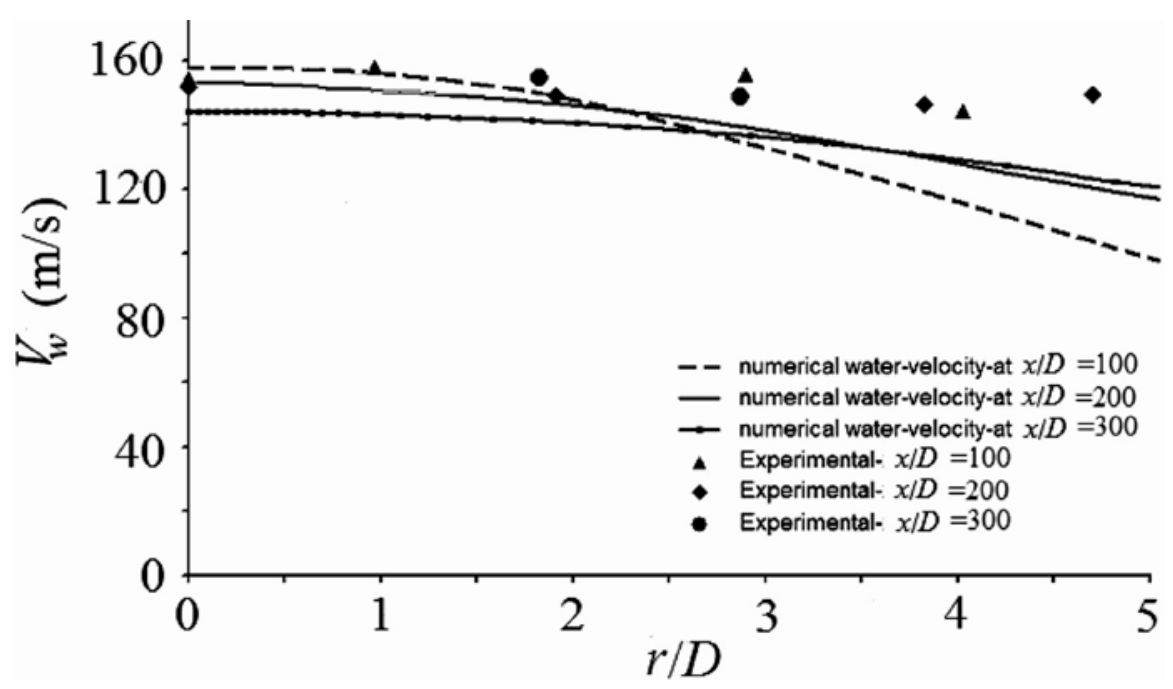

Figura 71. Distribución de velocidades para $x / D=100,200$ y 300 y comparación con los resultados de Rajaratnam et al. (1994) (Guha et al., 2010).

\begin{tabular}{|l|c|c|c|c|}
\hline \multicolumn{1}{|c|}{ Flujo } & Modelo $\boldsymbol{k}-\boldsymbol{\omega}$ & Modelo $\boldsymbol{k}-\boldsymbol{\varepsilon}$ & Modelo RNG $\boldsymbol{k}-\boldsymbol{\varepsilon}$ & Medido \\
\hline Extremo de estela & 0.326 & 0.256 & 0.290 & $0.320-0.400$ \\
\hline Capa de mezcla & 0.096 & 0.098 & 0.099 & $0.103-0.120$ \\
\hline Chorro plano & 0.108 & 0.109 & 0.147 & $0.100-0.110$ \\
\hline Chorro circular & 0.094 & 0.120 & 0.185 & $0.086-0.096$ \\
\hline Chorro radial & 0.099 & 0.094 & 0.111 & $0.096-0.110$ \\
\hline
\end{tabular}

Tabla 19. Ratios de expansión de flujo de corte libre para modelos de dos ecuaciones (Wilcox, 2010).

De los tres modelos analizados, $k$ - $\omega$ es el más cercano a las mediciones del ratio de expansión. De este modo, salvo para el caso del extremo de la estela que está un 6\% por debajo del umbral inferior, los valores obtenidos cayeron dentro del rango de valores medidos. Considerando el valor medio de los resultados medidos, la diferencia media con los valores obtenidos por el modelo $k-\omega$ fue del $6 \%$. La diferencia media entre los valores experimentales y los resultados del modelo $k$ - $\varepsilon$ fue del $17 \%$, siendo el chorro plano el único caso que calculó resultados dentro del rango de medidas. El modelo RNG $k-\varepsilon$ obtuvo diferencias aun mayores (una diferencia promedio del 36\%), con un valor el doble de los resultados medidos para el chorro circular.

Las figuras siguientes muestran que, en general, el modelo $k-\omega$ tiende a ajustarse adecuadamente a las distintas tipologías de chorros, el cual obtuvo una suave variación del perfil de velocidades cuando se aproxima a la zona exterior. El modelo $k-\varepsilon$ predijo una pendiente discontinua no física en el borde de algunos chorros, mientras que el modelo RNG obtuvo pendientes discontinuas para todos tipos de chorro analizados. 


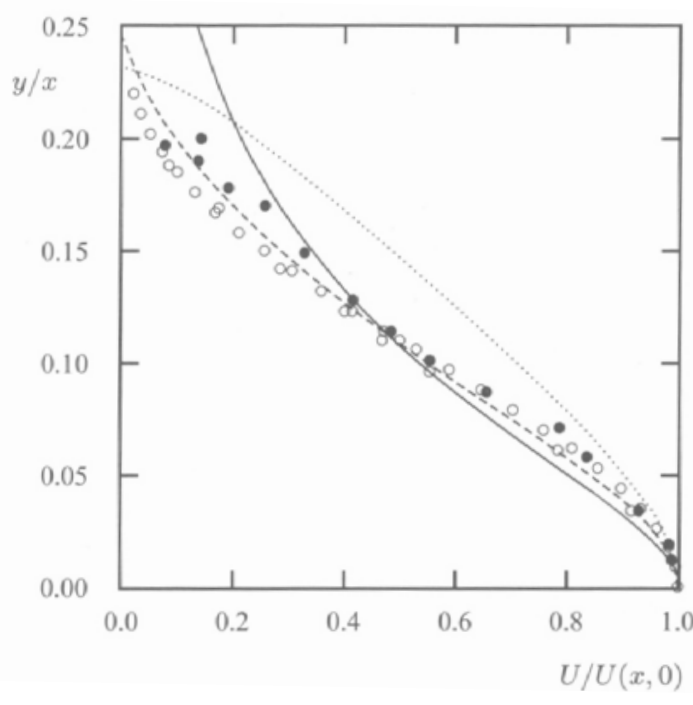

Figura 72. Comparación de los perfiles de velocidad medidos y calculados para el chorro plano: —— modelo $k-\omega$; - - - modelo $k-\varepsilon ; \cdots$ modelo $R N G k-\varepsilon ;$; Bradbury (1965); • Heskestad (1965) (Wilcox, 2010).

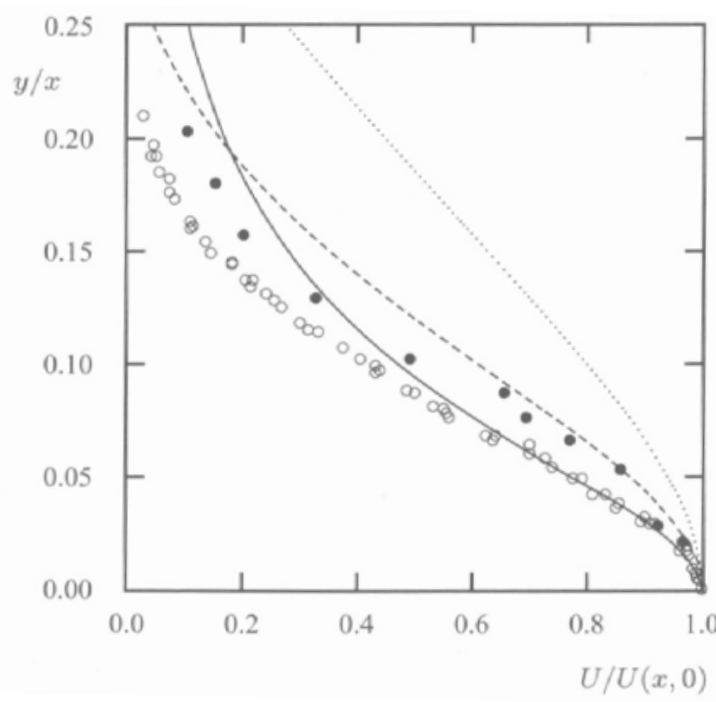

Figura 73. Comparación de los perfiles de velocidad medidos y calculados para el chorro circular: — modelo $k-\omega$; - - modelo $k-\varepsilon ; \cdot \cdots$ - modelo RNG k- $\varepsilon$; o Wygnanski y Fiedler (1969); • Rodi (1975) (Wilcox, 2010).

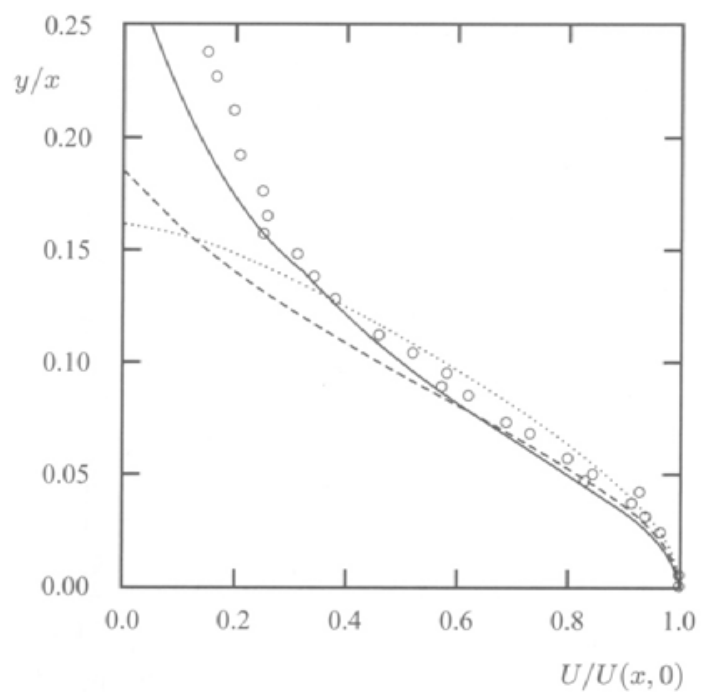

Figura 74. Comparación de los perfiles de velocidad medidos y calculados para el chorro radial: —— modelo $k-\omega$; - - - modelo $k-\varepsilon ; \cdots$ modelo $R N G k$ - $\varepsilon$; o Witze y Dwyer (1976)

(Wilcox, 2010).

Balabel et al. (2011) simularon con FLUENT toberas bidimensionales en cohetes aplicando seis modelos de turbulencia, y compararon las presiones estáticas en las paredes y en las zonas de choque con datos experimentales. Para diferentes presiones en la tobera (NPR), el modelo de turbulencia Shear Stress Transport (SST) obtuvo los mejores resultados tanto en tiempos de resolución como en precisión respecto las mediciones de laboratorio (Figura 75). 

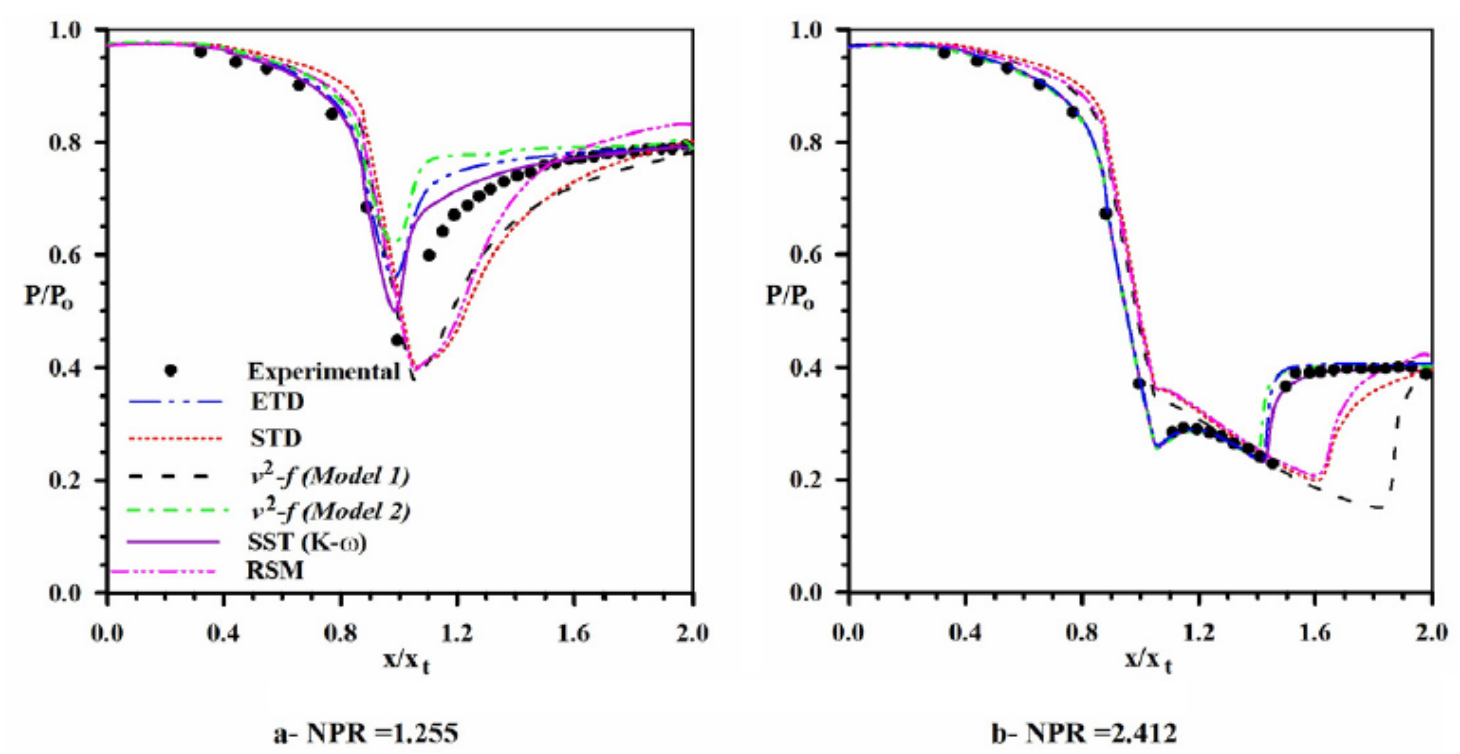

Figura 75. Comparación de los resultados de diferentes modelos de turbulencia con datos experimentales (Balabel et al., 2011).

Aziz y Khan (2011) simularon con FLOW-3D la evolución de las velocidades medias y la energía cinética de chorros planos verticales inyectados desde la solera en aguas someras, utilizando los modelos de turbulencia $k-\varepsilon$ y RNG. Los resultados se comparan con los resultados experimentales de Kuang et al. (2001). La Figura 76 muestra la evolución de la velocidad vertical central $w_{c}$, siendo $d$ el diámetro inicial del chorro, $z$ la distancia en el eje central del mismo y $x$ la distancia lateral. Los resultados de $w_{c}$ calculados con RNG fueron más precisos. Las velocidades laterales del chorro obtenidas con ambos modelos estaban ligeramente subestimadas, mientras que la expansión del chorro obtenida con $k-\varepsilon$ fue más real.

En la Figura 77 se muestran los perfiles de energía cinética $k$ para diferentes secciones de la zona de establecimiento del chorro, donde los resultados obtenidos con el modelo RNG se han desplazado 0.05 para una mejor comparación. El modelo $k-\varepsilon$ reprodujo de modo más fidedigno la forma y magnitud de la energía cinética, aunque la magnitud fue sobreestimada en la zona situada fuera del rango $-0.1 \leq x /\left(z-z_{0}\right) \leq 0.1$, especialmente cerca de la tobera $(z / d=10)$. Para la sección $z / d=24$, la energía cinética obtenida con el modelo RNG estaba sobreestimada un $30 \%$ en la zona central del chorro.

Los autores consideran que los resultados obtenidos con el modelo $k-\varepsilon$ son ligeramente más precisos. 


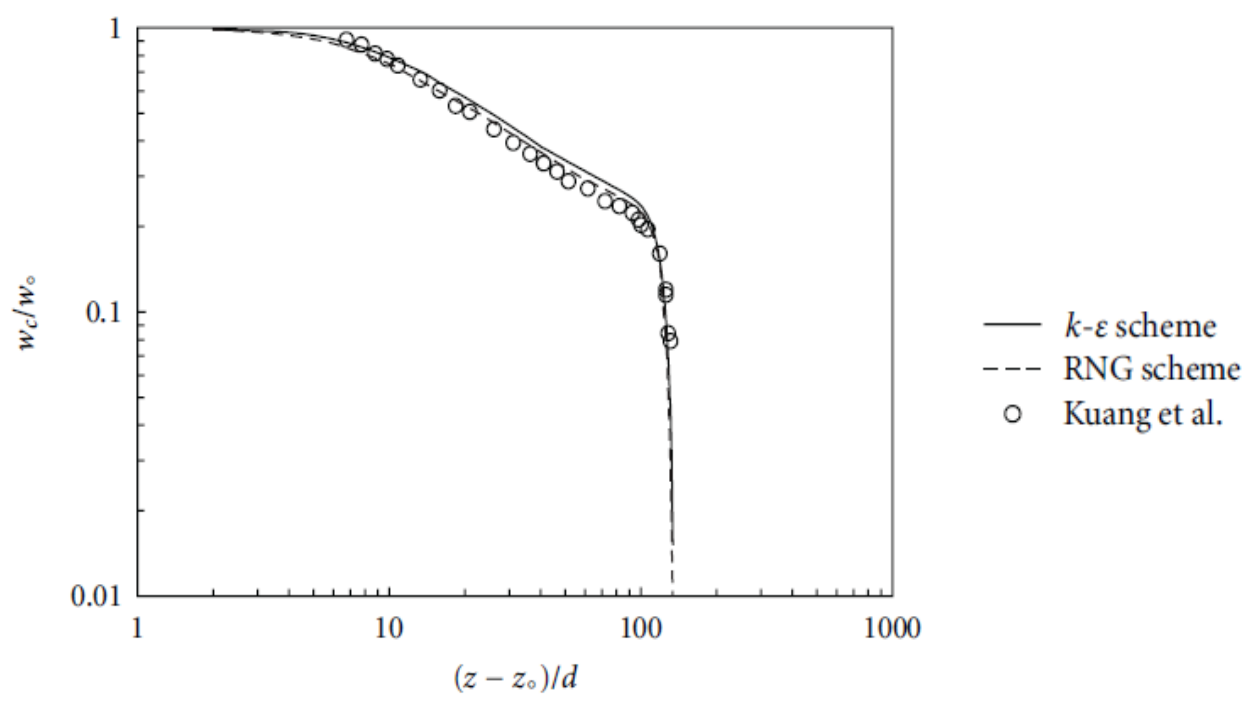

Figura 76. Variación de la velocidad en la línea central del chorro (Aziz y Khan, 2011).

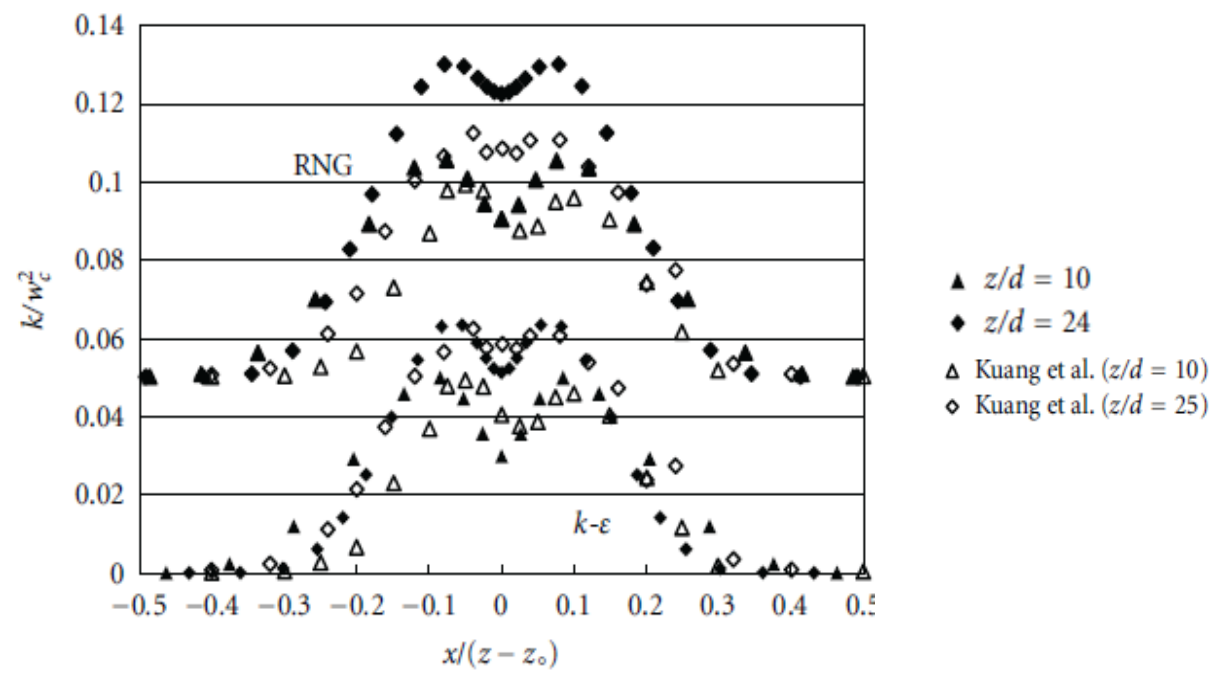

Figura 77. Perfiles de energía cinética turbulenta para distintas secciones (Aziz y Khan, 2011).

Kendil et al. (2012) compararon el campo de velocidades producido por chorros turbulentos verticales sumergidos en una piscina de agua, y generados con diferentes tasas de aireación. Las simulaciones se realizaron con ANSYS CFX 12.0, usando el modelo de turbulencia $k-\varepsilon$ y la aproximación Euleriana, mientras que los datos de laboratorio se obtuvieron con la técnica Particle Image Velocimetry (PIV).

Considerando un único fluido, los perfiles de velocidad radiales a distintas secciones fueron muy similares, mientras que sobre el eje se obtuvieron diferencias de velocidad de hasta $\pm 0.2 \mathrm{~m} / \mathrm{s}$. La Figura 78 muestra una comparación entre las simulaciones y los resultados experimentales de la velocidad axial del chorro, para cinco secciones transversales situadas por debajo de la zona de aplicación del chorro. 

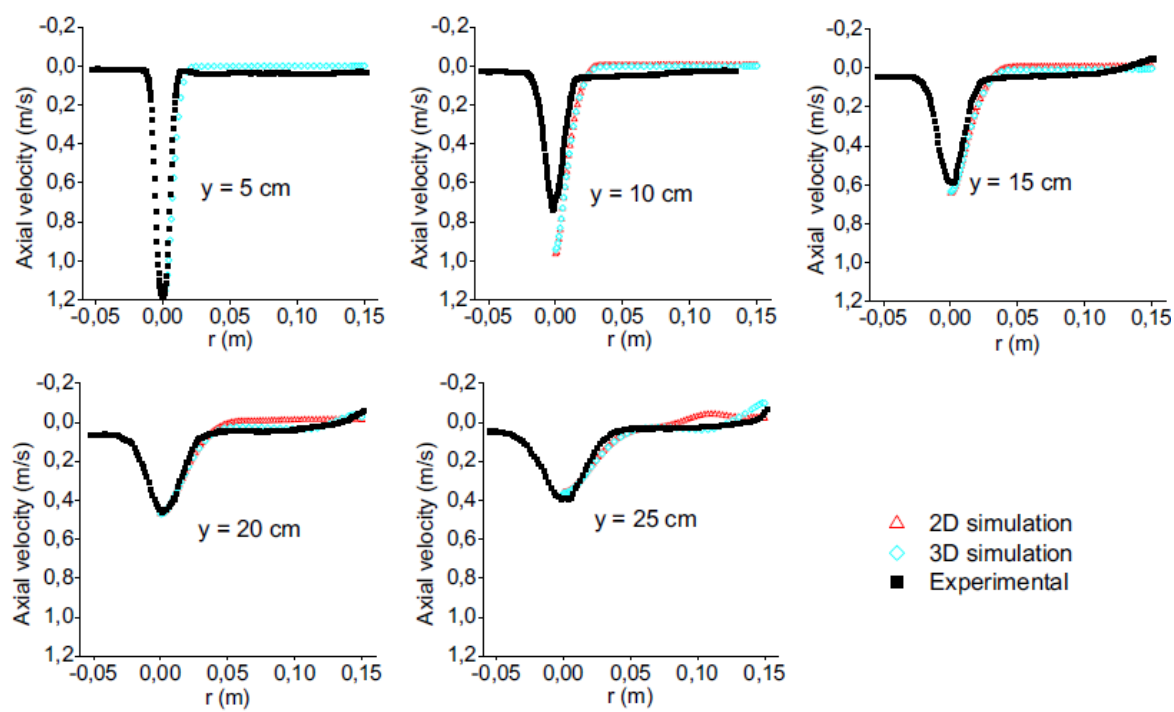

Figura 78. Distribución radial de la velocidad axial para flujo unifásico (Kendil et al., 2012).

La acción de la fuerza de sustentación sobre las pequeñas burbujas las mantiene en la región cercana a la línea central de chorro, mientras que las burbujas más grandes son empujadas hacia la pared del tanque, lejos de la línea central de chorro. Los autores investigaron el efecto del coeficiente de la fuerza de sustentación $C_{L}$ (Figura 79). Aplicando un coeficiente negativo para las burbujas grandes, obtuvieron una reducida estela, de modo que la fracción de aire se concentraba sobre el eje del chorro en contradicción con las observaciones experimentales. Sin embargo, para valores constantes positivos de $C_{L}$ observaron una buena difusión de la estela de burbujas.

La energía cinética simulada concordaba con las mediciones de laboratorio. Sin embargo, los remolinos transitorios no se resolvieron con la aproximación RANS de la turbulencia debido a que el modelo de turbulencia no considera la anisotropía.

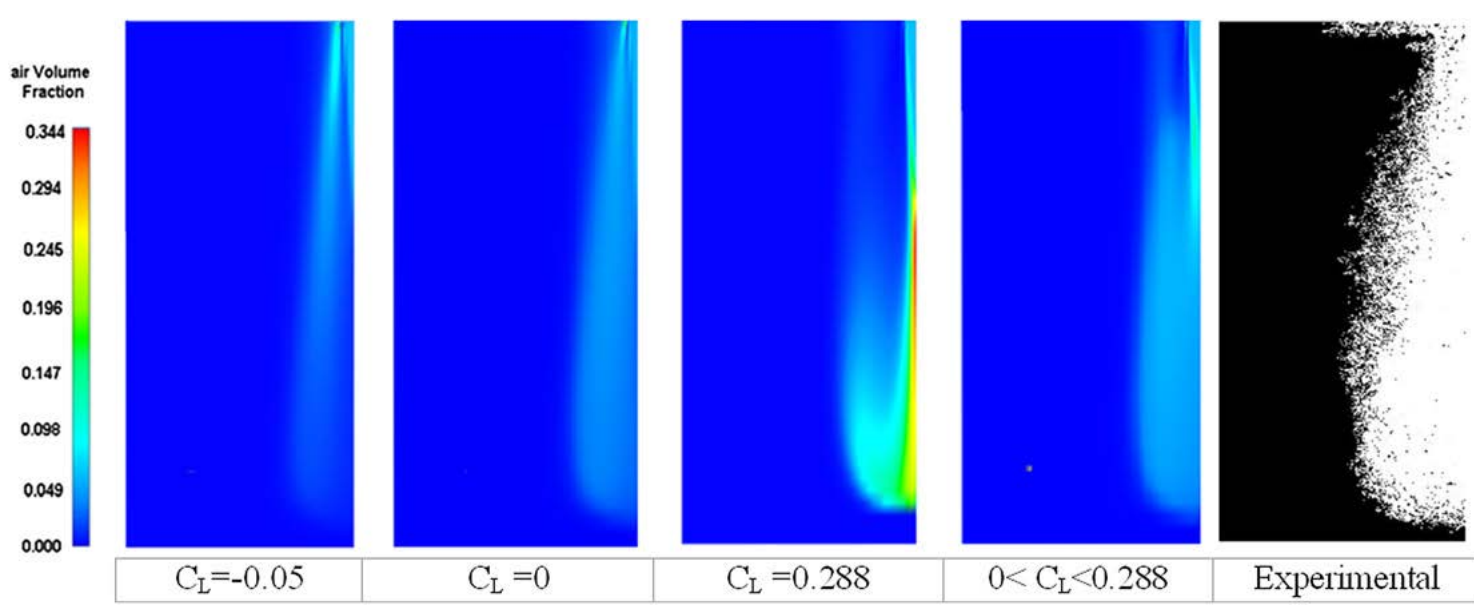

Figura 79. Forma de la estela para diferentes valores del coeficiente de la fuerza de sustentación $C_{L}$, considerando una concentración de aire $\alpha_{G}=5 \%$ (Kendil et al., 2012). 


\subsubsection{Futuros desafíos}

A pesar de los beneficios que ofrece la metodología CFD, existe un número de limitaciones que no permite realizar estudios fiables en el campo de la ingeniería hidráulica, y en concreto en su aplicación a aliviaderos. La Tabla 20 resume las limitaciones de estas simulaciones basada en la experiencia de análisis de aliviaderos (Ho y Riddette, 2010)

\begin{tabular}{|c|c|c|}
\hline Limitación & Progresos hasta la fecha & Sugerencia de futuros trabajos \\
\hline Cavitación & $\begin{array}{l}\text { Acertado cálculo de presiones para } \\
\text { un rango de formas de cresta. } \\
\text { Cavitación potencial determinada } \\
\text { con fórmulas empíricas. }\end{array}$ & $\begin{array}{l}\text { Modelación directa de cavitación para } \\
\text { propósitos de investigación. Investigación } \\
\text { de daños por cavitación con CFD para un } \\
\text { mejor conocimiento de las condiciones que } \\
\text { conducen a daños. }\end{array}$ \\
\hline $\begin{array}{l}\text { Efecto de entrada } \\
\text { de aire }\end{array}$ & $\begin{array}{l}\text { Acertada validación en el punto de } \\
\text { comienzo de aireación para un } \\
\text { número limitado de condiciones de } \\
\text { flujo. }\end{array}$ & $\begin{array}{l}\text { Validación de la cantidad de entrada de aire } \\
\text { y efectos de esponjamiento aguas abajo para } \\
\text { entrada superficial, entrada de aire en flujo } \\
\text { de chorros y dispositivos laterales/de fondo. }\end{array}$ \\
\hline $\begin{array}{l}\text { Transporte de } \\
\text { sedimentos }\end{array}$ & $\begin{array}{l}\text { Las publicaciones actuales tienen } \\
\text { éxito limitado usando ecuaciones de } \\
\text { transporte de sedimentos empíricas } \\
\text { discretizadas. }\end{array}$ & Desarrollo de algoritmos. \\
\hline Demanda de aire & No se ha estudiado. & $\begin{array}{l}\text { Validación de demanda de aire a lo largo } \\
\text { de chorros y bajo chorros de caída libre. }\end{array}$ \\
\hline $\begin{array}{l}\text { Flujos de pared, } \\
\text { poco calado y } \\
\text { elevada rugosidad }\end{array}$ & $\begin{array}{l}\text { Acuerdo cualitativo de velocidad } \\
\text { calculada. Dificultad de extraer } \\
\text { tensiones de corte debido a } \\
\text { conflictos entre elevada rugosidad y } \\
\text { pequeños tamaño de malla que } \\
\text { requieren los calados poco } \\
\text { profundos (problemas de longitudes } \\
\text { de escala diferentes). }\end{array}$ & $\begin{array}{l}\text { Estudio paramétrico para identificar los } \\
\text { límites de fiabilidad para una combinación } \\
\text { de lechos rugosos y calados de flujo. }\end{array}$ \\
\hline $\begin{array}{l}\text { Espesor de } \\
\text { chorros y longitud } \\
\text { de rotura }\end{array}$ & $\begin{array}{l}\text { Progresos limitados sólo en el } \\
\text { núcleo del chorro. Requiere una } \\
\text { resolución de malla muy fina. }\end{array}$ & $\begin{array}{l}\text { Extensión a modelos de dos fluidos para } \\
\text { incluir la entrada de aire y la longitud de } \\
\text { rotura. Validación de las presiones de } \\
\text { impacto frente a datos experimentales. }\end{array}$ \\
\hline $\begin{array}{l}\text { Presiones } \\
\text { fluctuantes en } \\
\text { aliviaderos/dientes } \\
\text { del cuenco }\end{array}$ & $\begin{array}{l}\text { Resultados obtenidos con modelos } \\
\text { de turbulencia RANS con mallas } \\
\text { relativamente gruesas. No hay datos } \\
\text { de validación. }\end{array}$ & $\begin{array}{l}\text { Obtención de datos experimentales fiables } \\
\text { para validación. Estudio paramétrico con } \\
\text { tamaños de malla y modelos de turbulencia } \\
\text { para identificar el nivel de fiabilidad de las } \\
\text { presiones calculadas. }\end{array}$ \\
\hline $\begin{array}{l}\text { Interacción } \\
\text { dinámica }\end{array}$ & $\begin{array}{l}\text { La formación de vórtices ha sido } \\
\text { capturada cualitativamente. No hay } \\
\text { datos de validación de la intensidad } \\
\text { de los vórtices. }\end{array}$ & $\begin{array}{l}\text { Obtención de datos fiables/datos de } \\
\text { prototipo para validación. Estudio } \\
\text { paramétrico con tamaños de mallado y } \\
\text { modelos de flujo. }\end{array}$ \\
\hline $\begin{array}{l}\text { Largos tiempos de } \\
\text { cálculo en modelos } \\
\text { complejos }\end{array}$ & $\begin{array}{l}\text { Uso de software de paralelización } \\
\text { para realizar el análisis en } \\
\text { ordenadores multi-CPU. Coste del } \\
\text { hardware y software como } \\
\text { consecuencia del análisis tipo } \\
\text { clúster. }\end{array}$ & $\begin{array}{l}\text { Software comercial para desarrollar } \\
\text { programas más eficientes, y suministro de } \\
\text { hardware para desarrollar ordenadores más } \\
\text { rápidos. }\end{array}$ \\
\hline
\end{tabular}

Tabla 20. Lista de limitaciones habituales y sugerencias para investigaciones futuras en CFD

(Ho y Riddette, 2010). 
El Comité Internacional de Grandes Presas (ICOLD, 2001), en su boletín n ${ }^{\circ} 122$ Computational procedures for dam engineering reliability and applicability, citado por Ho y Riddette (2010), considera cuatro índices de fiabilidad de las simulaciones para modelaciones hidráulicas con una serie de recomendaciones (Tabla 21):

- RI-1. Los fenómenos relacionados con la seguridad de la presa pueden ser analizados con fiabilidad por medio de modelos numéricos.

- RI-2. Los fenómenos relacionados con la seguridad de la presa pueden ser analizados por medio de modelos numéricos pero con algunas limitaciones y/o dificultades (simplificaciones en las hipótesis computacionales, falta 0 dificultada para tener datos experimentales fiables, coste del análisis, etc.).

- RI-3. Los fenómenos relacionados con la seguridad de la presa pueden ser analizados por medio de modelos numéricos, cuyos resultados pueden dar sólo indicios cuantitativos o cualitativos, como por ejemplo los resultados obtenidos cuando se deben realizar fuertes simplificaciones, etc.

- RI-4. Los fenómenos relacionados con la seguridad de la presa en la actualidad no pueden ser analizados por medio de modelos numéricos.

\begin{tabular}{|l|c|c|c|c|}
\hline \multicolumn{1}{|c|}{ Fenómeno } & Construcción & $\begin{array}{c}\text { Primer } \\
\text { llenado }\end{array}$ & Operación & $\begin{array}{c}\text { Índice de } \\
\text { fiabilidad }\end{array}$ \\
\hline Cavitación (presión) & $\sqrt{ }$ & $\sqrt{ }$ & $\sqrt{ }$ & 2 \\
\hline $\begin{array}{l}\text { Erosión (velocidades y contenido } \\
\text { de material sólido) }\end{array}$ & $\sqrt{ }$ & $\sqrt{ }$ & $\sqrt{ }$ & 4 \\
\hline $\begin{array}{l}\text { Movimiento de losas de aliviaderos } \\
\text { (debido a oscilaciones bajo presión) }\end{array}$ & No aplicable & $\sqrt{ }$ & $\sqrt{ }$ & 1 \\
\hline Caudales extremos (descarga) & $\sqrt{ }$ & $\sqrt{ }$ & 4 \\
\hline $\begin{array}{l}\text { Erosión en el pie de aguas abajo } \\
\text { (descarga y energía cinética) }\end{array}$ & No aplicable & $\sqrt{ }$ & $\sqrt{ }$ & $2-3$ \\
\hline $\begin{array}{l}\text { Sedimentación (transporte de } \\
\text { sedimentos) }\end{array}$ & No aplicable & No aplicable & & \\
\hline
\end{tabular}

Tabla 21. Resumen de la fiabilidad de los modelos numéricos en la modelación hidráulica (ICOLD, 2001).

Teniendo en cuenta los resultados positivos de estudios de validación y los proyectos en el mundo real que se han llevado a cabo usando modelos CFD, algunos de los índices de fiabilidad en el uso de modelaciones numéricas hidráulicas en ingeniería de presas que considera ICOLD deberían ser revisados. 


\section{ANÁLISIS Y DISCUSIÓN DE RESULTADOS}

\subsection{Campaña de laboratorio}

\subsubsection{Ensayos de laboratorio}

En este estudio se han empleado datos de aliviaderos de vertido libre obtenidos por diferentes autores en laboratorio. Los datos se han revisado y complementando mediante una campaña de laboratorio realizada en una nueva instalación. De este modo, se han comparado las presiones en la solera del cuenco de disipación obtenidas en tres infraestructuras de laboratorio diferentes (Castillo, 1989; Puertas, 1994; y en este estudio). Esto ha permitido reducir los errores de una instalación concreta, así como obtener ajustes con una mayor precisión.

Los datos de Castillo (1989) cubren un rango de $0.78 \leq H / L_{b} \leq 1.22$. Las alturas de caída $P_{l}$ van desde $1.60 \mathrm{~m}$ a $1.76 \mathrm{~m}$, siete colchones de agua $Y$ (desde el impacto directo hasta $0.25 \mathrm{~m}$ ) y tres caudales específicos $q$ (entre 0.033 y $0.050 \mathrm{~m}^{2} / \mathrm{s}$ ).

Los ensayos de Puertas (1994) tienen un rango de $0.42 \leq H / L_{b} \leq 3.06$, cuatro alturas de caída (de $1.85 \mathrm{~m}$ a $5.45 \mathrm{~m}$ ), diez colchones de agua (de $0.08 \mathrm{~m}$ a $0.80 \mathrm{~m}$ ), y un rango de caudales específicos entre 0.026 y $0.149 \mathrm{~m}^{2} / \mathrm{s}$.

Los registros de presiones de ambas tesis doctorales se obtuvieron mediante transductores de presión piezorresistivos instalados en la solera del cuenco de disipación. Se realizaron en torno a 200 ensayos, cada uno con 2400 datos, obtenidos a una frecuencia de muestreo de 20 datos por segundo.

Los datos obtenidos en este estudio cubren un rango de $0.60 \leq H / L_{b} \leq 2.02$. Se consideraron tres alturas de caída entre $1.70 \mathrm{~m}$ y $3.00 \mathrm{~m}$, siete colchones de agua (desde situación de impacto directo a $0.60 \mathrm{~m}$ ), y siete caudales específicos (entre 0.020 y 0.064 $\mathrm{m}^{2} / \mathrm{s}$ ). Cada uno de los 184 ensayos se midieron por triplicado, empleando una frecuencia de muestreo de $20 \mathrm{~Hz}$, y registrando 7200 datos en cada registro.

Para analizar los perfiles de velocidades en el cuenco de amortiguación se empleó un equipo Doppler. Se realizaron mediciones de velocidades instantáneas en distintas secciones situadas aguas abajo del punto de estancamiento del chorro.

Considerando una altura de vertedero de $2.35 \mathrm{~m}$, se ensayaron los resaltos sumergidos generados por 3 caudales de trabajo (0.023, 0.037 y $0.058 \mathrm{~m}^{2} / \mathrm{s}$ ), y 6 colchones de agua, analizándose un total de 18 resaltos. Para la correcta caracterización de la velocidad del flujo, el cuenco de disipación se dividió en secciones transversales espaciadas cada $10 \mathrm{~cm}$, tomando como sección de referencia el punto de estancamiento del chorro. En cada ensayo se registraron 5000 muestras a una frecuencia de $10 \mathrm{~Hz}$, 
obteniéndose un total de 1505 puntos muestreados. Siguiendo las investigaciones de Goring y Nikora (2002) y de Castillo (2008, 2009), se realizó el filtrado de los registros instantáneos aplicando los criterios de umbral de velocidad y umbral de aceleración.

Como alternativa del número de Weber que describe la importancia relativa de las fuerzas de inercia y de la tensión superficial, Kobus (1973) propone emplear el parámetro $Z \equiv\left(g \mu_{w}{ }^{4}\right) /\left(\rho \sigma_{w a}{ }^{3}\right)$, donde la viscosidad cinemática $\mu_{w}$, la densidad $\rho$ y la tensión superficial $\sigma_{w a}$ son las propiedades del fluido. La dependencia de la tasa específica de entrada de aire $\beta_{i}$ puede definirse como:

$$
\begin{gathered}
\beta_{i}=q_{a e} / q=f\left(\text { relación geométrica }, T_{u}, C_{p}, F_{i}, R_{e}, W_{e}\right) \\
\beta_{i}=q_{a e} / q=f\left(\text { relación geométrica }, T_{u}, C_{p}, F_{i}, R_{e}, Z\right)
\end{gathered}
$$

donde $q_{a e}$ es la tasa específica de entrada de aire, $q$ el caudal específico, $T_{u}$ la intensidad turbulenta y $C_{p}$ el coeficiente de presión.

$\mathrm{Al}$ evaluar modelos a escala que cumplen la semejanza de Froude, para un valor $Z$ dado, esta configuración de parámetros tiene la ventaja de concentrar los efectos de escala en el número de Reynolds. La Tabla 22 muestra el rango de valores de los chorros ensayados, junto a los números adimensionales obtenidos en la condición inicial (situada a una distancia vertical $h$ del labio del vertedero).

\begin{tabular}{|c|c|c|c|c|c|c|c|}
\hline $\begin{array}{c}\text { Altura de } \\
\text { vertedero } \\
\boldsymbol{P}_{\boldsymbol{I}}(\mathbf{m})\end{array}$ & $\begin{array}{c}\text { Caudal } \\
\text { específico } \\
\left.\boldsymbol{q} \mathbf{( m}^{\mathbf{2}} \mathbf{s}\right)\end{array}$ & $\begin{array}{c}\text { Velocidad } \\
\text { inicial } \\
\boldsymbol{V}_{\boldsymbol{i}}(\mathbf{m} / \mathbf{s})\end{array}$ & $\begin{array}{c}\text { Velocidad } \\
\text { incidente } \\
\boldsymbol{V}_{\boldsymbol{j}}(\mathbf{m} / \mathbf{s})\end{array}$ & $\begin{array}{c}\mathbf{N}^{\mathbf{0}} \text { de } \\
\text { Froude } \\
\boldsymbol{F}_{\boldsymbol{i}}\end{array}$ & $\begin{array}{c}\mathbf{N}^{\mathbf{0}} \mathbf{d e} \\
\text { Reynolds } \\
\boldsymbol{R}_{\boldsymbol{e}}\end{array}$ & $\begin{array}{c}\mathbf{N}^{\mathbf{0}} \text { de } \\
\text { Weber } \\
\boldsymbol{W}_{\boldsymbol{e}}\end{array}$ & $\begin{array}{c}\text { Parámetro } \\
\text { líquido } \\
\boldsymbol{Z}\end{array}$ \\
\hline \multirow{2}{*}{1.70} & 0.020 & 1.38 & 5.17 & 3.62 & 20225 & 384 & $2.56 \mathrm{E}-11$ \\
\cline { 2 - 8 } & 0.058 & 1.95 & 5.94 & 3.65 & 57335 & 1538 & $2.56 \mathrm{E}-11$ \\
\hline \multirow{2}{*}{2.35} & 0.017 & 1.31 & 6.03 & 3.42 & 17167 & 310 & $2.56 \mathrm{E}-11$ \\
\cline { 2 - 8 } & 0.057 & 1.93 & 6.93 & 3.71 & 56257 & 1494 & $2.56 \mathrm{E}-11$ \\
\hline \multirow{2}{*}{3.00} & 0.013 & 1.10 & 6.98 & 3.07 & 13253 & 201 & $2.56 \mathrm{E}-11$ \\
\cline { 2 - 8 } & 0.050 & 1.72 & 7.77 & 3.22 & 50035 & 1181 & $2.56 \mathrm{E}-11$ \\
\hline
\end{tabular}

Tabla 22. Rango de las condiciones de ensayo en laboratorio (valores mínimos y máximos).

En los ensayos realizados en la instalación de la Universidad Politécnica de Cartagena, las velocidades en el punto de incidencia fueron superiores a $5 \mathrm{~m} / \mathrm{s}$, mientras que los números de Reynolds en general superaron los 20000. De este modo, las fuerzas de inercia dominan a las fuerzas viscosas y tensión superficial. De los 184 ensayos, únicamente existen dos caudales específicos $\left(q<0.020 \mathrm{~m}^{2} / \mathrm{s}\right)$ con números de Reynolds que no cumplen esta condición (valores de 13000 y 17000), en los cuales los efectos de la tensión superficial no puede ser despreciada. Con algunas consideraciones, los valores de laboratorio pueden ser escalados aplicando semejanza de Froude. 


\subsubsection{Análisis general de los registros de presiones}

En la bibliografía existen estudios exhaustivos de mediciones de presiones en chorros circulares, obtenidos en modelos con velocidades superiores a $20 \mathrm{~m} / \mathrm{s}$ e intensidades turbulentas de hasta 5\% (Ervine et al., 1997; Bollaert, 2002; Manso et al., 2005, 2008; Federspiel, 2011). Sin embargo, los ensayos generalmente corresponden a relaciones de longitud de rotura $H / L_{b}<0.50$.

Castillo (2007) consideró cuatro tipos de chorros rectangulares. En este estudio, el límite entre colchón no efectivo y colchón efectivo se ha redefinido. De este modo, se puede establecer una nueva clasificación en base a: chorro no desarrollado y colchón no efectivo $\left(H / L_{b} \leq 1\right.$ e $\left.Y / B_{j} \leq 5.5\right)$; chorro no desarrollado y colchón efectivo $\left(H / L_{b} \leq 1 \mathrm{e}\right.$ $\left.Y / B_{j}>5.5\right)$; chorro desarrollado y colchón no efectivo $\left(H / L_{b}>1\right.$ e $\left.Y / B_{j} \leq 5.5\right)$; y chorro desarrollado y colchón efectivo $\left(H / L_{b}>1\right.$ e $\left.Y / B_{j}>5.5\right)$. En la Figura 80 se muestra el comportamiento que caracteriza cada grupo.

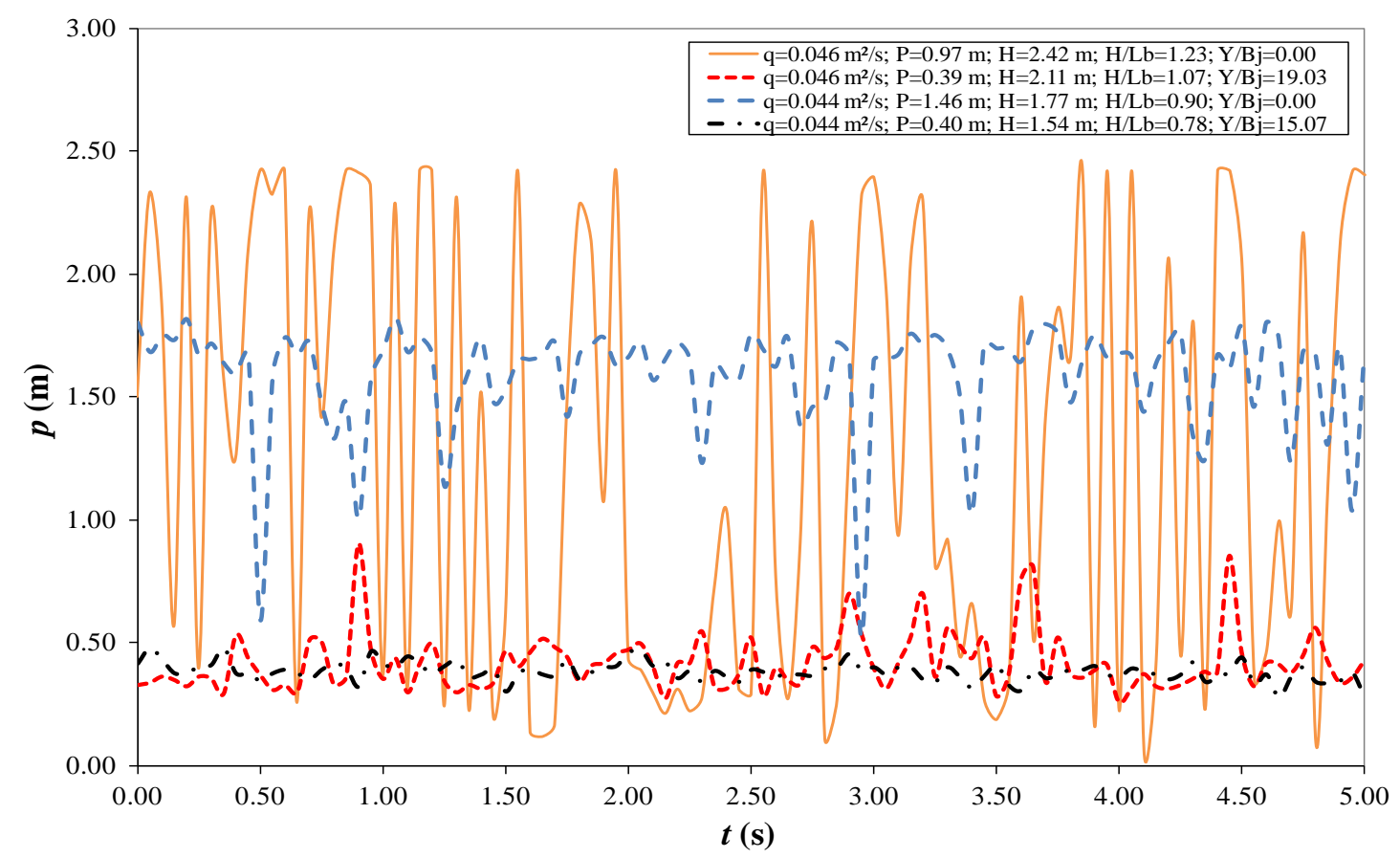

Figura 80. Patrones de presiones de los cuatro tipos de chorros rectangulares: Chorro no desarrollado y colchón no efectivo $\left(H / L_{b} \leq 1\right.$ e $\left.Y / B_{j} \leq 5.5\right)$; Chorro no desarrollado y colchón efectivo $\left(H / L_{b} \leq 1\right.$ e $\left.Y / B_{j}>5.5\right)$; Chorro desarrollado y colchón no efectivo $\left(H / L_{b}>1\right.$ e $Y / B_{j} \leq$ 5.5); Chorro desarrollado y colchón efectivo $\left(H / L_{b}>1\right.$ e $\left.Y / B_{j}>5.5\right)$. (adaptado de Castillo et al., 2014).

Los chorros no desarrollados $\left(H / L_{b} \leq 1\right)$ en general muestran un registro de presiones constante, con picos similares por encima y por debajo del valor medio. Los 
picos decrecen sustancialmente conforme se incrementa el colchón de agua (situaciones de colchón efectivo). Cuando el colchón es no efectivo, los efectos de la turbulencia de baja frecuencia se observan con claridad, con caídas de presión próximas al nivel de la presión atmosférica.

En el caso de chorros desarrollados $\left(H / L_{b}>1\right)$ se registran más fluctuaciones de la presión, debido a que la capa de corte turbulenta impacta en la solera del cuenco. Existe una alta entrada de aire en el chorro incidente, y se genera una zona de distribución de presiones superior a la del caso de chorros no desarrollados. El efecto conjunto es un incremento de la disipación de la energía y una reducción sustancial de la presión en la solera del cuenco. El efecto se incrementa con el aumento de la relación $H / L_{b}$ y con el colchón de agua.

La Figura 81 muestra la distribución de probabilidad de los cuatro tipos de registros analizados. Los patrones de las distribuciones son similares a la distribución de probabilidad normal, aunque se encuentran desplazados hacia la derecha en torno a $1.00\left(p-H_{m}\right) / H^{\prime}$, siendo $p$ la presión instantánea, $H_{m}$ la presión media del registro, y $H^{\prime}$ la raíz cuadrática media del registro de presiones.

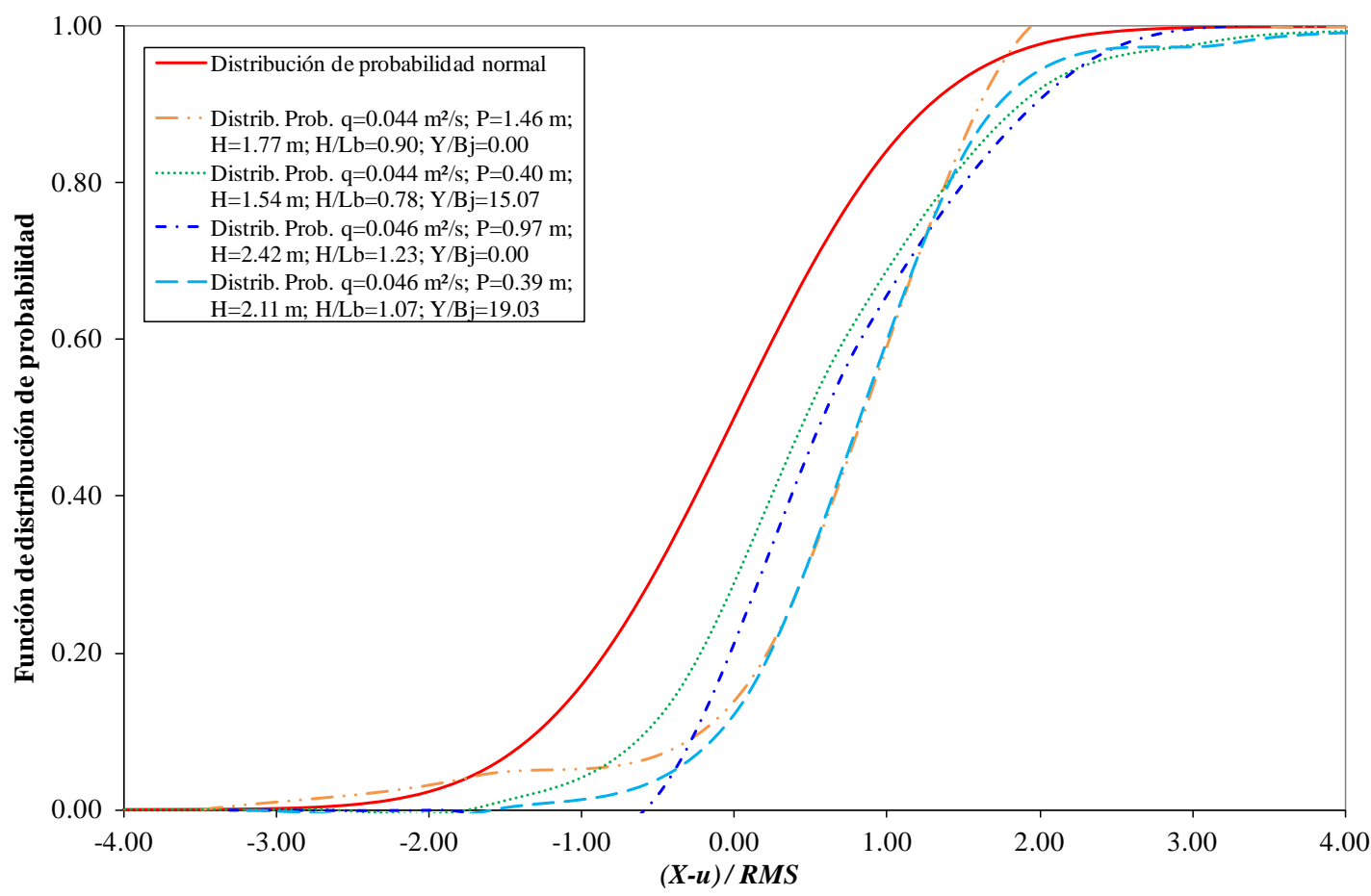

Figura 81. Función de distribución de probabilidad comparada con una distribución de Gauss. Chorros no desarrollados $\left(H / L_{b} \leq 1\right)$. Chorros desarrollados $\left(H / L_{b}>1\right)$. Colchón no efectivo $\left(Y / B_{j} \leq 5.5\right)$. Colchón efectivo $\left(Y / B_{j}>5.5\right)$. 
La función de densidad de probabilidad (probability density function, PDF) define la probabilidad de ocurrencia de los valores de presión en función de su desviación del valor de presión media. La Figura 82 muestra los cuatro registros de presiones en el dominio del tiempo en forma adimensional, comparados con la curva de la distribución normal.

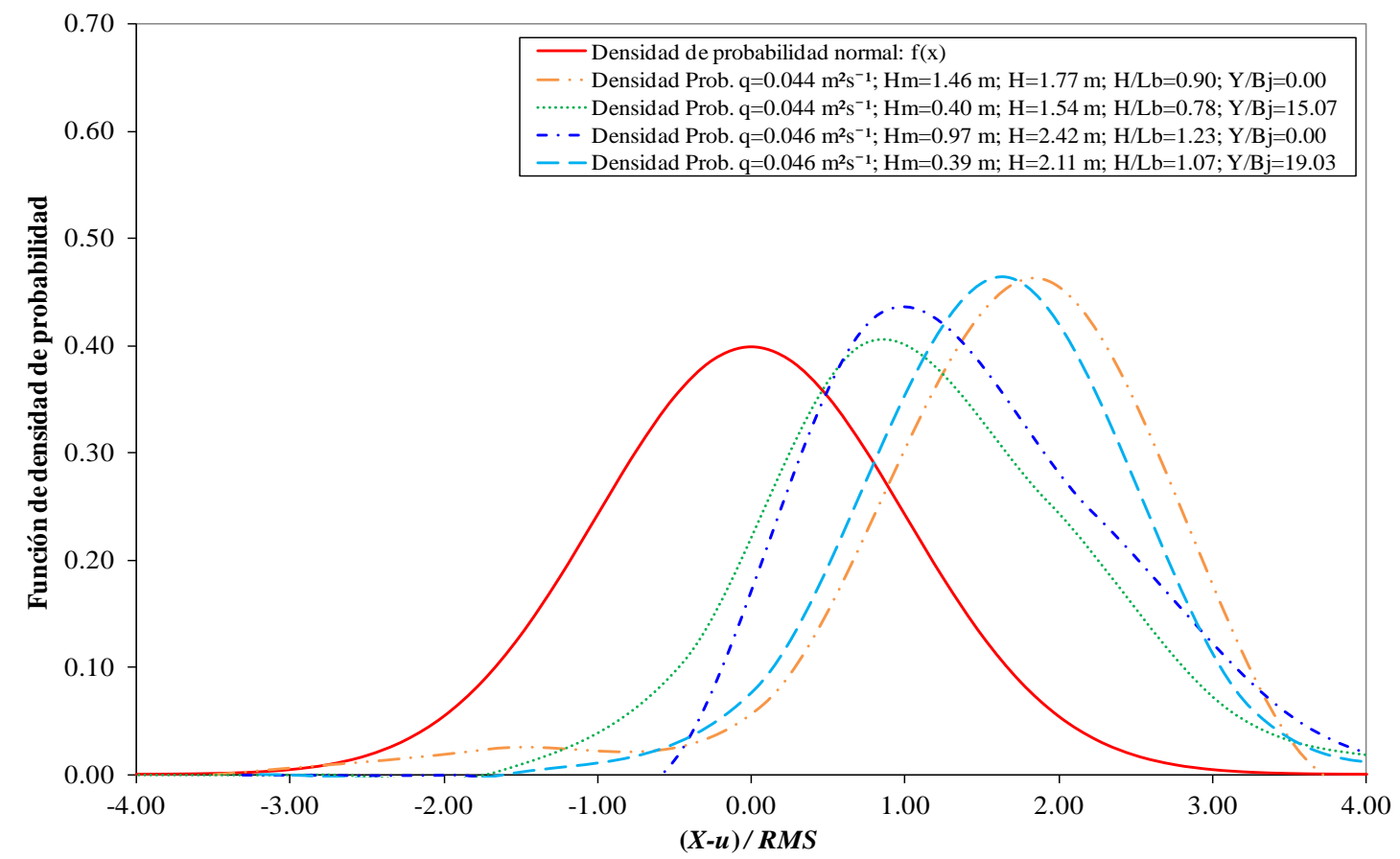

Figura 82. Función de densidad de probabilidad comparada con una distribución de Gauss. Chorros no desarrollados $\left(H / L_{b} \leq 1\right)$. Chorros desarrollados $\left(H / L_{b}>1\right)$. Colchón no efectivo $\left(Y / B_{j} \leq 5.5\right)$. Colchón efectivo $\left(Y / B_{j}>5.5\right)$. (Castillo et al., 2014).

Los chorros no desarrollados $\left(H / L_{b} \leq 1\right)$ y con colchón no efectivo $\left(Y / B_{j} \leq 5.5\right)$ se caracterizan por una PDF con sesgo negativo (-1.85), con un valor pico superior al de la curva de Gauss (0.46 en vez de 0.40), y una curtosis similar (2.92). Estos casos corresponden a las menores alturas de caída (menores velocidades del chorro incidente). Los colchones efectivos $\left(Y / B_{j}>5.5\right)$ generan una PDF similar a la curva de Gauss (curtosis $=2.62$ y valor máximo $=0.40$ ), aunque se caracterizan por un ligero sesgo positivo $(+0.80)$.

Los chorros no desarrollados $\left(H / L_{b}>1\right)$ y colchón no efectivo $\left(Y / B_{j} \leq 5.5\right)$ se caracterizan por una PDF con sesgo ligeramente positivo $(+0.50)$, que muestran un pico ligeramente superior al del la distribución de Gauss (0.43 en vez de 0.40). Con colchón efectivo $\left(Y / B_{j}>5.5\right)$, se observa una tendencia a aumentar el sesgo positivo de la curva PDF (+2.75) y la curtosis (14.44), aumentando el valor máximo hasta 0.48. 


\subsubsection{Metodología Paramétrica}

En este estudio se ha empleado la Metodología Paramétrica propuesta por Castillo (2006). Las formulaciones se han revisado y modificado con el fin de independizar de las condiciones iniciales. La Figura 83 muestra las variables más representativas de la Metodología empleada.

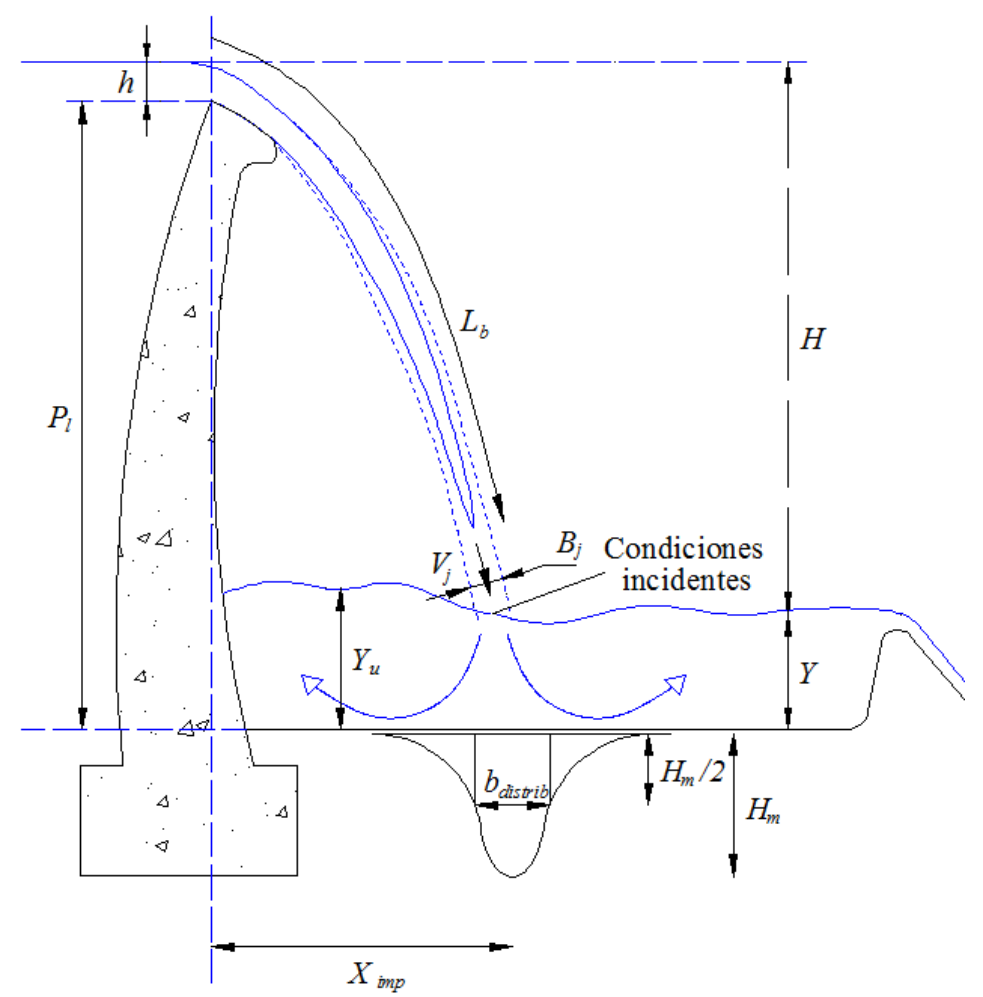

Figura 83. Esquema de parámetros relevantes en la formulación de la Metodología Paramétrica para análisis del sobrevertido de presas.

Las condiciones incidentes se definen por la velocidad media del chorro $V_{j}$, y el espesor del mismo $B_{j}$, donde:

$$
B_{j}=B_{g}+B_{s}=B_{g}+2 \xi
$$

siendo $B_{g}$ el espesor debido a condiciones gravitatorias, $B_{s}$ el espesor debido a la expansión lateral, y $\xi$ la distancia de expansión lateral debida al efecto de la aireación y turbulencia.

El tiempo $t$ para que el chorro caiga una cierta distancia se define como:

$$
t=\frac{V_{j}-V_{i}}{g}
$$


donde $V_{i}$ y $V_{j}$ son las velocidades medias en la condición inicial e incidente, respectivamente, y $g$ la aceleración de la gravedad.

Siguiendo a Ervine et al. (1997), y considerando la ecuación anterior, la expansión lateral del chorro por efectos de aireación y turbulencia puede ser obtenida como:

$$
\xi=k v^{\prime} t=k\left(\frac{v^{\prime}}{V_{i}}\right) V_{i} \frac{V_{j}-V_{i}}{g}
$$

donde $t$ es el tiempo para que el chorro caigua una determinada distancia, $v^{\prime}$ la componente turbulenta en la dirección principal del flujo, y $k$ un coeficiente de ajuste.

Considerando el parámetro turbulento $\varphi=k\left(v^{\prime} / V_{i}\right)=K_{\varphi} T_{u}$, con $K_{\varphi}$ un coeficiente del parámetro turbulento y $T_{u}$ la intensidad turbulenta, la expresión queda:

$$
\xi=K_{\varphi} T_{u} \sqrt{2 g(2 h)}\left[\frac{\sqrt{2 g H}-\sqrt{2 g(2 h)}}{g}\right]=2 K_{\varphi} T_{u} \sqrt{g h}\left[\frac{\sqrt{2 g H}-2 \sqrt{g h}}{g}\right]
$$

donde $H$ es la altura entre el nivel de agua aguas arriba del vertedero y el nivel de agua en el cuenco de disipación, y $h$ la carga de energía sobre el vertedero.

Reemplazando términos, el espesor del chorro en la condición incidente se puede obtener como:

$$
B_{j}=B_{g}+2 \xi=\frac{q_{j}}{\sqrt{2 g H}}+4 \varphi \sqrt{h}(\sqrt{2 H}-2 \sqrt{h})
$$

\subsubsection{Coeficientes de presión dinámica media}

Se puede definir el coeficiente de presión dinámica media $C_{p}$ en función de la relación colchón de agua/espesor del chorro incidente $\left(Y / B_{j}\right)$ y de la relación altura de caída/longitud de rotura $\left(H / L_{b}\right)$. De este modo, se pueden realizar diferentes ajustes a los registros de presiones instantáneos obtenidos en la solera de los cuencos de disipación de las infraestructuras empleadas por Castillo (1989), Puertas (1994) y en este estudio (recogido en Castillo et al., 2014).

Se han encontrado dos casos que muestran distinto comportamiento:

\section{Caso de colchón no efectivo $\left(Y \leq 5.5 B_{j}\right)$ :}

La desintegración de la parte compacta del chorro rectangular (zona de establecimiento del flujo) ocurre para un colchón aproximadamente de 5.5 veces el 
espesor del chorro incidente Para esta situación se pueden considerar dos comportamientos bien diferenciados (Figura 84):

- $\quad$ Si $H / L_{b}<1.00$ :

$$
C_{p}=1-0.0014 e^{5.755\left(H / L_{b}\right)}
$$

- $\quad$ Si $H / L_{b} \geq 1.00$ :

$$
C_{p}=14.643 e^{-3.244\left(H / L_{b}\right)}
$$

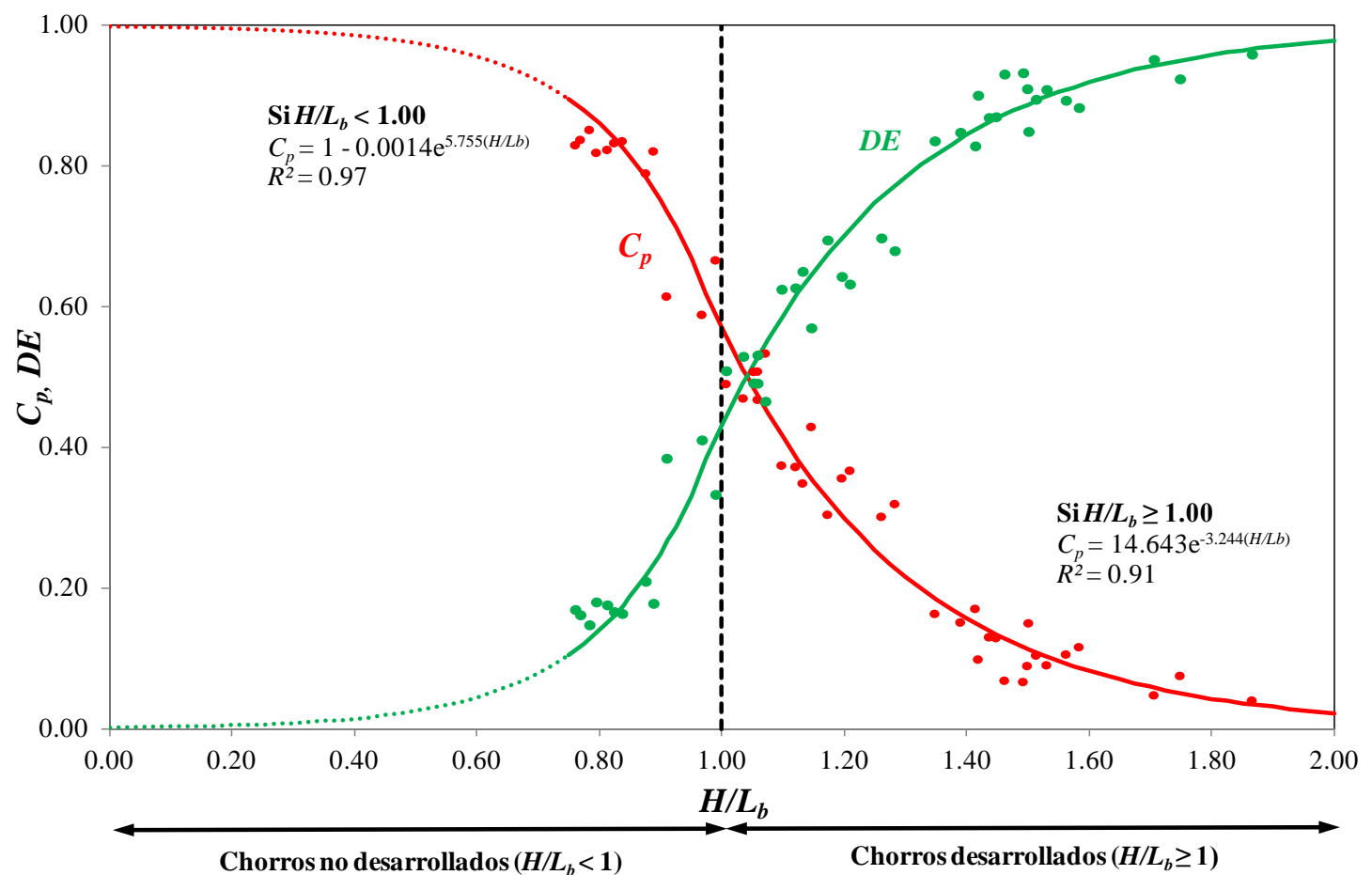

Figura 84. Coeficientes de presión dinámica media en la solera del cuenco de disipación y disipación de energía para $Y \leq 5.5 B_{j}$ (Castillo et al., 2014).

En estas situaciones, la energía disipada es debida al proceso de aireación que sufre el chorro durante su caída, y al efecto del calado de agua que se genera aguas arriba del punto de incidencia del chorro, mientras que el colchón de aguas abajo no influye en la disipación de energía. Con los ajustes exponenciales se obtienen coeficientes de correlación $R^{2}$ superiores al $90 \%$ para todo el rango $H / L_{b}$ ensayado.

La curva de energía disipada por efecto de la aireación durante la caída y del calado de recirculación aguas arriba del punto de estancamiento $D E$, puede ser obtenida como la diferencia entre la unidad y el valor correspondiente de $C_{p}$. 
En el caso de impacto directo, los resultados de laboratorio revelan que $C_{p}$ es muy sensible a la relación $H / L_{b}$ en el entorno entre 0.70 y 1.30 .

\section{Caso de colchón efectivo $\left(Y>5.5 B_{j}\right)$ :}

Se puede considerar que $C_{p}$ sólo depende de la relación $H / L_{b}$. Para esta situación, en la Figura 85 los datos se han comparado con la formulación obtenida por Cola (1965) para chorros rectangulares no aireados:

$$
C_{p}=\frac{H_{m}}{V_{j}^{2} / 2 g}=\frac{7.1822}{Y / B_{j}}
$$

donde $H_{m}$ es la presión media obtenida en la solera del cuenco, $V_{j}$ y $B_{j}$ son la velocidad media y el espesor del chorro en la condición incidente, respectivamente, e $Y$ es el calado en el cuenco.

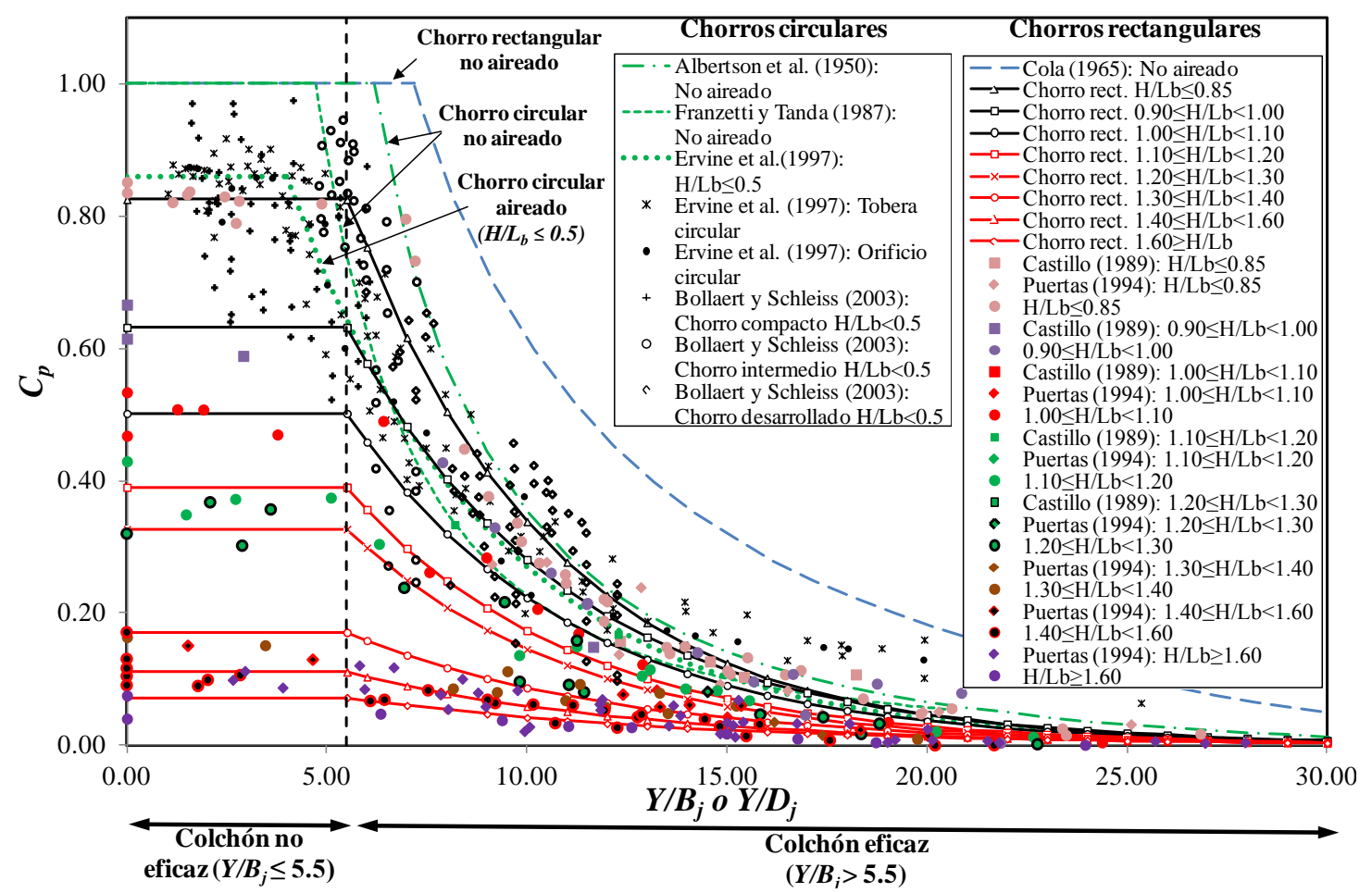

Figura 85. Coeficientes de presión dinámica media para diferentes formas de chorros y entradas de aire (Castillo et al., 2014).

Puesto que la fórmula de cola considera la presión media en vez de la presión dinámica media (a la presión media no se le ha restado el valor del colchón de agua), la 
curva se ha modificado con el fin de representar la relación $C_{p}=f\left(Y / B_{j}\right)$ sin tener en cuenta el colchón de agua.

La Figura 85 también muestra los ajustes de los chorros circulares aireados obtenidos por Ervine et al. (1997), y por Bollaert y Schleiss (2003), así como los ajustes obtenido para chorros circulares no aireados obtenidos por Albertson et al. (1950) y por Franzetty y Tanda (1987), citados por Bollaert y Schleiss (2003). De modo análogo a la operación realizada para el ajuste de Cola, la curva de Albertson et al. ha sido modificada para considerar la relación $C_{p}=f\left(Y / B_{j}\right)$ sin tener en cuenta el colchón de agua. Para relaciones $Y / B_{j}>5.5$ se observa que $C_{p}$ es función de las relaciones $Y / B_{j} \mathrm{y}$ $H / L_{b}$. La reducción de $C_{p}$ en chorros circulares es similar a la observada en chorros rectangulares de valores comparables.

Se han considerado ocho familias de curvas agrupadas en función de sus relaciones $H / L_{b}$, las cuales cumplen la relación:

$$
C_{p}=\frac{H_{m}-Y}{V_{j}^{2} / 2 g}=a e^{-b\left(Y / B_{j}\right)}
$$

Los parámetros $a$ y $b$ de la ecuación (186), y sus respectivos coeficientes de correlación $R^{2}$, se recogen en la Tabla 23.

\begin{tabular}{|c|c|c|c|}
\hline $\boldsymbol{H} / \mathbf{L}_{\boldsymbol{b}}$ & $\boldsymbol{a}$ & $\boldsymbol{b}$ & $\boldsymbol{R}^{\mathbf{2}}$ \\
\hline$\leq 0.85$ & 2.50 & 0.20 & 0.93 \\
\hline $0.90-1.00$ & 1.70 & 0.18 & 0.70 \\
\hline $1.00-1.10$ & 1.35 & 0.18 & 0.85 \\
\hline $1.10-1.20$ & 1.05 & 0.18 & 0.95 \\
\hline $1.20-1.30$ & 0.88 & 0.18 & 0.85 \\
\hline $1.30-1.40$ & 0.39 & 0.15 & 0.76 \\
\hline $1.40-1.60$ & 0.24 & 0.14 & 0.68 \\
\hline$\geq 1.60$ & 0.14 & 0.12 & 0.56 \\
\hline
\end{tabular}

Tabla 23. Parámetros del coeficiente de presión dinámica media cuando $Y>5.5 B_{j}$ (Castillo et al., 2014).

\subsubsection{Coeficientes de presión dinámica fluctuante}

La presión dinámica total es la suma de la presión dinámica media y la presión fluctuante. Se ha analizado la raíz cuadrática media de las fluctuaciones de presión considerando diferentes colchones de agua, caudales y alturas de caída. El coeficiente de presión dinámica fluctuante se define como: 


$$
C_{p}^{\prime}=\frac{H^{\prime}}{V_{j}^{2} / 2 g}
$$

donde $H^{\prime}$ es la raíz cuadrática media del registro de presiones instantáneas.

En el caso de chorros rectangulares, el mejor ajuste para cuantificar los coeficientes de presión dinámica fluctuante se han obtenido con dos tipos de ajustes en función de las relaciones $Y / B_{j}$ y $H / L_{b}$ (Figura 86 ):

- Para $Y / B_{j} \leq 14$, ajuste polinómico:

$$
C_{p}^{\prime}=a\left(\frac{Y}{B_{j}}\right)^{3}+b\left(\frac{Y}{B_{j}}\right)^{2}+c\left(\frac{Y}{B_{j}}\right)+d
$$

- $\quad$ Para $Y / B_{j}>14$, ajuste potencial:

$$
C_{p}^{\prime}=a e^{-b\left(Y / B_{j}\right)}
$$

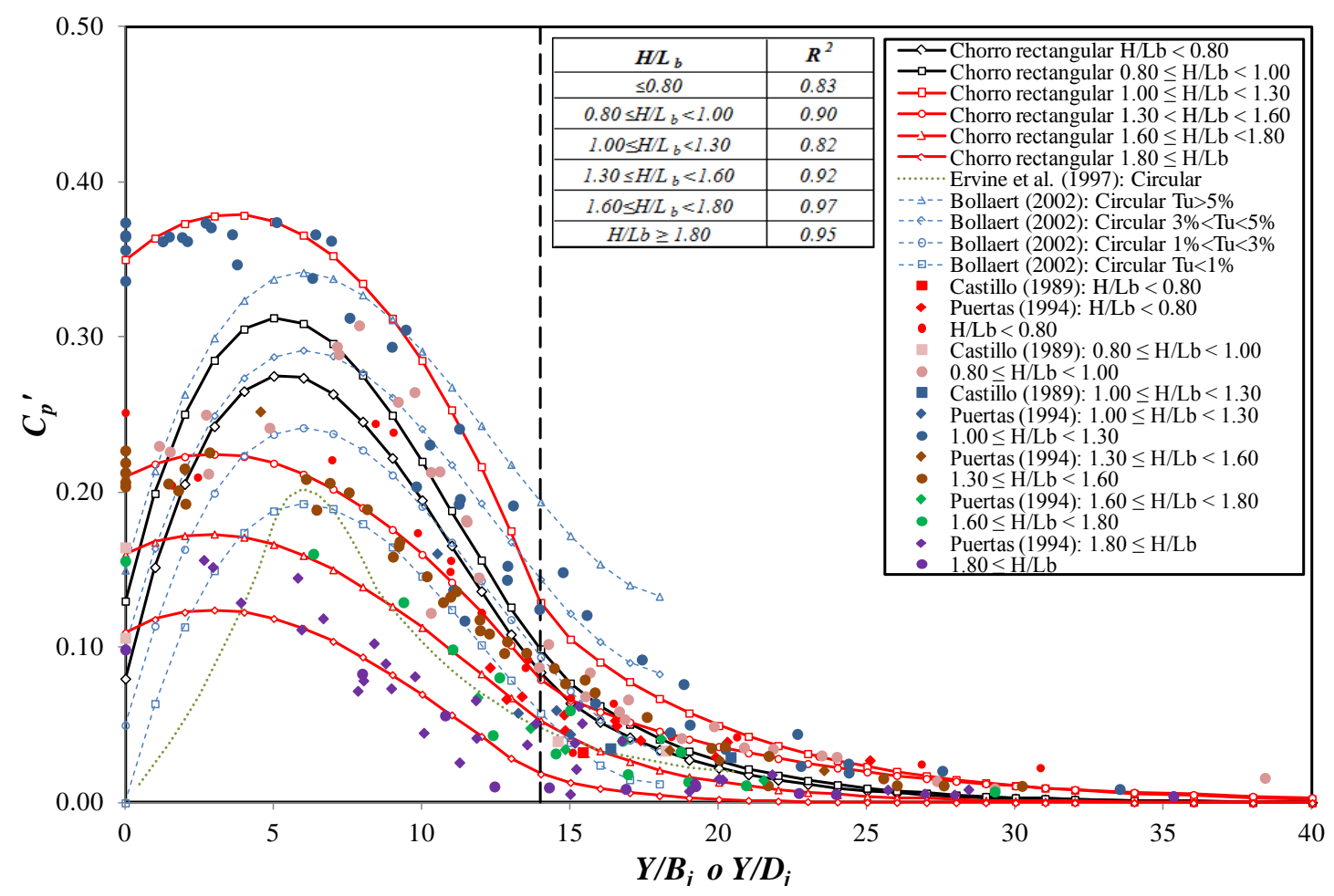

Figura 86. Variación del coeficiente de presión dinámica fluctuante con la relación colchón de agua/espesor del chorro (Castillo et al., 2014).

Los parámetros de las ecuaciones (188) y (189) se recogen en la Tabla 24 para los diferentes valores de $H / L_{b}$. 


\begin{tabular}{|c|c|c|c|c|c|c|}
\cline { 2 - 7 } \multicolumn{1}{c|}{} & \multicolumn{4}{c|}{$\boldsymbol{0} \leq \boldsymbol{Y} / \boldsymbol{B}_{\boldsymbol{j}} \leq \mathbf{1 4}$} & \multicolumn{2}{c|}{$\boldsymbol{Y}_{\boldsymbol{j}}>\mathbf{1 4}$} \\
\hline $\boldsymbol{H} / \mathbf{L}_{\boldsymbol{b}}$ & $\boldsymbol{a}$ & $\boldsymbol{b}$ & $\boldsymbol{c}$ & $\boldsymbol{d}$ & $\boldsymbol{a}$ & $\boldsymbol{b}$ \\
\hline$\leq 0.80$ & 0.00030 & -0.01000 & 0.0815 & 0.080 & 1.500 & 0.210 \\
\hline $0.80-1.00$ & 0.00030 & -0.01000 & 0.0790 & 0.130 & 1.800 & 0.210 \\
\hline $1.00-1.30$ & -0.000005 & -0.00220 & 0.0160 & 0.350 & 1.000 & 0.150 \\
\hline $1.30-1.60$ & 0.00003 & -0.00180 & 0.0100 & 0.210 & 0.400 & 0.120 \\
\hline $1.60-1.80$ & 0.00005 & -0.00195 & 0.0098 & 0.160 & 1.330 & 0.230 \\
\hline$\geq 1.80$ & 0.00005 & -0.00190 & 0.0100 & 0.110 & 2.500 & 0.350 \\
\hline
\end{tabular}

Tabla 24. Parámetros para el cálculo del coeficiente de presión dinámica fluctuante (Castillo et al., 2014).

Debido al efecto de la rotura del chorro, $C_{p}{ }^{\prime}$ tiende a incrementarse desde $H / L_{b} \leq$ 0.80 hasta obtener un máximo en el rango $1.00 \leq H / L_{b}<1.30$. El coeficiente de presión dinámica fluctuante tiende a reducirse para relaciones $H / L_{b}>1.30$ debido a que la influencia del chorro incidente se extiende sobre una superficie más amplia en la solera del cuenco.

Bollaert (2002) y Bollaert y Schleiss (2003) obtuvieron los valores de $C_{p}{ }^{\prime}$ para chorros circulares. Debido a que las velocidades del chorro en los ensayos fueron superiores a $20 \mathrm{~m} / \mathrm{s}$, los autores consideraron que sus estudios están exentos de efectos de escala, representando situaciones de prototipo.

La Tabla 25 resume los ajustes obtenidos para tanto chorros rectangulares como circulares. Aunque las velocidades de ensayo de los chorros rectangulares fueron menores de $10 \mathrm{~m} / \mathrm{s}$, los valores concuerdan con los resultados obtenidos por Bollaert (2002).

\begin{tabular}{|c|c|c|}
\hline \multicolumn{3}{|c|}{ Chorro rectangular } \\
\hline $\boldsymbol{H} / \boldsymbol{L}_{\boldsymbol{b}}$ & $\boldsymbol{Y}_{\boldsymbol{B}} \boldsymbol{B}_{\boldsymbol{j}}$ & $\boldsymbol{C}_{\boldsymbol{p}}{ }^{\prime}{ }_{\max }$ \\
\hline$\leq 0.80$ & 5 & 0.275 \\
\hline $0.80-1.00$ & 5 & 0.313 \\
\hline $1.00-1.30$ & 4 & 0.378 \\
\hline $1.30-1.60$ & 3 & 0.225 \\
\hline $1.60-1.80$ & 3 & 0.173 \\
\hline$\geq 1.80$ & 3 & 0.124 \\
\hline
\end{tabular}

\begin{tabular}{|c|c|c|c|}
\hline \multicolumn{4}{|c|}{ Chorro circular (Bollaert, 2002) } \\
\hline$H / L_{b}$ & $T_{u}$ & $Y / D_{j}$ & $C_{p}^{\prime} \max$ \\
\hline$<0.50$ & $<1 \%$ & 6 & 0.193 \\
\hline$<0.50$ & $1-3 \%$ & 6 & 0.242 \\
\hline$<0.50$ & $3-5 \%$ & 6 & 0.296 \\
\hline$<0.50$ & $>5 \%$ & 6 & 0.342 \\
\hline
\end{tabular}

Tabla 25. Variación del valor máximo del coeficiente de presión dinámica fluctuante para diferentes formas de chorro en función de la relación colchón de agua/espesor del chorro (Castillo et al., 2014).

Las curvas de ajuste de Bollaert muestran un valor máximo cuando $Y / D_{j} \approx 6$, incrementándose conforme la intensidad turbulenta aumenta (Figura 86). El valor promedio propuesto por Ervine et al. (1997) obtiene igualmente el máximo cuando $Y / D_{j}$ 
$\approx 6$, con un valor pico similar a los resultados de Bollaert con la menor turbulencia inicial. En el caso de chorros rectangulares, los valores máximos se obtienen para $Y / B_{j} \approx$ 5 cuando $H / L_{b}<1.00$ (chorros no desarrollados) y para $Y / B_{j} \approx 3$ cuando $H / L_{b}>1.30$ (chorros completamente desarrollados).

El coeficiente de presión dinámica fluctuante se ve influenciado por la relación entre la altura de caída y la longitud de rotura del mismo (Figura 87). Los resultados de Ervine et al. (1997) complementan los datos de este estudio.

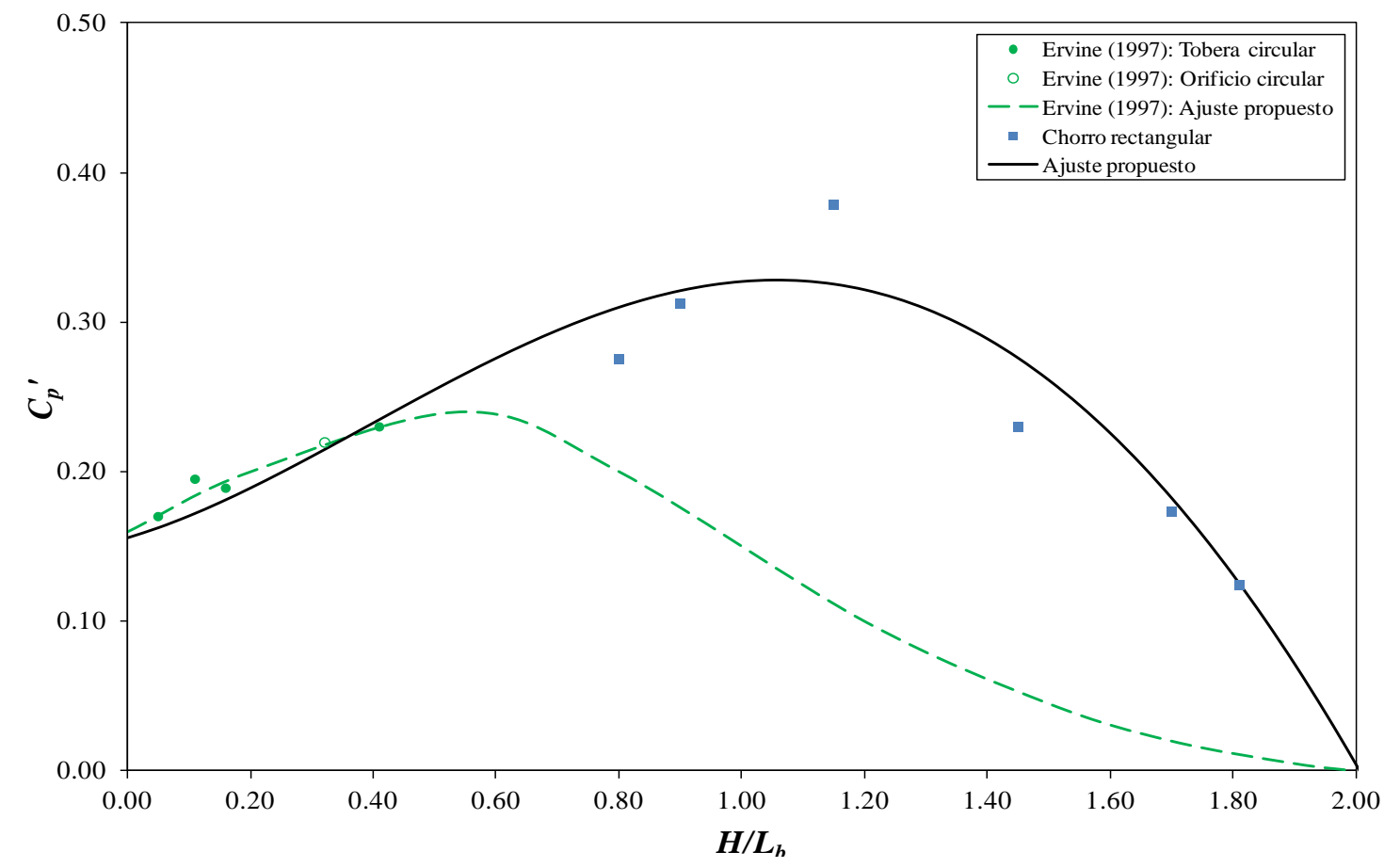

Figura 87. Variación del coeficiente de presión dinámica fluctuante con la longitud de rotura del chorro (Castillo et al., 2014).

El valor máximo del coeficiente de presión dinámica fluctuante se obtiene para una relación $H / L_{b}$ en torno a 1.00-1.20. Para chorros más cortos, el grado de turbulencia en el chorro es pequeño, por lo que $C_{p}{ }^{\prime}$ debería ser reducido. Alrededor de la longitud de rotura el grado de turbulencia del chorro aumenta, incrementándose el coeficiente de presión fluctuante. Cuando el chorro está complemente desarrollado, su influencia se extiende sobre una superficie más amplia, reduciéndose la magnitud de las fluctuaciones. Para alturas de caída con relaciones $H / L_{b}>2.0$, el coeficiente de presión dinámica fluctuante tiende a ser despreciable.

Con los datos existentes, se ha obtenido una curva de ajuste. La ecuación de la curva, con un coeficiente de correlación $R^{2}=0.94$, es: 


$$
C_{p}^{\prime}=0.0392\left(\frac{H}{L_{b}}\right)^{4}-0.2662\left(\frac{H}{L_{b}}\right)^{3}+0.2766\left(\frac{H}{L_{b}}\right)^{2}+0.1219\left(\frac{H}{L_{b}}\right)+0.1561
$$

\subsubsection{Coeficientes de presión dinámica extrema}

La aplicabilidad de las mediciones de valores extremos a escala de prototipo resulta cuestionable. La magnitud de la mediciones resulta altamente dependiente de las distribuciones de los valores extremos, que a su vez dependen de las características turbulentas existentes en el cuenco de disipación y de la longitud temporal de las observaciones. Lopardo (1988) recomienda el valor de corte límite con el percentil 1\% como una solución práctica para la fase de diseño.

A partir de las siguientes expresiones se han analizado las fluctuaciones máximas y mínimas definiéndolas en términos de coeficientes de presión:

$$
\begin{gathered}
C_{p}^{+}=\frac{p_{\max }-H_{m}}{V_{j}^{2} / 2 g} \\
C_{p}^{-}=\frac{H_{m}-p_{\min }}{V_{j}^{2} / 2 g}
\end{gathered}
$$

donde $p_{\max } \mathrm{y} p_{\min }$ son respectivamente los valores instantáneos máximos y mínimos del registro de presiones, considerando diferentes percentiles de corte, mientras que $H_{m}$ es la presión media obtenida en la solera del cuenco de disipación.

La Figura 88 muestra los valores del coeficiente de fluctuación máxima, mientras que la Figura 89 recopila los coeficiente de fluctuación mínima, donde se han considerado las parejas de percentiles 1-99\% y 5-95\%.

Los valores obtenidos no han permitido realizar un estudio en función de agrupamientos. Comparando los valores de la Figura 88 con la Figura 86 se observa que los valores máximos del coeficiente de presiones dinámicas máximas son en torno a dos-tres veces mayores que sus correspondientes RMS, mientras que los valores máximos del coeficiente de presiones mínimas están en torno al doble de dichos valores.

El pico de presiones positivas ocurre para relaciones $Y / B_{j} \approx 5-10$, mientras que las presiones máximas negativas se obtienen para relaciones $Y / B_{j}<5-7$ (colchón no efectivo). Los valores de $C_{p}{ }^{+}$y $C_{p}{ }^{-}$concuerdan con los resultados previamente publicados en chorros circulares (Ervine et al., 1997; Bollaert y Schleiss, 2003). 


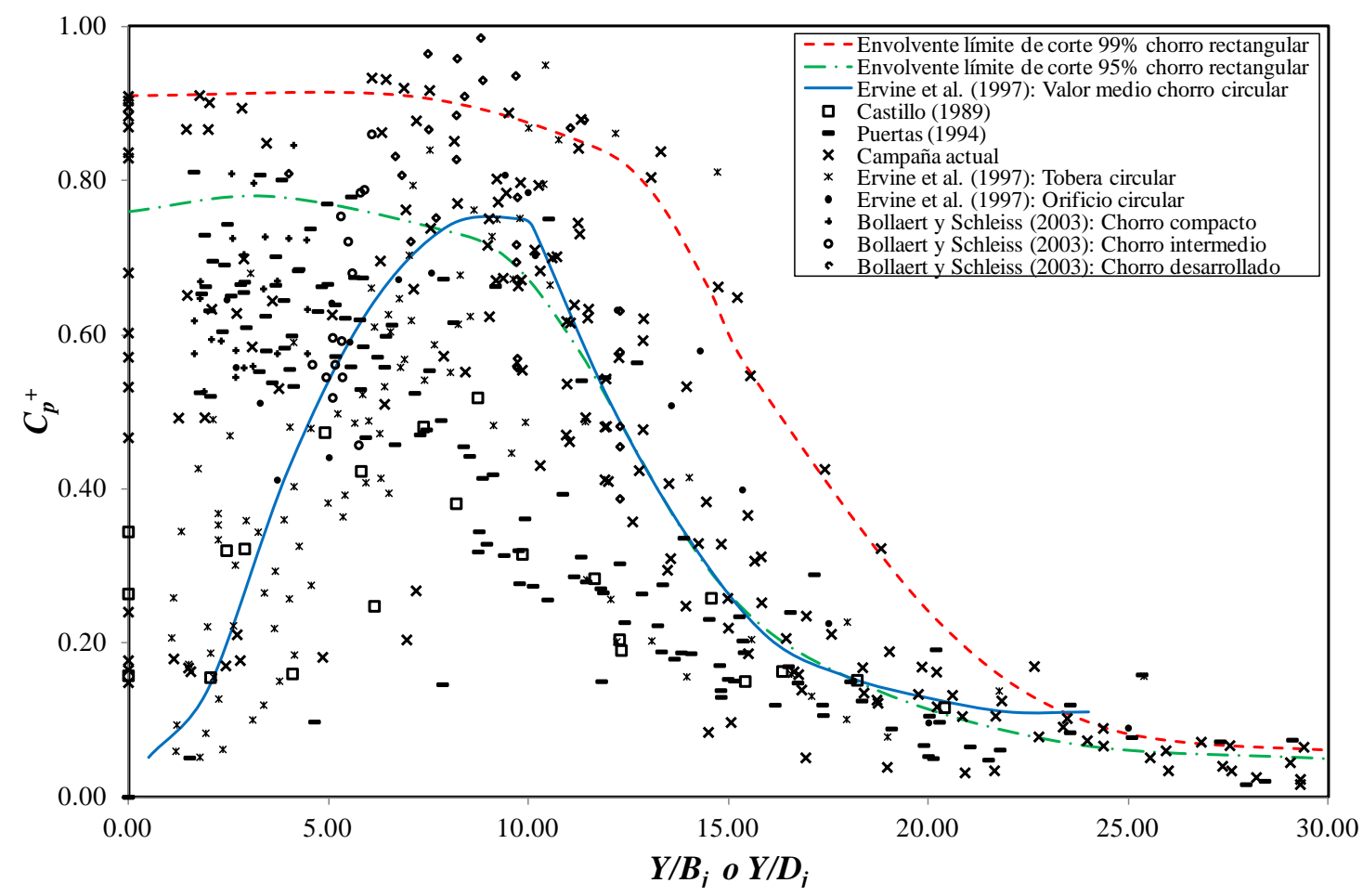

Figura 88. Variación del coeficiente de presión dinámica fluctuante máximo con el colchón de agua (Castillo et al., 2014).

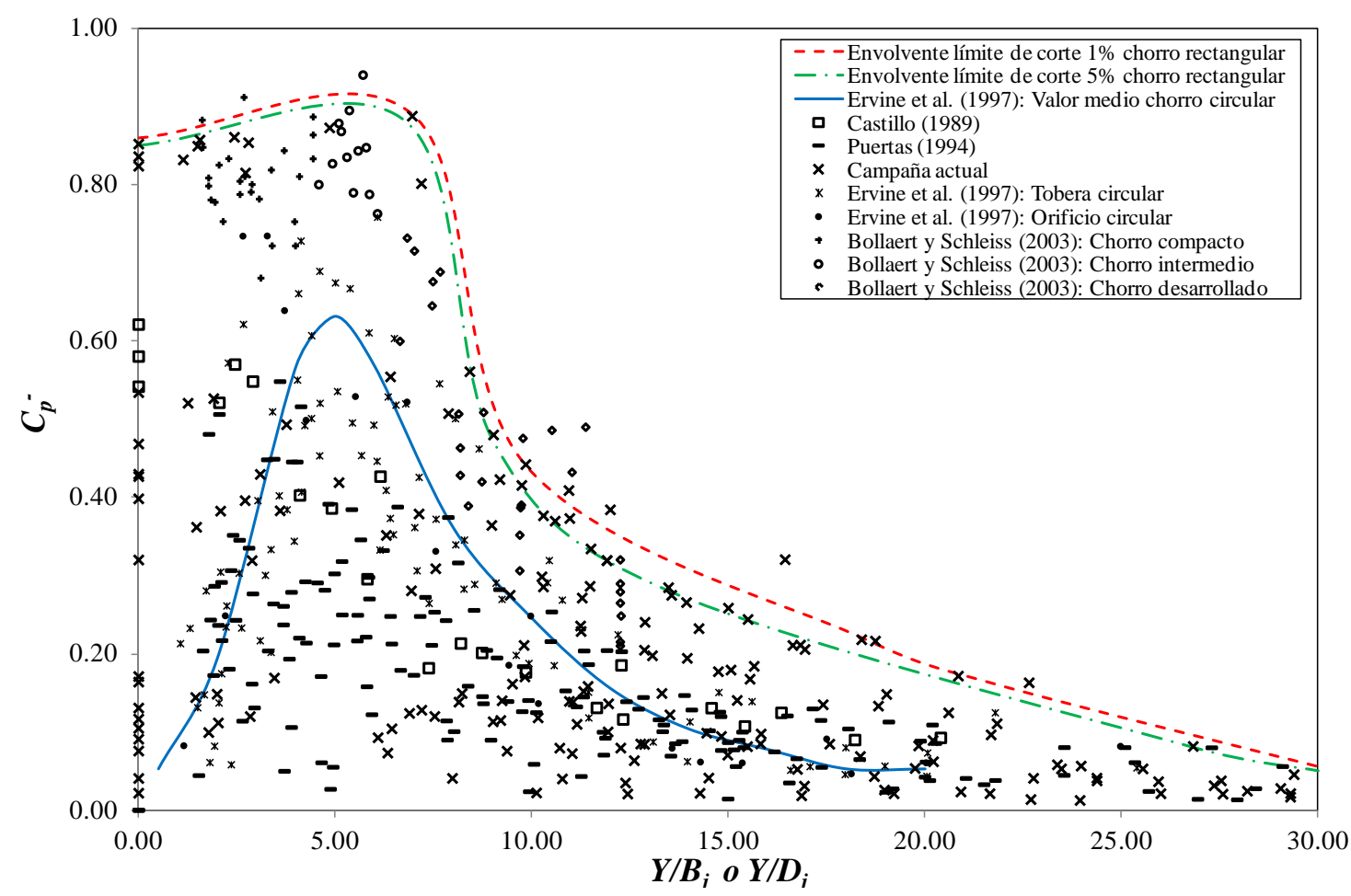

Figura 89. Variación del coeficiente de presión dinámica fluctuante mínimo con el colchón de agua (Castillo et al., 2014). 
Muchos de los valores en chorros rectangulares están por debajo de los resultados de chorros circulares. Esto puede ser debido al ensayo de chorros mucho más aireados (en chorros rectangulares $H / L_{b}>0.70$, mientras que en chorros circulares $H / L_{b}<0.50$ ).

\subsubsection{Distribución de presiones alrededor del punto de estancamiento}

Para el estudio de la distribución de presiones en el entorno del punto de estancamiento, Castillo (1989) analizó los resultados obtenidos por diversos autores. Se define $b_{\text {distrib }}$ como el ancho de la región de influencia de la presión (distancia entre los puntos situados aguas arriba y aguas abajo del punto de estancamiento donde la presión media es $p=H_{m} / 2$ ), mientras que $y_{k}$ es el calado necesario para que se desintegre el núcleo central del chorro en el colchón de agua.

Con el fin de determinar la distribución de presiones, se ha considerado la siguiente expresión:

$$
\frac{p}{H_{m}}=e^{-\phi\left(\frac{x}{b_{\text {distrib }}}\right)}
$$

donde $p$ es la presión dinámica media en la solera del cuenco, $H_{m}$ la presión dinámica media en el punto de estancamiento, $x$ la distancia horizontal desde el punto de estancamiento al punto considerado, e $Y$ el colchón de agua. El parámetro $\phi$ puede ser obtenido de la Tabla 26.

\begin{tabular}{|c|c|c|c|c|}
\hline Autor & $\phi$ & $b_{\text {distrib }}(m)$ & $y_{k}(m)$ & $H / L_{b}$ \\
\hline Cola (1966) & 40.51 & $0.13 Y$ & - & - \\
\hline \multirow{2}{*}{ Aki (1969) } & 12.56 & $0.13 Y$ & - & - \\
\hline & 12.56 & $0.23 Y$ & - & - \\
\hline \multirow{2}{*}{ Hartung y Häusler (1973) } & 19.60 & $0.13 Y$ & $5 B_{j}$ & - \\
\hline & 40.71 & $0.13 Y$ & $7.2 B_{j}$ & - \\
\hline Beltaos (1976) & 42.01 & $0.13 Y$ & - & - \\
\hline \multirow{4}{*}{ Presente estudio } & 10.00 & $0.13 Y$ & $5.5 B_{j}$ & $\leq 1$ (aguas abajo del punto de estancamiento) \\
\hline & 7.00 & $0.13 Y$ & $5.5 B_{j}$ & $>1$ (aguas abajo del punto de estancamiento) \\
\hline & 4.00 & $0.13 Y$ & $5.5 B_{j}$ & $\leq 1$ (aguas arriba del punto de estancamiento) \\
\hline & 3.00 & $0.13 Y$ & $5.5 B_{j}$ & $>1$ (aguas arriba del punto de estancamiento) \\
\hline
\end{tabular}

Tabla 26. Parámetros de la distribución de la presión dinámica media alrededor del punto de estancamiento (Castillo et al., 2014).

Se ha obtenido un adecuado ajuste entre las distintas formulaciones y los datos obtenidos por Castillo (1989), Puertas (1994) y en el presente estudio (Figura 90). 


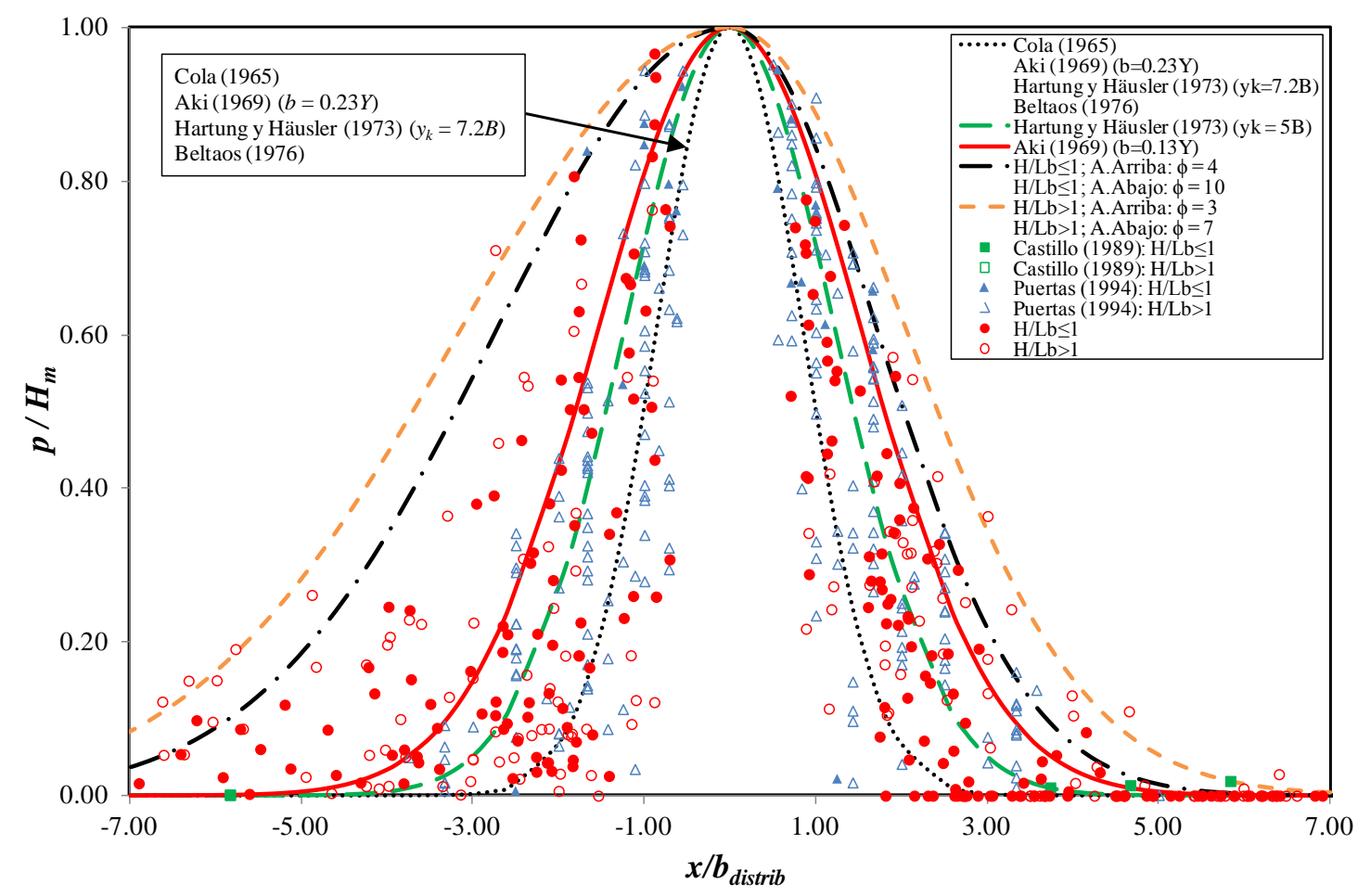

Figura 90. Distribución de presiones dinámicas medias alrededor del punto de estancamiento en función del parámetro $b_{\text {distrib }}$ (Castillo et al., 2014).

Los chorros desarrollados $\left(H / L_{b}>1\right)$ obtienen una región de distribución mayor que los chorros no desarrollados $\left(H / L_{b} \leq 1\right)$. El comportamiento del flujo es no simétrico debido a que el ángulo de incidencia del chorro es diferente de $90^{\circ} \mathrm{y}$ a la presencia de un contorno sólido aguas arriba del punto de incidencia (el cuenco de disipación vierte el agua por el extremo de aguas abajo).

De acuerdo a los resultados, la fórmula de Aki con $b_{\text {distib }}=0.13 Y$ obtiene unos valores superiores a los valores propuestos por los otros autores. Puesto que sus estudios se realizaron con chorros aireados, la envolvente de Aki está próxima a la envolvente de los datos de laboratorio para la situación de flujo simétrico. Las expresiones de Cola, Aki (considerando $b_{\text {distib }}=0.23 Y$ ), Hartung y Häusler (cuando $y_{k}=7.2 B_{j}$ ) y Beltaos están obtenidas con chorros no aireados. Sus resultados concuerdan con la envolvente inferior de los datos obtenidos en laboratorio (correspondiente a los datos con menor aireación). La fórmula de Hartung y Häusler (cuando $y_{k}=5 B_{j}$ ) obtiene resultados intermedios entre las propuestas por los otros autores.

Considerando la misma expresión general, se han obtenido diferentes curvas envolventes aguas arriba y aguas abajo del punto de estancamiento (Tabla 26). Cuando $H / L_{b} \leq 1$, se obtiene un parámetro $\phi=10$ aguas abajo del punto de estancamiento, y $\phi=$ 4 aguas arriba. Para $H / L_{b}>1$, los chorros están muy aireados y la región de influencia es mayor, obteniéndose $\phi=7$ aguas abajo y $\phi=3$ aguas arriba. 
Ervine et al. (1997) consideraron que la variación del coeficiente de presión dinámica media en la dirección longitudinal $C_{p}(x)$ puede ser obtenida con la siguiente expresión general:

$$
\frac{C_{p}(x)}{C_{p}}=e^{-K_{2}\left(\frac{x}{Y}\right)^{2}}
$$

donde $x$ es la distancia horizontal al punto de estancamiento e $Y$ el colchón de agua en el cuenco.

La distribución de Ervine et al. (válida para $H / L_{b} \leq 0.5$ ) concuerda con los resultados obtenidos en laboratorio con chorros rectangulares poco aireados (Figura 91).

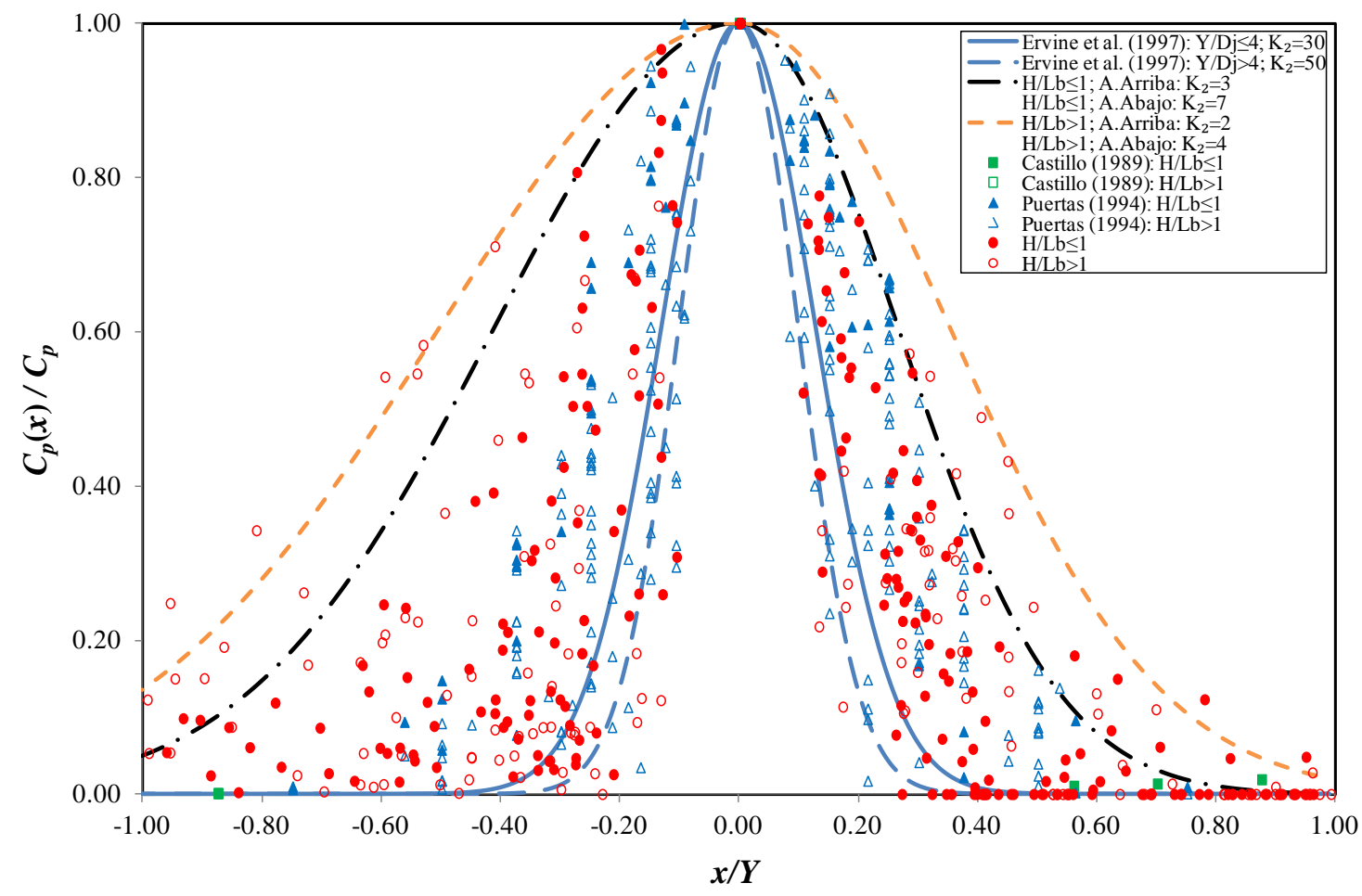

Figura 91. Distribución de presiones dinámicas medias alrededor del punto de estancamiento en función de la relación $x / Y$.

Empleando la misma fórmula general, se han obtenido diferentes envolventes superiores. Cuando $H / L_{b} \leq 1$, se obtiene un parámetro $K_{2}=7$ aguas abajo del punto de estancamiento, y $K_{2}=3$ aguas arriba. Para $H / L_{b}>1$, los chorros están muy aireados y la región de influencia es mayor, obteniéndose $K_{2}=4$ aguas abajo y $K_{2}=2$ aguas arriba.

De un modo similar, Bollaert (2002) consideró que la distribución longitudinal del coeficiente de presión dinámica media $C_{p}(x)$ puede ser obtenida en función de la relación adimensional $x / x_{\max }$ : 


$$
\frac{C_{p}(x)}{C_{p}}=e^{-K_{3}\left(\frac{x}{x_{\max }}\right)^{2}}
$$

donde $x_{\max }$ es la extensión longitudinal máxima del área influenciada por la capa de corte turbulenta del chorro incidente.

Considerando la expresión propuesta por Bollaert, en la Figura 92 se han obtenido las envolvente correspondientes al caso de chorros rectangulares.

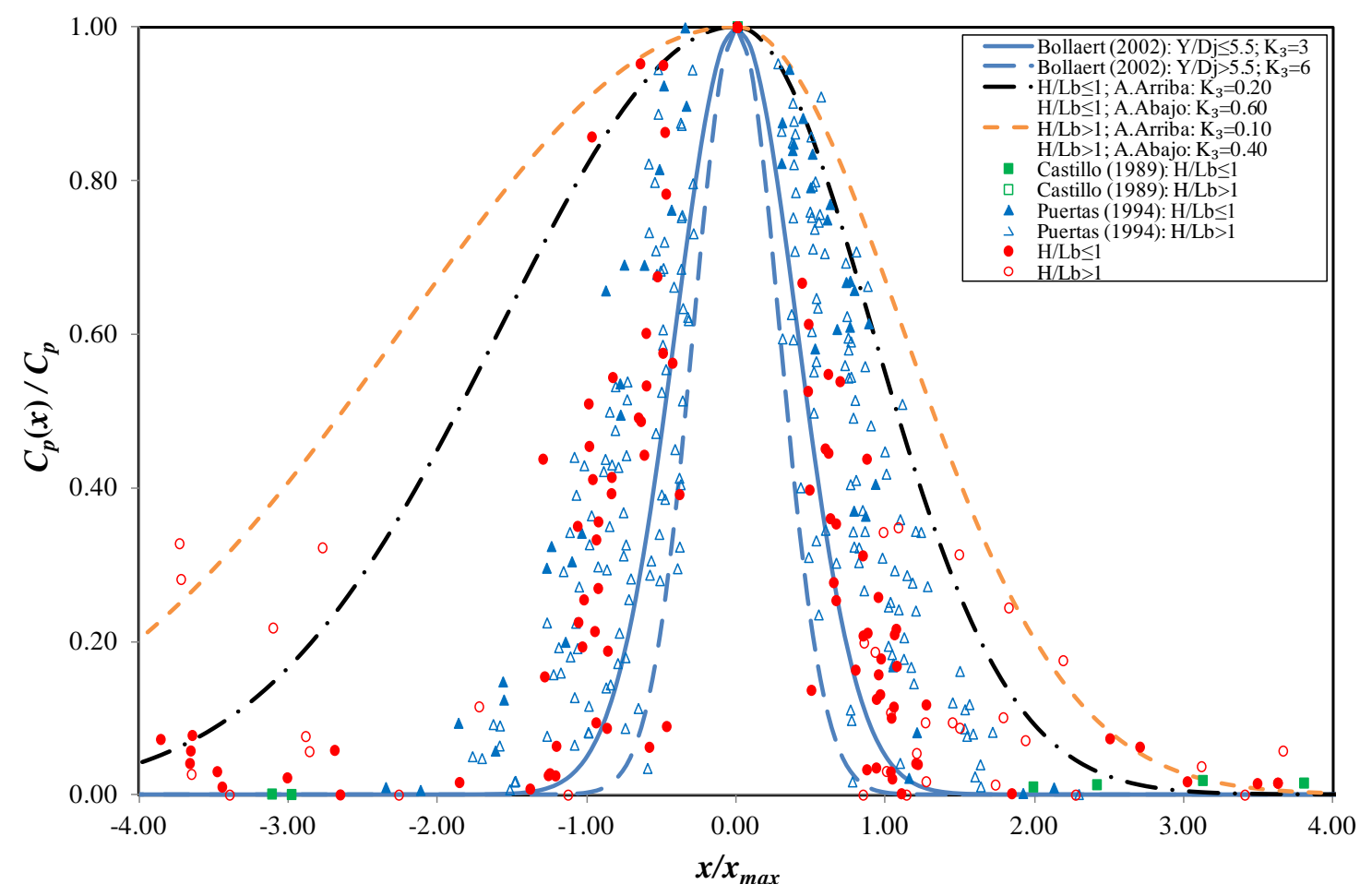

Figura 92. Distribución de presiones dinámicas medias alrededor del punto de estancamiento en función de la relación $x / x_{\max }$ (Castillo et al., 2014).

Como en el caso anterior, las distribuciones de Bollaert (válidas para $H / L_{b}<0.5$ ) concuerdan con los resultados de chorros rectangulares obtenidos cuando el chorro está poco aireado. Cuando $H / L_{b} \leq 1$, se obtiene un parámetro $K_{3}=0.6$ aguas abajo del punto de estancamiento, y $K_{3}=0.2$ aguas arriba. Para $H / L_{b}>1$, los chorros están muy aireados y la región de influencia es mayor, obteniéndose $K_{3}=0.4$ aguas abajo y $K_{3}=0.1$ aguas arriba.

Bollaert también obtuvo los coeficientes de distribución de presiones fluctuantes en torno al punto de estancamiento en chorros circulares. Al igual que en las distribuciones de presiones dinámicas medias, el comportamiento es no simétrico (Figura 93). 


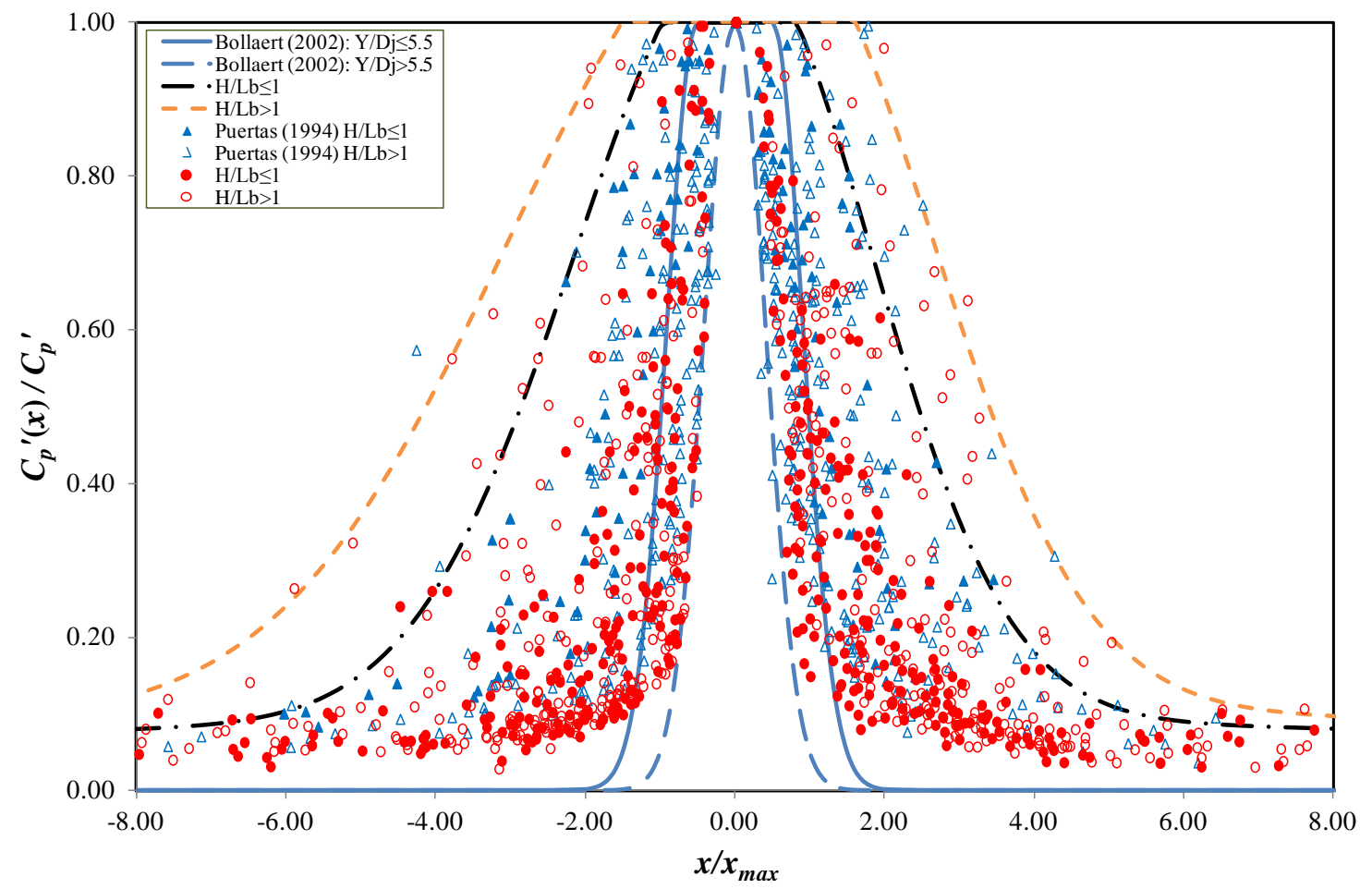

Figura 93. Distribución de presiones dinámicas fluctuantes alrededor del punto de estancamiento en función de la relación $x / x_{\max }$.

En la Tabla 27 se recogen las ecuaciones de ajuste empleadas como envolventes en la Figura 93 para chorros rectangulares y circulares.

\begin{tabular}{|c|c|c|}
\hline Tipo de chorro & \multicolumn{2}{|r|}{ Ajuste propuesto } \\
\hline $\begin{array}{l}\text { Circular desarrollado } \\
\text { (Bollaert, 2002) }\end{array}$ & \multicolumn{2}{|r|}{$\frac{C_{p}^{\prime}(x)}{C_{p}^{\prime}}=e^{-3\left(\frac{x}{x_{\max }}\right)^{2}}$} \\
\hline $\begin{array}{l}\text { Circular compacto } \\
\text { (Bollaert, 2002) }\end{array}$ & \multicolumn{2}{|r|}{$\frac{C_{p}^{\prime}(x)}{C_{p}^{\prime}}=\operatorname{mínimo}\left\{\begin{array}{c}1 \\
e^{-3\left(\frac{x}{x_{\max }}-0.5\right)^{2}}\end{array}\right.$} \\
\hline \multirow[t]{2}{*}{$\begin{array}{l}\text { Rectangular } \\
H / L_{b} \leq 1\end{array}$} & $\begin{array}{l}\text { Aguas } \\
\text { abajo }\end{array}$ & $\frac{C_{p}^{\prime}(x)}{C_{p}^{\prime}}=$ mínimo $\left\{\begin{array}{c}1 \\
e^{-0.15\left(\frac{x}{x_{\max }}\right)^{2}+0.10\left(1-\frac{x}{40 x_{\max }}\right)}\end{array}\right.$ \\
\hline & $\begin{array}{r}\text { Aguas } \\
\text { arriba }\end{array}$ & $\frac{C_{p}^{\prime}(x)}{C_{p}^{\prime}}=$ mínimo $\left\{\begin{array}{c}1 \\
e^{-0.11\left(\frac{x}{x_{\max }}\right)^{2}+0.10\left(1-\frac{x}{40 x_{\max }}\right)}\end{array}\right.$ \\
\hline \multirow[t]{2}{*}{$\begin{array}{l}\text { Rectangular } \\
H / L_{b}>1\end{array}$} & $\begin{array}{l}\text { Aguas } \\
\text { abajo }\end{array}$ & $\frac{C_{p}^{\prime}(x)}{C_{p}^{\prime}}=\operatorname{mínimo}\left\{\begin{array}{c}1 \\
e^{-0.12\left(\frac{x}{x_{\max }}-0.6\right)^{2}+0.12\left(1-\frac{x}{40 x_{\max }}\right)}\end{array}\right.$ \\
\hline & $\begin{array}{l}\text { Aguas } \\
\text { arriba }\end{array}$ & $\frac{C_{p}^{\prime}(x)}{C_{p}^{\prime}}=\operatorname{mínimo}\left\{\begin{array}{c}1 \\
e^{-0.55\left(\frac{x}{x_{\max }}\right)^{2}+0.12\left(1-\frac{x}{40 x_{\max }}\right)}\end{array}\right.$ \\
\hline
\end{tabular}

Tabla 27. Ajustes para el cálculo de la presión dinámica fluctuante alrededor del punto de estancamiento. 


\subsubsection{Colchón aguas arriba del punto de estancamiento}

Para el caso de chorros rectangulares, se puede calcular el colchón aguas arriba del punto de estancamiento $Y_{u}$ empleando la siguiente relación deducida de la ecuación de cantidad de movimiento:

$$
\frac{Y_{u}}{Y}=\sqrt{1-2 F_{D}\left[\left(V_{j} / V_{D}\right) \beta \cos \theta-1\right]}
$$

donde $Y$ es el colchón de agua efectivo en el cuenco de disipación, $F_{D}=V_{D}{ }^{2} /(g Y)$ es el cuadrado del número de Froude, $V_{D}$ es la velocidad del flujo en el colchón de aguas abajo, y $\theta$ es el ángulo de incidencia del chorro.

Considerando la distancia vertical desde el labio del vertedero hasta la solera del cuenco de disipación $P_{l}$, la Figura 94 muestra los resultados de laboratorio del coeficiente $\beta$ para la relación $Y / P_{l}$.

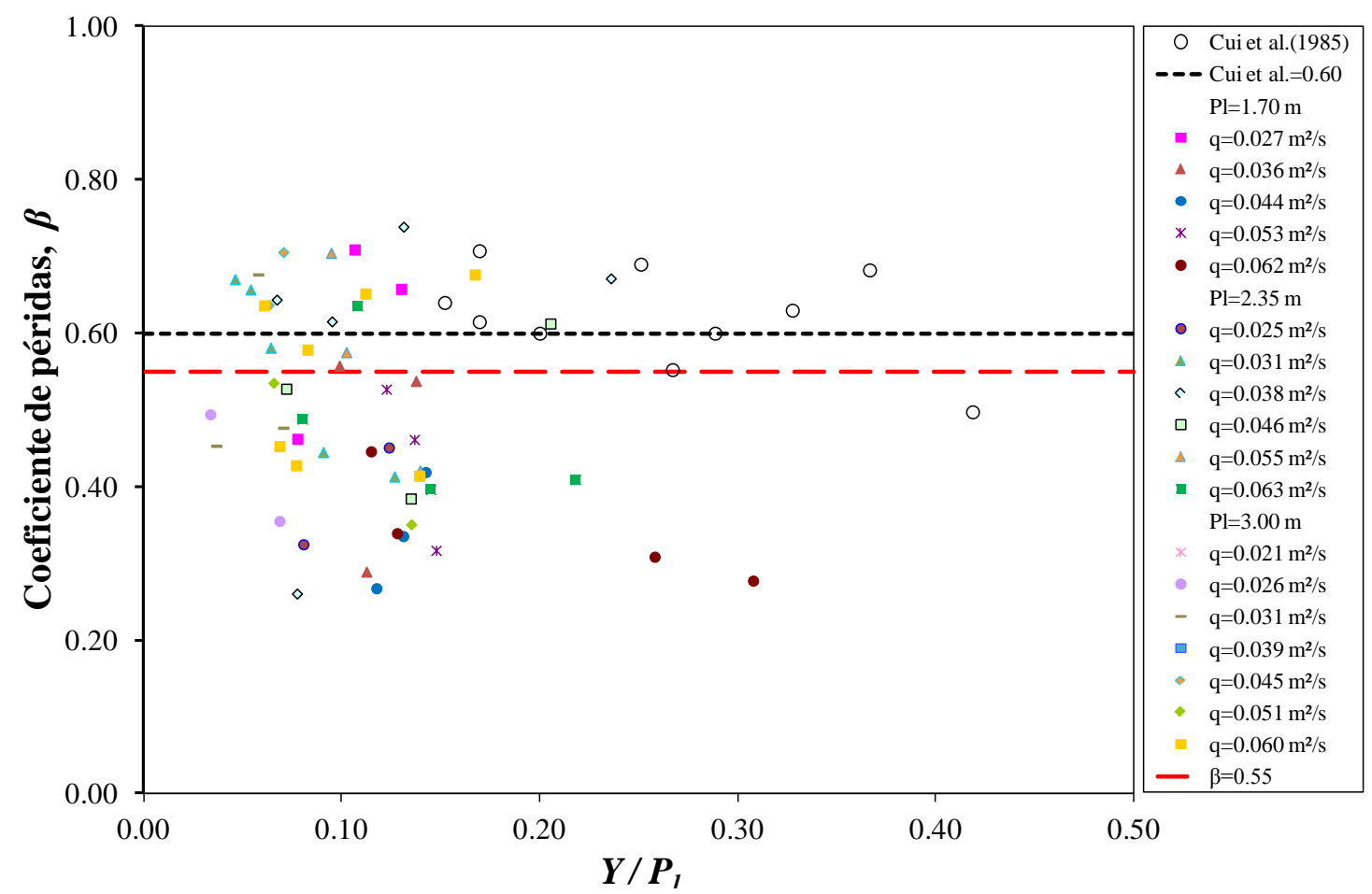

Figura 94. Coeficiente de pérdida de energía $\beta$ en función de la relación $Y / P_{l}$ (Castillo et al., 2014).

El coeficiente de pérdidas es aproximadamente 0.6 para el caso de chorro bidimensional (Cui Guang Tao et al., 1985) y 0.55 para el caso tridimensional (Castillo, 1989; revisado en Castillo y Carrillo, 2011). 


\subsubsection{Velocidades en el cuenco de amortiguación}

Para analizar los perfiles de velocidades aguas abajo del punto de incidencia del chorro se empleó un equipo Doppler. El cuenco de disipación se ha dividido en secciones transversales espaciadas cada $10 \mathrm{~cm}$, tomando como sección de referencia la zona del punto de estancamiento del chorro.

En la Figura 95 se han graficado las distribuciones de velocidad obtenidas en laboratorio, junto con las formulaciones propuestas por diversos autores. También se ha indicado el funcionamiento del cuenco frente a la forma clásica de resaltos sumergidos bajo compuerta. Los datos se ajustan a las distribuciones de los autores, aunque se observa una mayor dispersión cuando la relación $V_{x} / V_{\text {máx }}<0.60$. Esto se debe a que el chorro de entrada al colchón es casi vertical, cuando en resaltos sumergidos el chorro es horizontal. Se observa que los casos de mayor dispersión se deben a colchones con relaciones $Y / B_{j}>20$. Con estos datos se ha propuesto un nuevo ajuste:

$$
\frac{V}{V_{\text {máx }}}=1.48\left(\frac{y}{\delta_{l}}\right)^{1 / 7}\left(1-\operatorname{erf}\left(0.66 \frac{y}{\delta_{l}}\right)\right)
$$

donde $\delta_{l}$ es la longitud característica de la distribución de velocidades en el resalto hidráulico (calado $y$ donde $V_{x}=V_{\text {máx }} / 2$ ).

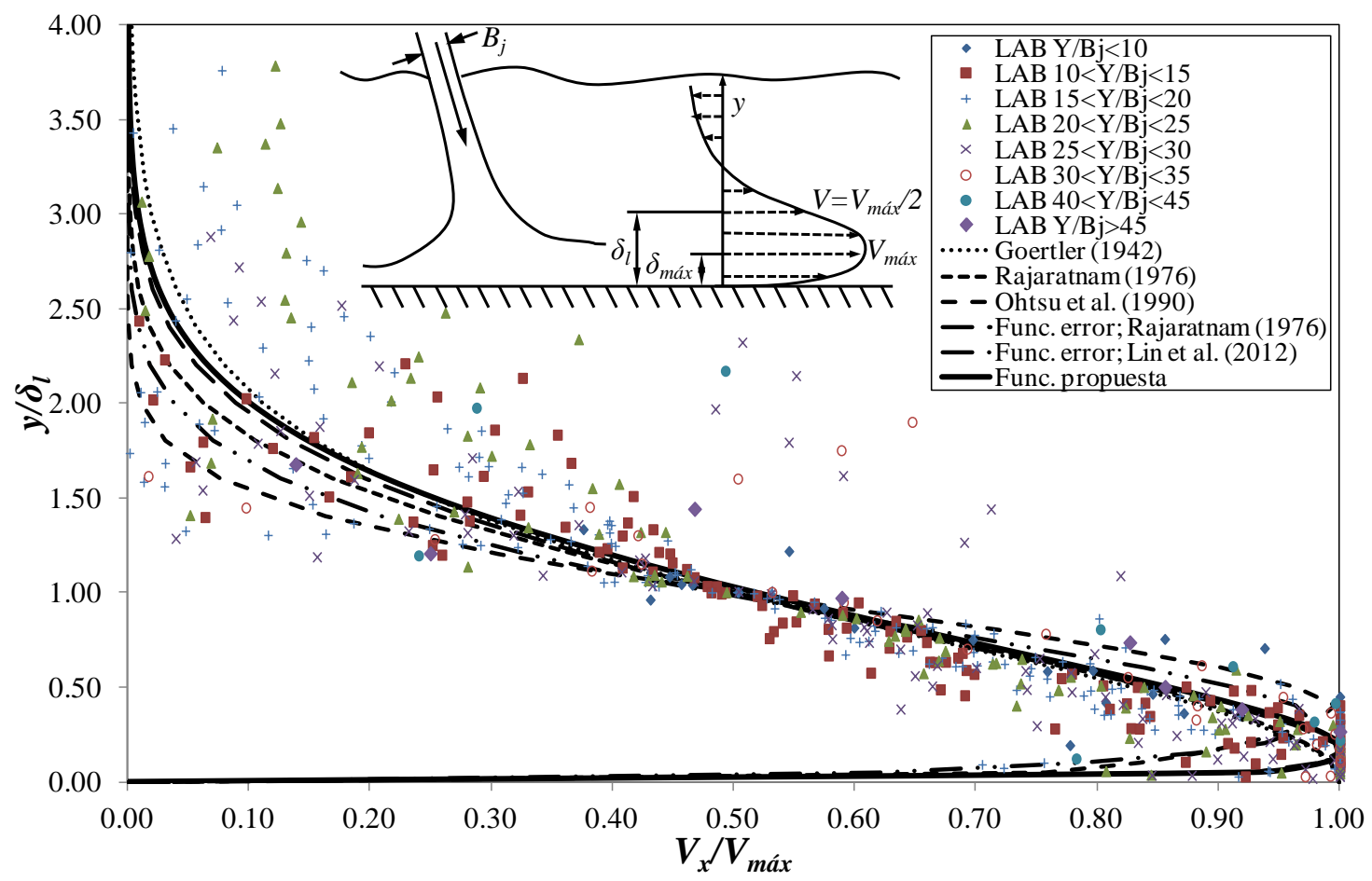

Figura 95. Perfil adimensional de velocidad media horizontal en el cuenco de disipación. 
Siguiendo los estudios de Wu y Rajaratnam (1995), en la Figura 96 se muestran los resultados de la longitud característica del campo de velocidades obtenidos en el cuenco de disipación, adimensionalizados en función del ancho del chorro incidente $B_{j}$.

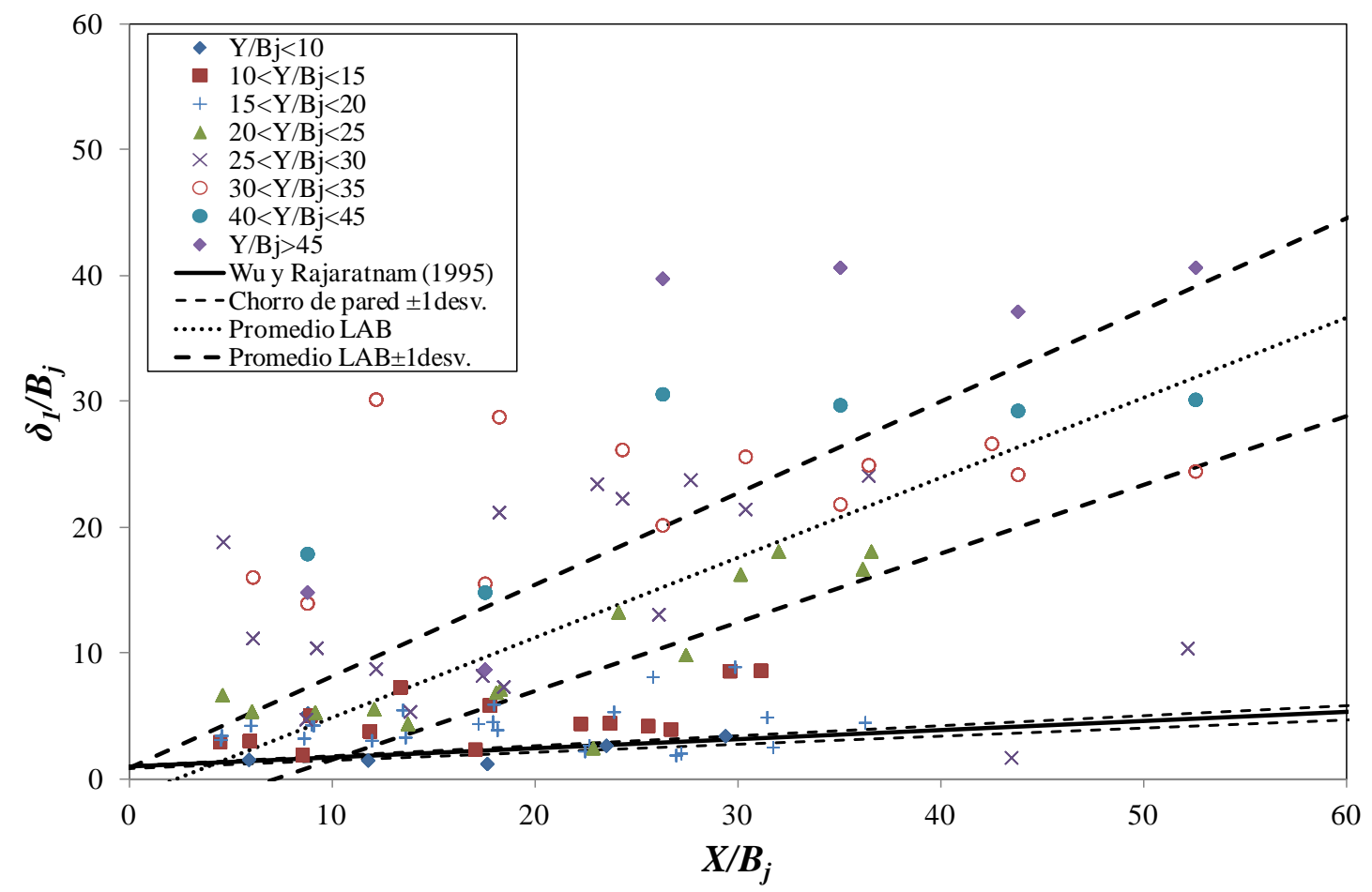

Figura 96. Variación de la longitud característica $\delta_{l}$ en resaltos sumergidos.

Para relaciones $Y / B_{j}$ hasta 20 se observa un comportamiento similar al del chorro de pared obtenido por Wu y Rajaratnam, sobre todo en la parte inicial de los resaltos con relaciones $X / B_{j}$ hasta 20 que es cuando se produce el corte con la banda del valor medio menos una desviación estándar. Los valores se ajustan por debajo de la banda delimitada por el valor medio más una desviación para colchones con $Y / B_{j}$ hasta 30 . Sin embargo, para colchones superiores la longitud característica es superior. En esta tipología donde el chorro vertiente incide en la lámina libre del colchón con un ángulo de casi $90^{\circ}$ se puede obtener una ley con los datos que caen en la banda definida por +/una desviación estándar.

$$
\frac{\delta_{l}}{B_{j}}=0.6367 \frac{X}{B_{j}}-1.5349
$$




\subsubsection{Disipación de energía en el cuenco de amortiguación}

Se ha analizado la pérdida de energía generada en los 18 resaltos hidráulicos empleados para el estudio de los perfiles de velocidad en el cuenco de disipación.

En la Figura 97 se muestra la relación entre la pérdida de energía y el número de Froude en la condición incidente del chorro $F_{j}$. Además, se han graficado las leyes teóricas de pérdida de energía obtenidas con la ecuación (71) en función de la relación entre el calado aguas arriba del resalto sumergido y el espesor del chorro incidente $\left(y_{3} / B_{j}\right)$. En general, los ensayos realizados muestran una pérdida de energía superior al $75 \%$ respecto a la energía del chorro incidente, la cual aumenta con la disminución de la relación $y_{3} / B_{j}$.

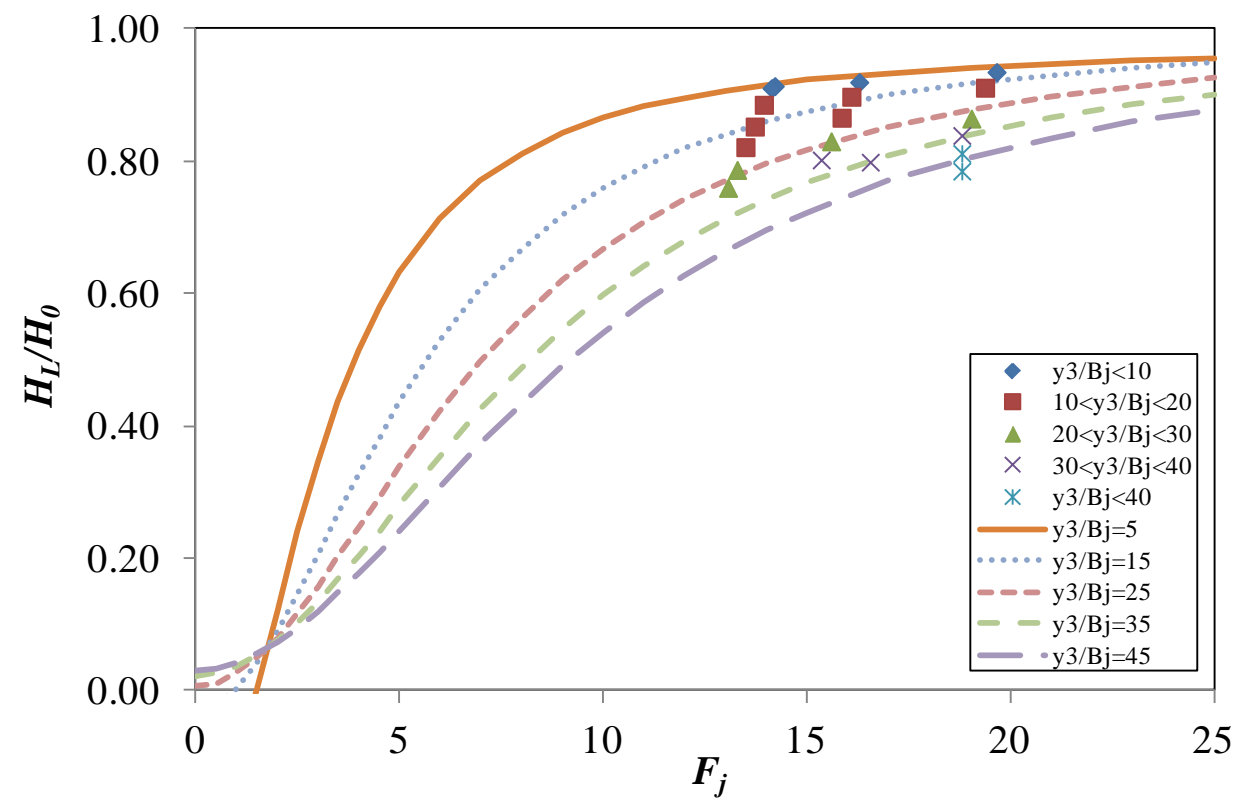

Figura 97. Pérdida de energía relativa en resaltos sumergidos en función del $n^{\circ}$ de Froude.

La Figura 98 muestra la pérdida de energía relativa en función de la relación $y_{3} / B_{j}$. Se observa que los datos de laboratorio siguen la tendencia marcada por las leyes teóricas obtenidas con la ecuación (71) en función del número de Froude del chorro incidente $F_{j}$. Las mayores eficiencias del resalto sumergido se obtienen con relaciones $y_{3} / B_{j}$ pequeñas, mientras que al aumentar el calado en el cuenco se tiende a perder efectividad en la disipación de energía. 


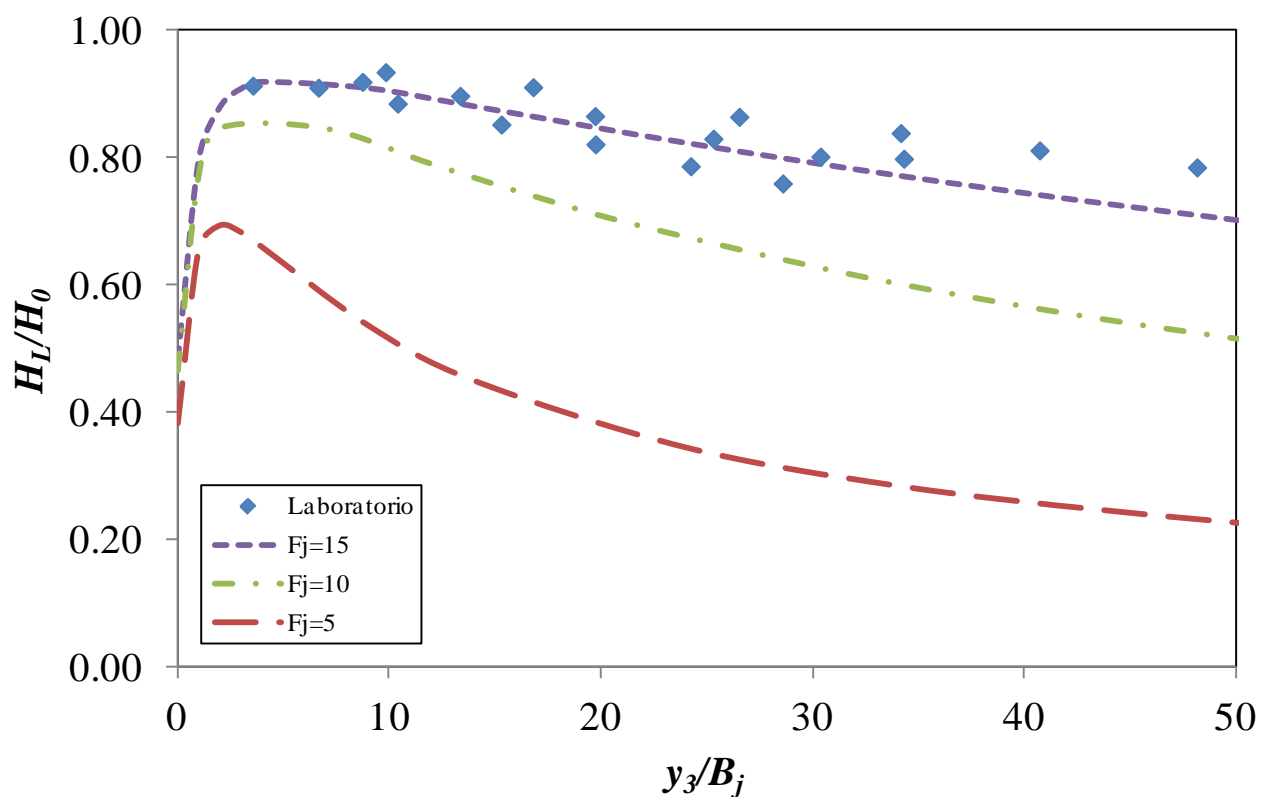

Figura 98. Pérdida de energía relativa en resaltos sumergidos en función de la relación $y_{3} / B_{j}$.

\subsection{Simulaciones numéricas}

En este apartado se realiza la validación de la metodología CFD en la simulación de un aliviadero de vertido libre frente a los resultados de laboratorio y las formulaciones empíricas.

Se han empleado dos paquetes informáticos comerciales de Dinámica de Fluidos Computacional:

- ANSYS CFX (versión 14.0). Este software de volúmenes finitos permite elegir entre una gran variedad de modelos de turbulencia: modelos laminares, modelos de viscosidad de remolino de una y dos ecuaciones (basados en $\varepsilon$ y en $\omega$ ), modelos de tensiones de Reynolds, Simulación de Gran Remolino (LES), etc. El volumen fluido se obtiene con un mallado no estructurado, lo que permite adaptar los elementos a cualquier geometría.

- FLOW-3D (versión 10.0). Se trata de un programa de diferencias finitas. Aunque la elección del modelo de turbulencia es bastante limitada, y diversos autores prevén un mayor desarrollo de la formulación basada en volúmenes finitos, este paquete informático está muy extendido en el mundo de hidráulica de presas. Presenta diversos modelos específicamente diseñados para el campo de la hidráulica: transporte de sedimentos, erosión, cavitación, precisión en el cálculo de la lámina libre, etc. 
Las simulaciones se han realizado reproduciendo las condiciones de contorno obtenidas en laboratorio (calados antes del aliviadero y en el cuenco de disipación, caudal circulante e intensidad turbulenta medida en la sección de entrada al modelo).

En ANSYS CFX se ha empleado un intervalo de paso fijo de 0.05 segundos, análoga a la frecuencia de muestreo empleada en las mediciones de presiones realizadas en el laboratorio $(20 \mathrm{~Hz})$. Por su parte, FLOW-3D requiere un intervalo de paso de resolución inferior, el cual se va adaptando por criterios de estabilidad internos. Aunque el intervalo de paso con que resuelve FLOW-3D está en torno a $10^{-4}$, los resultados se analizan con una frecuencia de muestreo de $20 \mathrm{~Hz}$.

Para juzgar la convergencia de la solución en un esquema de volúmenes finitos, un método muy extendido es monitorizar los residuos (Wasewar y Vijay Sarathi, 2008). Los residuos se definen como las inestabilidades obtenidas en cada iteración para cada ecuación de conservación. Se dice que la solución converge si los residuos son inferiores a un rango prefijado entre $10^{-3}$ y $10^{-6}$. En las simulaciones con ANSYS CFX se ha considerado un valor de $10^{-4}$ para todas las variables.

En el programa de diferencias finitas FLOW-3D, la convergencia de la solución se controla por criterios de estabilidad internos obtenidos en cada intervalo de paso, modificando dicho intervalo cuando es necesario.

Con el fin de obtener los registros de las distintas variables de interés, se han realizado simulaciones en régimen transitorio de 60 segundos. Los valores medios se calculan una vez que se han alcanzado las condiciones permanentes, tal y como se observa en la Figura 99.

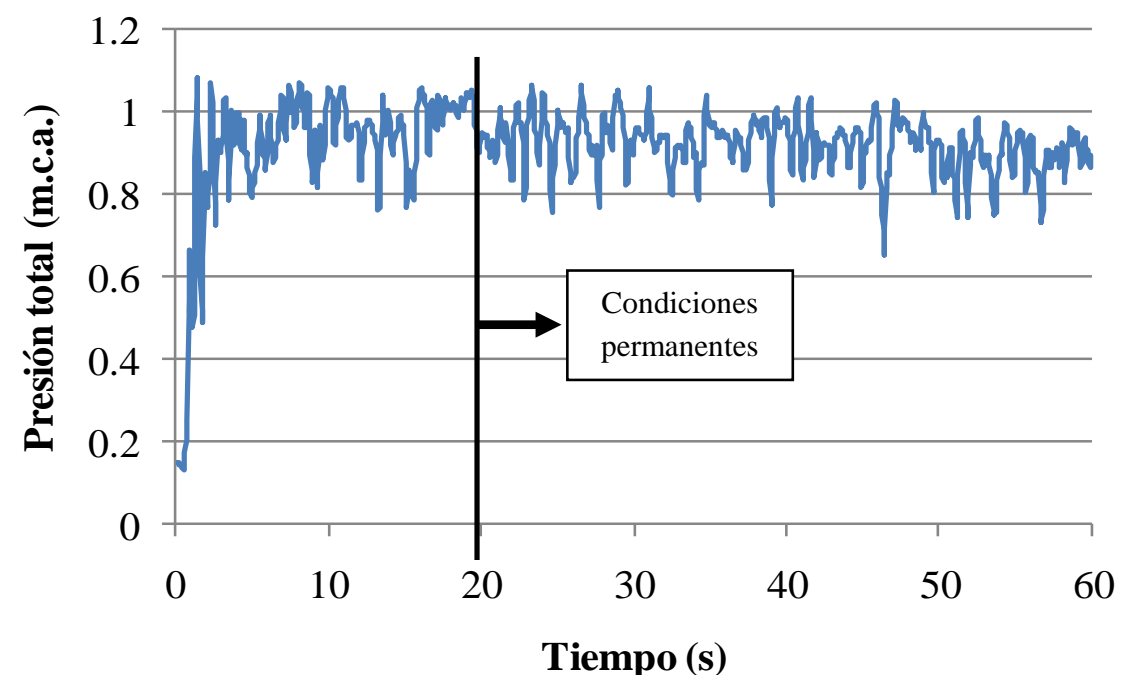

Figura 99. Transitorio de de la presión total simulada en el punto de estancamiento del cuenco de disipación. 


\subsubsection{Mallado}

En el estudio de los chorros turbulentos aparecen fenómenos de despegue del flujo y alta turbulencia que requieren elementos de mallado de gran calidad para su correcta resolución. Las características principales que diferencia a los distintos elementos son el número de nodos con el que resuelven las ecuaciones dentro de cada volumen de control y su distribución espacial. De este modo, a mayor número de nodos por elemento y con distribución espacial más regular, más precisa será su resolución interna. Por este motivo, tanto en ANSYS CFX como en FLOW-3D, se han empleado mallados con elementos hexagonales, obtenidos con sus respectivos módulos de mallado.

ANSYS CFX, al ser un programa de volúmenes finitos, admite diversas tipologías de elementos de malla. El módulo mallador semiautomático de ANSYS CFX, CFXMesh, realiza el mallado del dominio fluido con elementos tetraédricos, permitiendo emplear en las paredes elementos tipo cuña. Pese a su relativa automatización, los elementos tetraédricos pueden estar fuertemente deformados, lo que influye en los resultados obtenidos en la discretización de las ecuaciones de gobierno dentro de cada elemento de la malla.

De las cuatro tipologías de elementos que permite el módulo de resolución de ANSYS CFX, se optó por un mallado basado en elementos hexaédricos, puesto que la alta turbulencia que se genera en el fenómeno estudiado, junto al despegue del flujo, requieren una mayor precisión de cálculo. En las pruebas realizadas se ha comprobado que un mallado basado en elementos hexaédricos mejora el tiempo de cálculo y obtiene una solución más precisa de la lámina libre en un aliviadero de vertido libre.

Al ser un programa de diferencias finitas, FLOW-3D requiere la resolución de mallas estructuradas. Para definir los obstáculos, se emplea el método denominado Fractional Area/Volume Obstacle Representation (FAVOR), desarrollado por Hirt y Sicilian (1985). Este método permite emplear mallados completamente estructurados, mucho más fáciles de generar que los mallados deformados o no estructurados empleados por muchos códigos de CFD. A diferencia de otros métodos de elementos finitos o volúmenes finitos, no requiere un remallado ni distorsión del mallado durante el análisis transitorio, permitiendo aplicar un algoritmo de resolución sencillo.

El método FAVOR emplea una aproximación de primer orden para definir los obstáculos del flujo. Los elementos que obstaculizan totalmente al flujo tienen un valor de 0 , mientras que las celdas fuera del obstáculo tienen un valor de 1 . A los elementos que tienen un obstáculo parcial se les asigna un valor en función de la fracción del elemento que está obstaculizado. El método define el área de cada cara del elemento que el flujo puede atravesar, de modo que la superficie del obstáculo queda definida como una línea recta en un modelo bidimensional o como un plano en una simulación tridimensional. El esquema del método se representa en la Figura 100. 


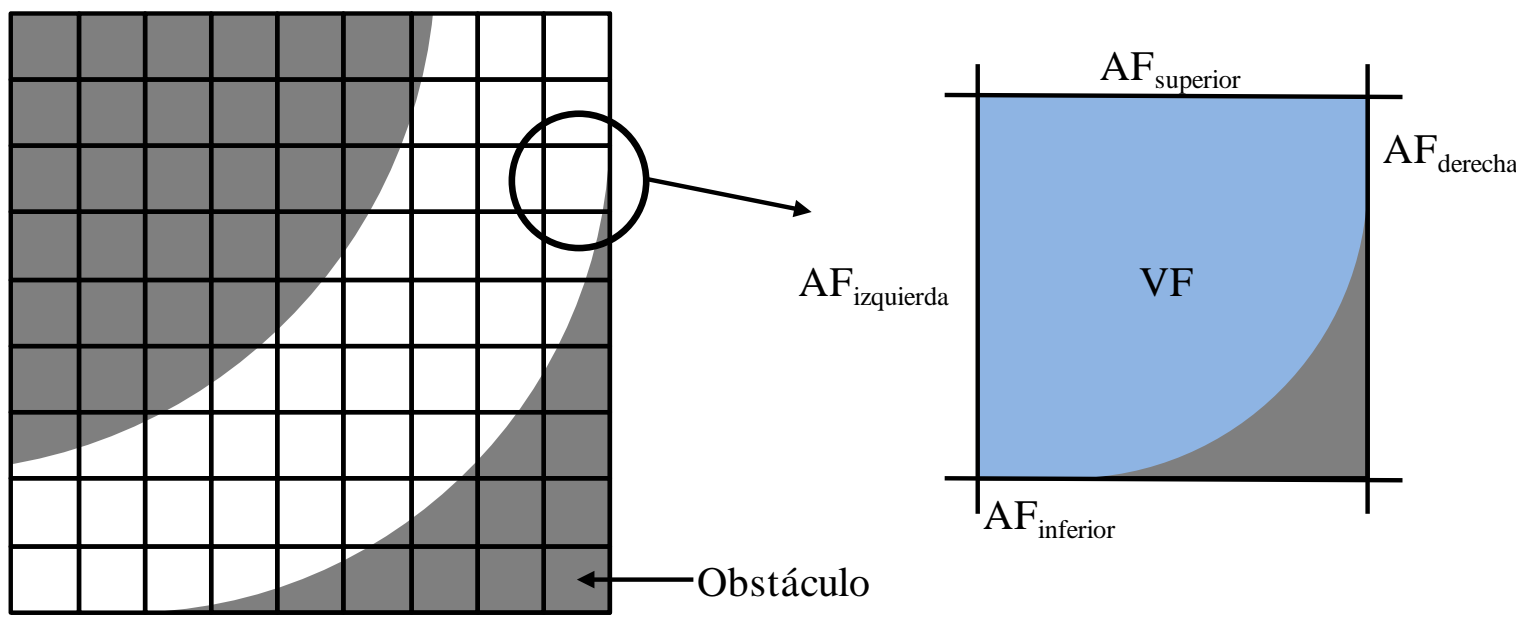

Figura 100. Esquema conceptual del método FAVOR.

siendo:

$$
V F=\frac{\text { Volumen abierto }}{\text { Volumen del elemento }} ; \quad A F=\frac{\text { Área abierta }}{\text { Área de la cara del elemento }}
$$

La fracción de volumen o área ocupada por el obstáculo y la fracción de fluido en cada elemento de la malla se definen al inicio del análisis.

Sin embargo, aunque la superficie curva de un obstáculo se puede aproximar con líneas rectas, esta suposición no deja de ser una aproximación. Se pueden cometer errores no despreciables cuando se realiza el mallado de zonas partes de pequeño tamaño. Estas situaciones obligan a emplear tamaños de mallado muy reducido para intentar aproximar el modelo a la geometría real.

\subsubsection{Modelos de turbulencia}

Para obtener la solución en tiempos adecuados se han empleado modelos de turbulencia, los cuales han sido diseñados para predecir los efectos de la turbulencia sin necesidad de resolver todas las escalas de turbulencia.

Como se comentó anteriormente, existen modelos de distintos grados de complejidad que van desde los modelos isotrópicos de dos ecuaciones como el clásico $k-\varepsilon$, hasta los modelos de cierre de segundo orden o modelos de tensiones de Reynolds (también conocidos como Second Moment Closure Models, SMC, o Reynolds Stress Models, RSM). Algunos modelos de turbulencia tienen aplicaciones específicas, 
mientras que otros pueden ser aplicados a una gran cantidad de flujos con un grado razonable de fiabilidad.

En general, se opta por los modelos de dos ecuaciones dada su gran estabilidad y eficiencia en la resolución. Sin embargo, los modelos isotrópicos dejan de ser válidos cuando se pretende conocer el campo turbulento, debiendo optarse por modelos de cierre de segundo orden que resuelven las componentes de la velocidad turbulenta.

Se debe tener en cuenta que el incremento del número de ecuaciones de transporte en los modelos SMC provoca una reducción de la robustez de la solución, requiriendo un mayor esfuerzo computacional.

Debido a que el flujo bifásico es anisotrópico, en este estudio se han considerado dos tipos de modelos:

- Modelos de cierre de primer orden:

Estudio del conjunto chorro + cuenco de disipación.

- Modelos de cierre de segundo orden:

Estudio de las componentes turbulentas del chorro.

\subsubsection{Lámina libre}

Se asume que los fluidos en flujo multifásico interactúan a escalas macroscópicas, muy superiores a las escalas moleculares. Algunos ejemplos son: burbujas de gas en un líquido, gotas de un líquido en un gas o en otro líquido inmiscible, etc. En estos casos, es necesario resolver los campos de velocidades, temperatura, etc. de cada fluido, los cuales interactúan entre ellos mediante fuerzas en la interfaz y transferencias de calor.

ANSYS CFX es un programa de volúmenes finitos. En cada volumen de control en que se divide el mallado, la suma de las fracciones volumétricas de todas las fases $r_{\alpha}$ es la unidad. Existen tres posibles valores de fracción volumétrica en cada elemento del mallado del dominio fluido:

- $r_{\alpha}=0$. El elemento no contiene la fase $\alpha$.

- $r_{\alpha}=1$. El elemento únicamente contiene la fase $\alpha$.

- $0<r_{\alpha}<1$. El elemento contiene la interfaz entre la fase $\alpha$ y otra o más fases fluidas.

De este modo, las fracciones volumétricas de las fases son igual a uno o cero en todas partes, excepto en la zona donde existe interacción entre los fluidos (flujo bifásico), pudiendo emplearse un único campo de velocidades. 
Para resolver la interfaz bifásica aire-agua, en ANSYS CFX se ha considerado el modelo homogéneo. Este modelo puede considerarse como un caso límite del modelo de flujo multifásico Euleriano-Euleriano en el que la tasa de transferencia en la interfaz es muy grande. Esto da lugar a que todos los fluidos compartan un campo de flujo común (velocidad, presión, temperatura, turbulencia, etc.).

Para un proceso de transporte dado, el modelo homogéneo asume que las cantidades transportadas son las mismas para todas las fases que contiene el elemento:

$$
\Phi_{\alpha}=\Phi \quad 1 \leq \alpha \leq N_{P}
$$

siendo $N_{P}$ el número total de fases.

Puesto que las cantidades transportadas son compartidas en el flujo multifásico homogéneo, el campo compartido se puede resolver con las ecuaciones de transporte en vez de usar una ecuación de transporte individual para cada fase. Las ecuaciones de transporte pueden ser derivadas sumando las ecuaciones de transporte individuales de cada fase para obtener una única ecuación de transporte. De este modo, la ecuación de cantidad de movimiento para el modelo homogéneo queda:

$$
\frac{\partial}{\partial t}(\rho \mathrm{U})+\nabla \cdot\left(\rho U \otimes U-\mu\left(\nabla \mathrm{U}+(\nabla \mathrm{U})^{\mathrm{T}}\right)\right)=S_{M}-\nabla \mathrm{P}
$$

donde:

$$
\begin{gathered}
\rho=\sum_{\alpha=1}^{N_{P}} r_{\alpha} \rho_{\alpha} \\
\mathrm{U}_{\alpha}=\mathrm{U} \quad 1 \leq \alpha \leq N_{P} \\
\mu=\sum_{\alpha=1}^{N_{P}} r_{\alpha} \mu_{\alpha}
\end{gathered}
$$

siendo $U$ la velocidad del flujo multifásico, $S_{M}$ el término fuente, $U_{\alpha}$ la velocidad de la fase $\alpha$ del fluido, $\rho$ la densidad del flujo multifásico, $\rho_{\alpha}$ la densidad de la fase $\alpha, \mu$ la viscosidad dinámica, $\mu_{\alpha}$ la viscosidad dinámica de la fase $\alpha, r_{\alpha}$ la fracción volumétrica de la fase $\alpha$, y $N_{p}$ el número total de fases en el fluido multifásico.

La transferencia en la interfaz no se tiene en cuenta en el modelo homogéneo, siendo esencialmente una ecuación de transporte de fase simple con densidad y viscosidad variable. 
Conjuntamente con el modelo homogéneo, se ha empleado el modelo de superficie libre que considera la diferencia de densidad entre los dos fluidos.

El modelo de superficie libre intenta resolver la interfaz entre los fluidos. Si únicamente existe dos fases en la simulación, se emplea la siguiente ecuación para obtener el área de la interfaz en contacto:

$$
A_{\alpha \beta}=\left|\nabla r_{\alpha}\right|
$$

donde $A_{\alpha \beta}$ es el área de la interfaz por unidad de volumen entre las fases $\alpha$ y $\beta$, y $r_{\alpha}$ es la fracción volumétrica de la fase $\alpha$.

Además, incluye unas características de discretización especiales del gradiente de presión y de los términos gravitatorios para asegurar que el flujo mantiene un buen comportamiento en la interfaz, aunque el método de discretización no queda definido de modo expreso.

Pese a todo, las fronteras entre las distintas fases no están claramente delimitadas, ya que en la zona próxima a la lámina libre se produce un cambio gradual de una fase a la otra (Figura 101).

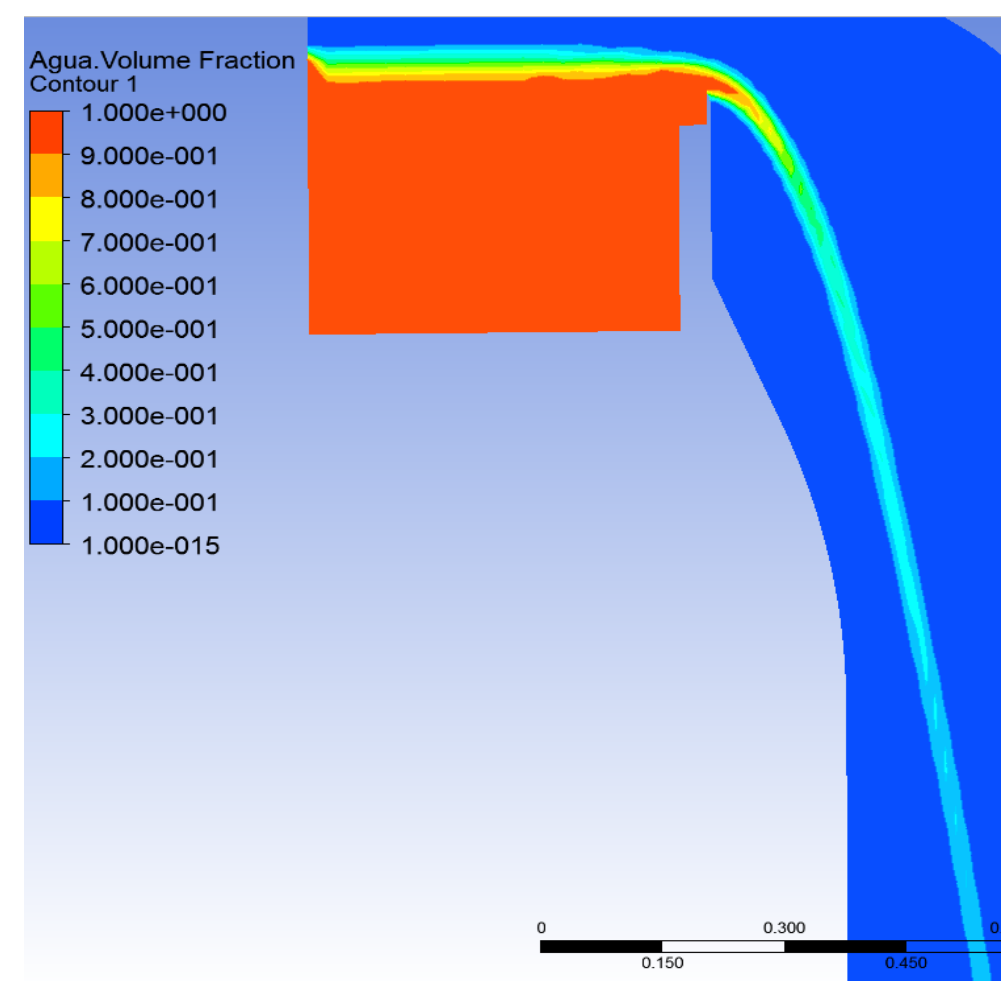

Figura 101. Interfaz del flujo bifásico aire-agua en un aliviadero de vertido libre. 
Generalmente, se asume que la lámina libre está delimitada por una fracción volumétrica de agua de 0.5 (fracción volumétrica de aire $=0.5$ ). Se ha empleado este valor para el cálculo de calados en los flujos en canal. Sin embargo, en la caída del chorro se observó que se produce una enorme entrada de aire, que comienza en la zona de despegue del flujo. Para delimitar el espesor del chorro y su longitud de rotura se ha empleado un valor de fracción volumétrica de agua igual a 0.2 (fracción volumétrica de aire $=0.8)$.

La Figura 102 muestra la lámina libre observada en la instalación de laboratorio y calculada con la metodología CFD cuando se han alcanzado condiciones de régimen permanente. Los perfiles son muy similares.
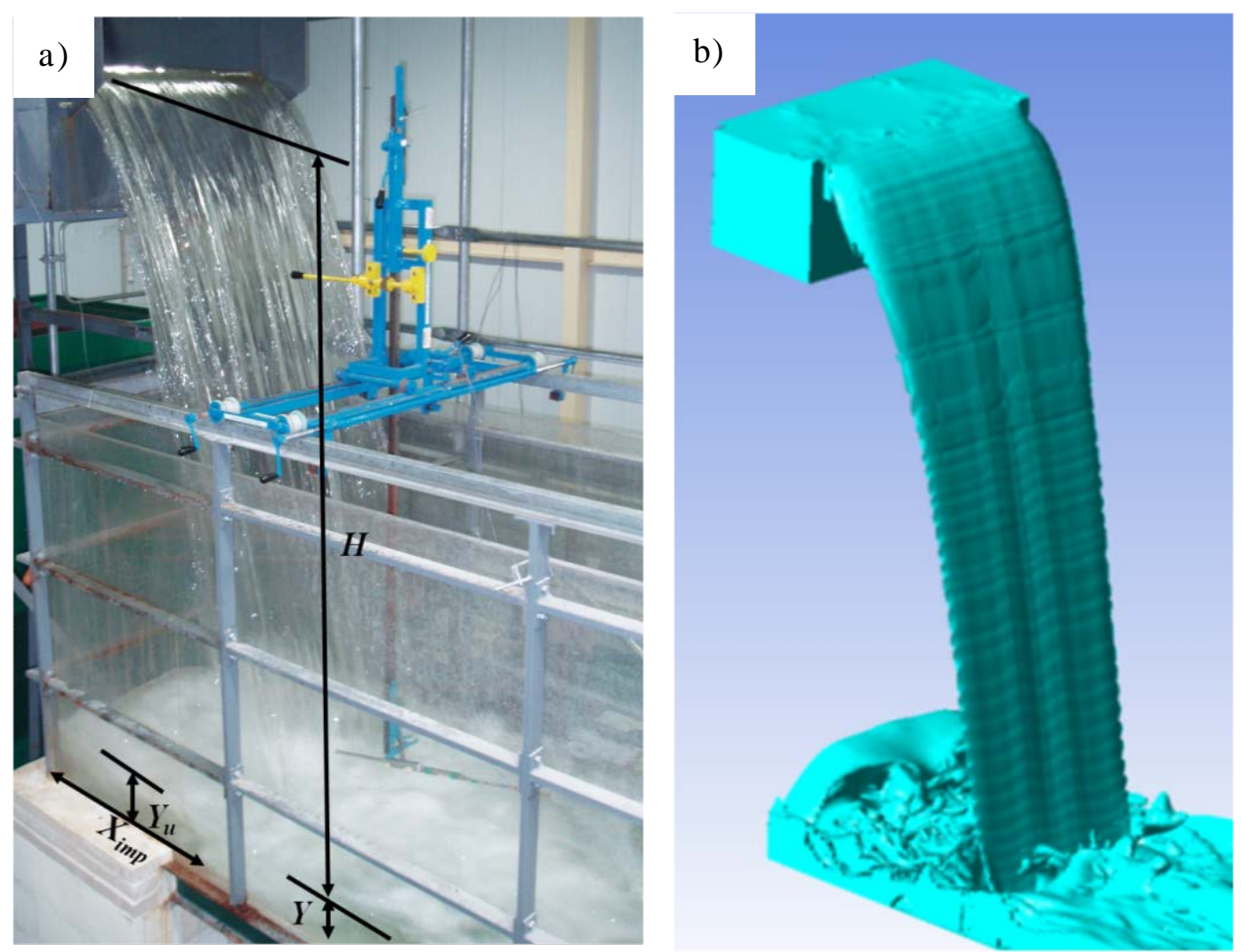

Figura 102. Lámina libre del chorro rectangular $\left(q=0.058 \mathrm{~m}^{2} / \mathrm{s}, H=2.27 \mathrm{~m}, h=0.095 \mathrm{~m}\right.$, $Y=0.17 \mathrm{~m}$ ): (a) Observación en laboratorio; (b) Resuelto con ANSYS CFX.

El método Volume of Fluid (VOF) empleado en FLOW-3D permite considerar únicamente la parte correspondiente al agua en el flujo (Hirt y Nichols, 1981). No se resuelve la dinámica de la fase gaseosa, sino que se trata como regiones de presión uniforme, utilizándose la presión como condición de contorno en la interfaz liquido-gas. Esto origina una teórica reducción del tiempo de simulación al no tener en cuenta la fase 
del aire. De este modo, se acota el cambio gradual en la interfaz aire-agua observado en otros programas de CFD.

Para encontrar, definir y aplicar correctamente las condiciones de contorno en la lámina libre, el método VOF define el volumen de fluido en cada elemento del mallado. El elemento recibe un valor de 0 si no contiene agua, mientras que si está lleno de agua recibe un valor de 1 . Si el elemento contiene la lámina libre, recibe un valor entre 0 y 1 en función de la fracción de volumen fluido que corresponde al agua en dicho elemento.

El ángulo de la superficie del agua en el elemento se determina en función de los valores de los elementos cercanos. De este modo, la localización de la superficie libre dentro de cada elemento se obtiene como una aproximación de primer orden (una línea recta en el caso bidimensional y una superficie plana en el espacio tridimensional).

El cálculo del campo fluido se realiza en cada intervalo de paso, recalculando la superficie libre en cada paso, lo que permite su movimiento temporal y espacial.

De los posibles sistemas de VOF disponibles en FLOW-3D, se ha optado por el método Split Lagrangian, también conocido como TruVOF. Este método obtiene buenos resultados en el rastreo de la interfaz aire-agua en movimientos tridimensionales complejos, y produce errores acumulados de volumen inferiores al resto de los métodos disponibles en el programa (FLOW Science Inc., 2011).

Teniendo en cuenta los métodos VOF y FAVOR, cada elemento puede tener uno de estos cinco valores: completamente sólido, parte sólida y fluida, completamente fluido, parcialmente fluido o completamente vacío.

Las ecuaciones de continuidad y cantidad de movimiento para un flujo no compresible, incluyendo los métodos VOF y FAVOR quedan:

$$
\begin{aligned}
& \frac{\partial}{\partial x}\left(u A_{x}\right)+\frac{\partial}{\partial y}\left(v A_{y}\right)+\frac{\partial}{\partial z}\left(w A_{z}\right)=0 \\
& \frac{\partial U_{i}}{\partial t}+\frac{1}{V_{F}}\left(U_{j} A_{j} \frac{\partial U_{i}}{\partial x_{j}}\right)=\frac{1}{\rho} \frac{\partial p}{\partial x_{i}}+g_{i}+f_{i}
\end{aligned}
$$

donde $V_{F}$ es la fracción volumétrica de agua en cada elemento, $A_{i}$ la fracción de área abierta al flujo en cada dirección, $p$ la presión, $g_{i}$ la fuerza gravitatoria, y $f_{i}$ representa las tensiones de Reynolds obtenidas por el modelo de turbulencia. En elementos completamente llenos de agua, $V_{F}$ y $A_{i}$ son igual a 1, quedando las ecuaciones básicas de flujo incompresible. 


\subsubsection{Estudios previos de la metodología CFD}

\subsubsection{1. $\quad$ Análisis de un aliviadero de presa hidrodinámico}

Previo al estudio de aliviaderos en vertido libre, se comprobó la capacidad del programa ANSYS CFX analizando un aliviadero de dos vanos con un perfil de vertedero de forma estándar tipo WES, definido por el U. S. Army Enginner Waterways Experimental Station, y una pila central tipo II. La modelación se realizó teniendo en cuenta una altura de diseño $H_{d}=4 \mathrm{~m}$, la cual define el perfil del aliviadero.

El mallado se realizó con el módulo de mallado ANSYS-Mesh. Para la obtención de una mayor precisión en el cálculo de la lámina libre, y aprovechando el cálculo en régimen permanente, en sucesivas iteraciones se refinó la malla en la zona en la que se sitúa la lámina libre de agua. En la Figura 103 se puede apreciar la diferente resolución de la malla empleada en la modelación del aliviadero.

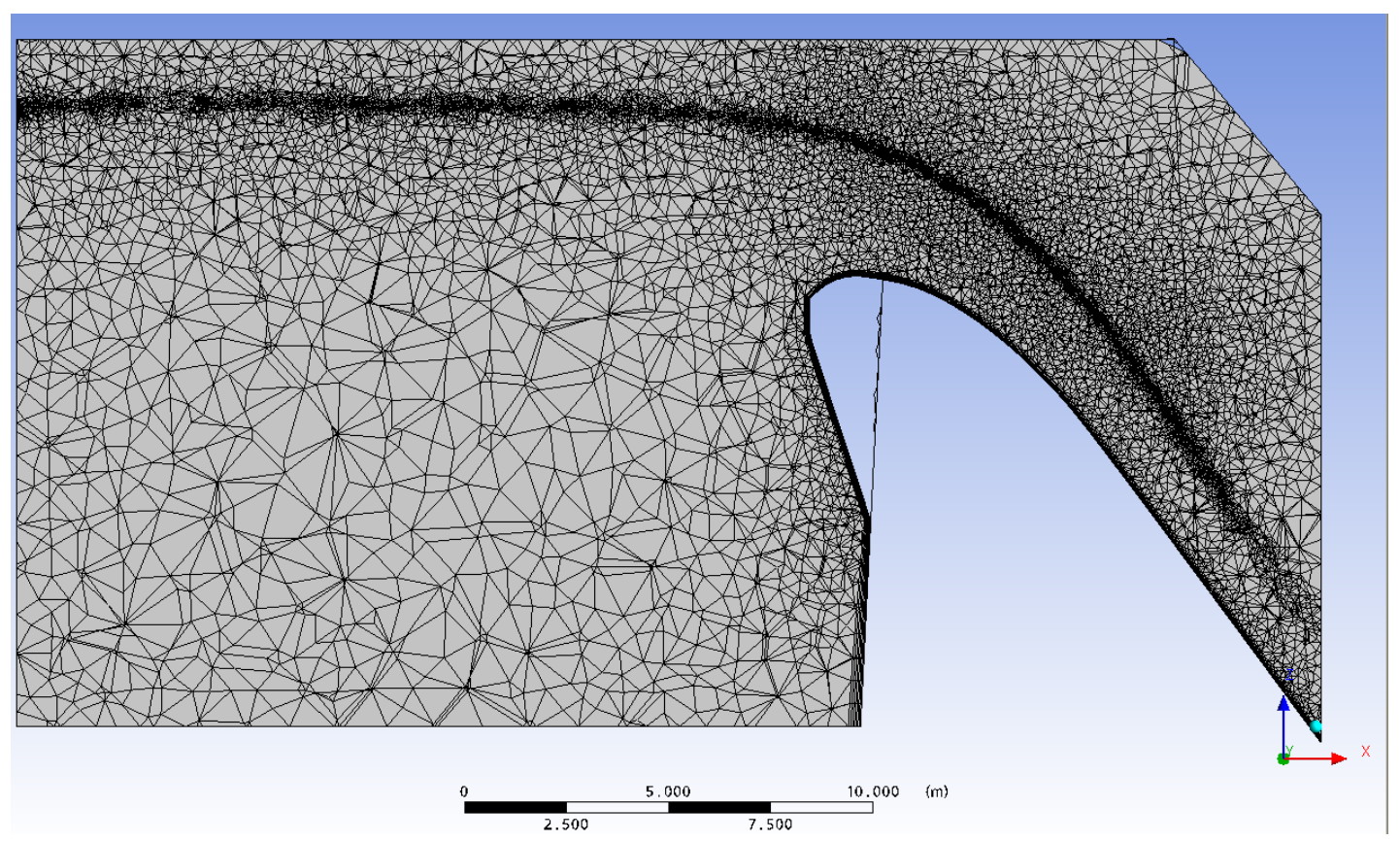

Figura 103. Adaptación del mallado en la zona de lámina libre (Carrillo y Castillo, 2010).

La Figura 104 muestra el adecuado ajuste entre los resultados obtenidos con la simulación numérica empleado el modelo de turbulencia SST y los valores experimentales correspondiente al perfil obtenido en el centro del vano. 


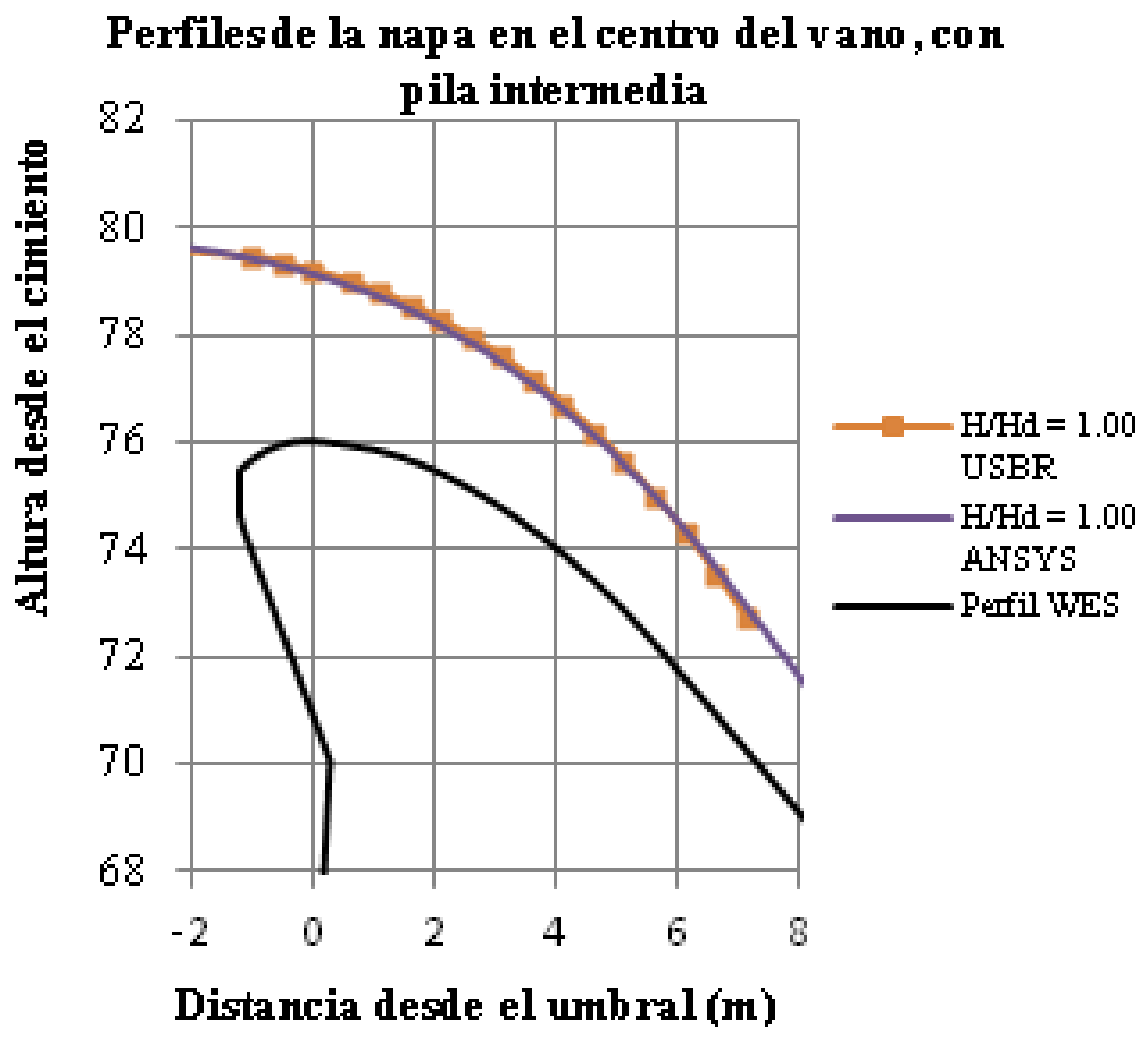

Figura 104. Comparación de resultados obtenidos en el centro del vano del aliviadero para la situación de Nivel Máximo Normal del embalse con $H_{d}=4$ m (Carrillo y Castillo, 2010).

\subsubsection{Análisis bidimensional de un aliviadero de vertido libre}

Previo al estudio tridimensional de un aliviadero de vertido libre, se realizó un estudio bidimensional con el fin de comprobar la capacidad de ANSYS CFX para analizar este tipo de estructuras.

La simulación consistió en un cálculo en régimen transitorio de 60 segundos empleando el modelo de turbulencia SST. En la Figura 105 se observa la gran cantidad de aire que entra en el cuenco de disipación.

Los resultados obtenidos de velocidad y ancho del chorro antes del impacto con el colchón de agua del cuenco de disipación, así como las presiones en el punto de estancamiento obtenidas se asemejaban a las mediciones de laboratorio y a los resultados obtenidos con la formulación propuesta por Castillo (2006, 2007). 


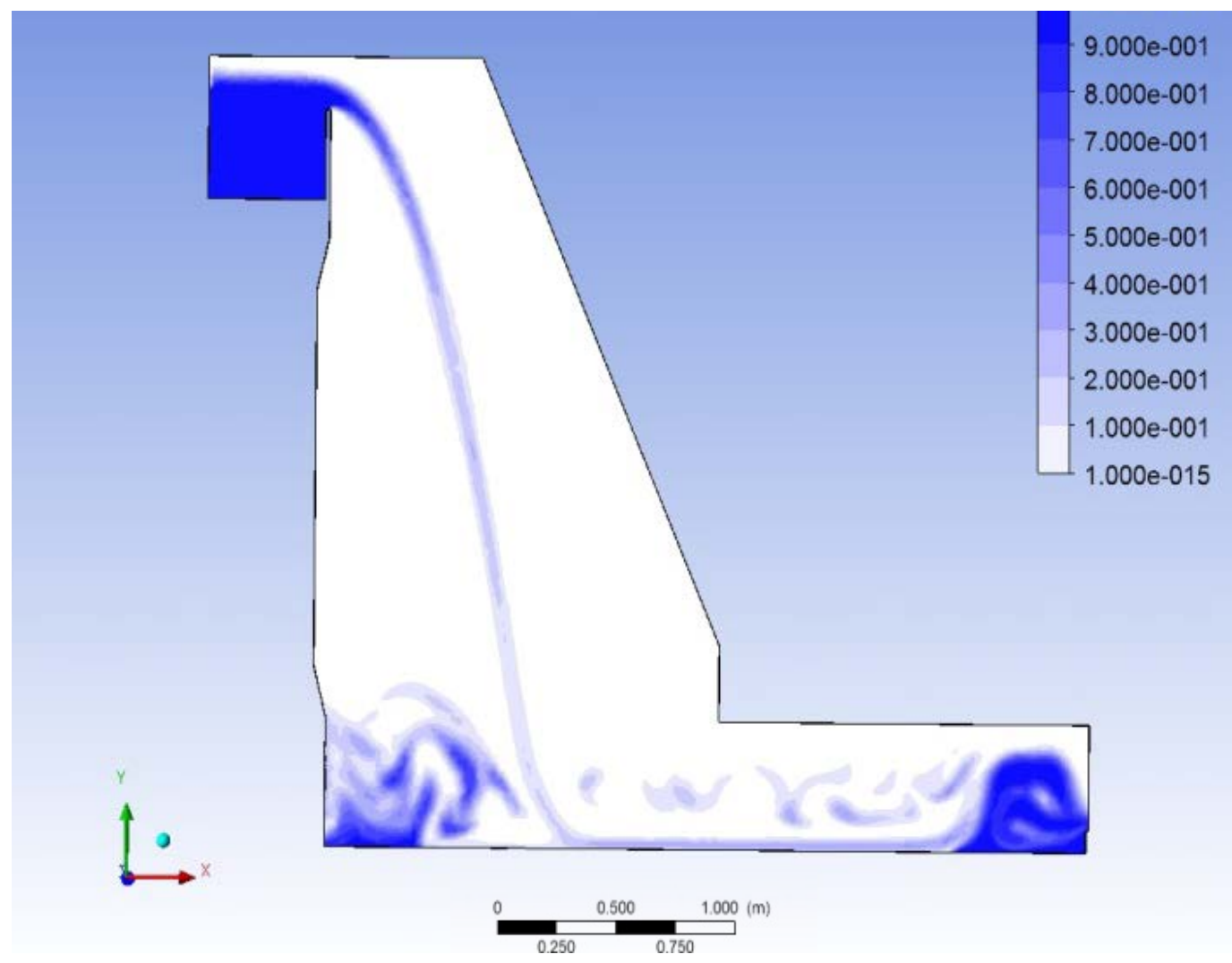

Figura 105. Perfil de la simulación bidimensional de un aliviadero de vertido libre (Castillo y Carrillo, 2010).

\subsubsection{Análisis tridimensional de aliviaderos de vertido libre y cuencos de disipación}

Puesto que la infraestructura del Laboratorio de Hidráulica de la Universidad Politécnica de Cartagena utilizada no es una estructura bidimensional (el ancho del vertedero no coincide con el ancho del cuenco de amortiguación), el estudio comparativo debe ser abordado con un modelo tridimensional.

Las condiciones de contorno del modelo corresponden al caudal, la turbulencia en la sección de entrada del flujo localizada a $0.50 \mathrm{~m}$ aguas arriba del vertedero (obtenida con ADV), los niveles de agua aguas arriba y aguas abajo, así como sus respectivas distribuciones de presiones hidrostáticas (Figura 106). 


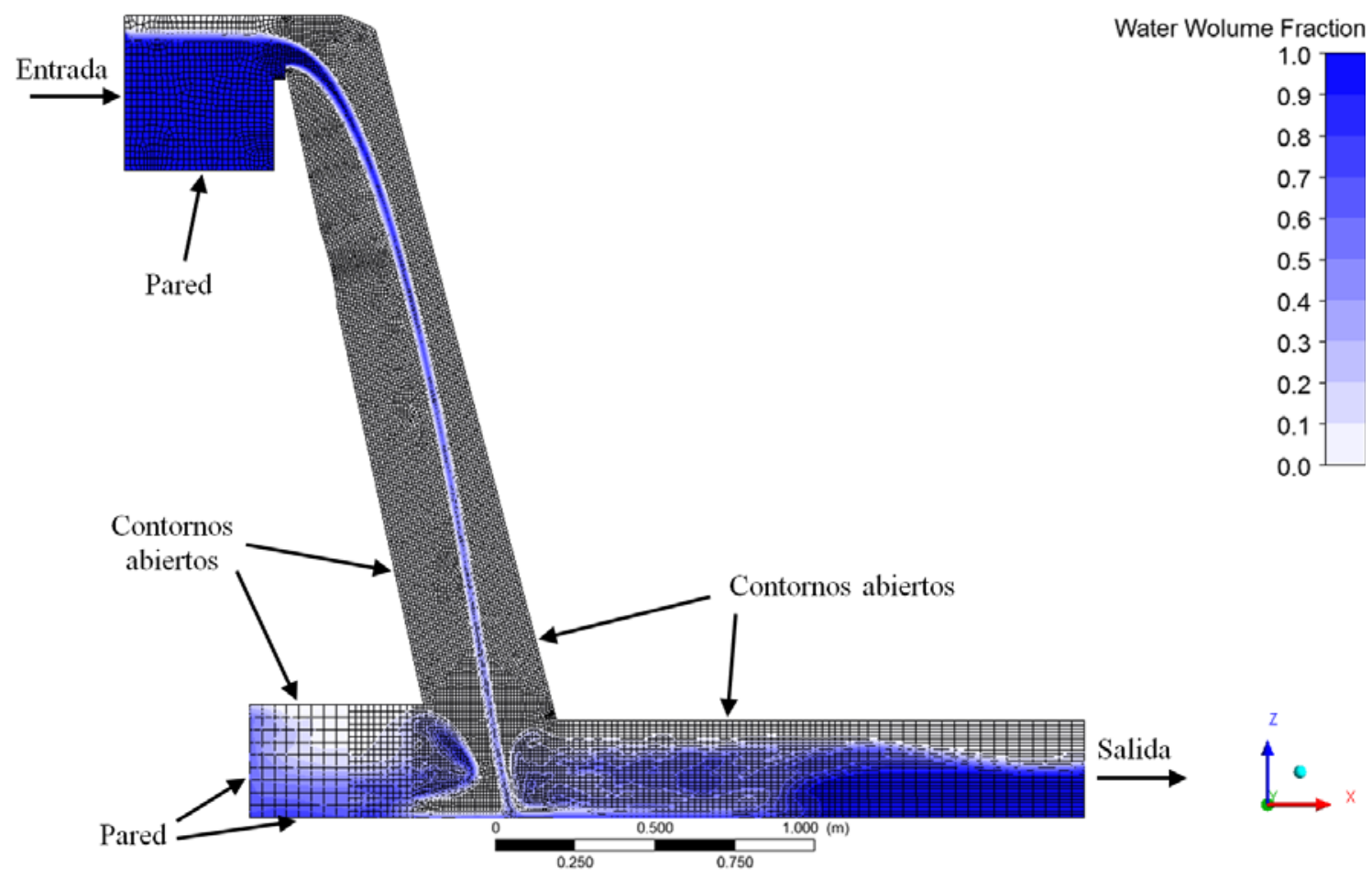

Figura 106. Esquema de las condiciones de contorno.

Por simplicidad y reducción de los tiempos de simulación, en las simulaciones donde ha sido posible se han considerado condiciones de simetría en el plano longitudinal del cuenco de disipación.

Las condiciones en la entrada consideran el caudal máximo en la dirección normal a la condición de contorno ( $\left.q=0.058 \mathrm{~m}^{2} / \mathrm{s}, q=0.037 \mathrm{~m}^{2} / \mathrm{s}, q=0.023 \mathrm{~m}^{2} / \mathrm{s}\right)$, la energía cinética turbulenta $\left(0.00036 \mathrm{~m}^{2} / \mathrm{s}^{2}\right.$ para $q=0.058 \mathrm{~m}^{2} / \mathrm{s}, 0.00019 \mathrm{~m}^{2} / \mathrm{s}^{2}$ para $q=0.037$ $\mathrm{m}^{2} / \mathrm{s}, 0.00011 \mathrm{~m}^{2} / \mathrm{s}^{2}$ para $q=0.023 \mathrm{~m}^{2} / \mathrm{s}$ ), y el nivel de agua aguas arriba del vertedero (2.445 m para $q=0.058 \mathrm{~m}^{2} / \mathrm{s}, 2.423 \mathrm{~m}$ para $q=0.037 \mathrm{~m}^{2} / \mathrm{s}, 2.397 \mathrm{~m}$ para $q=0.023$ $\left.\mathrm{m}^{2} / \mathrm{s}\right)$.

La salida se ha considerado como una condición de contorno abierta, con el flujo en dirección normal al contorno y distribución de presiones hidrostáticas. El nivel del agua en la condición de aguas abajo se ha modificado en función del colchón de agua en el cuenco observado en laboratorio.

Para las paredes del depósito superior, del vertedero y del cuenco de disipación, se han considerado condiciones de no deslizamiento y pared poco rugosa.

La atmósfera se ha simulado mediante una condición abierta, considerando una presión relativa de $0 \mathrm{~Pa}$, una concentración volumétrica de aire de 1 y una concentración de agua de 0. 


\subsubsection{Primera aproximación tridimensional}

Empleando el modelo de turbulencia SST y un mallado de elementos tetraédricos, se realizó la simulación tridimensional del modelo de laboratorio con ANSYS CFX, (Figura 107). El tamaño de la malla fue de $0.01 \mathrm{~m}$ en las zonas de interés (vuelo del chorro y zonas próximas al punto de estancamiento).
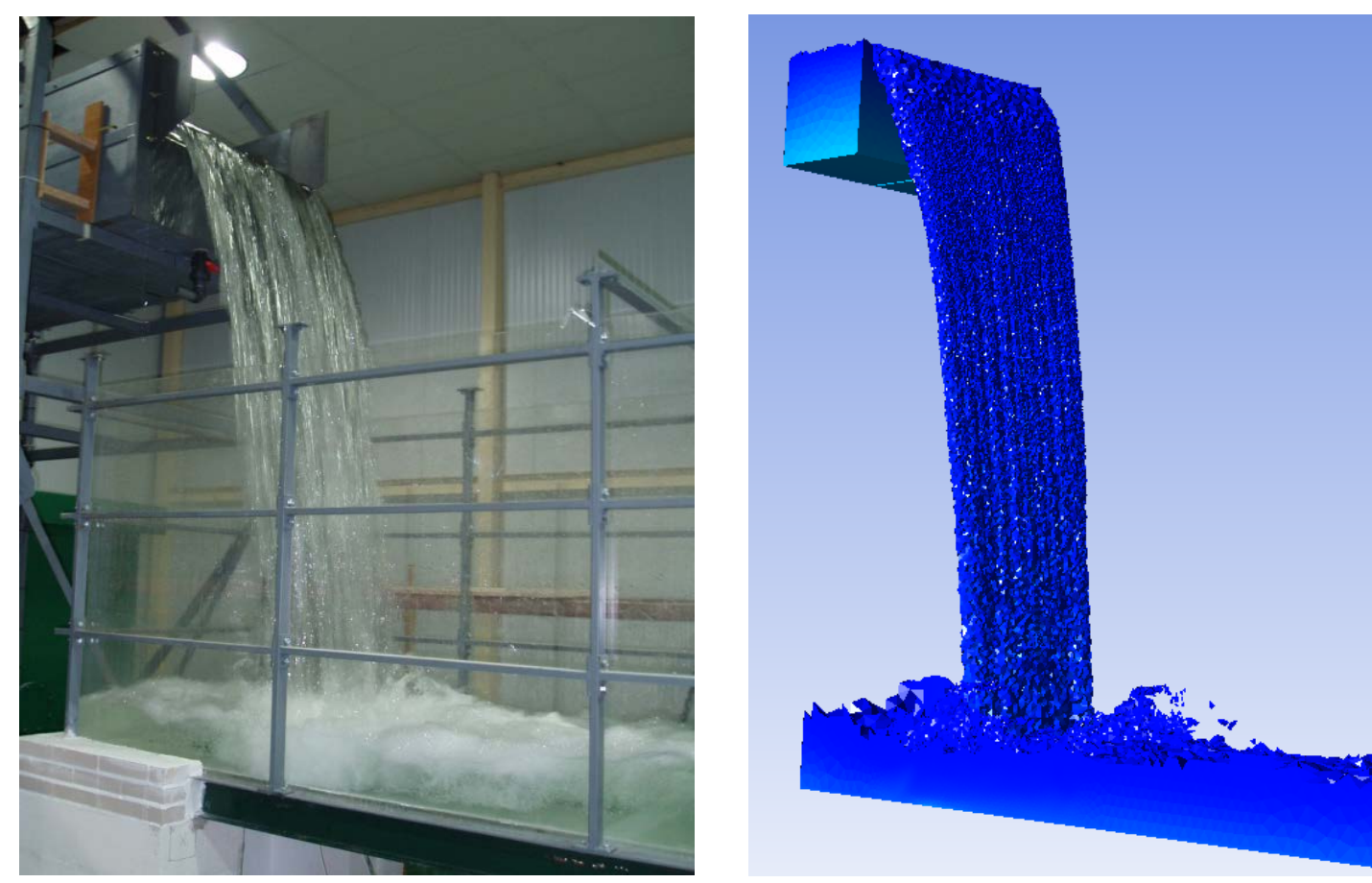

Figura 107. Comparación del vertido libre en laboratorio con la simulación numérica (Castillo y Carrillo, 2011).

El tiempo total de cálculo de la simulación en régimen transitorio de 60 segundos fue de $4.1 \times 10^{5} \mathrm{~s}$ (aproximadamente 4.7 días), paralelizando el cálculo en un ordenador de 8 núcleos.

Los resultados de la simulación se compararon con los datos medidos en laboratorio, y con la Metodología Paramétrica propuesta por Castillo (2006, 2007), obteniéndose resultados similares de presiones, espesores del chorro y velocidades para los dos caudales considerados, aunque se obtuvieron alcances del chorro alrededor de un $10 \%$ inferiores a los observados en laboratorio, posiblemente debidas al empleo de un mallado basado en elementos tetraédricos. 


\subsubsection{Influencia del mallado}

Debido al corto alcance obtenido en las simulaciones realizadas con un mallado basado en elementos tetraédricos, se consideró necesario realizar la comparativa con los resultados que obtiene ANSYS CFX al considerar un mallado basado en elementos hexaédricos (Figura 108).
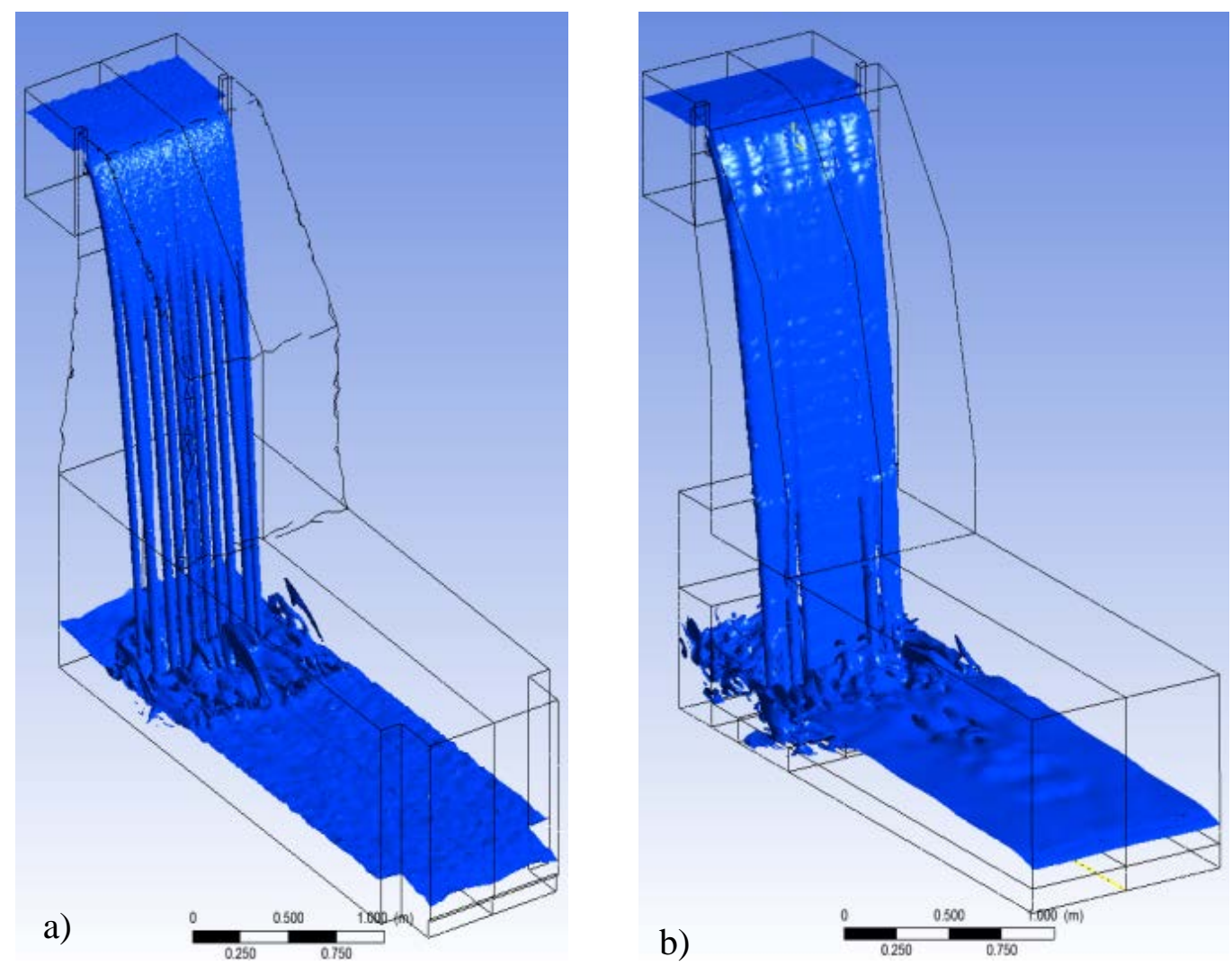

Figura 108. Simulación de la lámina de vertido libre: a) Mallado tetraédrico; b) Mallado hexaédrico (Carrillo y Castillo, 2011).

En la Tabla 28 se compara la distribución de las distintas tipologías de elementos para cada una de las mallas, así como el tiempo computacional y memoria requerida para realizar la simulación. Se comprueba que al emplear elementos hexaédricos del mismo tamaño que los tetraédricos se reduce considerablemente el número de elementos. Otra de las ventajas es que el tiempo de cálculo requerido para resolver la simulación se reduce a aproximadamente la mitad, sin requerir mayor memoria RAM. 


\begin{tabular}{|c|c|c|}
\hline & \multicolumn{2}{|c|}{ Mallado } \\
\hline & Tetraédrico & Hexaédrico \\
\hline $\mathrm{N}^{\mathrm{o}}$ Elementos & 3852769 & 414370 \\
\hline \% Tetraedros & 96.38 & 3.21 \\
\hline \% Pirámides & 0.00 & 6.74 \\
\hline \% Prismas & 3.62 & 0.69 \\
\hline \% Hexaedros & 0.00 & 89.36 \\
\hline $\begin{array}{l}\text { Tiempo de } \\
\text { cálculo (s) }\end{array}$ & $\begin{array}{l}3.081 \times 10^{6} \\
\approx 4.8 \text { días }\end{array}$ & $\begin{array}{l}1.330 \times 10^{6} \\
\approx 2.6 \text { días }\end{array}$ \\
\hline $\begin{array}{l}\text { Memoria RAM } \\
\text { necesaria (GHz) }\end{array}$ & 4 & 4 \\
\hline
\end{tabular}

Tabla 28. Número de elementos en las simulaciones del aliviadero de vertido libre y tiempo de cálculo requerido (Carrillo y Castillo, 2011).

Con el mallado de elementos tetraédricos se obtuvieron resultados adecuados de velocidades y calados, pero se observaba la formación de tubos en la lámina vertiente. Con el mallado basado en elementos hexaédricos se logró una lámina vertiente más real, así como una mejor precisión de los parámetros analizados. En la Tabla 29 se comparan los resultados obtenidos en los parámetros representativos en la teoría de aliviaderos de vertido libre, donde $y_{b}$ es el calado del flujo a su paso sobre el labio del aliviadero, $X_{\text {imp }}$ la distancia horizontal del aliviadero al punto de impacto, $H_{m}$ la presión media obtenida en el punto de estancamiento, y $C_{p}$ el coeficiente de presión dinámica media calculado como:

$$
C_{P}=\frac{H_{m}-Y}{H}
$$

siendo $H$ la diferencia entre la cota de la lámina libre aguas arriba del aliviadero y la superficie libre en el cuenco e $Y$ el calado en el cuenco de disipación.

\begin{tabular}{|l|c|c|c|}
\cline { 2 - 4 } \multicolumn{1}{c|}{} & \multicolumn{2}{c|}{ Mallado } & \multirow{2}{*}{ Laboratorio } \\
\cline { 2 - 4 } \multicolumn{1}{c|}{} & Tetraédrico & Hexaédrico & \\
\hline$y_{b}(\mathrm{~m})$ & 0.070 & 0.086 & 0.082 \\
\hline$X_{\text {imp }}(\mathrm{m})$ & 0.68 & 0.74 & 0.74 \\
\hline$H_{m}$ (m.c.a.) & 1.15 & 0.92 & 1.01 \\
\hline$C_{p}$ & 0.35 & 0.28 & 0.31 \\
\hline
\end{tabular}

Tabla 29. Comparativa de parámetros representativos de la teoría de vertido libre (Carrillo y Castillo, 2011).

En la Figura 109 se grafican las trayectorias de la lámina vertiente simulada con los dos tipos de mallado, junto a la solución de modelo de laboratorio propuesta por Scimemi (1930). El mallado hexaédrico obtiene una distancia de vuelo $X_{i m p}$ similar al 
observado en laboratorio, mientras que el mallado tetraédrico no es capaz de reproducir adecuadamente el despegue de la lámina de agua que se produce en el aliviadero de vertido libre.

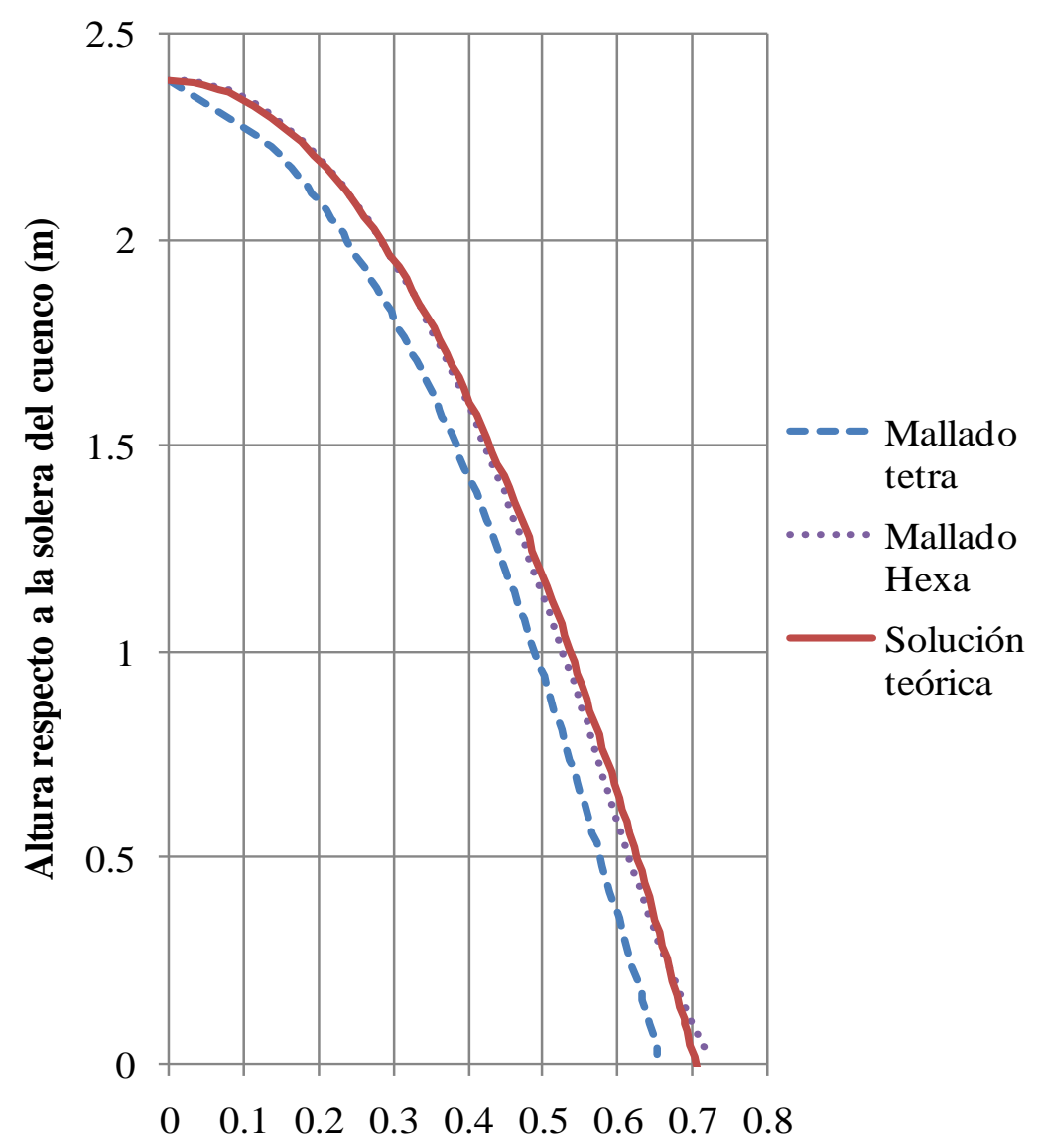

Distancia desde el aliviadero (m)

Figura 109. Trayectoria de la lámina libre (Carrillo y Castillo, 2011).

\subsubsection{Análisis de la turbulencia del chorro}

Debido al pequeño espesor del chorro no fue posible medir la componente turbulenta del mismo con la instrumentación de laboratorio. Sin embargo, teniendo en cuenta los resultados obtenidos con CFD por Castillo y Carrillo (2012, 2013), la expansión lateral del chorro rectangular ha sido estimada mediante simulaciones numéricas.

Con el fin de reproducir del modo más fidedigno posible las condiciones de laboratorio, se midió la turbulencia de entrada en el modelo de laboratorio (Figura 110). 


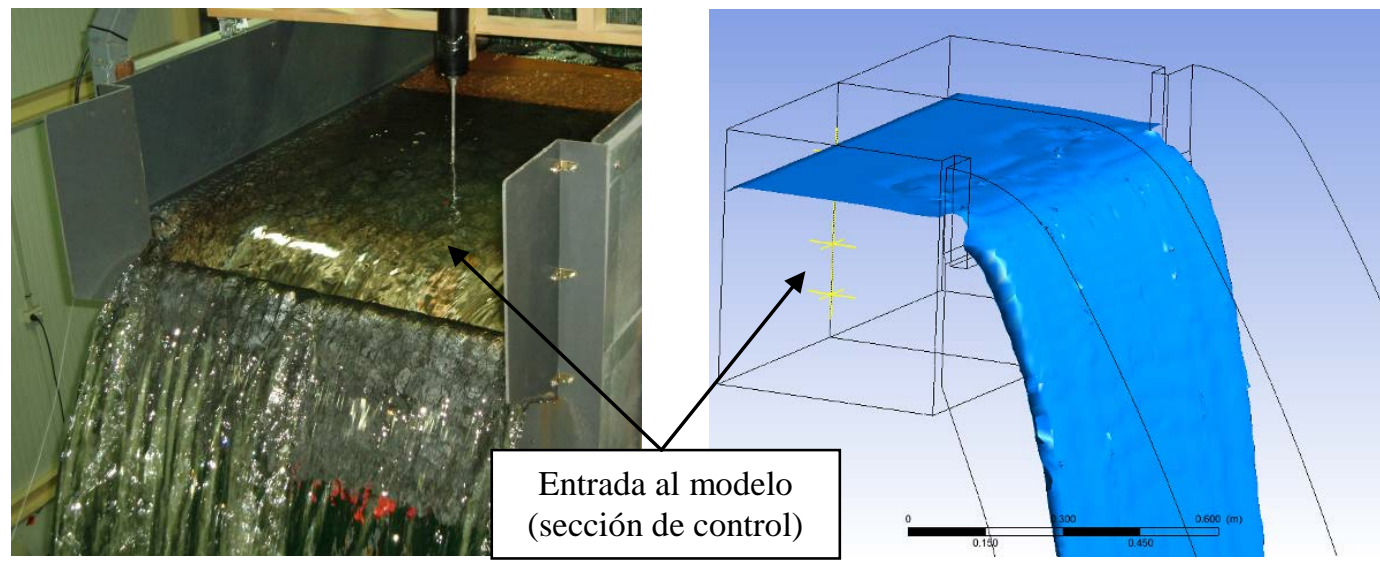

Figura 110. Sección de control de velocidades turbulentas en el modelo de laboratorio (Carrillo y Castillo, 2011).

La sección se situó $0.50 \mathrm{~m}$ aguas arriba del punto de vertido, suficientemente alejada del mismo para evitar las distorsiones debidas a la contracción y aceleración que el flujo sufre en las inmediaciones del aliviadero (carga máxima sobre vertedero, $h_{\text {máx }}=$ $0.095 \mathrm{~m}$ ). Las velocidades turbulentas obtenidas con esta metodología se emplearon como dato de entrada en las simulaciones numéricas realizadas.

De este modo, mediante el equipo Doppler se registró una elevada turbulencia en la condición de contorno de la entrada del modelo, siendo:

$$
T_{u}=\frac{\bar{V}^{\prime}}{V}=0.16
$$

donde $\bar{V}^{\prime}$ y $V$ son la raíz cuadrática media (RMS) y la velocidad media del flujo.

Las condiciones de contorno del modelo numérico corresponden a la turbulencia obtenida con equipo Doppler (condición de entrada localizada $0.50 \mathrm{~m}$ aguas arriba del vertedero), los niveles de agua de aguas arriba y aguas abajo, y sus distribuciones hidrostáticas de presiones.

Puesto que la turbulencia en el chorro vertiente influye en la entrada de aire así como en la longitud de rotura del chorro, se ha evaluado el funcionamiento de la turbulencia a lo largo del chorro. Mediante simulaciones en ANSYS CFX se han analizado las velocidades turbulentas, empleando el modelo de turbulencia de cierre de segundo orden Baseline Reynolds Stress.

A partir de la turbulencia calculadas en las simulaciones, se han obtenido las velocidades medias $(u, v, w)$ y turbulentas $\left(u^{\prime}, v^{\prime}, w^{\prime}\right)$ sobre una línea de corriente que parte del aliviadero. En las siguientes figuras se grafica la variación de las velocidades medias y turbulentas para distintos caudales. 

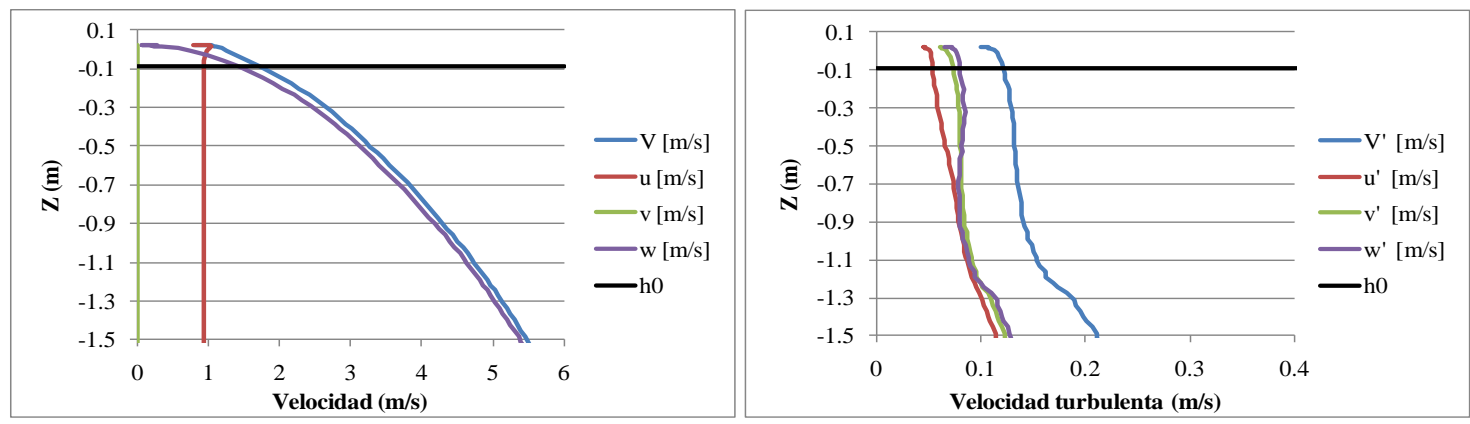

Figura 111. Velocidades medias y turbulentas para $q=0.058 \mathrm{~m}^{2} / \mathrm{s}$ y $\mathrm{h}=0.095 \mathrm{~m}$ (Carrillo y Castillo, 2011).

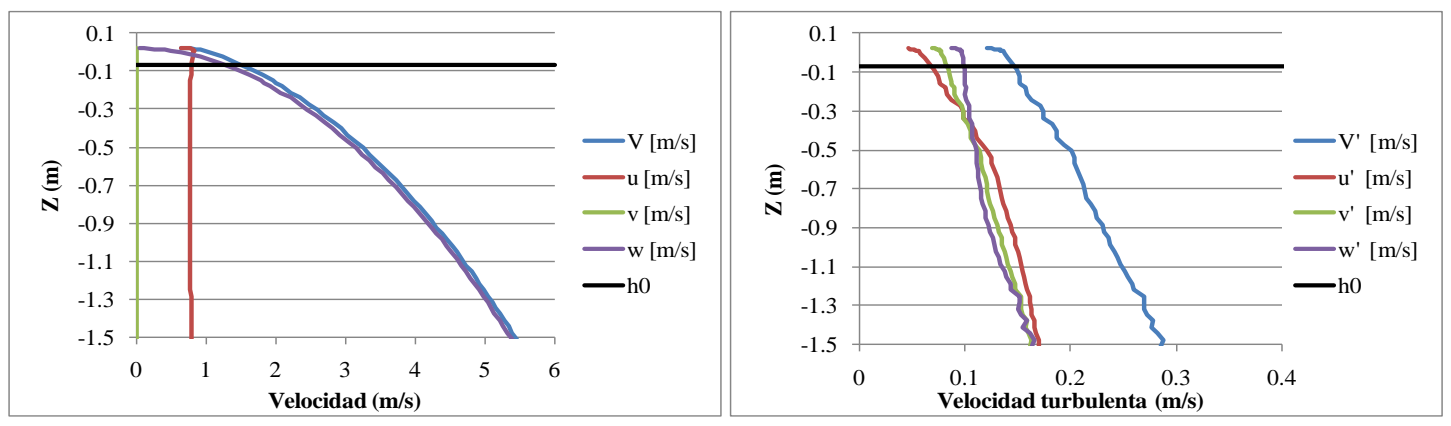

Figura 112. Velocidades y velocidades turbulentas para $q=0.037 \mathrm{~m}^{2} / \mathrm{s}$ y $\mathrm{h}=0.073 \mathrm{~m}$ (Carrillo y Castillo, 2011).
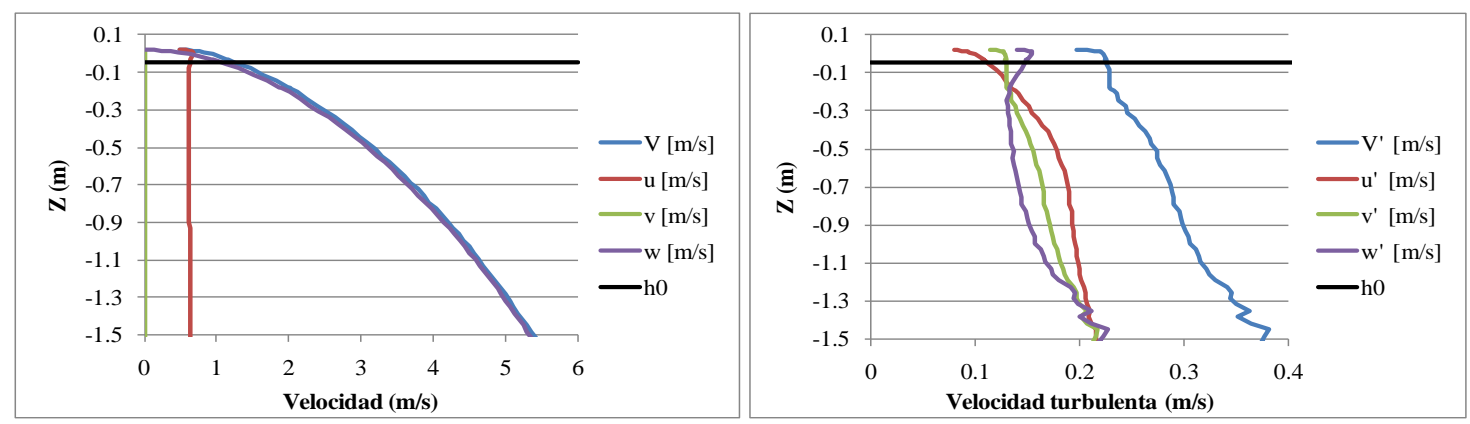

Figura 113. Velocidades medias y turbulentas para $q=0.023 \mathrm{~m}^{2} / \mathrm{s}$ y $\mathrm{h}=0.047 \mathrm{~m}$ (Carrillo y Castillo, 2011).

Las velocidades turbulentas tienden a igualarse a una cierta altura de caída, más alejada cuanto mayor es el caudal específico, mientras que las velocidades turbulentas se incrementan con la reducción del caudal específico.

En la Figura 114 se observa que, aunque el flujo llega al vertedero con una elevada intensidad de turbulencia, ésta se reduce rápidamente hasta alcanzar un valor aproximadamente constante. Este hecho se debe a que la aceleración del flujo por efecto de la gravedad es muy superior al incremento de la velocidad turbulenta en la caída. 


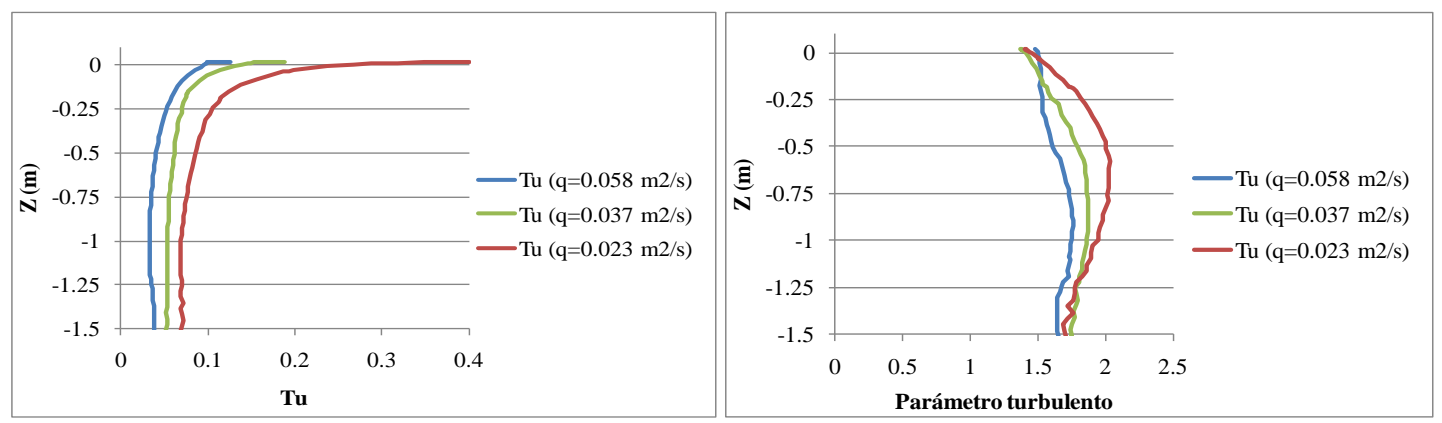

Figura 114. Evolución de la intensidad de turbulencia $T_{u} y$ del coeficiente del parámetro de turbulencia $K_{\varphi}$ para distintos caudales (Carrillo y Castillo, 2011).

También se observa que en la caída, el coeficiente del parámetro turbulento $K_{\varphi}$ tiende a un valor constante. Los caudales mayores presentan un chorro más compacto, tendiendo a alcanzar antes el valor constante.

Con el fin de determinar el efecto que tiene en las simulaciones numéricas la modificación de la turbulencia en la condición de entrada, se consideraron diferentes intensidades turbulentas en la entrada del modelo (0.01, 0.03, 0.05 y 0.15$)$.

Siguiendo una línea de corriente, la Figura 115 muestra la evolución de $T_{u}$ para distintas intensidades turbulentas en la condición de entrada cuando se considera un caudal específico de $0.058 \mathrm{~m}^{2} / \mathrm{s}$. Aunque la intensidad turbulenta es diferente, $T_{u}$ tendió a ser igual cuando existe suficiente distancia de caída.

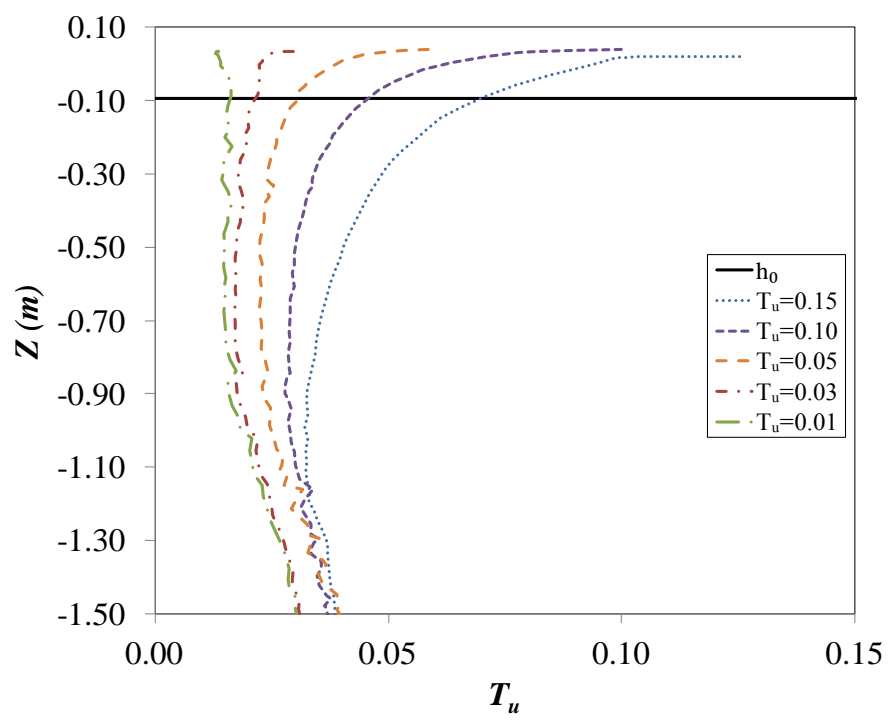

Figura 115. Evolución de la intensidad de turbulencia del chorro vertiente en función de la intensidad turbulenta en la condición de entrada (Castillo et al., 2014). 
La Tabla 30 muestra los resultados obtenidos en la condición inicial $(z=-h)$ y tras una caída de $1.50 \mathrm{~m}$.

\begin{tabular}{|c|c|c|c|c|c|}
\hline & \multicolumn{2}{|c|}{ Condición inicial $(z=-h)$} & \multicolumn{2}{|c|}{$z=-1.50 \mathrm{~m}$} \\
\hline & & $V(\mathrm{~m} / \mathrm{s})$ & $T_{u}(-)$ & $V(\mathrm{~m} / \mathrm{s})$ & $T_{u}(-)$ \\
\hline \multirow{5}{*}{$\begin{array}{c}T_{u} \text { en la } \\
\text { condición } \\
\text { de } \\
\text { entrada }\end{array}$} & 0.15 & 1.83 & 0.070 & 5.50 & 0.039 \\
\hline & 0.10 & 1.86 & 0.045 & 5.52 & 0.038 \\
\hline & 0.05 & 1.86 & 0.030 & 5.52 & 0.038 \\
\hline & 0.03 & 1.84 & 0.022 & 5.50 & 0.031 \\
\hline & 0.01 & 1.84 & 0.016 & 5.50 & 0.031 \\
\hline
\end{tabular}

Tabla 30. Velocidades turbulentas en función de la turbulencia en la condición de entrada para distintas alturas ( $q=0.058 \mathrm{~m}^{2} / \mathrm{s}, h=0.095 \mathrm{~m}$ ). (Castillo et al., 2014).

Aunque se ha modificado la turbulencia en la condición de entrada del modelo, la velocidad media no se ha visto afectada. En la condición inicial, $T_{u}$ tiende a decrecer cuando se reduce la turbulencia en la condición de entrada. Sin embargo, la intensidad de turbulencia tiende a un valor en torno a 0.03 cuando existe suficiente distancia de caída desde el vertedero.

Respecto al coeficiente del parámetro turbulento $K_{\varphi}$, se han obtenido los valores para el caso bidimensional y tridimensional. La Figura 116 muestra los resultados obtenidos considerando diferentes caudales e intensidades turbulentas en la condición de entrada.

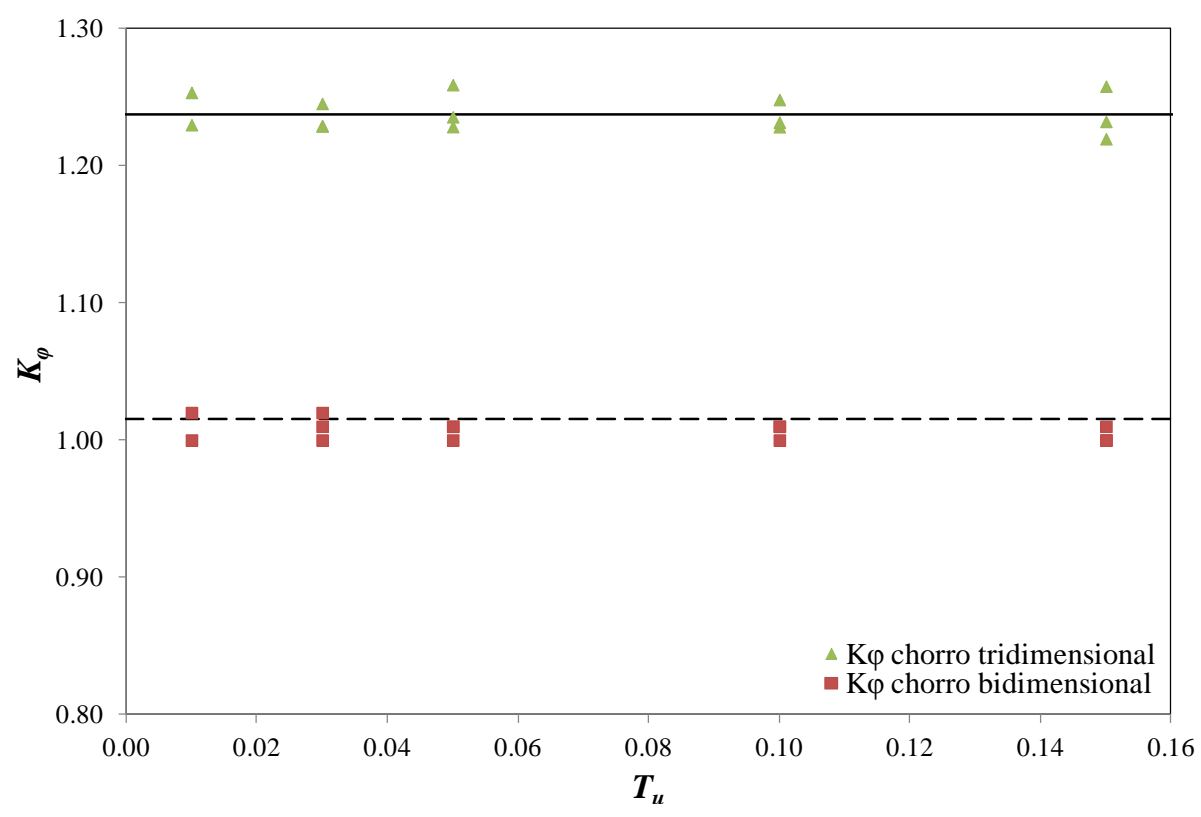

Figura 116. Coeficiente del parámetro turbulento $K_{\varphi}$ en función de la intensidad turbulenta en la condición inicial (Castillo et al., 2014). 
Para el caso bidimensional de chorro rectangular $K_{\varphi} \approx 1.01$. La velocidad turbulenta lateral está en torno a $0.18 v^{\prime}$, en vez de el valor de $0.38 v^{\prime}$ obtenido por Ervine y Falvey (1987) en chorros circulares. Para el chorro tridimensional rectangular, la velocidad turbulenta lateral está en torno a $0.18 v^{\prime}$, mientras que la velocidad turbulenta transversal es $0.71 v^{\prime}$, y $K_{\varphi} \approx 1.24$. De este modo, la expansión lateral en chorro rectangular tridimensional puede ser estimada como:

$$
\xi=k T_{u} V_{i} t=1.24 v^{\prime}
$$

\subsubsection{Estudio de la aireación y la velocidad del chorro}

Mediante equipo de fibra óptica se ha analizado la velocidad y la tasa de aireación en distintas secciones del chorro. Los datos obtenidos se han comparado con las simulaciones numéricas realizadas en ANSYS CFX y FLOW-3D.

La Figura 117 muestra los resultado de la lámina libre del chorro obtenidos con los dos programas de CFD para $q=0.058 \mathrm{~m}^{2} / \mathrm{s}$. Ambos programas obtienen una lámina libre similar a la observada en laboratorio.
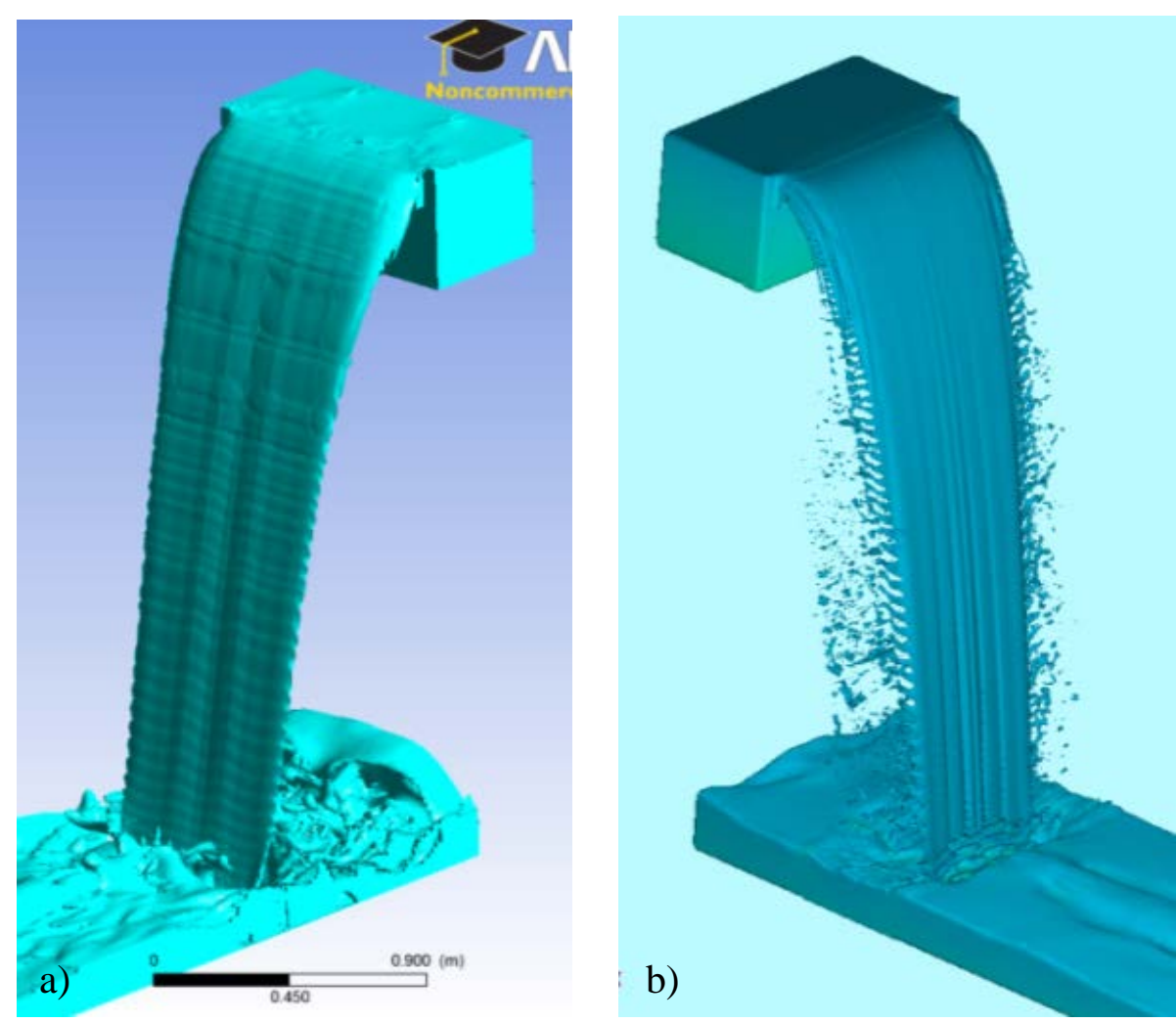

Figura 117. Lámina libre del chorro para $q=0.058 \mathrm{~m}^{2} / \mathrm{s}$ : a) ANSYS CFX; b) FLOW-3D (Castillo y Carrillo, 2012). 
La Figura 118 muestra las velocidades medidas y calculadas sobre una línea de corriente. ANSYS CFX sigue la evolución de las velocidades obtenidas con el equipo de fibra óptica. Por su parte, FLOW-3D obtiene velocidades erróneas ligeramente superiores a la velocidad teórica de caída libre, $V=\sqrt{2 g z}$, siendo $z$ la distancia de caída.

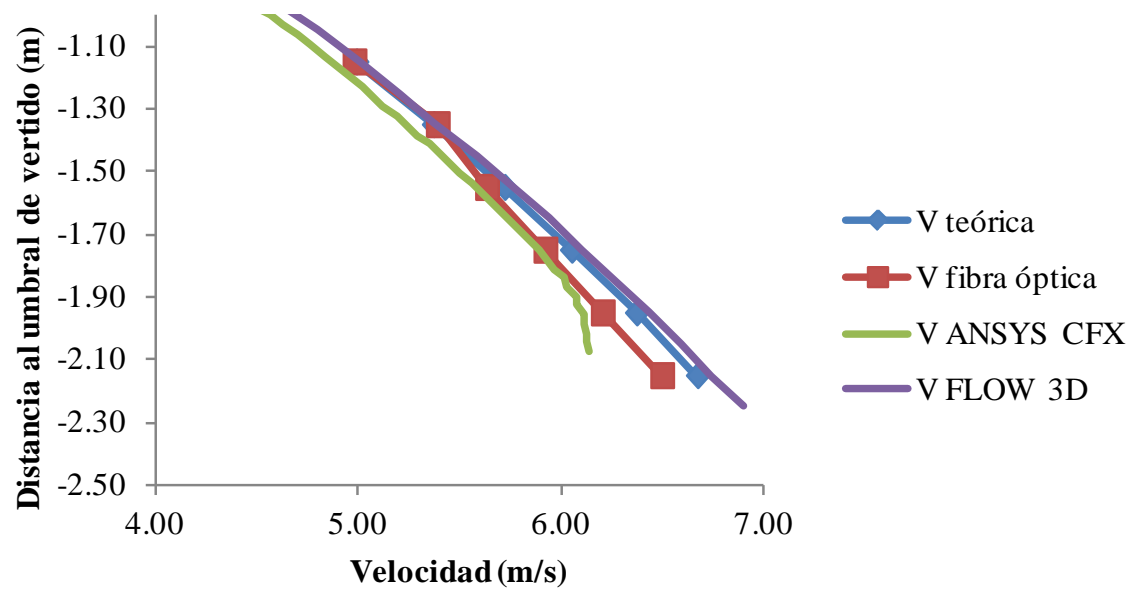

Figura 118. Velocidad en el chorro para $q=0.058 \mathrm{~m}^{2} / \mathrm{s}$ (Castillo y Carrillo, 2012).

La Figura 119 muestra la fracción volumétrica de agua $r_{\text {agua }}$ obtenida sobre una línea de corriente. ANSYS CFX sigue la forma observada con la fibra óptica, aunque considera que se produce una mayor entrada de aire en el chorro. Si bien, los resultados cualitativos son esperanzadores, las diferencias cuantitativas pueden ser debidas al empleo de un mallado excesivamente grueso para que el método Euleriano-Euleriano pueda ser capaz de reproducir la entrada de aire de modo correcto. El modelo homogéneo empleado no permite actuar sobre ningún parámetro de corrección de la aireación al no considerar la interacción entre las dos fases del flujo bifásico (agua-aire). Sería conveniente intentar abordar la aireación del chorro con un modelo inhomogéneo, el cual calcula la interacción entre las fases, aunque su coste computacional es mucho mayor.

FLOW-3D falla en el modelo de entrada de aire. Según han comentado los distribuidores de FLOW-3D consultados, en las últimas versiones se han realizado cambios en los modelos de turbulencia, de modo que el modelo de entrada de aire ha dejado de ser independiente de la malla. Una de las prioridades de Flow Science Inc. es remodelar el código para evitar que este efecto indeseable ocurra.

Al realizar las simulaciones de los caudales intermedios y bajos $(0.037$ y 0.023 $\mathrm{m}^{2} / \mathrm{s}$ ) con FLOW-3D se observó que se produce la rotura del chorro en puntos muy 
cercanos al umbral de vertido cuando se emplea un tamaño de malla de $0.005 \mathrm{~m}$. La Figura 120 muestra la rotura del chorro obtenida con el caudal $q=0.037 \mathrm{~m}^{2} / \mathrm{s}$, donde se observa que el modelo de turbulencia $k-\varepsilon$ tiende a producir la rotura del chorro un poco antes que el modelo RNG.

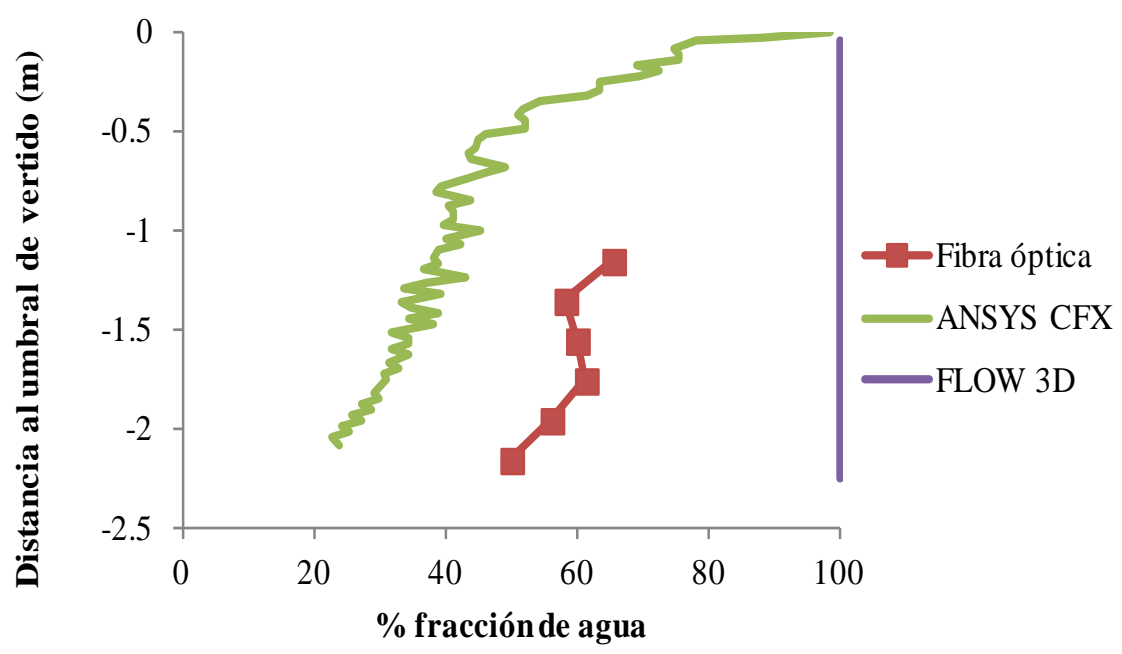

Figura 119. Fracción volumétrica de agua en el chorro $\left(r_{\text {agua }}\right)$ para $q=0.058 \mathrm{~m}^{2} / \mathrm{s}$ (Castillo y Carrillo, 2012).

a)

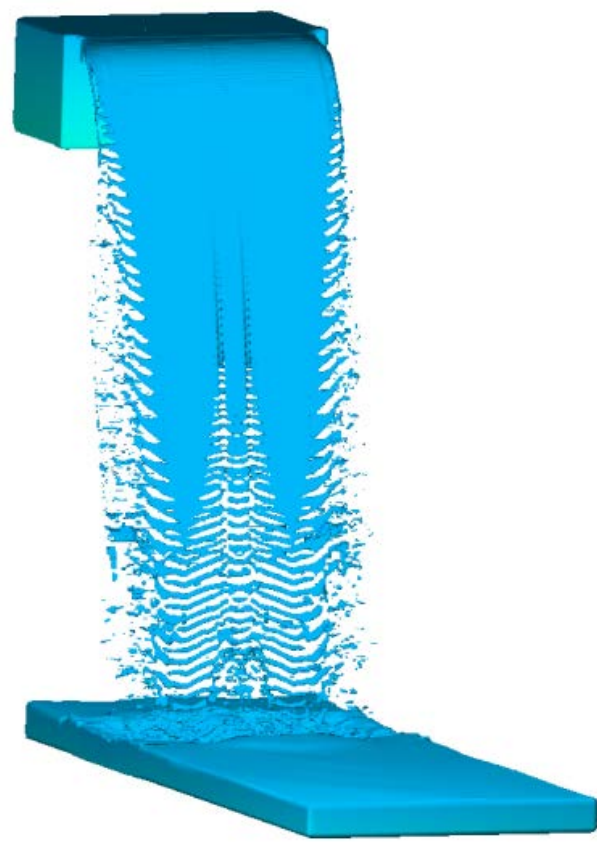

b)

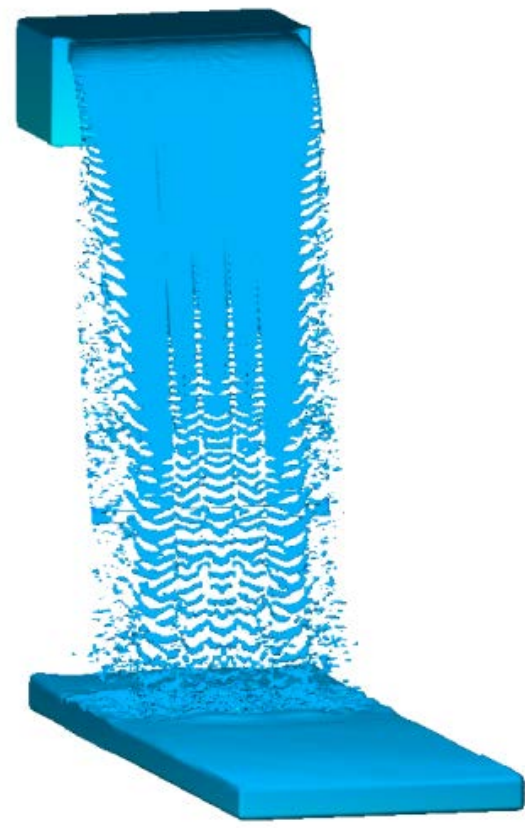

Figura 120. Lámina libre del chorro obtenida con FLOW-3D $\left(q=0.037 \mathrm{~m}^{2} / \mathrm{s}, H=2.30 \mathrm{~m}, \mathrm{~h}=\right.$ $0.073 \mathrm{~m}, Y=0.12 \mathrm{~m}$ ): a) Simulación con RNG; b) Simulación con $k-\varepsilon$ (Castillo y Carrillo, 2012). 
La Figura 121 muestra la lámina libre obtenida con ANSYS CFX empleando un tamaño de mallado de $0.01 \mathrm{~m}$, y FLOW-3D considerando dos tamaños de mallado (0.005 y $0.003 \mathrm{~m}$ ). Se observa que FLOW-3D mejora la simulación de la lámina libre al disminuir el tamaño de los elementos de la malla. Sin embargo, esta disminución aumenta el esfuerzo computacional y el tiempo requerido. De este modo, frente a las 2.0 horas que se requieren en ANSYS CFX para resolver un transitorio de 5 segundos de la lámina vertiente, FLOW-3D requiere 5.5 horas con el mallado de 0.005 m y 7.3 horas con el mallado de $0.003 \mathrm{~m}$.
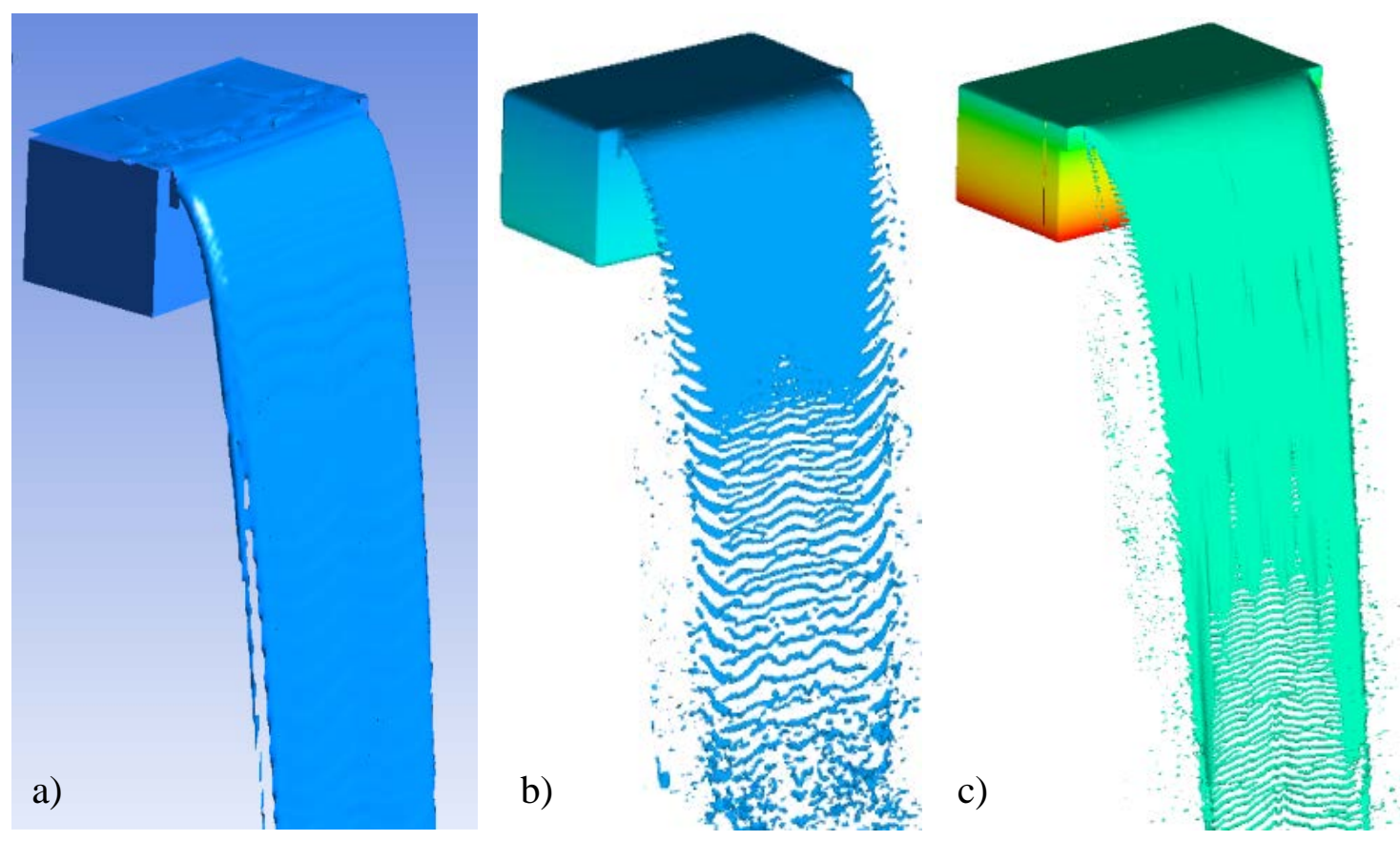

Figura 121. Lámina libre del chorro para $q=0.023 \mathrm{~m}^{2} / \mathrm{s}$ : a) ANSYS CFX; b) FLOW-3D con mallado de 0.005 m; c) FLOW-3D con mallado de 0.003 m (Castillo y Carrillo, 2012).

La Figura 122 muestra la fracción volumétrica de agua obtenida con los dos tamaños de mallado en FLOW-3D. La rotura del chorro ocurre cuando el espesor del chorro es similar a dos veces el tamaño del mallado.

La Figura 123 muestra las velocidades obtenidas con los dos paquetes informáticos sobre una línea de corriente para $q=0.023 \mathrm{~m}^{2} / \mathrm{s}$. Al igual que con el caudal grande, ANSYS CFX sigue la evolución de las velocidades obtenida con el equipo de fibra óptica, mientras que FLOW-3D obtiene velocidades ligeramente superiores a la velocidad teórica de caída libre. Los errores de truncamiento se disparan cuando se produce la rotura del chorro en FLOW-3D, imposibilitando obtener cualquier correlación con un experimento físico. 

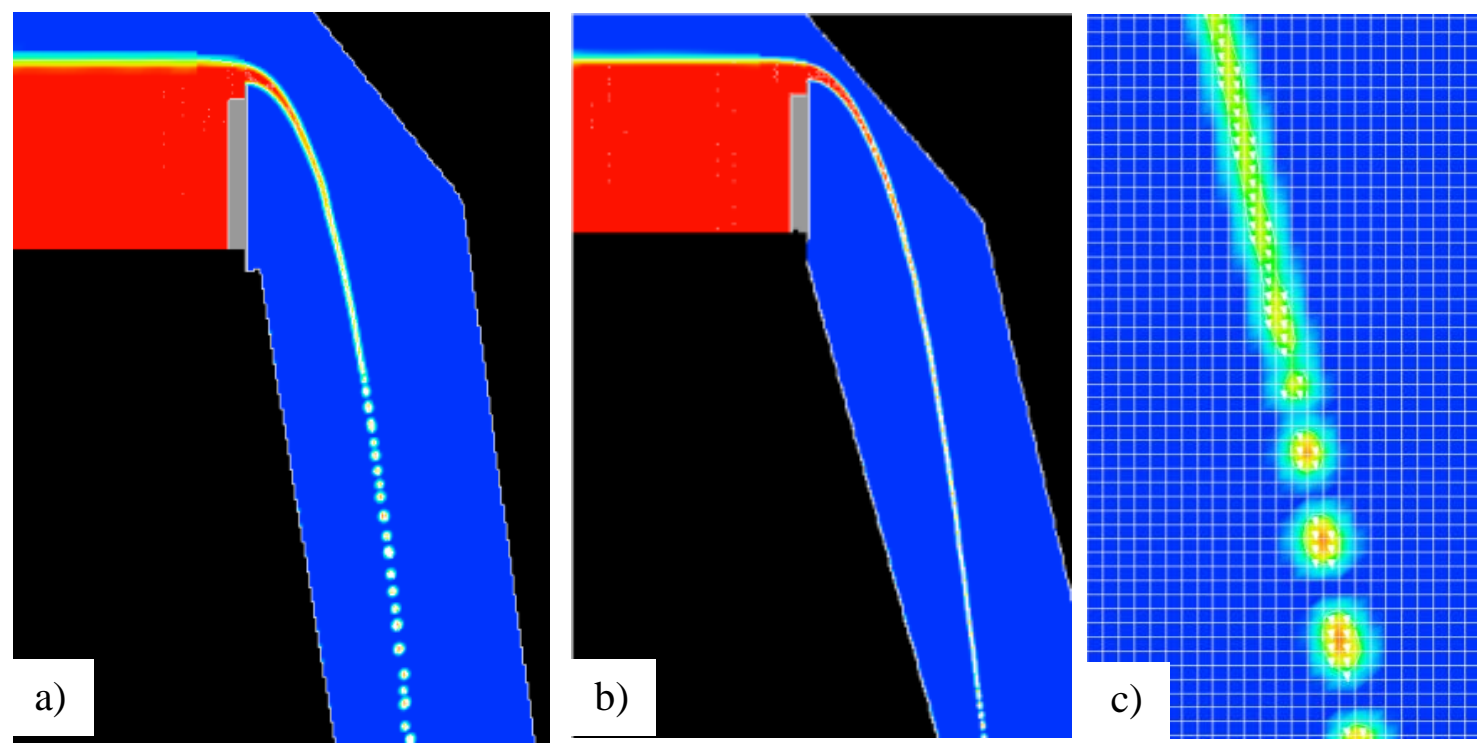

Figura 122. Fracción volumétrica de agua $r_{\text {agua }}$ para $q=0.023 \mathrm{~m}^{2} / \mathrm{s}$ : a) $\mathrm{FLOW}-3 \mathrm{D}$ con mallado de $0.005 \mathrm{~m}$; b) FLOW-3D con mallado de $0.003 \mathrm{~m}$; c) Detalle de la rotura del chorro (Castillo y Carrillo, 2012).
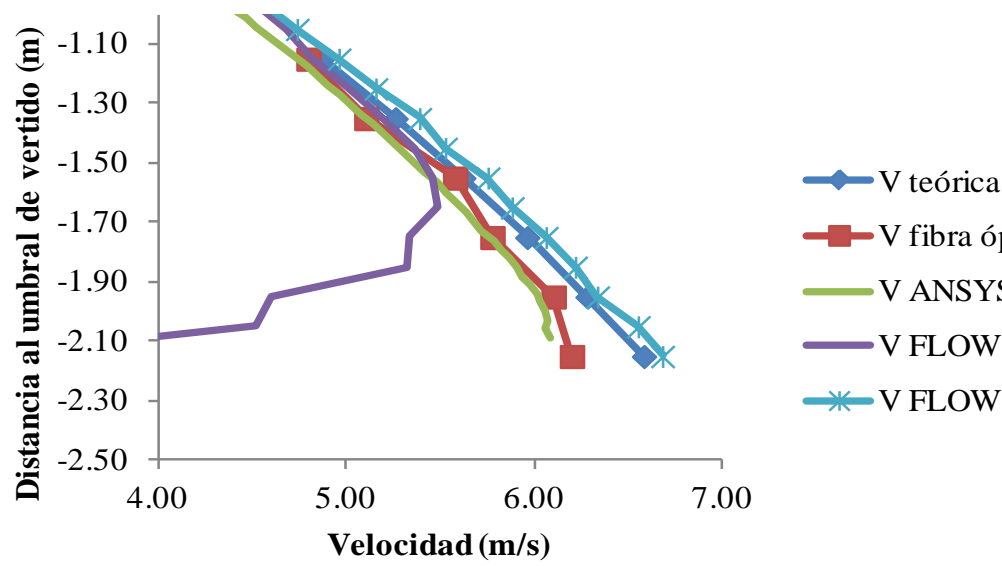

- V fibra óptica

$\longrightarrow$ V ANSYS CFX

$\longrightarrow$ FLOW 3D

*V FLOW 3D (3mm)

Figura 123. Velocidad en el chorro para $q=0.023 \mathrm{~m}^{2} / \mathrm{s}$ (Castillo y Carrillo, 2012).

La Figura 124 muestra la fracción volumétrica de agua obtenida sobre una línea de corriente para $q=0.023 \mathrm{~m}^{2} / \mathrm{s}$. Al igual que en el caso anterior, ANSYS CFX sigue la forma observada con la fibra óptica, aunque considera que se produce una mayor entrada de aire en el chorro. Por su parte, FLOW-3D no consigue reproducir la entrada la entrada de aire en el chorro hasta que se alcanza su rotura. 


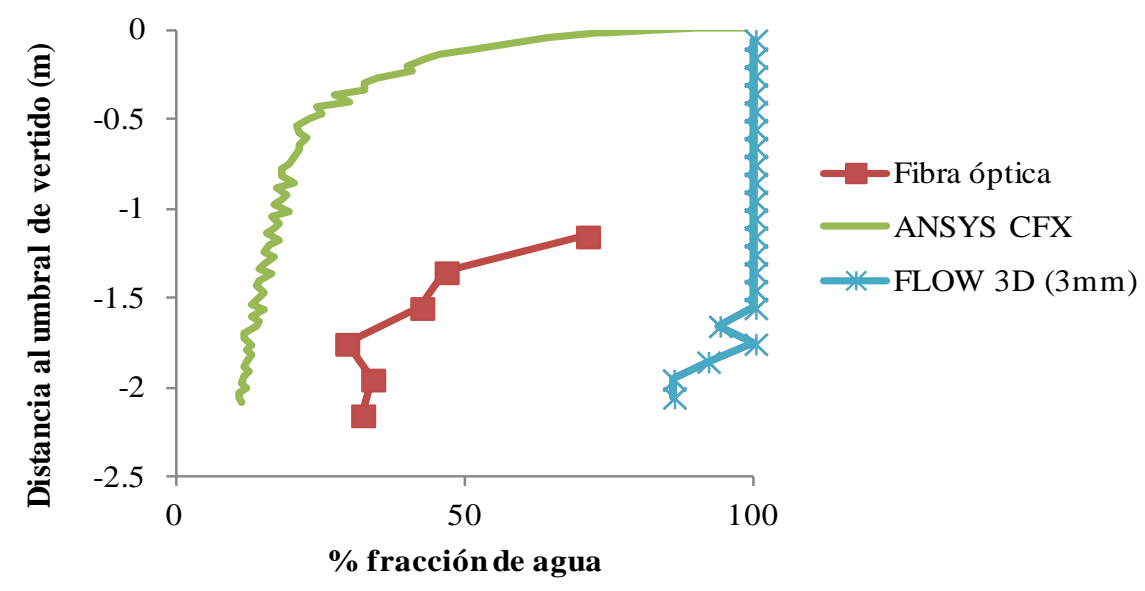

Figura 124. Fracción volumétrica de agua en el chorro $r_{\text {agua }}$ para $q=0.023 \mathrm{~m}^{2} / \mathrm{s}$ (Castillo y Carrillo, 2012).

Debido a la problemática observada en la simulación del chorro con FLOW-3D, se consideró conveniente valorar hasta qué punto influye la no alineación del chorro con el mallado en el esquema de diferencias finitas. En la Figura 125 se considera un mismo chorro con dos orientaciones distintas de la velocidad inicial y del vector gravedad.
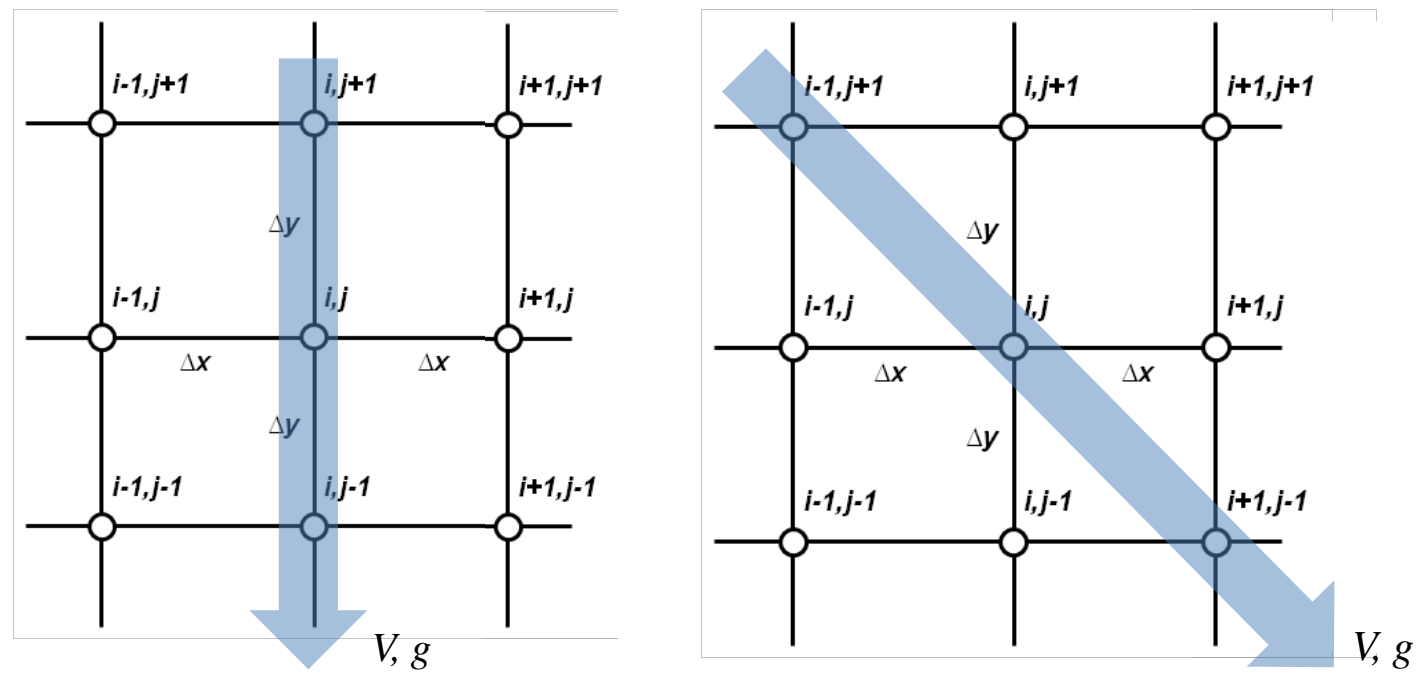

Figura 125. Orientación del chorro sobre el esquema de diferencias finitas (Castillo y Carrillo, 2012).

Para obtener las aproximaciones de segundo orden, el esquema de diferencias finitas emplea las siguientes expresiones: 


$$
\begin{gathered}
\left(\frac{\partial^{2} u}{\partial x^{2}}\right)_{i, j}=\frac{u_{i+1, j}-2 u_{i, j}+u_{i-1, j}}{(\Delta x)^{2}} \\
\left(\frac{\partial^{2} u}{\partial y^{2}}\right)_{i, j}=\frac{u_{i, j+1}-2 u_{i, j}+u_{i, j-1}}{(\Delta y)^{2}} \\
\left(\frac{\partial^{2} u}{\partial x \partial y}\right)_{i, j}=\frac{u_{i+1, j+1}-u_{i+1, j-1}-u_{i-1, j+1}+u_{i-1, j-1}}{4 \Delta x \Delta y}
\end{gathered}
$$

Según estas expresiones, el chorro queda definido por los nodos situados en la línea horizontal o vertical cuando el chorro está orientado con la malla. Sin embargo, en caso de un chorro oblicuo se emplean elementos fuera de la diagonal, por lo que para obtener resultados similares en el eje del chorro se requiere un mayor espesor.

La Figura 126 muestra los resultados obtenidos en una simulación de un chorro alineado con la malla. Se produce una distribución adecuada de caída libre sin que se produzca su rotura pese alcanzarse espesores similares a dos veces el tamaño de los elementos del mallado en la parte inferior del gráfico. Sin embargo, la entrada de aire en el chorro es muy reducida.

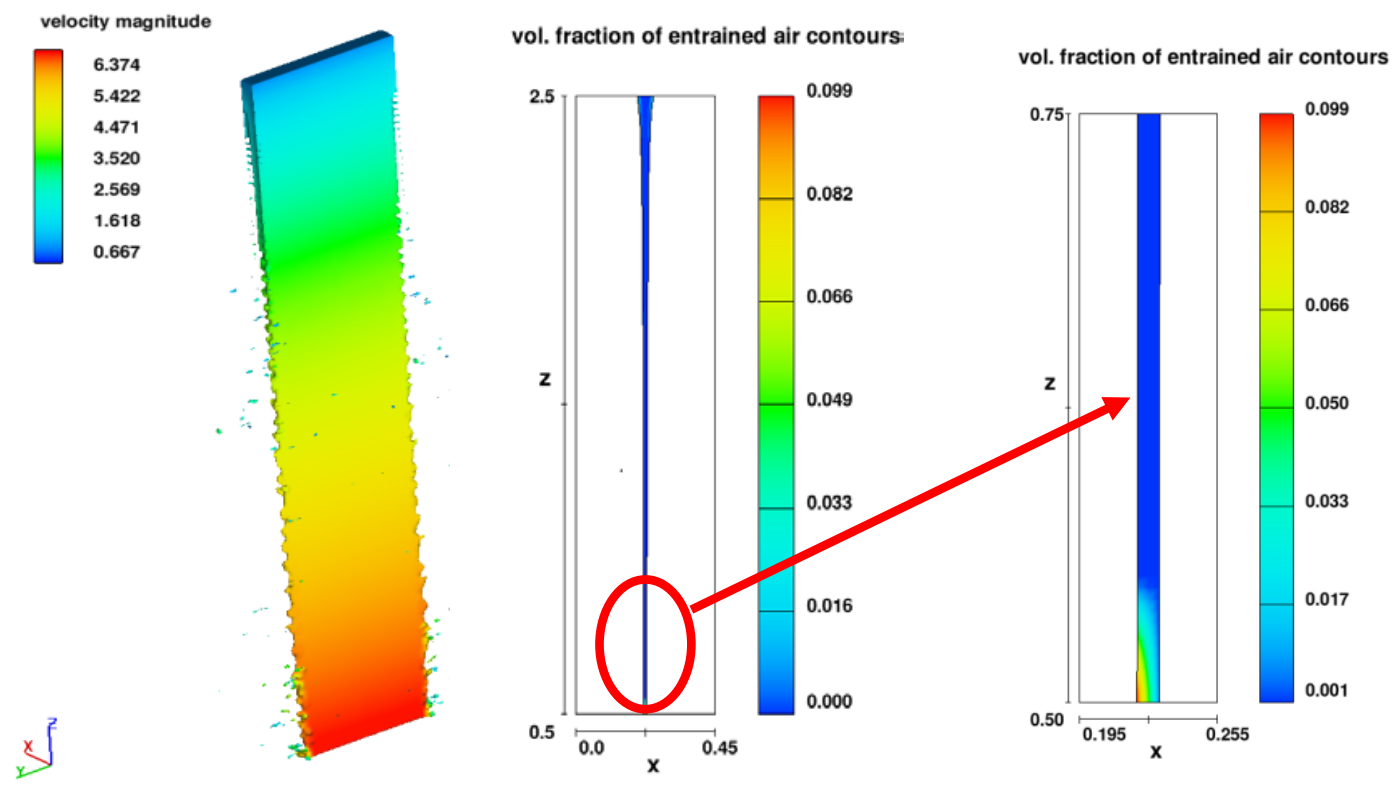

Figura 126. Chorro alineado con la malla en FLOW-3D (Castillo y Carrillo, 2012).

La Figura 127 muestra los resultados de un chorro que no está alineado con el mallado. Las perturbaciones que empiezan a aparecer aproximadamente a $1 \mathrm{~m}$ de 
iniciarse la caída, produciéndose la rotura del chorro a una distancia aproximada de 1.35 $\mathrm{m}$, frente a los 2 metros de caída sin perturbaciones que muestra el caso anterior.
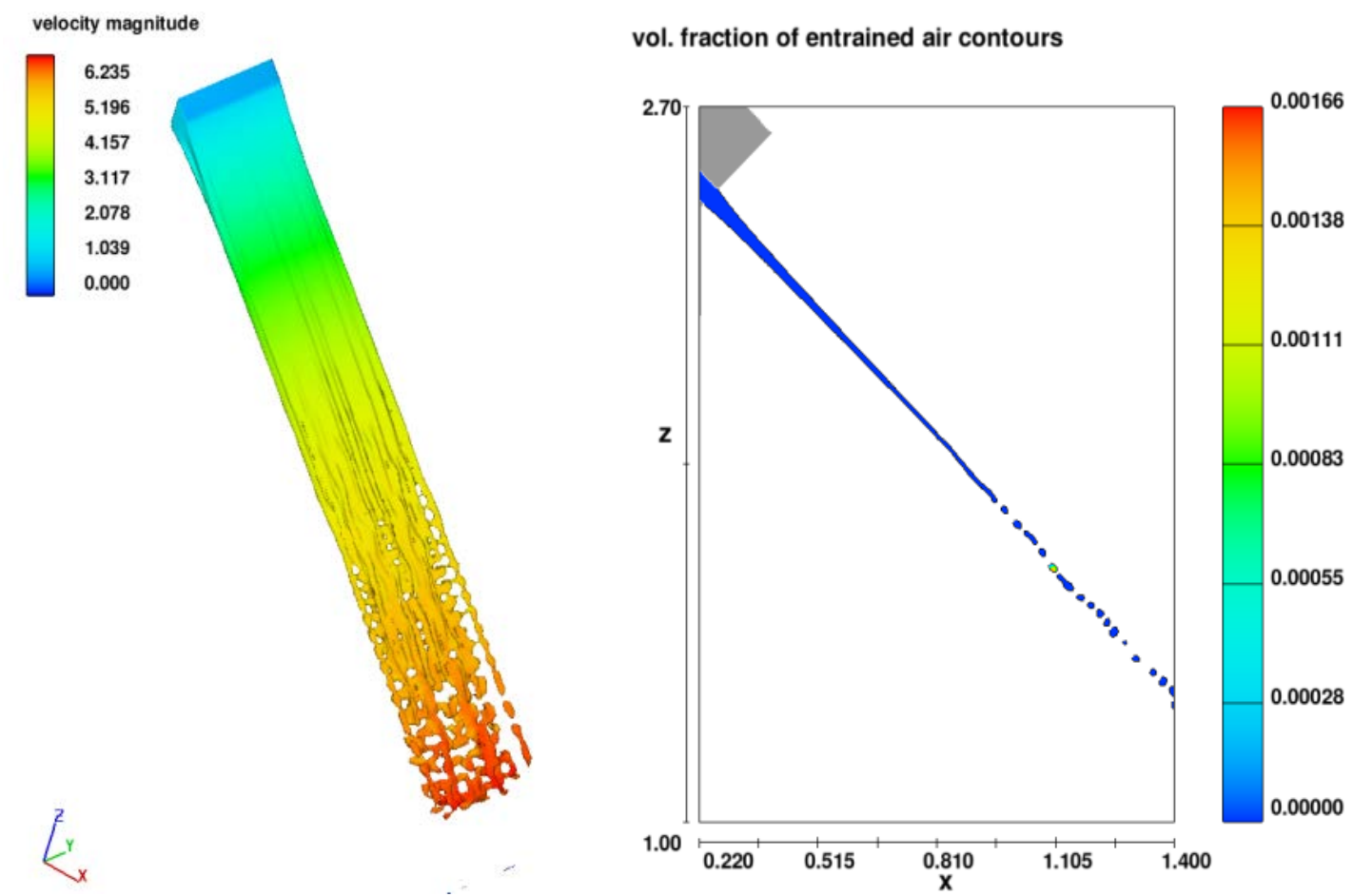

Figura 127. Chorro oblicuo a la malla en FLOW-3D (Castillo y Carrillo, 2012).

En el sobrevertido de presas se generan chorros que parten de una componente prácticamente horizontal en el punto de vertido y alcanzan el colchón de agua con una componente predominantemente vertical, por lo que el chorro en la fase de vuelo pasa por situaciones en que no está alineado con la dirección del mallado.

Para que no se produzca la rotura del chorro por fenómenos numéricos es necesario emplear un tamaño de mallado 2-3 veces menor que el espesor del chorro en chorros alineados con el mallado. Sin embargo, debido al esquema de diferencias finitas de segundo orden, en chorros oblicuos puede ser necesario emplear tamaños de mallado 5-6 veces menor que el espesor del chorro. Considerando que los espesores del chorro incidente en laboratorio están entre 0.025 y $0.01 \mathrm{~m}$, para su correcto cálculo se requerirían tamaños de mallado de entre 0.003 y $0.002 \mathrm{~m}$, incrementándose el coste computacional de la simulación.

Como se observa en las figuras anteriores, la reducción del tamaño del mallado no soluciona el problema de la aireación existente en la versión actual de FLOW-3D. Esto conlleva a que el chorro se resuelve sin considerar entrada de aire. Al no reproducir la 
zona emulsionada se obtienen menores espesores y no se produce la disipación de energía por aireación.

Para intentar acotar este problema, se ha realizado un análisis de sensibilidad de los modelos de aireación, y de turbulencia y del tamaño del mallado. Realizando una simulación de los $5 \mathrm{~cm}$ centrales del aliviadero de vertido libre, se puede analizar el funcionamiento de FLOW-3D. Considerado un mallado de $2 \mathrm{~mm}$ en todo el vuelo del chorro, la Figura 128 muestra la fracción volumétrica de agua del chorro vertiente, así como la entrada de aire en el tramo final de la caída, considerando el mallado de $2 \mathrm{~mm}$. Se observa que el chorro apenas se airea durante la caída.
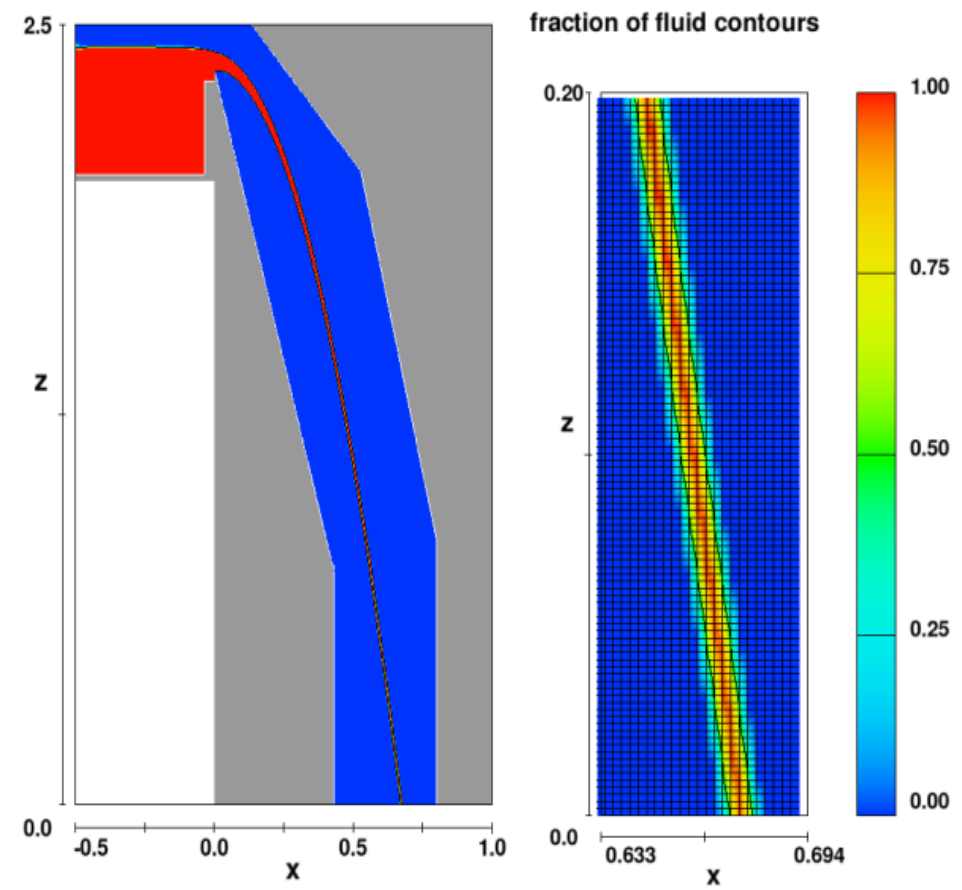

vol. fraction of entrained air contours

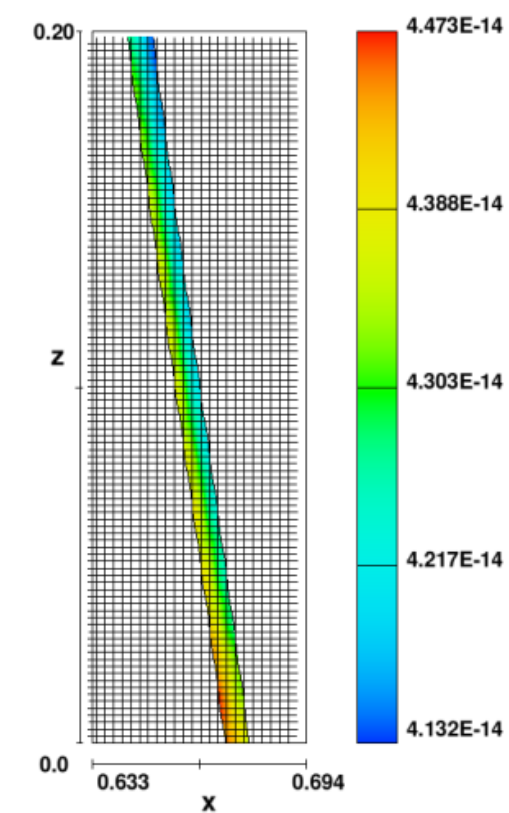

Figura 128. Fracción volumétrica de agua en el chorro vertiente simulada con FLOW-3D.

Si se analiza la energía cinética turbulenta en el chorro (Figura 129), se observa que las mayores zonas de energía cinética se encuentran en el labio del vertedero, mientras que en el vuelo se tiende a disipar la energía cinética turbulenta.

FLOW-3D no resuelve la mecánica de fluidos de la fase gaseosa, sino que sustituye su cálculo por condiciones de contorno de presión en las regiones de la lámina libre (FLOW Science, Inc., 2011). Al no existir contornos sólidos, y no aparecer la interacción con la fase gaseosa, el programa no es capaz de generar energía cinética turbulenta en el vuelo, por lo que no se produce el proceso de aireación.

Con los resultados observados en FLOW-3D, se consideró adecuado conocer la precisión del esquema numérico al resolver un problema de flujo bifásico distinto de los chorros turbulentos. Considerando los resultados obtenidos por Chanson y Brattberg 
(2000), se realizó la simulación numérica de un resalto hidráulico de iguales condiciones con FLOW-3D. Las condiciones de contorno se corresponden con el calado al inicio del resalto $y_{1}=0.014 \mathrm{~m}$, la velocidad de entrada $V_{1}=2.58 \mathrm{~m} / \mathrm{s}$, y el calado conjugado del resalto $y_{2}=0.119 \mathrm{~m}$. El número de Froude que caracteriza el resalto es $\mathrm{Fr}$ $=6.33$. Se han empleado los módulos de entrada de aire en el fluido, y se ha considerado con un tamaño de elementos de $0.01 \mathrm{~m}$.
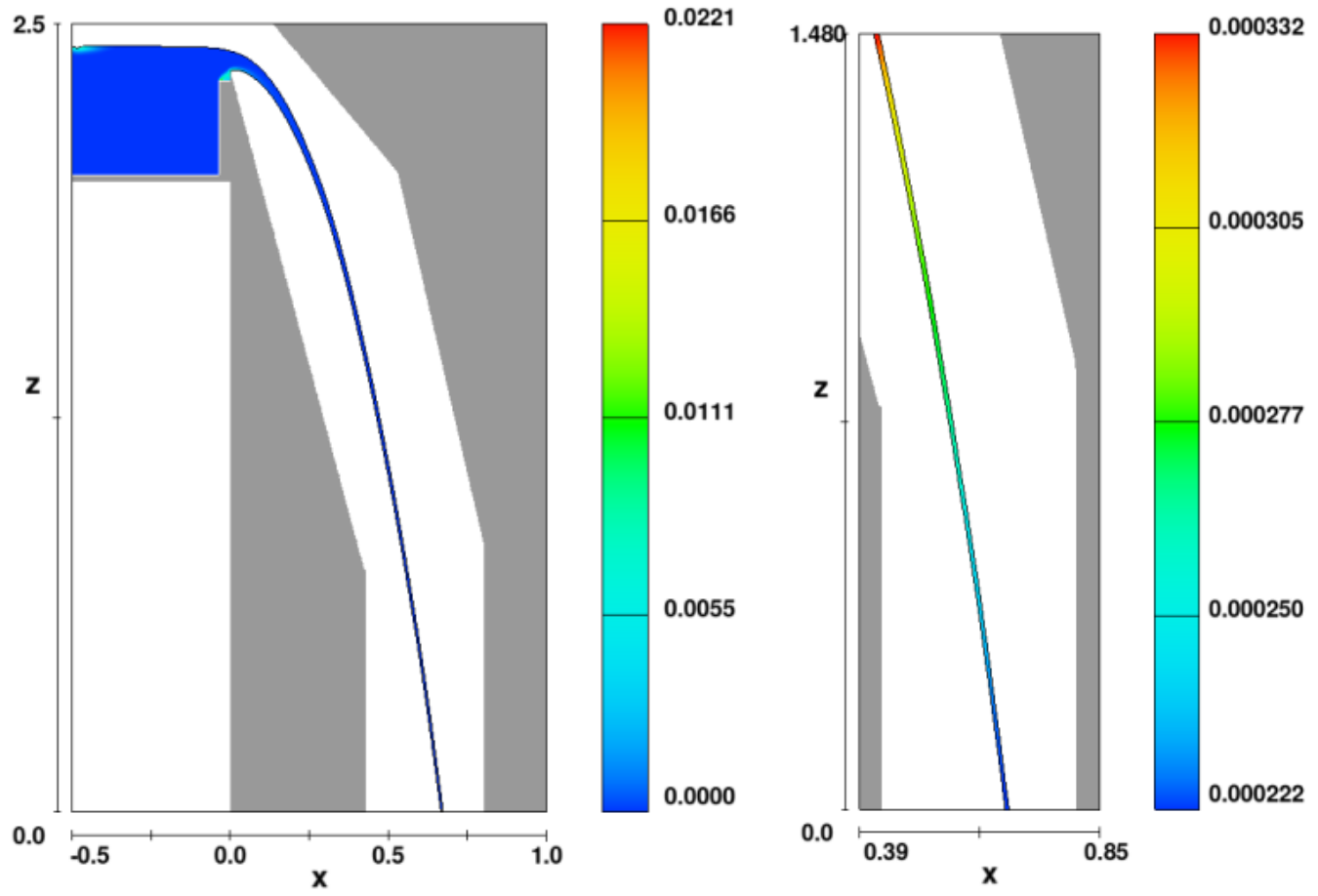

Figura 129. Energía cinética turbulenta en el chorro vertiente simulada con FLOW-3D.

La Figura 130 muestra los vectores de velocidad que el programa resuelve. La zona de flujo nulo en la parte central del resalto y la zona de recirculación en la parte superior del mismo quedan bien definidas.

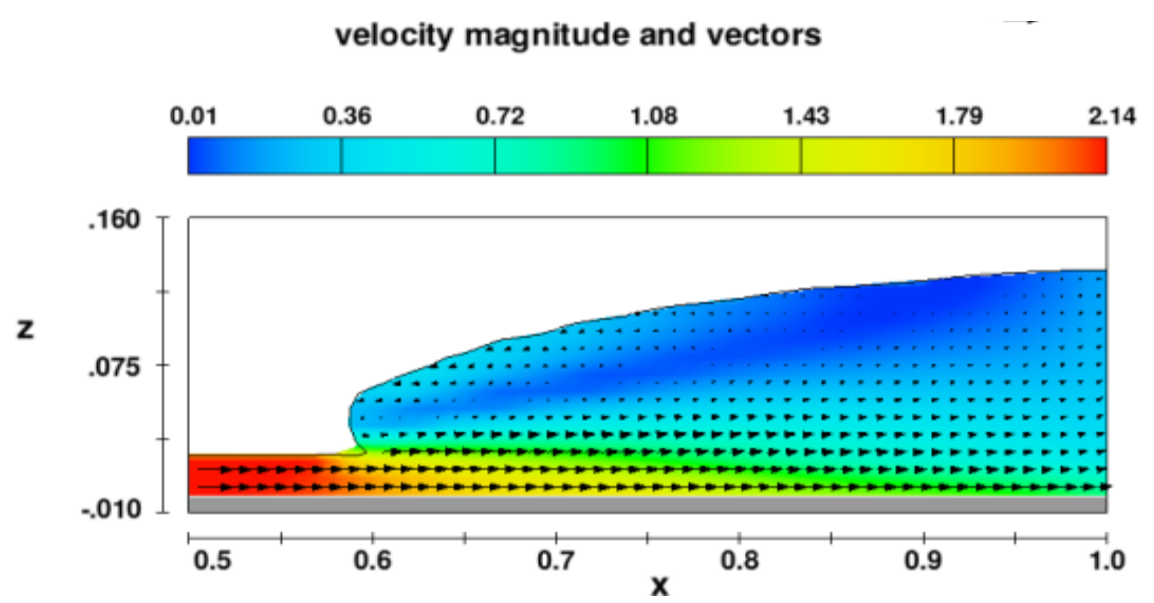

Figura 130. Vectores de velocidad en un resalto hidráulico simulado con FLOW-3D. 
La zona de mayor energía cinética turbulenta se concentra en la parte inicial de aguas arriba del resalto, localizándose en mitad de la columna de agua (Figura 131). Aguas abajo del resalto, la energía cinética tiende a disiparse.

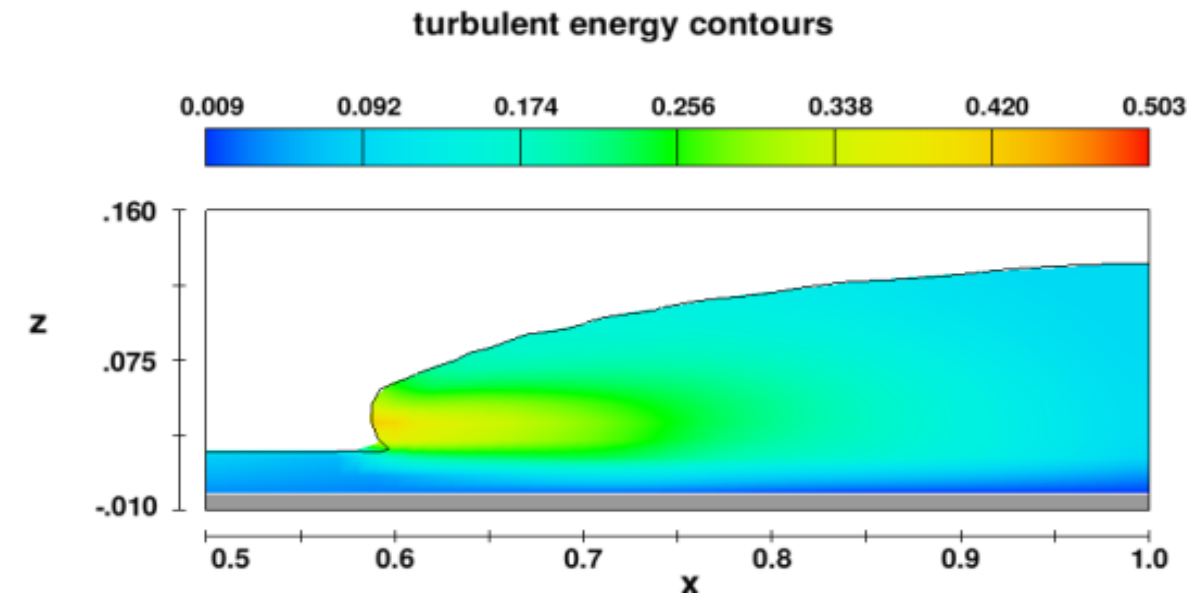

Figura 131. Energía cinética turbulenta en un resalto hidráulico simulado con FLOW-3D.

En este caso el modelo de entrada de aire sí que interacciona introduciendo aire en el líquido. La concentración de aire $C$ simulada alcanza el 50\% en la parte superior del resalto, mientras que la parte inferior del mismo está poco aireado (Figura 132).

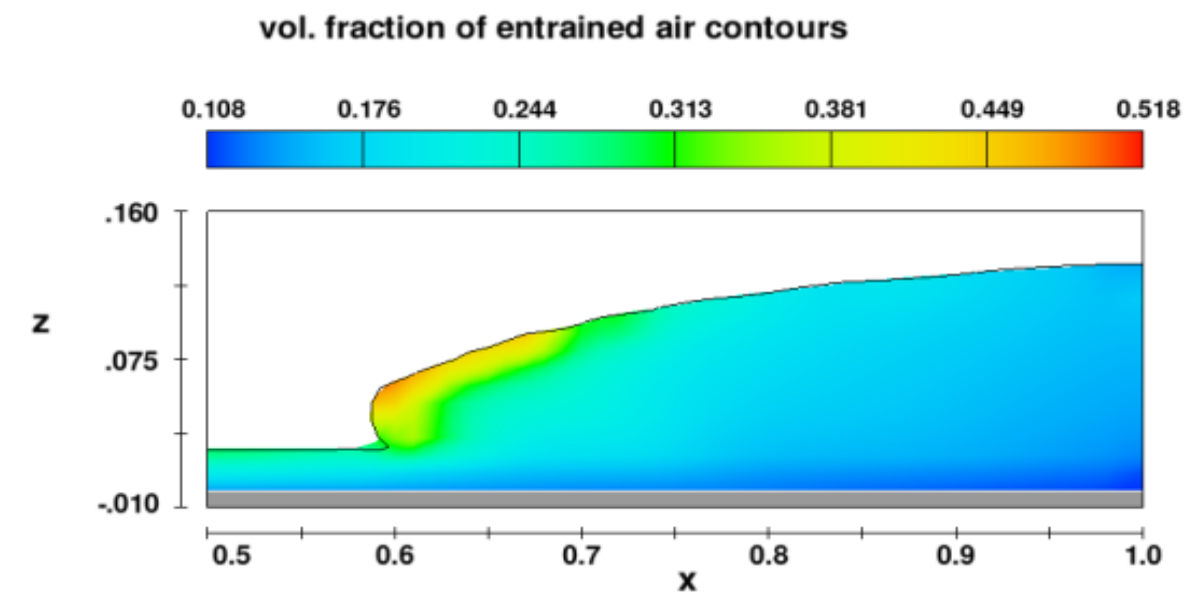

Figura 132. Fracción volumétrica de aire introducido en un resalto hidráulico simulado con FLOW-3D.

En la Figura 133 se comparan los perfiles de velocidad y concentración de aire obtenidos en las secciones transversales localizadas a 0.05 y $0.20 \mathrm{~m}$ del inicio del resalto, respectivamente. La simulación numérica se compara con los resultados de laboratorio obtenidos por Chanson y Brattberg (2000). 

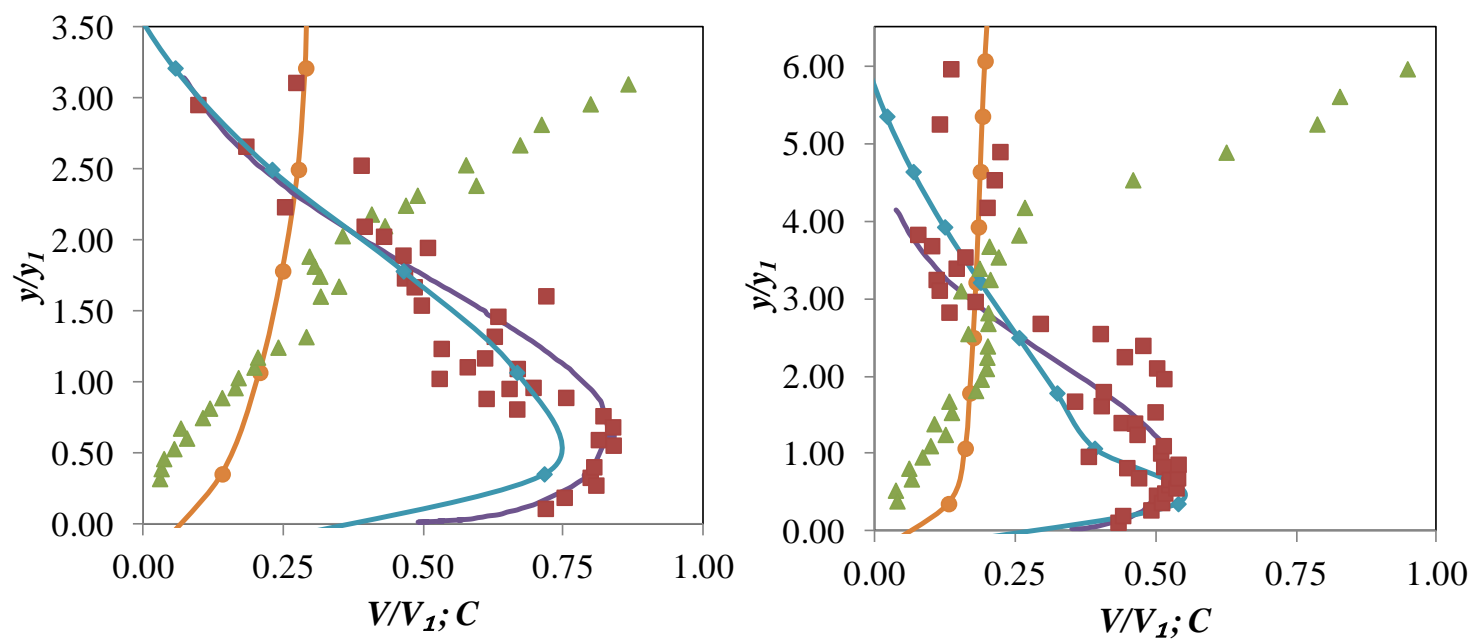

$\Delta$ Concentración aire; Chanson y Brattberg (2000) -Concentración aire; FLOW-3D V/V 1 ; Othsu et al. (1990)

a)

Figura 133. Comparación de resultados de laboratorio y numéricos en un resalto hidráulico: a) Sección a 0.05 m del inicio del resalto; a) Sección a 0.20 m del inicio del resalto.

En ambos casos, las velocidades adimensionalizadas $\left(V / V_{1}\right)$ de la simulación numérica son similares a los resultados de laboratorio, y al perfil teórico empleado por los autores, inicialmente propuesto por Othsu et al. (1990). En lo referente a la aireación, no se han obtenido en general buenos resultados. Los resultados concuerdan en la parte inferior del resalto hasta alcanzar valores de concentración de aire del 25\%. Las mayores diferencias se producen en la mitad superior del resalto, donde la variación de la lámina libre es muy acusada.

\subsubsection{5. $\quad$ Estudio del chorro + cuenco de disipación}

\subsection{Análisis en función del tamaño del mallado}

Con el fin de conocer su efecto sobre las distintas variables en ANSY CFX, se realizaron simulaciones con diferentes tamaños de mallado. La Tabla 31 muestra el número total de elementos hexaédricos empleados en cada simulación cuando se consideran diferentes tamaños de elementos en el entorno del chorro y del punto de estancamiento.

\begin{tabular}{|c|c|c|c|c|}
\hline Tamaño de mallado (mm) & 25.0 & 15.0 & 12.5 & 10.0 \\
\hline Número de elementos & 48854 & 160308 & 281586 & 456506 \\
\hline
\end{tabular}

Tabla 31. Número de elementos en función del tamaño del elemento de mallado empleado. 
Siguiendo una línea de corriente que parte del vertedero, la Figura 134 muestra la velocidad del flujo durante la caída del chorro cuando se consideran diferentes tamaños de elementos, mientras que la Figura 135 grafica la fracción volumétrica de aire. Los resultados se comparan con los valores medidos en laboratorio con un equipo de fibra óptica.
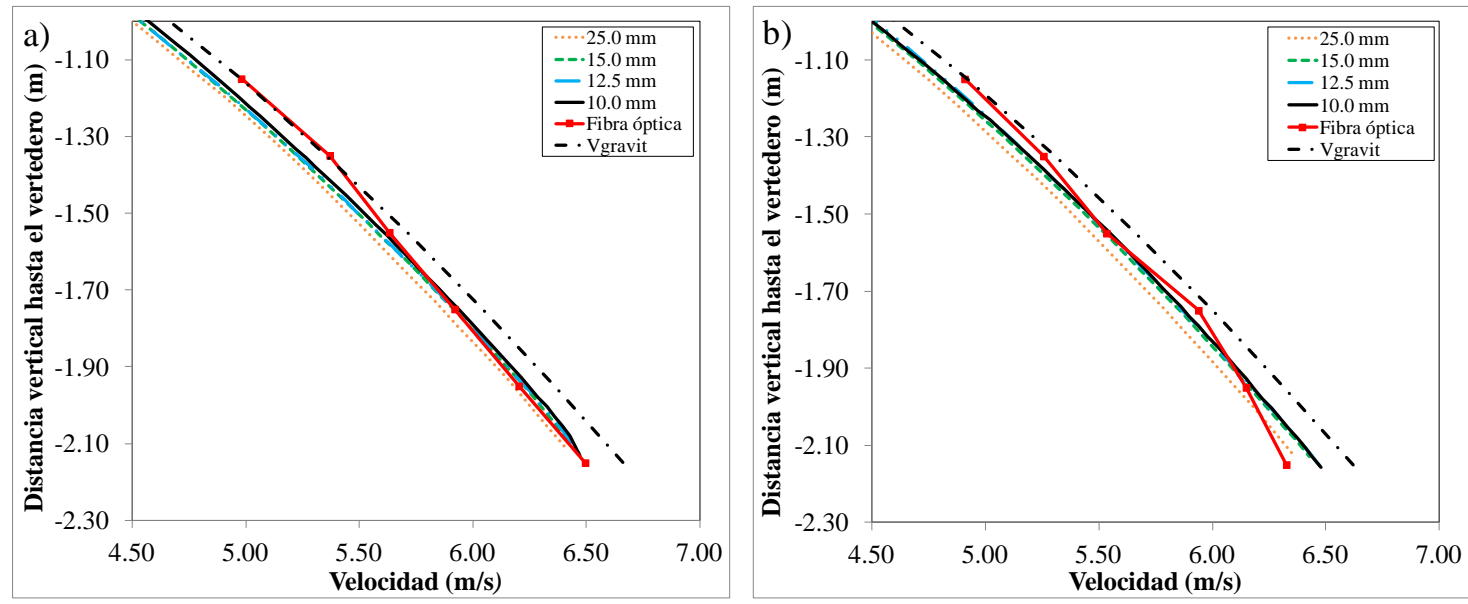

Figura 134. Velocidad del flujo en el chorro vertiente en función del tamaño de los elementos del mallado: a) $q=0.058 \mathrm{~m}^{2} / \mathrm{s}, h=0.095 \mathrm{~m}$; b) $q=0.037 \mathrm{~m}^{2} / \mathrm{s}, h=0.073 \mathrm{~m}$ (Castillo et al., 2014).
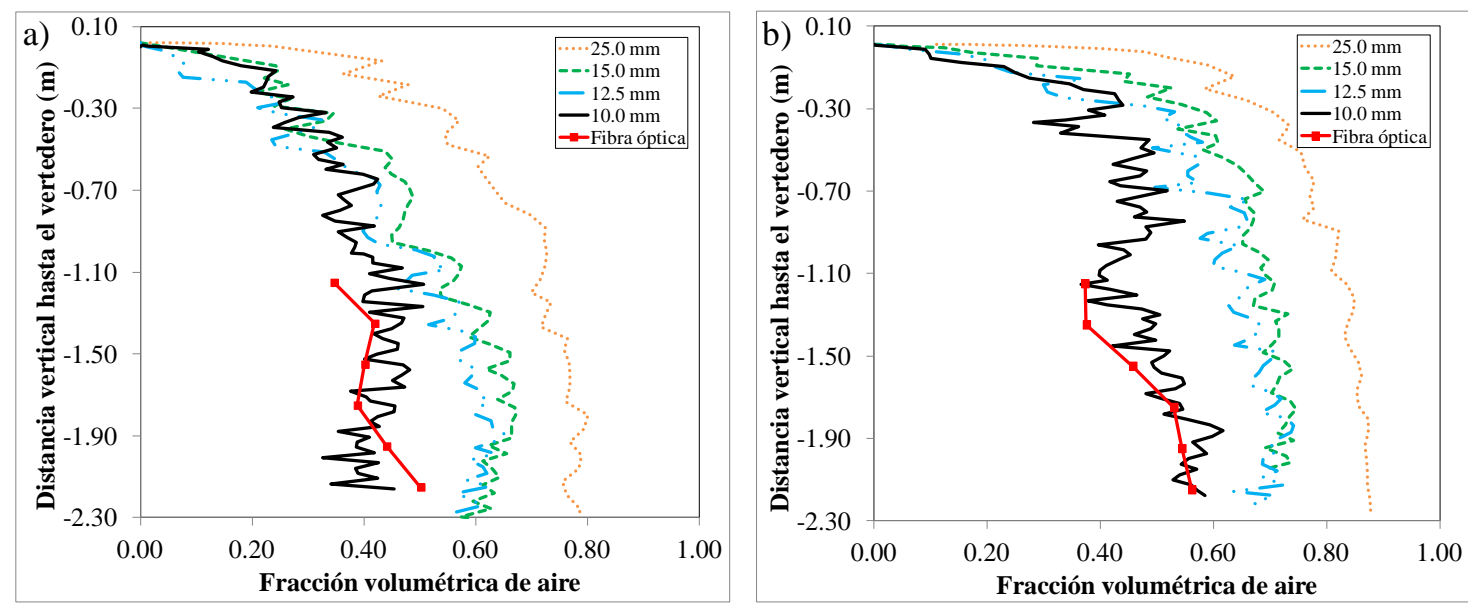

Figura 135. Fracción volumétrica de aire en el chorro vertiente en función del tamaño de los elementos del mallado: a) $q=0.058 \mathrm{~m}^{2} / \mathrm{s}, h=0.095 \mathrm{~m}$; b) $q=0.037 \mathrm{~m}^{2} / \mathrm{s}, h=0.073 \mathrm{~m}$ (Castillo et al., 2014).

El tamaño de los elementos del mallado tiene poca influencia en la velocidad del chorro vertiente. La velocidad obtenida en las simulaciones CFD y medida con el equipo de fibra óptica tendió a ser ligeramente inferior que la velocidad de caída libre 
debida exclusivamente al efecto de la gravedad, lo que está relacionado con el efecto de la fricción del aire en el flujo bifásico.

Para obtener la velocidad media, la fibra óptica requiere una cantidad mínima de aire dentro del chorro. De este modo se pueden obtener buenas correlaciones. En la Figura 134, la medición de la velocidad del chorro es más precisa cuando el chorro está suficientemente aireado. Esto ocurre cuando la altura de caída supera $1.40 \mathrm{~m}$ (velocidad superior a $5.30 \mathrm{~m} / \mathrm{s}$ ). En la parte final de la caída las simulaciones numéricas y las mediciones de laboratorio obtienen valores muy similares.

La fracción volumétrica de aire se muestra muy sensible al tamaño de los elementos del mallado. Cuando el tamaño del elemento es pequeño, los resultados tienden a ser más similares a los valores obtenidos con el equipo de fibra óptica. Sin embargo, los elementos pequeños conllevan un incremento en el esfuerzo computacional requerido, así como en el tiempo para resolver el mismo problema.

Para los dos caudales específicos considerados, los mejores resultados de fracción volumétrica de aire se obtuvieron considerando un tamaño de los elementos de $0.01 \mathrm{~m}$, aproximadamente la mitad del espesor del chorro en la condición incidente.

La Tabla 32 muestra los resultados de presión obtenidos en el punto de estancamiento con las simulaciones numéricas cuando se consideran diferentes tamaños de elementos. Los resultados de presiones en el caso de impacto directo concuerdan con los valores de fracción volumétrica de aire en el chorro.

\begin{tabular}{|c|c|c|c|c|c|}
\cline { 2 - 6 } \multicolumn{1}{c|}{} & \multicolumn{4}{c|}{ Tamaño del elemento del mallado (mm) } \\
\cline { 2 - 6 } \multicolumn{1}{c|}{} & LAB & 25.0 & 15.0 & 12.5 & 10.0 \\
\hline $\left.\boldsymbol{q} \mathbf{( m}^{2} / \mathbf{s}\right)$ & \multicolumn{5}{c|}{ Presión media $\boldsymbol{H}_{\boldsymbol{m}} \mathbf{( m )}$} \\
\hline $\mathbf{0 . 0 5 8}$ & 1.26 & 0.58 & 1.03 & 1.03 & 1.23 \\
\hline $\mathbf{0 . 0 3 7}$ & 0.97 & 0.33 & 0.69 & 0.79 & 1.06 \\
\hline
\end{tabular}

Tabla 32. Presión media en el punto de estancamiento en función del tamaño del elemento del mallado.

El mejor ajuste a los datos de laboratorio se ha obtenido cuando se considera un tamaño de elemento de $0.01 \mathrm{~m}$. Tamaños de elemento superiores producen presiones inferiores en el punto de estancamiento debido a que la aireación en el chorro no está simulada adecuadamente.

\subsection{Análisis en función de la turbulencia inicial}

Debido a que la turbulencia en la entrada del modelo del laboratorio está en torno al $16 \%$, frente a la turbulencia típica en prototipos de presas inferior al 3\%, se consideró 
necesario conocer cómo la variación de la turbulencia inicial afecta a la solución numérica de los diversos parámetros influyentes en la teoría de aliviaderos de vertido libre.

En este sentido, se realizaron simulaciones con ANSYS CFX, analizándose tres turbulencias distintas en la condición de contorno de entrada del flujo $\left(T_{u}=0.016 ; 0.03\right.$; 0.01 ) para el caudal específico $q=0.058 \mathrm{~m}^{2} / \mathrm{s}$ y colchón en el cuenco $Y=0.17 \mathrm{~m}$.

Se empleó el modelo de turbulencia SST. El mallado estaba basado en elementos hexaédricos, empleando un total de 750544 elementos, y considerando un tamaño de los elementos del mallado de $0.01 \mathrm{~m}$ en la zona de vuelo del chorro y alrededor del punto de estancamiento (Figura 136).

El tiempo medio de resolución de las simulaciones de 60 segundos, paralelizando el cálculo en un ordenador de 8 núcleos, fue de 4.1 x $10^{5} \mathrm{~s}$ ( $\approx 5$ días).

La Figura 137 muestra los registros de presiones obtenidos en el punto de estancamiento de las simulaciones numéricas y las mediciones realizadas en laboratorio, una vez se han alcanzado las condiciones de régimen permanente. Los resultados de ANSYS CFX están muy acotados y próximos al valor de presión media $H_{m}$ debido al tipo de promediado temporal que realiza el programa al emplear un modelo de turbulencia RANS.
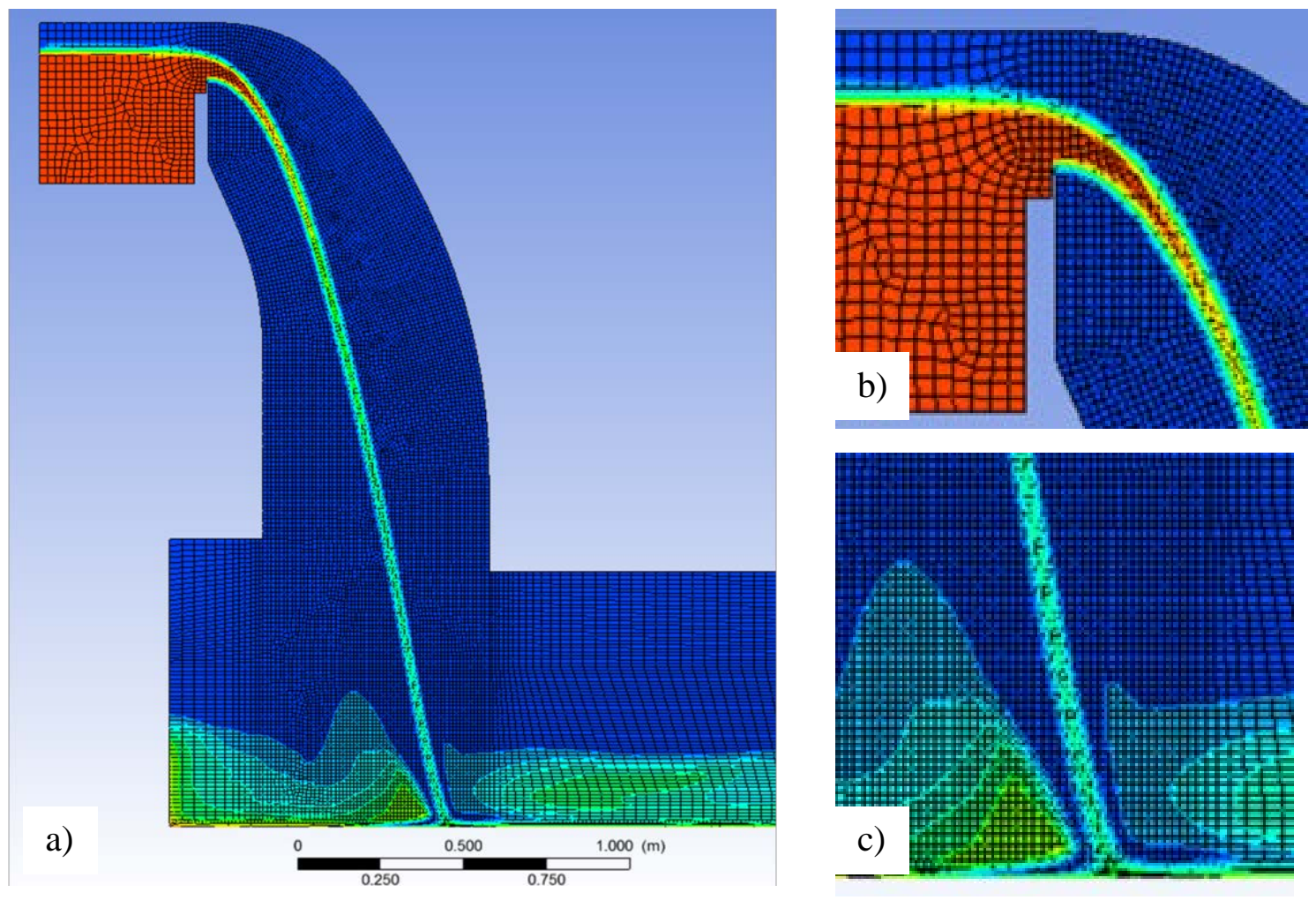

Figura 136. Mallado hexaédrico en ANSYS CFX: a) Vista general del modelo; b) Detalle del aliviadero; c) detalle del punto de incidencia y del punto de estancamiento. 


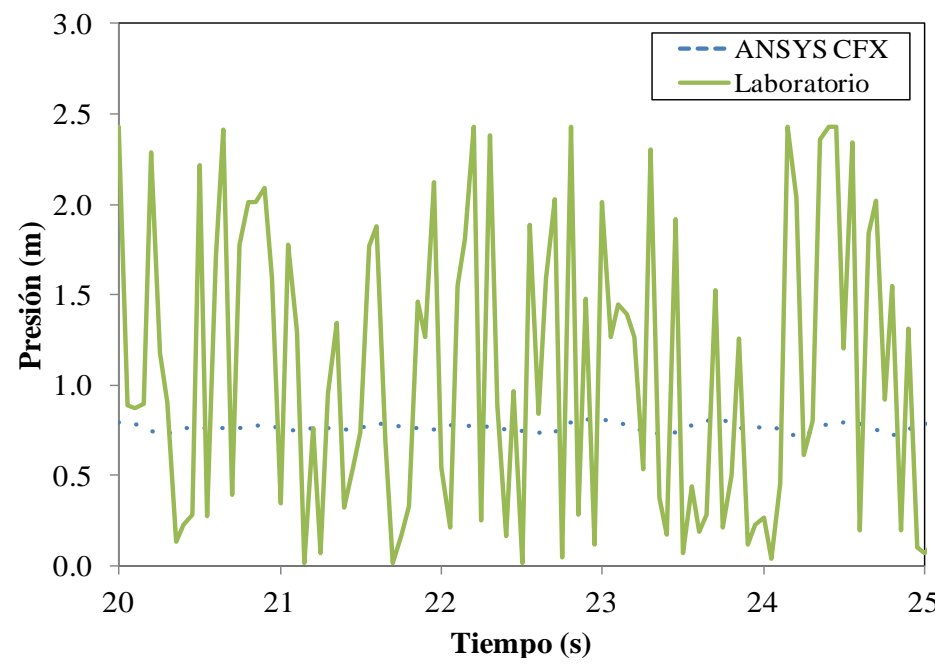

Figura 137. Distribución de presiones en el punto de estancamiento para $q=0.058 \mathrm{~m}^{2} / \mathrm{s}, H=$ $2.27 \mathrm{~m}, \mathrm{~h}=0.095 \mathrm{~m}$ e $Y=0.17 \mathrm{~m}$ (Castillo y Carrillo, 2012).

Los resultados se comparan con las mediciones realizadas en el laboratorio y la Metodología Paramétrica (Castillo, 2006, 2007, revisada en este trabajo), donde el coeficiente de presión dinámica media ha sido obtenido en las simulaciones numéricas y en laboratorio con la expresión:

$$
C_{p}=\frac{H_{m}-Y}{H}
$$

La Tabla 33 muestra una comparativa de los parámetros más importantes obtenidos en las simulaciones numéricas teniendo en cuenta diferentes intensidades turbulentas en la condición de entrada del flujo, donde $y_{b}$ es el calado sobre el labio del vertedero, $V_{0}$ la velocidad media en la sección del umbral de vertido, $V_{i}$ la velocidad media del chorro en la en la condición inicial (sección transversal localizada en la cota $z=-h$, siendo $z$ la componente vertical con origen en el labio del aliviadero y sentido ascendente), $B_{i}$ el ancho del chorro en la en la condición inicial, $V_{j}$ la velocidad media del chorro en la en la sección del chorro antes del impacto con el colchón de agua, $B_{j}$ el ancho del chorro en la en la sección del chorro antes del impacto con el colchón de agua, $L_{b}$ la longitud de rotura del chorro, $X_{i m p}$ la distancia del aliviadero al punto de estancamiento, $H_{m}$ la presión media en el punto de estancamiento, $Y_{u}$ el calado del colchón de agua aguas arriba del punto de impacto, $\theta$ el ángulo del chorro antes del impacto con el colchón de agua, y $C_{p}$ el coeficiente de presión dinámica media. La definición gráfica de las variables se recoge en la Figura 138. 


\begin{tabular}{|c|c|c|c|c|c|}
\cline { 2 - 6 } \multicolumn{1}{c|}{} & Lab. & $\begin{array}{c}\text { Metod. } \\
\text { Param. }\end{array}$ & $\begin{array}{c}\text { CFD } \\
\left(T_{u, x}=0.16\right)\end{array}$ & $\begin{array}{c}\text { CFD } \\
\left(T_{u, x}=0.03\right)\end{array}$ & $\begin{array}{c}\text { CFD } \\
\left(T_{u, x}=0.01\right)\end{array}$ \\
\hline$y_{b}(\mathrm{~m})$ & $\mathbf{0 . 0 8 2}$ & 0.083 & $\mathbf{0 . 0 7 8}$ & $\mathbf{0 . 0 7 7}$ & $\mathbf{0 . 0 7 8}$ \\
\hline$V_{0}(\mathrm{~m} / \mathrm{s})$ & - & $\mathbf{0 . 7 6}$ & $\mathbf{0 . 7 7}$ & $\mathbf{0 . 7 9}$ & $\mathbf{0 . 7 9}$ \\
\hline$V_{i}(\mathrm{~m} / \mathrm{s})$ & - & 1.54 & 1.78 & 1.75 & 1.75 \\
\hline$B_{i}(\mathrm{~m})$ & - & $\mathbf{0 . 0 5 3}$ & $\mathbf{0 . 0 4 8}$ & $\mathbf{0 . 0 4 8}$ & $\mathbf{0 . 0 4 7}$ \\
\hline$V_{j}(\mathrm{~m} / \mathrm{s})$ & 6.50 & 6.59 & 6.32 & 6.30 & 6.32 \\
\hline$B_{j}(\mathrm{~m})$ & - & $\mathbf{0 . 0 2 3}$ & $\mathbf{0 . 0 2 2}$ & $\mathbf{0 . 0 2 3}$ & $\mathbf{0 . 0 2 2}$ \\
\hline$L_{b}(\mathrm{~m})$ & - & 3.23 & $>H$ & $>H$ & $>H$ \\
\hline$X_{i m p}(\mathrm{~m})$ & $\mathbf{0 . 7 4}$ & 0.75 & $\mathbf{0 . 7 5}$ & $\mathbf{0 . 7 5}$ & $\mathbf{0 . 7 4}$ \\
\hline $\begin{array}{c}H_{m} \\
(\text { w.c.m. })\end{array}$ & $\mathbf{1 . 1 5}$ & 1.17 & 1.07 & $\mathbf{1 . 1 3}$ & $\mathbf{1 . 1 4}$ \\
\hline$Y_{u}(\mathrm{~m})$ & $\mathbf{0 . 1 6}$ & 0.17 & 0.12 & 0.10 & 0.115 \\
\hline$\theta\left(^{\circ}\right)$ & 81 & $\mathbf{8 1 . 5 8}$ & $\mathbf{8 2 . 7 5}$ & $\mathbf{8 2 . 4 2}$ & $\mathbf{8 2 . 4 9}$ \\
\hline$C_{p}$ & $\mathbf{0 . 4 3}$ & 0.44 & 0.40 & 0.42 & $\mathbf{0 . 4 3}$ \\
\hline
\end{tabular}

Tabla 33. Comparación de las principales variables medidas y calculadas con diferentes turbulencias en la condición de entrada para $q=0.058 \mathrm{~m}^{2} / \mathrm{s}, H=2.27 \mathrm{~m}, \mathrm{~h}=0.095 \mathrm{me}$ $Y=0.17 \mathrm{~m}$ (Castillo y Carrillo, 2012).

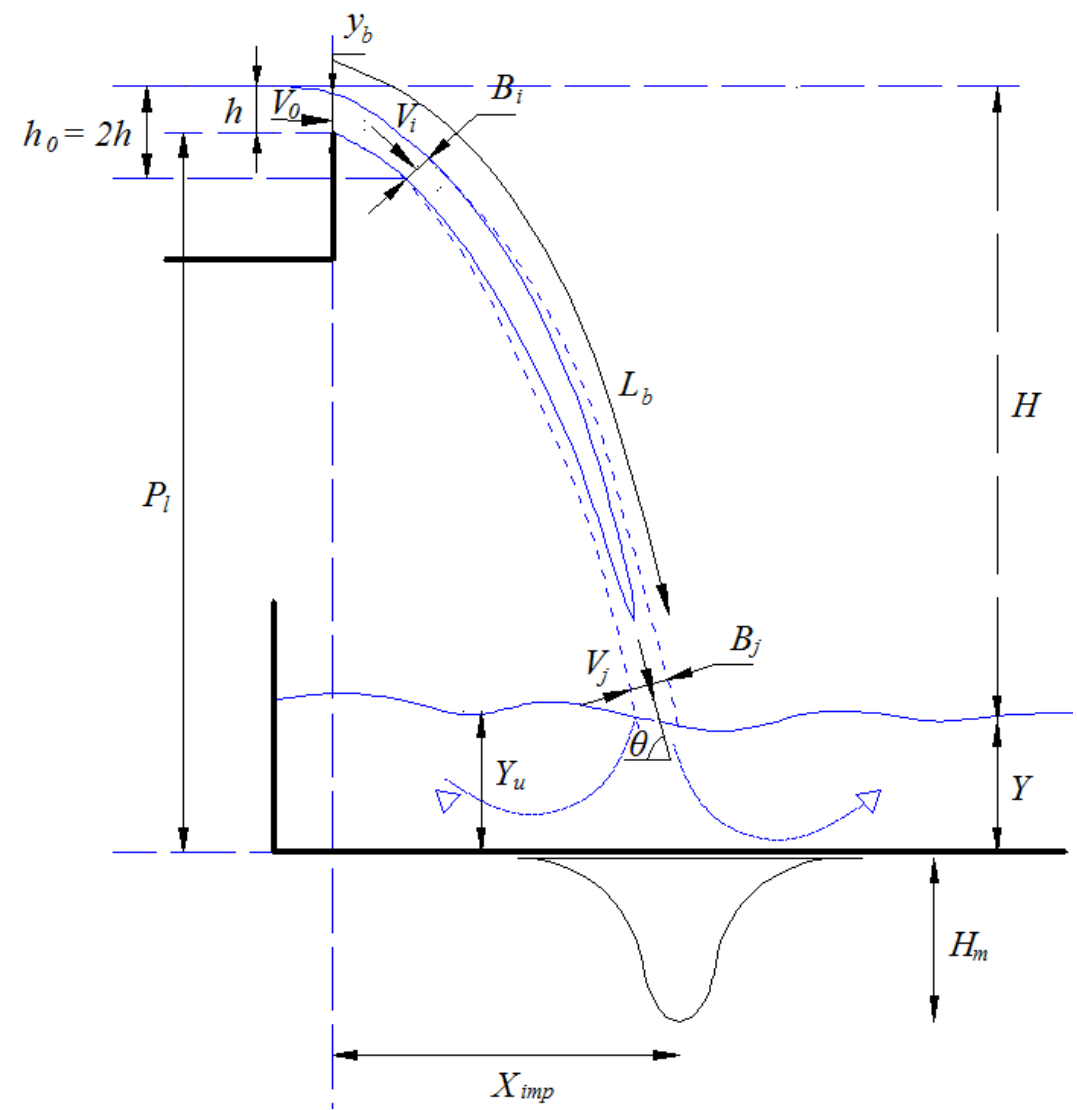

Figura 138. Esquema de variables en vertido libre. 
En la tabla anterior se puede ver que en general con los 3 métodos se obtienen resultados similares. En todos los casos el chorro todavía mantiene un núcleo de agua compacto en la zona de incidencia puesto que no existe distancia suficiente de caída para que se produzca la disgregación total del chorro $\left(H<L_{b}\right)$, y también en todos los casos, se dispone de un colchón efectivo $\left(Y>4 B_{j}\right)$.

Las principales diferencias corresponden al colchón aguas arriba del punto de estancamiento obtenidos en las simulaciones. Los resultados no son muy acertados debido a que la alta tasa de aireación en esa zona dificulta la determinación de la frontera líquida con el tamaño de mallado considerado.

En las simulaciones, la reducción de la turbulencia en la condición inicial de los modelos tiende a aumentar ligeramente el coeficiente de presión dinámica media en el punto de estancamiento, asemejándose al valor obtenido en laboratorio y con la Metodología Paramétrica.

\subsection{Análisis de la influencia del factor de escala}

Aceptando el hecho de que la aireación es un efecto de escala que presenta difícil solución, se consideraron diferentes factores de escala que cumplen la ley de semejanza de Froude. De este modo, las presiones debidas al impacto de un chorro cuya velocidad se ha adquirido por efectos gravitatorios mantendrán una relación proporcional. Los factores de escala considerado se recogen en la Tabla 34.

\begin{tabular}{|l|c|c|c|c|c|}
\cline { 3 - 6 } \multicolumn{2}{c|}{} & \multicolumn{4}{c|}{ Escala } \\
\hline Variable & Factor de escala & $\mathbf{1 : 1}$ & $\mathbf{1 : 1 0}$ & $\mathbf{1 : 2 0}$ & $\mathbf{1 : 4 0}$ \\
\hline Longitud & $k_{l}$ & 1 & 10 & 20 & 40 \\
\hline Tiempo & $\sqrt{k_{l}}$ & 1 & 3.16 & 4.47 & 6.32 \\
\hline Velocidad & $\sqrt{k_{l}}$ & 1 & 3.16 & 4.47 & 6.32 \\
\hline Aceleración & 1 & 1 & 1 & 1 & 1 \\
\hline Caudal & $k_{l}^{5 / 2}$ & 1 & 316.23 & 1788.85 & 10119.29 \\
\hline Fuerza & $k_{l}{ }^{3}$ & 1 & 1000 & 8000 & 64000 \\
\hline Presión & $k_{l}$ & 1 & 10 & 20 & 40 \\
\hline
\end{tabular}

Tabla 34. Factores de escala con la ley de Froude para diferentes variables.

En las simulaciones numéricas, a escala de laboratorio 1:1 se han considerado elementos hexaédricos con un tamaño del $0.01 \mathrm{~m}$ en la zona del chorro vertiente y del punto de estancamiento. Para el resto de las simulaciones, el mallado se ha modificado teniendo en cuenta el correspondiente factor de escala $k_{l}$. 
En las siguientes tablas se comparan los resultados obtenidos con las tres metodologías (simulaciones numéricas, resultados de laboratorio escalados y Metodología Paramétrica), considerando diferentes colchones de agua en el cuenco de disipación. Las condiciones de contorno $(q, H, h$ e $Y$ ) se muestran con el fin de conocer sus valores tras el proceso de escalado.

\begin{tabular}{|c|c|c|c|c|c|c|c|c|c|c|c|c|}
\hline \multirow[b]{3}{*}{ Variable } & \multicolumn{12}{|c|}{ Factor de escala } \\
\hline & \multicolumn{3}{|c|}{$1: 1$} & \multicolumn{3}{|c|}{$1: 10$} & \multicolumn{3}{|c|}{$1: 20$} & \multicolumn{3}{|c|}{$1: 40$} \\
\hline & CFD & Lab. & Param. & CFD & Lab. & Param. & CFD & Lab. ${ }^{*}$ & Param. & CFD & Lab. $^{*}$ & Param. \\
\hline$q\left(\mathrm{~m}^{2} / \mathrm{s}\right)$ & 0.058 & 0.058 & 0.058 & 1.83 & 1.83 & 1.83 & 5.19 & 5.19 & 5.19 & 14.63 & 14.63 & 14.63 \\
\hline$H(\mathrm{~m})$ & 2.41 & 2.41 & 2.41 & 24.11 & 24.11 & 24.11 & 48.22 & 48.22 & 48.22 & 96.44 & 96.44 & 96.44 \\
\hline$h(\mathrm{~m})$ & 0.091 & 0.091 & 0.091 & 0.91 & 0.91 & 0.91 & 1.82 & 1.82 & 1.82 & 3.64 & 3.64 & 3.64 \\
\hline$Y(\mathrm{~m})$ & 0.03 & 0.03 & 0.03 & 0.30 & 0.30 & 0.30 & 0.60 & 0.60 & 0.60 & 1.20 & 1.20 & 1.20 \\
\hline$L_{b}(\mathrm{~m})$ & $>\mathrm{H}$ & - & 2.32 & $>\mathrm{H}$ & - & 23.24 & $>\mathrm{H}$ & - & 46.49 & $>\mathrm{H}$ & - & 92.98 \\
\hline$H_{m}(\mathrm{~m})$ & 1.13 & 1.26 & 1.27 & 11.14 & 12.60 & 12.66 & 21.62 & 25.20 & 25.32 & 43.67 & 50.40 & 50.65 \\
\hline$C_{p}(-)$ & 0.46 & 0.51 & 0.51 & 0.45 & 0.51 & 0.51 & 0.44 & 0.51 & 0.51 & 0.44 & 0.51 & 0.51 \\
\hline
\end{tabular}

Tabla 35. Comparación de las principales variables medidas y calculadas (a escala de laboratorio $q=0.058 \mathrm{~m}^{2} / \mathrm{s}, Y=0.03 \mathrm{~m}$ ).

\begin{tabular}{|c|l|l|l|l|l|l|l|l|l|l|l|l|l|}
\cline { 2 - 16 } \multicolumn{1}{c|}{} & \multicolumn{10}{c|}{$1: 10$} & \multicolumn{3}{c|}{ Factor de escala } \\
\cline { 2 - 16 } \multicolumn{1}{c|}{} & \multicolumn{3}{c|}{$1: 1$} & \multicolumn{3}{c|}{$1: 20$} & \multicolumn{3}{c|}{$1: 40$} \\
\hline Variable & CFD & Lab. & Param. & CFD & Lab. & Param. & CFD & Lab. $^{*}$ & Param. & CFD & Lab. $^{*}$ & Param. \\
\hline$q\left(\mathrm{~m}^{2} / \mathrm{s}\right)$ & 0.058 & 0.058 & 0.058 & 1.83 & 1.83 & 1.83 & 5.19 & 5.19 & 5.19 & 14.63 & 14.63 & 14.63 \\
\hline$H(\mathrm{~m})$ & 2.28 & 2.28 & 2.28 & 22.76 & 22.76 & 22.76 & 45.52 & 45.52 & 45.52 & 91.04 & 91.04 & 91.04 \\
\hline$h(\mathrm{~m})$ & 0.091 & 0.091 & 0.091 & 0.91 & 0.91 & 0.91 & 1.82 & 1.82 & 1.82 & 3.64 & 3.64 & 3.64 \\
\hline$Y(\mathrm{~m})$ & 0.165 & 0.165 & 0.165 & 1.65 & 1.65 & 1.65 & 3.30 & 3.30 & 3.30 & 6.60 & 6.60 & 6.60 \\
\hline$L_{b}(\mathrm{~m})$ & $>\mathrm{H}$ & - & 2.32 & $>\mathrm{H}$ & - & 23.24 & $>\mathrm{H}$ & - & 46.49 & $>\mathrm{H}$ & - & 92.98 \\
\hline$H_{m}(\mathrm{~m})$ & 1.07 & 1.15 & 1.17 & 10.89 & 11.50 & 11.38 & 21.86 & 23.00 & 22.77 & 42.12 & 46.00 & 45.53 \\
\hline$C_{p}(-)$ & 0.40 & 0.43 & 0.44 & 0.41 & 0.43 & 0.43 & 0.41 & 0.43 & 0.43 & 0.39 & 0.43 & 0.43 \\
\hline
\end{tabular}

Tabla 36. Comparación de las principales variables medidas y calculadas (a escala de laboratorio $q=0.058 \mathrm{~m}^{2} / \mathrm{s}, Y=0.17 \mathrm{~m}$ ).

\begin{tabular}{|c|l|l|l|l|l|l|l|l|l|l|l|l|l|}
\cline { 2 - 15 } \multicolumn{1}{c|}{} & \multicolumn{9}{c|}{$1: 10$} & \multicolumn{3}{c|}{ Factor de escala } \\
\cline { 2 - 16 } \multicolumn{1}{c|}{} & \multicolumn{3}{c|}{$1: 1$} & \multicolumn{3}{c|}{$1: 20$} & \multicolumn{3}{c|}{$1: 40$} \\
\hline Variable & CFD & Lab. & Param. & CFD & Lab. $^{*}$ & Param. & CFD & Lab. $^{*}$ & Param. & CFD & Lab. $^{*}$ & Param. $^{2}$ \\
\hline$q\left(\mathrm{~m}^{2} / \mathrm{s}\right)$ & 0.058 & 0.058 & 0.058 & 1.83 & 1.83 & 1.83 & 5.19 & 5.19 & 5.19 & 14.63 & 14.63 & 14.63 \\
\hline$H(\mathrm{~m})$ & 2.19 & 2.19 & 2.19 & 22.11 & 22.11 & 22.11 & 44.22 & 44.22 & 44.22 & 88.44 & 88.44 & 88.44 \\
\hline$h(\mathrm{~m})$ & 0.091 & 0.091 & 0.091 & 0.91 & 0.91 & 0.91 & 1.82 & 1.82 & 1.82 & 3.64 & 3.64 & 3.64 \\
\hline$Y(\mathrm{~m})$ & 0.25 & 0.25 & 0.25 & 2.50 & 2.50 & 2.50 & 5.00 & 5.00 & 5.00 & 10.00 & 10.00 & 10.00 \\
\hline$L_{b}(\mathrm{~m})$ & $>\mathrm{H}$ & - & 2.32 & $>\mathrm{H}$ & - & 23.24 & $>\mathrm{H}$ & - & 46.49 & $>\mathrm{H}$ & - & 92.98 \\
\hline$H_{m}(\mathrm{~m})$ & 0.88 & 0.73 & 0.72 & 9.51 & 7.30 & 6.72 & 18.90 & 14.60 & 13.44 & 37.94 & 29.20 & 26.87 \\
\hline$C_{p}(-)$ & 0.29 & 0.22 & 0.21 & 0.32 & 0.22 & 0.19 & 0.32 & 0.22 & 0.19 & 0.32 & 0.22 & 0.19 \\
\hline
\end{tabular}

Tabla 37. Comparación de las principales variables medidas y calculadas (a escala de laboratorio $q=0.058 \mathrm{~m}^{2} / \mathrm{s}, Y=0.25 \mathrm{~m}$ ). 


\begin{tabular}{|c|c|c|c|c|c|c|c|c|c|c|c|c|}
\hline \multirow[b]{3}{*}{ Variable } & \multicolumn{12}{|c|}{ Factor de escala } \\
\hline & \multicolumn{3}{|c|}{$1: 1$} & \multicolumn{3}{|c|}{$1: 10$} & \multicolumn{3}{|c|}{$1: 20$} & \multicolumn{3}{|c|}{$1: 40$} \\
\hline & CFD & Lab. & Param. & CFD & Lab. ${ }^{*}$ & Param. & CFD & Lab. $^{*}$ & Param. & CFD & Lab. ${ }^{*}$ & Param. \\
\hline$q\left(\mathrm{~m}^{2} / \mathrm{s}\right)$ & 0.058 & 0.058 & 0.058 & 1.83 & 1.83 & 1.83 & 5.19 & 5.19 & 5.19 & 14.63 & 14.63 & 14.63 \\
\hline$H(\mathrm{~m})$ & 2.11 & 2.11 & 2.11 & 21.11 & 21.11 & 21.11 & 42.22 & 42.22 & 42.22 & 84.44 & 84.44 & 84.44 \\
\hline$h(\mathrm{~m})$ & 0.091 & 0.091 & 0.091 & 0.91 & 0.91 & 0.91 & 1.82 & 1.82 & 1.82 & 3.64 & 3.64 & 3.64 \\
\hline$Y(\mathrm{~m})$ & 0.35 & 0.35 & 0.35 & 3.50 & 3.50 & 3.50 & 7.00 & 7.00 & 7.00 & 14.00 & 14.00 & 14.00 \\
\hline$L_{b}(\mathrm{~m})$ & $>\mathrm{H}$ & - & 2.32 & $>\mathrm{H}$ & - & 23.24 & $>\mathrm{H}$ & - & 46.49 & $>\mathrm{H}$ & - & 92.98 \\
\hline$H_{m}(\mathrm{~m})$ & 0.56 & 0.55 & 0.56 & 5.70 & 5.50 & 5.37 & 11.13 & 11.00 & 10.75 & 22.51 & 22.00 & 21.50 \\
\hline$C_{p}(-)$ & 0.10 & 0.10 & 0.10 & 0.11 & 0.10 & 0.09 & 0.10 & 0.10 & 0.09 & 0.10 & 0.10 & 0.09 \\
\hline
\end{tabular}

Tabla 38. Comparación de las principales variables medidas y calculadas (a escala de laboratorio $q=0.058 \mathrm{~m}^{2} / \mathrm{s}, Y=0.35 \mathrm{~m}$ ).

La longitud de rotura del chorro $L_{b}$ no queda bien definida en las simulaciones numéricas. Con el tamaño de mallado empleado, la aproximación Euleriana no puede seguir la desintegración del chorro en gotas de agua dispersas. Este resultado concuerda con los estudios de Ho y Riddette (2010), quienes consideran un progreso limitado hasta la fecha puesto que $L_{b}$ requiere una resolución de mallado muy fina.

El coeficiente $C_{p}$ es un parámetro adimensional. Por este motivo, las diferentes relaciones de escala no le afectan. Debido a que el tamaño de los elementos del mallado se ha escalado con la misma semejanza de Froude, ANSYS CFX tiende a escalar todos los parámetros siguiendo la ley de semejanza. La Metodología Paramétrica obtiene valores similares tanto en el valor de presión media en el punto de estancamiento $H_{m}$, como en el coeficiente de presión dinámica media $C_{p}$.

Las siguientes figuras muestran los resultados obtenidos con las diversas escalas. Los resultados están clasificados como resultados de simulaciones (CFD), mediciones de laboratorio (LAB), Metodología Paramétrica (Param.), y el escalado de los datos de laboratorio (LAB escalado).

Considerando que el calado sobre un vertedero de pared delgada es $y_{b} \approx 0.85 h$, la Figura 139 muestra los resultados obtenidos. Los valores de la simulación numérica son muy similares a los valores obtenidos con la formulación anterior. Sin embargo, los calados medidos en laboratorio tienden a ser ligeramente superiores puesto que la pared del vertedero en la instalación no es estrictamente plana por el lado de aguas arriba.

Debido a la inestabilidad del chorro, los espesores en la condición incidente no pudieron ser obtenidos con precisión usando fotografías del perfil longitudinal. En la Figura 140 se comparan los resultados obtenidos en la simulaciones numéricas, con los valores obtenidos con la Metodología Paramétrica a escala laboratorio y posteriormente escalados, y empleando la Metodología Paramétrica directamente con las condiciones escaladas. Los resultados obtenidos son muy similares para las diversas escalas consideradas. 


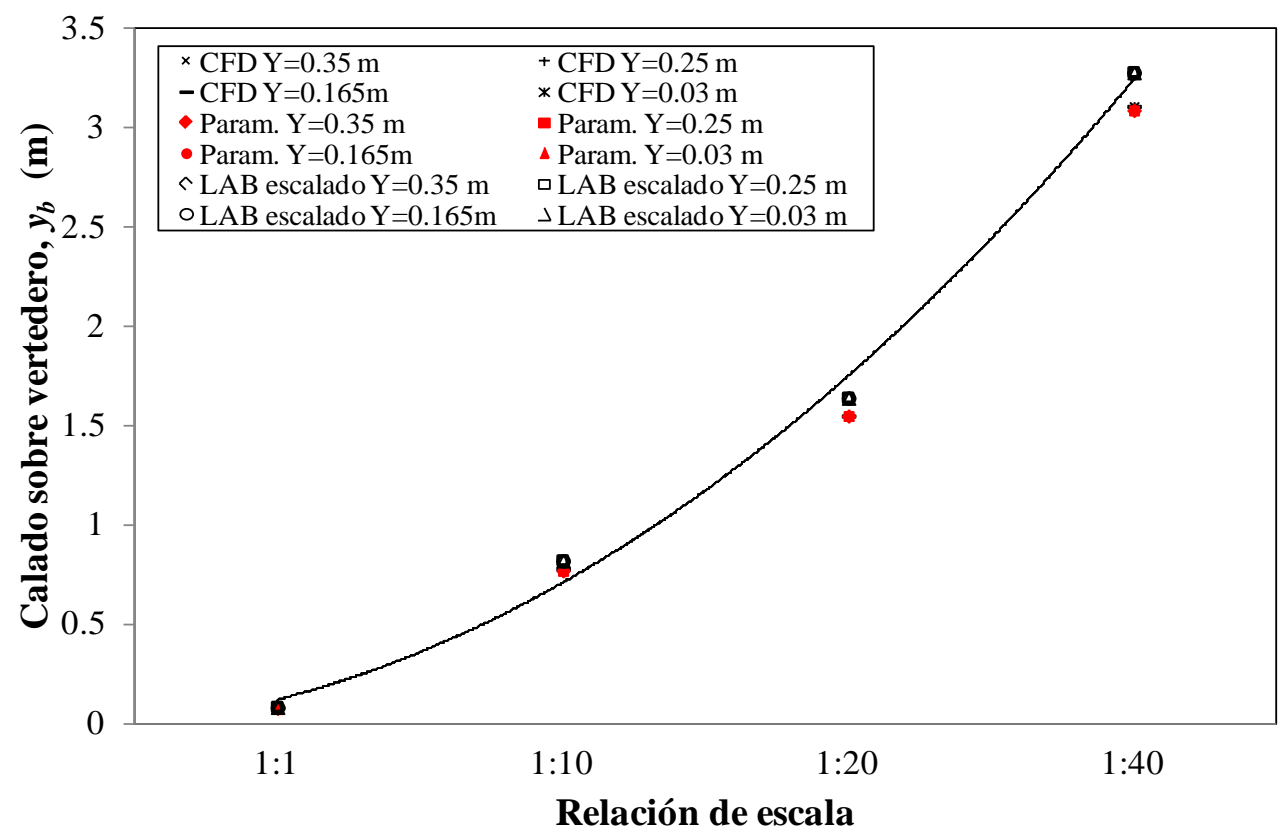

Figura 139. Calado sobre vertedero $y_{b}$ para distintas relaciones de escala.

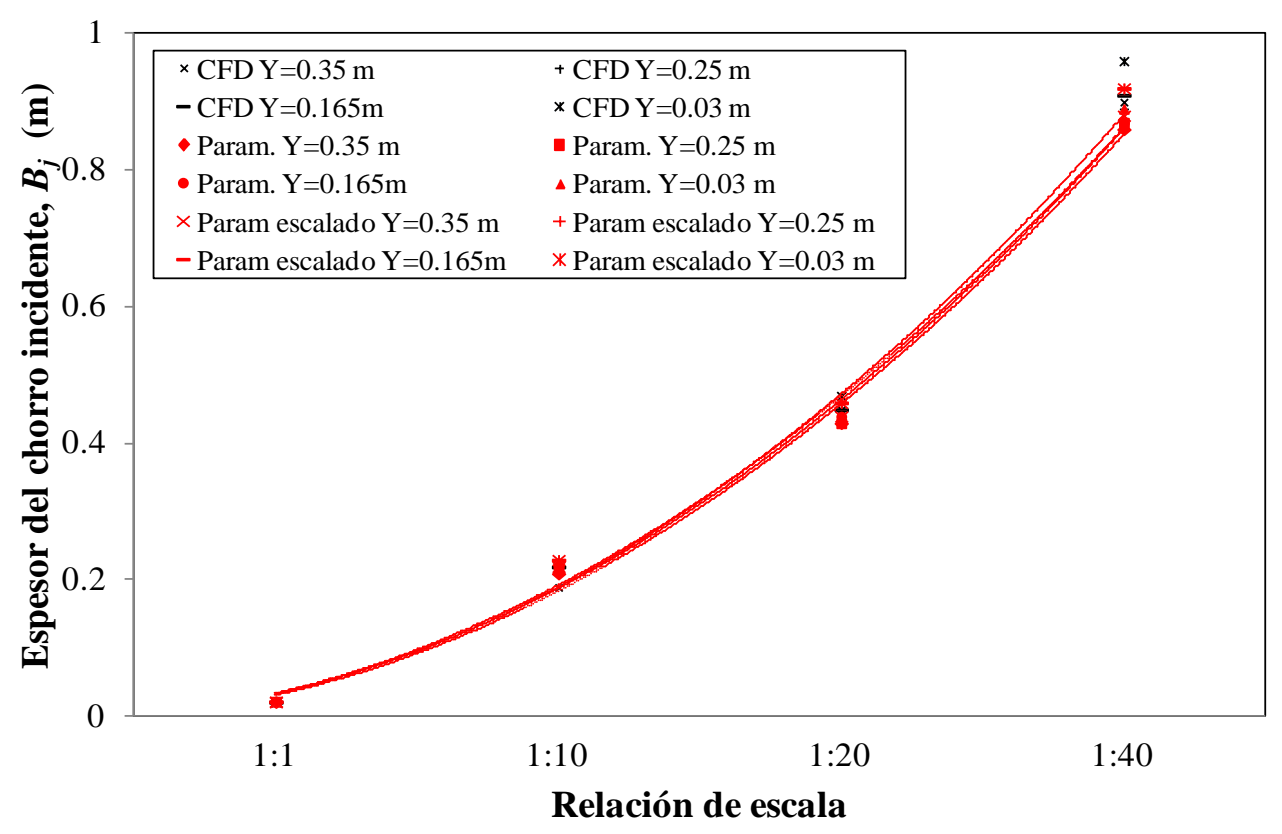

Figura 140. Espesor del chorro incidente $B_{j}$ para distintas relaciones de escala.

Para la velocidad incidente (Figura 141), la Metodología Paramétrica considera la velocidad de caída libre sin tener en cuenta la deceleración sufrida por la aireación en el vuelo. Las mediciones de laboratorio se han obtenido con equipo de fibra óptica, por lo que la velocidad es un poco inferior a la anterior. Las velocidades calculadas por ANSYS CFX son un poco inferiores a las obtenidas con la fibra óptica. 
En la Figura 142 se observa que la Metodología Paramétrica obtiene mayores distancias desde el labio del vertedero al punto de estancamiento $X_{\text {imp }}$, mientras que ANSYS CFD obtiene valores similares a los resultados de laboratorio escalados. Estos resultados concuerdan con las velocidades incidentes del chorro obtenidas.

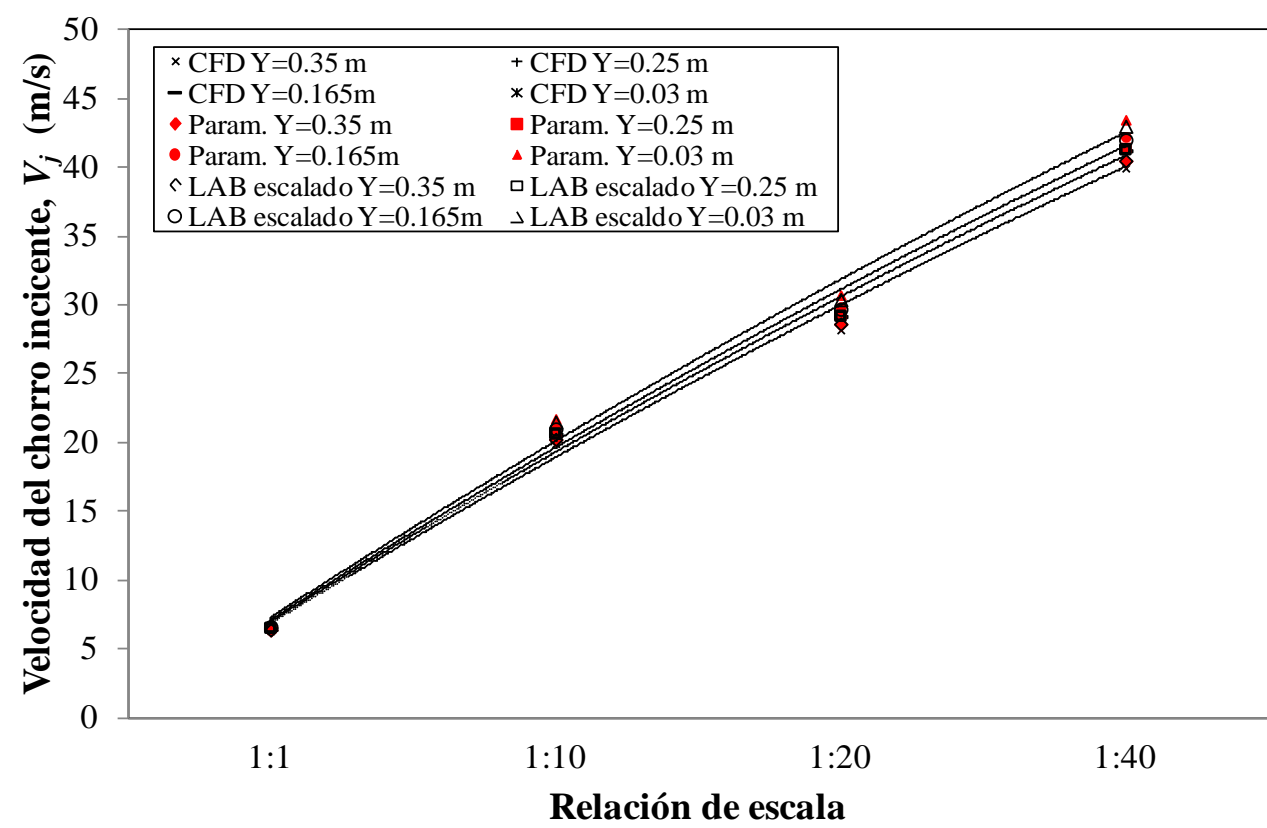

Figura 141.Velocidad del chorro incidente $V_{j}$ para distintas relaciones de escala.

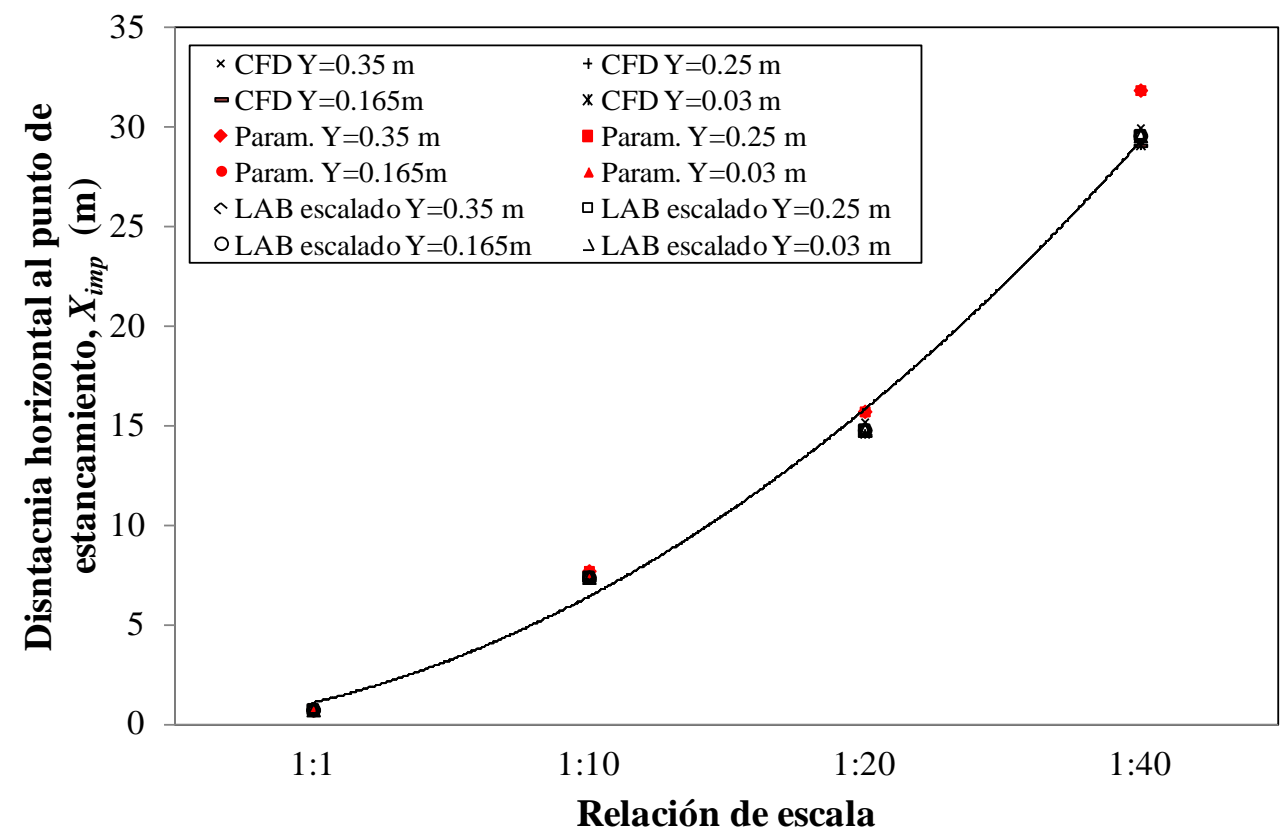

Figura 142. Distancia horizontal al punto de estancamiento $X_{\text {imp }}$ para distintas relaciones de escala. 
La Figura 143 muestra los valores de $C_{p}$ obtenidos en el caso de un chorro de impacto directo $\left(Y / B_{j}=1.30\right)$. Además, en la Metodología Paramétrica se han considerado diferentes intensidades turbulentas en la condición inicial del método. Los valores de las simulaciones son ligeramente inferiores a los obtenidos en laboratorio y con la Metodología Paramétrica. Por otro lado, la turbulencia en la condición inicial tiene un efecto muy significativo en el valor de $C_{p}$. Considerando valores usuales en prototipo, las diferencias pueden ser muy importantes: si $T_{u}=0.6 \%, C_{p}=0.47$; sin embargo, si $T_{u}=1.0 \%, C_{p}=0.10$.

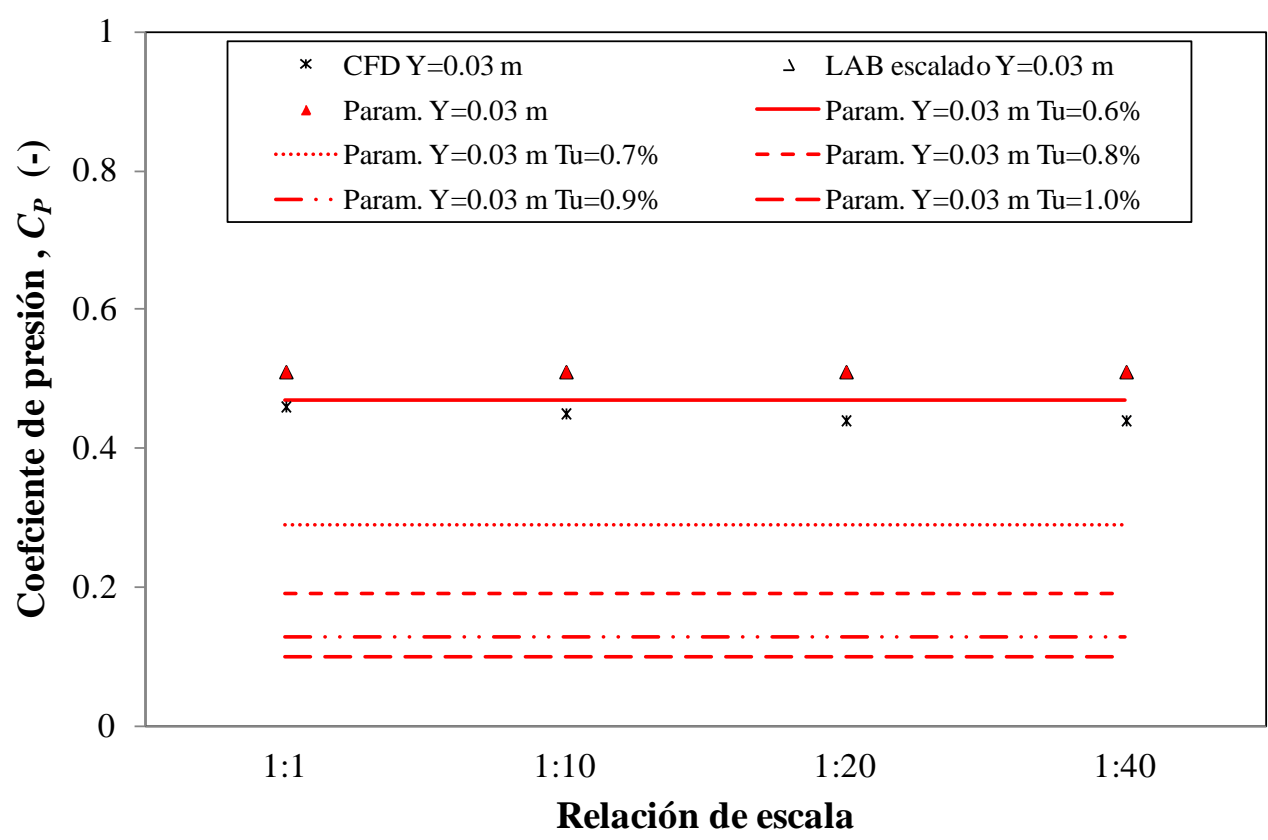

Figura 143. Coeficiente de presión dinámica media $C_{p}$ para distintas relaciones de escala. Caso $Y / B_{j}=1.30$.

De este modo, queda de manifiesto la importancia de conocer las condiciones iniciales del chorro para la adecuada caracterización hidrodinámica de los disipadores de energía, y en concreto de su intensidad turbulenta $T_{u}$. Se sugiere calcular la intensidad turbulenta con la Metodología Paramétrica a escala de modelo (por ejemplo, a escala 1:40), y mantener ese valor para el cálculo a escala de prototipo.

Finalmente, la Figura 144 muestra el calado en el cuenco aguas arriba del punto de estancamiento. Debido a la alta aireación que se produce en esta zona del modelo, resulta muy complicado discretizar la lámina libre en las simulaciones numéricas. Por este motivo, los resultados de las simulaciones CFD son más dispersos. 


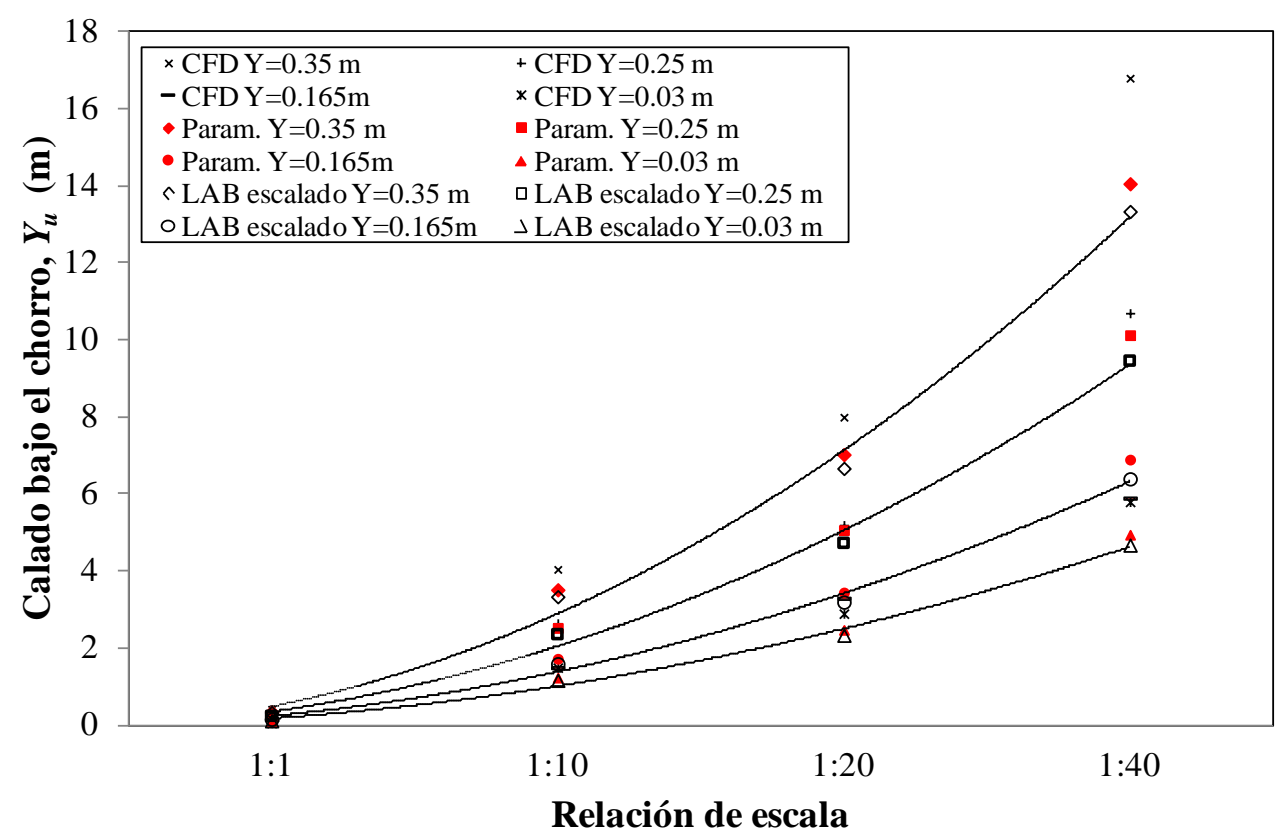

Figura 144. Calado bajo el chorro $Y_{u}$ para distintas relaciones de escala.

\subsection{Análisis de la influencia del modelo de turbulencia}

Debido a la variedad de modelos de turbulencia, se consideró necesario comprobar si su elección tiene efectos destacables sobre los diferentes parámetros considerados en el vertido libre.

En las simulaciones realizadas con ANSY CFX se han considerado tres modelos de turbulencia de dos ecuaciones: el modelo estándar $k-\varepsilon$, el modelo renormalizado $k-\varepsilon$ (RNG), y el modelo Shear Stress Transport basado en el modelo $k$ - $\omega$ (SST). El mallado está conformado por elementos hexaédricos de $0.01 \mathrm{~m}$. El tiempo medio de cálculo requerido fue de $2.605 \times 10^{5} \mathrm{~s}$ ( $\approx 3.0$ días), parelizando el cálculo en 8 procesos con un equipo que dispone de dos procesadores Intel ${ }^{\circledR}$ Xeon ${ }^{\circledR}$ E5620 (Quad Core, $2.40 \mathrm{GHz}$, $12 \mathrm{MB}$ Cache). Los resultados se comparan con datos obtenidos en laboratorio y con los nuevos ajustes propuestos de la Metodología Paramétrica.

La Tabla 39 compara los resultados obtenidos con los tres modelos de turbulencia, considerando $q=0.058 \mathrm{~m}^{2} / \mathrm{s}, H=2.27 \mathrm{~m}$ e $Y=0.17 \mathrm{~m}$. Todos los casos consideran que existe un colchón efectivo de agua $\left(Y>5.5 B_{j}\right.$ ) en el cuenco de disipación, y que $H<L_{b}$.

La Tabla 40 compara los resultados obtenidos con $q=0.037 \mathrm{~m}^{2} / \mathrm{s}, H=2.30 \mathrm{~m}$ e $Y$ $=0.12 \mathrm{~m}$, donde todos los casos consideran un colchón efectivo de agua $\left(Y>5.5 B_{j}\right)$. Los tres modelos de turbulencia obtienen $H<L_{b}$. Sin embargo, la Metodología Paramétrica obtiene la disgregación del chorro antes del impacto $\left(H>L_{b}\right)$.

La Tabla 41 recoge los resultados obtenidos considerando $q=0.023 \mathrm{~m}^{2} / \mathrm{s}, H=2.28$ m e $Y=0.12 \mathrm{~m}$. En todos los caso se produce la disgregación del chorro antes del 
impacto $\left(L_{b}<H\right)$. En las simulaciones realizadas con este caudal no se ha podido calcular el espesor del chorro antes del impacto puesto que en esa zona existen fracciones volumétricas de agua inferiores al 0.2 (criterio aplicado para calcular los espesores del chorro y su longitud de rotura).

\begin{tabular}{|l|c|c|c|c|c|}
\cline { 2 - 6 } \multicolumn{1}{c|}{} & SST & $k-\varepsilon$ & RNG & LAB & PARAM \\
\hline$V_{i}(\mathrm{~m} / \mathrm{s})$ & 1.78 & 1.75 & 1.78 & - & 2.03 \\
\hline$B_{i}(\mathrm{~m})$ & 0.048 & 0.049 & 0.047 & - & 0.056 \\
\hline$V_{j}(\mathrm{~m} / \mathrm{s})$ & 6.37 & $\mathbf{6 . 4 7}$ & 6.59 & $\mathbf{6 . 5 0}$ & 6.69 \\
\hline$b_{j}(m)$ & $\mathbf{0 . 7 0}$ & 0.67 & 0.69 & $\mathbf{0 . 7 7}$ & - \\
\hline$B_{j}(\mathrm{~m})$ & 0.022 & 0.022 & 0.023 & - & 0.023 \\
\hline$L_{b}(\mathrm{~m})$ & $>H$ & $>H$ & $>H$ & - & 2.32 \\
\hline$X_{i m p}(\mathrm{~m})$ & $\mathbf{0 . 7 3}$ & 0.69 & $\mathbf{0 . 7 3}$ & $\mathbf{0 . 7 4}$ & 0.75 \\
\hline$H_{m}(\mathrm{~m})$ & $\mathbf{1 . 0 7}$ & 1.23 & 1.25 & $\mathbf{1 . 1 5}$ & 1.17 \\
\hline$Y_{u}(\mathrm{~m})$ & 0.15 & 0.15 & $\mathbf{0 . 1 7}$ & $\mathbf{0 . 1 7}$ & 0.18 \\
\hline$\theta\left(^{\circ}\right)$ & 77.05 & 78.74 & $\mathbf{7 9 . 2 1}$ & $\mathbf{8 1}$ & 81.58 \\
\hline$C_{p}(-)$ & $\mathbf{0 . 4 0}$ & 0.47 & 0.48 & $\mathbf{0 . 4 3}$ & 0.44 \\
\hline
\end{tabular}

Tabla 39. Comparación de las principales variables medidas y calculadas $\left(q=0.058 \mathrm{~m}^{2} / \mathrm{s}, H=\right.$ $2.27 \mathrm{~m}, \mathrm{~h}=0.095 \mathrm{~m}, \mathrm{e} Y=0.17 \mathrm{~m})$.

\begin{tabular}{|l|c|c|c|c|c|}
\cline { 2 - 6 } \multicolumn{1}{c|}{} & SST & $k-\varepsilon$ & RNG & LAB & PARAM \\
\hline$V_{i}(\mathrm{~m} / \mathrm{s})$ & 1.42 & 1.38 & 1.51 & - & 1.76 \\
\hline$B_{i}(\mathrm{~m})$ & $\mathbf{0 . 0 4 1}$ & $\mathbf{0 . 0 4 2}$ & 0.039 & - & $\mathbf{0 . 0 4 2}$ \\
\hline$V_{j}(\mathrm{~m} / \mathrm{s})$ & $\mathbf{6 . 2 7}$ & 6.15 & 6.40 & $\mathbf{6 . 3 3}$ & 6.72 \\
\hline$b_{j}(\mathrm{~m})$ & $\mathbf{0 . 6 4}$ & $\mathbf{0 . 6 4}$ & $\mathbf{0 . 6 4}$ & $\mathbf{0 . 6 7}$ & - \\
\hline$B_{j}(\mathrm{~m})$ & $\mathbf{0 . 0 1 7}$ & 0.018 & $\mathbf{0 . 0 1 7}$ & - & $\mathbf{0 . 0 1 7}$ \\
\hline$L_{b}(\mathrm{~m})$ & $>H$ & $>H$ & $>H$ & - & 1.98 \\
\hline$X_{\text {imp }}(\mathrm{m})$ & $\mathbf{0 . 6 3}$ & 0.56 & 0.60 & $\mathbf{0 . 6 4}$ & 0.65 \\
\hline$H_{m}(\mathrm{~m})$ & 0.71 & 0.69 & 0.89 & 0.81 & 0.79 \\
\hline$Y_{u}(\mathrm{~m})$ & $\mathbf{0 . 1 0}$ & 0.17 & 0.17 & $\mathbf{0 . 1 2}$ & 0.13 \\
\hline$\theta\left(^{\circ}\right)$ & 78.33 & 79.16 & $\mathbf{7 9 . 1 3}$ & $\mathbf{8 2}$ & 84.82 \\
\hline$C_{p}(-)$ & $\mathbf{0 . 2 6}$ & 0.25 & $\mathbf{0 . 3 4}$ & $\mathbf{0 . 3 0}$ & 0.29 \\
\hline
\end{tabular}

Tabla 40. Comparación de las principales variables medidas y calculadas ( $q=0.037 \mathrm{~m}^{2} / \mathrm{s}, H=$ $2.30 \mathrm{~m}, \mathrm{~h}=0.073 \mathrm{~m}$, e $Y=0.12 \mathrm{~m}$ ).

\section{Espesores del chorro}

Las simulaciones numéricas siguieron la evolución del chorro con un adecuado grado de ajuste. Considerando una fracción volumétrica de aire de 0.80 , el espesor del chorro obtenido en las condiciones incidentes fue muy similar al calculado empleado la Metodología Paramétrica en los caudales $q=0.058$ y $0.037 \mathrm{~m}^{2} / \mathrm{s}$. Con el mismo criterio de relación agua-aire, el ancho transversal del chorro fue algo inferior al observado en 
laboratorio. El modelo de turbulencia SST fue ligeramente más preciso en la resolución de las dimensiones del chorro.

\begin{tabular}{|l|c|c|c|c|c|}
\cline { 2 - 6 } \multicolumn{1}{c|}{} & SST & $k-\varepsilon$ & RNG & LAB & PARAM \\
\hline$V_{i}(\mathrm{~m} / \mathrm{s})$ & 1.20 & 1.21 & 1.21 & - & 1.46 \\
\hline$B_{i}(\mathrm{~m})$ & $\mathbf{0 . 0 3 4}$ & $\mathbf{0 . 0 3 4}$ & 0.035 & - & $\mathbf{0 . 0 3 1}$ \\
\hline$V_{j}(\mathrm{~m} / \mathrm{s})$ & 6.02 & 6.03 & $\mathbf{6 . 2 0}$ & $\mathbf{6 . 2 0}$ & 6.63 \\
\hline$b_{j}(\mathrm{~m})$ & - & - & - & 0.62 & - \\
\hline$B_{j}(\mathrm{~m})$ & - & - & - & - & 0.012 \\
\hline$L_{b}(\mathrm{~m})$ & 1.08 & 1.09 & 1.48 & - & 1.79 \\
\hline$X_{i m p}(\mathrm{~m})$ & $\mathbf{0 . 5 0}$ & 0.47 & 0.48 & $\mathbf{0 . 5 4}$ & 0.49 \\
\hline$H_{m}(\mathrm{~m})$ & $\mathbf{0 . 3 5}$ & 0.31 & 0.40 & $\mathbf{0 . 3 7}$ & 0.42 \\
\hline$Y_{u}(\mathrm{~m})$ & 0.08 & 0.08 & 0.08 & 0.12 & 0.12 \\
\hline$\theta\left(^{\circ}\right)$ & 78.70 & 79.05 & 79.53 & $\mathbf{8 3}$ & 85.40 \\
\hline$C_{p}(-)$ & $\mathbf{0 . 1 0}$ & 0.08 & $\mathbf{0 . 1 2}$ & $\mathbf{0 . 1 1}$ & 0.13 \\
\hline
\end{tabular}

Tabla 41. Comparación de las principales variables medidas y calculadas ( $q=0.023 \mathrm{~m}^{2} / \mathrm{s}, H=$ $2.28 \mathrm{~m}, \mathrm{~h}=0.047 \mathrm{~m}, \mathrm{e} Y=0.12 \mathrm{~m}$ ).

\section{Velocidades del chorro}

Las velocidades obtenidas en la condición incidente tendieron a ser algo inferiores a las obtenidas con el equipo de fibra óptica, excepto las resueltas con el modelo RNG que tendieron a ser algo superiores, aunque inferiores a las que se calculan con la velocidad de caída libre sin tener en cuenta pérdidas por fricción con el aire. Estas velocidades concuerdan con los resultados de presiones medias obtenidos en el punto de estancamiento, $H_{m}$.

\section{Ángulos incidentes del chorro}

Existen pequeñas diferencias entre los ángulos incidentes del chorro calculados con los tres modelos de turbulencia, los cuales obtuvieron valores similares a los medidos en laboratorio y calculados con la Metodología Paramétrica.

\section{Distancia horizontal al punto de estancamiento}

Respecto a la distancia horizontal desde el vertedero al punto de estancamiento $X_{\text {imp }}$, los resultados obtenidos con el modelo de turbulencia SST fueron los más similares a los datos de laboratorio y a los valores calculados con la fórmula de Scimeni (1930), mientras que los obtenidos con el modelo $k-\varepsilon$ fueron hasta $8 \mathrm{~cm}$ inferiores de los resultados de laboratorio. 


\section{Coeficiente de presión dinámica media}

El coeficiente de presión dinámica media de las mediciones de laboratorio se calculó mediante la ecuación (214). Para los tres caudales específicos considerados, la presión dinámica media $H_{m}$, y el coeficiente de presión dinámica media $C_{p}$, obtenidos con los tres modelos de turbulencia fueron similares a las mediciones de laboratorio y a los resultados de la Metodología Paramétrica. En diferencias absolutas, el modelo de turbulencia SST fue ligeramente más preciso que los otros dos. El modelo SST tendió a subestimar el valor de $C_{p}$ en $0.01-0.04$ (diferencias del 7-13\% respecto a los resultados de laboratorio), mientras que el modelo RNG $k-\varepsilon$ tendió a sobreestimarlos en $0.01-0.05$ (diferencias del 10-13\%), y el modelo $k$ - $\varepsilon$ varió entre -0.05 y +0.04 (diferencias entre el -9 y el 17\%).

Las diferencias entre los resultados de laboratorio y la Metodología Paramétrica están en torno al 2-3\%.

\section{Relación entre el cochón aguas arriba y aguas abajo del punto de estancamiento}

Debido a la recirculación del flujo, ANSY CFX introduce una gran cantidad de aire en el colchón bajo el chorro, lo que dificulta realizar una adecuada discretización de la lámina libre aguas arriba del punto de impacto para comparar con los datos de laboratorio y con los estudios de Cui Guang Tao et al. (1985) y Castillo et al. (2014). Esta situación podría mejorar en caso de considerar un menor tamaño de mallado en la zona bajo el chorro, pero conllevaría un incremento en el coste computacional y en el tiempo de resolución.

Para el mayor caudal específico, los resultados de los tres modelos de turbulencia fueron muy similares a los resultados de laboratorio y a la formulación empírica. Sin embargo, en los caudales medio e inferior las diferencias entre los calados pueden llegar a los $5 \mathrm{~cm}$ debido a la mezcla de aire-agua que el programa resuelve.

\section{Distribución de presiones en el entorno del punto de estancamiento}

Con el fin de comparar las presiones obtenidas en torno al punto de estancamiento, en la Figura 145 se grafica la presión media calculada en un plano vertical paralelo a la línea central del modelo, junto a los resultados obtenidos en laboratorio.

Las simulaciones siguen la tendencia marcada por los puntos registrados en laboratorio. Aguas arriba y aguas abajo del punto de estancamiento, los tres modelos de turbulencia obtienen resultados similares, con una ligera mejor aproximación del modelo SST en la zona de aguas abajo del caudal intermedio. 


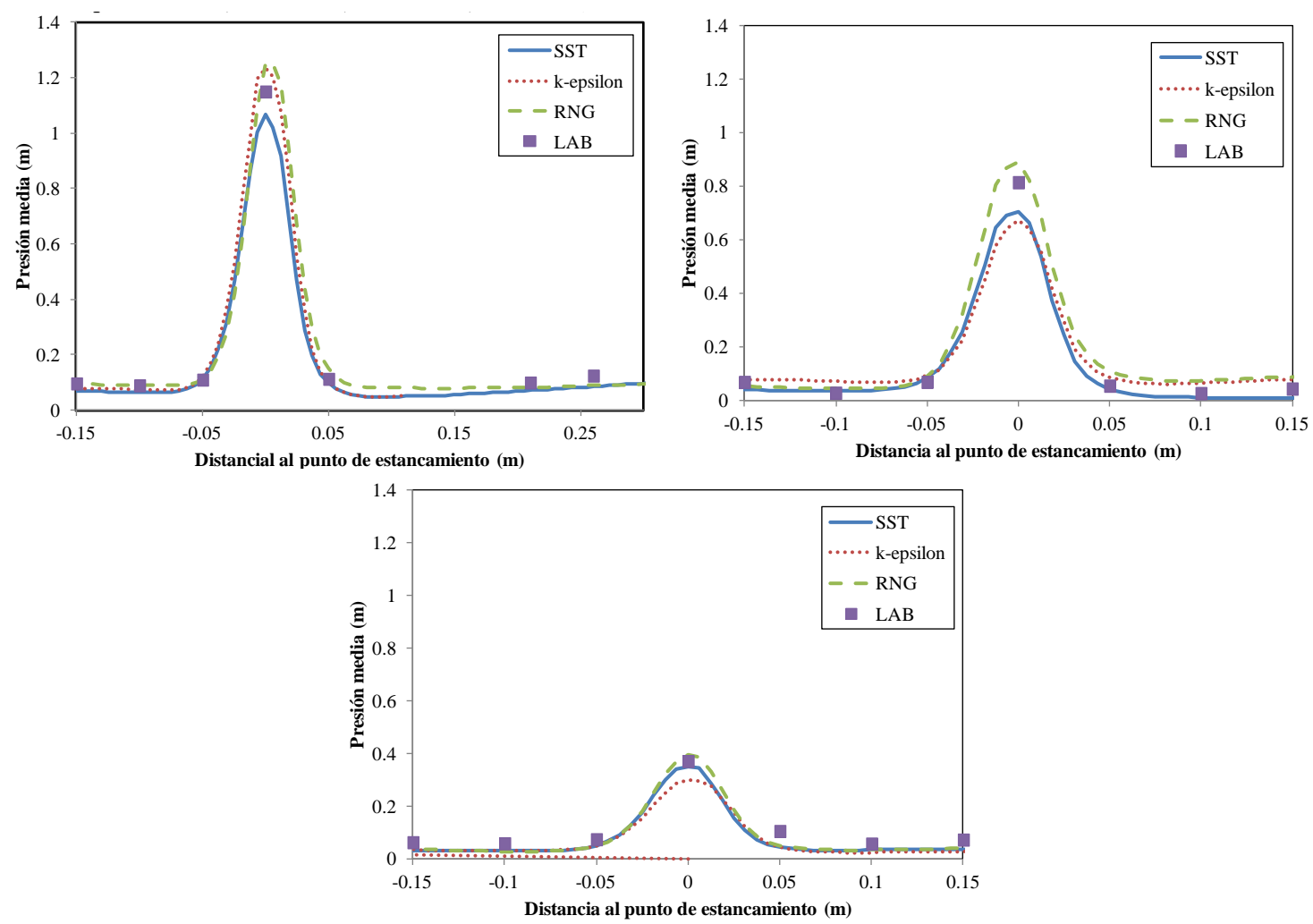

Figura 145. Distribución de presiones medias en torno al punto de estancamiento.

En cuanto a la presión media en el punto de estancamiento, el modelo RNG tiende a obtener valores ligeramente superiores, mientras que el modelo SST muestra valores ligeramente por debajo, aunque levemente más próximos a los obtenidos en laboratorio.

La Figura 146 muestra las distribuciones de presiones obtenidas en laboratorio y resueltas con CFD, comparándolas con las formulaciones empíricas tradicionales. Se han considerado dos caudales específicos, con sus correspondientes colchones.

De acuerdo a los resultados, las formulaciones de Cola, Aki (con $b_{\text {distrib }}=0.23 Y$ ), Hartung y Häusler (con $y_{k}=7.2 B_{j}$ ), y Beltaos son equivalentes. Estas formulaciones, que se han obtenido con chorros no aireos, están en concordancia con los datos obtenidos en laboratorio y en las simulaciones numéricas para el caso del mayor caudal específico (caso $q=0.058 \mathrm{~m}^{2} / \mathrm{s}$, poco aireado con $H / L_{b}=0.98$ ).

La fórmula de Aki con $b_{\text {distrib }}=0.13 Y$ obtiene una envolvente superior de la distribución de presiones dinámicas medias propuestas por los otros autores. Puesto que se obtuvo con chorros aireados, la formulación actúa como envolvente de los datos de laboratorio y de las simulaciones numéricas analizadas.

La expresión de Hartung y Häusler (con $y_{k}=5 B_{j}$ ) obtiene un resultado intermedio entre las definidas por el resto de autores. Sus valores concuerdan con los datos de 
laboratorio y los resultados de CFD para el caso del menor caudal específico (caso $q=$ $0.037 \mathrm{~m}^{2} / \mathrm{s}$, aireado con $H / L_{b}=1.16$ ).
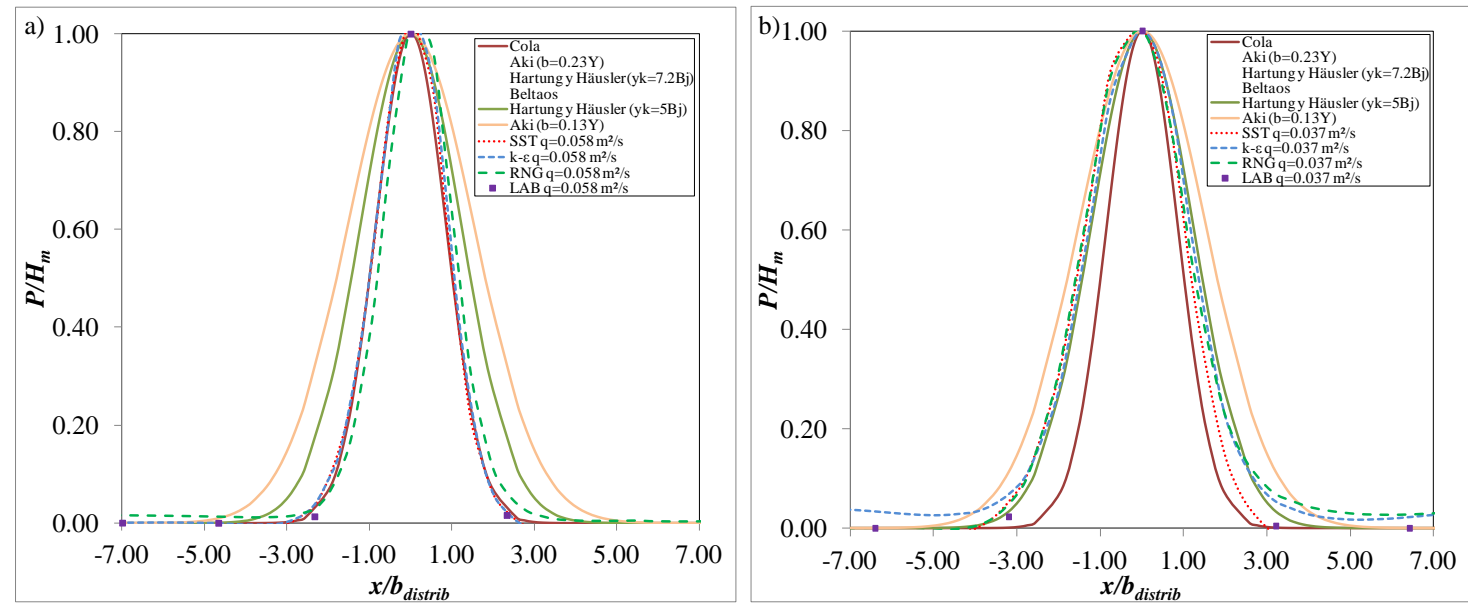

Figura 146. Distribución de presiones medias en torno al punto de estancamiento en función de las formulaciones clásicas: a) $q=0.058 \mathrm{~m}^{2} / \mathrm{s}, h=0.095 \mathrm{~m}, H=2.27 \mathrm{~m}, Y=0.17 \mathrm{~m}$; b) $q=$ $0.037 \mathrm{~m}^{2} / \mathrm{s}, h=0.073 \mathrm{~m}, H=2.30 \mathrm{~m}, Y=0.12 \mathrm{~m}$.

En ambos casos, el modelo de turbulencia SST ha obtenido el mejor ajuste de las simulaciones numéricas. Para el caudal específico mayor, los tres modelos de turbulencia obtuvieron resultados similares. Con el menor caudal específico, el modelo de turbulencia $k-\varepsilon$ tendió a sobreestimar la presión aguas arriba del punto de estancamiento, mientras que aguas abajo los modelos RNG y $k-\varepsilon$ tendieron a sobreestimar las mediciones de laboratorio.

La Figura 147 muestra las distribuciones de la presión dinámica media obtenidas con dos caudales específicos. Las simulaciones se comparan con la formulación propuesta por Ervine et al. (1997), basada en la distancia radial adimensional $x / Y$.
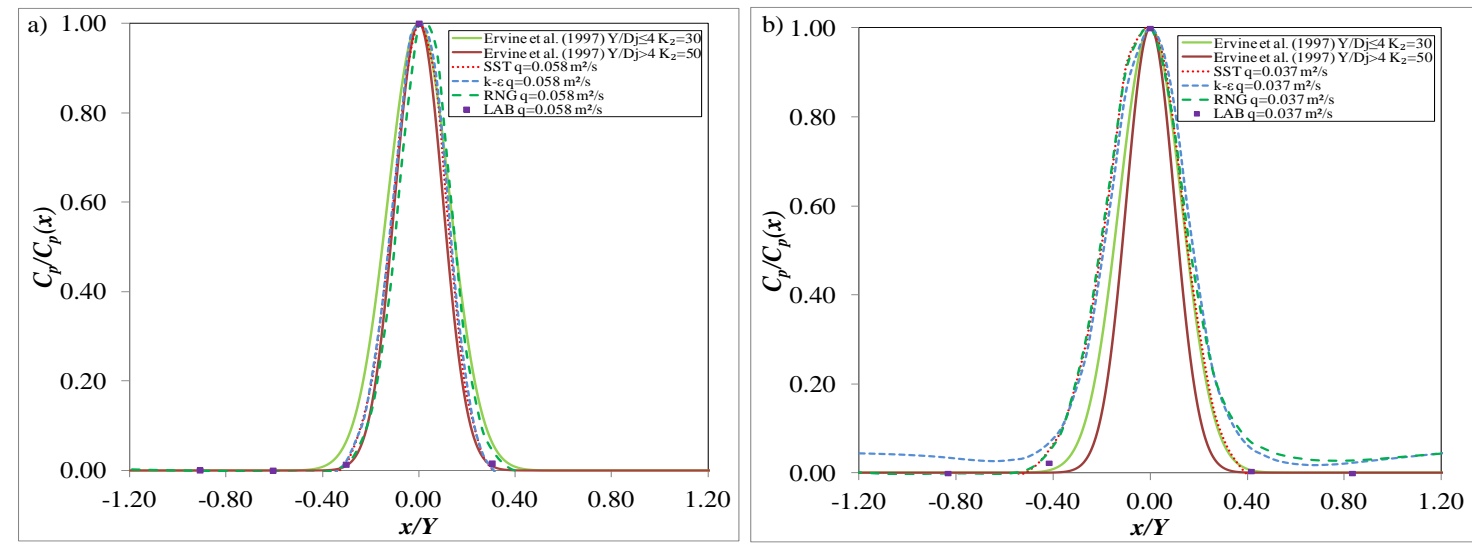

Figura 147. Distribución de presiones medias en torno al punto de estancamiento en función de la relación $x / Y$ : a) $q=0.058 \mathrm{~m}^{2} / \mathrm{s}, h=0.095 \mathrm{~m}, H=2.27 \mathrm{~m}, Y=0.17 \mathrm{~m}$; b) $q=0.037 \mathrm{~m}^{2} / \mathrm{s}, h=$ $0.073 \mathrm{~m}, H=2.30 \mathrm{~m}, Y=0.12 \mathrm{~m}$ 
Las simulaciones muestran un comportamiento no simétrico entre los lados de aguas arriba y de aguas abajo, lo que concuerda con la distribución esperable en chorros vertientes.

La distribución de Ervine et al., obtenida para chorros circulares y válida para $H / L_{b}$ $\leq 0.5$, considera un parámetro $K_{2}=30$ para calados poco profundos y 50 para colchones grandes. La distribución concuerda con los resultados numéricos y los datos de laboratorio en el caso de chorros poco aireados $\left(q=0.058 \mathrm{~m}^{2} / \mathrm{s}, H / L_{b}=0.98\right)$. Cuando el chorro está más airado, la distribución de Ervine et al. actúa como una envolvente inferior de los datos. Los resultados obtenidos se pueden representar considerando $K_{2}=$ 20 en el tramo de aguas arriba y 15 en el de aguas abajo, en vez de los valores sugeridos para chorros circulares.

Aguas arriba, los modelos de turbulencia SST y RNG obtuvieron valores similares a los de laboratorio, mientras que el modelo $k-\varepsilon$ tendió a sobreestimar la presión. Aguas abajo, los modelos $k-\varepsilon$ y RNG tendieron a sobreestimar los resultados de laboratorio.

La Figura 148 muestra las distribuciones de la presión dinámica media considerando la expresión exponencial propuesta por Bollaert (2002), basada en la distancia radial adimensional $x / x_{\max }$. Se han considerado dos caudales específicos.

En ambos casos, las simulaciones numéricas muestran un comportamiento no simétrico entre las distribuciones de aguas arriba y de aguas abajo (más claramente identificable en el caudal específico menor). Este comportamiento concuerda con la distribución esperable en chorros vertientes, donde la presión de aguas arriba tiende a ser ligeramente superior debido al efecto de la recirculación del flujo.
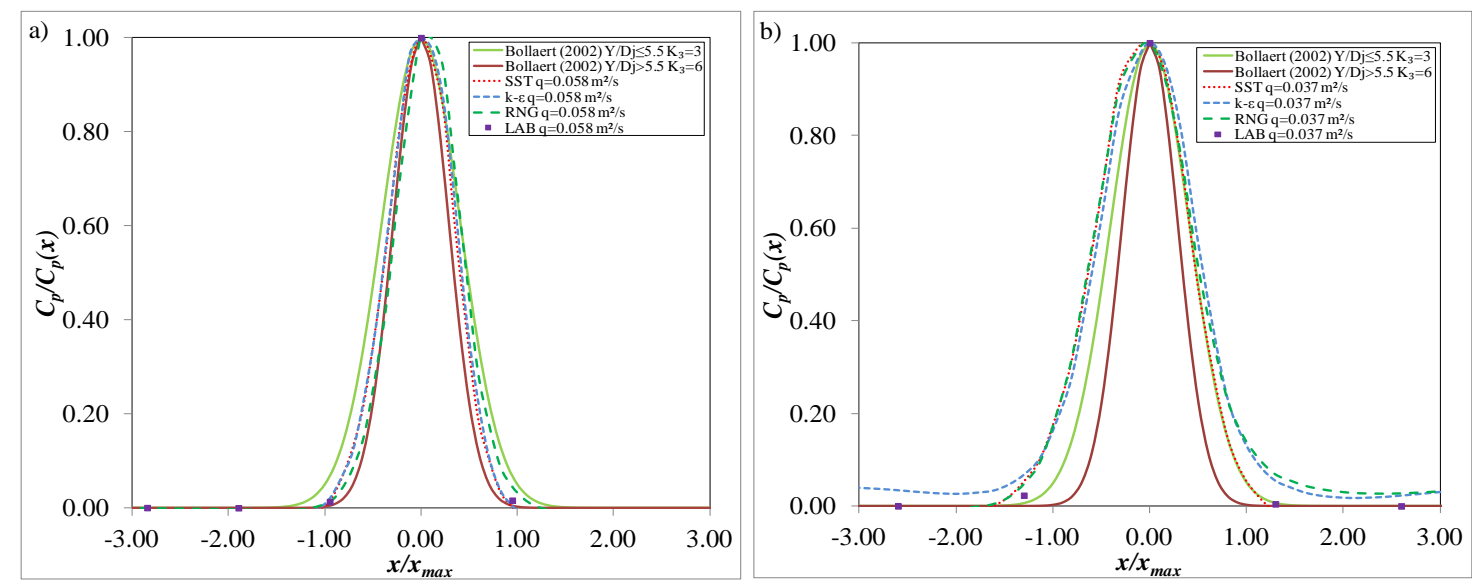

Figura 148. Distribución de presiones medias en torno al punto de estancamiento en función de la relación $x / x_{\max }$ : a) $q=0.058 \mathrm{~m}^{2} / \mathrm{s}, h=0.095 \mathrm{~m}, H=2.27 \mathrm{~m}, Y=0.17 \mathrm{~m}$; b) $q=0.037 \mathrm{~m}^{2} / \mathrm{s}, h$ $=0.073 \mathrm{~m}, H=2.30 \mathrm{~m}, Y=0.12 \mathrm{~m}$. 
La distribución de Bollaert, obtenida para chorros circulares y válida para $H / L_{b} \leq$ 0.5, considera un parámetro $K_{3}=6$ en el caso de colchones efectivos. Esta distribución concuerda con los resultados numéricos y los datos de laboratorio en el caso de chorros poco aireados $\left(q=0.058 \mathrm{~m}^{2} / \mathrm{s}, H / L_{b}=0.98\right)$. Cuando el chorro está más airado $(q=$ $0.037 \mathrm{~m}^{2} / \mathrm{s}, H / L_{b}=1.16$ ), la distribución de Bollaert actúa como una envolvente inferior de la distribución de presiones. En este caso, los resultados se pueden representar considerando $K_{3}=1.5$ en el tramo de aguas arriba y $K_{3}=2.0$ en el de aguas abajo, en vez de los valores sugeridos para chorros circulares $\left(K_{3}=3\right.$ para colchón no efectivo, $K_{3}$ $=6$ para colchón efectivo).

Aguas arriba, los modelos de turbulencia SST y RNG obtuvieron valores ligeramente inferiores a los de laboratorio, mientras que el modelo $k$ - $\varepsilon$ tendió a sobreestimar la presión. Aguas abajo del punto de estancamiento, los modelos $k-\varepsilon \mathrm{y}$ RNG tendieron a sobreestimar los resultados de laboratorio.

\section{Comportamiento global}

Respecto al comportamiento global de los modelos de turbulencia, existen pocas diferencias entre los resultados obtenidos con los tres modelos. El modelo $k$ - $\varepsilon$ tiende a obtener los resultados más dispares, mientras que el modelo SST muestra los valores más próximos a las mediciones de laboratorio y a la Metodología Paramétrica en la mayoría de las variables analizadas.

\subsection{Estudio sistemático}

Una vez analizados los diversos resultados comentados con anterioridad, se procedió a realizar con ANSYS CFX el estudio sistemático de tres caudales específicos (0.023, 0.037 y $0.058 \mathrm{~m}^{2} / \mathrm{s}$ ), considerando diferentes colchones de agua. Los resultados se comparan con los valores obtenidos en laboratorio y con la Metodología Paramétrica. La Tabla 42 recopila los resultados obtenidos en el punto de estancamiento.

En general se obtiene un adecuado ajuste entre los coeficientes de presión dinámica media y las presiones dinámicas medias de las tres metodologías. Las máximas diferencias entre los resultados numéricos con los otros métodos corresponden a situaciones de impacto directo, donde existe un cambio busco de energía cinética a potencial en una zona muy reducida, generándose un fuerte gradiente entre los elementos próximos del mallado.

Considerando la diferencia de altura entre el nivel de aguas arriba del vertedero y el colchón de agua en el cuenco de disipación de cada ensayo, las diferencias entre las simulaciones y los datos de laboratorio muestran un error máximo entre -4 y +7\%. 


\begin{tabular}{|c|c|c|c|c|c|c|c|c|}
\cline { 4 - 10 } \multicolumn{2}{c|}{} & \multicolumn{2}{c|}{ CFD } & \multicolumn{2}{c|}{ LABORATORIO } & \multicolumn{2}{c|}{ PARAM. } \\
\hline$q\left(\mathrm{~m}^{2} / \mathrm{s}\right)$ & $Y(\mathrm{~m})$ & $H(\mathrm{~m})$ & $H_{m}(\mathrm{~m})$ & $C_{p}(-)$ & $H_{m}(\mathrm{~m})$ & $C_{p}(-)$ & $H_{m}(\mathrm{~m})$ & $C_{p}(-)$ \\
\hline 0.023 & 0.02 & 2.377 & 0.36 & 0.14 & 0.41 & 0.16 & 0.45 & 0.18 \\
\hline 0.023 & 0.11 & 2.277 & 0.35 & 0.10 & 0.34 & 0.10 & 0.35 & 0.11 \\
\hline 0.023 & 0.22 & 2.177 & 0.22 & 0.00 & 0.29 & 0.03 & 0.29 & 0.03 \\
\hline 0.023 & 0.30 & 2.097 & 0.30 & 0.00 & 0.31 & 0.00 & 0.32 & 0.01 \\
\hline 0.037 & 0.02 & 2.397 & 1.06 & 0.45 & 0.97 & 0.39 & 0.70 & 0.28 \\
\hline 0.037 & 0.12 & 2.297 & 0.71 & 0.26 & 0.81 & 0.30 & 0.79 & 0.29 \\
\hline 0.037 & 0.24 & 2.177 & 0.44 & 0.09 & 0.43 & 0.08 & 0.44 & 0.09 \\
\hline 0.037 & 0.33 & 2.087 & 0.32 & 0.00 & 0.39 & 0.03 & 0.41 & 0.04 \\
\hline 0.058 & 0.03 & 2.411 & 1.23 & 0.46 & 1.26 & 0.51 & 1.24 & 0.50 \\
\hline 0.058 & 0.17 & 2.276 & 1.07 & 0.40 & 1.15 & 0.43 & 1.17 & 0.44 \\
\hline 0.058 & 0.25 & 2.191 & 0.88 & 0.29 & 0.73 & 0.22 & 0.78 & 0.24 \\
\hline 0.058 & 0.35 & 2.091 & 0.56 & 0.10 & 0.55 & 0.10 & 0.57 & 0.11 \\
\hline
\end{tabular}

Tabla 42. Estudio sistemático con CFD de presiones en el punto de estancamiento.

Para el estudio de los perfiles de velocidades medias horizontal y vertical, y de energía cinética turbulenta en el cuenco de disipación, se han considerado tres caudales específicos. Los perfiles se han medido con equipo Doppler en distintas secciones del cuenco, localizadas a una distancia $X$ aguas abajo del punto de estancamiento. En las siguientes figuras se comparan las variables considerando tres colchones efectivos.
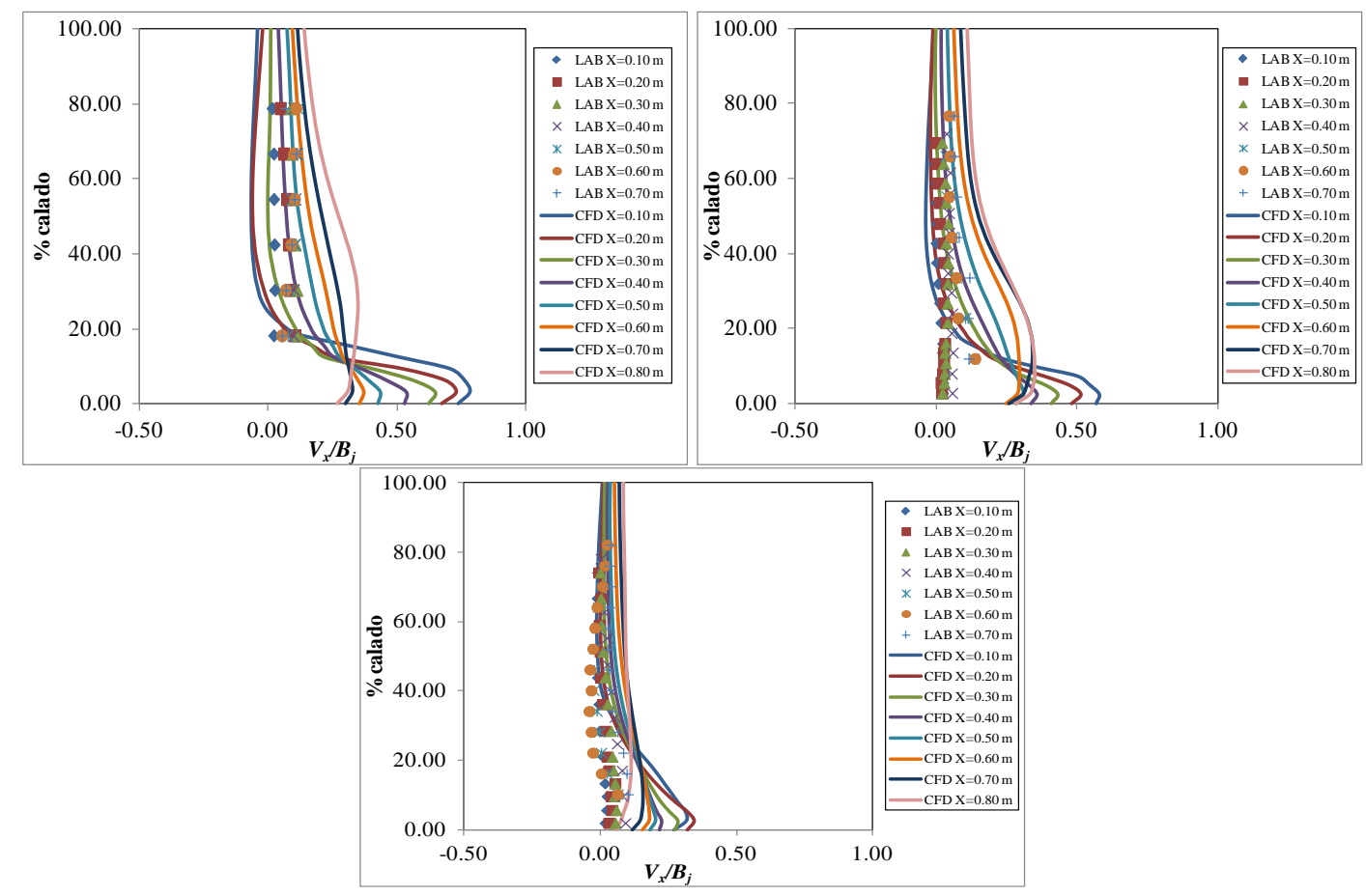

Figura 149. Perfiles de velocidades medias horizontales en el cuenco de disipación aguas abajo del punto de estancamiento: a) $q=0.058 \mathrm{~m}^{2} / \mathrm{s}, H=2.27 \mathrm{~m}, Y=0.17 \mathrm{~m}$; $b$ ) $q=0.058 \mathrm{~m}^{2} / \mathrm{s}, H=$ $2.20 \mathrm{~m}, Y=0.25 \mathrm{~m}$; c) $q=0.058 \mathrm{~m}^{2} / \mathrm{s}, H=2.10 \mathrm{~m}, Y=0.35 \mathrm{~m}$. 

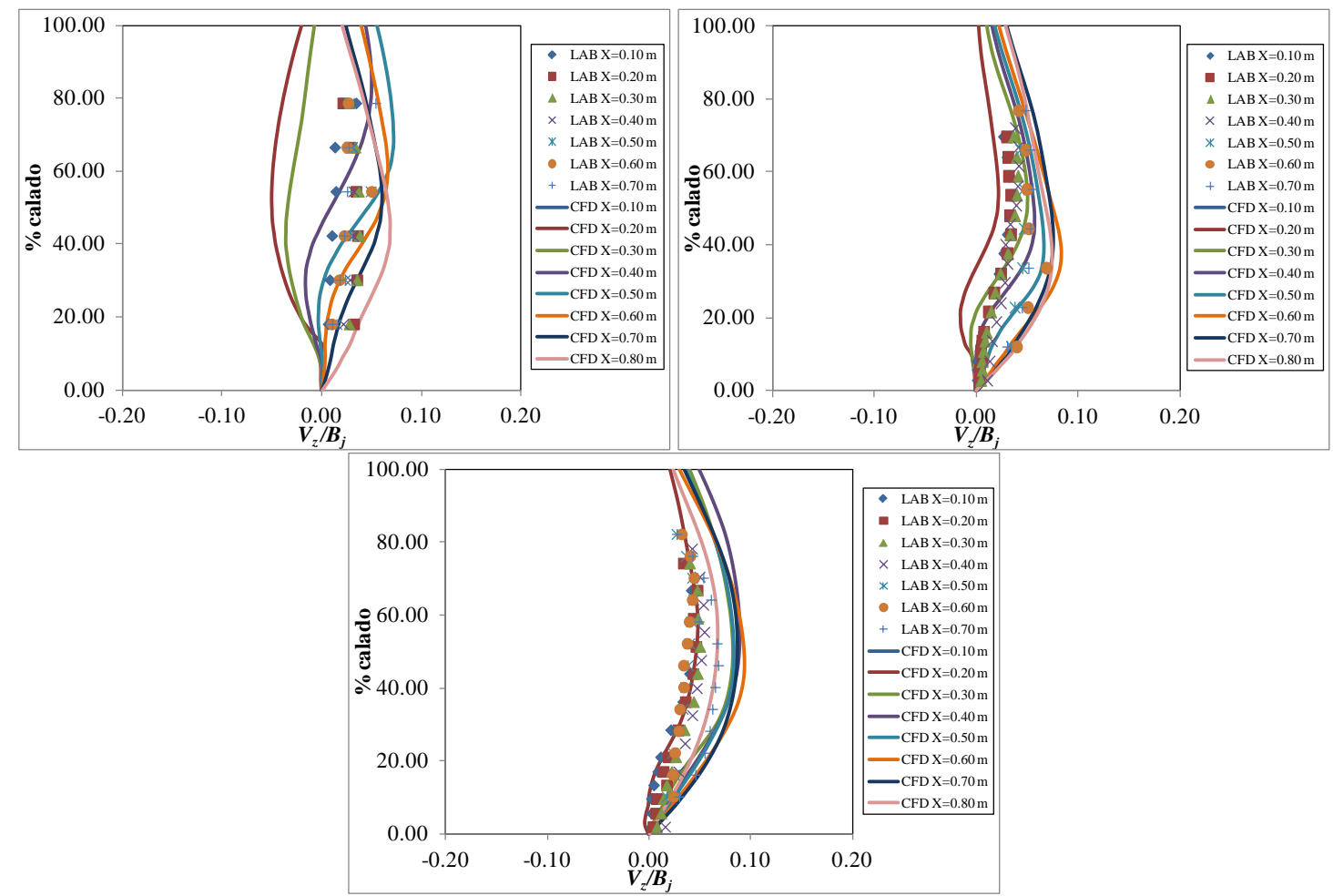

Figura 150. Perfiles de velocidades medias verticales en el cuenco de disipación aguas abajo del punto de estancamiento: a) $q=0.058 \mathrm{~m}^{2} / \mathrm{s}, H=2.27 \mathrm{~m}, Y=0.17 \mathrm{~m}$; b) $q=0.058 \mathrm{~m}^{2} / \mathrm{s}, H=$ $2.20 \mathrm{~m}, Y=0.25 \mathrm{~m}$; c) $q=0.058 \mathrm{~m}^{2} / \mathrm{s}, H=2.10 \mathrm{~m}, Y=0.35 \mathrm{~m}$.

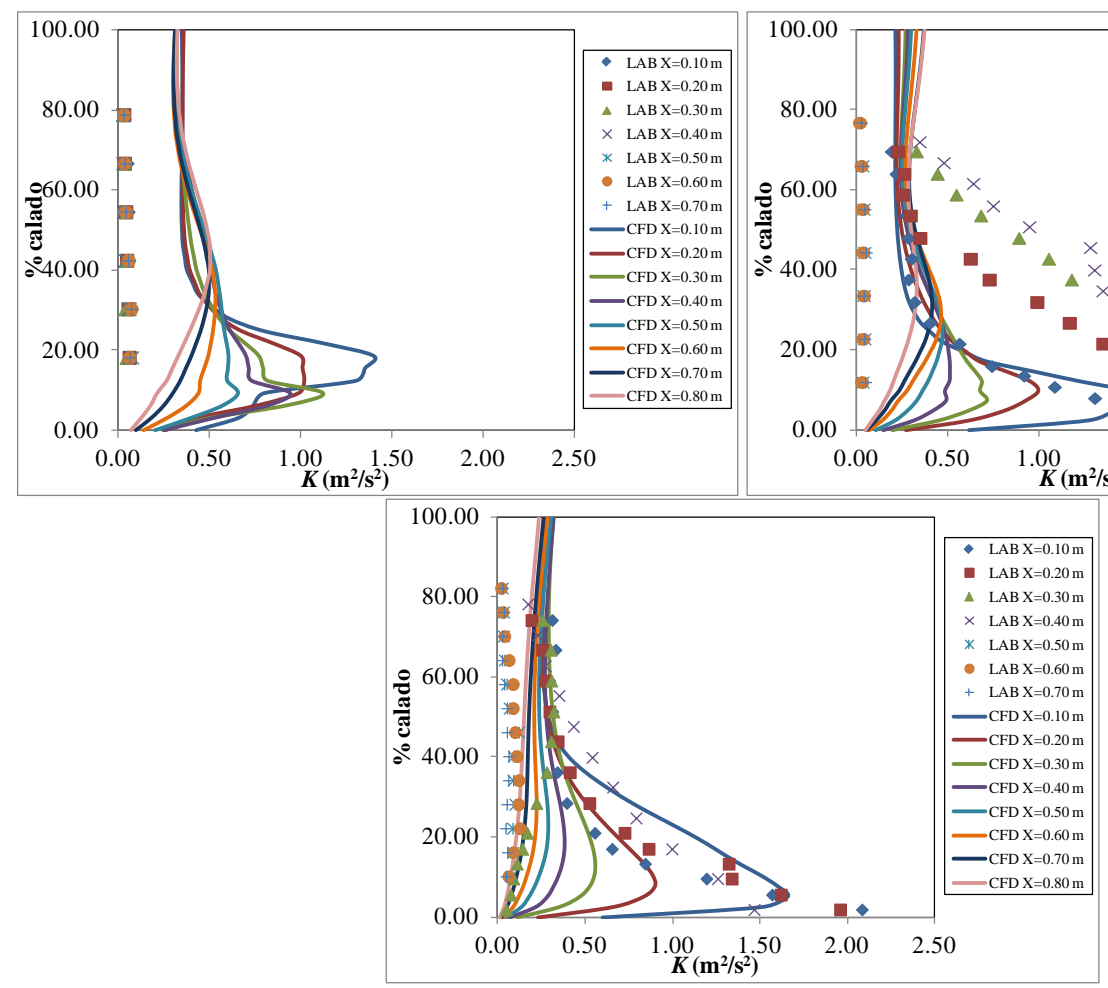

Figura 151. Perfiles de energía cinética turbulenta K en el cuenco de disipación aguas abajo del punto de estancamiento: a) $q=0.058 \mathrm{~m}^{2} / \mathrm{s}, H=2.27 \mathrm{~m}, Y=0.17 \mathrm{~m}$; b) $q=0.058 \mathrm{~m}^{2} / \mathrm{s}, H=$ $2.20 \mathrm{~m}, Y=0.25 \mathrm{~m}$; c) $q=0.058 \mathrm{~m}^{2} / \mathrm{s}, H=2.10 \mathrm{~m}, Y=0.35 \mathrm{~m}$. 


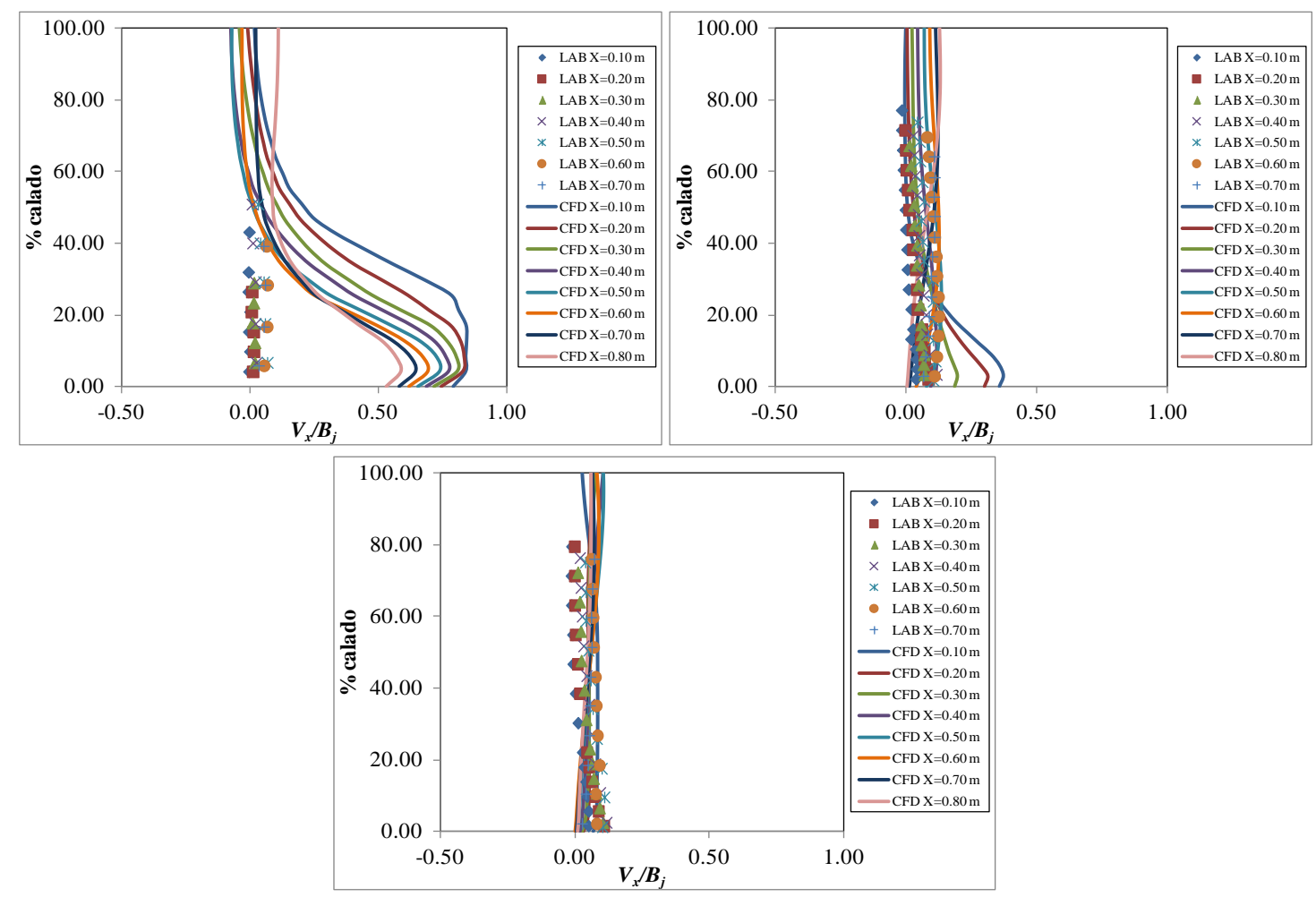

Figura 152. Perfiles de velocidades medias horizontales en el cuenco de disipación aguas abajo del punto de estancamiento: a) $q=0.037 \mathrm{~m}^{2} / \mathrm{s}, H=2.30 \mathrm{~m}, Y=0.12 \mathrm{~m}$; b) $q=0.037 \mathrm{~m}^{2} / \mathrm{s}, H=$ $2.20 \mathrm{~m}, Y=0.24 \mathrm{~m}$; c) $q=0.037 \mathrm{~m}^{2} / \mathrm{s}, H=2.12 \mathrm{~m}, Y=0.33 \mathrm{~m}$.

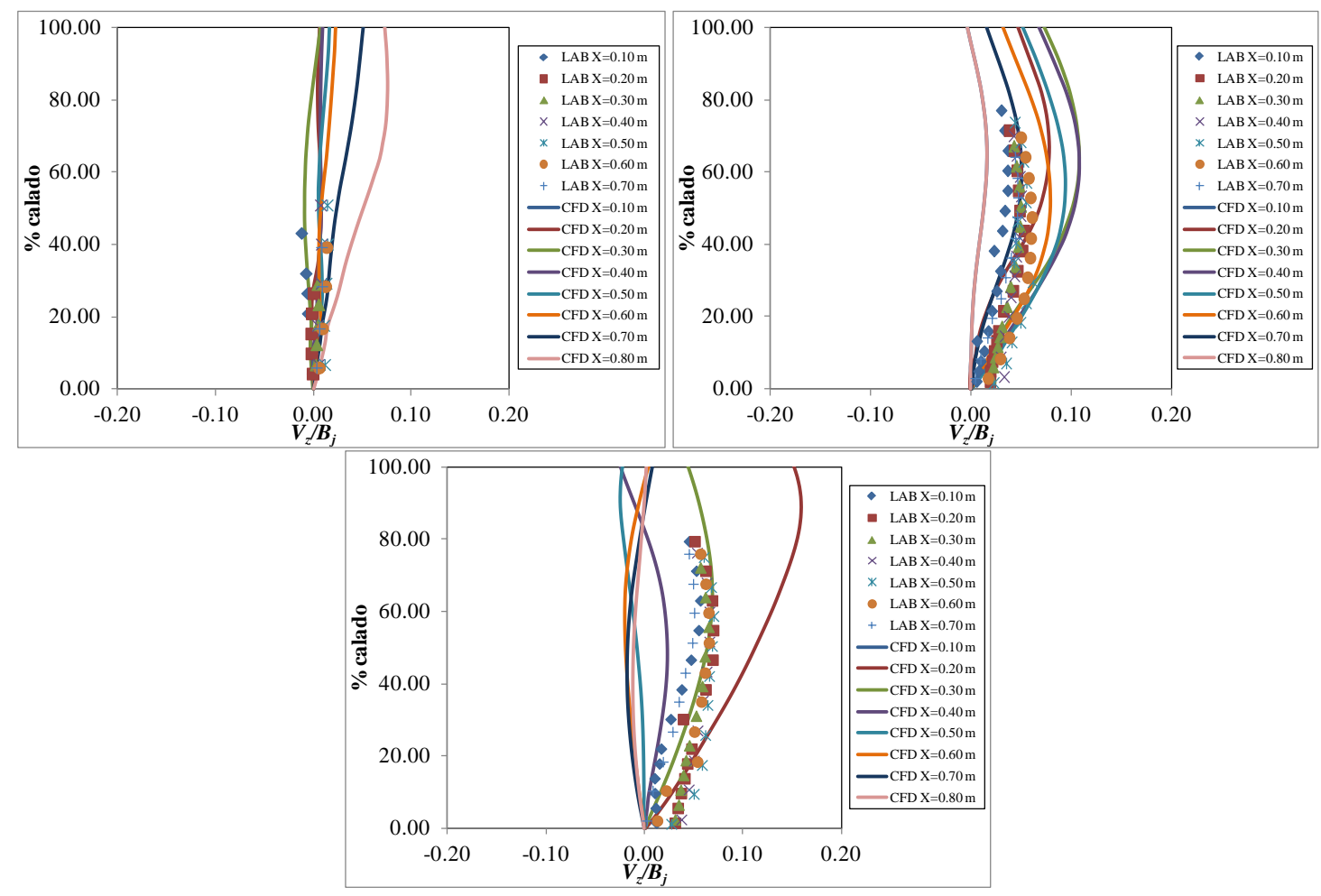

Figura 153. Perfiles de velocidades medias verticales en el cuenco de disipación aguas abajo del punto de estancamiento: a) $q=0.037 \mathrm{~m}^{2} / \mathrm{s}, H=2.30 \mathrm{~m}, Y=0.12 \mathrm{~m}$; b) $q=0.037 \mathrm{~m}^{2} / \mathrm{s}, H$ $=2.20 \mathrm{~m}, Y=0.24 \mathrm{~m}$; c) $q=0.037 \mathrm{~m}^{2} / \mathrm{s}, H=2.12 \mathrm{~m}, Y=0.33 \mathrm{~m}$. 

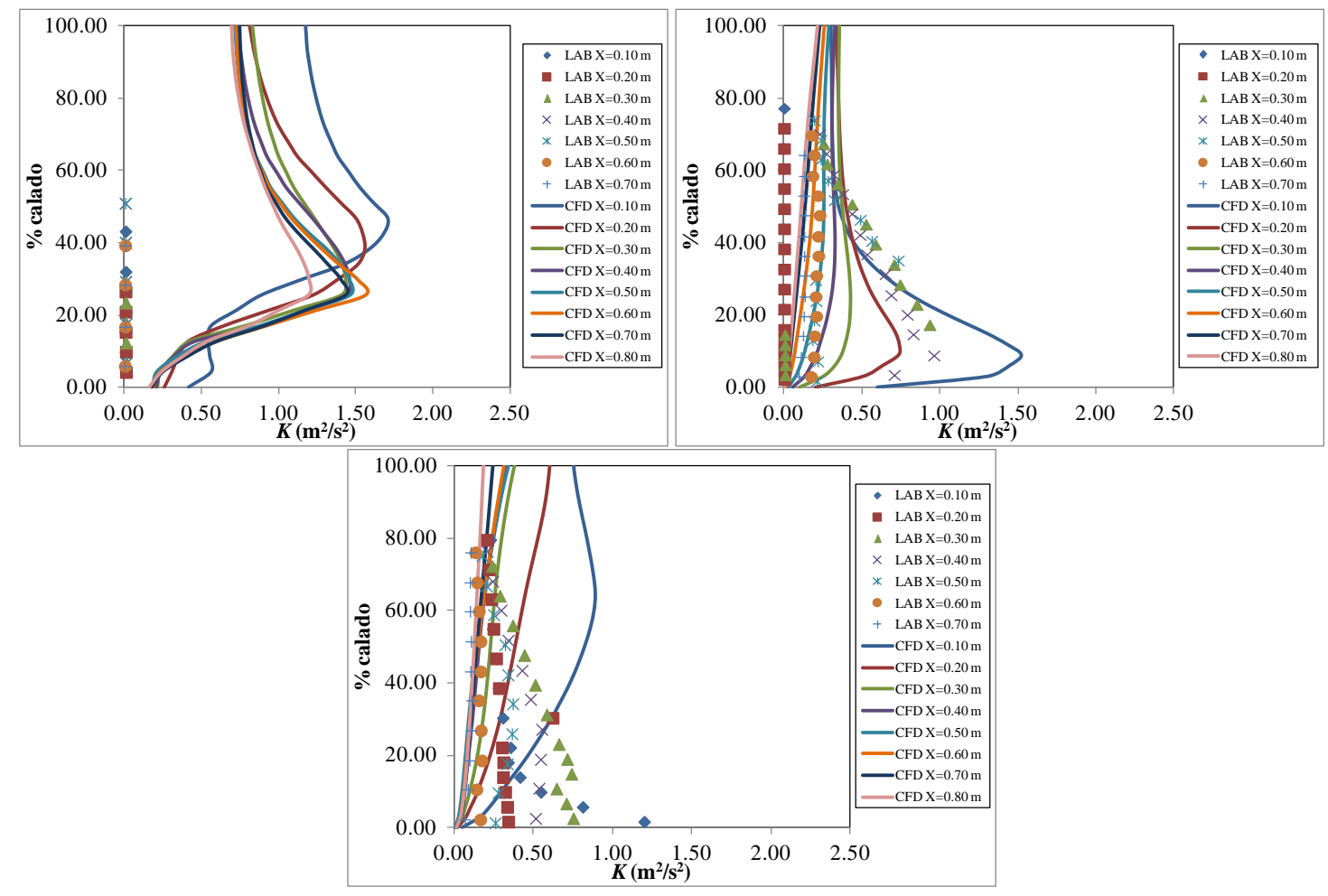

Figura 154. Perfiles de energía cinética turbulenta K en el cuenco de disipación aguas abajo del punto de estancamiento: a) $q=0.037 \mathrm{~m}^{2} / \mathrm{s}, H=2.30 \mathrm{~m}, Y=0.12 \mathrm{~m}$; b) $q=0.037 \mathrm{~m}^{2} / \mathrm{s}, H$ $=2.20 \mathrm{~m}, Y=0.24 \mathrm{~m}$; c) $q=0.037 \mathrm{~m}^{2} / \mathrm{s}, H=2.12 \mathrm{~m}, Y=0.33 \mathrm{~m}$.

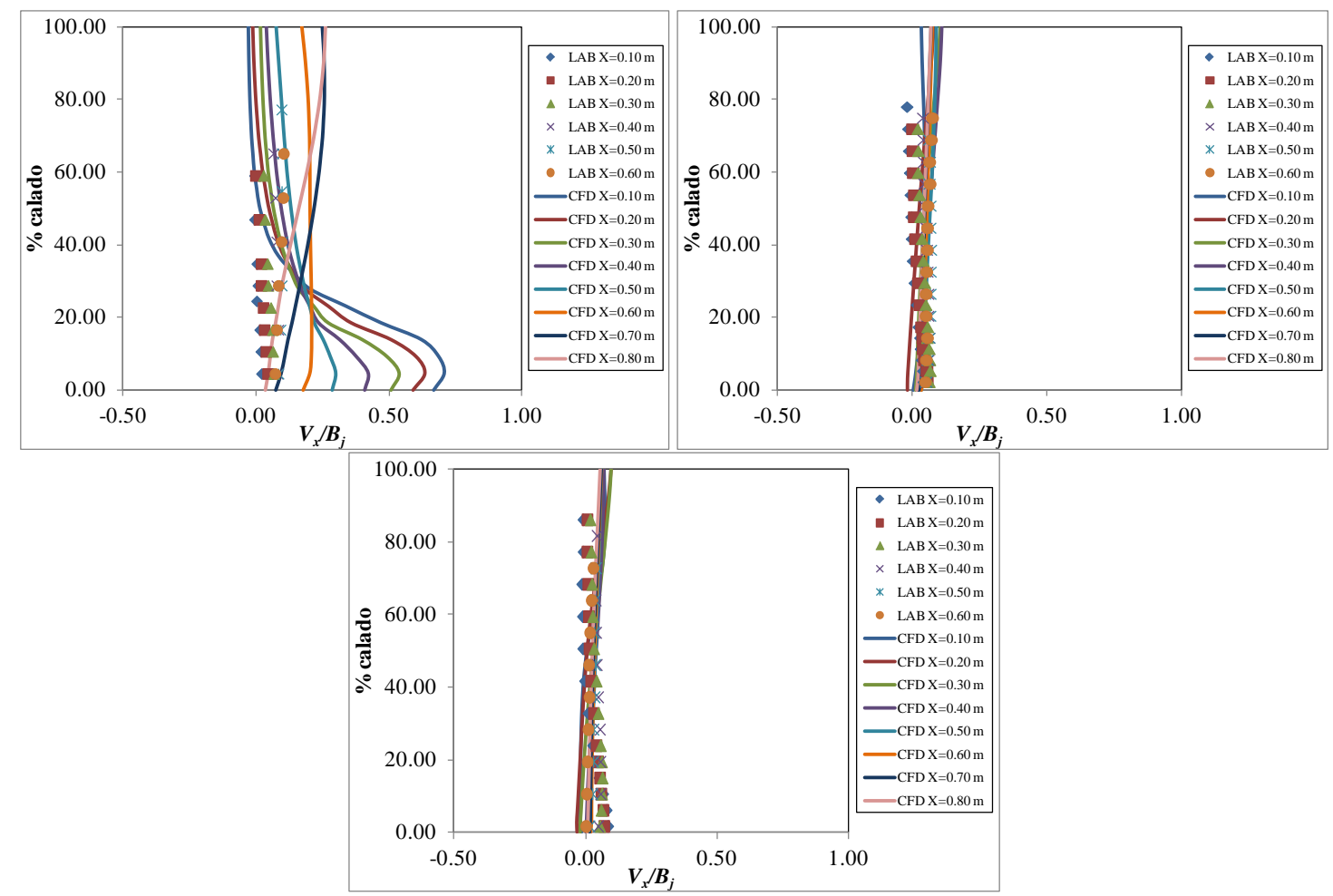

Figura 155. Perfiles de velocidades medias horizontales en el cuenco de disipación aguas abajo del punto de estancamiento: a) $q=0.023 \mathrm{~m}^{2} / \mathrm{s}, H=2.29 \mathrm{~m}, Y=0.12 \mathrm{~m}$; b) $q=0.020 \mathrm{~m}^{2} / \mathrm{s}, H$ $=2.18 \mathrm{~m}, Y=0.22 \mathrm{~m}$;c) $q=0.023 \mathrm{~m}^{2} / \mathrm{s}, H=2.10 \mathrm{~m}, Y=0.30 \mathrm{~m}$. 

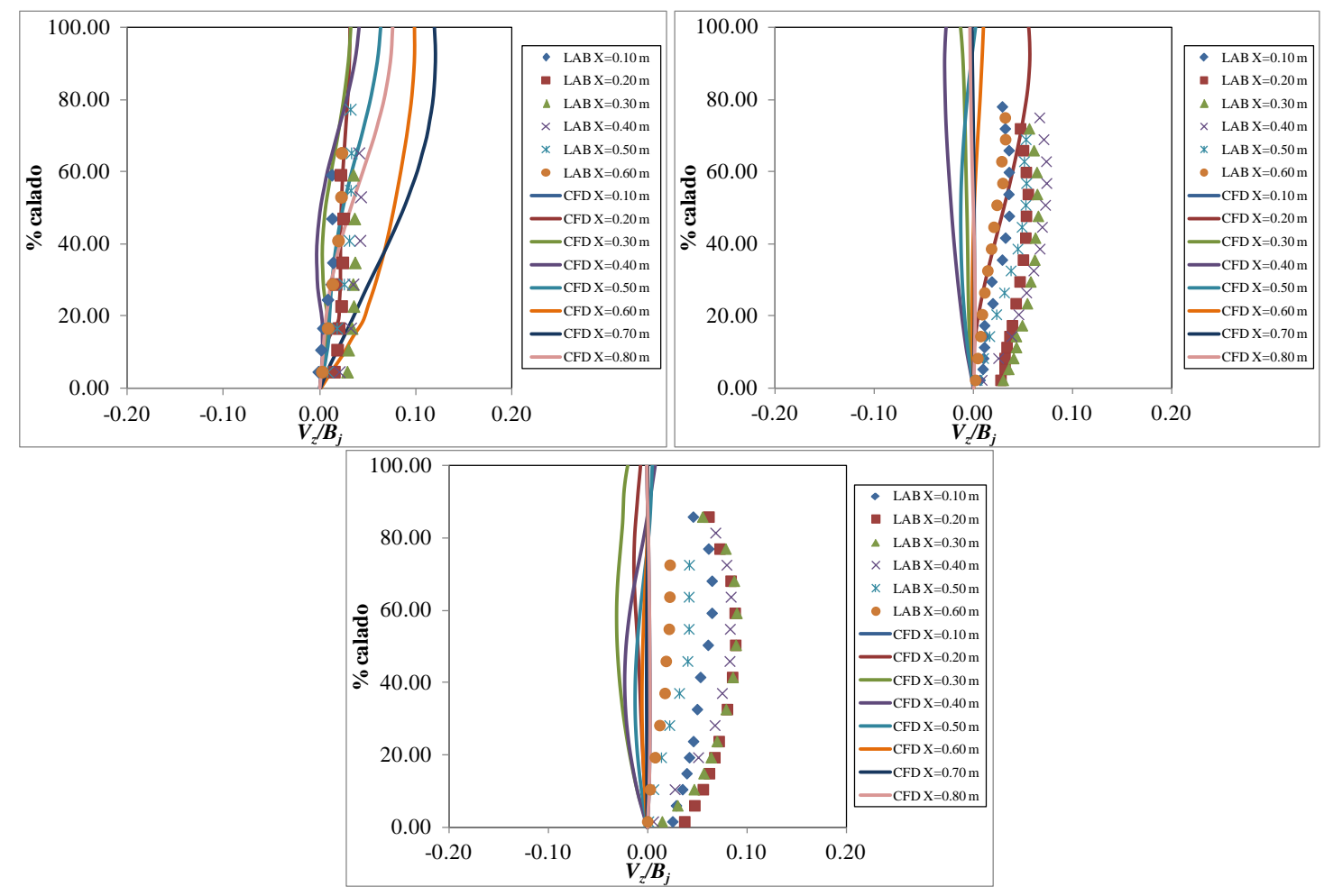

Figura 156. Perfiles de velocidades medias verticales en el cuenco de disipación aguas abajo del punto de estancamiento: a) $q=0.023 \mathrm{~m}^{2} / \mathrm{s}, H=2.29 \mathrm{~m}, Y=0.12 \mathrm{~m}$; b) $q=0.020 \mathrm{~m}^{2} / \mathrm{s}, H$ $=2.18 \mathrm{~m}, Y=0.22 \mathrm{~m}$;c) $q=0.023 \mathrm{~m}^{2} / \mathrm{s}, H=2.10 \mathrm{~m}, Y=0.30 \mathrm{~m}$.

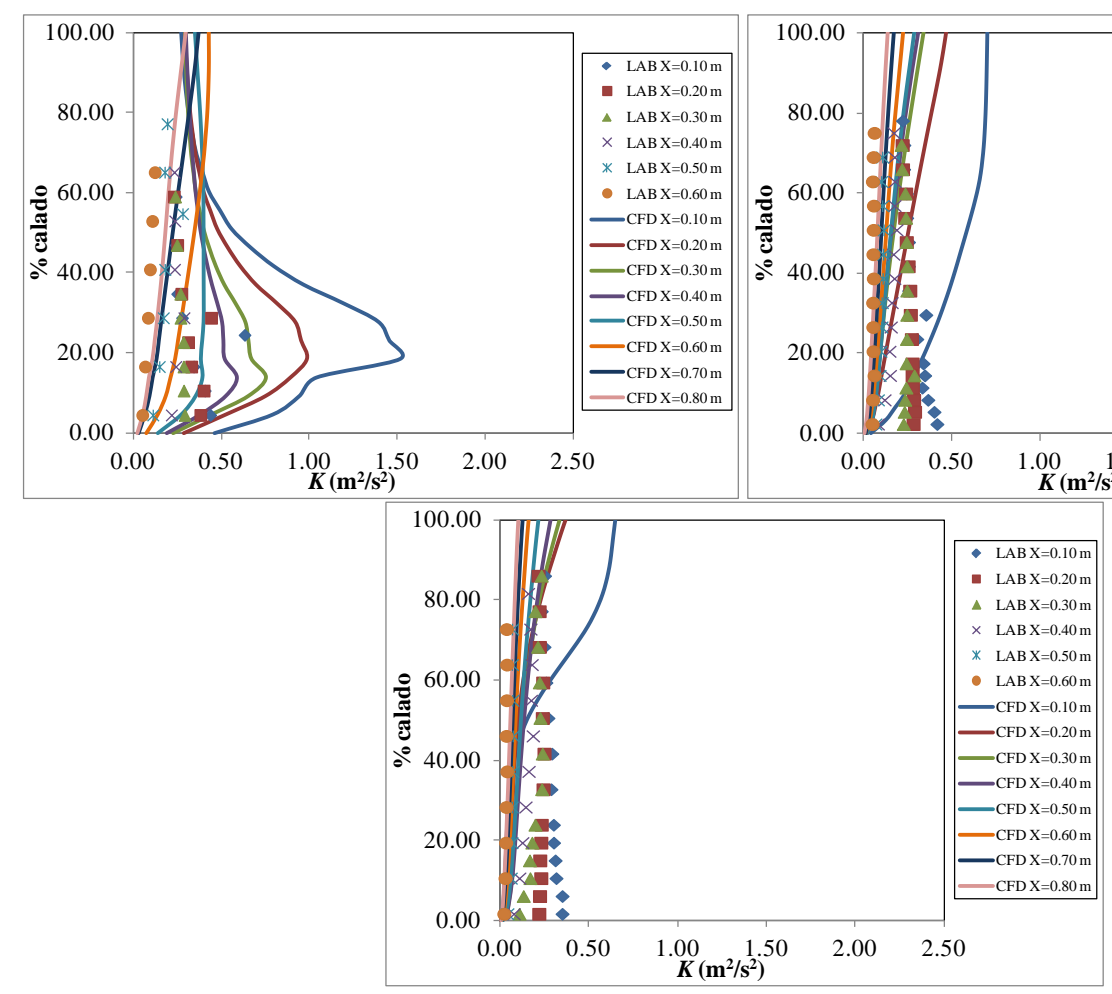

Figura 157. Perfiles de energía cinética turbulenta K en el cuenco de disipación aguas abajo del punto de estancamiento: a) $q=0.023 \mathrm{~m}^{2} / \mathrm{s}, H=2.29 \mathrm{~m}, Y=0.12 \mathrm{~m}$; b) $q=0.020 \mathrm{~m}^{2} / \mathrm{s}, H$ $=2.18 \mathrm{~m}, Y=0.22 \mathrm{~m}$;c) $q=0.023 \mathrm{~m}^{2} / \mathrm{s}, H=2.10 \mathrm{~m}, Y=0.30 \mathrm{~m}$. 
En general, las simulaciones numéricas obtenidos en los distintos perfiles situados a una distancia $X$ del punto de estancamiento siguen el patrón observado en las mediciones realizadas con el equipo Doppler, con diferencias en las velocidades inferiores al $10 \%$ de la velocidad incidente. Sin embargo, existen algunas configuraciones en las que las simulaciones divergen de los datos de laboratorio. Las discrepancias son notables en los perfiles de velocidades obtenidos en las inmediaciones del punto de estancamiento con pequeños colchones. En estas situaciones, se produce una gran entrada de aire en el colchón de agua (Figura 158), lo que dificulta las mediciones con equipos Doppler. Puesto que la señal del equipo Doppler se transmite por el medio líquido, el chorro de fondo no queda bien recogido en las mediciones de laboratorio cercanas al punto de estancamiento. Del mismo modo, las energías cinéticas turbulentas obtenidas con colchones pequeños tienden a ser muy reducidas comparadas con los valores registrados en los colchones superiores y con los resultados de las simulaciones numéricas.

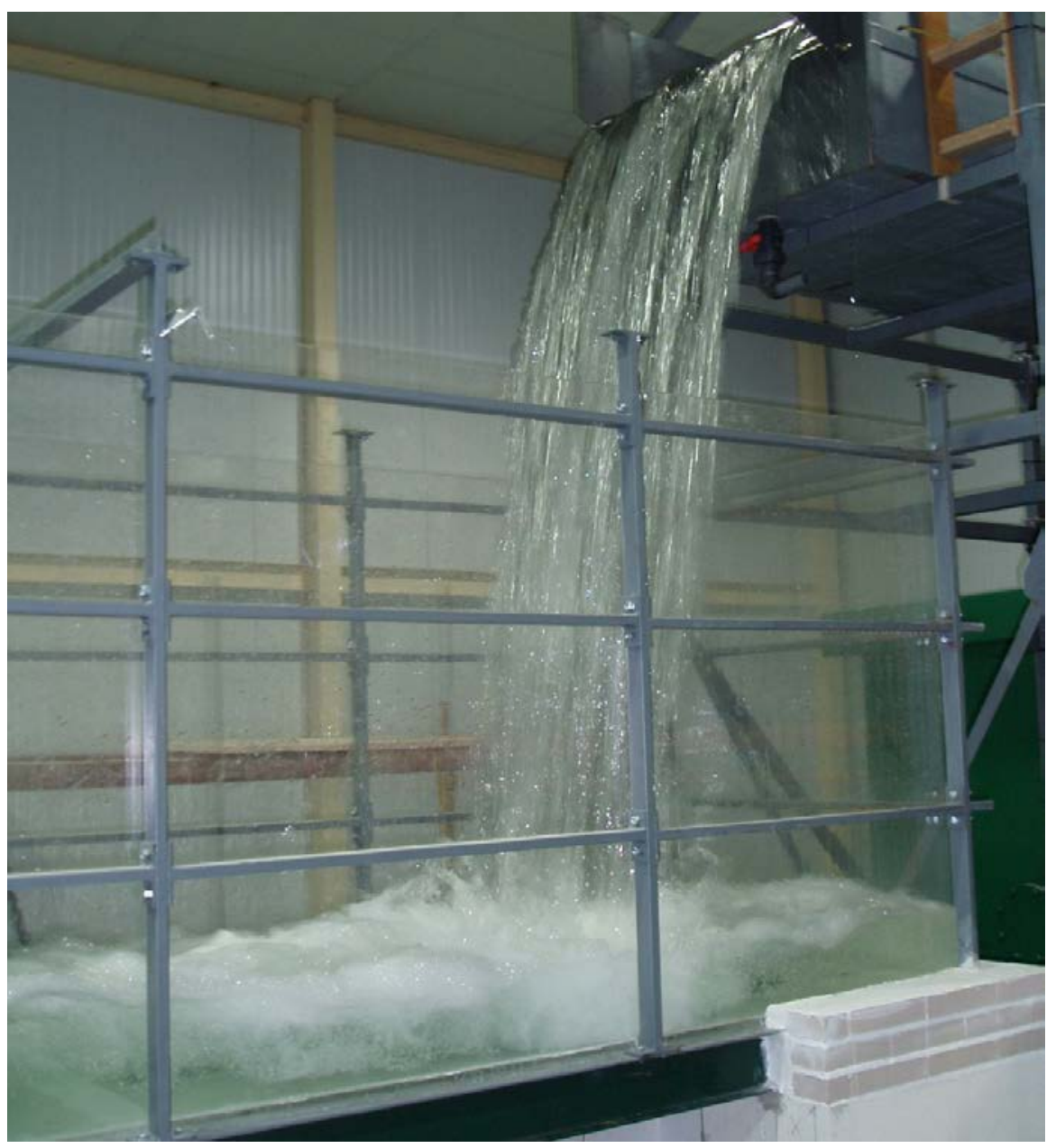

Figura 158. Entrada de aire en el cuenco de disipación observada en laboratorio ( $q=0.058$ $\mathrm{m}^{2} / \mathrm{s}, H=2.27 \mathrm{~m}, Y=0.17 \mathrm{~m}$ ). 
En la Figura 159 se muestras las distribuciones de velocidades adimensionalizadas obtenidas con las simulaciones numéricas. La Figura 160 muestra los resultados obtenidos tanto en laboratorio como con las simulaciones numéricas. En ambos casos, los resultados son muy similares a las leyes teóricas propuestas por diferentes autores y en este trabajo en el apartado de análisis de resultados de laboratorio. Debido al efecto de recirculación que aparece en la parte superior de los resaltos sumergidos analizados, se observa una zona de dispersión para relaciones $V_{x} / V_{\text {máx }}<0.40$.

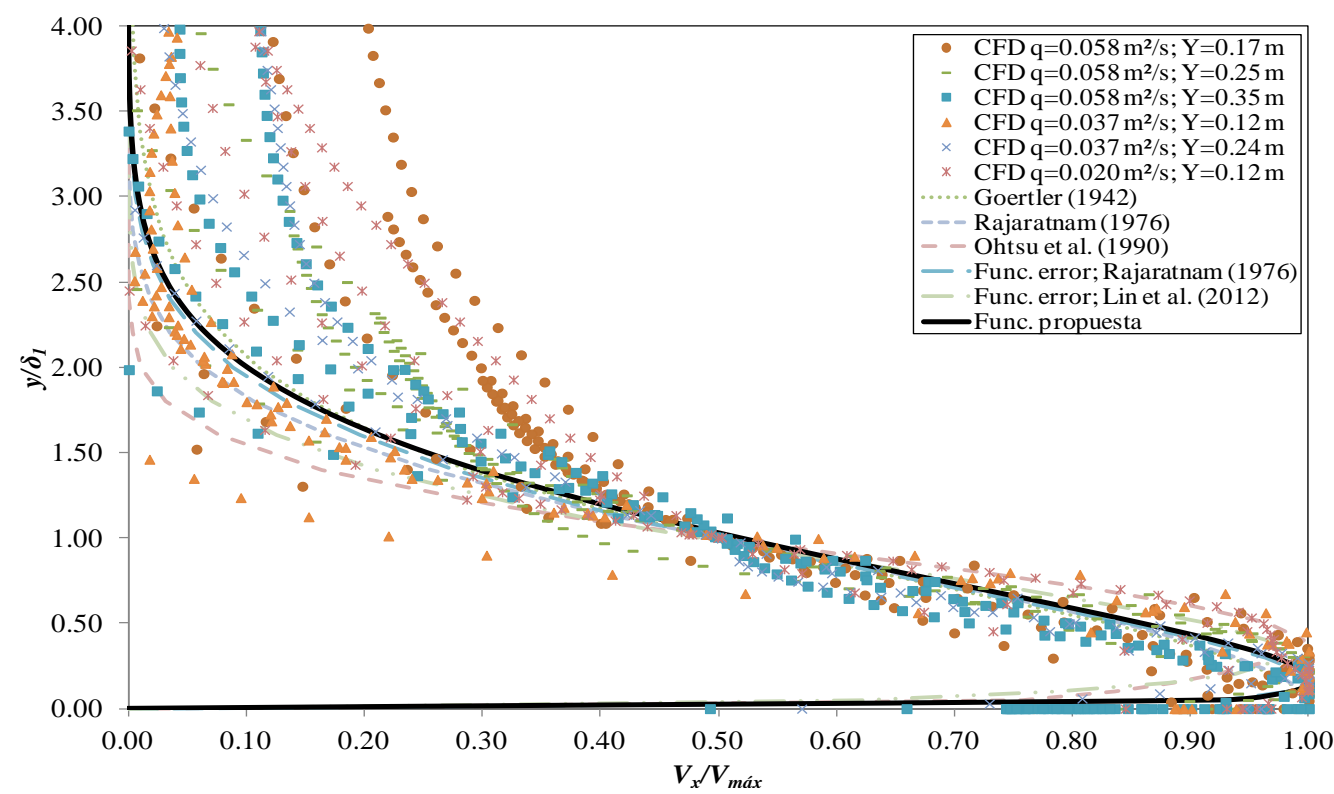

Figura 159. Distribución de velocidades obtenidas aguas abajo del punto de estancamiento con simulaciones numéricas.

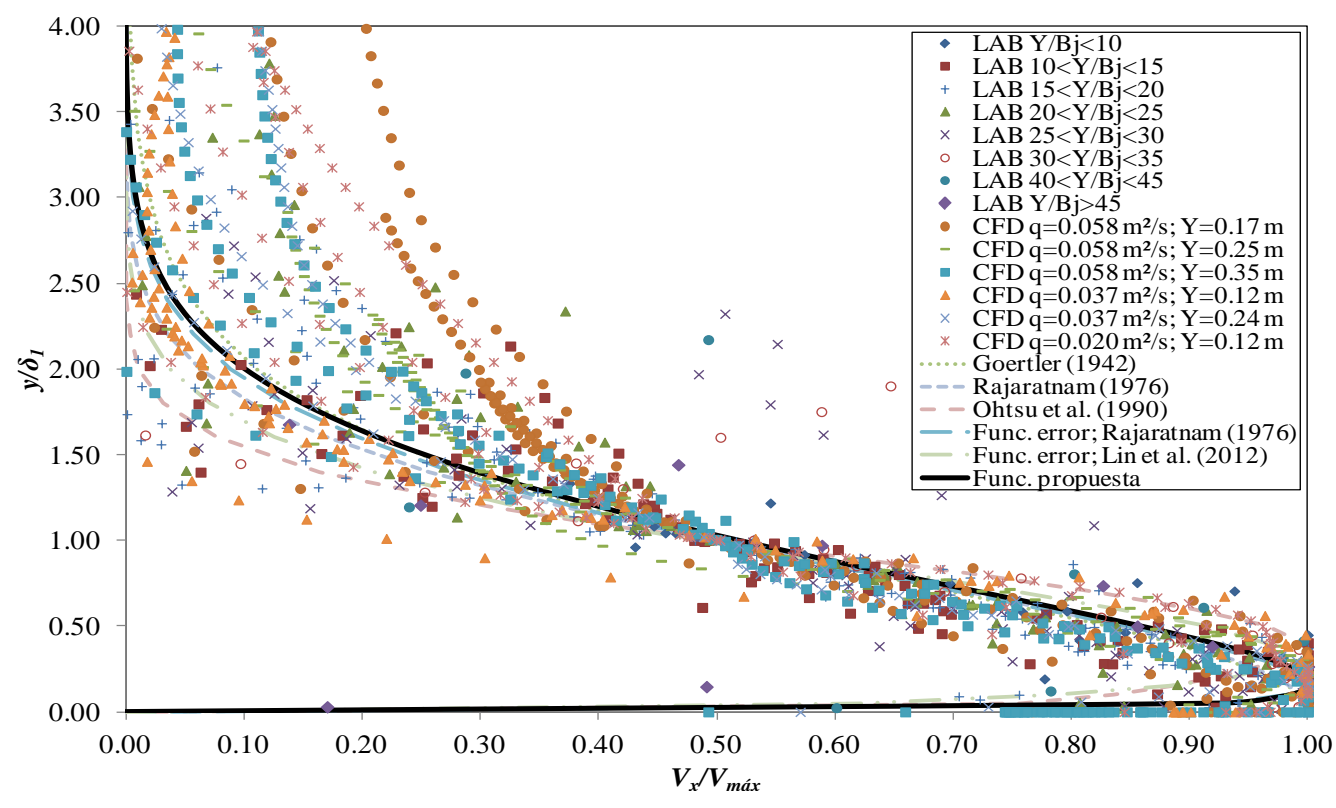

Figura 160. Distribución de velocidades obtenidas aguas abajo del punto de estancamiento con simulaciones numéricas y mediciones de laboratorio. 


\section{ESTUDIO DE APLICACIÓN}

En la parte final de este proyecto de investigación se tuvo la oportunidad de aplicar los resultados obtenidos en un caso de una presa en fase de diseño. Las simulaciones numéricas con CFD fueron comprobadas y validadas en un estudio de socavación. Se realizó la comparación con formulaciones empíricas obtenidas en modelos hidráulicos y prototipos, y con la metodología semiempírica propuesta por Annandale (1995, 2006), en la cual se ha mejorado la caracterización de las fluctuaciones de presión con los resultados anteriormente expuestos, y publicados en Castillo y Carrillo (2013, 2014).

\subsection{Características de la presa}

El estudio analizó los cambios esperados en el río Paute (Ecuador) como resultado de la construcción de la presa Paute-Cardenillo (propiedad de Celec Ep-Hidropaute). La presa forma parte del Sistema Eléctrico Nacional de Ecuador. Para evaluar la estabilidad y la seguridad de la estructura, se determinó la forma y dimensiones de la erosión generada aguas abajo de la presa.

Se trata de una presa arco de doble curvatura con una altura máxima de $135 \mathrm{~m}$ sobre los cimientos y una longitud de coronación de 270 metros aproximadamente (Figura 161). La coronación se sitúa a la cota 926 metros. El embalse tiene una longitud de 2.98 km cuando el nivel del agua está en la cota 924 m (nivel máximo normal).

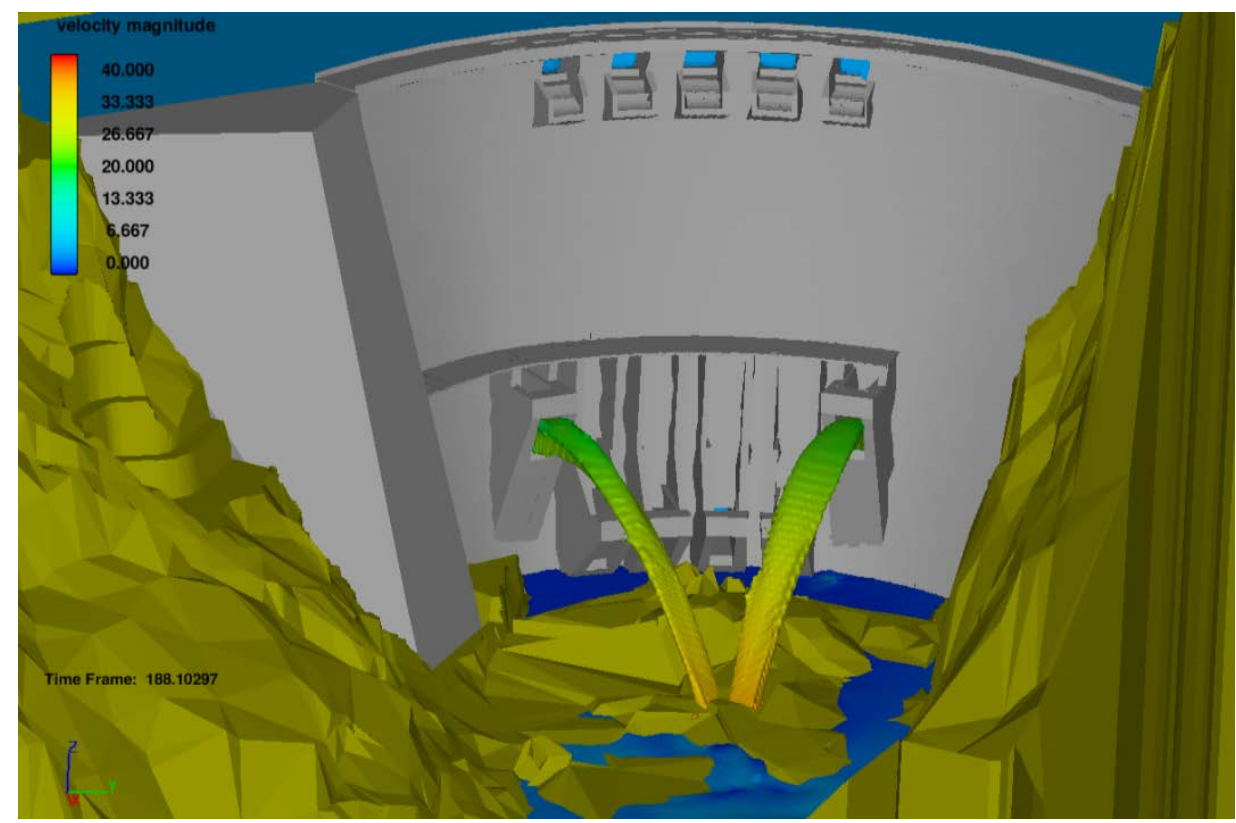

Figura 161. Presa y cerrada del proyecto Paute-Cardenillo (Ecuador). 
Los órganos de desagüe consisten en un aliviadero de vertido libre controlado por clapetas con capacidad para trasegar un caudal de $Q_{4}=700 \mathrm{~m}^{3} / \mathrm{s}$ (período de retorno $T R$ = 4 años), y un desagüe de medio fondo constituido por con dos conductos de sección 5.00 x $5.80 \mathrm{~m}$. Considerando el nivel máximo normal (924 m), los desagües intermedios tienen una capacidad de $Q_{40}=1760 \mathrm{~m}^{3} / \mathrm{s}$. Considerando el funcionamiento conjunto del aliviadero de superficie y los desagües de medio fondo, la capacidad de descarga es $Q_{100}$ $=2340 \mathrm{~m}^{3} / \mathrm{s}$. Si además se considera la descarga adicional de los 4 desagües de fondo, la capacidad de descarga de la presa sería $Q_{10000}=5520 \mathrm{~m}^{3} / \mathrm{s}$.

El lecho del río está conformado por una capa de $24 \mathrm{~m}$ de material aluvial, debajo de la cual se dispone una capa de $10 \mathrm{~m}$ de roca meteorizada. La roca sana se sitúa a 34 m de la cota original del terreno.

\subsection{Estudio de la socavación con fórmulas empíricas}

La mayor parte de las ecuaciones existentes han sido obtenidas mediante análisis dimensional y análisis estadístico de datos obtenidos en modelos reducidos construidos bajo leyes de semejanza de Froude para el cálculo de la socavación aguas abajo de un salto de esquí. Algunas formulaciones están basadas en mediciones de prototipos.

Se han seleccionado veintinueve ecuaciones y se ha determinado la profundidad de socavación en la zona de impacto para caudales de diferentes períodos de retorno. La expresión general de las formulaciones se puede expresar como:

$$
D_{s}=h+D=K \frac{q^{x} H_{n}^{y} h^{w}}{g^{v} d^{z}}
$$

donde $D_{s}$ es la profundidad de socavación medida desde la superficie del agua, $h$ la profundidad del agua hasta el lecho del cauce (calado aguas abajo), $D$ la profundidad de socavación desde el lecho del cauce original, $K$ un coeficiente experimental, generalmente relacionado a la resistencia del material, $q$ el caudal específico, $H_{n}$ la carga de energía neta, y $d$ el diámetro característico del material. El resto de las variables se definen en la Figura 162.

La Tabla 43 muestra los coeficientes correspondientes de las fórmulas simplificadas propuestas por diversos autores, mientras que la Tabla 44 recoge las expresiones que no se adaptan a la forma general. 


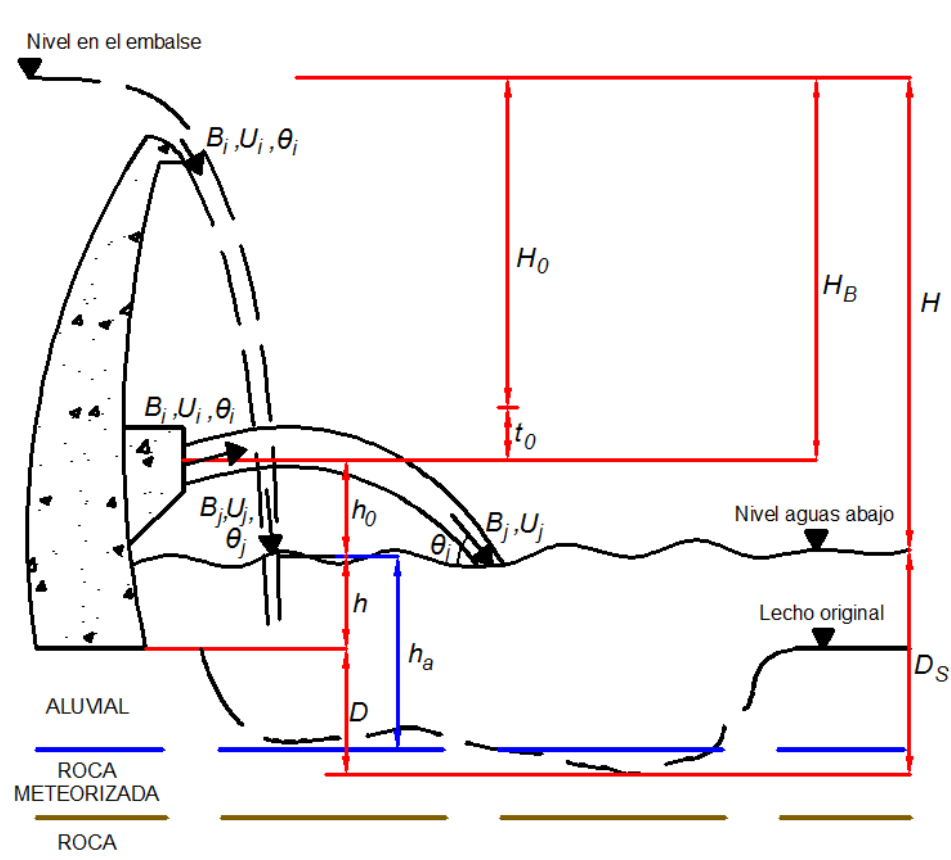

$x, y, w, v, z$. Exponentes empíricos.

$t_{0}=$ pérdidas de energía en el conducto.

$H_{n}=H_{0}=H_{B}-t_{0}$. Energía neta en la salida del conducto.

$H_{n}=H$. Altura de caída desde el nivel del embalse.

$h_{0}$. Distancia vertical entre la salida del desagüe y el nivel del cuenco

$h_{a}$. Distancia vertical entre el nivel del cuenco y el fondo.

$B_{i}, U_{i}, \theta_{i}$. Espesor, velocidad y ángulo del chorro en las condiciones iniciales.

$B_{j}, U_{j}, \theta_{j}$. Espesor, velocidad y ángulo del chorro en las condiciones incidentes.

Figura 162. Esquema de socavación en la presa Paute-Cardenillo (Castillo y Carrillo, 2014).

\begin{tabular}{|l|c|c|c|c|c|}
\hline \multicolumn{1}{|c|}{ Autor (año) } & $\boldsymbol{k}$ & $\boldsymbol{x}$ & $\boldsymbol{y}$ & $\boldsymbol{z}$ & $\boldsymbol{d}$ \\
\hline Schoklitsch (1932) & 0.521 & 0.57 & 0.20 & 0.32 & $d_{90}$ \\
\hline Veronese-A (1937) & 0.202 & 0.54 & 0.225 & 0.42 & $d_{m}$ \\
\hline Veronese-B (1937) & 1.900 & 0.54 & 0.225 & 0 & - \\
\hline Eggenburger (1944) & 1.440 & 0.60 & 0.50 & 0.40 & $d_{90}$ \\
\hline Hartung (1959) & 1.400 & 0.64 & 0.36 & 0.32 & $d_{85}$ \\
\hline Frake (1960) & 1.130 & 0.67 & 0.50 & 0.50 & $d_{90}$ \\
\hline Damle-A (1966) & 0.652 & 0.50 & 0.50 & 0 & - \\
\hline Damle-B (1966) & 0.543 & 0.50 & 0.50 & 0 & - \\
\hline Damle-C (1966) & 0.362 & 0.50 & 0.50 & 0 & - \\
\hline Chee y Padiyar (1969) & 2.126 & 0.67 & 0.18 & 0.063 & $d_{m}$ \\
\hline Bisaz y Tschopp (1972) & 2.760 & 0.50 & 0.25 & 1.00 & $d_{90}$ \\
\hline Chee y Kung (1974) & 1.663 & 0.60 & 0.20 & 0.10 & $d_{m}$ \\
\hline Martins-B & 1.500 & 0.60 & 0.10 & 0 & - \\
\hline Taraimovich (1978) & 0.633 & 0.67 & 0.25 & 0 & - \\
\hline Machado (1980) & 1.350 & 0.50 & 0.3145 & 0.0645 & $d_{90}$ \\
\hline SOFRELEC (1980) & 2.300 & 0.60 & 0.10 & 0 & - \\
\hline INCYTH (1981) & 1.413 & 0.50 & 0.25 & 0 & - \\
\hline Suppasri (2007) & 0.150 & 0.38 & 0.75 & 0 & - \\
\hline
\end{tabular}

Tabla 43. Coeficientes de formulaciones de socavación simplificadas (Castillo y Carrillo, 2014). 


\begin{tabular}{|l|l|}
\hline \multicolumn{1}{|c|}{ Autor (año) } & \multicolumn{1}{c|}{ Fórmula } \\
\hline Jaeger (1939) & $D_{S}=0.6 q^{0.5} H_{n}^{0.25}\left(h / d_{m}\right)^{0.333}$ \\
\hline Mikhalev (1960) & $D_{S}=\frac{1.804 q \cdot \sin \theta_{T}}{1-0.215 \cdot \cot \theta_{T}}\left(\frac{1}{d_{90}^{0.33} h^{0.50}}-\frac{1.126}{H_{n}}\right)$ \\
\hline $\begin{array}{l}\text { Rubinstein } \\
\text { (1965) }\end{array}$ & $D_{S}=h+0.19\left(\frac{H_{n}+h}{d_{90}}\right)^{0.75}\left(\frac{q^{1.20}}{H_{n}^{0.47} h^{0.33}}\right)$ \\
\hline $\begin{array}{l}\text { Mirskhulava } \\
\text { (1967) }\end{array}$ & $D_{S}=\left(\frac{0.97}{\left.\sqrt{d_{90}}-\frac{1.35}{\sqrt{H_{n}}}\right) \frac{q \cdot \sin \theta_{T}}{1-0.175 \cdot \cot \theta_{T}}+0.25 h}\right.$ \\
\hline $\begin{array}{l}\text { Martins-A (1973) } \\
\text { Mason-A (1985) }\end{array}$ & $D_{S}=0.14 N-0.73 \frac{h^{2}}{N}+1.7 h ; N=\left(Q^{3} H_{n}^{1.5} / d_{m}^{2}\right)^{1 / 7}$ \\
\hline $\begin{array}{l}\text { Mason-B (1989) } \\
\text { Bombardelli and } \\
\text { Gioia (2006) }\end{array}$ & $D_{S}=3.27 \frac{q^{0.60} H^{0.05} h^{0.15}}{g^{0.30} d^{0.10}}$ \\
\hline $\begin{array}{l}\text { Modified } \\
\text { Veronese (1994) }\end{array}$ & $D_{S}=1.90 h^{0.225} q^{0.54} \cdot \sin \theta_{T}$ \\
\hline$g^{0.30} d^{0.06}$ \\
\hline $\begin{array}{l}g^{0.33} d^{0.33} \\
\text { Hoffmans (1998) }\end{array}$
\end{tabular}

Tabla 44. Formulaciones de socavación generales (Castillo y Carrillo, 2014).

En la Tabla $44 d_{m}(\mathrm{~m})$ es el tamaño medio de partícula del material de fondo, $\theta_{T}\left({ }^{\circ}\right)$ el ángulo del chorro de impacto con el nivel de aguas abajo, $d_{90}(\mathrm{~m})$ el tamaño del material del fondo en el cual el $90 \%$ en peso es más pequeño, $g\left(9.81 \mathrm{~m} / \mathrm{s}^{2}\right)$ la aceleración de la gravedad, $\beta$ la relación aire-agua, $u_{T}(\mathrm{~m} / \mathrm{s})$ la velocidad del chorro de impacto en la superficie de aguas abajo, $c_{2 v}$ un coeficiente relacionado con el $d_{90}$, $k_{t}$ el factor hidráulico, $k_{e}$ el coeficiente de socavación de la roca, $t_{T}(\mathrm{~m})$ el espesor del chorro en el punto de incidencia con el nivel de aguas abajo, $m$ un coeficiente del calado del colchón de agua, $\rho$ la densidad del agua, y $\rho_{s}$ la densidad del sedimento.

La Tabla 45 recoge los valores de socavación generados por el aliviadero de vertido libre y el desagüe de medio fondo, obtenidos con diversos caudales de funcionamiento.

En el caso del funcionamiento del aliviadero de vertido libre, en valor medio la socavación alcanzaría una profundidad de unos $15 \mathrm{~m}$ para el caudal máximo de diseño $\left(Q_{40}=700 \mathrm{~m}^{3} / \mathrm{s}\right)$, con lo cual todavía quedarían $9 \mathrm{~m}$ para alcanzar la roca meteorizada. 
Sin embargo, si se considera el valor medio +1 desviación estándar, el caudal de 500 $\mathrm{m}^{3} / \mathrm{s}$ alcanzaría el estrato de la roca meteorizada (socavación en torno a $30 \mathrm{~m}$ ).

\begin{tabular}{|c|c|c|c|c|c|c|c|}
\cline { 3 - 8 } \multicolumn{1}{c|}{} & \multicolumn{4}{c|}{ Aliviadero de vertido libre } & \multicolumn{3}{c|}{ Desagüe de medio fondo } \\
\cline { 2 - 8 } & $\begin{array}{c}\text { Caudal } \\
\left(\mathrm{m}^{3} / \mathrm{s}\right)\end{array}$ & $\begin{array}{c}\text { Valor medio } \\
-1 \text { desviación }\end{array}$ & $\begin{array}{c}\text { Valor } \\
\text { medio }\end{array}$ & $\begin{array}{c}\text { Valor medio } \\
+1 \text { desviación }\end{array}$ & $\begin{array}{c}\text { Valor medio } \\
-1 \text { desviación }\end{array}$ & $\begin{array}{c}\text { Valor } \\
\text { medio }\end{array}$ & $\begin{array}{c}\text { Valor medio } \\
+1 \text { desviación }\end{array}$ \\
\hline \multirow{4}{*}{$\begin{array}{c}\text { Socavación } \\
(\mathrm{m})\end{array}$} & 500 & 0 & 10 & 30 & 4 & 9 & 14 \\
\cline { 2 - 9 } & 700 & 0 & 15 & 34 & 7 & 13 & 20 \\
\cline { 2 - 8 } & 1320 & - & - & - & 14 & 32 & 46 \\
\hline
\end{tabular}

Tabla 45. Cálculo de la socavación del aliviadero de vertido libre y del desagüe de medio fondo con las formulaciones empíricas. Valores medios y dispersión de +/- 1 desviación estándar (Castillo y Carrillo, 2014).

Las socavaciones producidas por el desagüe de medio fondo, en valor medio, erosionarían el aluvial del cauce totalmente a partir de un caudal de $Q_{22}=1320 \mathrm{~m}^{3} / \mathrm{s}$. Sin embargo, no se alcanzaría la roca meteorizada con el caudal de diseño $Q_{40}=1760$ $\mathrm{m}^{3} / \mathrm{s}$, llegando la erosión hasta una profundidad de unos $32 \mathrm{~m}$ desde la cota actual de cauce. Si se considera el valor medio +1 desviación estándar, el caudal de $830 \mathrm{~m}^{3} / \mathrm{s}$ ya alcanzaría el estrato de la roca meteorizada (socavación en torno a $24 \mathrm{~m}$ ), mientras que el caudal de $1250 \mathrm{~m}^{3} / \mathrm{s}$ alcanzaría la roca sana.

\subsection{Estudio de socavación con el método semiempírico de fluctuaciones de presión e índices de erosionabilidad}

El índice de erosionabilidad se basa en un umbral de erosión que relaciona la magnitud de la capacidad erosiva del agua con la capacidad relativa de un material (natural o artificial) para resistir la erosión. Existe una relación entre la potencia de escurrimiento o la magnitud de la capacidad erosiva del agua $P$ y una función matemática $f(K)$ que representa la capacidad relativa del material para resistir la erosión. En el umbral de erosión, la relación se puede escribir como $P=f(K)$. Cuando $P>f(K)$ se supera el umbral de erosión y el material es erosionado.

Según Annandale (2006), el proceso de erosión es debida a las fluctuaciones de presión, más que a un proceso de corte. La cuantificación de las fluctuaciones de presión de los chorros incidentes en cuencos de disipación ha sido objeto de estudio por diversos autores, y ha formado también parte de este trabajo.

Las presiones dinámicas de los chorros se relacionan con la intensidad de la turbulencia en las condiciones de descarga, la longitud del vuelo del chorro, el diámetro 
(chorros circulares) o el espesor (chorros rectangulares) en las condiciones incidentes y la profundidad del colchón de agua en el cuenco de disipación.

Annandale (1995, 2006) resume y establece una relación entre la potencia de escurrimiento y el índice de erosionabilidad para una amplia variedad de materiales y condiciones de flujo. La potencia de escurrimiento por unidad de área disponible en el chorro incidente se puede expresar como:

$$
P_{\text {chorro }}=\frac{\gamma Q H}{A}
$$

donde $\gamma$ es el peso específico del agua, $Q$ el caudal, $H$ la altura de caída o la carga de energía aguas arriba, y $A$ el área del chorro en la superficie de impacto.

El índice de erosionabilidad se define como:

$$
K=M_{s} \cdot K_{b} \cdot K_{d} \cdot J_{s}
$$

donde $M_{s}$ es el número de resistencia de masa, $K_{b}$ el número del tamaño del bloque, $K_{d}$ el número de resistencia al corte en contorno de la discontinuidad, y $J_{s}$ el número de estructura relativa del grano. La Tabla 46 resume las formulaciones empleadas para

\begin{tabular}{|c|c|c|}
\hline Material & Fórmula & Parámetros \\
\hline Roca & $\begin{array}{l}\text { Cuando UCS } \leq 10 \mathrm{MPa}: \\
\qquad \begin{array}{r}M_{S}=0.78 C_{r} \mathrm{UCS}^{1.05} \\
\text { Cuando UCS > } 10 \mathrm{MPa}: \\
\qquad M_{S}=C_{r} \mathrm{UCS} \\
C_{r}=\frac{g \rho_{r}}{\gamma_{r}}\end{array}\end{array}$ & $\begin{array}{l}\text { UCS. Coeficiente de resistencia a compresión no } \\
\text { confinada } \\
C_{r} \text {. Coeficiente de la densidad relativa } \\
\rho_{r} \text {. Densidad de la roca } \\
g . \text { Aceleración de la gravedad } \\
\gamma_{r}=27.10^{3} \mathrm{~N} / \mathrm{m}^{3} \text {. Peso específico de referencia de } \\
\text { la roca }\end{array}$ \\
\hline $\begin{array}{l}\text { Granular no } \\
\text { cohesivo }\end{array}$ & \multicolumn{2}{|c|}{$\begin{array}{l}\text { La magnitud relativa se obtiene a partir de los resultados del test de penetración estándar } \\
\text { SPT. Cuando este valor excede a } 80 \text {, el material granular no cohesivo se considera } \\
\text { equivalente a una roca. }\end{array}$} \\
\hline Roca & $K_{b}=\frac{\mathrm{RQD}}{J_{n}}$ & $\begin{array}{l}\text { RQD varía entre } 5 \text { y } 100 \\
J_{n} \text { varía entre } 1 \text { y } 5 \\
K_{b} \text { varía entre } 1 \text { y } 100\end{array}$ \\
\hline $\begin{array}{l}\text { Granular no } \\
\text { cohesivo }\end{array}$ & $K_{b}=1000 D^{3}$ & $D$. Diámetro del bloque medio \\
\hline Roca & $K_{d}=\frac{J_{r}}{J_{a}}$ & \\
\hline $\begin{array}{l}\text { Granular no } \\
\text { cohesivo }\end{array}$ & $K_{d}=\tan \phi$ & $\begin{array}{l}\phi \text {. Ángulo de fricción residual o interna del } \\
\text { material granular }\end{array}$ \\
\hline
\end{tabular}
obtener los distintos parámetros.

Tabla 46. Parámetros del índice de erosionabilidad (adaptado de Annandale, 2006). 
El umbral de resistencia de la roca frente a la potencia de escurrimiento, expresado en $\mathrm{kW} / \mathrm{m}^{2}$, se calcula en función del índice de erosionabilidad $K$ :

$$
\begin{array}{ll}
P_{\text {roca }}=0.48 K^{0.44} & \text { para } \mathrm{K} \leq 0.1 \\
P_{\text {roca }}=K^{0.75} & \text { para } K>0.1
\end{array}
$$

La presión dinámica en la solera del cuenco de disipación se puede dividir en dos componentes: el coeficiente de presión dinámica media $C_{p} \mathrm{y}$ el coeficiente de presión dinámica fluctuante $C_{p}$ '. Los coeficientes de presión dinámica se utilizan como estimadores de los coeficientes de reducción del potencial de escurrimiento. De este modo se considera el efecto de la desintegración del chorro en el aire y su difusión en el cuenco de amortiguación.

Las presiones dinámicas están relacionadas con la relación altura de caída/longitud de rotura del chorro, $H / L_{b}$, y con la relación colchón de agua/ancho del chorro incidente, $Y / B_{j}$. La presión dinámica total se puede expresar como:

$$
P_{\text {total }}=C_{p}\left(\frac{Y}{B_{j}}\right) P_{\text {chorro }}+F C_{p}{ }^{\prime}\left(\frac{Y}{B_{j}}\right) P_{\text {chorro }}
$$

donde $C_{p}\left(Y / B_{j}\right)$ es el coeficiente medio de presión dinámica, $C_{p}{ }^{\prime}\left(Y / B_{j}\right)$ el coeficiente de presión dinámica fluctuante, $P_{\text {chorro }}$ la potencia de escurrimiento por unidad de área, y $F$ el factor de reducción del coeficiente de presión dinámica fluctuante.

Para el caso de chorros rectangulares, las fórmulas se han ajustado mediante el uso de nuevos datos de laboratorio, cuyos valores pueden obtenerse en Figura $85\left(C_{p}\left(Y / B_{j}\right.\right.$, $\left.\left.H / L_{b}\right)\right)$, Figura $86\left(C_{p}^{\prime}\left(Y / B_{j}, H / L_{b}\right)\right)$ y Figura $87\left(C_{p}{ }^{\prime}\left(H / L_{b}\right)\right)$.

La Tabla 47 muestra los valores de las diferentes variables consideradas y su respectivo cálculo en la metodología semiempírica.

\begin{tabular}{|l|c|l|l|}
\hline Ángulo de fricción interna de la roca, SPT $\left({ }^{\circ}\right)$ & 38 & $\mathrm{~N}^{\circ}$ de juntas del sistema (calculado), $J_{n}$ & 1.83 \\
\hline Peso específico $\left(\mathrm{KN} / \mathrm{m}^{3}\right)$ & 27.64 & Espac. de discontinuidades, $J_{x}, J_{y}, J_{z}(\mathrm{~m})$ & 0.5 \\
\hline Resist. a compresión no confinada, UCS $(\mathrm{MPa})$ & 50 & Diámetro medio de bloque (calc.), $D(\mathrm{~m})$ & 0.5 \\
\hline Coeficiente de densidad relativa, $C_{r}$ & 1.024 & Grado de rugosidad, $J_{r}$ & 2 \\
\hline RQD (calculado) & 82.66 & Grado de alteración, $J_{a}$ & 1 \\
\hline
\end{tabular}

Tabla 47. Datos de entrada y valores calculados en la metodología semiempírica.

La Tabla 48 muestra los resultados obtenidos en los tres tipos de material existentes en el lugar de la cerrada. 


\begin{tabular}{|l|c|c|c|}
\cline { 2 - 4 } \multicolumn{1}{c|}{} & Aluvial & Roca meteorizada & Roca sana \\
\hline $\boldsymbol{M}_{\boldsymbol{s}}$ & 0.19 & 0.41 & 51.19 \\
\hline $\boldsymbol{K}_{\boldsymbol{b}}$ & 11.39 & 125 & 49.18 \\
\hline $\boldsymbol{K}_{\boldsymbol{d}}$ & 0.78 & 0.78 & 2 \\
\hline $\boldsymbol{J}_{\boldsymbol{s}}$ & 1 & 1 & 0.6 \\
\hline Índice de erosionabilidad, $\boldsymbol{K}$ & 1.69 & 40.04 & 3020.77 \\
\hline Potencial de escurrimiento, $\boldsymbol{P}_{\text {roca }}\left(\mathbf{k w} / \mathbf{m}^{\mathbf{2}}\right)$ & 1.48 & 15.92 & 407.46 \\
\hline
\end{tabular}

Tabla 48. Índices de erosionabilidad K y umbral de la potencia de escurrimiento $P_{\text {roca }}$ en el perfil estratigráfico correspondiente a la zona aguas abajo de la presa Paute-Cardenillo.

Para el funcionamiento del aliviadero de vertido libre, considerando un colchón de agua de $24 \mathrm{~m}$ (erosión de $18 \mathrm{~m}$ ), el caudal de $500 \mathrm{~m}^{3} / \mathrm{s}$ ya dispondría de la potencia necesaria para erosionar la roca meteorizada, aunque el caudal de diseño de $700 \mathrm{~m}^{3} / \mathrm{s}$ no dispondría de la potencia suficiente para erosionar la roca sana (Tabla 49). Estos resultados confirman que la erosión máxima del aliviadero de vertido libre podría ser del orden de $34 \mathrm{~m}$.

\begin{tabular}{|c|c|c|c|c|c|}
\hline $\begin{array}{c}Q_{1 \text { vano }} \\
\left(\mathrm{m}^{3} / \mathrm{s}\right)\end{array}$ & $\begin{array}{c}Q_{5 \text { vanos }} \\
\left(\mathrm{m}^{3} / \mathrm{s}\right)\end{array}$ & $\begin{array}{c}P_{\text {chorro }} \\
\left(\mathrm{Kw} / \mathrm{m}^{2}\right)\end{array}$ & $\begin{array}{c}\text { Aluvial } \\
\left(P_{\text {roca }}=1.48 \mathrm{KW} / \mathrm{m}^{2}\right)\end{array}$ & $\begin{array}{c}\text { Roca meteorizada } \\
\left(P_{\text {roca }}=15.92 \mathrm{KW} / \mathrm{m}^{2}\right)\end{array}$ & $\begin{array}{c}\text { Roca sana } \\
\left(P_{\text {roca }}=407.46 \mathrm{KW} / \mathrm{m}^{2}\right)\end{array}$ \\
\hline 25 & 125 & 0.40 & No erosiona & No erosiona & No erosiona \\
\hline 50 & 250 & 0.72 & No erosiona & No erosiona & No erosiona \\
\hline 100 & 500 & 21.66 & Si erosiona & Si erosiona & No erosiona \\
\hline 140 & 700 & 111.75 & Si erosiona & Si erosiona & No erosiona \\
\hline
\end{tabular}

Tabla 49. Cálculo de la socavación del aliviadero de vertido libre en función del índice de erosionabilidad de los materiales.

Considerando el desagüe de medio fondo, suponiendo un colchón de agua de $24 \mathrm{~m}$ correspondiente a una socavación del lecho $D_{s}=22 \mathrm{~m}$, en la Tabla 50 se observa que el umbral de potencia de escurrimiento de la roca meteorizada no resiste el caudal de período de retorno anual $\left(Q_{m a}=136 \mathrm{~m}^{3} / \mathrm{s}\right)$. La roca sana podría resistir hasta un caudal de período de retorno de 6 años $\left(Q_{6}=920 \mathrm{~m}^{3} / \mathrm{s}\right)$.

Para evitar la continuación de la socavación, se propone la construcción de una losa de hormigón de $2 \mathrm{~m}$ de espesor, cimentada sobre la roca meteorizada (la solera del cuenco queda a $22 \mathrm{~m}$ bajo el nivel del cauce natural). La potencia del caudal de diseño $\left(Q_{40}=1760 \mathrm{~m}^{3} / \mathrm{s}\right)$ es inferior al umbral de potencia de una losa de hormigón con resistencia característica de $20 \mathrm{MPa}\left(P_{\text {hormigón }}=788 \mathrm{KW} / \mathrm{m}^{2}\right)$. 


\begin{tabular}{|c|c|c|c|c|c|}
\hline $\begin{array}{c}Q_{\text {desagües }} \\
\left(\mathrm{m}^{3} / \mathrm{s}\right)\end{array}$ & $\begin{array}{c}P_{\text {chorro }} \\
\left(\mathrm{KW} / \mathrm{m}^{2}\right)\end{array}$ & $\begin{array}{c}\text { Aluvial } \\
\left(P_{\text {roca }}=1.48\right. \\
\left.\mathrm{KW} / \mathrm{m}^{2}\right)\end{array}$ & $\begin{array}{c}\text { Roca meteorizada } \\
\left(P_{\text {roca }}=15.92\right. \\
\left.\mathrm{KW} / \mathrm{m}^{2}\right)\end{array}$ & $\begin{array}{c}\text { Roca sana } \\
\left(P_{\text {roca }}=407.46\right. \\
\left.\mathrm{KW} / \mathrm{m}^{2}\right)\end{array}$ & $\begin{array}{c}\text { Losa de hormigón } \\
\left(P_{\text {roca }}=788\right. \\
\left.\mathrm{KW} / \mathrm{m}^{2}\right)\end{array}$ \\
\hline 136.3 & 43.30 & Si erosiona & Si erosiona & No erosiona & No erosiona \\
\hline 400 & 132.04 & Si erosiona & Si erosiona & No erosiona & No erosiona \\
\hline 920 & 404.00 & Si erosiona & Si erosiona & No erosiona & No erosiona \\
\hline 1180 & 670.79 & Si erosiona & Si erosiona & Si erosiona & No erosiona \\
\hline 1760 & 746.83 & Si erosiona & Si erosiona & Si erosiona & No erosiona \\
\hline 1820 & 795.02 & Si erosiona & Si erosiona & Si erosiona & Si erosiona \\
\hline
\end{tabular}

Tabla 50. Cálculo de la socavación del desagüe de medio fondo en función del índice de erosionabilidad de los materiales.

\subsection{Modelación matemática tridimensional de la socavación}

Una vez realizado el estudio de socavación a pie de presa con los métodos empíricos y semiempíricos, se pudo realizar la simulación con modelos matemáticos tridimensionales.

Mediante FLOW-3D se ha abordado la simulación numérica del comportamiento hidráulico y de las socavaciones generadas en el cauce por la acción del aliviadero superior y los desagües intermedios. Este programa incorpora modelos transporte de sedimentos y un modelo empírico de erosión del lecho (Guo, 2002; Mastbergen y von den Berg, 2003).

El sedimento en suspensión se transporta por advección, teniendo en cuenta el balance entre las fuerzas de sedimentación y de arrastre de cada especie considerada. El programa emplea la ecuación de Meyer-Peter y Müller (1948) para resolver el transporte de sedimentos de fondo, mientras que el espesor de la capa en que se produce el transporte de fondo se obtiene con las relaciones propuestas por Van Rijn (1984).

El cambio en el lecho $Z_{b}$ se calcula a partir de la ecuación general de balance de masa para el transporte de fondo (ecuación de Exner):

$$
\left(1-p_{m}\right) \frac{\partial Z_{b}}{\partial t}+\frac{\partial Q_{b s}}{\partial s}+\frac{\partial Q_{b n}}{\partial n}=0
$$

donde $p_{m}$ es la porosidad del material del lecho, $Q_{b s}$ y $Q_{b n}$ son el caudal de transporte de fondo en la dirección principal s y en la dirección transversal $n$ del flujo, respectivamente. Estos caudales calculan a partir de la ecuación de transporte de fondo que no está en equilibrio:

$$
\frac{\partial\left(Q_{b} \alpha_{b s}\right)}{\partial s}+\frac{\partial\left(Q_{b} \alpha_{b n}\right)}{\partial s}=-\frac{1}{L_{s}}\left(Q_{b}-Q_{e}\right)
$$


donde $\alpha_{b s}$ y $\alpha_{b n}$ son los cosenos de los vectores de dirección que determinan las componentes del transporte de fondo en las direcciones $s$ y $n$, respectivamente. El modelo considera que el no equilibrio es proporcional a la diferencia entre el trasporte de fondo que no está en equilibrio $Q_{b}$ y el trasporte de fondo en equilibrio $Q_{e}$, estando relacionado con la longitud de adaptación del no equilibrio $L_{s}$.

En el embalse, las condiciones se corresponden a un nivel de lámina de agua con la cota correspondiente al nivel de operación normal del embalse (924 m).

En el lecho se ha considerado un nivel de agua correspondiente a un calado de $6 \mathrm{~m}$, similar al calado del río en régimen natural para un caudal de $2340 \mathrm{~m}^{3} / \mathrm{s}$, correspondiente al funcionamiento conjunto del aliviadero de vertido libre y los desagües intermedios. Sin embargo este calado resulta irrelevante en el análisis de socavación ya que el foso de erosión sobrepasa los $22 \mathrm{~m}$. La condición de contorno de aguas abajo se sitúa a más de $300 \mathrm{~m}$ de la presa, suficientemente alejado de la zona de influencia de los chorros incidentes y de la recirculación generada en el cuenco de erosión formado.

En los apartados anteriores se ha comprobado que los vertidos en caída libre simulados en FLOW-3D requieren un mallado muy fino en el entorno del chorro. Para que el esquema en diferencias finitas pueda resolver adecuadamente el chorro se requiere un tamaño de elementos del mallado de entre 2 y 3 veces menor que el espesor del chorro. En este sentido, se han considerado tamaños de elementos en el entorno de los chorros vertientes de $0.30 \mathrm{~m}$. Sin embargo, se observa que los chorros tienden a romperse a una determinada altura de caída (Figura 163).
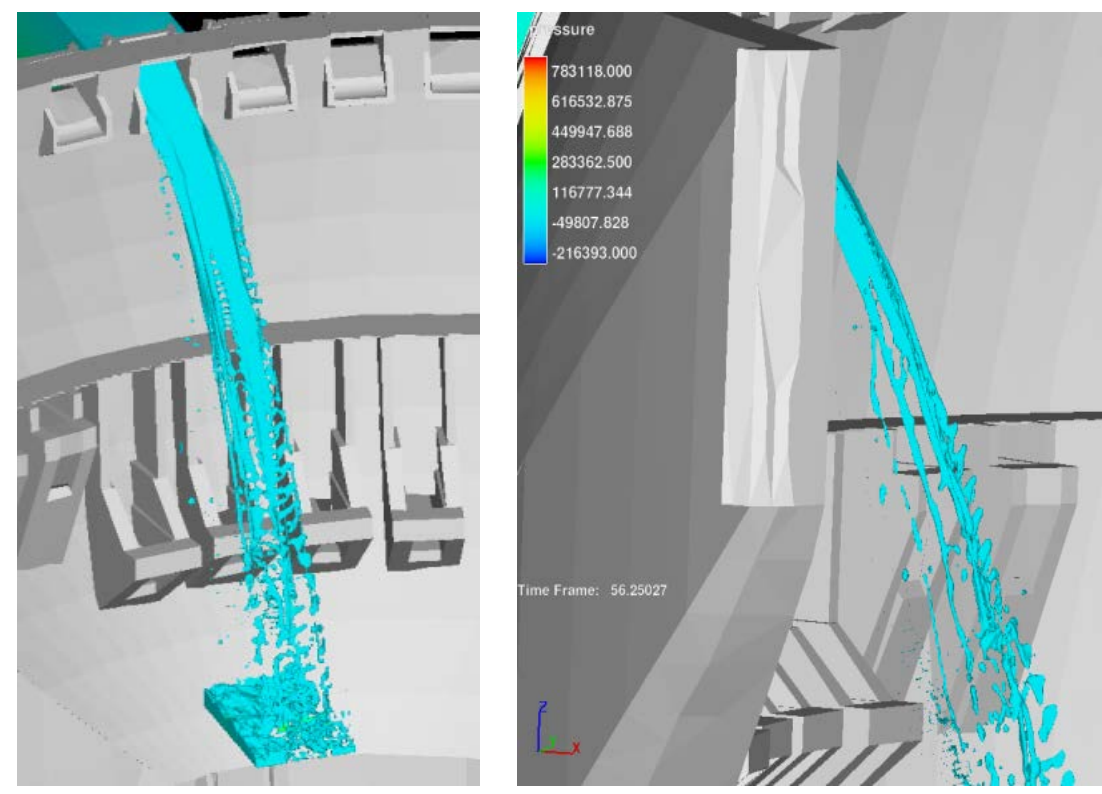

Figura 163. Detalle de la rotura del chorro en la simulación de un vano del aliviadero de vertido libre obtenido con FLOW-3D. 
En la Figura 164 se observa que la rotura del chorro se produce cuando el espesor del chorro es aproximadamente el doble del tamaño del mallado, sin que se reproduzca adecuadamente la aireación del chorro vertiente.

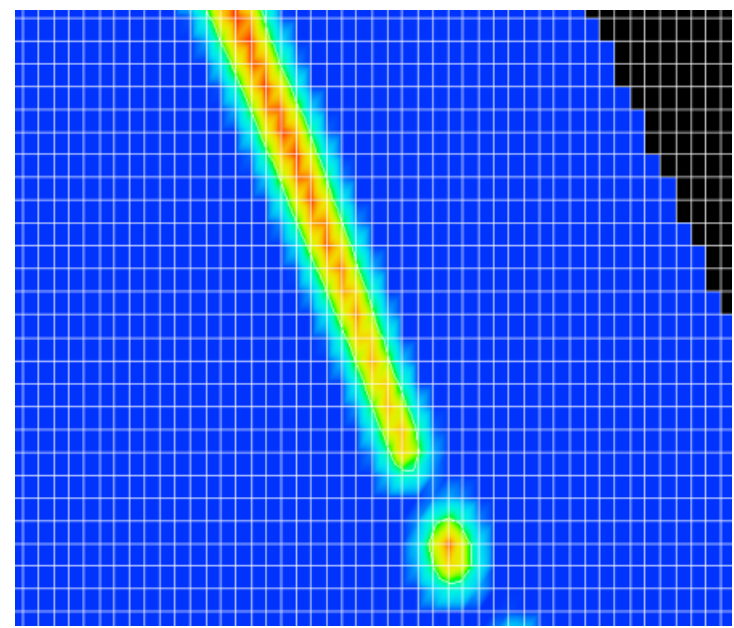

Figura 164. Detalle de la rotura del chorro de un vano del aliviadero.

Con el fin de simular el adecuado funcionamiento del aliviadero de vertido libre, se realizó un análisis de sensibilidad de diversos parámetros: modelos de aireación y modelo de turbulencia, tamaño del mallado, y criterios de resolución, entre otros (Figura 165 y Tabla 51).
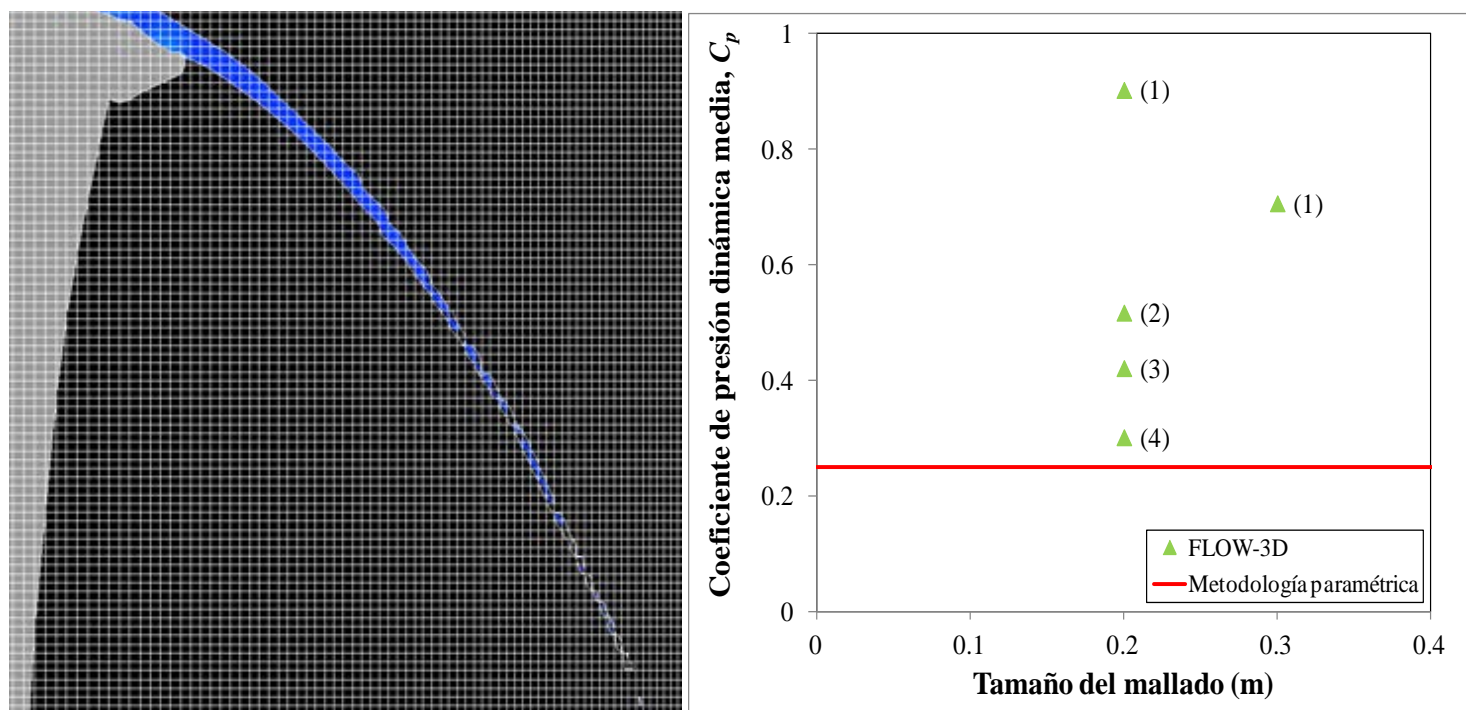

Figura 165. Mallado del aliviadero de vertido libre de la presa Paute-Cardenillo y análisis de sensibilidad en FLOW-3D (Castillo y Carrillo, 2014). 


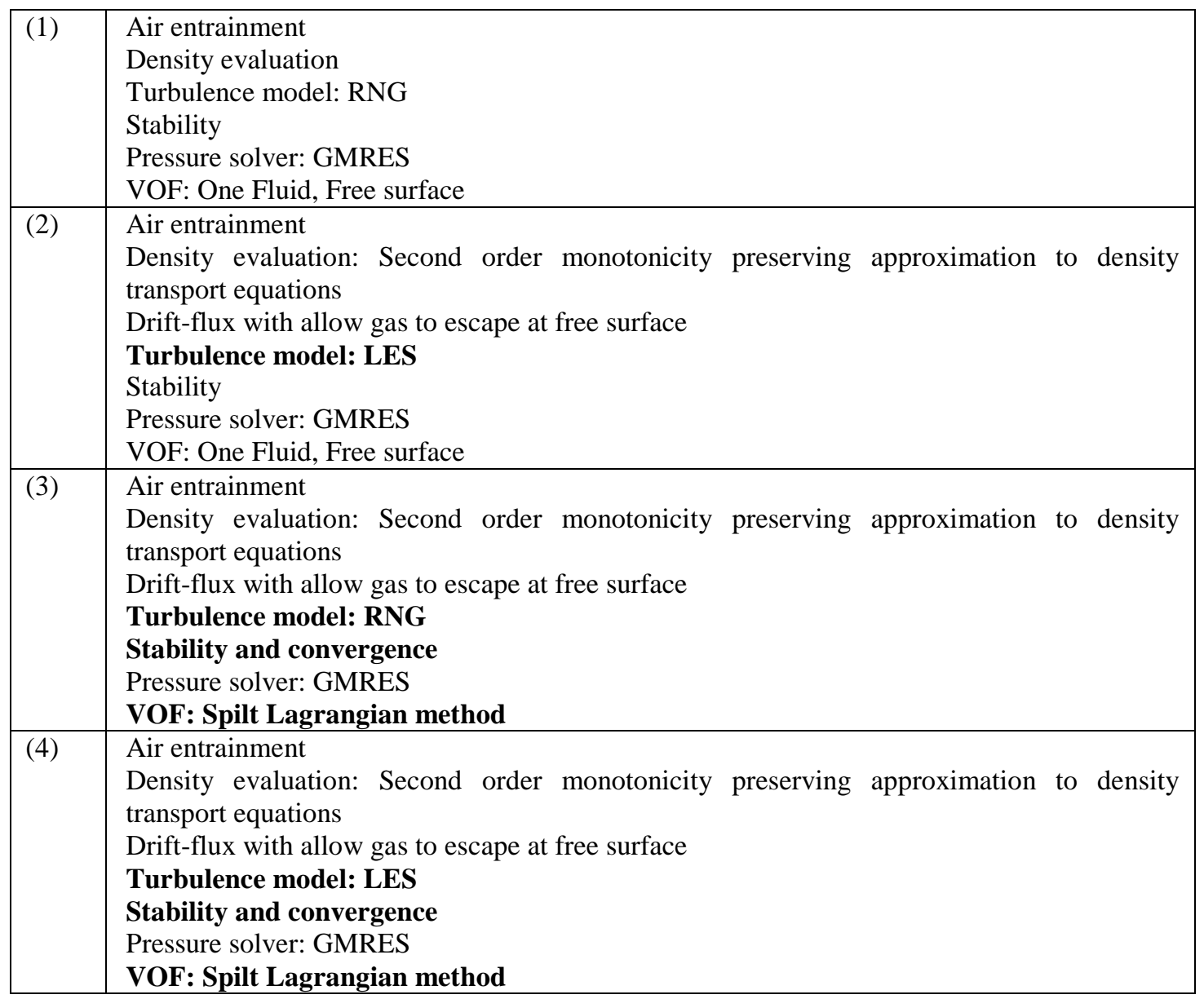

Tabla 51. Análisis de sensibilidad en FLOW-3D para el aliviadero de vertido libre de la presa Paute-Cardenillo.

Los resultados no son buenos cuando se combinan el modelo de turbulencia RNG $k-\varepsilon$ con los métodos de un fluido y lámina libre. De este modo, cuando el tamaño de la malla se reduce de $0.30 \mathrm{~m}$ a $0.20 \mathrm{~m}$, el valor de $C_{p}$ aumenta de 0.71 a 0.90 . Sin embargo, cuando se utiliza el modelo RNG $k-\varepsilon$ con el método Split Lagrangian para resolver la superficie libre los resultados son más similares al valor esperado.

Los mejores resultados se obtuvieron con un tamaño de malla de $0.2 \mathrm{~m}$ y el modelo de turbulencia LES. Como criterio de resolución se seleccionó la opción de estabilidad y convergencia, mientras que la lámina libre se resolvió con el método Split Lagrangian. Considerando esta configuración, se ha obtenido un registro de presiones en el punto de estancamiento y un valor del coeficiente de presión dinámica media similares a los obtenidos con la Metodología Paramétrica (Figura 166 y Tabla 52). 


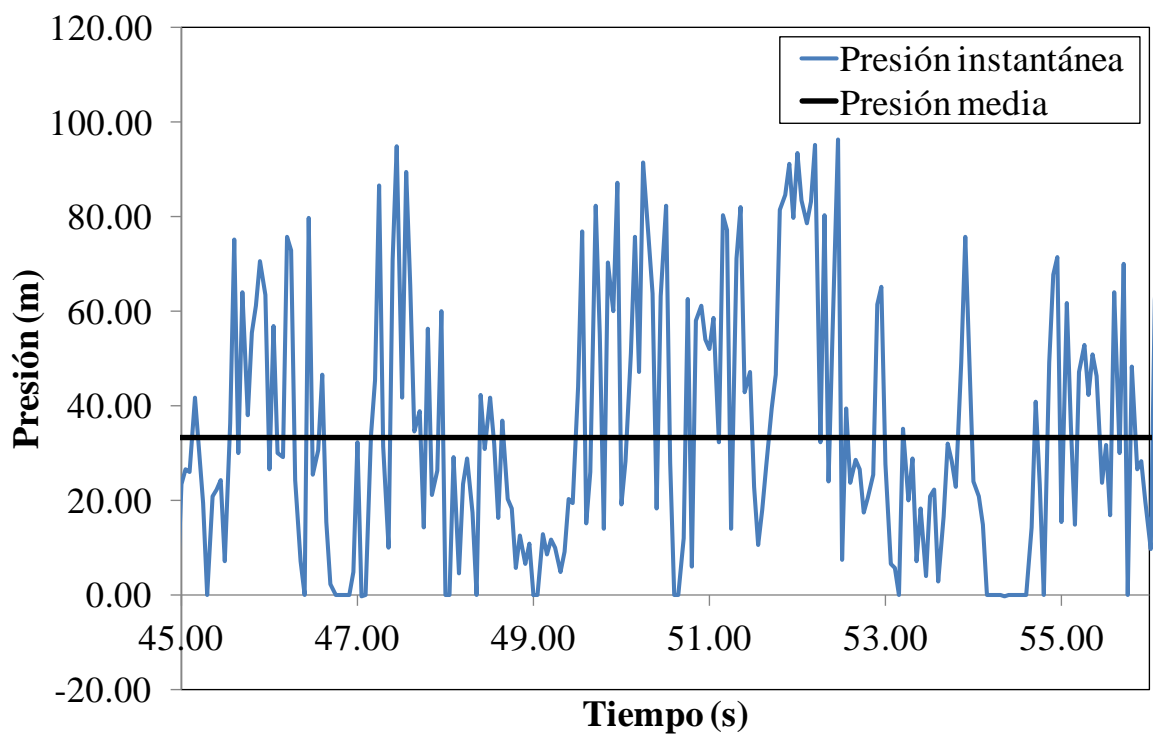

Figura 166. Registro de presiones en el punto de estancamiento.

La Tabla 52 compara la presión media y el coeficiente de presión dinámica media obtenidos para la situación de colchón de agua no efectivo considerando la mejor configuración. Los resultados fueron similares a los calculados con la Metodología Paramétrica. La presión dinámica media y el coeficiente de $C_{p}$ obtenidos con FLOW-3D son algo mayores que los valores de la Metodología Paramétrica. Esto es debido a que el modelo de aireación no resuelve todavía de modo eficiente la aireación en el chorro.

\begin{tabular}{|l|c|c|}
\cline { 2 - 3 } \multicolumn{1}{c|}{} & Metodología paramétrica & FLOW-3D \\
\hline Altura de caída (m) & 102.00 & 102.00 \\
\hline Presión dinámica media (m) & 30.56 & 33.44 \\
\hline Coeficiente de presión dinámica media (-) & 0.28 & 0.31 \\
\hline
\end{tabular}

Tabla 52. Comparación de presiones y $C_{p}$ en la presa Paute-Cardenillo considerando un colchón de agua de $2 \mathrm{~m}$.

Una vez calibrado el programa con la configuración (4) definida en la Figura 165 y en la Tabla 51, se procedió a simular el vertido libre del aliviadero superior para el nivel máximo probable en el embalse (924 msnm). Con este nivel de embalse se tiene un caudal circulante de $700 \mathrm{~m}^{3} / \mathrm{s}$ (140 m³ por cada uno de los 5 vanos).

En lo que se refiere a la socavación, los chorros del aliviadero de vertido libre tendieron a incidir en una misma zona. El borde de aguas arriba del cuenco de socavación se produjo aproximadamente a $35 \mathrm{~m}$ aguas abajo del pie de presa. La socavación en planta tenía unas dimensiones aproximadas de $34 \mathrm{~m}$ de largo y $48 \mathrm{~m}$ de ancho (Figura 167). La erosión alcanza la cota 798 msnm, generándose un foso de $22 \mathrm{~m}$ 
de profundidad respecto de la cota $820 \mathrm{msnm}$ del terreno original. Este valor es un poco superior al calculado con la media de las fórmulas empíricas y similar al obtenido con la metodología semiempírica de Annandale (1995, 2006).

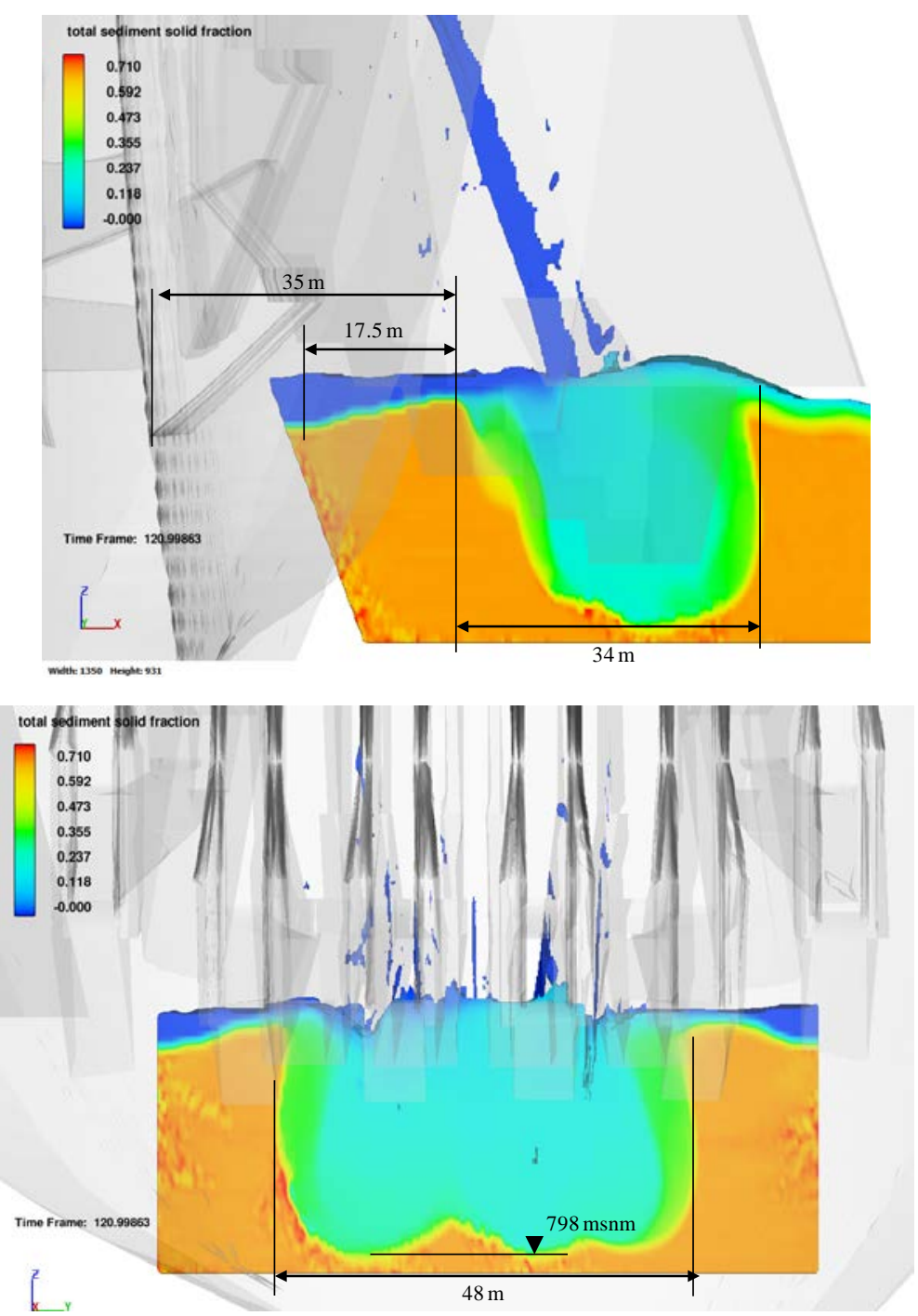

Figura 167. Perfil longitudinal y transversal de la socavación debida al aliviadero de vertido libre.

La socavación de los desagües de medio fondo resultó más sencilla debido a las mayores dimensiones de los conductos y del chorro vertiente. El mallado se realizó teniendo en cuenta las dimensiones de los desagües intermedios (sección de paso rectangular con dimensiones $5.00 \mathrm{~m}$ x $5.80 \mathrm{~m}$ ). Previo análisis de sensibilidad, se consideró adecuado emplear un mallado con un tamaño de celdas de $1 \mathrm{~m}$, el cual captura adecuadamente el funcionamiento de los desagües intermedios (Figura 168). 


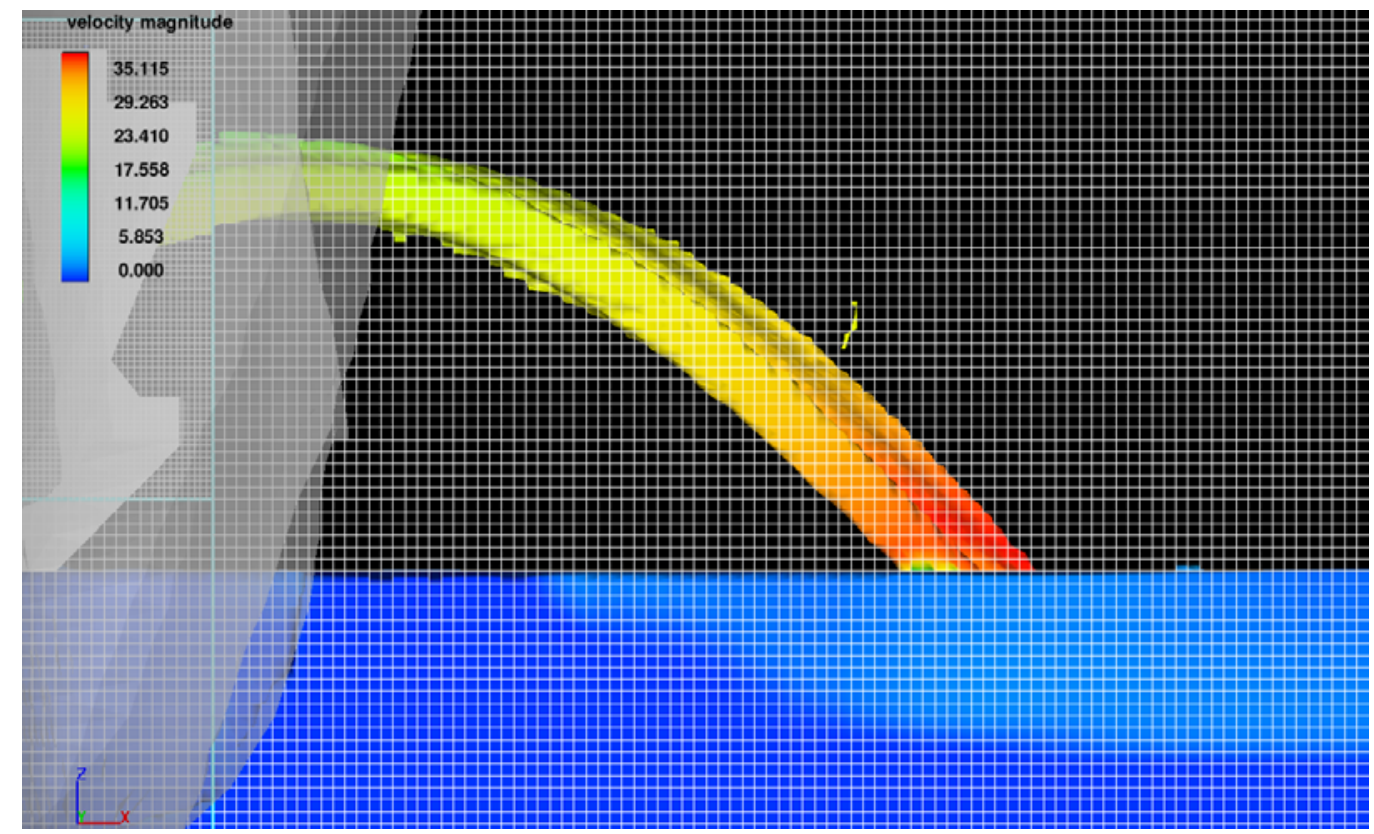

Figura 168. Mallado de los desagües intermedios.

La socavación se limitó a las capas de material aluvial y roca meteorizada, considerando que se cimienta una losa de hormigón de $2 \mathrm{~m}$ de espesor sobre el estrato competente de la roca meteorizada.

Para el caudal descargado por el desagüe intermedio con el nivel máximo normal en el embalse $\left(Q=1760 \mathrm{~m}^{3} / \mathrm{s}\right)$, la distancia horizontal de caída del chorro hasta el impacto de los dos chorros se encuentra alrededor de 90 m (Figura 169).

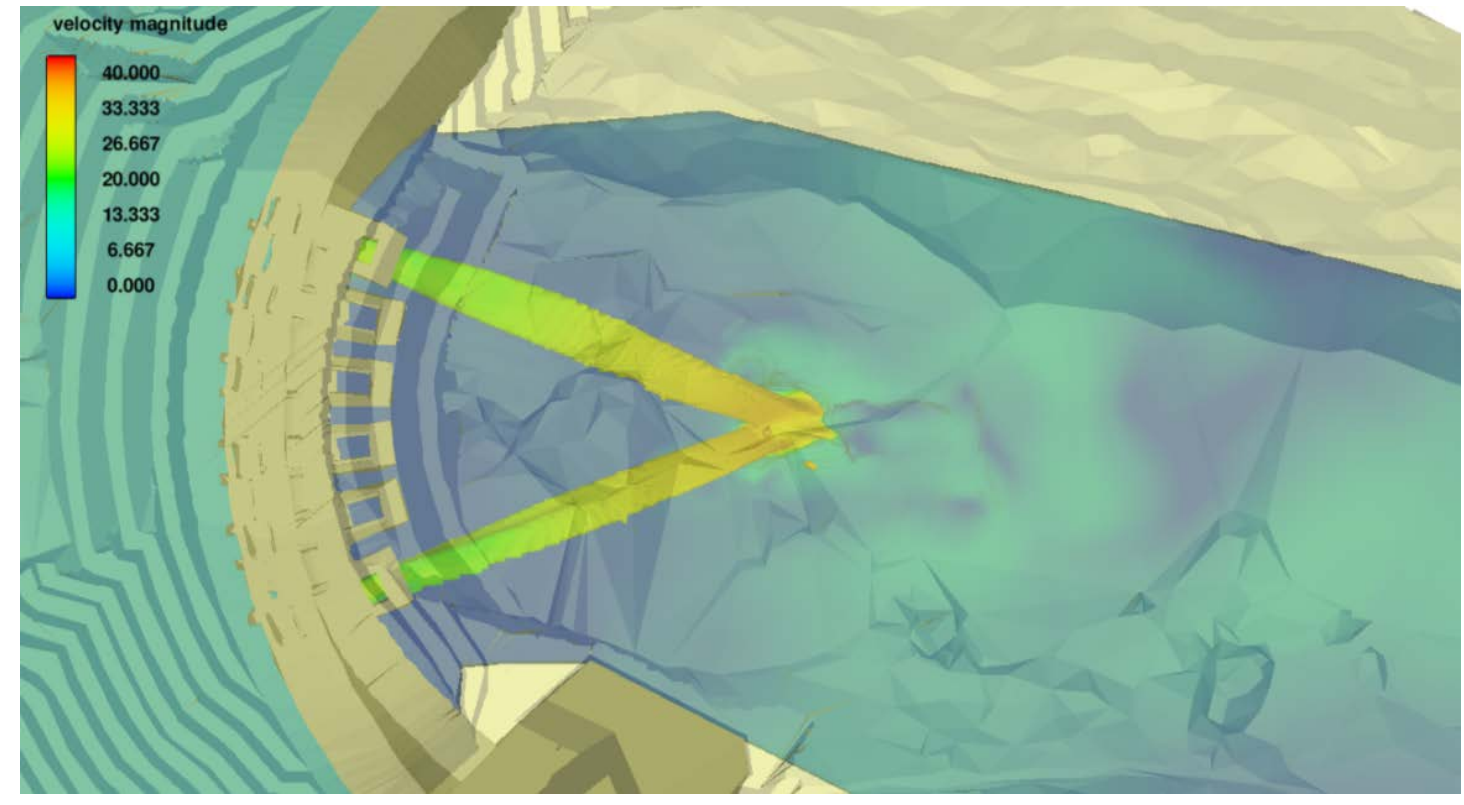

Figura 169. Funcionamiento conjunto de los dos desagües intermedios (caudal total de 1760 $\left.\mathrm{m}^{3} / \mathrm{s}\right)$. Vista en planta. 
El espesor de los chorros incidentes está en el entorno de $6.00 \mathrm{~m}$. La velocidad de incidencia está en el entorno de $36 \mathrm{~m} / \mathrm{s}$, mientras que se ha calculado un ángulo de incidencia de $45.25^{\circ}$ (Figura 170). La velocidad a la salida del cuenco está en el entorno de los $5 \mathrm{~m} / \mathrm{s}$, observándose el fenómeno de disipación de energía en el cuenco erosionado.

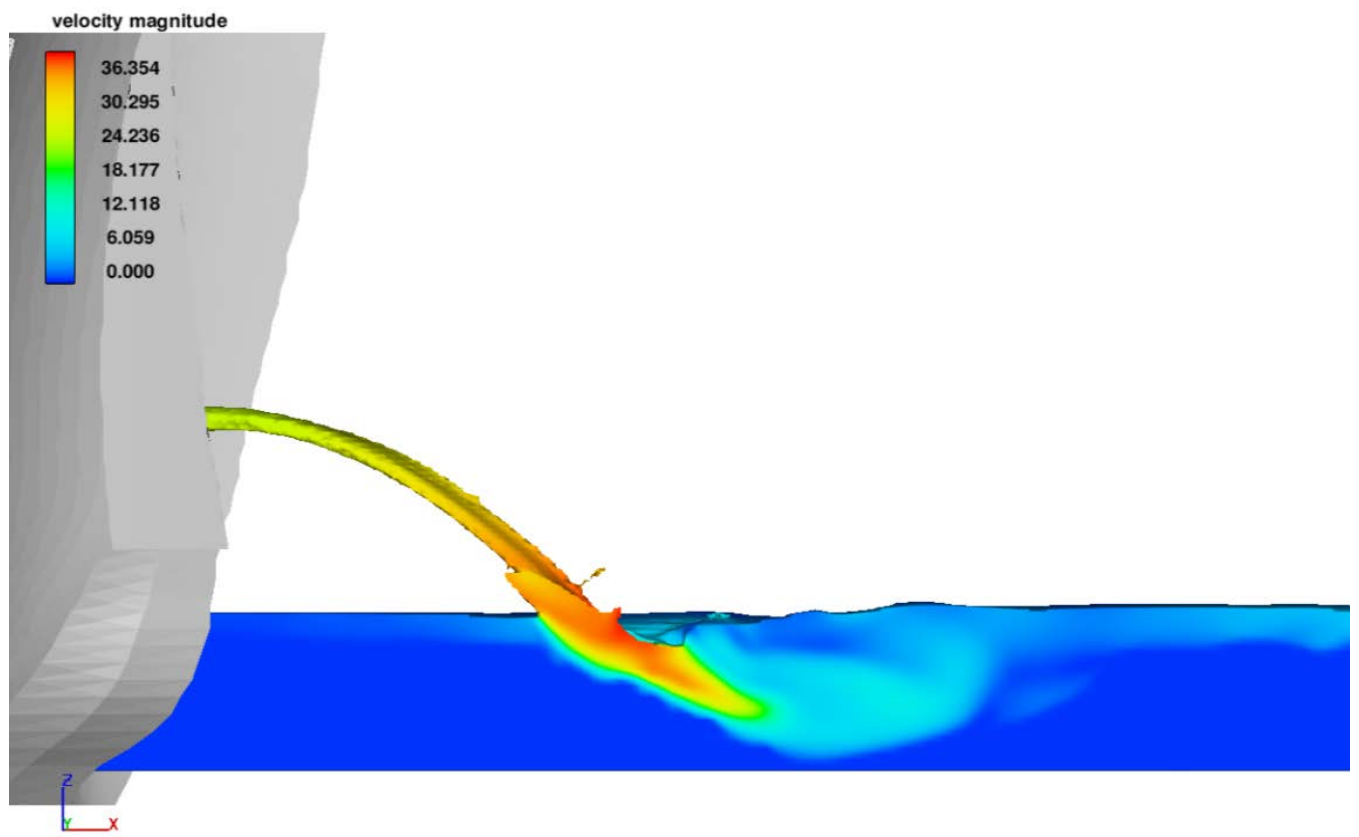

Figura 170. Perfil longitudinal de las velocidades en el cuenco erosionado.

Los siguientes gráficos muestran la forma del cuenco conformado, una vez que se ha erosionado la capa de $24 \mathrm{~m}$ de aluvial, alcanzándose la solera de una futura losa de hormigón situada a la cota 798 msnm.

La Figura 171 muestra la forma del cuenco conformado, una vez que se ha erosionado la capa de $24 \mathrm{~m}$ de aluvial, alcanzándose el estrato de roca meteorizada. El cuenco erosionado tiene un ángulo de aguas arriba en torno a 39\% mientras que la pendiente de aguas abajo está en el entorno de $70^{\circ}$. El inicio del cuenco se encuentra a una distancia aproximada de $100 \mathrm{~m}$ del pie de presa. Se ha erosionado una longitud de $80 \mathrm{~m}$ sobre el estrato de roca meteorizada, mientras que la longitud del cuenco en el nivel del terreno original es de $108 \mathrm{~m}$. 


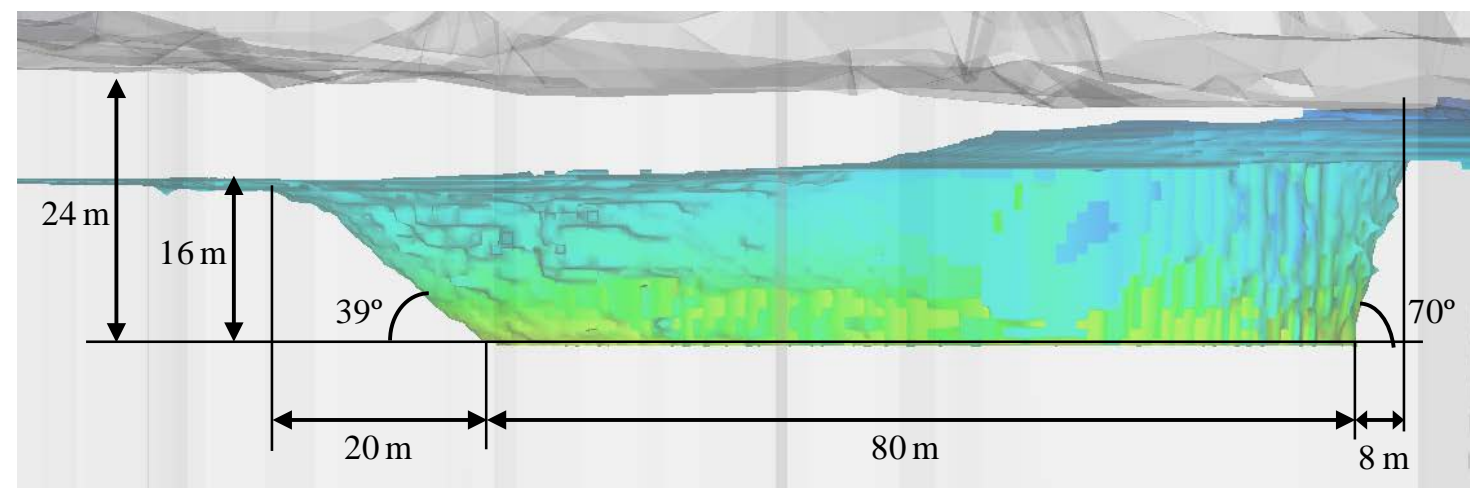

Figura 171. Perfil del cuenco de socavación generado en la capa aluvial por el desagüe intermedio.

La forma en planta del cuenco erosionado se muestra en la Figura 172. El ancho del cuenco está en torno a 50 m en la cota del terreno original.

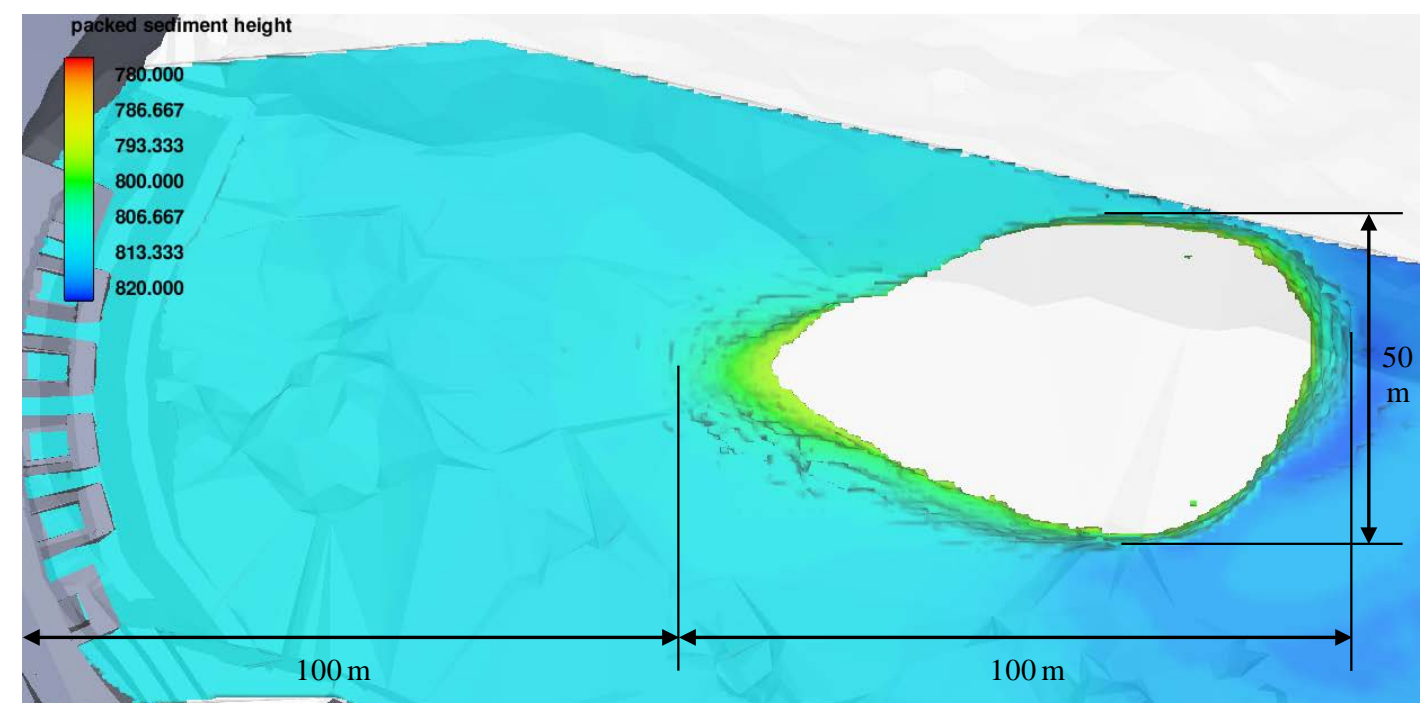

Figura 172. Planta del cuenco de socavación generado en la capa aluvial por el desagüe intermedio.

La sección transversal (Figura 173) muestra taludes muy verticales a ambos lados del cuenco. 


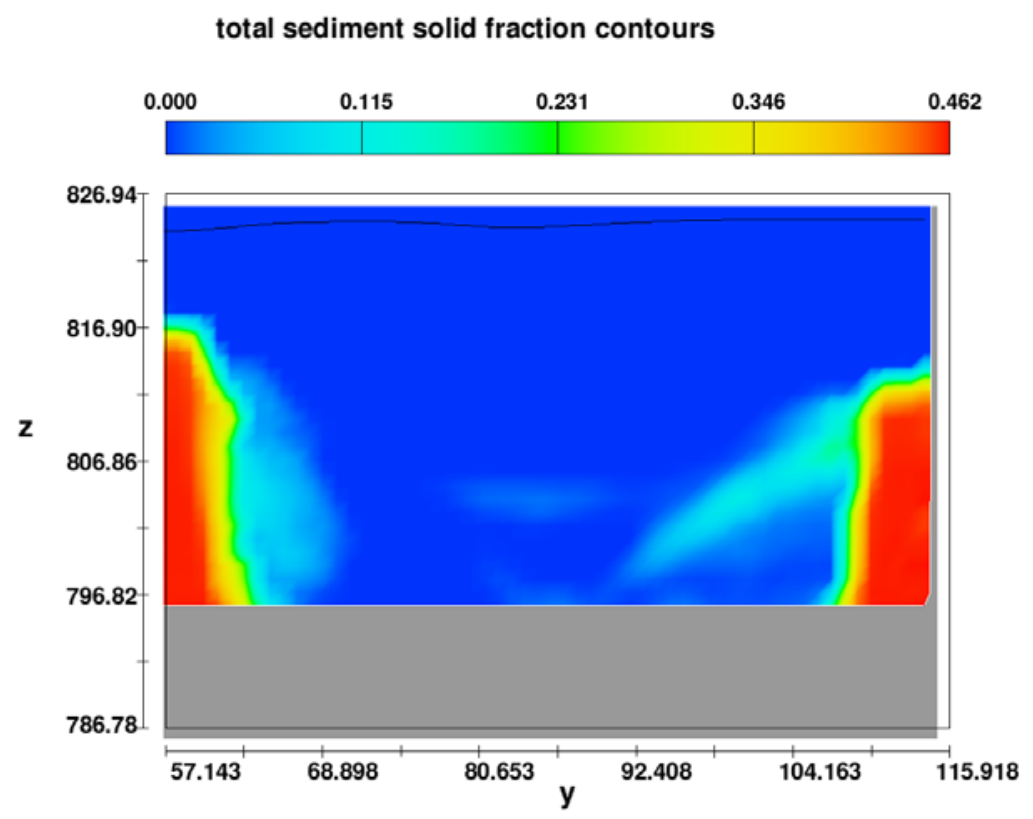

Figura 173. Sección trasversal del cuenco de socavación generado por el desagüe intermedio.

En la Figura 174 se indican las zonas de impacto e influencia de los diferentes fosos de socavación formados con los distintos escenarios considerados.

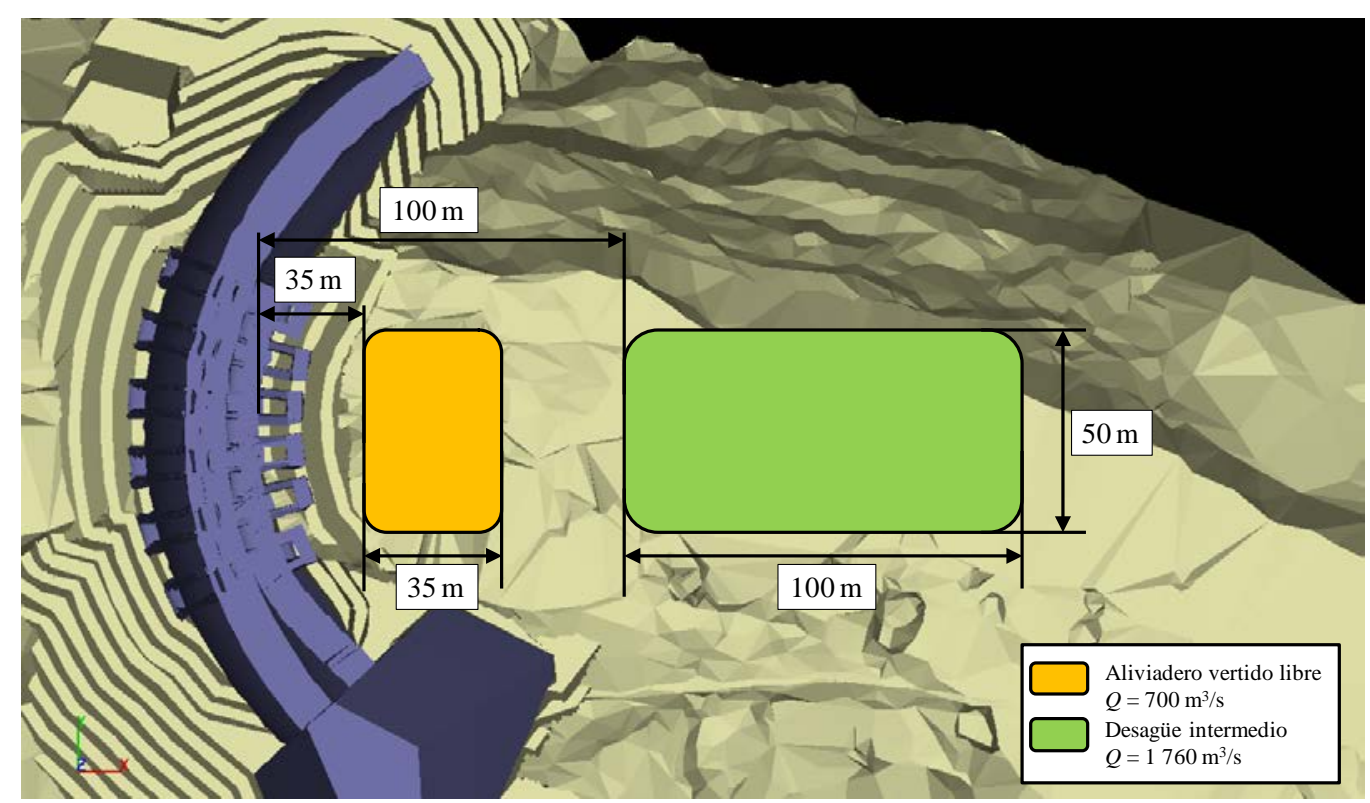

Figura 174. Localización de los cuencos erosionados generados en los distintos escenarios. 
Tras el estudio de los diferentes cuencos de socavación generados por los aliviaderos superiores, desagües intermedios y desagües de fondo, se propone un diseño de cuenco pre-excavado consistente en:

- Losa de hormigón en masa de 2 m de espesor, con cimiento ubicado a la cota 796 msnm.

- Longitud de cuenco de $60 \mathrm{~m}$ y acho de 50 m. Losa de aproximación al cuenco de unos $100 \mathrm{~m}$ y $50 \mathrm{~m}$ de ancho.

- Taludes aguas arriba y en las dos márgenes con pendientes 1:1, mientras que en la zona de aguas abajo se ha considerado una pendiente de 1:2 para conseguir una transición suave del flujo en su retorno al cauce natural.

- Taludes laterales con pendiente similar a la ladera de la margen izquierda.

La forma y dimensiones del cuenco se han obtenido en función de las dimensiones de los cuencos erosionados y de la forma de la cerrada aguas abajo de la presa. En la zona de implantación de la presa los chorros del desagüe intermedio salen esviados hacia la ladera izquierda, atacando peligrosamente el pié de talud de la montaña. La forma en planta del cuenco, así como su longitud se han tenido que modificar para adaptarse a la topografía existente. En la Figura 175 se indica la forma del cuenco preexcavado y su disposición en el terreno existente.

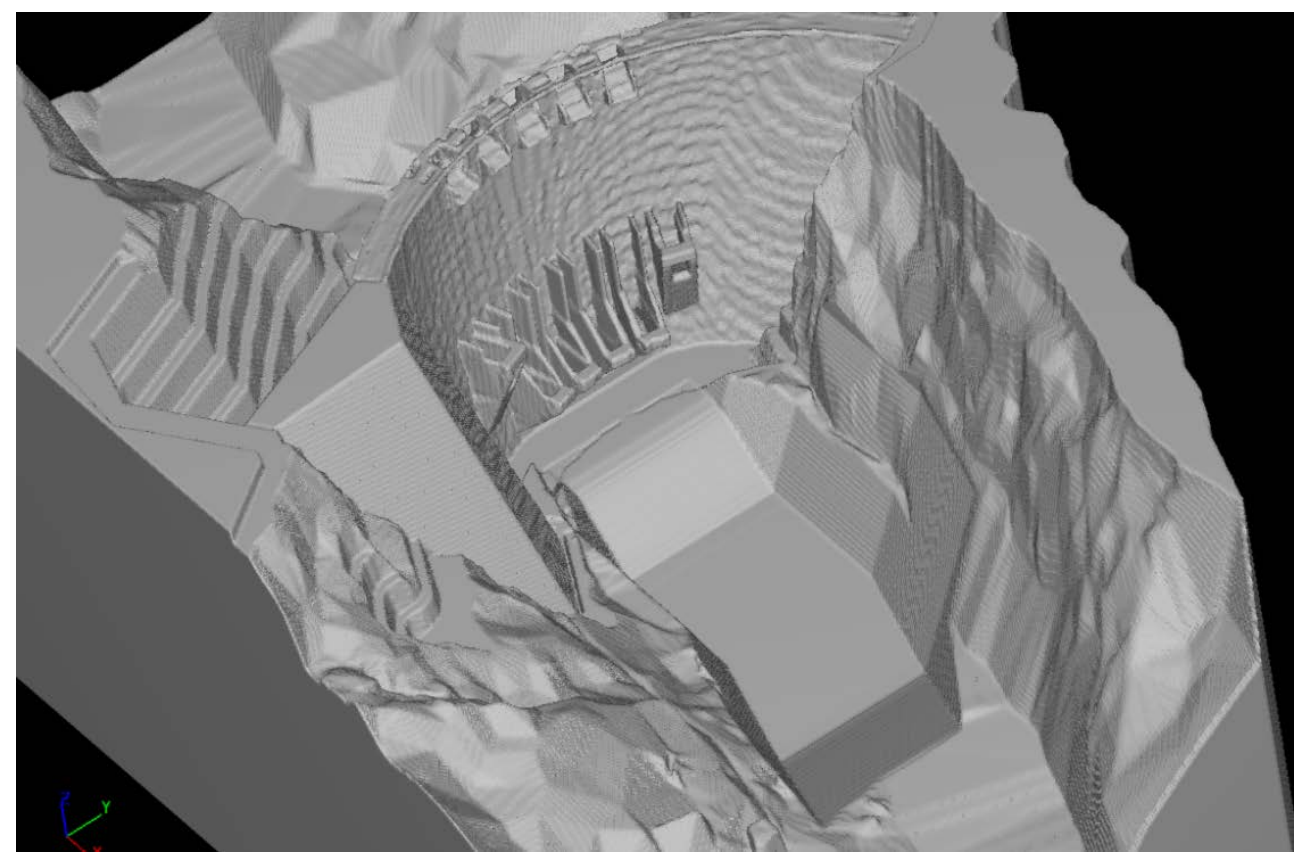

Figura 175. Cuenco pre-excavado propuesto y su disposición en el terreno. 
En la Figura 176 se observa el adecuado comportamiento del aliviadero de vertido libre en el cuenco de amortiguación. Los chorros inciden en la proximidad del talud de aguas arriba del cuenco pre-excavado. Aunque la velocidad de incidencia está próxima a los $44 \mathrm{~m} / \mathrm{s}$, se produce una rápida difusión en el cuenco pre-excavado. El funcionamiento del cuenco permite que el flujo retorne al cauce natural a velocidades inferiores a $3 \mathrm{~m} / \mathrm{s}$.

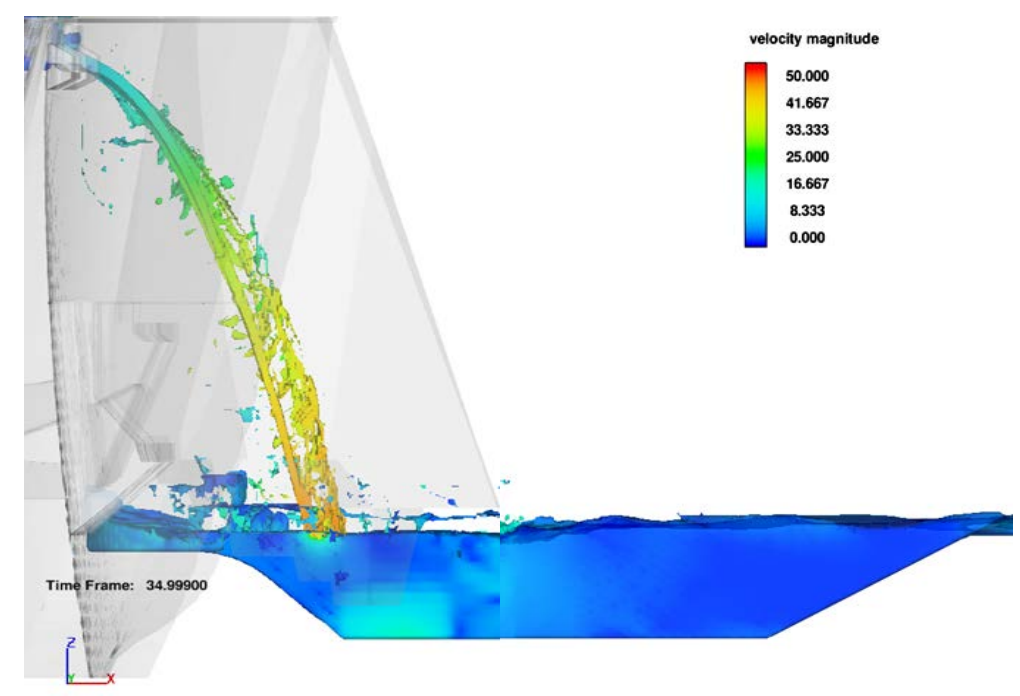

Figura 176. Funcionamiento del cuenco pre-excavado para el caudal de $700 \mathrm{~m}^{3} / \mathrm{s}$ descargado por el aliviadero superior.

Puesto que los chorros se difuminan totalmente antes de alcanzar el fondo, las presiones totales instantáneas en la solera se mantienen en la presión hidrostática del colchón de agua de 28 m (Figura 177).

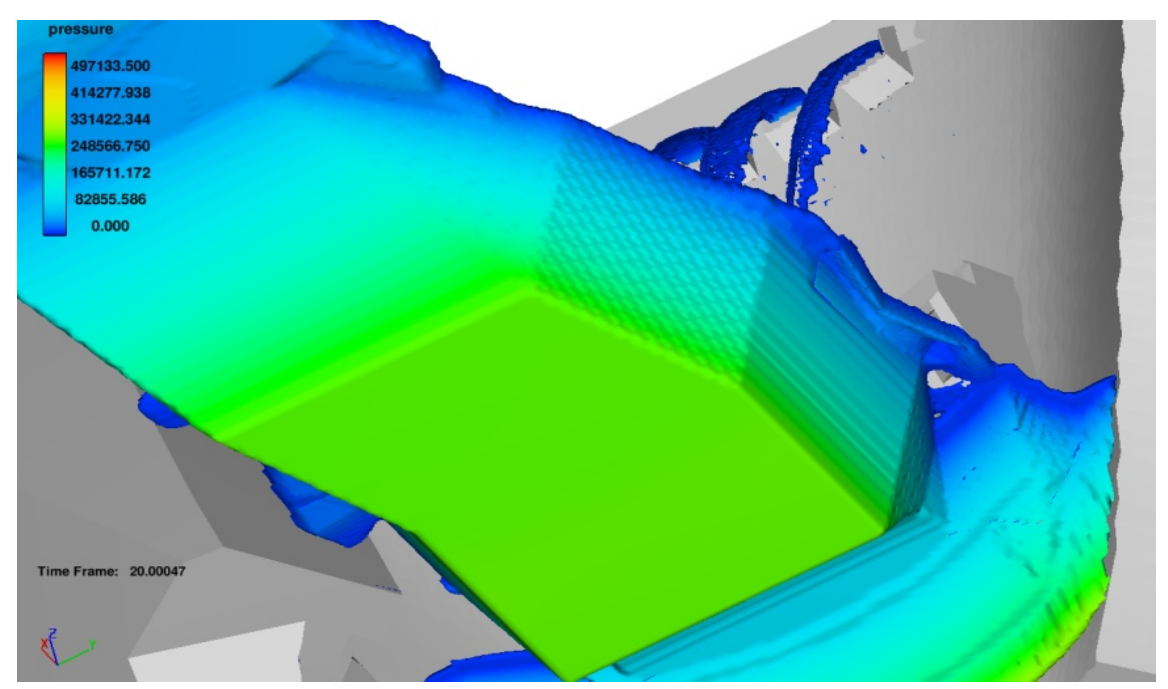

Figura 177. Distribución de presiones en solera del cuenco pre-excavado para el caudal de 700 $\mathrm{m}^{3} / \mathrm{s}$ descargado por el aliviadero de superficie. 
En la Figura 178 se observa el funcionamiento del cuenco pre-excavado con el caudal de $1760 \mathrm{~m}^{3} / \mathrm{s}$ desaguado por los desagües intermedios. La velocidad de incidencia de los chorros está en torno a $36 \mathrm{~m} / \mathrm{s}$. La difusión en el cuenco permite que las velocidades desciendan hasta valores por debajo de $10 \mathrm{~m} / \mathrm{s}$, obteniéndose velocidades en el retorno al cauce en torno a $6 \mathrm{~m} / \mathrm{s}$ con el caudal de 40 años de periodo de retorno.

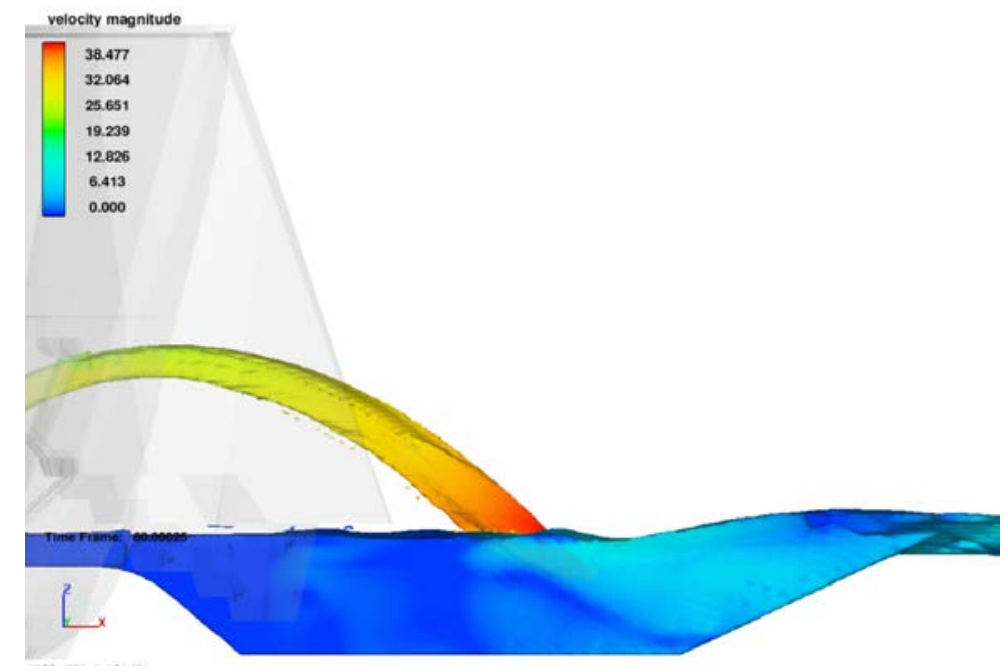

Figura 178. Distribución de velocidades dentro del cuenco pre-excavado para un caudal de $1760 \mathrm{~m}^{3} / \mathrm{s}$ que pasa por los desagües intermedios.

En las siguientes figuras se muestran los vectores de velocidad obtenidos en el cuenco de disipación. El colchón de agua produce una rápida difusión de la velocidad, mientras que los remolinos se concentran en la parte de aguas abajo del cuenco de disipación, sin alcanzar la zona de transición con el cauce natural.

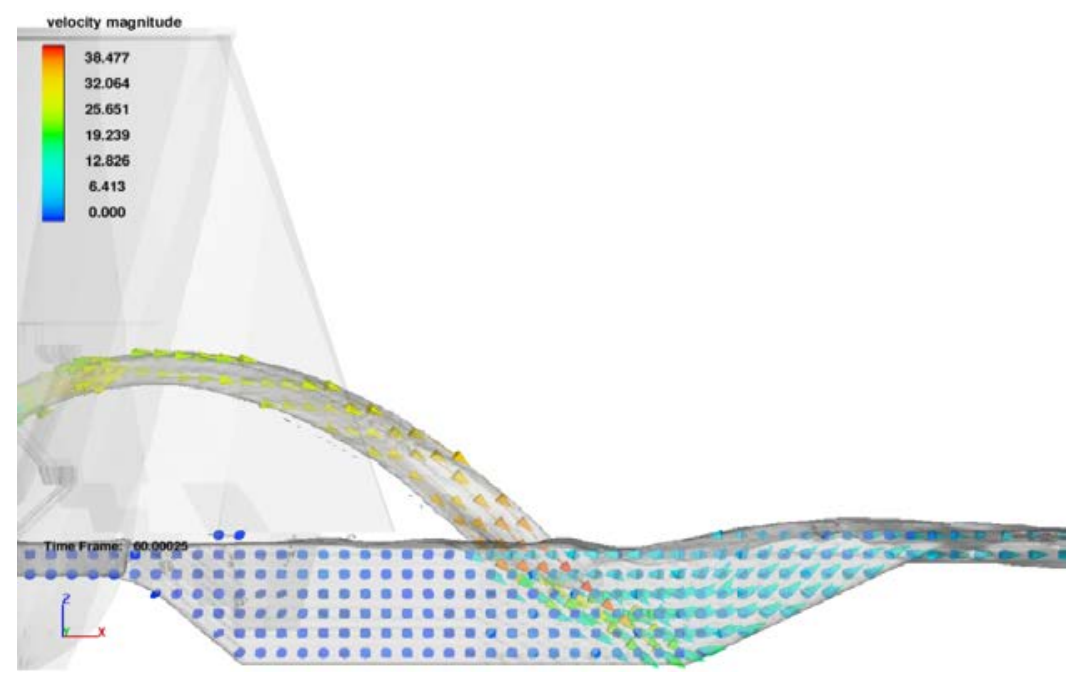

Figura 179. Vectores de velocidades dentro del cuenco pre-excavado para un caudal de 1760 $\mathrm{m}^{3} / \mathrm{s}$ que pasa por los desagües intermedios. Vista en perfil. 


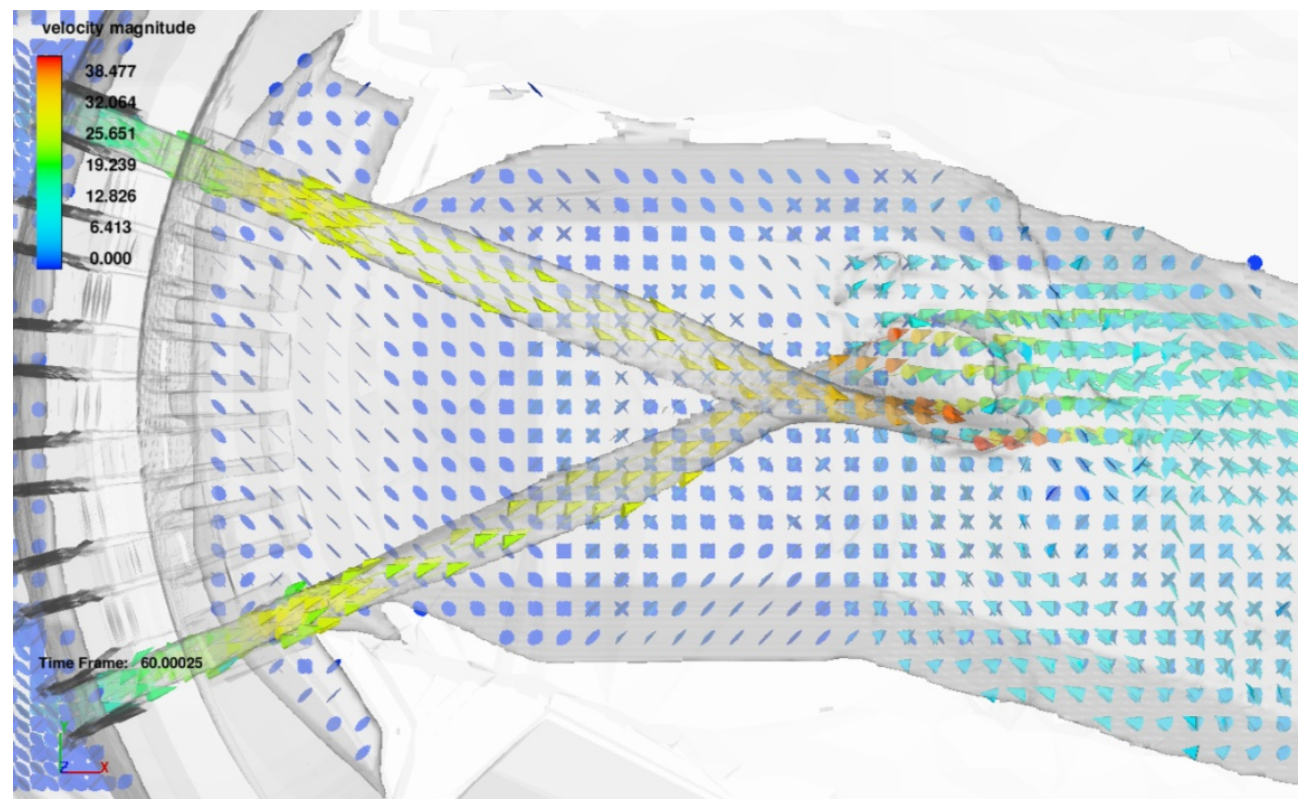

Figura 180. Vectores de velocidades dentro del cuenco pre-excavado para un caudal de 1760 $\mathrm{m}^{3} / \mathrm{s}$ que pasa por los desagües intermedios. Vista en planta.

La distribución de presiones en la solera se muestra en la Figura 181. Debido a que se ha propuesto un cuenco de amortiguación de menor longitud para adaptarse a la forma de la cerrada, las presiones totales máximas en la parte final del mismo están en el entorno de $45 \mathrm{~m}$, considerando un calado en el cuenco de $24 \mathrm{~m}$.

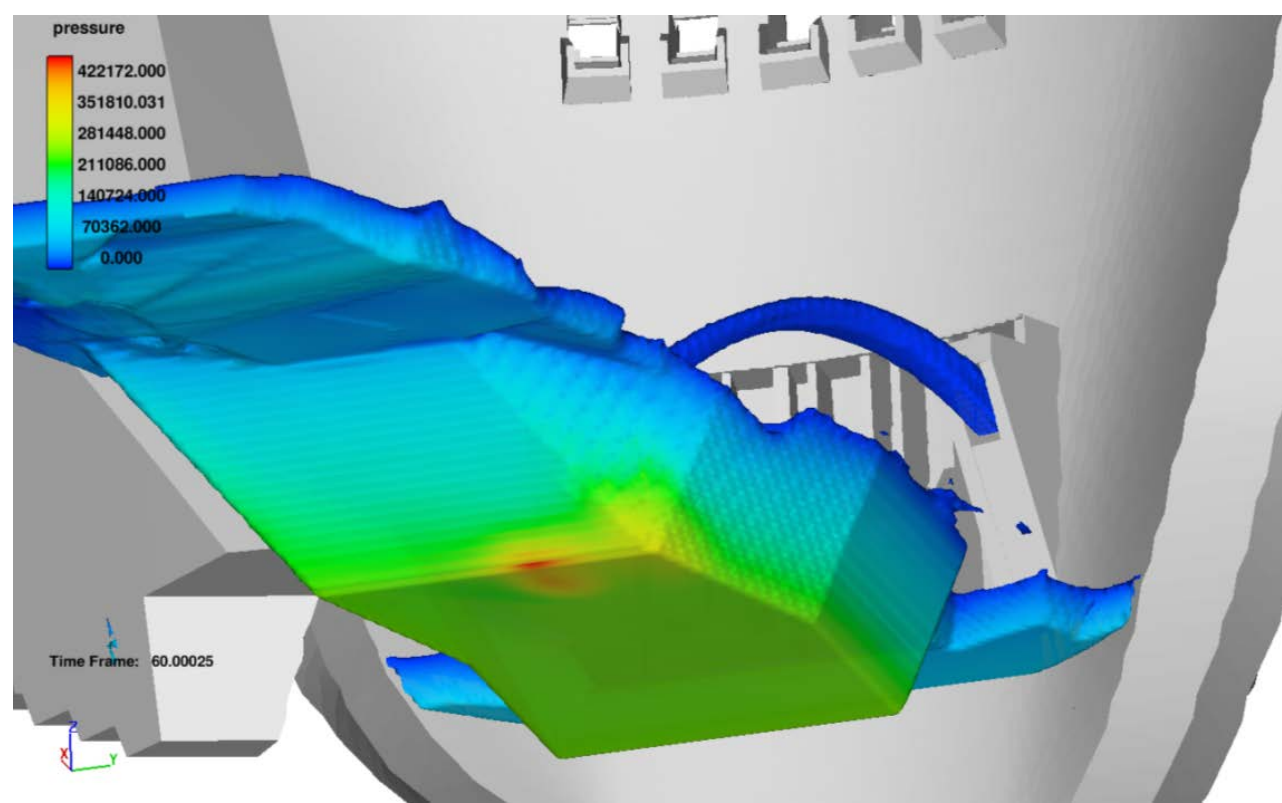

Figura 181. Distribución de presiones en la solera del cuenco pre-excavado para un caudal de $1760 \mathrm{~m}^{3} / \mathrm{s}$ que pasa por los desagües intermedios. 
En la Figura 182 se observa el funcionamiento de los 4 desagües de fondo cuando el embalse se encuentra en la cota $924 \mathrm{msnm}$, los cuales tienen una capacidad de $4737.91 \mathrm{~m}^{3} / \mathrm{s}$. Los chorros inciden con una velocidad en torno a $40 \mathrm{~m} / \mathrm{s}$.

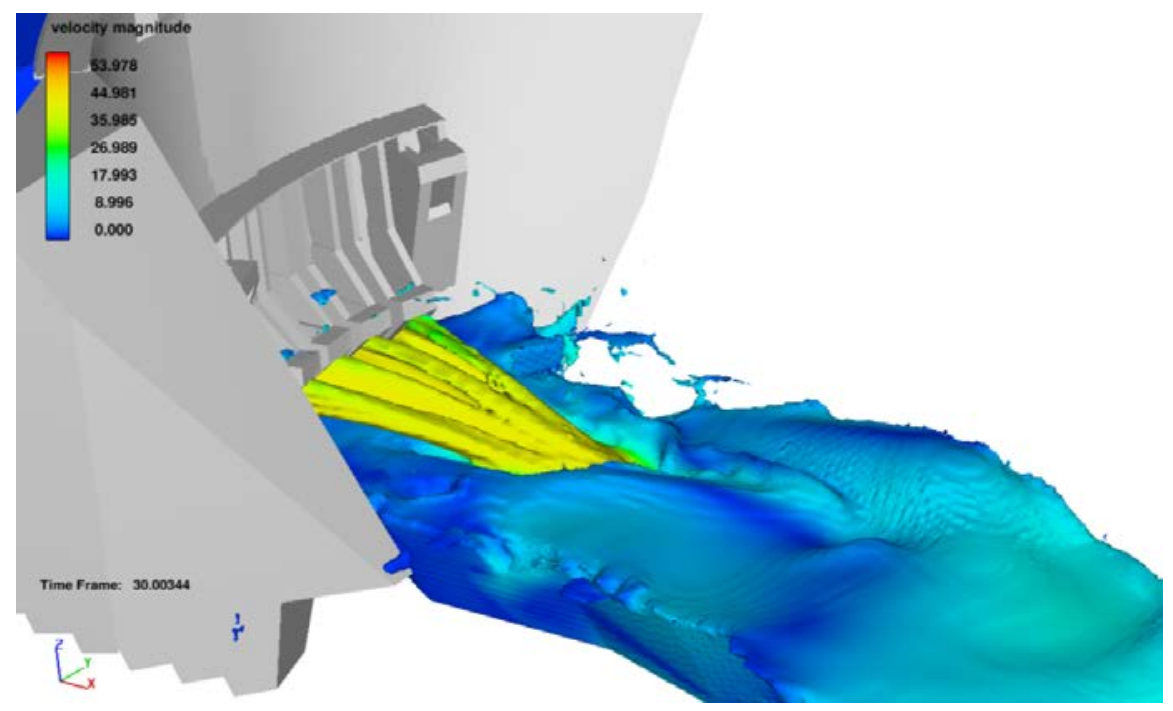

Figura 182. Funcionamiento del cuenco pre-excavado para un caudal de $4737.91 \mathrm{~m}^{3} / \mathrm{s}$ por los desagües de fondo.

En la solera del cuenco se genera un chorro de fondo que va perdiendo intensidad conforme avanza hacia aguas abajo, aunque el chorro de fondo no llega a disiparse totalmente debido a menor longitud del cuenco (Figura 183). La velocidad a la salida del cuenco está en torno a los $8 \mathrm{~m} / \mathrm{s}$, para un caudal correspondiente a un periodo de retorno semejante a 5000 años.

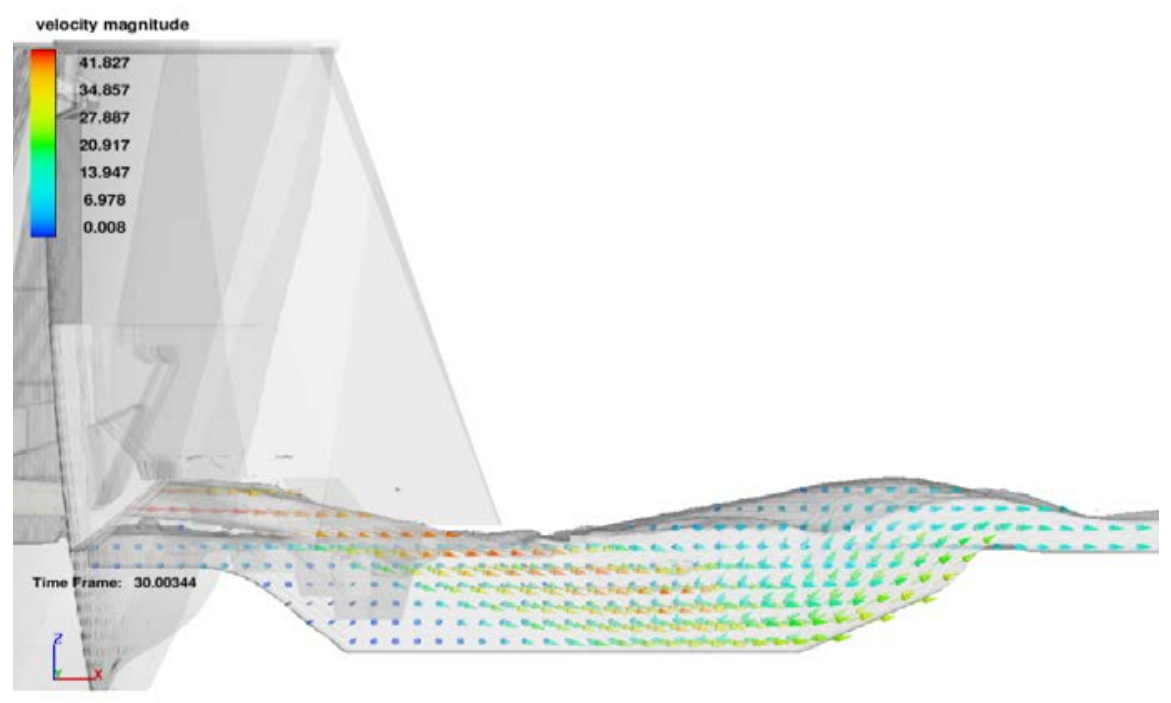

Figura 183. Vectores de velocidades dentro del cuenco pre-excavado para un caudal de $4737.91 \mathrm{~m}^{3} / \mathrm{s}$ por los desagües de fondo. 
En la Figura 184 se muestran las velocidades para el funcionamiento conjunto de todos los órganos de desagüe, mientras que la Figura 185 muestra las presiones en la solera del cuenco pre-excavado. El caudal de $7197.91 \mathrm{~m}^{3} / \mathrm{s}$ corresponde a un periodo de retorno semejante a 3500 años. Las velocidades a la salida del cuenco de disipación están en torno a los $10 \mathrm{~m} / \mathrm{s}$, mientras que las presiones máximas en la solera no superan los $50 \mathrm{~m}$.
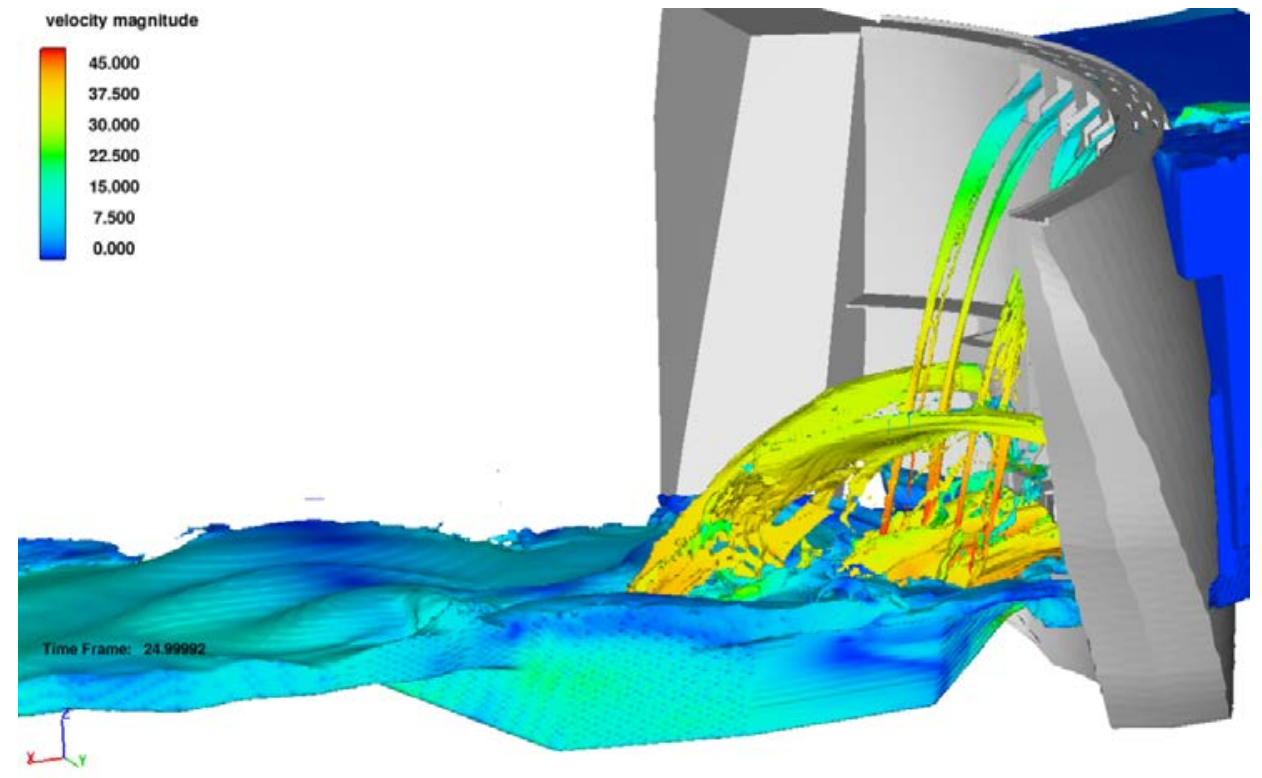

Figura 184. Funcionamiento del cuenco pre-excavado para un caudal de $7197.91 \mathrm{~m}^{3} / \mathrm{s}$ (aliviadero de superficie + desagüe de medio fondo + desagüe de fondo).

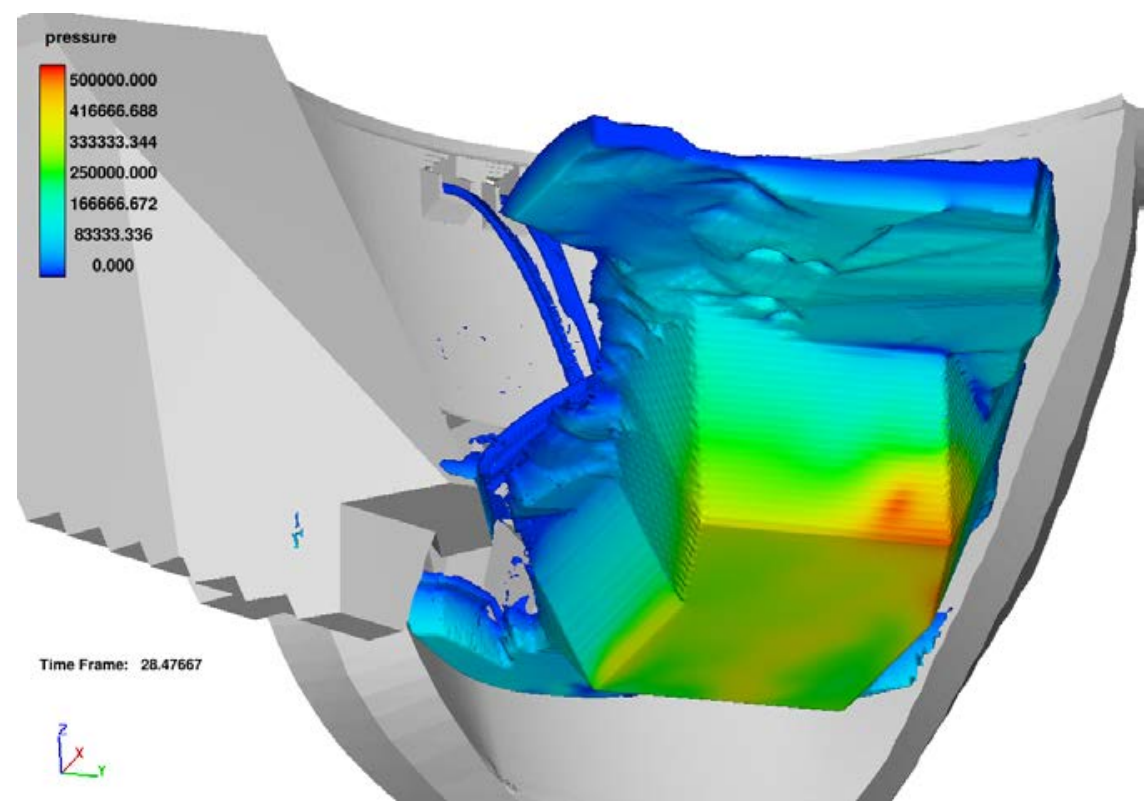

Figura 185. Distribución de presiones en la solera del cuenco pre-excavado para un caudal de $7197.91 \mathrm{~m}^{3} / \mathrm{s}$ (aliviadero de superficie + desagüe de medio fondo + desagüe de fondo). 


\section{CONCLUSIONES}

Esta Tesis ha consistido en el estudio de las acciones hidrodinámicas en cuencos de disipación situados aguas abajo de un aliviadero de vertido libre. Se ha realizado la caracterización en una infraestructura de laboratorio específicamente diseñada para tal fin. Además, se ha comprobado la adecuación de los programas de Dinámica de Fluidos Computacional para analizar este tipo de estudios.

Se han realizado nuevos estudios experimentales en laboratorio con el fin de mejorar el diseño de las estructuras de disipación y avanzar en el conocimiento de los chorros turbulentos, donde se han caracterizando simultáneamente el fenómeno de aireación del chorro junto a medidas de presión, velocidades y tasas de aireación. Estos datos permiten complementar los estudios previos y validar los códigos de CFD.

Para el estudio de presiones en la solera del cuenco se analizaron tres modelos experimentales diferentes. Los datos de las Tesis Doctorales de Castillo (1989) y Puertas (1994) se complementaron con 190 ensayos obtenidos en una nueva instalación de laboratorio. Las casi 400 configuraciones abarcan un rango de $0.42 \leq H / L_{b} \leq 3.06$ para el estudio de presión en el punto de estancamiento y distribuciones de presiones en el entorno del punto de estancamiento.

Autores como Bollaert y Schleiss (2003) consideran una región de transición entre las situaciones de colchón no efectivo y colchón efectivo (chorros compactos y desarrollados) para chorros circulares, que se encuentra entre $4<Y / D_{j}<6$. En chorros rectangulares se ha considerado que el nivel de agua necesario para obtener un colchón de agua eficaz es $Y / B_{j} \sim 5.5$. El valor máximo del coeficiente de presión dinámica es $C_{p}$ $=0.86$ en el caso de chorros circulares, mientras que se reduce a $C_{p}=0.83$ para el caso de chorros rectangulares. Considerando un colchón de agua eficaz, las tendencias obtenidas son similares en ambos tipos de chorros (ver Figura 85).

En chorros rectangulares se han considerado ocho familias de curvas que describen el comportamiento de $C_{p}$ en función de la relación $H / L_{b}$, válidas para el rango $0.80 \leq$ $H / L_{b} \leq 1.60$. Los resultados concuerdan con los datos bien documentados de chorros circulares poco aireados $\left(H / L_{b} \leq 0.50\right)$ obtenidos por diversos autores.

Los valores de los coeficientes presión dinámica fluctuante de los chorros rectangulares son muy similares a los obtenidos en chorros circulares. El valor máximo de estos coeficientes de presión se encuentra para relaciones $H / L_{b}$ entre 1.00 y 1.20 . Se ha obtenido una relación entre el coeficiente de presión fluctuante y el grado de rotura del chorro (ver Figura 86). 
Los valores de los coeficientes de presión dinámicas extremas no han permitido establecer una clara agrupación. Los datos obtenidos concuerdan con los resultados publicados en el caso de chorros circulares (ver Figura 88 y Figura 89).

Se han obtenido diversos ajustes para caracterizar la distribución de la presión en el entorno del punto de estancamiento. Los chorros desarrollados $\left(H / L_{b}>1\right)$ distribuyen la presión en una región mayor que los chorros no desarrollados $\left(H / L_{b} \leq 1\right)$. El comportamiento es asimétrico debido a la inclinación del chorro incidente y a la influencia de la pared situada aguas arriba. Los chorros aireados rectangulares muestran una región de distribución de la presión mayor que los chorros circulares, puesto que presentan un mayor grado de aireación durante la caída. Se han obtenido nuevos parámetros de ajuste con el fin de determinar las envolventes de los chorros rectangulares desarrollados y no desarrollados (ver Figura 90, Figura 91 y Figura 92).

En la revisión bibliográfica previa se ha comprobado que existen resultados acertados de modelaciones numéricas de aliviaderos con perfil hidrodinámico. Sin embargo, el estudio de aliviaderos de vertido libre y cuencos de disipación no ha sido suficientemente abordado.

En la aproximación de volúmenes finitos, los elementos tetraédricos resultan adecuados para la resolución de la mayoría de problemas de estudio. Sin embargo, en el caso de un aliviadero de vertido libre, donde existen fenómenos de despegue y alta turbulencia en el cálculo bifásico, es preferible emplear mallados basados en elementos hexaédricos.

En general, los resultados obtenidos con ANSYS CFX muestran una buena concordancia con las mediciones de laboratorio y con la Metodología Paramétrica. Sin embargo, dado que se utilizaron modelos de turbulencia RANS, el programa obtiene un registro de presión promediado en contraste con la variabilidad natural del fenómeno, lo que limita el posible análisis de las presiones dinámicas fluctuantes simuladas.

ANSYS CFX sigue la evolución de las velocidades en el chorro registradas en laboratorio, mientras que FLOW-3D obtiene velocidades similares a la velocidad teórica de caída libre. Las velocidades del chorro obtenidas con ANSYS CFX y con el equipo de fibra óptica muestran el efecto de la fricción aire-agua durante la caída. Aunque el tamaño de los elementos del mallado tuvo una pequeña influencia en la velocidad de caída del chorro, la fracción volumétrica del aire se muestra muy sensible a su tamaño en ANSYS CFX. El código de la versión 10.0 de FLOW-3D no resuelve de modo adecuado la entrada de aire en el chorro vertiente.

El modelo homogéneo empleado no permite actuar sobre la aireación al no considerar la interacción entre las dos fases. Sería conveniente intentar abordar el estudio de aireación del chorro con un modelo inhomogéneo, el cual resuelva la interacción entre las fases, aunque su coste computacional es mayor. 
La longitud de rotura $L_{b}$ no queda bien definida con ninguno de los dos enfoques que usan los programas empleados. El modelo Euleriano-Euleriano de ANSYS CFX no puede simular la formación de las pequeñas gotas a menos que se emplee un tamaño de mallado muy reducido. Por su parte, FLOW-3D provoca la rotura cuando el espesor del chorro está en el entorno de dos veces el tamaño del mallado, lo que dispara los errores de truncamiento e imposibilita su utilización.

En el esquema en diferencias finitas de FLOW-3D se ha comprobado que existe una gran disparidad entre los resultados obtenidos por un chorro alineado a la malla y un chorro oblicuo a la misma, apareciendo inestabilidades cuando el flujo del chorro no está orientado con el mallado.

Para que no se produzca la rotura del chorro por fenómenos numéricos, en FLOW3D es necesario emplear un tamaño de mallado 2-3 veces menor que el espesor del chorro en el caso de flujos alineados con el mallado. Sin embargo, debido al esquema de diferencias finitas, en chorros oblicuos puede ser necesario emplear tamaños de mallado 5-6 veces menor que el espesor del chorro (Figura 125 y ecuación (213)). Para situaciones de laboratorio se requieren tamaños de mallado de entre 0.003 y $0.002 \mathrm{~m}$, incrementándose el coste computacional de la simulación.

La turbulencia en el chorro se analizó mediante simulaciones numéricas, las cuales fueron calibradas y validadas mediante la medición de las velocidades y la tasa de aireación del chorro, y de las presiones en el punto de estancamiento. De esta manera se obtuvo una mejor caracterización de los coeficientes de presión dinámica de laboratorio (medio, fluctuante, máximo y mínimo).

A pesar de que se modificó la turbulencia en la condición de entrada al modelo numérico, la turbulencia se reduce rápidamente debido a que, en la caída, la aceleración por efecto de la gravedad es muy superior al incremento de la velocidad turbulenta. $T_{u}$ tiende a un valor próximo a 0.03 cuando existe una suficiente distancia de caída (Figura 115).

La presión media modelizada en el punto de estancamiento concuerda con los resultados de la aireación y de la velocidad del chorro. Los mejores resultados se obtuvieron al emplear un tamaño de elementos de aproximadamente la mitad del espesor de chorro en la condición incidente. Tamaños de elemento mayores producen presiones más bajas en el punto de estancamiento debido a que la aireación no se modeliza adecuadamente (Figura 135 y Tabla 32).

En lo que respecta a la comparación de los modelos de turbulencia, se observa poca variación entre los modelos utilizados. El modelo de turbulencia SST obtuvo resultados ligeramente más precisos al realizar la comparación con los datos de laboratorio y con la Metodología Paramétrica. 
Se ha obtenido un buen ajuste de los coeficientes de presión dinámica media y la presión entre los resultados de laboratorio, la Metodología Paramétrica y las simulaciones numéricas con el modelo de turbulencia SST. Teniendo en cuenta la diferencia de altura entre el nivel del agua aguas arriba y aguas abajo de cada ensayo, las diferencias entre las simulaciones y los datos de laboratorio mostraron un error máximo entre $-4 \%$ y $+7 \%$ (Tabla 42 ).

En las distribuciones en el entorno del punto de estancamiento, el modelo de turbulencia SST parece obtener resultados ligeramente más precisos. Con el caudal específico más pequeño, el modelo $k-\varepsilon$ tiende a sobreestimar la distribución presiones aguas arriba y aguas abajo, mientras que el modelo RNG tiende a sobrestimar los resultados aguas abajo.

Aunque el análisis de chorro rectangular y aireados es complejo, este estudio ofrece una metodología de diseño práctico para evaluar este tipo de flujos. Los estudios se llevaron a cabo con velocidades incidentes moderadas. Para extrapolar los resultados a escala de prototipo, el diseñador debe tener en cuenta los posibles efectos de escala. Del lado de la seguridad, se puede considerar que los efectos de escala no serían importantes hasta una relación de escala de Froude 1:10. 


\section{FUTUROS DESARROLLOS}

Este estudio se ha centrado en el análisis temporal de presiones y velocidades obtenidas en laboratorio y con simulaciones numéricas de CFD a escala reducida.

Como futuros desarrollos de esta línea de investigación se plantean los siguientes análisis:

- Análisis frecuencial para obtener la densidad espectral de los números de Strouhal, presiones y velocidades.

- Análisis en detalle de la aireación del chorro, así como de la distribución de la aireación en el seno del cuenco de disipación.

- Estudio de la distribución de velocidades y aguas arriba del punto de estancamiento.

- Evaluación de la turbulencia en el chorro vertiente y su evolución durante la caída.

- Análisis de presiones de chorros rectangulares con altas velocidades incidentes y distintas tasas de aireación con el fin de independizar los resultados de los efectos de escala.

- Empleo de modelos no homogéneos para analizar la interacción de las dos fases, aire y agua, en el estudio de cuencos de disipación.

- Validación de programas numéricos de código abierto de enfoque euleriano como OpenFOAM, y con enfoque lagrangiano como Smoothed-Particle Hydrodynamics (SPH). 


\section{BIBLIOGRAFÍA}

Aki, S. (1969). Study of the efficiency of the water cushions in free falling jets. Journal of the Central Electric Research Institute, (in Japanese).

Albertson, M. L., Dai, Y. B., Jenson, R. A., \& Rouse, H. (1950). Diffusion of submerged jets. Proceedings ASCE, 74.

American Institute of Aeronautics and Astronautics (AIAA). (1998). Guide for the verification and validation of computational fluid dynamics simulations. AIAA G-077-1998.

Amorin, J. C., Rodrigues, C. R., \& Marques, M. G. (2004). A numerical and experimental study of hydraulic jump stilling basin. En S. S. Wang (Ed.), Advances in Hydro-Science and-Engineering, Volume VI: Proceedings of Abstracts and Papers of the 6th International Conference on Hydro-Science and-Engineering. 6. Brisbane, Australia: University of Mississippi, Oxford, MS.

Annandale, G. W. (1995). Erodibility. Journal of Hydraulic Research, 33(4), 471-494.

Annandale, G. W. (2006). Scour technology. Mechanism and engineering practice. McGraw-Hill.

ANSYS, Inc. (2010). ANSYS CFX Reference Guide. Release 13.0.

ANSYS, Inc. (2010). ANSYS CFX-Solver Modeling Guide. Release 13.0.

ANSYS, Inc. (2010). ANSYS CFX-Solver Theory Guide. Release 13.0.

Armengou, J. (1991). Vertido libre por coronación de presas bóveda. PhD Thesis. Universitat Politècnica de Catalunya, Spain, (in Spanish).

Aziz, T. N., \& Khan, A. A. (2011). Simulation of vertical plane turbulent jet in shallow water. Advances in Civil Engineering, 2011, 1-10.

Aziz, T. N., Raiford, J. P., \& Khan, A. A. (2008). Numerical simulation of turbulent jets. Engineering application of Computational Fluid Mechanics, 2(2), 234-243.

Balabel, A., Hegab, A., Nasr, M., \& El-Behery, S. (2011). Assessment of turbulence modeling for gas flow in two-dimensional convergent-divergent rocket nozzle. Applied Mathematical Modelling, 35, 3408-3422.

Bardina, J. E., Huang, P. G., \& Coakley, T. J. (1997). Turbulence Modeling Validation, Testing, and Development. NASA Technical Memorandum 110446.

Baron, T. (1949). Technical Report No4. University of Illinois.

Bazin, H. E. (1896). Expériences nouvelles sur l'écoulement en déversoir. Annales des Ponts et Chaussées, 7, 249-357, (in French). 
Beltaos, S. (1976). Oblique impingement of circular turbulent jets. Journal of Hydraulic Research, 14(1), 17-36.

Beltaos, S., \& Rajaratnam, N. (1973). Plane turbulent impinging jets. Journal of Hydraulic Research, 11(1), 29-59.

Beltaos, S., \& Rajaratnam, N. (1974). Impinging circular turbulent jets. Journal of the Hydraulics Division, ASCE, 100(HY10), 1313-1328.

Bin, A. K. (1993). Gas entrainment by plunging liquid jets. Chem. Eng. Sci., 48(3), 585630.

Bischof, C. H., Bücker, H. M., \& Rasch, A. (2004). Sensibility analysis of turbulence models using automatic differentiation. SIAM Journal on Scientific Computing (SISC), 26(2), 510-522.

Blanco, E. (2007). Simulación numérica de flujos (CFD) con el programa FLUENT. Universidad de Oviedo, Spain, (in Spanish).

Boes, R., \& Hager, W. H. (1998). Fiber-optical experimentation in two-phase cascade flow. Proceedings of the International RCC Dams Seminar. Denver, USA: K. HANSEN.

Bollaert, E. F. (2002). Communication $N^{o} 13$. Transient water pressures in joints and formation of rock scour due to high-velocity jet impact. (A. J. Schleiss, Ed.) Laboratory of Hydraulic Constructions (LHC), École Polytechnique Fédérale de Lausanne (EPFL), Switzerland.

Bollaert, E. F., \& Schleiss, A. (2001). A new approach for better assessment of rock scouring due to high velocity jets at dams spillways. Proceedings of the ICOLD European Symposium. Geiranger, Norway.

Bollaert, E. F., \& Schleiss, A. (2001). Air bubble effects on transient water pressures in rock fissures due to high velocity jet impact. Proceedings of the XXICth IAHR Congress. Beijing, China.

Bollaert, E. F., \& Schleiss, A. (2003). Scour of rock due to the impact of plunging high velocity jets. Part 1: A state-of-the-art review. Journal of Hydraulic Research, 41(5), 451-464.

Bollaert, E. F., \& Schleiss, A. (2003). Scour of rock due to the impact of plunging high velocity jets. Part 2: Experimental result of dynamic pressures at pool bottoms and in one- and two-dimensional closed end rock joints. Journal of Hydraulic Research, 41(5), 465-480.

Bormann, E., \& Julien, P. Y. (1991). Scour downstream of grade-control structures. Journal of Hydraulic Engineering, ASCE, 117(5), 579-594. 
Bos, M. G. (1989). Discharge measurement structures (Third revised ed.). International Institute for Land Reclamation and Improvement/ILRI.

Bouhadji, L. (2004). Three Dimensional Numerical Simulation of Turbulent Flow Over Spillways. ASL-AQFlow Inc., Sidney, British Columbia, Canada.

Bradbury, L. J. (1965). The structure of a self-preserving turbulence plane jet. Journal of Fluid Mechanics, 23, 31-64.

Bradshaw, P. (1972). The understanding and prediction of turbulent flow. The Aeronautical Journal, 76(739), 403-418.

Brattberg, T., \& Chanson, H. (1998). Air entrapment and air bubble dispersion at twodimensional plunging water jets. Chem. Eng. Sci., 53(41), 13-27.

Bretschneider, H. (1971). Kopfbauwerke bei SchuBrinnen. Wasserwirtschaft No.5. (in German).

Carrillo, J. M., \& Castillo, L. G. (2010). Análisis del flujo turbulento y bifásico en un aliviadero de presa mediante simulación numérica tridimensional. Jornadas de Introducción a la Investigación de la UPCT, 3, 99-101, (in Spanish).

Carrillo, J. M., \& Castillo, L. G. (2011). Consideraciones del mallado aplicadas al cálculo de flujos bifásicos con las técnicas de Dinámica de Fluidos Computacional. Jornadas de Introducción a la Investigación de la UPCT, 4, 3335, (in Spanish).

Carrillo, J. M., \& Castillo, L. G. (2011). Simulación de flujos bifásicos con metodología CFD y contrastación de resultados con medidas de laboratorio. II Jornadas de Ingeniería del Agua, JIA 2011. Barcelona, Spain, (in Spanish).

Carrillo, J. M., \& Castillo, L. G. (2011). Simulación numérica en aliviaderos de vertido libre. Seminario de la Red de Laboratorios de Hidráulica de España, RLHE. Madrid, Spain, (in Spanish).

Carrillo, J. M., \& Castillo, L. G. (2012). Calibración de dos programas CFD. Aplicación a aliviaderos de vertido libre. Jornadas de Introducción a la Investigación de la UPCT, 5, 33-35, (in Spanish).

Carrillo, J. M., \& Castillo, L. G. (2013). Validación de herramientas CFD para el estudio de sobrevertido de presas. Jornadas de Introducción a la Investigación de la UPCT, 6, 37-39, (in Spanish).

Carrillo, J. M., \& Castillo, L. G. (2014). Validación de simulaciones de flujo bifásico en programas de CFD. Accepted for publication in the Journal Jornadas de Introducción a la Investigación de la UPCT, 7, (in Spanish). 
Castillo, L. G. (1989). Metodología experimental y numérica para la caracterización del campo de presiones en los disipadores de energía hidráulica. Aplicación al vertido libre en presas bóveda. PhD Thesis. Universitat Politècnica de Catalunya, Spain, (in Spanish).

Castillo, L. G. (1990). Comprobación y unificación de las formulaciones de la presión dinámica media de un chorro incidente en el punto de estancamiento y la zona de influencia. XIV Congreso Latinoamericano de Hidráulica, 1, págs. 383-392. Montevideo, Uruguay, (in Spanish).

Castillo, L. G. (2002). Parametrical analysis of the ultimate scour and mean dynamic pressures at plunge pools. En A. A. Balkema, Rock scour due to falling highvelocity jets (págs. 95-104). A. J. Schleiss and E. F. R. Bollaert.

Castillo, L. G. (2006). Aerated jets and pressure fluctuation in plunge pools. The 7th International Conference on Hydroscience and Engineering (ICHE-2006), IAHR, ASCE. Philadelphia, USA: Drexel University. College of Engineering. DSpace Digital Lybrary. DU Haggerty Library.

Castillo, L. G. (2007). Pressure characterization of undeveloped and developed jets in shadow and deep pool. Proceedings of XXXII IAHR Congress. 2, págs. 645-655. Venice, Italy: International Association for Hydro-Environment Engineering and Research (IAHR).

Castillo, L. G. (2008). Validation of instantaneous velocities measurements with ADV equipment in turbulent high two-phase flows. The Eight International Conference on Hydro-Science and Engineering (ICHE). Nagoya, Japan.

Castillo, L. G. (2009). Filtering and validation of velocities obtained with ADV equipment inside of hydraulic jumps. International Workshop on Environmental Hydraulics, IWHE. Valencia, Spain.

Castillo, L. G. (2009). Filtrado de velocidades y caracterización de parámetros dentro de resaltos hidráulicos libres y sumergidos. I Jornadas de Ingeniería del Agua. Madrid, Spain, (in Spanish).

Castillo, L. G. (2009). Measurement of velocities and characterization of some parameters of free and submerged hydraulic jumps. 33rd International Association of Hydraulic Engineering \& Research Congress, IAHR. Vancouver, Canada.

Castillo, L. G. (2011). Aireación natural y forzada de flujos en vertido libre por coronación de presas y su difusión en cuencos de disipación de energía hidráulica. Memoria técnica. BIA 2011-28758-C03-02. Ministerio de Economía y Competitividad, Spain, (in Spanish). 
Castillo, L. G., \& Carrillo, J. M. (2010). Simulación numérica y validación de las acciones hidrodinámicas en cuencos de disipación de energía. Seminario de la Red de Laboratorios de Hidráulica de España, RLHE. Valencia, Spain, (in Spanish).

Castillo, L. G., \& Carrillo, J. M. (2011). Numerical simulation and validation of hydrodynamics actions in energy dissipation devices. 34th International Association of Hydraulic Engineering \& Research Congress, IAHR, (págs. 4416-4423). Brisbane, Australia.

Castillo, L. G., \& Carrillo, J. M. (2012). Caracterización del fenómeno de vertido libre frente a variación del índice de turbulencia inicial con metodología CFD. $X X V$ Congreso Latinoamericano de Hidráulica. San José, Costa Rica, (in Spanish).

Castillo, L. G., \& Carrillo, J. M. (2012). Hydrodynamics characterization in plunge pools. Simulation with CFD methodology and validation with experimental measurements. 2nd IAHR European Congress. Munich, Germany.

Castillo, L. G., \& Carrillo, J. M. (2012). Medidas de velocidades en chorros rectangulares y contraste con simulaciones CFD. Proyecto EMULSIONA. Seminario de la Red de Laboratorios de Hidráulica de España, RLHE. Madrid, Spain, (in Spanish).

Castillo, L. G., \& Carrillo, J. M. (2013). Analysis of the scale ratio in nappe flow case by means of CFD numerical simulation. Proceedings of the 35th IAHR World Congress. Chengdu, China.

Castillo, L. G., \& Carrillo, J. M. (2013). Estudio de socavación aguas abajo de la presa Paute-Cardenillo. Proceedings of the III Jornadas de Ingeniería del Agua, 2, págs. 237-244. Valencia, Spain, (in Spanish).

Castillo, L. G., \& Carrillo, J. M. (2013). Influencia de la escala en simulaciones numéricas de aliviaderos de vertido libre. Proyecto EMULSIONA. Proceedings of the III Jornadas de Ingeniería del Agua, 2, págs. 245-252. Valencia, Spain, (in Spanish).

Castillo, L. G., \& Carrillo, J. M. (2013). Influencia de la relación de escala en la simulación numérica de aliviaderos de vertido libre. Seminario de la Red de Laboratorios de Hidráulica de España. Madrid, Spain, (in Spanish).

Castillo, L. G., \& Carrillo, J. M. (2014). Scour analysis downstream of Paute-Cardenillo Dam. Accepted for publication in the Proceedings of the 3rd IAHR Europe Congress. Oporto, Portugal. 
Castillo, L. G., \& Carrillo, J. M. (2014). Scour estimation of the Paute-Cardenillo Dam. Proceedings of the International Perspectives on Water Resources \& the Environment 2014 conference. Quito, Ecuador.

Castillo, L. G., Carrillo, J. M., \& Blázquez, A. (2014). Plunge pool mean dynamic pressures: a temporal analysis in nappe flow case. Accepted for publication in the Journal of Hydraulic Research.

Castillo, L. G., Carrillo, J. M., \& Sordo-Ward, A. (2014). Simulation of overflow nappe impingement jets. Accepted for publication in the Journal of Hydroinformatics.

Castillo, L. G., Dolz, J., \& Polo, J. (1991). Acquisition and analysis of data to characterize dynamic actions in hydraulic energy dissipators. XXIV IAHR Congress, $D$, págs. 273-280. Madrid, Spain.

Castillo, L. G., Molina, B., \& Carrillo, J. M. (2010). Caracterización de velocidades y ley de variación del número de Froude dentro de resaltos hidráulicos. Seminario de la Red de Laboratorios de Hidráulica de España. Valencia, Spain, (in Spanish).

Castillo, L. G., Puertas, J., \& Dolz, J. (1996). Análisis conjunto de las formulaciones de socavación y presión dinámica media al pié de presas. XVII Congreso Latinoamericano de Hidráulica, IAHR, 5, págs. 179-190. Guayaquil, Ecuador, (in Spanish).

Castillo, L. G., Puertas, J., \& Dolz, J. (1999). Discussion of "Pressures fluctuations on plunge pool floors" by D. A. Ervine, H. T. Falvey and W. A. Withers. Journal of Hydraulic Research, 37(2), 272-277.

Castillo, L. G., Puertas, J., \& Dolz, J. (2007). Discussion of "Scour of rock due to the impact of plunging high velocity jets. Part I: A state-of-the-art review" by E. F. R. Bollaert and A. J. Schleiss. Journal of Hydraulic Research, 45(6), 853-858.

Castillo, L. G., Vigueras-Rodríguez, A., \& Carrillo, J. M. (2013). Análisis espectral de presiones en aliviaderos de vertido libre: aplicación al filtrado de medidas. Seminario de la Red de Laboratorios de Hidráulica de España. Madrid, Spain, (in Spanish).

Chandler, K., Gill, D., Maher, B., Macnish, S., \& Roads, G. (2003). Coping with probable maximum flow - an alliance project delivery for Wivenhoe Dam. Proceedings of the 43rd ANCOLD Conference. Hobart, Tasmania, Australia.

Chanel, P. G., \& Doering, J. C. (2007). An evaluation of Computational Fluid Dynamics for spillway modelling. 16th Australian Fluid Mechanics Conference, (págs. 1201-1206). Gold Coast, Australia. 
Chanel, P. G., \& Doering, J. L. (2008). Assessment of spillway modelling using Computational Fluid Dynamics. Canadian Journal of Civil Engineering, 35, 1481-1485.

Chanson, H. (1995). Air bubble diffusion in supercritical open channel flow. Proceedings of the 12th Australasian Fluid Mechanics Conference. 2, págs. 707710. Sydney, Australia: Bilger RW.

Chanson, H. (1996). Air bubble entrainment in free surface turbulent shear flows. London: Academic Press.

Chanson, H. (1996). Energy loss at drops - Discussion. Journal of Hydraulic Research, 34(2), 273-278.

Chanson, H., \& Brattberg, T. (2000). Experimental study of the air-water shear flow in a hydraulic jump. International Journal of Multiphase Flow, 26(4), 583-607.

Chanson, H., \& Manasseh, R. (2003). Air entrainment processes in a circular plunging jet: void-fraction and acoustic measurements. Journal of Fluid Engineering, 125, 910-921.

Chee, S. P., \& Yuen, E. M. (1985). Erosion of unconsolidated gravel beds. Canadian Journal of Civil Engineering, 12, 559-566.

Cola, R. (1965). Energy dissipation of a high-velocity vertical jet entering a basin. Proceedings of the 11th Congress of the IAHR. Leningrad.

Cola, R. (1966). Diffusione di un getto piano verticale in un bacino d'acqua d'altezza limitata. L'energia elettrica, 11, 649-664, (in Italian).

Comité Nacional Español de Grandes Presas (CNEGP). (1997). Guía Técnica de Seguridad de Presas $N^{\circ} 5$. Aliviaderos y Desagües. CNEGP.

Comité Nacional Español de Grandes Presas (CNEGP). (1997). Guía Técnica de Seguridad de Presas $N^{\circ}$ 5. Aliviaderos y Desagües. Comité Nacional Español de Grandes Presas (CNEGP).

Cook, C. B., \& Richmond, M. C. (2001). Simulation of tailrace hydroinformatics using Computational Fluid Dynamics models. Springfield, VA, USA: US Army Corp of Engineers Portland District Report PNNL-13467. US Dept of Commerce, National Technical Information Service.

Cook, C. B., Richmond, M. C., Serkowski, J. A., \& Ebner, L. L. (2002). Free-surface Computational Fluid Dynamics modelling of a spillway and a tailrace: case study of the Dalles Project. Proceedings of Hydrovision 2002 Conference. Portland, OR, USA. 
Creager, W. P. (1917). Engineering of Masonry Dams. New York, USA: John Wiley \& Sons.

Cui-Guang-Tao, Lin-Ji-Yong, \& Liang-Xing-Rong. (1985). Study on the force and effect of the Arch dam overflow water tongue on the river bed. Journal of Hydraulic Engineering, 8, 53-68, (in Chinese).

Cummings, P., \& Chanson, H. (1997). Air entrainment in the developing flow region of plunging jets. Part I: theoretical developments. Journal of Fluids Engineering, 119, 597-602.

D'Alpaos, L., \& Ghetti, A. (1984). Some new experiments on surface tension and viscosity effects on the trajectory of a falling jet. Symposium on Scale Effects in Modelling Hydraulic Structures, (págs. 1-8). Esslingen.

Davidson, L. (2011). An introduction to turbulence models (Vol. 97/2). Göteborg, Sweden: Department of Thermo and Fluid Dynamics. Chalmers University of Technology.

Davies, J. T. (1972). Turbulence Phenomena. Academic Press, New York and London.

Edwards, J. L. (2006). The analysis of spillways using computational fluid dynamics. BEng thesis. University of Sydney.

El Hammoumi, M., Achard, J. L., \& Davoust, L. (2002). Measurements of air entrainment by vertical plunging liquid jets. Experiments in Fluids, 32(6), 24-38.

Ervine, D. A. (1976). The entrainment of air in water. Water Power and Dam Construction, 28(12), 27-30.

Ervine, D. A. (1998). Air entrainment in hydraulic structures: a review. Proceedings of the Institution of Civil Engineers Wat., Marit. \& Energy, 130, págs. 142-153.

Ervine, D. A., \& Falvey, H. R. (1987). Behaviour of turbulent jets in the atmosphere and plunge pools. Proceedings of the Institutions of Civil Engineers, 83 (2), págs. 295-314.

Ervine, D. A., Falvey, H. R., \& Withers, W. (1997). Pressure fluctuations on plunge pool floors. Journal of Hydraulic Research, 35(2), 257-279.

Ervine, D. A., McKeogh, E., \& Elsawy, E. M. (1980). Effect of turbulence intensity on the rate of air entrainment by plunging water jets. Proceedings of the Inst. Civ. Eng., Part 2, (págs. 425-445).

Falvey, H. T., \& Ervine, D. A. (1988). Aeration in jets and high velocity flows. Proceedings of Model-prototype Correlation of Hydraulic Structures. Colorado.

Felderspiel, M. P. (2011). Response of an embedded block impacted by high-velocity jets. PhD Thesis. École Polytechnique Fédérale de Lausanne, Suisse. 
Flow Science, Inc. (2011). FLOW-3D. Theory v10.0.

Flow Science, Inc. (2011). FLOW-3D. User manual v10.0.

Franzetti, S., \& Tanda, M. G. (1984). Getti deviati a simmetria assiale. Report of Istituto di Idraulica e Costruzioni Idrauliche. Politecnico di Milano, Italy, (in Italian).

Franzetti, S., \& Tanda, M. G. (1987). Analysis of turbulent pressure fluctuation caused by a circular impinging jet. Proceedings of the International Conference on New Technology in Model Testing in Hydraulic Research, (págs. 85-91). India.

Fuamba, M., Brousseau, G., Mainville, E., Colameo, S., \& Sparks, D. (2006). Role and behavior of surge chamber in hydropower: case of the Robert Bourassa Hydroelectric Power Plant in Quebec, Canada. (L. B. al., Ed.) Dams and Reservoirs, Society and Environment in the 21st Century.

Gendzelevich, W., Baryla, A., Groenveld, J., \& McNeil, D. (2009). Red River Floodway Expansion Project-Design and Construction of the Outlet Structure. Canadian Dam Association 2009 Annual conference. Whistler, BC, Canada.

Gessler, D. (2005). CFD modeling of spillway performance. EWRI 2005: Impacts of global climate change, Proceedings of the 2005 World Water and Environmental Resources Congress. Anchorage, Alaska, USA.

Gessler, D., \& Rasmussen, B. (2005). Before the flood. Desktop Engineering.

Gill, M. A. (1979). Hydraulics of Rectangular Vertical Drop Structures. Journal of Hydraulic Research, 17(4), 289-302.

Goring, G., \& Nikora, V. (2002). Despiking acoustic Doppler velocimeter data. Journal of Hydraulic Engineering, 128(1), 117-126.

Görtler, H. (1942). Berechnung von Aufgaben der freien Turbulenz auf Grund eines neuen Näherungsansatzes. Journal of Applied Mathematics and Mechanics / Zeitschrift für Angewandte Mathematik und Mechanik - ZAMM, 22(5), 244-254, (in German).

Groeneveld, J., Sweeney, C., Mannheim, C., Simonsen, C., Fry, S., \& Moen, K. (2007). Comparison of intake pressures in physical and numerical models of the Cabinet Gorge Dam Tunnel. Waterpower XV. Chattanooga, TN, USA.

Guha, A., Barron, R. M., \& Balachandar, R. (2010). Numerical simulation of highspeed turbulent water jets in air. Journal of Hydraulic Research, 48(1), 119-124.

Guo, J. (2002). Hunter Rouse and Shields diagram. Proceedings of the 1th IAHR-APD Congress, 2, págs. 1069-1098. Singapore. 
Guo, Z. Z. (1982). Principle and design of energy dissipation and protect scour. Beijing: Science Press.

Gutmark, E., \& Wygnanski, I. (1976). The planar turbulent jet. Journal of Fluid Mechanics, 73, 465-495.

Hager, W. H. (1992). Energy dissipators and hydraulic jump (Vol. 8). Dordrecht, The Netherlands: Kluwer Academic Publ. Water Science and Technology Library.

Hager, W. H., \& Schwalt, M. (1994). Broad-crested weir. Journal of Irrigation and Drainage, 120(1), 13-26.

Hargreaves, D. M., Morvan, H. P., \& Wright, N. G. (2007). Validation of the Volume of Fluid Method for free surface calculation: the broad-crested weir. Engineering Applications of Computational Fluid Mechanics, 1(2), 136-146.

Harlow, F. H., \& Nakayama, P. I. (1968). Transport of turbulence energy decay rate. Los Álamos Sci. Lab. University of California.

Hartung, F., \& Häusler, E. (1973). Scours, stilling basins and downstream protection under free overfall jets at dams. Proceedings of the 11th Congress on Large Dams, (págs. 39-56). Madrid, Spain.

Häusler, E. (1966). Dynamishe Wassserdrücke auf Tosbeckenplatten infolge freier Uberfall-strahlen bei Talsperren. Die Wasserwirtschaft, 2, (in German).

Heskestad, G. (1965). Hot-wire measurements in a plane turbulent jet. Journal of Applied Mechanics, 32(4), 721-734.

Hinze, J. O. (1975). Turbulence (Second ed.). New York: McGraw-Hill.

Hirsch, C. (2007). Numerical Computation of Internal and External Flows. Volume 1 Fundamentals of Computational Fluid Dynamics (Second ed.). Elsevier.

Hirt, C. W., \& Nichols, B. D. (1981). Volume of fluid (VOF) method for the dynamics of free boundaries. Journal of Computational Physics, 39, 201-225.

Hirt, C. W., \& Sicilian, J. M. (1985). A porosity technique for the definition of obstacles in rectangular cell meshes. Proceedings of the 4th International Conference on Numerical Ship Hydrodynamics. Washington, D.C.: National Academy of Science.

Ho, D. K., \& Riddette, K. M. (2010). Application of computational fluid dynamics to evaluate hydraulic performance of spillways in Australia. Australian Journal of Civil Engineering, 6(1), 81-104.

Ho, D. K., Boyes, K. M., Donohoo, S. M., \& Cooper, B. W. (2003). Numerical flow analysis for spillways. Proceeding of the 43th ANCOLD Conference. Hobart, Tasmania, Australia. 
Ho, D. K., Cooper, B. W., Riddette, K. M., \& Donohoo, S. M. (2006). Application of numerical modelling to spillways in Australia. En L. B. al. (Ed.), Proceedings of the International Symposium on Dams in the Societies of the 21st Century, 22nd International Congress on Large Dams (ICOLD) (págs. 951-959). Barcelona, Spain: Taylor \& Francis Group, London, UK.

Ho, D. K., Donohoo, S. M., Boyes, K., Riddette, K. M., \& Lock, C. C. (2003). Numerical analysis and the Real World: it looks pretty but is it right? Proceedings of the NAFEMS World Congress, (págs. 27-31). Orlando, Florida, USA.

Homma, M. (1953). An experimental study on water fall. Proceedings of the Minnesota International Hydraulics Conference. United Stated.

Horeni, P. (1956). Disintegration of a free jet of water in air. Vyzkumny Ustav Vodohospodarsky Prace a Studie, Sesit 93. Prague, Czechoslovakia, (in Czech).

International Committee On Large Dams (ICOLD). (2001). Computational procedures for dam engineering reliability and applicability. Bulletin 122.

Johnson, M. C., \& Savage, B. (2006). Physical and numerical comparison of flow over ogee spillway in the presence of tailwater. Journal of Hydraulic Engineering, ASCE, 132(12), 1353-1357.

Kendil, F. Z., Danciu, D. V., Schmidtke, M., Salah, A. B., Lucas, D., Krepper, E., \& Mataoui, A. (2012). Flow field assessment under a plunging liquid jet. Progress in Nuclear Energy, 56, 100-110.

Kenny, R. J. (2007). Computational fluid dynamics modelling of an uncontrolled ogee spillway crest using Fluent software. MEng Thesis. Queensland University of Technology.

Kiger, K. T., \& Duncan, J. H. (2012). Air-entrainment mechanisms in plunging jets and breaking waves. Annual Review of Fluid Mechanics, 44, 563-596.

Kindsvater, C. E., \& Carter, R. W. (1957). Discharge characteristics of rectangular thinplate weirs. Journal of the Hydraulics Division of the ASCE, 83(No. HY 6).

Kobus, H. (1973). Bemessungsgrundlagen and Anwendungen für Luftschleier im Wasserbau. Heft 7. (E. S. Verlag, Ed.) Schriftenreihe Wasser und Abwasser in Forschung und Praxis, (in German).

Kolmogorov, A. N. (1941). Local structure of turbulence in incompressible viscous fluid for very large Reynolds number. Doklady Akademiya Nauk SSSR, 30, 299303.

Kotate, S., \& Hijikata, K. (1993). Numerical Simulations of Heat Transfer and Fluid Flow on a Personal Computer. Elsevier. 
Krothapalli, A., Baganoff, D., \& Karamcheti, K. (1981). On the mixing of a rectangular jet. Journal of Fluid Mechanic, 107, 201-220.

Kuang, J., Hsu, C. T., \& Qiu, H. (2001). Experiments on vertical turbulent plane jets in water of finite depth. Journal of Engineering Mechanics, 127(1), 18-26.

Launder, B. E., \& Sharma, B. I. (1974). Application of the energy dissipation model of turbulence to the calculation of flow near a spinning disc. Heat and Mass Transfer, 1(2), 131-138.

Leach, S. J., Walker, G. L., Smith, A. V., Farmer, I. W., \& Taylor, G. (1966). Some aspects of rock cutting by high-speed water jets. Philosophical Transactions of the Royal Society A: Physical, Mathematical and Engineering Sciences, 260(1110), 295-310.

Lencastre, A. (1961). Descarregadores de lâmina livre. Lisbon, Portugal: LNEC, (in Portuguese).

Lesleighter, E., McPherson, B., Riddette, K., \& Williams, J. (2008). Modelling procedures used for the spillway upgrade for Lake Manchester Dam. Proceedings of the 2008 ANCOLD Conference. Australian Committee On Large Dams.

Lewis, T. M. (1996). Prediction of velocities within jets formed by overtopping steep dams. MsC Thesis. Colorado State University. United Stated.

Lin, C., Hsieh, S.-C., Lin, I.-J., Chang, K.-A., \& Raikar, R. V. (2012). Flow property and self-similarity in steady hydraulic jumps. Experiments in Fluids, 53, 15911616.

Liu, P., Gao, J., \& Li, Y. (1998). Experimental investigation of submerged impinging jets in a plunge pool downstream of large dams. Science in China, 41(4), 357365.

Lopardo, R. (1988). Stilling basin pressure fluctuations. Proceedings of the International Conference on model-prototype correlation in hydraulic structures, (págs. 56-73). Colorado Springs, Colorado.

Ma, J., Oberai, A. A., Drew, D. A., Lahey, R. T., \& Moraga, F. J. (2009). A quantitative sub-grid air entrainment model for bubbly flows: plunging jets. Computers and Fluids, 39, 77-86.

Manso, P. A., Bollaert, E., \& Schleiss, A. (2005). Dynamic pressures generated by plunging jets in confined pools under extreme flood discharges. Proceedings of the XXXI IAHR Congress, 1, págs. 2848-2860. Seoul. 
Manso, P. A., Bollaert, E., \& Schleiss, A. (2008). Evaluation of high-velocity plunging jet-issuing characteristics as a basis for plunge pool analysis. Journal of Hydraulic Research, 46(2), 147-157.

Marques, M. G., Ollermann, G., Weiller, C., \& Endres, L. A. (1998). Perda de carga no interior de um ressalto hidráulico a jusante do vertedouro. Congresso Latino Americano de Hidráulica. Oaxaca, México, (in Portuguese).

Mastbergen, D. R., \& Von den Berg, J. H. (2003). Breaching in fine sands and the generation of sustained turbidity currents in submarine canyons. Journal of Sedimentology, 50, 625-637.

Maynord, S. T. (1985). General spillway investigation. Technical report (U.S. Army Engineer Waterways Experiment Station) -- HL-85-1. US Army Corps of Engineers, Dept. of the Army, Washington, DC, USA.

McKeogh, E. (1978). A study of air entrained using plunging water jets. PhD Thesis. Queens University Belfast, Ireland.

McKeogh, E., \& Elsawy, E. (1980). Air retained in pool by plunging water jet. Journal of the Hydraulics Division, 106(10), 1577-1593.

Melo, J. F., Pinheiro, A. N., \& Ramos, C. M. (2006). Forces on plunge pool slabs: Influence of joints location and width. Journal of Hydraulic Engineering, 132(1), 49-60.

Menter, F. R. (1994). Eddy Viscosity Transport Equations and their Relation to the $\kappa-\varepsilon$ Model. NASA Technical Memorandum 108854.

Menter, F. R. (1994). Two-equation eddy-viscosity turbulence models for engineering applications. AIAA-Journal, 32(8), 1598 - 1605.

Menter, F. R. (2012). Large eddy simulation and hybrid RANS-LES turbulence modeling. ANSYS Germany GmbH.

Meyer-Peter, E., \& Müller, R. (1948). Formulations of the bed-load transport. Proceedings of the II IAHR, (págs. 39-64). Estocolmo, Suecia.

Molina, B., Carrillo, J. M., \& Castillo, L. G. (2010). Análisis y validación de velocidades instantáneas en flujos altamente turbulentos y aireados. Jornadas de Introducción a la Investigación de la UPCT, 106-108, (in Spanish).

Moore, W. L. (1943). Energy loss at the base of a free overfall. Transactions American Society of Civil Engineering, 108, 1343-1360.

Naudascher, E. (1984). Scale effects in model tests. Proceedings of the IAHR Symposium on Scale Effects in Modeling Hydraulic Structures. Esslingen, Germany. 
Naudascher, E. (2000). Hidráulica de canales. Limusa. Noriega editores.

Ohtsu, I. O., Yasuda, Y., \& Awazu, S. (1990). Free and submerged hydraulic jumps in rectangular channels. Nihon University, Japan. Report of Research Inst. of Science and Technology, No. 35.

Orszag, S. A., Yakhot, V., Flannery, W. S., Boysan, F., Choudhury, D., Maruzewski, J., \& Patel, B. (1993). Renormalization-Group modeling and turbulence simulations. ELSEVIER SCIENCE PUBL B V, 1031-1046.

Poggensee, H. (1942). Die Druckverteilung am Hackenschütz bei verschiedener Ausbildung der Krone. Dissertation. Universität Karlsruhe, (in German).

Poreh, M., \& Hefez, E. (1967). Initial scour and sediment motion due to an impinging submerged jet. Proceedings of the 12th IARH Congress. Fort Collons.

Puertas, J. (1994). Criterios hidráulicos para el diseño de cuencos de disipación de energía en presas bóveda con vertido libre por coronación. PhD Thesis. Universitat Politècnica de Catalunya, Spain, (in Spanish).

Puertas, J., \& Dolz, J. (2002). Pressure fields due to the impingement of free falling jets on a riverbed. En A. J. Schleiss, \& E. F. Bollaert (Edits.), Rock scour due to falling high-velocity jets (págs. 105-114). The Netherlands: A. A. Balkema Swets \& Zeitlinger.

Puertas, J., \& Dolz, J. (2005). Plunge pool pressures due to a falling rectangular jet. Journal of Hydraulic Engineering, 131(5), 404-407.

Rahimzadeh, H., Maghsoodi, R., Sarkardeh, H., \& Tavakkol, S. (2012). Simulating flow over circular spillways by using different turbulence models. Engineering Applications of Computational Fluid Mechanics, 6(1), 100-109.

Raiford, J. P., \& Khan, A. A. (2006). Numerical modeling of internal flow structure in submerged hydraulic jumps. Proceedings of World Water and Environmental Resources Congress 2006, ASCE. Omaha, NE, USA.

Rajaratnam, N. (1965). Submerged hydraulic jump. Journal of the Hydraulic Division, 91 (HY4), 71-96.

Rajaratnam, N. (1965). The hydraulic jump as a wall jet. Journal of the Hydraulic Division, 91 (HY5) , 107-132.

Rajaratnam, N. (1976). Turbulent jets. Amsterdam: Elsevier.

Rajaratnam, N., \& Chamani, M. R. (1995). Energy loss at drops. Journal of Hydraulic Research, 33(3), 373-384. 
Rajaratnam, N., Steffler, P. M., Rizvi, S. A., \& Smy, P. R. (1994). Experimental study of very high velocity circular water jets in air. Journal of Hydraulics Research, 32(3), 461-470.

Ramos, C. M. (1988). Hydrodynamic actions on hydraulic structures. LNEC-65/53/315.

Rand, W. (1955). Flow geometry at straight drop spillways. Proceedings of the American Society of Civil Engineering, 81, paper 791, págs. 1-13.

Ranga Raju, K. G., \& Asawa, G. L. (1977). Viscosity and surface tension effects on weir flow. Journal of the Hydraulics Division, 103(10), 1227-1231.

Reda, M. A. (2011). 2D-3D modeling of flow over sharp-crested weir. Journal of Applied Sciences Research, 7(12), 2495-2505.

Rodi, W. (1975). A new method of analyzing hot-wire signals in highly turbulent flows and its evaluation in round jets. Disa Information, $\mathrm{N}^{\mathrm{o}} .17$.

Sarker, M. A., \& Rhodes, D. G. (2004). Calculation of free-surface profile over a rectangular broad-crested weir. Flow Measurement and Instrumentation, 15, 215-219.

Savage, B. M., \& Johnson, M. C. (2001). Flow over ogee spillway: physical and numerical model case study. Journal of Hydraulic Engineering, ASCE, 127(8), 640-649.

Savage, B. M., Johnson, M. C., \& Towler, B. (2009). Hydrodynamic forces on a spillway: can we calculate them? Proceedings of the 2009 Annual Conference, Association of State Dam Safety Officials (ASDSO). Hollywood, FL, USA.

Schauer, J. J., \& Eustis, R. H. (1963). The flow development and heat transfer characteristics of plane turbulent impinging jets. Techical Report. Dept. Mech. Eng., Stanford, CA.

Scimemi, E. (1930). Sulla forma delle vene tracimanti. L'Energia Elettrica, 7(4), 293305, (in Italian).

Sfeir, A. A. (1976). The velocity and temperature fields of rectangular jets. International Journal or Heat Mass and Transfer, 19, 1289-1297.

Smagorinsky, J. (1963). General Circulation Experiments with the Primitive Equations. Journal of Monthly Weather Review, 93, 99-165.

Stutz, B., \& Reboud, J. L. (1997). Experiment on unsteady cavitation. Experiments in Fluids, 22, 191-198.

Stutz, B., \& Reboud, J. L. (1997). Two-phase flow structure of sheet cavitation. Phys. Fluids, 9(12), 3678-3686. 
Taylor, G. I. (1935). Statistical theory of turbulence IV - Diffusion in a turbulent air stream. Proceedings of the Royal Society of London Series a-mathematical and physical sciences. 151 (A873), págs. 0465-0478. Royal Society of London.

Tennekes, H., \& Lumley, J. L. (1983). A First Course in Turbulence. MIT Press, Cambridge, MA.

Toso, J., \& Bowers, E. C. (1988). Extreme pressures in hydraulic jump stilling basin. Journal of Hydraulic Engineering, 114(HY8), 829-843.

Tota, P. V. (2009). Turbulent flow over a backward-facing step using the RNG $\kappa-\varepsilon$ model. Flow Science, Inc.

United States Bureau of Reclamation (USBR). (2007). Diseño de pequeñas presas (3 ed.). Bellisco. Ediciones técnicas y científicas.

van de Sande, E., \& Smith, J. M. (1976). Jet break-up and air entrainment by low velocity turbulent water jets. Chemical Engineering Science, 31, 219-224.

Van Rijn, L. C. (1984). Sediment transport, Part I: Bed load transport. Journal of Hydraulic Engineering, 110(10), 1431-1456.

Varshney, D. V. (1977). Model scale and the discharge coefficient. Water Power and Dam Construction, 4, 48-52.

Von Kármán, T. (1937). On the statistical theory of turbulence. Proceedings of the National Academy of Sciences of the United States of America. 23, págs. 98-105. National Academy of Sciences.

Wahl, T. L., Frizell, K. H., \& Cohen, E. A. (2008). Computing the Trajectory of Free Jets. Journal of Hydraulic Engineering, 134(2), 256-260.

Wasewar, L., \& Vijay Sarathi, J. (2008). CFD modelling and simulation of jet mixed tanks. Engineering Applications in Computational Fluid Mechanics, 2(2), 155171.

Weilin, X., Huasheng, L., Yongquan, Y., \& Chigong, W. (2002). Turbulent flow and energy dissipation in plunge pool of high arch dam. Journal of Hydraulic Research, 40(4), 471-476.

Wilcox, D. C. (2010). Turbulence Modeling for CFD (Third ed.). DCW Industries, Inc.

Withers, W. (1991). Pressure fluctuations in the plunge pool of an impinging jet spillway. PhD Thesis. University of Glasgow.

Witze, P. O., \& Dwyer, H. A. (1976). The turbulent radial jet. Journal of Fluid Mechanics, 75, 401-417.

Wu, S., \& Rajaratnam, N. (1995). Free jumps, submerged jumps and wall jets. Journal of Hydraulic Research, 33(2), 197-212. 
Wygnanski, I., \& Fiedler, H. E. (1969). Some measurements in the self-preserving jet. Journal of Fluid Mechanics, 38, 577-612.

Xu Duo-Ming. (1983). Pressao no fundo de um canal devido ao choque de um jacto plano, e suas caracteristicas de fluctuacao, Translation from chinese by J. A. Pinto de Campos. Lisbon, Portugal, (in Portuguese).

Yakhot, V., \& Orszag, S. A. (1986). Renormalization Group analysis of turbulence. I. Basic Theory. Journal of Scientific Computing, 1(1), 3-51.

Yang, J., \& Johansson, N. (1998). Determination of spillway discharge capacity - CFD modeling and experiment verification. Proceedings of the 3rd International Conference on Advantages in Hydroscience and Engineering. Cottbus, Germany.

Zamora, B. (2008). Notas sobre simulación numérica de flujos de fluidos. Universidad Politécnica de Cartagena, Spain, (in Spanish). 
BIBLIOGRAFíA 